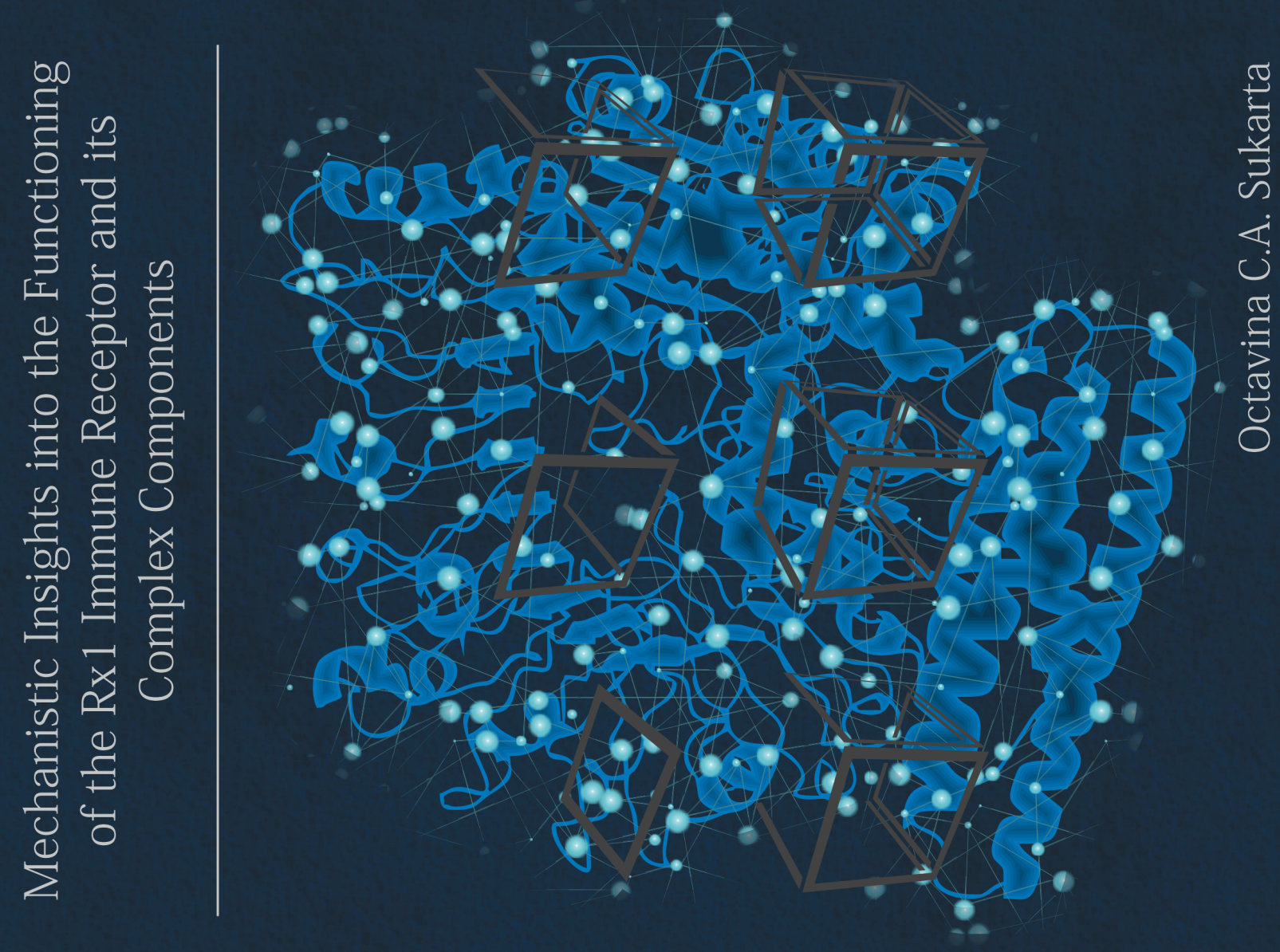

Mechanistic Insights into the Functioning of Rx1 and its Complex Components

O.C.A. Sukarta

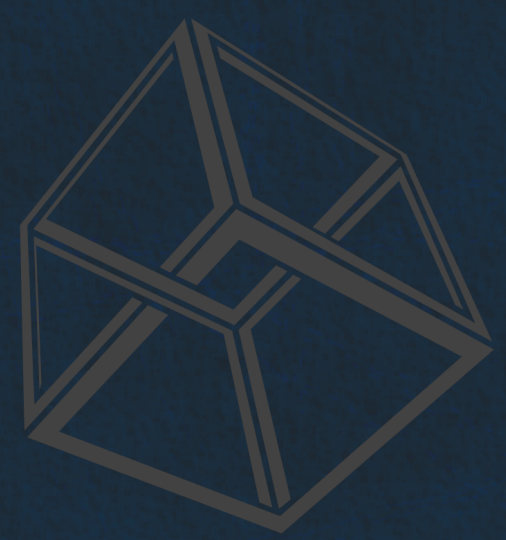




\section{Propositions}

1. The co-factor RanGAP2 acts as a bait, which is involved in but not required for effector recognition by $\mathrm{R} \times 1$ and Gpa2 (this thesis)

2. Functional compatibility between the $C C$ and LRR domains is a constraint for the successful engineering of $\mathrm{Rx} 1$ and its homologs (this thesis)

3. The need to publish in peer-reviewed journals stifles scientific progress.

4. Improving methods for drug delivery will more efficiently combat problems with antibiotic resistance than identifying novel drugs.

5. The altmetrics of a scientist is a more accurate measure of performance than his/her impact factor.

6. Humanity undermines itself by entertaining the idea that Artificial Intelligence can reach a point of technological singularity.

7. A workplace should be run like a seven-eleven; you can come when you need to.

Propositions belonging to the thesis entitled:

"Mechanistic Insights into the Functioning of the Rx1 Immune Receptor and its

Complex Components",

Octavina Citra Ayudhany Sukarta

Wageningen, 4 December 2020 
Mechanistic Insights into the Functioning of the Rx1 Immune Receptor and its Complex Components

Octavina Citra Ayudhany Sukarta 
Thesis Committee

Promotor

Prof. Dr Geert Smant

Professor of Nematology

Wageningen University \& Research

\section{Co-promotor}

Dr Aska Goverse

Associate Professor, Laboratory of Nematology

Wageningen University \& Research

\section{Other members}

Prof. Dr Yuling Bai, Wageningen University \& Research

Dr Thomas Kroj, French National Institute of Agriculture, Food and Environment, Montpellier, France

Dr Mathieu Joosten, Wageningen University \& Research

Dr Wladimir Tameling, Keygene N.V., Wageningen

This research was conducted under the auspices of the Graduate School Experimental Plant Sciences 


\title{
Mechanistic Insights into the Functioning of the Rx1 Immune Receptor and its Complex Components
}

\author{
Octavina Citra Ayudhany Sukarta
}

\section{Thesis}

submitted in fulfillment of the requirements for the degree of doctor

at Wageningen University

by the authority of the Rector Magnificus,

Prof. Dr A.P.J. Mol,

in the presence of the

Thesis Committee appointed by the Academic Board

to be defended in public

on Friday 4 December 2020

at 11:00 A.M in the Aula. 
Octavina Citra Ayudhany Sukarta

Mechanistic Insights into the Functioning of the Rx1 Immune Receptor and its Complex Components

PhD thesis, Wageningen University, Wageningen, the Netherlands (2020)

With references and with summaries in English and Dutch

DOI: https://doi.org/10.18174/531084

ISBN: 978-94-6395-540-9 
To my family 


\section{Table of Contents}

$\begin{array}{llr}\text { Chapter } 1 & \text { General Introduction } & 9\end{array}$

Chapter 2 Structure-Informed Insights for NLR Functioning in Plant Immunity 29

Chapter 3 A Tomato Homolog of the Potato CC-NB-LRR Immune Receptor Rx1 Differs in Domain Cooperativity and Complex Formation Despite Sharing High Sequence Similarity

Chapter 4 The RanGTPase Activating Protein 2 (RanGAP2) is a Common Target of the Cognate Effectors of its Bound Receptors, Gpa2 and Rx1

Chapter 5 A DNA-Binding Bromodomain-Containing Protein Interacts with and Reduces Rx1-Mediated Immune Response to Potato Virus X

Chapter 6 The Glycine-Rich RNA-Binding Protein 7 (GRP7) is a Novel Component belonging to the Resistance Complexes of the Potato CC-NB-LRRs Rx1 and Gpa2

Chapter 7 General Discussion

Summary

Acknowledgments

About the Author

List of Publications

Training and Education Statement 


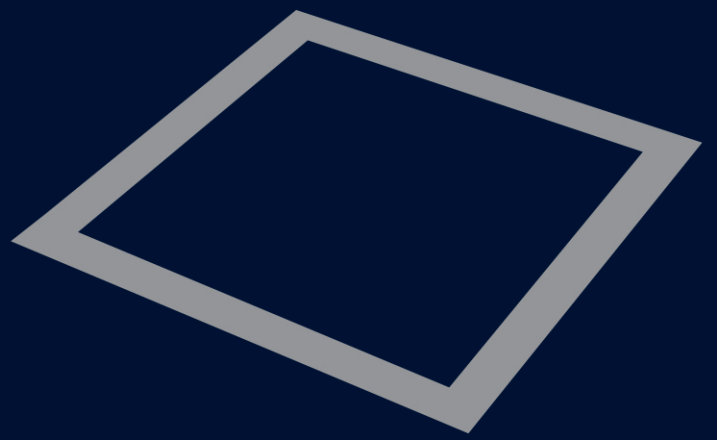

Octavina C. A Sukarta

Laboratory of Nematology, Wageningen University \& Research, Wageningen, the Netherlands. 


\section{Chapter}

1

General Introduction 


\section{PREFACE}

All living organisms must share their space, which puts them at constant risk of being invaded by microbes with the potential to cause disease. The ability to distinguish self from non-self, therefore, represents an important life trait. As such, all forms of life are endowed with a genetic 'battle plan' to sense danger and spare the host from further injury. Commonly referred to as the 'immune system', this organization of built-in resistance mechanisms is present in protozoans and vertebrates alike (Turvey et al. 2010). In mammals, the concept of immunity is characterized by both innate and adaptive responses. The former is often viewed as a primitive, first-line form of defence that bars intrusion of most microbes seeking to colonize the host (Menezes et al. 2002). In an exquisite manner, mammals can further refine this defence response and establish sustained immunological memory by virtue of specialized antigen-specific cells. This constitutes the 'adaptive' arm of immunity, which builds upon basal resistance mechanisms. Unlike mammals, however, plants must survive solely on innate defences alone as they lack specialized immune cells and a circulatory system (van Wersch et al. 2020). Plant innate immunity may thus appear to be seemingly simpler. However, in their 400 million years of evolutionary history, plants have shown remarkable plasticity to cope with microbial infections (Menezes et al. 2002). Many would, therefore, argue that immunity in plants is composed of an elaborate, multi-faceted system with subtleties existing at the molecular and biochemical levels (Bezerra-Neto et al. 2020, Menezes et al. 2002, van Wersch et al. 2020, Lolle et al. 2020). At the heart of this complexity lies the plants' surveillance machinery. This consists of receptor proteins that play an instrumental role in perceiving and translating foreign signals into defence outputs. My dissertation focuses on a particular group of immune receptors termed the NB-LRRs that mediate a specific, inducible branch of plant innate immunity. With this chapter, I begin by detailing the core paradigms that shape our understanding of plant defence before focusing on the molecular aspects of NB-LRR function. Knowledge gaps that drive the direction of this thesis are further discussed.

\section{Plant Innate Immunity}

Plants are sedentary beings and, as such, incapable of escaping from an impending threat. However, each plant cell is hardwired with immunogenic capabilities (van Wersch et al. 2020, Bezerra-Neto et al. 2020). The plant surface constitutes the first interface which a pathogen encounters and is 
thus, well-guarded by both mechanical and chemical barriers. To establish infection, an invading pathogen must initially overcome a formidable obstacle, the rigid plant cell wall (Bezerra-Neto et al. 2020). The plant cell wall is further bejeweled with a waxy cuticle and trichomes, which together effectively barricade microbial entry into the host. Breaching these physical obstructions (e.g. by wounding or through natural openings) then exposes the pathogen to a cocktail of antimicrobial compounds.

In the event that a pathogen is able to contest these preformed barriers, the inducible, non-self-recognition system of the plant is set in motion. Classically, this is viewed to consist of two interconnected branches, both of which hinges on the activity of specialized receptor proteins (Fig. 1A) (Han 2019, Jones et al. 2006). These receptors work to detect, relay, and respond to the message of non-self. Starting at the surface, membrane-bound Pattern Recognition Receptors (PRRs) survey the host extracellular space for signs of invasion (Zipfel 2014, Jones et al. 2006). This may refer to microbial-derived structures that are conserved throughout whole pathogenic classes, formally termed as Pathogen-Associated Molecular Patterns (PAMPs). PAMPs are diverse, ranging from proteinaceous components such as the bacterial flagellin to carbohydrate-based features such as the fungal chitin (Felix et al. 1999, Zipfel 2014). A generic trait shared by PAMPs, however, is that they are indispensable for the survival of a pathogen. In addition, PRRs may also recognize immunogenic factors derived from the host itself as a consequence of microbial invasion (e.g. remnants of the plant cell wall released upon pathogen-induced degradation), termed Damage-Associated Molecular Patterns (DAMPs) (Zipfel 2014, Jones et al. 2006). In either case, DAMP/PAMP perception induces a concoction of cellular responses that can restrict infection by a wide range of pathogenic classes. These responses include the production of Reactive Oxygen Species (ROS), $\mathrm{Ca}^{2+}$ fluxes, and dynamic transcriptional changes, which are collectively coined as PAMP-Triggered Immunity (PTI). PTI provides the plant's first, inducible form of defence, also referred to as basal immunity.

If a pathogen is unable to evade PTI-mediated defences of the plant, it can no longer secure its resources and proliferate effectively. Selection pressure then forces the pathogen to adapt by evolving effector molecules (Jones et al. 2006). Effectors are introduced into the host space in a vast variety of forms, including as proteases, transcription factors, or chemical mimics of plant hormones (Toruño et al. 2016). Essentially, however, they all function by tampering with the 
host cellular machinery and/or defence response to promote colonization in a process referred to as Effector-Triggered Susceptibility (ETS) (Fig. 1A). In a complementary fashion, the host plant can revamp its defence armory by evolving Resistance $(R)$ genes. $R$-genes mediate host-specific resistance by encoding immune receptors that detect one or a few effector molecules (Jones et al. 2006). Referred to as Effector Triggered Immunity (ETI), R-gene mediated defences largely overlap with PTI but are more amplified, sustained, and restricted to a few pathogenic classes (Lolle et al. 2020). The collective suit of response are referred to as the Hypersensitive Response (HR), which often manifests in local cell death of the infected tissue that prevents further spreading of the pathogen by limiting nutrient availability (Hammond-Kosack et al. 1996). Despite lacking circulatory cells, plants can disseminate these local defence responses to naïve plant cells via mobile chemical signals such as salicylic acid and its methylated derivatives in a process termed Systematic Acquired Resistance (SAR) (Dong 2001).

The evolutionary interplay between the host and pathogen, as described above, is genetically conceptualized in Flor's gene-for-gene model, which postulates that each $R$ gene of the plant has a matching avirulence (Avr) gene derived from the pathogen (Flor 1971). In this setting, however, there is selection pressure put on both the pathogen and plant to evade and expand recognition, respectively. The consequence of this evolutionary arms-race drives pathogens to specialize in their catalog of effector proteins and plants in their recognition repertoire. More importantly, this molecular warfare has far-reaching implications on global agriculture and food security (Jones et al. 2006). It is reported that an estimate of $13 \%$ in crop yield succumbs to plant infections each year (Nelson et al. 2018). It is, therefore, unsurprising that many breeding efforts are dedicated to improving disease resistance. Though effective, chemical control is neither a financially nor environmentally friendly solution. $R$ genes thus represent a sustainable alternative with biotechnological potential (Bezerra-Neto et al. 2020). This potential has long been recognized, propelling the identification, isolation, and breeding of many $R$ genes from wild plant species. A bottleneck of $R$-gene based resistances, however, is that pathogens are dynamic and fast-evolving by nature, which enables them to rapidly render formerly-resistant varieties useless in the field (Nelson et al. 2018). A landmark goal is, therefore, to develop durable and broad-spectrum resistance. This ushered an exciting period of innovative breeding, including the design of $\mathrm{R}$ proteins with novel and tailored recognition specificities. However, this hinges on a solid understanding of the molecular and mechanistic basis of 
the function of immune receptors and their associated responses, which will facilitate the deployment of rationale breeding strategies based on $R$ genes.

\section{Structure and Function of a Molecular Sentinel}

$R$-gene products constitute a highly diverse group of proteins with at least five classes having been described in plants (Hammond-Kosack et al. 1997). The vast majority belongs to the family of Nucleotide-Binding Leucine-Rich Repeat (NB-LRR) type of R proteins, which also forms the subject focus of this thesis. The first NB-LRR receptors were isolated in the early 1990s, and since then, the genomes of many plant species are predicted to house a huge repertoire of NB-LRR-encoding genes (Whitham et al. 1994, Mindrinos et al. 1994, Bent et al. 1994, Meyers et al. 2003). Five years after their discovery in plants, NB-LRRs were described in animals and humans, which were thought to have arisen by convergent evolution (Ting et al. 2008, Han 2019,, Urbach et al. 2017). Plant and mammalian NB-LRRs are analogous in their domain organizations, pinpointing at similar downstream pathways and activation mechanisms. Indeed, though differences do exist, many parallels can be drawn between the two systems (Han 2019, Bentham et al. 2017). Primarily, the function of both plant and mammalian NB-LRRs revolves around the central tenet of a 'molecular switch' model, which offers a mechanistic basis of how an NB-LRR receptor can detect and transduce exogenous signals into quick defence outputs (Takken et al. 2006). In mammalian innate immunity, the switch function is activated by PAMPs, whereas plant NB-LRRs recognize specific effector molecules (Bentham et al. 2017). In both cases, however, elicitor recognition prompts a rapid cell death response, which is generally used as an indication for NB-LRR-activity.

In plants, the modular built of a stereotypical NB-LRR receptor is composed of three well-defined parts (Fig. 1B). Sitting at the core is an NB-ARC region, so-called after the three apoptotic proteins from which the subdomains were first reported, namely: APAF-1, Resistance-Proteins and CED-4 (van der Biezen et al. 1998). The plant NB-ARC features signature motifs for nucleotide binding, consistent with studies demonstrating that NB-LRRs can preferentially associate and hydrolyze ATP/ADP (Tameling et al. 2002). The switch function of the receptor is thought to correlate directly with the status of bound-nucleotide, enabling the receptor to flip between two configurations: an ADP-bound OFF state and ATP-bound ON state. The nature of nucleotide associated with the NB-ARC, 
in turn, affects the conformation of the adjacent receptor domains. Positioned at the C-terminus is a Leucine-Rich Repeat (LRR) region, which takes the form of a horseshoe-shaped structure adorned by solvent-exposed, hypervariable residues on the surface (McHale et al. 2006). As these residues have been shown to undergo positive selection, they are thought to reflect a function of the LRR in effector recognition processes. At the opposite end, the N-terminus of a plant NB-LRR is typically a coiled-coil (CC) or Toll-Interleukin Like (TIR) domain, which broadly categorizes these receptors into two classes: the CC-NB-LRR and TIR-NB-LRR (van Wersch et al. 2020). The N-terminal moieties are commonly associated with immune signaling and heterocomplex formation. This is evidenced by experimental work indicating that the N-termini of plant NB-LRRs can self-associate, which is thought to provide a scaffold for downstream interactions (Bentham et al. 2017). Although the three modules of an NB-LRR receptor were initially thought to fulfill separate functions (as described in this section), there is accumulating evidence that they may fulfill overlapping roles (more extensively reviewed in Chapter 2).

In the cell, the building blocks of an NB-LRR receptor assemble to occupy various compartments (Lolle et al. 2020). For instance, some NB-LRRs span membranous structures such as the plasma membrane and endomembranes, while others can simultaneously reside in multiple locations such as the nucleus and cytoplasm. Diverse subcellular distribution patterns of NB-LRRs are thought to grant the plant with broad surveillance of pathogens with different infection strategies (Bezerra-Neto et al. 2020, Lolle et al. 2020). The promiscuity of these receptors also suggests distinct signaling pathways, although compartment-specific activity is not always clear. Irrespective of these differences, in these compartments, NB-LRRs fulfill their roles as molecular sentinels (Fig. 1C). In line with the gene-to-gene hypothesis, plant NB-LRRs are known to have a direct, receptor-ligand model of recognition. However, this may be an oversimplification as cases in which detection occurs indirectly are more frequently reported. This includes the perception of effector-induced modification inflicted on a host target or mimics thereof (van Wersch et al. 2020). Thus, indirect effector recognition is postulated to provide the host with leverage over the fast-evolving pathogens (van Wersch et al. 2020, van der Hoorn et al. 2008). In any case, the detection of a cognate effector activates the NB-LRR, which is ensued by substantial structural rearrangements, and in some cases, spatial subcellular redistribution of the receptor molecule. For example, both the CC-NB-LRR and TIR-NB-LRR MLA10 and RPS4 traffic into the nucleus, possibly to direct transcriptional reprogramming towards defence 
(Shen et al. 2007, Wirthmueller et al. 2007). However, whether the re-localization of an NB-LRR receptor is at all required to initiate immune signalling remains an open question. Likewise, how the diversity by which NB-LRRs can undergo recognition, activation and immune signaling ultimately feed into a common defence output remains to be explored (Lolle et al. 2020). 
A.
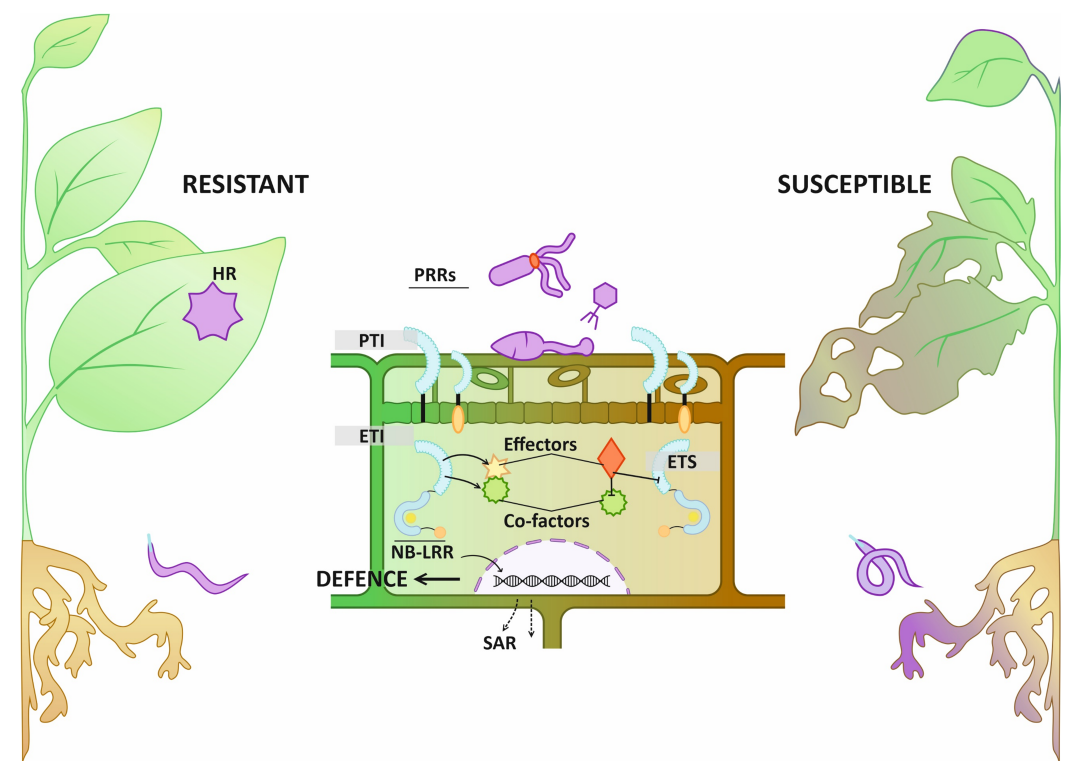

B.

CC/TIR

NB-ARC

LRR

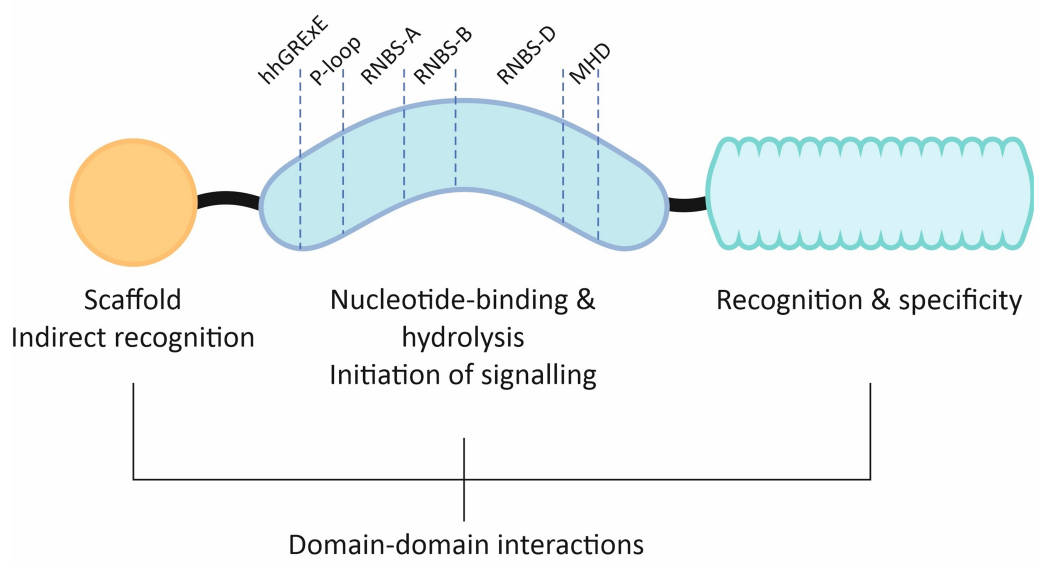

C.

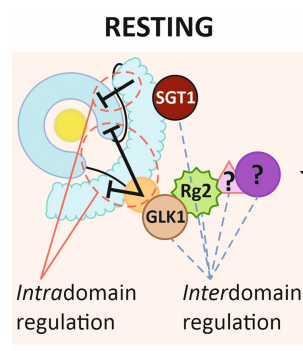

Chapter 3 Chapters 5 \& 6

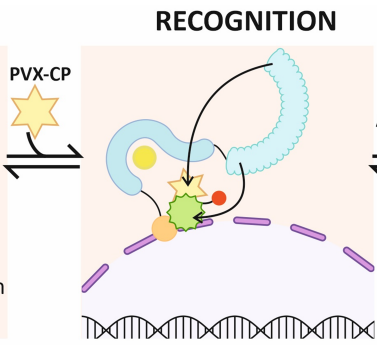

Chapter 4
SIGNALLING

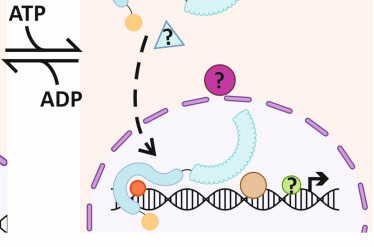

Chapters 5 \& 6

Fig. 1: Intracellular NB-LRRs immune receptors mediate a specialized branch of plant innate immunity. A) The plant innate immune system comprises two spatially divergent branches: PTI 
(Figure legend continued) and ETI that are mediated by PRRs and Resistance Proteins, the latter of which includes NB-LRR receptors. Effectors produced by the pathogen can suppress the host defence mechanisms, giving rise to ETS and rendering the plant susceptible. In resistant plants, effectors or their activity can be recognized, which activates downstream signaling towards immunity that often culminates in cell death. This local defence response can be disseminated to naïve cells in a process termed SAR.

B) Schematic representation of the domain architecture of a stereotypical plant NB-LRR receptor and associated functions. NB-LRR immune receptors are thought to fulfill a role as a molecular switch unit according to a general framework depicted in (C) using the potato Rx1 model system as an example. In the absence of a pathogen, both intra- and intermolecular interactions help to maintain $\mathrm{Rx} 1$ in a repressed state. The presence of the cognate PVX-CP effector is speculated to be perceived indirectly via an intermediary host-component. The prevailing theory is that this refers to the cytoplasmic co-factor, RanGAP2, although the physical interaction of RanGAP2 with PVX-CP and the exact effector-induced modifications inflicted on RanGAP2 remains to be determined. Upon effector recognition, $\mathrm{R} \times 1$ is predicted to undergo a conformational change that initiates downstream signaling. The thesis chapters related to these individual processes are included in the scheme.

\section{Regulation of a Molecular Switch}

As is the case for all living organisms, a plant must balance its energy for growth, development, and defence. This becomes particularly prominent in the context of NB-LRR-mediated resistance, where there is high-cost associated with receptor misfiring (van Wersch et al. 2020). Mutations that trigger the constant activation of immune responses (termed autoimmunity or autoactivity) can lead to retarded developmental phenotypes or even prove fatal (van Wersch et al. 2016). In unchallenged plants, NB-LRRs are thus kept in an autoinhibited state while also being readily stimulated when necessary. Thus, a crucial aspect of NB-LRR research is understanding how these receptors are regulated in the cell. As erroneous activation of the receptor is unfavorable for fitness, these receptors are subject to strict homeostasis. This begins from the very moment NB-LRRs are expressed. The transcripts of some NB-LRRs are maintained at constitutively low levels, putting the plant in a constant state of vigilance to scan for effectors or their activities (Bezerra-Neto et al. 2020). However, this 
can have high metabolic costs, which drives other $R$ genes to be expressed only in the event of a microbial infection (Wu et al. 2014). This regulation can be further restricted to specific tissues and organs (van Wersch et al. 2020). Extensive studies have shown that the modulation of $R$-gene transcripts is the combined outcome of pre- and post-transcriptional regulatory processes that are aimed to maximize immune output with minimal fitness cost (Lai et al. 2018). While the components associated with these processes are beginning to emerge, there are considerable knowledge gaps in their synergistic/antagonistic actions for the accurate spatial and temporal regulation of NB-LRR transcripts.

Once expressed, $R$ gene products are maintained in a resting, signaling-competent state granted by interactions between the receptor domains (Fig. 1C) (Monteiro et al. 2018). Within this receptor complex, the three parts of an NB-LRR protein form a perfect fit, providing an elegant form of intrinsic control that constraints improper assembly and functioning. Activation of the receptor requires the relief of this auto-repressed state. In some systems, for example, it is vital that regions in the NB-ARC and LRR subdomains are matching to keep the receptor in an autoinhibited state (Slootweg et al. 2013, Rairdan et al. 2006). Deleting domain fragments or inappropriate pairings can result in spurious or dampened immune responses. Due to the strict requirements needed to maintain the receptor module, changes in one region are, therefore, often reflected in the co-evolution of adjacent subdomains. This pinpoints that targeting specific contact points between domains can alter the overall sensitivity of the receptor protein i.e. how easily it adopts an active conformation (Monteiro et al. 2018). Tinkering intramolecular interactions underlying NB-LRR proteins can, therefore, provide an important basis for resistance engineering. However, this relies on precise knowledge of how the receptor domains interact, which is currently fragmentary over a few NB-LRR systems (more extensively reviewed in Chapter 2).

Beyond the receptor unit, the stability and activity of NB-LRRs are tightly controlled by proteins existing as a macromolecular complex with the receptor (Fig. 1C) (Sun et al. 2020). These co-factors are co-opted to modulate the immune output, mainly by maintaining the receptor in a dormant state, regulating receptor maturation, and/or downstream response. Efforts to screen NB-LRR associated proteins by genetic or biochemical approaches have uncovered a pool of NB-LRR interacting proteins from molecular chaperones (e.g. SGT1 and HsP90), transcription factors to so-called 'helper' NB-LRRs (Shirasu 2009). Despite the growing catalog of NB-LRR interacting 
partners, however, identifying these components is still a challenge due to the complex nature of in vivo systems. Hence, signaling partners that immediately link activated NB-LRRs receptors to downstream events remain poorly defined (Sun et al. 2020). A long-withstanding interest in the field is, therefore, to elucidate the composition and dynamics of NB-LRR complexes pre- and post-activation. This will help pinpoint specific activation and signaling mechanisms involved in NB-LRR defence, which can, in turn, provide new avenues for fine-tuning the defence response.

\section{The Potato CC-NB-LRR immune receptor Rx1}

The scope and complexity of plant NB-LRR function provide ample opportunities for research. Hereby, the potato CC-NB-LRR Rx1 has emerged as a convenient model system to decipher key molecular events associated with plant NB-LRR function. Since it was first identified in Solanum tuberosum spp. andigena and introgressed into potato, $\mathrm{R} \times 1$ has been extensively used in studies that are aimed to elucidate mechanisms of plant-pathogen interactions (Ross 1986, Querci et al. 1995). For this purpose, it is useful that $\mathrm{R} \times 1$ is fully operational in heterologous model systems, including Nicotiana benthamiana (Bendahmane et al. 1999). Epitope-tagged versions of the full-length receptor, or its domains, can be expressed stably in planta, enabling studies at the molecular, cellular, and biochemical levels. Expressing the independent domains of Rx1 separately in trans can also reconstitute a functional receptor unit in planta, facilitating structure-functional studies aiming to resolve domain-domain interactions (Moffett et al. 2002). More importantly, however, the relationship between $\mathrm{Rx} 1$ and its cognate pathogen is well characterized. $\mathrm{R} \times 1$ confers relatively durable resistance to Potato Virus $X(P V X)$, which itself is a centerpiece in many host-virus studies. PVX is a single-stranded RNA virus and a member of the family of Alphaflexiviridae (Martelli et al. 2007). Its genome harbors 5 open reading frames (ORFs), the products of which encode for an RNA-dependent RNA Polymerase, a coat protein, and triple-gene block movement proteins that collectively function to hijack the host cellular machinery to support viral replication. Despite expressing only a few proteins, PVX is a widespread pathogen impacting some of agriculture's most important Solanaceous crops, leading to the introgression of Rx1 in several commercial cultivars (Ross 1986).

Although Rx1 and PVX fit within the framework of a general NB-LRR/effector model, the resistance phenotypes triggered by Rx1 deviate from those typically described (Bendahmane et al. 1999). 
Reverse genetics and transient experiments show that Rx1 detects a 110 amino acid fragment of the coat protein (CP) of all PVX strains (Bendahmane et al. 1999, Moffett et al. 2002). An exception is that of the resistance breaker PVX-HB, which has amassed mutations at positions 121 and 127 of its CP (Bendahmane et al. 1995).In the case of Rx1, however, recognition of its cognate effector does not induce the development of cell death lesions characteristic of HR. Instead, PVX-CP recognition prompts a rapid, asymptomatic response that effectively restricts viral accumulation to initially infected cells termed Extreme Resistance (ER) (Bendahmane et al. 1999). Unlike HR, Rx1-mediated ER is effective in isolated protoplasts and against taxonomically-unrelated viruses such as Tobacco Mosaic Virus (Bendahmane et al. 1995). The underlying mechanisms, however, are unclear, although ER has been shown to involve suppression of viral translation in a process involving the activity of Argonaute-like proteins (Bhattacharjee et al. 2009). Intriguingly, Rx1 can also trigger HR typical of a classical NB-LRR under certain experimental conditions such as when PVX-CP is overexpressed in heterologous assays (Bendahmane et al. 1999). The prevailing view is, therefore, that $\mathrm{HR}$ is an epistatic pathway that occurs when ER fails to effectively contain the virus. More extensive research is needed to illustrate whether distinct genetic and molecular requirements indeed uncouple these processes. Taken together, however, the existence of ER and HR constitutes an important theme in studies aiming to elucidate the functionality of $R \times 1$.

Perhaps, a more relevant feature of $\mathrm{R} \times 1$ is that it belongs to a small gene cluster in chromosome XII of potato with three other neighboring homologs (van der Vossen et al. 2000). The most well-studied of these is Gpa2, which shares high sequence similarity with $\mathrm{R} \times 1$ ( $88 \%$ at the protein level). Yet, unlike $\mathrm{R} \times 1, \mathrm{Gpa} 2$ acts belowground to confer defence against the potato cyst nematode Globodera pallida. In potato plants harboring Gpa2, the nematodes can still infect the roots and initiate a complex feeding structure (termed the feeding site). However, the surrounding tissues will eventually collapse after a couple of days, limiting the supply of nutrients needed by the nematode to complete its lifecycle (van der Vossen et al. 2000). Transient assays in N. benthamiana demonstrate that Gpa2 can trigger relatively mild cell death when co-expressed with Gp-RBP1 effectors produced in the dorsal glands of the nematode (Sacco et al. 2009). In hindsight, it is remarkable how the highly homologous receptors Rx1 and Gpa2 are characterized by distinct resistance phenotypes against unrelated pathogens. This is the more intriguing when considering that recognition specificity of these receptors can be exchanged by switching only a small portion of the receptor's LRR C-terminal end 
(Slootweg et al. 2017). This pinpoints that activation of defence signaling by the receptor domains is independent of the plant organs under attack and lifestyle of the pathogen. Thus, despite eliciting dissimilar resistance responses, Rx1 and Gpa2 have shared commonalities in their downstream signaling. Indeed, like $\mathrm{R} \times 1$, the cell death response of $\mathrm{Gpa} 2$ also relies on its interaction with the RanGTPase activating protein 2 (RanGAP2) (Tameling et al. 2007, Sacco et al. 2007, Sacco et al. 2009). Moreover, the domain exchange study described for Rx1 and Gpa2 also demonstrates a bifurcation in the role of the LRR in recognition and intramolecular regulation (Rairdan et al. 2006, Slootweg et al. 2013). Particularly, an operational $\mathrm{R} \times 1 / \mathrm{Gpa} 2$ receptor cannot be achieved when exchanging a larger fragment of the LRR as this compromises compatibility with the ARC2 region. Having two immune receptors that are highly alike but have distinct recognition profiles is, therefore, useful to study mechanisms underlying $R$-gene evolution, recognition and activation.

Aside from structure-functional studies, $\mathrm{R} \times 1$ has helped to shape our understanding of the molecular dialogue linking effector recognition and disease output. Rx1 localizes to the nucleus and cytoplasm, and both pools of the receptor are required for full immunity (Slootweg et al. 2010). The recognition of PVX-CP occurs in the cytoplasm, whereby a fraction of cytoplasmic $R \times 1$ is tethered to the nuclear envelope by virtue of the association of the receptor with RanGAP2 (Tameling et al. 2010). Until recently, the function of $\mathrm{R} \times 1$ in the nucleus has remained obscure. A combination of biochemical and functional studies have shown, however, that the Rx1-NB-ARC can directly associate with and deform DNA upon CP-elicitation, though this interaction appears to lack specificity (Fenyk et al. 2015). This phenomenon was also described for the tomato CC-NB-LRR I2, offering new light on a novel and potentially general feature of activated plant NB-LRRs (Fenyk et al. 2015). How aspecific binding of $\mathrm{R} \times 1$ to DNA links to the precise mobilization of an immune response is not fully understood. Rx1, however, was recently shown to recruit and modulate the DNA binding of the Golden2-like transcription factor (GLK1), giving rise to a model in which nuclear-associated factors guide $R \times 1$ to specific sites in the genome (Townsend et al. 2018). Though our knowledge of how Rx1 behaves in the cell has advanced, the identity, dynamics, and co-operative function of Rx1-associated components remain largely unknown. 


\section{Scope of this Thesis}

In this thesis, I explore the breadth and depth of plant NB-LRR function by performing heterologous studies of the Rx1 receptor in $N$. benthamiana. One overarching objective was to explore the role of intradomain interactions in modulating complex-formation and defence activation. A second pillar of the thesis focuses on interdomain interactions by characterizing pre-existing and novel host-proteins associating with the Rx1 complex at the molecular and cellular levels. By addressing both aims, the thesis adds to the mechanistic understanding of upstream and downstream events regulating NB-LRR folding, recognition, and activation. The findings of these studies may resonate to other R-proteins that share similar structural characteristics.

Recent years have witnessed rapid and exciting advancements in the field of plant NB-LRR biology. We, therefore, begin the thesis by reviewing current insights on NB-LRR function in Chapter 2 , with an emphasis on structural and molecular aspects. By drawing on the extensive body of works available, we unveil the immense diversity in the manner by which these receptors can perceive effectors and are activated to relay the message of intrusion. This review serves as an essential foundation in delineating the remainder of the thesis chapters.

In Chapter 3, we probe further at the extent by which intra-domain associations regulating NB-LRR activity are conserved between homologous $\mathrm{R}$ genes originating from closely related species. To that end, we have identified a homolog of Rx1 from tomato (Rslyc) that does not confer defence against PVX. We aimed to resolve molecular determinants underlying differences in PVX recognition and immune activation of Rx1 and Rslyc using trans-complementation approaches in $\mathrm{N}$. benthamiana. We discovered that although these receptors bear broad similarities, they demand distinct structural requirements for complex formation and activation. This, in turn, illustrates a level of functional constraint, which shapes the recognition of $\mathrm{R}$ genes and their activation sensitivity.

In Chapter 4, we step beyond the R protein and shift our attention towards the intermolecular regulation of NB-LRRs by their associated host factors. We start by examining an established co-factor, RanGAP2, whose exact role in Rx1/Gpa2-mediated resistance is not well-defined. However, based on artificial-tethering studies to the GpRBP-1 effector, it was previously implicated that RanGAP2 may partake in effector recognition (Sacco et al. 2009). Here, we present evidence that RanGAP2 can, in fact, form complexes with the cognate effectors of Rx1 and Gpa2 in planta, and that 
effector targeting contributes to the virulence of both PVX in N. benthamiana and cyst nematodes in Arabidopsis. We infer that the interaction PVX-CP/Gp-RBP1 with RanGAP2 is not the sole determining factor in mediating the recognition of these effectors as the physical association expands to both virulent and avirulent factors. A conceptual model hypothesizing the role of RanGAP2 as bait in recognition by Rx1 and Gpa2 is proposed.

We move from recognition events in the cytoplasm to function in the nucleus by resolving the complex of Rx1 at the chromatin in Chapter 5. In a Yeast-2-Hybrid screening, a N. benthamiana homolog of the DNA-Binding Bromo-Domain Containing Protein ( $\mathrm{NbDBCP}$ ) was identified as a novel interactor of the Rx1-CC domain. We demonstrate that NbDBCP can likewise form a complex with full-length $\mathrm{R} \times 1$ in planta and acts as a suppressor of defences mediated by Rx1 and its co-factor GLK1. Targeted mutation demonstrates that these immune-related functions of NbDBCP depends on an intact bromodomain. More detailed biochemical studies show that the capacity of NbDBCP to bind DNA is impaired by the presence of Rx1 pre and post-elicitation by PVX-CP. Together, our data illustrate that Rx1 can co-op and regulate the activity of nuclear-associated factors with DNA-binding activities for immunity.

As only a handful of Rx1 and Gpa2-interacting proteins has been reported, in Chapter 6, we expanded our screening efforts to identify additional interactors by Co-Immunoprecipitation/Mass-Spectrometry analysis in N. benthamiana. The Glycine Rich RNA Binding Protein 7 (NbGRP7) was originally found to co-precipitate with the Gpa2-CC domain, but we demonstrate that it is also a novel component belonging to the Rx1 complex. Functional analysis reveals that NbGRP7 positively regulates defence responses conferred by both receptors, which relies on its RNA binding activity. Interestingly, in subsequent expression analyses, we show that ectopic expression of NbGRP7 impacts the steady-state abundance of Rx1 pre- and post-activation. The interaction of Rx1/Gpa2 with NbGRP7 is an illustrative example that plant NB-LRRs can recruit a diverse plethora of host components with housekeeping functions including those involved in post-transcriptional regulation for their defence functions.

In Chapter 7, I close the thesis by placing my main findings in the framework of current scientific discussions related to Rx1 and plant NB-LRRs in general. By drawing parallels with other systems, I derive at an integrated working model for the functioning of $\mathrm{Rx} 1$ as a pathogen detector and molecular switch of plant immune responses. A list of prospective questions that may shape the direction of future research concludes the chapter. 


\section{References}

Bendahmane, A., Kanyuka, K., and Baulcombe, D. C. (1999), The Rx gene from potato controls separate virus resistance and cell death responses. Plant Cell. 11.

Bendahmane, A., Köhm, B. A., Dedi, C., and Baulcombe, D. C. (1995), The coat protein of potato virus X is a strain-specific elicitor of Rx1-mediated virus resistance in potato. The Plant Journal. 8(6): p. 933-941.

Bent, A., Kunkel, B., Dahlbeck, D., Brown, K., Schmidt, R., Giraudat, J., Leung, J., and Staskawicz, B. (1994), RPS2 of Arabidopsis thaliana: a leucine-rich repeat class of plant disease resistance genes. Science. 265(5180): p. $1856-1860$.

Bentham, A., Burdett, H., Anderson, P. A., Williams, S. J., and Kobe, B. (2017), Animal NLRs provide structural insights into plant NLR function. Annals of Botany. 119(5): p. 698-702.

Bezerra-Neto, J. P., Araújo, F. C., Ferreira-Neto, J. R. C., Silva, R. L. O., Borges, A. N. C., Matos, M. K. S., Silva, J. B., Silva, M. D., Kido, E. A., and Benko-Iseppon, A. M. (2020), NBS-LRR genes - Plant health sentinels: Structure, roles, evolution and biotechnological applications. In: Applied Plant Biotechnology for Improving Resistance to Biotic Stress. Ed. by P. Poltronieri and Y. Hong. Academic Press, p. 63-120.

Bhattacharjee, S., Zamora, A., Azhar, M. T., Sacco, M. A., Lambert, L. H., and Moffett, P. (2009), Virus resistance induced by NB-LRR proteins involves Argonaute4-dependent translational control. The Plant Journal. 58(6): p. 940-951.

Van der Biezen, E. A. and Jones, J. D. G. (1998), The NB-ARC domain: A novel signalling motif shared by plant resistance gene products and regulators of cell death in animals. Current Biology. 8(7): R226-R227.

Dong, X. (2001), Genetic dissection of systemic acquired resistance. Current Opinion in Plant Biology. 4(4): p. 309-314.

Felix, G., Duran, J. D., Volko, S., and Boller, T. (1999), Plants have a sensitive perception system for the most conserved domain of bacterial flagellin. The Plant Journal. 18(3): p. 265-276.

Fenyk, S., Townsend, P. D., Dixon, C. H., Spies, G. B., de San Eustaquio Campillo, A., Slootweg, E. J., Westerhof, L. B., Gawehns, F. K. K., Knight, M. R., Sharples, G. J., Goverse, A., Pålsson, L.-O., Takken, F. L. W., and Cann, M. J. (2015), The Potato Nucleotide-binding Leucine-rich Repeat (NLR) Immune Receptor Rx1 Is a Pathogen-dependent DNA-deforming Protein. The Journal of biological chemistry. 290(41): p. 24945-24960. ppublish.

Flor, H. H. (1971), Current status of the gene-for-gene concept. Annual Review of Phytopathology. 9: p. 297-323.

Hammond-Kosack, K. E. and Jones, J. D. (1996), Resistance gene-dependent plant defense responses. The Plant Cell. 8(10): p. 1773-1791.

- (1997), Plant Disease Resistance Genes. Annu Rev Plant Physiol Plant Mol Biol. 48: p. 575-607.

Han, G.-Z. (2019), Origin and evolution of the plant immune system. New Phytologist. 222(1): p. 70-83.

Van der Hoorn, R. A. and Kamoun, S. (2008), From Guard to Decoy: a new model for perception of plant pathogen effectors. Plant Cell. 20(8): p. 2009-17.

Jones, J. D. and Dangl, J. L. (2006), The plant immune system. Nature. 444(7117): p. 323-9.

Lai, Y. and Eulgem, T. (2018), Transcript-level expression control of plant NLR genes. Molecular Plant Pathology. 19(5): p. $1267-1281$.

Lolle, S., Stevens, D., and Coaker, G. (2020), Plant NLR-triggered immunity: from receptor activation to downstream signaling. Current Opinion in Immunology. 62: p. 99-105.

Martelli, G. P., Adams, M. J., Kreuze, J. F., and Dolja, V. V. (2007), Family Flexiviridae: A Case Study in Virion and Genome Plasticity. Annual Review of Phytopathology. 45(1): p. 73-100.

McHale, L., Tan, X., Koehl, P., and Michelmore, R. W. (2006), Plant NBS-LRR proteins: adaptable guards. Genome Biology. 7(4): p. 212. 
Menezes, H. and Jared, C. (2002), Immunity in plants and animals: common ends through different means using similar tools. Comparative Biochemistry and Physiology Part C: Toxicology \& Pharmacology. 132(1): p. 1-7.

Meyers, B. C., Kozik, A., Griego, A., Kuang, H., and Michelmore, R. W. (2003), Genome-wide analysis of NBS-LRR-encoding genes in Arabidopsis. Plant Cell. 15(4): p. 809-34.

Mindrinos, M., Katagiri, F., Yu, G. L., and Ausubel, F. M. (1994), The A. thaliana disease resistance gene RPS2 encodes a protein containing a nucleotide-binding site and leucine-rich repeats. Cell. 78(6): p. 1089-99.

Moffett, P., Farnham, G., Peart, J., and Baulcombe, D. C. (2002), Interaction between domains of a plant NBS-LRR protein in disease resistance-related cell death. The EMBO Journal. 21(17): p. 4511-4519.

Monteiro, F. and Nishimura, M. T. (2018), Structural, Functional, and Genomic Diversity of Plant NLR Proteins: An Evolved Resource for Rational Engineering of Plant Immunity. Annu Rev Phytopathol. 56: p. 243-267.

Nelson, R., Wiesner-Hanks, T., Wisser, R., and Balint-Kurti, P. (2018), Navigating complexity to breed disease-resistant crops. Nature Reviews Genetics. 19(1): p. 21-33.

Querci, M., Baulcombe, D. C., Goldbach, R. W., and Salazar, L. F. (1995), Analysis of the resistance-breaking determinants of potato virus $X(P V X)$ strain $\mathrm{HB}$ on different potato genotypes expressing extreme resistance to PVX. Phytopathology. 85(9): p. 1003-1010.

Rairdan, G. J. and Moffett, P. (2006), Distinct Domains in the ARC Region of the Potato Resistance Protein Rx Mediate LRR Binding and Inhibition of Activation. Plant Cell. 18(8): p. 2082-2093.

Ross, H. (1986), Potato breeding - problems and perspectives. Berlin: V.P. Parey.

Sacco, M. A., Koropacka, K., Grenier, E., Jaubert, M. J., Blanchard, A., Goverse, A., Smant, G., and Moffett, P. (2009), The cyst nematode SPRYSEC protein RBP-1 elicits Gpa2- and RanGAP2-dependent plant cell death. PLoS Pathog. 5.

Sacco, M. A., Mansoor, S., and Moffett, P. (2007), A RanGAP protein physically interacts with the NB-LRR protein Rx, and is required for Rx-mediated viral resistance. Plant J. 52(1): p. 82-93.

Shen, Q.-H., Saijo, Y., Mauch, S., Biskup, C., Bieri, S., Keller, B., Seki, H., Ülker, B., Somssich, I. E., and Schulze-Lefert, P. (2007), Nuclear Activity of MLA Immune Receptors Links Isolate-Specific and Basal Disease-Resistance Responses. Science. 315(5815): p. 1098.

Shirasu, K. (2009), The HSP90-SGT1 Chaperone Complex for NLR Immune Sensors. Annual Review of Plant Biology. 60(1): p. 139-164.

Slootweg, E., Roosien, J., Spiridon, L. N., Petrescu, A. J., Tameling, W., Joosten, M., Pomp, R., van Schaik, C., Dees, R., Borst, J. W., Smant, G., Schots, A., Bakker, J., and Goverse, A. (2010), Nucleocytoplasmic distribution is required for activation of resistance by the potato NB-LRR receptor $\mathrm{Rx} 1$ and is balanced by its functional domains. Plant Cell. 22(12): p. 4195-215.

Slootweg, E., Koropacka, K., Roosien, J., Dees, R., Overmars, H., Lankhorst, R. K., van Schaik, C., Pomp, R., Bouwman, L., Helder, J., Schots, A., Bakker, J., Smant, G., and Goverse, A. (2017), Sequence Exchange between Homologous NB-LRR Genes Converts Virus Resistance into Nematode Resistance, and Vice Versa. Plant physiology. 175(1): p. 498-510.

Slootweg, E. J., Spiridon, L. N., Roosien, J., Butterbach, P., Pomp, R., Westerhof, L., Wilbers, R., Bakker, E., Bakker, J., Petrescu, A.-J., Smant, G., and Goverse, A. (2013), Structural Determinants at the Interface of the ARC2 and Leucine-Rich Repeat Domains Control the Activation of the Plant Immune Receptors Rx1 and Gpa2. Plant Physiology. 162(3): p. 1510-1528.

Sun, Y., Zhu, Y.-X., Balint-Kurti, P. J., and Wang, G.-F. (2020), Fine-Tuning Immunity: Players and Regulators for Plant NLRs. Trends in Plant Science. 25(7): p. 695-713. 
Takken, F. L. W., Albrecht, M., and Tameling, W. I. L. (2006), Resistance proteins: molecular switches of plant defence. Curr Opin Plant Biol. 9(4): p. 383-90.

Tameling, W. I. L. and Baulcombe, D. C. (2007), Physical association of the NB-LRR resistance protein Rx with a Ran GTPase-activating protein is required for extreme resistance to Potato virus X. Plant Cell. 19(5): p. 1682-94.

Tameling, W. I. L., Elzinga, S. D. J., Darmin, P. S., Vossen, J. H., Takken, F. L. W., Haring, M. A., and Cornelissen, B. J. C. (2002), The tomato R gene products I-2 and Mi-1 are functional ATP binding proteins with ATPase activity. Plant Cell. 14(11): p. 2929-2939.

Tameling, W. I. L., Nooijen, C., Ludwig, N., Boter, M., Slootweg, E., Goverse, A., Shirasu, K., and Joosten, M. (2010), RanGAP2 Mediates nucleocytoplasmic partitioning of the NB-LRR immune receptor Rx in the solanaceae. Thereby dictating Rx function. Plant Cell. 22.

Ting, J. P., Lovering, R. C., Alnemri, E. S., Bertin, J., Boss, J. M., Davis, B. K., Flavell, R. A., Girardin, S. E., Godzik, A., Harton, J. A., Hoffman, H. M., Hugot, J. P., Inohara, N., Mackenzie, A., Maltais, L. J., Nunez, G., Ogura, Y., Otten, L. A., Philpott, D., Reed, J. C., Reith, W., Schreiber, S., Steimle, V., and Ward, P. A. (2008), The NLR gene family: a standard nomenclature. Immunity. 28(3): p. 285-7.

Toruño, T. Y., Stergiopoulos, I., and Coaker, G. (2016), Plant-Pathogen Effectors: Cellular Probes Interfering with Plant Defenses in Spatial and Temporal Manners. Annual Review of Phytopathology. 54(1): p. 419-441.

Townsend, P. D., Dixon, C. H., Slootweg, E. J., Sukarta, O. C. A., Yang, A. W. H., Hughes, T. R., Sharples, G. J., Pålsson, L. O., Takken, F. L. W., Goverse, A., and Cann, M. J. (2018), The intracellular immune receptor Rx1 regulates the DNA-binding activity of a Golden2-like transcription factor. J Biol Chem. 293(9): p. 3218-3233.

Turvey, S. E. and Broide, D. H. (2010), Innate immunity. J Allergy Clin Immunol. 125(2 Suppl 2): S24-32.

Urbach, J. M. and Ausubel, F. M. (2017), The NBS-LRR architectures of plant R-proteins and metazoan NLRs evolved in independent events. Proceedings of the National Academy of Sciences of the United States of America. 114(5): p. 1063-1068.

Van der Vossen, E. A. G., van der Voort, J., Kanyuka, K., Bendahmane, A., Sandbrink, H., Baulcombe, D. C., Bakker, J., Stiekema, W. J., and Klein-Lankhorst, R. M. (2000), Homologues of a single resistance-gene cluster in potato confer resistance to distinct pathogens: a virus and a nematode. Plant J. 23.

Van Wersch, R., Li, X., and Zhang, Y. (2016), Mighty Dwarfs: Arabidopsis Autoimmune Mutants and Their Usages in Genetic Dissection of Plant Immunity. Frontiers in Plant Science. 7(1717).

Van Wersch, S., Tian, L., Hoy, R., and Li, X. (2020), Plant NLRs: The Whistleblowers of Plant Immunity. Plant Communications. 1(1): p. 100016.

Whitham, S., Dinesh-Kumar, S. P., Choi, D., Hehl, R., Corr, C., and Baker, B. (1994), The product of the tobacco mosaic virus resistance gene N: Similarity to toll and the interleukin-1 receptor. Cell. 78(6): p. 1101-1115.

Wirthmueller, L., Zhang, Y., Jones, J. D. G., and Parker, J. E. (2007), Nuclear Accumulation of the Arabidopsis Immune Receptor RPS4 Is Necessary for Triggering EDS1-Dependent Defense. Current Biology. 17(23): p. 2023-2029.

Wu, L., Chen, H., Curtis, C., and Fu, Z. Q. (2014), Go in for the kill: How plants deploy effector-triggered immunity to combat pathogens. Virulence. 5(7): p. 710-721.

Zipfel, C. (2014), Plant pattern-recognition receptors. Trends in Immunology. 35(7): p. 345-351. 


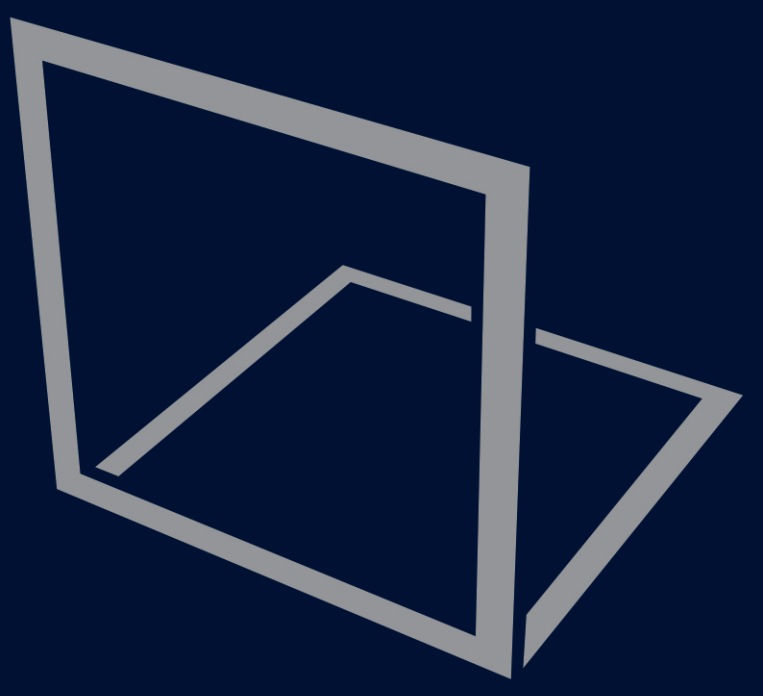

Octavina C. A Sukarta, a

Erik J. Slootweg ${ }^{1, \text { a }}$

Aska Goverse $^{1}$

${ }^{1}$ Laboratory of Nematology, Wageningen University \& Research, Wageningen, the Netherlands.

${ }^{a}$ These authors have equal contributions in writing this manuscript 


\section{Chapter}

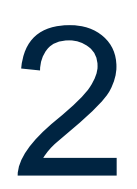

Structure-Informed Insights for NLR

Functioning in Plant Immunity

*This is a slightly modified version of the manuscript published in

Seminars in Cell \& Developmental Biology; 2016,

Volume 56, pages: 134-149. 


\section{ABSTRACT}

To respond to foreign invaders, plants have evolved a cell autonomous multi-layered immune system consisting of extra- and intracellular immune receptors. Nucleotide binding and oligomerization domain (NOD)-like receptors (NLRs) mediate recognition of pathogen effectors inside the cell and trigger a host specific defence response, often involving controlled cell death. NLRs consist of a central Nucleotide-Binding domain, which is flanked by an N-terminal CC or TIR domain and a C-terminal Leucine-Rich Repeat domain (LRR). These multidomain proteins function as a molecular switch and their activity is tightly controlled by intra and inter-molecular interactions. In contrast to metazoan NLRs, the structural basis underlying NLR functioning as a pathogen sensor and activator of immune responses in plants is largely unknown. However, the first crystal structures of a number of plant NLR domains were recently obtained. In addition, biochemical and structure-informed analyses revealed novel insights in the cooperation between NLR domains and the formation of pre- and post activation complexes, including the coordinated activity of NLR pairs as pathogen sensor and executor of immune responses. Moreover, the discovery of novel integrated domains underscores the structural diversity of NLRs and provides alternative models for how these immune receptors function in plants. In this review, we will highlight these recent advances to provide novel insights in the structural, biochemical and molecular aspects involved in plant NLR functioning.

\section{INTRODUCTION}

Plants have evolved a cell autonomous multi-layered immune system consisting of extra- and intracellular immune receptors encoded by so called resistance $(R)$ genes, which trigger host specific defence responses upon recognition of pathogen-derived effectors (Jones et al. 2006, Dodds et al. 2010). The prevalent type of intracellular immune receptor is the Nucleotide-Binding Leucine-Rich Repeat protein (NLR). Plant NLR immune receptors have a modular architecture with a central nucleotide binding domain. In higher plants, the N-terminal domain is most commonly either a coiled coil (CC) domain or a Toll Interleukin-1-like receptor (TIR) domain. Almost invariably, a Leucine-rich repeat structure (LRR) is present at the C-terminus that often has a complex dual function in autoinhibition and pathogen perception. These domains act in concert as a molecular switch, which is able to toggle between a signaling-competent resting state in the absence of a 
pathogen and an active immune signaling state in the presence of specific pathogens (Takken et al. 2012).

Invading pathogens release proteins called effectors into the cell that target host cell components to promote virulence (Mukhtar et al. 2011, Asai et al. 2015). NLRs may directly or indirectly recognize these effectors, resulting in the activation of defence responses. Often, a controlled cell death response or so called Hypersensitive Response (HR) is induced, which also limits spreading of the pathogen (Feechan et al. 2015). However, the molecular mechanisms underlying this complex and multistep process are still poorly understood. In the last decade, structure-informed analyses have contributed significantly to understand the cooperation between domains in NLR functioning as a molecular switch that integrates pathogen detection and immune activation. For an increasing number of plant NLRs, the crystal structures of individual $\mathrm{N}$ - terminal domains have been resolved. However, structural insights in the nucleotide-binding and LRR domains still depend on homology modeling. Pre- and post-activation complex formation and cooperation between NLRs have emerged as novel mechanisms important for NLR functioning. Finally, the discovery of NLRs with a variety of integrated domains has also contributed to the development of novel recognition and activation models.

In this review, we describe recent advances in the structural and molecular basis of NLR functioning in plants. We will focus first on the structural diversity of NLRs in plants (Section 1), followed by a brief description of the individual domains (Section 2). We will highlight how these domains contribute to the molecular switch function of NLRs in plants (Section 3), as well as their role in pathogen detection (Section 4) and downstream signaling (Section 5). Based on this information, an integrated picture will be provided on the structural components underlying the functioning of NLRs as molecular switch and pathogen detectors in plants (Section 6).

\section{The shaping of Multidomain NLRs in Plants}

Although there is little evidence supporting a common origin of the immune systems (Maekawa et al. 2011a, Yue et al. 2012), the presence of common domains and the overall modular architectures in immune receptors of plants and metazoans suggest that a surprisingly small set of protein structures meets the functional demands required for sensing pathogens and subsequently initiating 
an appropriate defence response. NLRs can be divided in two subclasses, either CNL or TNL, based on their N-terminal domain (Fig. 1). Whereas both classes are present in dicots, the group of TNL receptors is lost during evolution in monocots (Meyers et al. 1999). The presence of both classes in moss and liverworts, which diverged from the lineage of higher plants around 450-480 million years ago, indicates that the basic building blocks of the plant immune system were in place very early on in the evolution of plants (Xue et al. 2012, Tanigaki et al. 2014). Prior to the early land plants, the individual NB-ARC, LRR and TIR domains are found, but not yet combined in a single protein (Yue et al. 2012).

Even though the TNL and CNL architectures form the two most prevalent classes of intracellular immune receptors in plants, many alternative architectures exist in which the small set of core domains is rearranged in different configurations or additional domains have been incorporated (Fig. 1). Some of these evolved after the divergence of specific plant families. For example, the subclass of SD-CNLs have a unique domain N-terminal to their CC and has been found only in Solanaceous plant species (Milligan et al. 1998, van Ooijen et al. 2008b, Salmeron et al. 1996). In poplar, NLRs containing a BED Zn-finger DNA-binding domain are highly enriched Kohler et al. 2008). Most likely, these independent proteins from which the NB-LRR domains originate cooperate with each other in a functional complex. Fusion into a single protein would have reduced the entropy of the interaction and make the cooperation more efficient (Rosetta stone principle, (Marcotte et al. 1999). Non-canonical domains contribute to most aspects of NLR functioning, including activation (Section 3), pathogen detection (Section 4) and signaling (Section 5). Integrated effector targets or decoys of effector targets occur in about 3.5\% of the NLRs and analysis of these domains can point to important immune signaling components or susceptibility factors (Kroj et al. 2016, Sarris et al. 2016).

Apart from canonical TNL and CNL proteins, plant genomes harbor a large set of truncated NLR-like proteins (Fig. 1). In the Arabidopsis genome for example, which encodes $\sim 150$ full-length NLRs, 58 genes encode NLR-like proteins lacking the LRR or even NB-ARC sequences (Meyers et al. 2002, Meyers et al. 2003). Until recently it was unclear if these truncated proteins had a role in plant immunity and if they could act as immune receptors. Although RPW8 only consists of a CC domain and lacks NB-ARC and LRR sequences, it confers broad-spectrum resistance to powdery mildew pathogens in Arabidopsis thaliana (Xiao et al. 2001). However, RPW8 appears to function 
more as a general enhancer of salicylic-acid dependent basal defences than as a specific immune receptor (Wang et al. 2007b). Interestingly, sequence relationships of the TIR and NB-ARC domains of truncated forms of TIR-NB-ARC (TN) or TIR-X (TX) proteins indicate that many of them evolved independently from the canonical TNLs (Meyers et al. 2003, Meyers et al. 2002, Nandety et al. 2013). In monocots, not only all TNLs have been lost, but most TN and TX proteins as well, suggesting that they cooperate in resistance in dicots. In Arabidopsis a TN protein, TN2, has been found to activate the typical TNL signaling pathway in response to modifications of a component of the exocyst complex, consistent with a role as guard of host proteins (Zhao et al. 2015). Alternatively, TN and TX proteins might function in TNL pathways in a role analogous to adaptor TIR proteins like MyD88 in downstream signal transduction from Toll-like receptors in animal innate immunity (O'Neill et al. 2007).

\section{Structural Aspects of the Multidomain architecture of NLRs}

Here, we will focus on the main structural elements of plant NLR proteins, which together form a signaling competent immune receptor. Although no complete structure is yet available for a plant NLR immune receptor, significant progress has been made in the last decade in resolving the experimental structures of various $\mathrm{N}$-terminal domains ( $\mathrm{CC}$ and $\mathrm{TIR}$ ). The availability of suitable templates from structurally related proteins has allowed the in silico homology modeling of NB-ARC and LRR domains. This enabled structure-informed analysis of NLR functioning and the identification of structural determinants involved in controlling their activity (Section 3), but also pathogen recognition (Section 4) and defence signaling (Section 5).

\subsection{CC domains}

Although CC domains are usually characterized by a heptad repeat pattern and a predicted propensity to form a coiled coil structure, their amino acid sequence is not strongly conserved. The negatively charged EDVID motif (consensus WLXxVRELAYDAEDVLDx) forms an exception to this lack of conservation and is present in the majority of $\mathrm{CC}$ domains and defines the $\mathrm{CC}_{\mathrm{EDVID}}$ subclass (Mazourek et al. 2009, Rairdan et al. 2008). A second, less prevalent subclass (CCR) was defined on RPW8, which is a R protein consisting of a CC only that lacks an EDVID motif (Collier et al. 2009, 
Collier et al. 2011). CNLs containing a CCR also differ from $C_{\text {EDVID }}-N B-L R R s$ in the sequence of their NB-ARC and form an ancient clade within the NLRs (Cannon et al. 2002, Meyers et al. 2003). For two $\mathrm{CC}_{\mathrm{EDVID}}$ domains the crystal structure has been resolved and surprisingly, despite sharing similar sequences, they adopt distinct overall topologies. The CC of the Mla10 immune receptor from barley forms homodimers and the interlocking $\alpha$-helices from both protomers form an elongated 4-helix bundle (Maekawa et al. 2011b). The individual domains consist of $3 \alpha$-helices connected by loops. The $\mathrm{CC}$ of the potato immune receptor $\mathrm{Rx} 1$ does not dimerize, but forms a complex with the WPP (Trp-Pro-Pro) domain of RanGAP2, a co-factor involved in Rx1 functioning (Sacco et al. 2007, Tameling et al. 2007). It adopts a more compact fold consisting of four $\alpha$-helices in a helix bundle. Aromatic residues from the fourth helix constitute the main interface with the three-helix bundle of the WPP domain (Hao et al. 2013). In both CC structures the acidic residues of the EDVID motif are exposed to the solvent and in case of Rx1 they are not part of the interface with RanGAP2. Mutagenesis of the EDVID motif of $\mathrm{Rx} 1$ demonstrated its requirement for the intramolecular interaction with the NB-ARC and LRR, and the functionality of the full-length protein (Rairdan et al. 2008). 


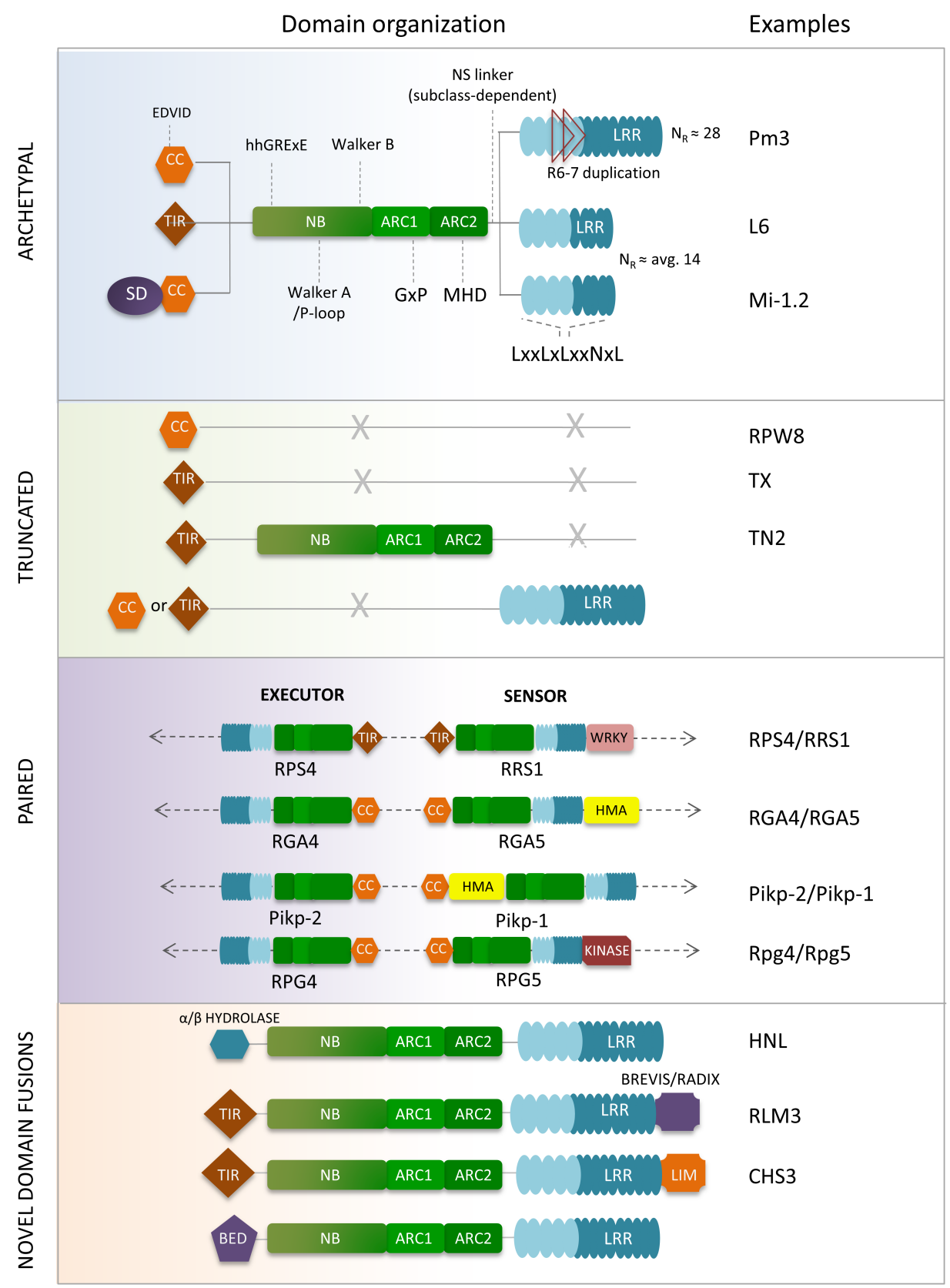

Fig. 1: Schematic representations of the diversity in NLR architectures described in this review. The archetypal plant NLR is composed of three domains: a conserved central NB-ARC domain flanked by an N-terminal response (either a CC or TIR domain) and an irregular hypervariable LRR at the C-terminus (NR refers to repeat numbers), which contain conserved motifs involved in the cooperation and regulation of the molecular switch function of NLRs. The LRR domain may arise from 
(Figure legend continued) duplication and deletion events (indicated by red triangles) as exemplified in Pm3 allelic variants. It also has a dual function that can be correlated with a strict charge separation as observed for Rx1 (indicated by different shades of blue). A subclass of plant NLRs contains an additional Solaneceous Domain (SD) at their N-terminus. Truncated NLRs may lack the canonical domain subunits (indicated with a cross) or alternatively, contain unknown domains. NLR pairs consisting of an executor and sensor protein are encoded by genes, which are located in a head-to-head position in plant genomes to ensure coordinated expression. They often contain distinct integrated decoy domains, which may contribute to the switch function and pathogen detection. Finally, NLRs with novel domain fusions incorporated at their $\mathrm{N}$ or $\mathrm{C}$ termini have also been reported. Here, we show only a few examples which are described in this review. These domain fusions may have potential signaling capacities or roles as integrated decoys, although evidence for such subtypes are still limited to a few studies.

\subsection{TIR domains}

The TIR domain is thought to function mainly as an adaptor domain mediating protein-protein interactions. Often these interactions involve either self-association or homotypic interactions with other TIR domains (Ve et al. 2015, Burch-Smith et al. 2007). The crystal structures of several plant TIR domains are determined in the last few years (reviewed in (Ve et al. 2015)). TIR domains adopt a flavodoxin-like fold in which a five-stranded parallel $\beta$-sheet is in general surrounded by five $\alpha$-helices. The region between the $4^{\text {th }}$ and $5^{\text {th }}$ beta-strand deviates from the structures found in animal and bacterial TIRs, namely; instead of one or two helices the TIR domains contain three well defined helices as shown for the TNLs L6 (Bernoux et al. 2011a), RPS4 (Williams et al. 2014) and AtTIR (Chan et al. 2010). Only the TIR of RRS1 contains a single helix in this region due to a deletion (Williams et al. 2014). Interestingly, some variation has been observed in the interfaces involved in the homotypic interactions of plant TIR domains. The TIR domain of L6 dimerizes via a symmetric interface which is required for signaling (Bernoux et al. 2011a). In crystals of the homoand heterodimers of the RPS4 and RRS1 TIR and the homodimer of AtTIR the interface between the TIRs differs from the one found for the L6 TIR dimer (Chan et al. 2010, Williams et al. 2014). Adjacent serine and histidine residues stabilize the interface between the TIR domains of RPS4 and RRS1, 
and this SH motif appears to be conserved in many plant TIR domains (Ve et al. 2015, Williams et al. 2014).

\subsection{NB-ARC subdomains}

The central NB-ARC domain as found in plant NLRs and several metazoan apoptosis regulators consist of three structural subunits that all contribute to the nucleotide-binding pocket (van der Biezen et al. 1998). The first subunit (NB) is characterized by a central parallel $\beta$-sheet P-loop NTPase structure, which is one of the most common protein domains overall (Leipe et al. 2004, Wendler et al. 2012). The phosphate-binding loop (P-loop, kinase 1a, Walker A) and the kinase 2 (Walker B) motif are located at the tips of the first and third strand, respectively, and are involved in coordinating the $\beta$ - and $\gamma$-phosphates of ATP and a water activating magnesium cation, which in turn is coordinated by the $\beta$ - and $\gamma$-phosphates of ATP (see Wendler et al. 2012 for a detailed review). The ARC domain, which stands for Apaf-1, Resistance proteins, CED4, consists of two subunits. The ARC1 subunit is a helical bundle formed by $4 \alpha$-helices. The conserved GXP (GLPL) motif in the ARC1 and the hhGRExE motif upstream of the P-loop emerged at the same point in evolution and are among the main motifs that define the STAND (Signal Transduction ATPases with Numerous Domains) class of ATPases to which the NB-ARC of plant NLRs belong. Both motifs contribute to the coordination of the adenine group of the nucleotide (Danot et al. 2009, Neuwald et al. 1999). The third subdomain (ARC2) has a winged-helix fold and contains the conserved MHD (Met-His-Asp) motif. The histidine in this motif interacts with the $\beta$-phosphate of the nucleotide (Reubold et al. 2011). In plant NLRs, a flexible linker connects the ARC2 directly to the LRR domain. In metazoan immune receptors in the STAND class a second helical domain (ARC3 or HD2) is positioned in between the ARC2 and the various C-terminal structures that serve as sensor and inhibitor domain (Leipe et al. 2004, Albrecht et al. 2006). In addition to the plant and metazoan intracellular immune receptors and apoptosis regulators, the STAND class of ATPases also includes proteins with signaling functions in bacteria like the maltotriose transcription regulator MalT protein from E. coli (Snider et al. 2008, Marquenet et al. 2010). 


\subsection{LRR domains}

The LRR domains present at the C-terminus of plant NLRs are defined by a repeating motif in the sequence in which hydrophobic residues (often leucine) alternate with hydrophilic residues in a fixed pattern ( $L x x L x L x x N x L)$. No structures of $L R R$ domains from plant NLRs have yet been resolved, but LRR structures from other proteins or from metazoan NLRs are available (Hong et al. 2012, Reubold et al. 2014, Hu et al. 2013). The repeating leucine-rich motifs form a parallel $\beta$-sheet at the concave side of the arc-shaped structure with the leucines forming the hydrophobic core and the residues in between exposed to the surface. The sequences linking the repeats form the convex side and can take a variety of secondary structures. LRRs in plant NLRs are often quite irregular with a large variation in the length of the individual repeats and in the number of repeats (Padmanabhan et al. 2009). The N-terminal repeats and the linker connecting them to the NB-ARC exhibit conserved motifs that are NLR lineage specific and which are required for NLR functioning (Meyers et al. 2003). The LRR structure with a large exposed and adaptable surface on a conserved backbone is especially suited for protein-protein interactions and often plays a dual role: in autoinhibition via intramolecular interactions with the NB-ARC or N-terminal domains and in recognition as a pathogen sensor domain. Hypervariability and patterns of positive selection on surface exposed positions in this domain indicate the ability to rapidly develop new recognition specificities (see Section 4.1). Sequence exchange, visible as mosaic patterns in sequence alignments reshuffles variation and speed up divergence (Smith et al. 2005). In silico modeling of NLR LRR structures has provided insights in the surfaces determining intramolecular interactions and pathogen detection. For example, structure-informed analysis of the LRR of Gpa2 revealed conserved basic residues required for the intramolecular interaction of the LRR with the NB-ARC and opposite charge distributions of the $\mathrm{N}$ and $\mathrm{C}$ terminal regions, consistent with the dual role of LRR domains in controlling NLR activity and pathogen detection (Slootweg et al. 2013). Likewise, the LRRs of Lr10 and Pm3 showed that stacking interactions between aromatic residues in consecutive repeats stabilize the LRR structure and allowed the mapping of positions under positive selection on the surface (Sela et al. 2012, Sela et al. 2014). Finally, plant and animal NLRs depend on a specialized chaperone complex consisting of SGT1, RAR1 and HSP90 to regulate folding, protein stability and localization, and this chaperone system associates with the NLR via the LRR domain (Hoser et al. 2013, Bieri et al. 2004, Leister et al. 2005, Kud et al. 2013, Kadota et al. 2012. 


\section{Molecular Basis Underlying NLR Activation}

In the absence of pathogens, NLRs exist in an autoinhibited conformation in the plant cell, the 'off'-state. This is tightly controlled by inter- and intramolecular interactions to avoid the autoactivation of immune responses which lead to cell death. Recognition of the pathogen coincides with a change in the conformation to an 'on'-state, which can initiate defence responses. In this section, we discuss the molecular mechanisms of NLR activation and how different structural domains contribute to the regulation of NLR activity in plants.

\subsection{Nucleotide binding and ATPase activity involved in NLR activation}

NB-LRR proteins can be seen as a molecular switch with the ability to change between distinct conformational states (Takken et al. 2006). The NB-ARC is the main determinant of the switch as it can adopt strikingly different conformations depending on the bound nucleotide (Fig. 2A). In the 'off'-state, the NB-ARC has a compact structure forming a nucleotide-binding pocket which encloses a bound ADP (Maekawa et al. 2011b, Williams et al. 2011, Tameling et al. 2006). The change to an active conformation requires the partial opening of the structure to allow the exchange of ADP for ATP. In the ATP-bound conformation, as seen in crystal structures of several metazoan STAND ATPases, the ARC2 is rotated away from the NB and ARC1, resulting in a more open structure (Zhou et al. 2015, Yan et al. 2005, Reubold et al. 2011).

ATPase activity has been observed for a few plant NLRs (e.g. I-2, Mi-1.2) and in these proteins mutations in the kinase2 motif that abolish ATPase activity lead to autoactivity (Tameling et al. 2006, Tameling et al. 2002). This indicates that their NB-ARC can bind ATP in the absence of a pathogen, but that they are able to return to an ADP bound conformation via their ATPase activity in the absence of a pathogen effector. The TNL M protein from flax preferentially binds ADP, but a mutation in the MHD motif (D>V) in the ARC2 domain shifts the preference to ATP (Williams et al. 2011). Equivalent mutations in the MHD motif cause constitutive activation for many NLRs. Mutations in for example the P-loop of the NB that strongly reduce the affinity for nucleotides almost invariably result in a loss-of-function of the NLR (Tameling et al. 2002, Williams et al. 2011). Thus, without nucleotide bound in the NB-ARC the protein cannot adopt an active state. Mutagenesis of the downstream NLR NRC1 revealed 11 gain-of-function mutants with mutations in the nucleotide-binding pocket 
and in motifs that contact different parts of the nucleotide (Sueldo et al. 2015). Thus, autoactivity and loss of function can be considered as the extreme ends of an activation spectrum for NLRs as determined by the nucleotide-bound state of the NB-ARC. Characteristics of the NB-ARC determine the affinity of the subdomains for the bound nucleotide and each other, and thereby determine the sensitivity of the switch for activation. The close link between the trigger sensitivity of the NB-ARC switch and recognition specificity is further discussed in Section 4.4 .

\subsection{Cooperation between domains to control NLR activity}

Although the NB-ARC is the core of the molecular switch, the flanking domains also contribute to the functioning of the switch via their interactions with the NB-ARC subdomains and each other. Sequence exchanges between closely related NLR proteins have been instrumental in determining the fine-tuned cooperation between domains involved in autoinhibition and activation. Coevolution between cooperating regions of NLR domains ensures that changes in one region that would, for instance, render the protein overly sensitive for activation are compensated by changes elsewhere in the protein. The high cost of a constitutively active NLR means there is strong selection on the correct regulation of the switch. Combining segments of closely related proteins partially negates this intramolecular coevolution, leading to either inactive or constitutively active chimeric proteins. For example, sequence exchanges between the CNLs Gpa2 and Rx1 from potato revealed that the C-terminal half of the ARC2 domain and the N-terminal repeats of the LRR cooperate with each other (Slootweg et al. 2013, Rairdan et al. 2006) (Fig. 2B; 1). Replacing the LRR of RPS5 by the LRR of RPS2 leads to autoactivity and even the removal of the complete LRR of RPS5 yields an autoactive CC-NB-ARC construct. Stepwise truncation of the LRR showed that the N-terminal 4 repeats are the minimal region that can maintain the inactive state of the CC-NB-ARC of RPS5; deletion of the 4th repeat activates the protein (Qi et al. 2012). Likewise, an intergenic recombination event between the CNL resistance genes Rp1-D and Rp1-dp2 against Puccinia sorghi in corn (Zea mays) resulted in the chimeric protein Rp1-D21, which is constitutively active due to a mismatch between the ARC2 and the LRR (Hu et al. 1996, Smith et al. 2010, Wang et al. 2015a). The responsible region in the ARC2 of Rp1 is almost identical to the region in the Gpa2/Rx1 chimeras. For Rp1, however, it is the $\mathrm{C}$-terminal region of the LRR that has to match the ARC2 for proper autoinhibition instead of the N-terminal repeats like in Gpa2 and Rx1 (Fig. 2B; 1). The residues in the ARC2 from 
Rp1-dp2 that cause autoactivity in the context of the Rp1-D protein carry largely opposite charges in comparison to Rp1-D, changing electrostatic characteristics of the ARC2 surface (Wang et al. 2015a). Similar complementary patches with charged residues are present on the surface of the ARC2 and the conserved lateral side of the LRR of Gpa2 and Rx1. Replacing charged residues in these patches by uncharged/neutral residues of similar dimensions, led to a decrease in binding between the CC-NB-ARC and LRR and a loss-of-function of the coexpressed domains, whereas the full-length proteins harboring these substitutions are not autoactive and still exhibit wild-type levels of elicitor-dependent activation (Slootweg et al. 2013). A mismatch between the ARC2 and LRR can also result in the inability of an NLR to switch upon elicitor binding. In chimeric constructs of the TNLs RPP1-NdA and RPP1-WsB the direct association of a specific elicitor to the RPP1-NdA LRR could not activate the protein unless the LRR was combined with the matching ARC2 domain (Yan et al. 2005). Thus, the ARC2 and LRR form a functional co-evolving complex which keeps the NLR protein in an autoinhibited state and bridge the switch and sensor functionalities of the NLR (Fig. 2B; 1).

\subsection{Switching on and off: a dynamic multilayered regulation mechanism}

Dissociation of the LRR from the CC-NB-ARC is one of the initial steps in elicitor-dependent release of the autoinhibitory state for Rx1 and Gpa2 (Slootweg et al. 2013, Rairdan et al. 2006). Exchange of a single residue in the ARC2 domain reduced elicitor-dependent activation of full-length $R \times 1$ while increasing the affinity of the CC-NB-ARC for the LRR expressed in trans as observed in a co-immunoprecipitation experiment. Interestingly, in silico homology modeling revealed that this substitution was positioned in the interface of the ARC2 with the NB in the ADP-bound conformation. The nucleotide-bound state of the NB-ARC might thus be coupled to its interaction with the LRR (Slootweg et al. 2013) (Fig. 2B; 2). Surprisingly, the mutation of the P-loop, preventing nucleotide-binding, did not block the interaction of the CC-NB-ARC with the LRR or their elicitor-dependent dissociation (Rairdan et al. 2006). Deletion of the ARC2 and LRR domain results in a slight autoactive cell death response for which only the NB is required and which is independent of nucleotide-binding (Bendahmane et al. 2002, Rairdan et al. 2008). 
Important insights in the mechanism linking effector perception (Section 4) and the activation of the NLR have been gained recently through studies of the TNL L proteins from flax, which confer resistance to flax rust (Melampsora lini). Previously, it was unclear if the perception of the effector forced a conformational change in the NLR that leads to nucleotide-exchange and the "on"- state or if a conformational change has to precede recognition. L 5 and L6 have been shown previously to interact with variants of the flax rust effector AvrL567 in a yeast-two hybrid assay (Dodds et al. 2006). Sequence exchanges between $L 5$ and $L 6$ indicate that intramolecular interactions between the NB-ARC and LRR influence the ability of the protein to interact with the elicitor AvrL567 from Melampsora lini and specificity for AvrL567 variants is determined by the LRR (Fig. 2B; 2)(Ravensdale et al. 2012). A difference in activity between $L 6$ and L7, which have identical NB-ARC and LRR sequences and exhibit similar recognition specificities, could be explained by the interplay between a few polymorphic residues in the TIR and NB domain (Bernoux et al. 2016). The combination of residues presents in the TIR and NB of L7 lead to a higher ADP occupancy of the protein, a lower elicitor-dependent activity and a lower affinity for the elicitor in comparison to L6. Altering either position in the TIR domain or the NB domain alleviated the enhanced ADP-binding and led to L6 levels of activity and elicitor-binding. The data support a model in which the ATP-bound conformation of the protein has a higher affinity for the elicitor than the ADP-bound conformation and that cooperation between the TIR and NB plays a regulatory role in the nucleotide-exchange (Fig. 2C). Most importantly, it suggests that the protein is in an equilibrium between an ADP-and an ATP-bound state and that the elicitor binds and stabilizes specifically the ATP-bound form, thereby shifting the equilibrium to the "on"-state (Bernoux et al. 2016). It will be interesting to know if the regulation of the activity of other NLRs follows a similar equilibrium model. In the bacterial transcription factor MalT, the ARC3 mediates a comparable regulatory function in maintaining an equilibrium between conformational states of the protein; in the absence of the elicitor it stabilizes the closed conformation of the NB-ARC, but after an initial low affinity interaction of the elicitor to the sensor it can contribute to a higher affinity binding of the elicitor which competes with its inhibitory interaction with the nucleotide-binding domain (Danot 2015). In plant NLRs, the N-terminal region of the LRR and ARC2 might function analogously and contribute to two subsequent layers of inhibitory regulation. 
An additional layer of regulation of the switch function involves the interaction between the $\mathrm{CC}$ and NB-ARC as shown for Rp1. The Rp1 CC domain can dimerize and signal a cell death response autonomously, which is suppressed when the NB domain is present in the same construct or when the NB-ARC is coexpressed (Wang et al. 2015a). The signaling capacity of the TIR domain of L6 is similarly suppressed when fused to the NB-ARC (Bernoux et al. 2011a) (Fig. 2B; 3). A mutation in the EDVID motif (D82N) of Rp1-D did not reduce its ability to cause an HR as a separate domain, but does lead to a loss in activity of the full-length Rp1-D21 and a strong reduction in the interaction between the CC and the NB-ARC (Wang et al. 2015a). This supports a model in which the NLR protein has to adopt a 'primed' state, analogous to setting a mousetrap, before triggering its activity (Collier et al. 2009). The cooperation between the C-terminus of the LRR and the ARC2 functions as a trigger that can translate a recognition event via the LRR in a conformational change of the NB-ARC or even stabilize an active ATP-bound conformation of the NB-ARC.

Some of these regulatory functions of the core domains are taken over by additional domains integrated into canonical CNLs and TNLs like the SD domain (Fig. 1 \& Fig. 2B; 4). For example, chimeric proteins between the tomato SD-CNL Mi-1.2 and the non-functional homolog Mi-1.1 revealed cooperation between the $\mathrm{N}$-terminal half of the SD domain and the $\mathrm{C}$-terminus of the LRR in autoinhibition (Hwang et al. 2003, Hwang et al. 2000, van Ooijen et al. 2008a). While the N-terminal SD1 domain is required for autoinhibition in cooperation with the LRR, the SD2 and adjacent CC domain act as positive regulators of Mi-1.2 cell death signaling (Lukasik-Shreepaathy et al. 2012). Mi-1.2 has been one of the few plant NLRs for which ATPase activity has been demonstrated (Tameling et al. 2002). Autoactivating mutations in P-loop and kinase 2 motifs that are likely to affect the ATPase activity show a different dependence on the positive regulatory role of the LRR than autoactivating mutations in the MHD motif of the ARC2 that are likely to augment the affinity for ATP over ADP. Both require the presence of the LRR for signaling cell death, but although the MHD mutants exhibit a faster and stronger response in the full-length protein, the co-expression of SD-CC-NB-ARC carrying the MHD mutants and the LRR does not result in cell death (van Ooijen et al. 2008a), whereas the autoactivating mutations in the NB do function in trans, even when the SD and CC are expressed as separate proteins as well (Lukasik-Shreepaathy et al. 2012).

The NLR protein Prf from tomato harbors a large unique domain, N-terminal to its SD and CC domains. This domain binds to the kinase Pto and interacts with the LRR via an intramolecular interaction (Saur 
et al. 2015, Gutierrez et al. 2010). Transphosphorylation of Pto affects part of the binding interface of the N-terminal domain with Pto and thereby, disrupts the interaction of the $\mathrm{N}$-terminal domain with the LRR (Ntoukakis et al. 2013, Du et al. 2012). This in turn is likely to lift intramolecular autoinhibitory interactions with the NB-ARC and allow the switch to adopt its active conformation. The dominant negative effect on elicitor-dependent and independent activation by the coexpression of a separate Prf LRR concurs with an inhibitory role of the LRR (Du et al. 2012). These results indicate that the $\mathrm{N}$ - and C-terminal domains are coupled to the nucleotide-dependent conformational switch of the NB-ARC at different levels with both positive and inhibitory roles (Fig. 2B; 4). 
$2 \mathrm{~A}$

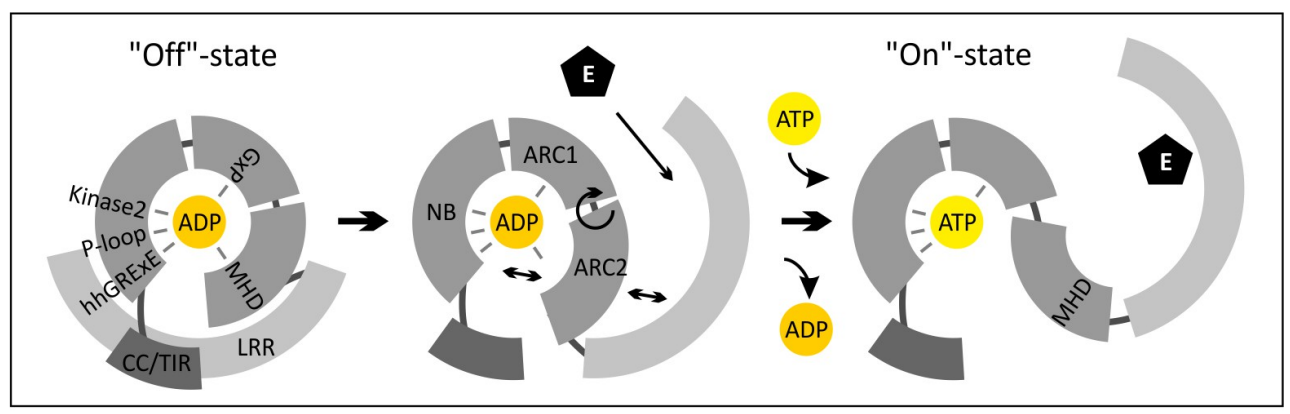

2B

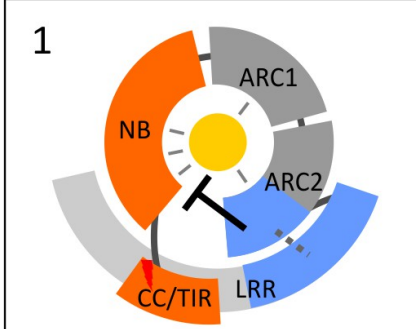

2

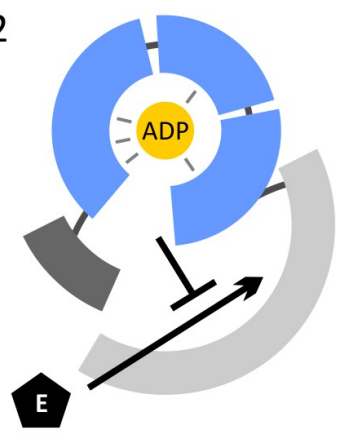

5

4

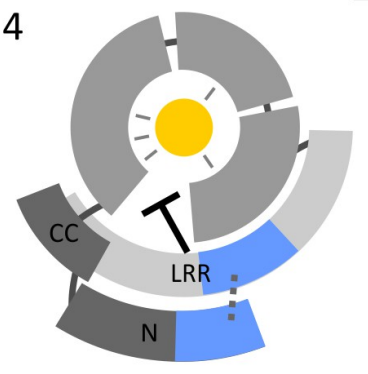

3

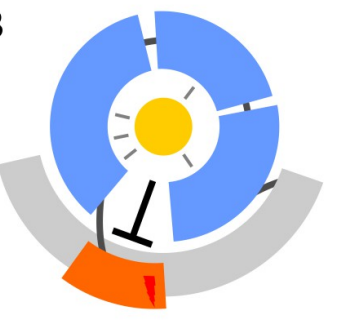

$2 \mathrm{C}$

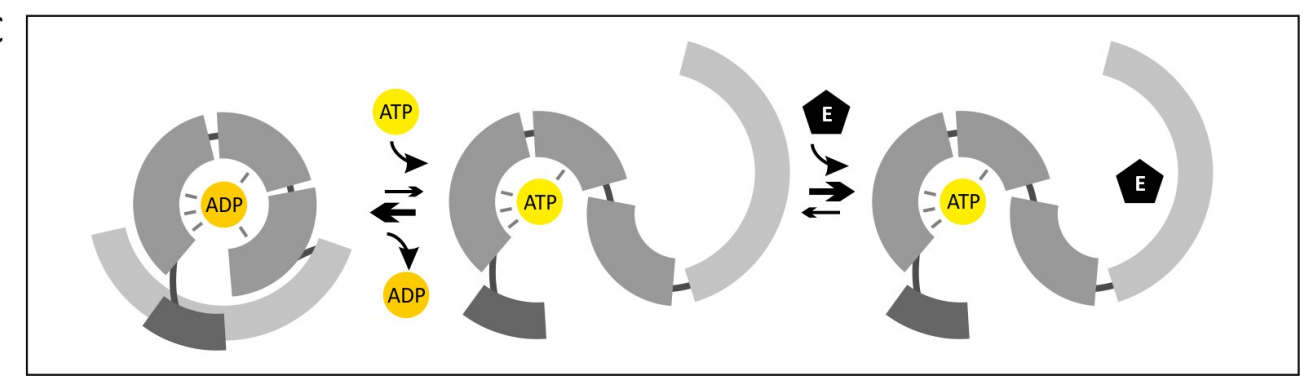

Fig. 2: Schematic representations of the conformational changes and cooperations between domains that regulate the activation of NLRs. $2 A$ ) An NLR is kept in an inactive state by interactions of conserved motifs (including the hhGRExE, P-loop, Kinase2, GXP and MHD motifs) in the NB-ARC subunits (NB, ARC1, ARC2) with ADP and by interactions of the NB-ARC with the N-terminal CC or TIR domains and the C-terminal LRR domain, stabilizing the ADP-binding pocket. Activation entails 
(Figure legend continued) the release of inhibitory interactions (marked by double-headed arrows) which allows the exchange of ADP for ATP. In the active ATP-bound state, the ARC2 is rotated away from the NB-ARC1 subunits. Hydrolysis of the ATP might return the structure to a closed ADP-bound form, allowing the protein to switch between the "off"- and "on"-state. An effector $(E)$ is shown as interacting with the LRR, either directly or indirectly, but other domains have been implicated in the recognition of effectors as well.

2B) Various examples of distinct intramolecular inhibitory interactions that regulate the activity of NLRs. Domains with an inhibitory function are shown in blue. Domains with autonomous signaling activity are colored orange. Dotted lines mark interactions of cooperating domains.

1. Segments of the LRR and the ARC2 (blue) cooperate closely in suppressing inadvertent signaling by the N-terminal domains (orange), linking the sensor and switch functions of the NLR as shown for Rx/Gpa2, Rp1, RPS5, RPP1.// 2. The nucleotide-bound state of the NB-ARC (blue) affects the accessibility of the LRR for elicitor-binding as shown for Rx and L6.

3. In the 'off'-state, the NB-ARC (blue) suppresses signaling via the N-terminal domains (orange) as shown for Rp1, L6, among others.

4. Additional domains $\mathrm{N}$-terminal to the $\mathrm{CC}$ have been shown to cooperate with the LRR in maintaining the 'off'-state. Changes in this interaction can function as a trigger to activate the protein as shown for example for Mi-1.2 and Prf.

5. In oligomeric NLR complexes, the partners regulate each other's activity. In heteromeric pairs, the sensor NLR inhibits the activity of the executor NLR via interactions of the N-terminal domains, but releases this inhibition upon sensing an effector which targets an integrated decoy ( $D$, green) like the WRKY domain in RRS1 or the HMA domain in RGA5.

2C) The alternative equilibrium model as described for the TNL $L$ proteins from flax posits that the NLR is in an equilibrium between the ADP and ATP-bound state even in the absence of a pathogen effector, but with the equilibrium shifted (indicated by the thicker arrow) to the ADP-bound form. The ATP-bound state interacts preferentially with the effector and is stabilized by this interaction, thereby shifting the equilibrium to the active ATP-bound conformation in the presence of a pathogen effector. 


\subsection{Cross talk: cooperation between NLRs in regulating the switch function}

NLRs that, like Prf, are in an oligomeric state prior to activation might regulate each other's activity via intermolecular interactions. For example, coexpression of the functional Rp1-dp2 variant with the autoactive chimeric Rp1-D21 suppresses the constitutive response, suggesting the Rp1 variants regulate each other (Wang et al. 2015a). In heteromeric NLR pairs, the two protomers often have distinct functions in autoinhibition, recognition and signaling. Usually one of the partner NLRs (sensor) is fused to an integrated effector target (Section 4) whereas the second partner (executor) has a canonical CNL or TNL configuration (Fig. 1, Fig 2B; 5). One could speculate that the integration of the decoy hampers the signaling of an NLR which is compensated by delegating this function to a second NLR. The well-studied heteromeric NLR pairs of the rice proteins RGA4 (CNL) with the non-canonical RGA5 (CNL-RATX1) and of Arabidopsis RPS4 (TNL) with RRS1 (TNL-WRKY) share mechanistic characteristics. RGA4 is autoactive in the absence of RGA5, and RGA5 is able to suppress its activity via a direct interaction (Césari et al. 2014b). The degenerate MHD motif in the ARC2 of RGA4 (Thr-Tyr-Gly) might explain its constitutive activity; if the residues in the motif are substituted for the canonical sequence MHD, RGA4 loses its autoactivity. Nucleotide-binding is required for its function, as a mutation in the P-loop also abolishes its activity completely. Surprisingly the RGA5 protein does not require a functional nucleotide-binding pocket for either suppression of RGA4 or activation of RGA4 after effector-recognition (Césari et al. 2014b). The TIR domain of RRS1 also suppresses the activity of the TIR of RPS4 via a direct interaction and in the cell RPS4 and RRS1 are present as a signaling competent complex in which RRS1 acts as sensor and RPS4 as executor. RPS4 requires a functional P-loop for activation, but RRS1 does not (Williams et al. 2014, Sohn et al. 2014). Interestingly, the autoactivity of overexpressed RPS4 is also partially dependent on RRS1, so it might have a positive contribution as well to the activation of RPS4 or contribute to signaling (Heidrich et al. 2013). The coiled-coil domains of RGA4 and RGA5 can homo- and heterodimerize and RGA5 requires the CC to suppress the activity of RGA4 in the absence of an elicitor. Deletion of the C-terminal RATX1 domain does not affect the ability of RGA5 to suppress RGA4, but if the linker between RATX1 and the LRR is also absent the suppression is lost (Césari et al. 2014b). The RGA4/RGA5 and RPS4/RRS1 heterodimers of RGA4 and RGA5 do not seem to dissociate in the presence of the elicitor indicating that conformational changes in the heteromeric complex lead to the activation of RGA4 (Williams et al. 2014, Césari et al. 2014b). 
The genes encoding NLRs that operate in pairs are often located in close proximity in the genome as head-to-head pairs with a shared promoter region ensuring coordinated transcriptional regulation (Fig. 1) (Okuyama et al. 2011, Saucet et al. 2015, Sinapidou et al. 2004). The distribution of sensor, switch and signaling functions between the protomers of heteromeric NLR pairs can only function if a fine-tuned interaction is maintained through coevolution, analogous to the cooperation between the domains within an NLR (Wang et al. 2015a, Slootweg et al. 2013). Close proximity in the genome is advantageous as it lowers the risk of mispairing due to cross-over events. Hybrid necrosis, the occurrence of autoimmune responses due to epistatic interactions between natural genetic variants, can reveal cooperation between proteins in immunity. Many hotspots for hybrid necrosis in plant genomes encode NLRs. The DM1 locus in Arabidopsis encodes a TNL and its uk-3 allele leads to autoimmune response if combined with the $u k-1$ allele of the DM2 locus (Bomblies et al. 2007). An extensive study of hybrid incompatibility among Arabidopsis accessions has shown that the highly variable RPP1-like NLR genes of the DM2 locus can cause incompatibilities with a range of genes encoding proteins with distinct functions (Chae et al. 2014), including other NLRs in the DM1 locus (SSI4) and DM4 locus (RPP8). Incompatible interactions between the DM6 (RPP7 TNL) and DM7 (RPW8) or between alleles of the DM8 (RPP4/5) locus also point to close cooperation between R proteins regulating each other's activity (Chae et al. 2014).

Conversely, resistance signaling by the CNL Pm8 in wheat can be suppressed if the homologous protein Pm3 is expressed in the same plant and even the HR of an autoactive Pm8 with a mutation in the MHD motif is inhibited by coexpression of Pm3 (Hurni et al. 2014, Stirnweis et al. 2014b). Pm8 and Pm3 can form a heteromeric complex which is unable to signal, but some Pm3 variants (Pm3-1B) can interact with Pm8 without inhibitory effects. Multiple domains have a role in the formation of the heteromeric complex, but the LRR domain was identified as the domain conferring the dominant-negative effect (Stirnweis et al. 2014a).

\section{Sensing the Intruder: A Cornucopia of Models for Effector Recognition}

This section will focus on the role of the various NLR subdomains in pathogen recognition. Here, we define recognition as an early event in NLR function (sensing), which includes the interaction dynamics between the immune receptor and its cognate effector during pathogen detection. The 
gene-for-gene hypothesis, which postulates that for every NLR R gene in plants there is a matching pathogen avirulence gene (Flor 1971), supports a Receptor-Ligand model involving a direct physical association between the R-Avr pair. However, there is ample evidence that NLRs encoded by $R$ genes may also recognize effectors encoded by Avr genes indirectly by sensing perturbations of host targets (Bernoux et al. 2011a). The targeted host factors act as co-factor in recognition either as a 'guardee' or 'decoy' (Bernoux et al. 2011b). Guardees are thought to be actual virulence targets guarded by R proteins whereas the Decoy model postulates that a guarded target mimics the real target. In the Bait-and-switch model, no function is ascribed other than as a co-factor (molecular bait) to assist in perceiving the pathogen (Collier et al. 2009). To date, indirect sensing has emerged as the most prevalent model for pathogen detection by NLRs. Intermediary models also exist such as those including functional NLR pairs (Césari et al. 2014a, Khan et al. 2016, Le Roux et al. 2015, Nishimura et al. 2015, Wu et al. 2015b). In this section, we highlight existing experimental and biochemical evidence supporting these models. In the later part of this section, we will address functional aspects related to recognition specificity and how this could be exploited to engineer disease resistance with modified recognition specificities.

\subsection{Direct effector recognition via the LRR subdomains}

In several cases, the N-terminal half of the LRR has been found to act as inhibitor of NLR activity in the absence of a pathogen in cooperation with the ARC2 subdomain (Section 3.2). However, it also has a large surface area that harbors hypervariable surface-exposed residues (e.g. those found in the barley L6), pinpointing to a function in pathogen sensing. Combined, this suggests a dual role of the LRR domain in NLR functioning as inhibitor and sensor. Indeed, modification of these residues in the variable regions of the LRR has been demonstrated to influence the specificity of disease resistance (Sela et al. 2012, Farnham et al. 2006, Bendahmane et al. 2002). However, relatively few effectors have been shown to interact directly with the LRR, suggesting that this is not the sole determinant involved in effector recognition. In addition, a direct interaction of an effector to the LRR does not necessarily lead to the activation of the NLR. The nematode effector SPRYSEC19 binds to the seven C-terminal repeats of the LRR of the SD-CNL Sw5-F from tomato, but this interaction does not lead to the activation of the receptor (Postma et al. 2012). However, introduction of mutations 
that in general lead to autoactivation also did not trigger a cell death response, suggesting that the functionality of this immune receptor is compromised.

Different NLR allelic variants may have evolved different modes of sensing the pathogen, which is possibly reflected in structural changes in the LRR region (Sela et al. 2014). Chimeric exchanges between Arabidopsis RPP1 receptor alleles mapped distinct interacting surfaces of the LRR domain contributing to allele-specific recognition of the ATR1 effector from the oomycete Hyaloperonospora arabidopsidis (Steinbrenner et al. 2015). Interestingly, similar studies involving the flax L5 and L6 allelic variants indicate that both the first four and last seven repeats of the LRR contribute to resistance specificity, which could be linked to a direct interaction between the LRR and the flax rust effectors (Ravensdale et al. 2012). It is conceivable that such interface arrangements may contribute to increased affinity for receptor-ligand interactions, effectively 'clamping' the effector to facilitate the recognition process (Sela et al. 2014). Similar interaction hotspots were also reported for the cereal mildew resistance protein Pm3 that recognize Avr-PM3 whereby elicitor-binding is predicted to occur between repeats 11 and 26 (Sela et al. 2014). Herein, the internal LRR repeat 24 was thought to undergo conformational rearrangements, destabilizing the inhibitory LRR/NB-ARC interaction (Section 3). It is worth noting that Pm3 is characterized by an unusually large LRR domain, arising from duplication events (Fig. 1). It also belongs to a subtype of LRR-containing proteins (including plant NLRs) with 'island domains'; interstrand regions that may form loops extending outside the canonical LRR unit (Matsushima et al. 2012). The recognition functions of these regions have been demonstrated for cell-surface LRR receptors such as BRI1 whereby mutations in the island domains impair the perception of its cognate elicitor brassinolide (BL) (Hothorn et al. 2011). An additional case is the intervening islands of the vertebrate innate immune receptor TLR5, which have been correlated with ligand binding of the bacterial flagellins (Sela et al. 2014, Yoon et al. 2012). However, the functional role or structures of these island domain in pathogen sensing by intracellular NLRs have not been studied extensively.

In some cases, however, non-LRR mediated recognition is reported and could be attributed to proteins with a non-classical NLR domain architecture such as the truncated TIR-X and TIR-NLR proteins (Fig. 1) (Zhao et al. 2015). Like NOD2, the mammalian NLRP1 binds to L-muramyl dipeptide (MDP) and the crystal structure of its LRR has been resolved (Reubold et al. 2014). Interestingly, however, homology modeling reveals that no conserved exposed surface residues could be mapped 
for the NLRP1 LRR at positions involved in MDP binding. The authors concluded that ligand binding is mediated by an alternative domain, which has yet to be determined.

\subsection{Indirect recognition: access to NLRs with decoys or guardees as bait}

In contrast to direct interactions, there is more empirical support for indirect modes of pathogen detection (van der Hoorn et al. 2008). A yeast two-hybrid screen of $A$. thaliana-derived immune proteins (including the N-terminal domains of plant NLRs) and effectors from Pseudomonas syringae and Hyaloperonospora arabidopsidis indicates that merely 2 out of the 30 NLR fragments tested interact directly with a pathogen effector, whereas 24 out of the 52 NLR interactors were known effector targets (Mukhtar et al. 2011). The N-terminal domain of NLRs often plays a prominent role as a platform for interactions with host-proteins that serve as a bait. Indeed, the majority of recognition co-factors reported to date all physically interact to the N-terminal domains of NLRs (Lukasik et al. 2009). As the N-terminal domains of NLRs tend to have a higher degree of conservation between systems, this suggested that the C-terminal part of NLRs may still confer recognition specificity. The co-factor would thus have to be positioned close to the C-terminal half of the LRR, facilitating surveillance for any effector-induced alternations of the bait or facilitating direct contact of the LRR with the effector. This could in turn be mediated by transient low-affinity direct interactions by the LRR. Such a model is supported by the Arabidopsis NLR RPS5, in which the LRR domain specifically monitors the cleavage of the guarded PBS1 kinase by the Pseudomonas effector AvrPphB. PBS1 binds via the CC domain of RPS5, but the LRR is required to sense the small conformational change in the kinase caused by the cleavage of its activation loop (Qi et al. 2012). Moreover, it can distinguish between PBS1 and several homologous kinases via a sequence motif at the opposite site of the protein from the cleaved activation loop (DeYoung et al. 2012, Qi et al. 2014). The conformational change caused by the proteolysis can be mimicked by inserting a 5 aa sequence at the position of the cleavage site (DeYoung et al. 2012). It will be interesting to know how such a subtle change in the conformation of the guardee can relieve the inhibitory effect of the LRR in RPS5 (Section 3). However, the finding that one of the 21 TIR-NB proteins in A. thaliana Col-0, TIR-NBS2 (TN2, At1g17615) directly interacts with the exocyst component EXO70B1 of the host and is activated by loss-of-function mutations in this protein shows that it might monitor the integrity of EXO70B1 as an 
immune receptor even though it lacks a LRR domain (Zhao et al. 2015), pointing out a role for other domains in indirect recognition.

Other examples show that NLRs can accessorize with multiple host factors to maximize their surveillance capacity. A prime example is the CNL ZAR1 that monitors the integrity of Receptor-like cytoplasmic kinase (RLCK) type-proteins such as ZED1, PBL2 and RSK1 (Lewis et al. 2013, Lewis et al. 2010, Wang et al. 2015b). Acetylation of ZED1 by the Pseudomonas effector HopZ1a triggers ZAR1-mediated resistance. In this case, ZED1 most likely functions as a decoy as it has no intrinsic kinase activity nor does it affect virulence of Pseudomonas when knocked out. A recent survey reports that ZAR1 may also guard PBL2 (Wang et al. 2015b). Interestingly, however, this guarding function does not occur directly but instead, through the intermediary RLCK, RKS1. ZAR1 and RKS1 exist in a preformed complex independently of elicitation, wherein the latter acts as an extended scaffold to expand recognition specificity of ZED1 against Xanthomonas. Activation of the recognition complex is achieved by dual-uridylation of PBL2 by the Xanthomonas effector AvrAC.

In addition, effectors may compete for the same interaction sites as a host factor on the receptor and thereby activate it, without modifying the host factor itself. For instance, both the virulent IPI-O4 and avirulent IPI-O1 effectors from Phytophthora infestans physically bind in planta to the CC domain of the CNL RB (Rpi-blb1). The CC domain is required for receptor dimerization and activity of RB (Chen et al. 2012). It was therefore postulated that binding of IPI-O to RB inhibits oligomerization of the immune receptor or access to pro-activating accessory proteins, for instance, by reducing availability of signaling scaffolds. Likewise, a competition for a shared interacting surface may also underlie the indirect effector sensing by the Prf/Pto complex (Ntoukakis et al. 2014, Ntoukakis et al. 2013). The kinase Pto acts as a decoy of the cytoplasmic kinase domains of Pattern Recognition Receptors (PRRs) targeted by the effectors AvrPto and AvrPtoB from Pseudomonas (Ntoukakis et al. 2014). The crystal structures of AvrPto and AvrPtoB in complex with Pto show that both effectors bind directly to the catalytic kinase domain of Pto on overlapping regions, inhibiting the Pto kinase activity (Dong et al. 2009, Xing et al. 2007, Mathieu et al. 2014). Residues within this region of Pto are also required to maintain the SD-NLR Prf in an inhibited configuration prior to elicitation. Such overlap suggests that the effectors and R protein may compete for the same interacting surfaces on Pto, although this has yet to be demonstrated experimentally (Mucyn et al. 2009). Effector binding to a Pto molecule results in transphosphorylation of the inhibited Pto by a second Pto in the oligomeric complex. Such 
transphosphorylation events induce a partial loss of interaction to the unique $\mathrm{N}$-terminal domain of Prf (Section 3.4) (Saur et al. 2015, Ntoukakis et al. 2013). The transphosphorylation of the Pto kinases requires a functional Prf for the induced proximity in the complex (Ntoukakis et al. 2013). This unique oligomerization arrangement is believed to act as a molecular trap, capturing the effector effectively to facilitate recognition.

\subsection{Brothers-in-arms: NLR pairs of sensors and executors}

There is an emerging paradigm that several NLR receptors function in heteromeric pairs to perceive pathogens, as first documented for the Arabidopsis TNLs RPP2A/RPP2B (Sinapidou et al. 2004). In such a complex, a functional bifurcation into sensor and executor roles is maintained as described in Section 3.4 (Fig. 1 \& Fig. 2B; 5). A similar recognition mechanism has been reported for the mouse NLRC4, which recruits both NAIP2 and NAIP5 as sensors to broaden specificity (Kofoed et al. 2011). The observation that a single executor NLR interacts with multiple sensors indicates genetic flexibility of the metazoan NLR system, compared to the 1:1 genetic relationship observed so far for functional plant NLR pairs. However, a similar signaling role of a single NLR in relaying pathogen recognition via multiple NLRs may also occur in plants as suggested by the observation that members of the small ADR1 family of CNLs in Arabidopsis function as helper NLRs downstream of other CNLs and TNLs (Bonardi et al. 2011).

Sensor NLRs in NLR pairs are often characterized by atypical domains that may be integrated before, within or after the archetypal receptor structure (Kroj et al. 2016). Examples include the LIM, WRKY and Heavy Metal Associated (HMA) domains, which have been independently integrated into multiple NLR proteins (Fig. 1). More interestingly, many of these unconventional domain fusions are predicted to be related to putative virulence targets and exhibit coevolutionary dynamics with the effectors (Césari et al. 2014a, Khan et al. 2016, Wang et al. 2015b). Combined, this gave rise to the 'integrated decoy hypothesis' (Césari et al. 2014a). For instance, RRS1-R is a sensor that is constitutively associated with the Arabidopsis TNL RPS4 to mediate recognition of both PopP2 from Ralstonia solanacearum and AvrRps4 from Pseudomonas syringae. RRS1-R has an integrated WRKY motif, enabling it to bind to a W-box cis-element in DNA. PopP2 acetylates RRS1-R in this motif, triggering a loss of DNA binding. This was later demonstrated to be insufficient for defence 
activation, but must occur in conjunction with overall change in conformation of the RPS4/RRS1 complex (Sarris et al. 2015, Le Roux et al. 2015).

Detection of an elicitor by sensor NLRs has in some cases, been reported to occur by direct physical binding. In both RGA4/RGA5 and Pik-2/Pik-1 CNL pairs from rice, a direct interaction between the Magnaporthae effector and the HMA (Heavy-Metal Associated Domain) is required for receptor activation (Cesari et al. 2013, Maqbool et al. 2015). In Pik-1, the HMA domain is integrated between the CC and NB-ARC domain and in RGA5, it is integrated in the C-terminal to the LRR. A crystal structure of the Pikp-1 HMA and Avr-PikD complex shows how characteristics of the integrated HMA domain determine recognition specificity of the Pik-1/Pik-2 pair for the Magnaporthae AvrPik effectors (Maqbool et al. 2015). Although the HMA domain forms a dimer in the complex, the majority of the interface with the effector is mediated by one of the two HMA monomers (Maqbool et al. 2015). The HMA adopts a structure in which a four-stranded antiparallel $\beta$-sheet and two $\alpha$-helices pack in a $\alpha / \beta$ sandwich (HMA-domain fold, Pfam PF00403). The Pikp-HMA lacks the cysteine residues that allow this type of domain to bind metal ions, which suggests they lost their original function and therefore supports a role as an integrated decoy. Variation between Pik alleles can be mapped on the HMA structure and indicates a hotspot for Avr-Pik recognition in the HMA/AvrPik interface. It will be interesting to see how escaping recognition by the integrated HMA in Pik-1 trades off against the ability of AvrPik to interact with and modify its potential intended host targets. Several scenarios could be envisioned for how such interaction might affect the existing interaction within the NLR complex, for example by competing with interactions of NLR domains to the HMA or by bridging and stabilizing interdomain interactions (Maqbool et al. 2015).

\subsection{Recognition specificity: the sum of NLR activation and pathogen detection?}

In addition to the different modes of action in pathogen detection, an intriguing next question is how this relates to the differences observed in recognition specificity of NLRs ranging from highly specific to broad spectrum. There is accumulating evidence that detection of effectors and specificity of the resistance response are the combined efforts of the individual receptor domains. For instance, the presence of residues under positive selection in other domains than the LRR, like for example the ARC2 subdomain (Ravensdale et al. 2012), further support the co-evolution and cooperation between 
distinct NLR subdomains (Section 3). Moreover, exchanging just the TIR domains of L6 and L2 shifted the specificity of the chimeric protein (Luck et al. 2000). However, interaction with the effectors still depends on the LRR region, indicating that recognition relies on both the $\mathrm{N}$ - and $\mathrm{C}$-terminal domains acting in concert. In addition, introducing mutations at a conserved lysine residue in the P-loop of the L6 NB domain has also been shown to interfere with effector binding in yeast (Dodds et al. 2006). The contribution of the NB-ARC domain in pathogen detection is further supported in a stepwise artificial evolution study involving Rx1 (Farnham et al. 2006). Random mutagenesis of the LRR resulted in an Rx1 variant with a broadened recognition specificity beyond its cognate pathogen PVX. One mutant with a substitution in the LRR gained recognition specificity for Poplar Mosaic Virus (PopMV), but did not block viral replication sufficiently to stop spreading of the virus to neighboring cells, resulting in a trailing necrosis. However, subsequent random mutagenesis of the NB-ARC yielded four Rx mutants showing enhanced sensitivity in the activation by PopMV due to substitutions in the nucleotide-binding pocket (Harris et al. 2013). Moreover, a single mutation (I141N) in the $\mathrm{N}$-terminal CC domain, sensitized I-2 to effectors from Fusarium oxysporum f. sp. lycopersici, but also broadened the recognition to include Phytophthora infestans strains (Giannakopoulou et al. 2015). This mutation was initially based on two autoactive mutants of the potato orthologue R3a $\left(\mathrm{R} 3 \mathrm{a}^{\mathrm{I} 148 \mathrm{~F}}\right.$ and $\mathrm{R} 3 \mathrm{a}^{\mathrm{N} 336 \mathrm{Y}}$ ). However, the complementary I-2 mutants were either non-functional or autoactive possibly due to compromised interactions between the CC and LRR domains. The I-2/141N mutant is thought to be a sensitized version with a reduced activation threshold, hypothesized to arise from modified ATP-binding capacities. These data show that different structural domains of NLRs contribute to the activation sensitivity and recognition spectrum. Combined, this corroborates a model in which recognition specificity is the sum of pathogen detection and activation of the NLR immune receptor (Fig. 3), blurring the lines between NLR functions. This knowledge enables the structure-informed modulation of NLR sensitivity and the engineering of synthetic receptor variants with different recognition spectra. 

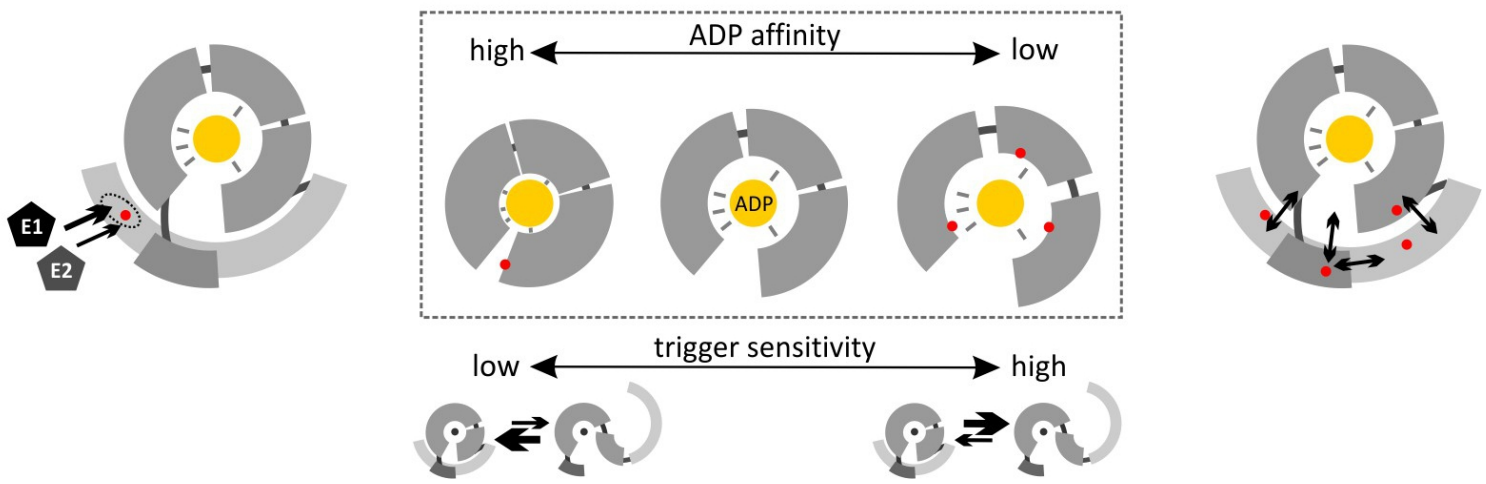

Fig. 3: A schematic representation of a model in which the recognition specificity of NLRs is the sum of pathogen detection and activation of the NLR immune receptor. Left panel: Surface characteristics of the sensor (often the LRR) determine the spectrum of effectors it can perceive (E1, E2, etc). Central panel: changes in the nucleotide-binding pocket (indicated by red dots) that affect the affinity for ADP or (right panel) the interaction between the subunits of the NB-ARC affect the rate at which the nucleotide can be exchanged, thereby shifting the equilibrium between ADP- and ATP-bound state as described in Fig. 2A and Fig 2C. Intramolecular interactions, effector-binding and nucleotide-binding are thus interdependent parameters and together, they determine the trigger sensitivity of the NLR.

\section{Molecular Interactions Involved in NLR-Induced Downstream Signaling}

Following effector-mediated activation, molecular re-arrangements in NLRs (Section 3) are thought to result in the exposure of novel surface regions that facilitate downstream interactions culminating in defence signaling. Various approaches were used to identify NLR domains involved in signaling and their interacting or downstream acting partners, which together constitute a signaling competent immune complex. In this section, we will focus on the series of intra- and intermolecular interactions plant NLRs undergo with their signaling components, which may be compartment specific events. However, as will be discussed, experimental proof of how NLRs and their signaling components interact is lacking. 


\subsection{Rallying recruits: formation of higher-order complexes}

Metazoan STAND proteins have the propensity to multimerize following activation, forming large cellular assemblies known as inflammosomes that are detectable by light microscopy (Lechtenberg et al. 2014). Effector-induced homotypic interactions have also been documented for plant NLRs, for example for the tobacco TNL N, although formation of similar super-structures is not yet reported (Mestre et al. 2006, Qi et al. 2013). Both CNLs and TNLs may engage in homotypic interactions to initiate downstream signaling. Mutants of the barley Mla10 in which the CC domains fail to dimerize are impaired in their cell-death inducing function (Maekawa et al. 2011b). This cannot be generalized, however, as dimerization is not required for the autoactive signaling of the CC domain of Rp1 (Wang et al. 2015a). The tobacco N protein oligomerizes in the presence of the Tobacco Mosaic Virus p50 protein when it targets the host protein NRIP1. Effector-induced oligomerization of $\mathrm{N}$ involves the TIR domain and requires an intact P-loop. Surprisingly, mutagenesis of the RNSB-A motif does not affect dimerization, but strongly affects N-mediated resistance (Mestre et al. 2006). The interaction of the TIR domain with NRIP1 prior to activation and the dependency on the nucleotide-binding state for oligomerization after effector recognition suggest an intricate interplay between the nucleotide-binding state of the NB-ARC and intermolecular interactions in the regulation of TIR-TIR oligomerization (Caplan et al. 2008). Dimerization of the L6 TIR domain is also important for downstream signaling, as homo-dimerization is negatively regulated via intramolecular interactions with the NB-ARC in the absence of an effector. Effector binding to the LRR might compete with the LRR-mediated regulation of the NB-ARC conformation, allowing dimerization to occur (Bernoux et al. 2011a, Bernoux et al. 2016, Wang et al. 2007a).

Receptor oligomerization may introduce new interacting scaffolds for signaling partners. Proof of direct interaction between an NLR receptor and its signaling component is scarce. However, a recent study demonstrates that CC domains with homology to the tomato I-2 could directly interact with the chloroplastic protein Thylakoid Formation 1 (THF1) by Co-IP and bimolecular fluorescence complementation assay (Hamel et al. 2016). Silencing and transient overexpression data pinpoint THF1 as a negative regulator of cell death. Particularly, the interaction of the CC of the NLR N' destabilizes THF1, which correlates with the cell death activity. Other types of signaling components recruited include NDR1 that is often associated as a hub in CNL-mediated defence responses, whereas TNL-mediated defence responses are dependent on the lipase-like proteins EDS1 and 
PAD4 (Aarts et al. 1998, Shapiro et al. 2001, Parker et al. 2000). In addition, overexpression of at least 5 Arabidopsis TN and TX genes led to the induction of cell death and EDS1-dependent resistance pathways (Nandety et al. 2013), demonstrating that even though they form evolutionary distinct clades from the TNLs they share common signaling pathways. Although no evidence for direct interaction with TNLs exists, there is considerable structural data for the functional interactions of EDS1 with SAG101 and PAD4 (Wagner et al. 2013). Crystallization studies of these complexes reveal distinct PAD4 and SAG101 binding interfaces, thus allowing signaling to occur exclusively by one or the other. This is thought to enable fine-tuning of the defence response via competition between the two heterodimer complex types. Detailed analysis predicts further bifurcation, in which the $\mathrm{N}$-terminal domain is involved in complex formation with SAG101/PAD4 whereas an exposed cavity on the opposite site of the heterodimer could act as an additional ligand-docking platform. Interestingly, this region contains several conserved EDS1 residues, possibly enabling the protein to mediate multiple overlapping interactions, for instance with the TIR domains of various TNL receptors.

NLRs may also recruit so-called 'helper NLRs' to form the final active signalosome. Helper NLRs provide a non-canonical route to resistance, for instance by complementing sensor NLRs that do not possess apparent signaling domains. They often transduce signaling of multiple immune receptors and are associated with basal defences, thereby acting as a molecular bridge between the two plant immune layers. Prominent examples include the Arabidopsis ADR1 family, of which three members (ADR1, ADR1-L1 and ADR1-L2) co-operate synergistically in various TNL signal transduction routes (Bonardi et al. 2011). In addition, the requirement of the RPW8-like CNL NRG1 downstream of the TNL N and the loss of NRG1 like proteins from plant lineages that have lost TNLs suggest a role as a specialist signaling protein in TNL mediated resistance (Collier et al. 2011). Similar signaling hubs have also been reported for CNLs, such as the Nicotiana benthamianaNRC3, demonstrated to be required for Pto and to a lesser-extent Cf4- mediated cell death responses (Wu et al. 2015a). The activity of helper NLRs is often demonstrated as being P-loop independent, further discriminating their role from the classical molecular switch receptors. It is imaginable that recruitment of helper NLRs may be convenient for NLR variants that lack the structural capacity to signal (e.g. truncated receptors or those with degenerate P-loop) or to link signaling between non-nuclear receptors. 


\subsection{Integrated signaling components: leads to pathways involved in defence?}

Certain NLR subtypes seem to have signaling components integrated directly in the receptor structure (Fig. 1). Such domains may constitute the primary signaling unit, thereby minimizing the need to engage in heterotypic interactions, enabling a rapid defence output. A recent survey of the moss/bryophyte Physcomitrella patens genome identified a new class of NLR proteins coined the PK-NB-LRRs (PNLs) with an N-terminal protein kinase domain (Xue et al. 2012, Tanigaki et al. 2014). However, further research is required to determine whether kinase activity is retained in these proteins. Interestingly, this subclass, including truncated versions lacking for example the LRR domain, was more prevalent than the CNLs and TNLs. Other examples include NB-LRRs from the liverwort Marchantia polymorpha with domains homologous to $\alpha / \beta$-hydrolase $(\mathrm{HNL})$ at their N-terminus (Xue et al. 2012).

The RLM3 resistance gene against Leptosphaeria maculans and other necrotrophic fungi in Arabidopsis encodes a TNL with three repeats of a BREVIS RADIX domain in its C-terminal domain (Staal et al. 2008). BRX domains have been shown to mediate homo- and heterotypic protein interaction. Further, the protein BRX in Arabidopsis has been shown to translocate to the nucleus and to be involved in the transcriptional auxin response (Briggs et al. 2006, Scacchi et al. 2009). CHS3 (chilling sensitive 3), is a nuclear localized atypical TNL in Arabidopsis in which a LIM domain and LIM associated DUF3633 domain are linked to the C-terminus of the LRR (Yang et al. 2010, Zhao et al. 2014, Bi et al. 2011). Mutations close to the LIM domain cause the protein to become autoactive (Yang et al. 2010, Bi et al. 2011). The LIM domain itself contains two zinc-finger structures in which conserved cysteines and histidines coordinate two $\mathrm{Zn} 2+$ cations and is well characterized in proteins from animals (Perez-Alvarado et al. 1994). It is mainly involved in protein-protein interactions. The combination of a single LIM and the associated domain of unknown function appears unique for plants and is found in DA1, a regulator of seed and organ size (Li et al. 2008, Zhao et al. 2014).

It is unclear how and why these domain fusions were integrated to the archetypal NLR structure. However, based on homologies to existing signaling components, it is tempting to speculate that these fusions existed as independent structures before associating with NLR receptors (Xue et al. 2012). They may then have undergone different structural changes throughout evolution, acquiring 
new functions (e.g. in immune signaling) or alternately, compromising existing ones. In the latter case, these domain fusions may then have acted as integrated decoys, which would enable them to be retained throughout evolution. An example is the barley CNL Rpg5, which confers resistance against Puccinia graminis. Rpg5 has a serine/threonine protein kinase domain integrated C-terminal to the LRR, which is required for its function and has homology to protein kinases involved in resistance like Pto from tomato (Brueggeman et al. 2008). Such homology suggests a function as an integrated bait for the kinase in Rpg5, and has indeed been reported to function as a pair with Rpg4 to confer resistance against wheat stem rust in barley (Wang et al. 2013). Another well-characterized example is RRS1 which has an integrated WRKY motif that is also present in transcription factors implicated in defence (Heidrich et al. 2013). Hence, by directly functioning as transcription factors this could conceivably bypass the need to recruit signaling components. However, it appears that this domain is targeted and acetylated by PopP2 pointing out its alternative role as an integrated decoy (Section 4). A recent genomic screening of more than 19 crop species revealed that integrated domains are prevalent across angiosperms with remarkable diversity in the types of non-classical fusions than previously ever described (265 distinct types in 750 NLR proteins tested). These domain fusions overlap with previously found host targets, which supports their role as baits for effector recognition (Section 4.2) (Sarris et al. 2016). This offers exciting new possibilities for identifying potential host targets or susceptibility factors using integrated domains as baits. However, for a majority of the integrated domains described here, it remains to be proven whether they fulfill a genuine signaling function or mimic existing immune signaling components and act as integrated decoys.

\subsection{Nuclear activity of NLRs: transcription factors and DNA binding}

While certain NLRs have incorporated transcription-factor like motifs in their structure, others are known to directly recruit components involved in transcriptional reprogramming of the cell following activation of immune responses (Bhattacharjee et al. 2013). For instance, the CC domain of barley MLA10 interacts with WRKY1/2 in yeast, antagonizing their transcription-repressor activities, allowing for MYB6 activation. This requires dimerization of MLA10 via its CC domain as substitutions in the hydrophobic interface also disrupt the interaction of the CC with WRKY transcription factors, even when the substitutions are located beyond the minimal region required for the WRKY interaction. This indicates that the dimerization interface might indirectly affect the accessibility of the binding 
site for transcription factors on the CCs (Chang et al. 2013, Maekawa et al. 2011b). Interaction of WRKY2 with the full-length receptor was reported to occur in the nucleus and only upon effector-dependent activation (Chang et al. 2013). Furthermore, the $\mathrm{N}$ protein forms a complex with the SPL6 (squamosa promoter binding protein (SBP)-domain) transcription factor within distinct nuclear bodies (Padmanabhan et al. 2013) and SNC1 interacts with TPR1 (Topless-related 1), which functions as a repressor of negative defence regulators (Xu et al. 2014, Zhu et al. 2010). Hence, direct recruitment of transcription factors by CNLs and TNLs may be a common mechanism to regulate defence gene expression and downstream immune responses.

Consistent with a nuclear function, some NLRs harbor canonical nuclear localization signals (NLS), including N, SNC1 and RPS4. In these cases, forced cytoplasmic targeting of the receptors interfere with defence functions of these receptors (Shen et al. 2007). However, other NLRs with non-canonical localization signals may also show a nucleocytoplasmic distribution. The nucleocytoplasmic distribution of Rx1 is SGT1-dependent, showing compromised nuclear localization in SGT1-silenced plants (Hoser et al. 2014, Slootweg et al. 2010). SGT1 forms a complex with the LRR and nucleocytoplasmic distribution may occur via a 'piggy-back' mechanism as proposed for the translocation of the TNL N protein (Hoser et al. 2013). Nuclear shuttling of $\mathrm{N}$ depends on the phosphorylation status of SGT1. Phosphorylation of SGT1 by activated SIPK kinase (salicylic-acid induced protein kinase) at a highly conserved SGS (SGT1 specific sequence) motif results in increased nuclear accumulation. Likewise, phosphomimic mutants exhibited pronounced nuclear localizations. The nuclear shuffling of phosphorylated SGT1 at specific residues simultaneously shifts the balance of $\mathrm{N}$ into the nucleus, which impaired resistance against TMV. The nuclear pool of $\mathrm{N}$ is also indispensable to trigger p50-mediated $\mathrm{HR}$ as complete exclusion abrogates this response. These data point to a close relationship between structural conformations, complex formation and subcellular localization in NLR functioning.

Recently, structural modeling indicated that both $\mathrm{R} \times 1$ and $\mathrm{I}-2$ share significant structural homologies with the mammalian DNA replication origin-binding Cdc6 and Orc1 proteins, which contact and deform double stranded DNA via the C-terminal ARC domain (Chen et al. 2008, Dueber et al. 2007, Feng et al. 2000). Strong conservation was observed in the NB and ARC domains, raising the possibility that both CNLs could contact DNA in a similar way (Fenyk et al. 2016, Fenyk et al. 2015). Indeed, in vitro DNA binding experiments have shown that $\mathrm{Rx} 1$ and $\mathrm{I}-2$ can bind and distort duplex 
DNA like Cdc6/Orc1, although with slightly different preferences. Such findings indicate novel DNA binding and melting activities that may provide a rationale for the shuttling of some NLRs into the nucleus. This depends on the ATPase activity of the receptors and occurs only upon elicitation by the cognate effectors, thereby linking receptor-DNA interactions with the activated state of the protein. Further, the inactive Rx1 K176R P-loop mutant is also defective in its capacity to distort nucleic acids in vitro, indirectly demonstrating the biological relevance of DNA binding by Rx1. Interestingly, the same I-2 mutant showed comparable DNA-interaction patterns as its wildtype counterpart, according to EMSA experiments suggesting similar affinities to DNA (Fenyk et al. 2015). However, spatial analysis by fluorescence anisotropy revealed overall conformational differences of the binding complex, which depends on the intact P-loop. Combined, these findings indicate specificities in the mode of action of binding that may vary on the nucleic acid type. However, the exact mode of the interaction is still unknown and may involve other specific interacting components like transcription factors as reported for other NLRs.

\section{Conclusions}

In this review, we have highlighted recent advances in the structural, biochemical and molecular aspects involved in NLR functioning in plants. Recent progress in the field of NLR structural biology has expanded our understanding of how these receptors function as pathogen sensors and molecular switches involved in the activation of immune responses. Although plant NLRs share a core set of modular structures involved in the molecular switch function, multifunctionality of the receptor domains has been demonstrated in activation and pathogen recognition, resulting in the discovery of novel activation and regulation mechanisms. Several important new paradigms have emerged. For example, changes in the sensitivity of NLRs can modulate the recognition spectrum of the immune receptor. Furthermore, it appears that bait proteins have an integral role in priming NB-LRR proteins to be functional and retain an inactive conformation until a structural modification triggers release of autoinhibition (Collier et al. 2009). Thus, resistance engineering should take cooperative polymorphisms in the various domains into account when engineering for more sensitized, 'trigger-happy' versions or immune receptors with novel recognition specificities. Moreover, the emergence of atypical NLR architectures, which include other integrated domains, add to the structural and functional diversity of NLRs in plants to counteract a wide range of invading 
pathogens and pests. However, a number of recurring themes surface. A full-length receptor structure at atomic resolution (with and without its matching effector) remain largely enigmatic, but would be crucial to verify existing 3D model predictions. In addition, the identification as well as detailed biochemical and structural analysis of NLR-signaling complexes is hampered, due to the low abundance of associated signaling components and/or dynamic nature of these complexes. Hence, it will be interesting to known how domain cooperation and protein complex formation take place in real time and how conformational changes contribute to the specific subcellular targeting of NLR proteins. Moreover, the role of post-translational modification in NLR functioning is an emerging topic. These studies combined may give insight on existing fundamental questions such as how defence signaling is relayed between different cellular compartments such as the cytoplasm and nucleus. Finally, advanced proteomics, spectroscopic techniques and molecular simulation analysis (with complementary in planta assays) provide a promising complement to existing structural studies to bridge the current gaps in knowledge.

\section{ACKNOWLEDGEMENTS}

This work benefited from interactions through the COST Action FA1208. 


\section{References}

Aarts, N., Metz, M., Holub, E., Staskawicz, B. J., Daniels, M. J., and Parker, J. E. (1998), Different requirements for EDS1 and NDR1 by disease resistance genes define at least two R gene-mediated signaling pathways in Arabidopsis. Proceedings of the National Academy of Sciences of the United States of America. 95(17): p. 10306-10311.

Albrecht, M. and Takken, F. L. W. (2006), Update on the domain architectures of NLRs and R proteins. Biochem Biophys Res Commun. 339(2): p. 459-62.

Asai, S. and Shirasu, K. (2015), Plant cells under siege: plant immune system versus pathogen effectors. Current Opinion in Plant Biology. 28: p. 1-8.

Bendahmane, A., Farnham, G., Moffett, P., and Baulcombe, D. C. (2002), Constitutive gain-of-function mutants in a nucleotide binding site-leucine rich repeat protein encoded at the Rx locus of potato. Plant Journal. 32(2): p. 195-204.

Bernoux, M., Burdett, H., Williams, S. J., Zhang, X., Chen, C., Newell, K., Lawrence, G. J., Kobe, B., Ellis, J. G., Anderson, P. A., and Dodds, P. N. (2016), Comparative Analysis of the Flax Immune Receptors L6 and L7 Suggests an Equilibrium-Based Switch Activation Model. Plant Cell. 28(1): p. 146-59.

Bernoux, M., Ve, T., Williams, S., Warren, C., Hatters, D., Valkov, E., Zhang, X., Ellis, J. G., Kobe, B., and Dodds, P. N. (2011a), Structural and functional analysis of a plant resistance protein TIR domain reveals interfaces for self-association, signaling, and autoregulation. Cell Host Microbe. 9(3): p. 200-11.

Bernoux, M., Ellis, J. G., and Dodds, P. N. (2011b), New insights in plant immunity signaling activation. Current Opinion in Plant Biology. 14(5): p. 512-518.

Bhattacharjee, S., Garner, C. M., and Gassmann, W. (2013), New clues in the nucleus: transcriptional reprogramming in effector-triggered immunity. Front Plant Sci. 4: p. 364.

Bi, D., Johnson, K. C., Zhu, Z., Huang, Y., Chen, F., Zhang, Y., and Li, X. (2011), Mutations in an Atypical TIR-NB-LRR-LIM Resistance Protein Confer Autoimmunity. Front Plant Sci. 2(71).

Bieri, S., Mauch, S., Shen, Q. H., Peart, J., Devoto, A., Casais, C., Ceron, F., Schulze, S., Steinbiss, H. H., Shirasu, K., and Schulze-Lefert, P. (2004), RAR1 positively controls steady state levels of barley MLA resistance proteins and enables sufficient MLA6 accumulation for effective resistance. Plant Cell. 16(12): p. 3480-3495.

Van der Biezen, E. A. and Jones, J. D. G. (1998), The NB-ARC domain: A novel signalling motif shared by plant resistance gene products and regulators of cell death in animals. Current Biology. 8(7): R226-R227.

Bomblies, K., Lempe, J., Epple, P., Warthmann, N., Lanz, C., Dangl, J. L., and Weigel, D. (2007), Autoimmune response as a mechanism for a Dobzhansky-Muller-type incompatibility syndrome in plants. PLoS Biol. 5(9): e236.

Bonardi, V., Tang, S., Stallmann, A., Roberts, M., Cherkis, K., and Dangl, J. L. (2011), Expanded functions for a family of plant intracellular immune receptors beyond specific recognition of pathogen effectors. Proc Natl Acad Sci U S A. 108(39): p. 16463-8.

Briggs, G. C., Mouchel, C. F., and Hardtke, C. S. (2006), Characterization of the Plant-Specific BREVIS RADIX Gene Family Reveals Limited Genetic Redundancy Despite High Sequence Conservation. Plant Physiology. 140(4): p. $1306-1316$.

Brueggeman, R., Druka, A., Nirmala, J., Cavileer, T., Drader, T., Rostoks, N., Mirlohi, A., Bennypaul, H., Gill, U., Kudrna, D., Whitelaw, C., Kilian, A., Han, F., Sun, Y., Gill, K., Steffenson, B., and Kleinhofs, A. (2008), The stem rust resistance gene Rpg5 encodes a protein with nucleotide-binding-site, leucine-rich, and protein kinase domains. Proc Natl Acad Sci U S A. 105(39): p. 14970-5.

Burch-Smith, T. M., Schiff, M., Caplan, J. L., Tsao, J., Czymmek, K., and Dinesh-Kumar, S. P. (2007), A novel role for the TIR domain in association with pathogen-derived elicitors. PLoS Biol. 5(3): e68. 
Cannon, S. B., Zhu, H., Baumgarten, A. M., Spangler, R., May, G., Cook, D. R., and Young, N. D. (2002), Diversity, distribution, and ancient taxonomic relationships within the TIR and non-TIR NBS-LRR resistance gene subfamilies. Journal of molecular evolution. 54(4): p. 548-562. ppublish.

Caplan, J. L., Mamillapalli, P., Burch-Smith, T. M., Czymmek, K., and Dinesh-Kumar, S. P. (2008), Chloroplastic protein NRIP1 mediates innate immune receptor recognition of a viral effector. Cell. 132(3): p. 449-462.

Cesari, S., Thilliez, G., Ribot, C., Chalvon, V., Michel, C., Jauneau, A., Rivas, S., Alaux, L., Kanzaki, H., Okuyama, Y., Morel, J.-B., Fournier, E., Tharreau, D., Terauchi, R., and Kroj, T. (2013), The rice resistance protein pair RGA4/RGA5 recognizes the Magnaporthe oryzae effectors AVR-Pia and AVR1-CO39 by direct binding. The Plant cell. 25(4): p. 1463-1481. ppublish.

Césari, S., Bernoux, M., Moncuquet, P., Kroj, T., and Dodds, P. N. (2014a), A novel conserved mechanism for plant NLR protein pairs: the "integrated decoy" hypothesis. Front Plant Sci. 5(606).

Césari, S., Kanzaki, H., Fujiwara, T., Bernoux, M., Chalvon, V., Kawano, Y., Shimamoto, K., Dodds, P., Terauchi, R., and Kroj, T. (2014b), The NB-LRR proteins RGA4 and RGA5 interact functionally and physically to confer disease resistance. The EMBO Journal. 33(17): p. 1941-1959.

Chae, E., Bomblies, K., Kim, S.-T., Karelina, D., Zaidem, M., Ossowski, S., Martín-Pizarro, C., Laitinen, R. A. E., Rowan, B. A., Tenenboim, H., Lechner, S., Demar, M., Habring-Müller, A., Lanz, C., Rätsch, G., and Weigel, D. (2014), Species-wide Genetic Incompatibility Analysis Identifies Immune Genes as Hot Spots of Deleterious Epistasis. Cell. 159(6): p. 1341-1351.

Chan, S. L., Mukasa, T., Santelli, E., Low, L. Y., and Pascual, J. (2010), The crystal structure of a TIR domain from Arabidopsis thaliana reveals a conserved helical region unique to plants. Protein Sci. 19(1): p. 155-61.

Chang, C., Yu, D., Jiao, J., Jing, S., Schulze-Lefert, P., and Shen, Q.-H. (2013), Barley MLA Immune Receptors Directly Interfere with Antagonistically Acting Transcription Factors to Initiate Disease Resistance Signaling. The Plant Cell Online. 25(3): p. 1158-1173.

Chen, Y., Liu, Z., and Halterman, D. A. (2012), Molecular Determinants of Resistance Activation and Suppression by Phytophthora infestans Effector IPI-O. PLoS Pathog. 8(3): e1002595.

Chen, Z., Speck, C., Wendel, P., Tang, C., Stillman, B., and Li, H. (2008), The architecture of the DNA replication origin recognition complex in Saccharomyces cerevisiae. Proceedings of the National Academy of Sciences. 105(30): p. 10326-10331.

Collier, S. M., Hamel, L. P., and Moffett, P. (2011), Cell Death Mediated by the N-Terminal Domains of a Unique and Highly Conserved Class of NB-LRR Protein. Mol Plant Microbe Interact. 24(8): p. 918-31.

Collier, S. M. and Moffett, P. (2009), NB-LRRs work a "bait and switch" on pathogens. Trends in Plant Science. 14(10): p. 521-529.

Danot, O. (2015), How 'arm-twisting' by the inducer triggers activation of the MalT transcription factor, a typical signal transduction ATPase with numerous domains (STAND). Nucleic Acids Res. 43(6): p. 3089-99.

Danot, O., Marquenet, E., Vidal-Ingigliardi, D., and Richet, E. (2009), Wheel of Life, Wheel of Death: A Mechanistic Insight into Signaling by STAND Proteins. Structure. 17(2): p. 172-82.

DeYoung, B. J., Qi, D., Kim, S. H., Burke, T. P., and Innes, R. W. (2012), Activation of a plant nucleotide binding-leucine rich repeat disease resistance protein by a modified self protein. Cell Microbiol. 14(7): p. 1071-84.

Dodds, P. N., Lawrence, G. J., Catanzariti, A. M., Teh, T., Wang, C. I., Ayliffe, M. A., Kobe, B., and Ellis, J. G. (2006), Direct protein interaction underlies gene-for-gene specificity and coevolution of the flax resistance genes and flax rust avirulence genes. Proc Natl Acad Sci U S A. 103(23): p. 8888-93. 
Dodds, P. N. and Rathjen, J. P. (2010), Plant immunity: towards an integrated view of plant-pathogen interactions. Nat Rev Genet. 11(8): p. 539-48.

Dong, J., Xiao, F., Fan, F., Gu, L., Cang, H., Martin, G. B., and Chai, J. (2009), Crystal structure of the complex between Pseudomonas effector AvrPtoB and the tomato Pto kinase reveals both a shared and a unique interface compared with AvrPto-Pto. Plant Cell. 21(6): p. 1846-59.

Du, X., Miao, M., Ma, X., Liu, Y., Kuhl, J. C., Martin, G. B., and Xiao, F. (2012), Plant programmed cell death caused by an autoactive form of Prf is suppressed by co-expression of the Prf LRR domain. Mol Plant. 5(5): p. 1058-67.

Dueber, E. L. C., Corn, J. E., Bell, S. D., and Berger, J. M. (2007), Replication Origin Recognition and Deformation by a Heterodimeric Archaeal Orc1 Complex. Science. 317(5842): p. 1210-1213.

Farnham, G. and Baulcombe, D. C. (2006), Artificial evolution extends the spectrum of viruses that are targeted by a disease-resistance gene from potato. Proc Natl Acad Sci U S A. 103(49): p. 18828-33.

Feechan, A., Turnbull, D., Stevens, L., Engelhardt, S., Birch, P. J., Hein, I., and Gilroy, E. (2015), The Hypersensitive Response in PAMP- and Effector-Triggered Immune Responses. In: Plant Programmed Cell Death. Ed. by A. N. Gunawardena and P. F. McCabe. Springer International Publishing. Chap. 10, p. 235-268.

Feng, L., Wang, B., Driscoll, B., and Jong, A. (2000), Identification and characterization of Saccharomyces cerevisiae Cdc6 DNA-binding properties. Mol Biol Cell. 11(5): p. 1673-85.

Fenyk, S., Dixon, C. H., Gittens, W. H., Townsend, P. D., Sharples, G. J., Pålsson, L.-O., Takken, F. L. W., and Cann, M. J. (2016), The Tomato Nucleotide-Binding Leucine-Rich Repeat (NLR) Immune Receptor I-2 Couples DNA-Binding to Nucleotide-Binding Domain Nucleotide Exchange. Journal of Biological Chemistry. 291(3): p. 1137-1147.

Fenyk, S., Townsend, P. D., Dixon, C. H., Spies, G. B., de San Eustaquio Campillo, A., Slootweg, E. J., Westerhof, L. B., Gawehns, F. K. K., Knight, M. R., Sharples, G. J., Goverse, A., Pålsson, L.-O., Takken, F. L. W., and Cann, M. J. (2015), The Potato Nucleotide-binding Leucine-rich Repeat (NLR) Immune Receptor Rx1 Is a Pathogen-dependent DNA-deforming Protein. The Journal of biological chemistry. 290(41): p. 24945-24960. ppublish.

Flor, H. H. (1971), Current status of the gene-for-gene concept. Annual Review of Phytopathology. 9: p. 297-323.

Giannakopoulou, A., Steele, J. F. C., Segretin, M. E., Bozkurt, T. O., Zhou, J., Robatzek, S., Banfield, M. J., Pais, M., and Kamoun, S. (2015), Tomato I2 Immune Receptor Can Be Engineered to Confer Partial Resistance to the Oomycete Phytophthora infestans in Addition to the Fungus Fusarium oxysporum. Molecular Plant-Microbe Interactions: MPMI-07-15-0147-R.

Gutierrez, J. R., Balmuth, A. L., Ntoukakis, V., Mucyn, T. S., Gimenez-lbanez, S., Jones, A. M. E., and Rathjen, J. P. (2010), Prf immune complexes of tomato are oligomeric and contain multiple Pto-like kinases that diversify effector recognition. The Plant Journal. 61(3): p. 507-518.

Hamel, L. P., Sekine, K. T., Wallon, T., Sugiwaka, Y., Kobayashi, K., and Moffett, P. (2016), The chloroplastic protein THF1 interacts with the coiled-coil domain of the disease resistance protein N' and regulates light-dependent cell death. Plant Physiol. 7(00234).

Hao, W., Collier, S. M., Moffett, P., and Chai, J. (2013), Structural basis for the interaction between the potato virus $X$ resistance protein (Rx) and its cofactor Ran GTPase-activating protein 2 (RanGAP2). The Journal of biological chemistry. 288(50): p. 35868-35876.

Harris, C. J., Slootweg, E. J., Goverse, A., and Baulcombe, D. C. (2013), Stepwise artificial evolution of a plant disease resistance gene. Proceedings of the National Academy of Sciences. 110(52): p. 21189-21194.

Heidrich, K., Tsuda, K., Blanvillain-Baufume, S., Wirthmueller, L., Bautor, J., and Parker, J. E. (2013), Arabidopsis TNL-WRKY domain receptor RRS1 contributes to temperature-conditioned RPS4 auto-immunity. Front Plant Sci. 4: p. 403. 
Hong, M., Yoon, S. I., and Wilson, I. A. (2012), Structure and functional characterization of the RNA-binding element of the NLRX1 innate immune modulator. Immunity. 36(3): p. 337-47.

Van der Hoorn, R. A. and Kamoun, S. (2008), From Guard to Decoy: a new model for perception of plant pathogen effectors. Plant Cell. 20(8): p. 2009-17.

Hoser, R., Lichocka, M., Zurczak, M., Hennig, J., and Krzymowska, M. (2014), Emerging role of SGT1 as a regulator of NB-LRR-receptor nucleocytoplasmic partitioning. Plant Signal Behav. 9(4).

Hoser, R., Zurczak, M., Lichocka, M., Zuzga, S., Dadlez, M., Samuel, M. A., Ellis, B. E., Stuttmann, J., Parker, J. E., Hennig, J., and Krzymowska, M. (2013), Nucleocytoplasmic partitioning of tobacco N receptor is modulated by SGT1. New Phytol. 200(1): p. 158-71.

Hothorn, M., Belkhadir, Y., Dreux, M., Dabi, T., Noel, J. P., Wilson, I. A., and Chory, J. (2011), Structural basis of steroid hormone perception by the receptor kinase BRI1. Nature. 474(7352): p. 467-71.

Hu, G., Richter, T. E., Hulbert, S. H., and Pryor, T. (1996), Disease Lesion Mimicry Caused by Mutations in the Rust Resistance Gene rp1. Plant Cell. 8(8): p. 1367-1376.

Hu, Z., Yan, C., Liu, P., Huang, Z., Ma, R., Zhang, C., Wang, R., Zhang, Y., Martinon, F., Miao, D., Deng, H., Wang, J., Chang, J., and Chai, J. (2013), Crystal Structure of NLRC4 Reveals Its Autoinhibition Mechanism. Science. 341(6142): p. 172-175.

Hurni, S., Brunner, S., Stirnweis, D., Herren, G., Peditto, D., Mclntosh, R. A., and Keller, B. (2014), The powdery mildew resistance gene Pm8 derived from rye is suppressed by its wheat ortholog Pm3. The Plant Journal. 79(6): p. 904-913.

Hwang, C. F., Bhakta, A. V., Truesdell, G. M., Pudlo, W. M., and Williamson, V. M. (2000), Evidence for a role of the N terminus and leucine-rich repeat region of the Mi gene product in regulation of localized cell death. Plant Cell. 12(8): p. 1319-1329.

Hwang, C. F. and Williamson, V. M. (2003), Leucine-rich repeat-mediated intramolecular interactions in nematode recognition and cell death signaling by the tomato resistance protein Mi. Plant Journal. 34(5): p. 585-593.

Jones, J. D. and Dangl, J. L. (2006), The plant immune system. Nature. 444(7117): p. 323-9.

Kadota, Y. and Shirasu, K. (2012), The HSP90 complex of plants. Biochim Biophys Acta. 3: p. 689-97.

Khan, M., Subramaniam, R., and Desveaux, D. (2016), Of guards, decoys, baits and traps: pathogen perception in plants by type III effector sensors. Current Opinion in Microbiology. 29: p. 49-55.

Kofoed, E. M. and Vance, R. E. (2011), Innate immune recognition of bacterial ligands by NAIPs determines inflammasome specificity. Nature. 477(7366): p. 592-5.

Kohler, A., Rinaldi, C., Duplessis, S., Baucher, M., Geelen, D., Duchaussoy, F., Meyers, B. C., Boerjan, W., and Martin, F. (2008), Genome-wide identification of NBS resistance genes in Populus trichocarpa. Plant molecular biology. 66(6): p. 619-636. ppublish.

Kroj, T., Chanclud, E., Michel-Romiti, C., Grand, X., and Morel, J. B. (2016), Integration of decoy domains derived from protein targets of pathogen effectors into plant immune receptors is widespread. New Phytol. 5(10): p. 13869.

Kud, J., Zhao, Z., Du, X., Liu, Y., Zhao, Y., and Xiao, F. (2013), SGT1 interacts with the Prf resistance protein and is required for Prf accumulation and Prf-mediated defense signaling. Biochem Biophys Res Commun. 431(3): p. 501-5.

Le Roux, C., Huet, G., Jauneau, A., Camborde, L., Trémousaygue, D., Kraut, A., Zhou, B., Levaillant, M., Adachi, H., Yoshioka, H., Raffaele, S., Berthomé, R., Couté, Y., Parker, J. E., and Deslandes, L. (2015), A Receptor Pair with an Integrated Decoy Converts Pathogen Disabling of Transcription Factors to Immunity. Cell. 161(5): p. 1074-1088.

Lechtenberg, B. C., Mace, P. D., and Riedl, S. J. (2014), Structural mechanisms in NLR inflammasome signaling. Curr Opin Struct Biol. 29: p. 17-25. 
Leipe, D. D., Koonin, E. V., and Aravind, L. (2004), STAND, a class of P-loop NTPases including animal and plant regulators of programmed cell death: Multiple, complex domain architectures, unusual phyletic patterns, and evolution by horizontal gene transfer. Journal Of Molecular Biology. 343(1): p. 1-28.

Leister, R. T., Dahlbeck, D., Day, B., Li, Y., Chesnokova, O., and Staskawicz, B. J. (2005), Molecular genetic evidence for the role of SGT1 in the intramolecular complementation of Bs2 protein activity in Nicotiana benthamiana. Plant Cell. 17(4): p. 1268-1278.

Lewis, J. D., Lee, A. H., Hassan, J. A., Wan, J., Hurley, B., Jhingree, J. R., Wang, P. W., Lo, T., Youn, J. Y., Guttman, D. S., and Desveaux, D. (2013), The Arabidopsis ZED1 pseudokinase is required for ZAR1-mediated immunity induced by the Pseudomonas syringae type III effector HopZ1a. Proc Natl Acad Sci U S A. 110(46): p. 18722-7.

Lewis, J. D., Wu, R., Guttman, D. S., and Desveaux, D. (2010), Allele-specific virulence attenuation of the Pseudomonas syringae HopZ1a type III effector via the Arabidopsis ZAR1 resistance protein. PLoS Genet. 6(4): e1000894.

Li, Y., Zheng, L., Corke, F., Smith, C., and Bevan, M. W. (2008), Control of final seed and organ size by the DA1 gene family in Arabidopsis thaliana. Genes Dev. 22(10): p. 1331-6.

Luck, J. E., Lawrence, G. J., Dodds, P. N., Shepherd, K. W., and Ellis, J. G. (2000), Regions outside of the leucine-rich repeats of flax rust resistance proteins play a role in specificity determination. Plant Cell. 12(8): p. 1367-1377.

Lukasik, E. and Takken, F. L. W. (2009), STANDing strong, resistance proteins instigators of plant defence. Current Opinion in Plant Biology. 12(4): p. 427-436.

Lukasik-Shreepaathy, E., Slootweg, E., Richter, H., Goverse, A., Cornelissen, B. J., and Takken, F. L. (2012), Dual regulatory roles of the extended $\mathrm{N}$ terminus for activation of the tomato MI-1.2 resistance protein. Mol Plant Microbe Interact. 25(8): p. 1045-1057.

Maekawa, T., Kufer, T. A., and Schulze-Lefert, P. (2011a), NLR functions in plant and animal immune systems: So far and yet so close. Nature Immunology. 12(9): p. 818-826.

Maekawa, T., Cheng, W., Spiridon, L. N., Töller, A., Lukasik, E., Saijo, Y., Liu, P., Shen, Q.-H., Micluta, M. A., Somssich, I. E., Takken, F. L. W., Petrescu, A.-J., Chai, J., and Schulze-Lefert, P. (2011b), Coiled-Coil Domain-Dependent Homodimerization of Intracellular Barley Immune Receptors Defines a Minimal Functional Module for Triggering Cell Death. Cell Host \& Microbe. 9(3): p. 187-199.

Maqbool, A., Saitoh, H., Franceschetti, M., Stevenson, C., Uemura, A., Kanzaki, H., Kamoun, S., Terauchi, R., and Banfield, M. (2015), Structural basis of pathogen recognition by an integrated HMA domain in a plant NLR immune receptor. eLife. 4.

Marcotte, E. M., Pellegrini, M., Ng, H. L., Rice, D. W., Yeates, T. O., and Eisenberg, D. (1999), Detecting protein function and protein-protein interactions from genome sequences. Science (New York, N.Y.) 285(5428): p. 751-753. ppublish.

Marquenet, E. and Richet, E. (2010), Conserved motifs involved in ATP hydrolysis by MalT, a signal transduction ATPase with numerous domains from Escherichia coli. J Bacteriol. 192(19): p. 5181-91.

Mathieu, J., Schwizer, S., and Martin, G. B. (2014), Pto kinase binds two domains of AvrPtoB and its proximity to the effector E3 ligase determines if it evades degradation and activates plant immunity. PLoS Pathog. 10(7).

Matsushima, N. and Miyashita, H. (2012), Leucine-Rich Repeat (LRR) Domains Containing Intervening Motifs in Plants. Biomolecules. 2(2): p. 288-311.

Mazourek, M., Cirulli, E. T., Collier, S. M., Landry, L. G., Kang, B. C., Quirin, E. A., Bradeen, J. M., Moffett, P., and Jahn, M. M. (2009), The fractionated orthology of Bs2 and Rx/Gpa2 supports shared synteny of disease resistance in the Solanaceae. Genetics. 182(4): p. 1351-64. 
Mestre, P. and Baulcombe, D. C. (2006), Elicitor-mediated oligomerization of the tobacco N disease resistance protein. Plant Cell. 18(2): p. 491-501.

Meyers, B. C., Dickerman, A.W., Michelmore, R.W., Sivaramakrishnan, S., Sobral, B.W., and Young, N.D. (1999), Plant disease resistance genes encode members of an ancient and diverse protein family within the nucleotide-binding superfamily. Plant Journal. 20(3): p. 317-332.

Meyers, B. C., Kozik, A., Griego, A., Kuang, H., and Michelmore, R. W. (2003), Genome-wide analysis of NBS-LRR-encoding genes in Arabidopsis. Plant Cell. 15(4): p. 809-34.

Meyers, B. C., Morgante, M., and Michelmore, R. W. (2002), TIR-X and TIR-NBS proteins: two new families related to disease resistance TIR-NBS-LRR proteins encoded in Arabidopsis and other plant genomes. Plant Journal. 32(1): p. 77-92.

Milligan, S. B., Bodeau, J., Yaghoobi, J., Kaloshian, I., Zabel, P., and Williamson, V. M. (1998), The Root Knot Nematode Resistance Gene Mi from Tomato Is a Member of the Leucine Zipper, Nucleotide Binding, Leucine-Rich Repeat Family of Plant Genes. Plant Cell. 10(8): p. 1307-1320.

Mucyn, T. S., Wu, A. J., Balmuth, A. L., Arasteh, J. M., and Rathjen, J. P. (2009), Regulation of tomato Prf by Pto-like protein kinases. Mol Plant Microbe Interact. 22(4): p. 391-401.

Mukhtar, M. S., Carvunis, A.-R., Dreze, M., Epple, P., Steinbrenner, J., Moore, J., Tasan, M., Galli, M., Hao, T., Nishimura, M. T., Pevzner, S. J., Donovan, S. E., Ghamsari, L., Santhanam, B., Romero, V., Poulin, M. M., Gebreab, F., Gutierrez, B. J., Tam, S., Monachello, D., Boxem, M., Harbort, C. J., McDonald, N., Gai, L., Chen, H., He, Y., Consortium, E. U. E., Vandenhaute, J., Roth, F.P., Hill, D. E., Ecker, J. R., Vidal, M., Beynon, J., Braun, P., and Dangl, J. L. (2011), Independently Evolved Virulence Effectors Converge onto Hubs in a Plant Immune System Network. Science. 333(6042): p. 596-601.

Nandety, R. S., Caplan, J. L., Cavanaugh, K., Perroud, B., Wroblewski, T., Michelmore, R. W., and Meyers, B. C. (2013), The role of TIR-NBS and TIR-X proteins in plant basal defense responses. Plant Physiol. 162(3): p. 1459-72.

Neuwald, A. F., Aravind, L., Spouge, J. L., and Koonin, E. V. (1999), AAA(+): A class of chaperone-like ATPases associated with the assembly, operation, and disassembly of protein complexes. Genome Research. 9(1): p. 27-43.

Nishimura, M. T., Monteiro, F., and Dangl, J. L. (2015), Treasure your exceptions: unusual domains in immune receptors reveal host virulence targets. Cell. 161(5): p. 957-60.

Ntoukakis, V., Balmuth, A. L., Mucyn, T. S., Gutierrez, J. R., Jones, A. M., and Rathjen, J. P. (2013), The tomato Prf complex is a molecular trap for bacterial effectors based on Pto transphosphorylation. PLoS Pathog. 9(1): e1003123.

Ntoukakis, V., Saur, I. M.-L., Conlan, B., and Rathjen, J. P. (2014), The changing of the guard: the Pto/Prf receptor complex of tomato and pathogen recognition. Curr Opin Plant Biol. 20: p. 69-74.

O'Neill, L. A. J. and Bowie, A. G. (2007), The family of five: TIR-domain-containing adaptors in Toll-like receptor signalling. Nat Rev Immunol. 7(5): p. 353-364.

Okuyama, Y., Kanzaki, H., Abe, A., Yoshida, K., Tamiru, M., Saitoh, H., Fujibe, T., Matsumura, H., Shenton, M., Galam, D. C., Undan, J., Ito, A., Sone, T., and Terauchi, R. (2011), A multifaceted genomics approach allows the isolation of the rice Pia-blast resistance gene consisting of two adjacent NBS-LRR protein genes. Plant J. 66(3): p. 467-79.

Van Ooijen, G., Mayr, G., Albrecht, M., Cornelissen, B. J. C., and Takken, F. L. W. (2008a), Transcomplementation, but not Physical Association of the CC-NB-ARC and LRR Domains of Tomato R Protein Mi-1.2 is Altered by Mutations in the ARC2 Subdomain. Mol Plant. 1(3): p. 401-410. 
Van Ooijen, G., Mayr, G., Kasiem, M. M. A., Albrecht, M., Cornelissen, B. J. C., and Takken, F. L. W. (2008b), Structure-function analysis of the NB-ARC domain of plant disease resistance proteins. J. Exp. Bot. 59(6): p. 1383-1397.

Padmanabhan, M., Cournoyer, P., and Dinesh-Kumar, S. P. (2009), The leucine-rich repeat domain in plant innate immunity: a wealth of possibilities. Cell Microbiol. 11(2): p. 191-8.

Padmanabhan, M. S., Ma, S., Burch-Smith, T. M., Czymmek, K., Huijser, P., and Dinesh-Kumar, S. P. (2013), Novel positive regulatory role for the SPL6 transcription factor in the N TIR-NB-LRR receptor-mediated plant innate immunity. PLoS pathogens. 9(3): e1003235. ppublish.

Parker, J. E., Feys, B. J., van der Biezen, E. A., Noel, L., Aarts, N., Austin, M. J., Botella, M., Frost, L. N., Daniels, M. J., and Jones, J. D. G. (2000), Unravelling R gene-mediated disease resistance pathways in Arabidopsis. Molecular Plant Pathology. 1(1): p. 17-24.

Perez-Alvarado, G. C., Miles, C., Michelsen, J. W., Louis, H. A., Winge, D. R., Beckerle, M. C., and Summers, M. F. (1994), Structure of the carboxy-terminal LIM domain from the cysteine rich protein CRP. Nat Struct Biol. 1(6): p. 388-98.

Postma, W. J., Slootweg, E. J., Rehman, S., Finkers-Tomczak, A., Tytgat, T. O., van Gelderen, K., Lozano-Torres, J. L., Roosien, J., Pomp, R., van Schaik, C., Bakker, J., Goverse, A., and Smant, G. (2012), The Effector SPRYSEC-19 of Globodera rostochiensis Suppresses CC-NB-LRR-Mediated Disease Resistance in Plants. Plant Physiology. 160(2): p. 944-954.

Qi, D., DeYoung, B. J., and Innes, R. W. (2012), Structure-Function Analysis of the Coiled-Coil and Leucine-Rich Repeat Domains of the RPS5 Disease Resistance Protein. Plant Physiol. 158(4): p. 1819-32.

Qi, D., Dubiella, U., Kim, S. H., Sloss, D. I., Dowen, R. H., Dixon, J. E., and Innes, R. W. (2014), Recognition of the protein kinase AVRPPHB SUSCEPTIBLE1 by the disease resistance protein RESISTANCE TO PSEUDOMONAS SYRINGAE5 is dependent on s-acylation and an exposed loop in AVRPPHB SUSCEPTIBLE1. Plant Physiol. 164(1): p. 340-51.

Qi, D. and Innes, R. W. (2013), Recent advances in plant NLR structure, function, localization and signaling. Frontiers in Immunology. 4.

Rairdan, G. J., Collier, S. M., Sacco, M. A., Baldwin, T. T., Boettrich, T., and Moffett, P. (2008), The Coiled-Coil and Nucleotide Binding Domains of the Potato Rx Disease Resistance Protein Function in Pathogen Recognition and Signaling. Plant Cell. 20(3): p. 739-751.

Rairdan, G. J. and Moffett, P. (2006), Distinct Domains in the ARC Region of the Potato Resistance Protein Rx Mediate LRR Binding and Inhibition of Activation. Plant Cell. 18(8): p. 2082-2093.

Ravensdale, M., Bernoux, M., Ve, T., Kobe, B., Thrall, P. H., Ellis, J. G., and Dodds, P. N. (2012), Intramolecular Interaction Influences Binding of the Flax L5 and L6 Resistance Proteins to their AvrL567 Ligands. PLoS Pathog. 8(11): e1003004

Reubold, T. F., Hahne, G., Wohlgemuth, S., and Eschenburg, S. (2014), Crystal structure of the leucine-rich repeat domain of the NOD-like receptor NLRP1: implications for binding of muramyl dipeptide. FEBS Lett. 588(18): p. 3327-32.

Reubold, T. F., Wohlgemuth, S., and Eschenburg, S. (2011), Crystal structure of full-length Apaf-1: how the death signal is relayed in the mitochondrial pathway of apoptosis. Structure. 19(8): p. 1074-83.

Sacco, M. A., Mansoor, S., and Moffett, P. (2007), A RanGAP protein physically interacts with the NB-LRR protein Rx, and is required for Rx-mediated viral resistance. Plant J. 52(1): p. 82-93. 
Salmeron, J.M., Oldroyd, G. E., Rommens, C.M., Scofield, S. R., Kim, H.S., Lavelle, D. T., Dahlbeck, D., and Staskawicz, B. J. (1996), Tomato Prf is a member of the leucine-rich repeat class of plant disease resistance genes and lies embedded within the Pto kinase gene cluster. Cell. 86(1): p. 123-33.

Sarris, P. F., Cevik, V., Dagdas, G., Jones, J. D., and Krasileva, K. V. (2016), Comparative analysis of plant immune receptor architectures uncovers host proteins likely targeted by pathogens. BMC Biol. 14(1): p. 016-0228.

Sarris, P. F., Duxbury, Z., Huh, S. U., Ma, Y., Segonzac, C., Sklenar, J., Derbyshire, P., Cevik, V., Rallapalli, G., Saucet, S. B., Wirthmueller, L., Menke, F. L., Sohn, K. H., and Jones, J. D. (2015), A Plant Immune Receptor Detects Pathogen Effectors that Target WRKY Transcription Factors. Cell. 161(5): p. 1089-100.

Saucet, S. B., Ma, Y., Sarris, P. F., Furzer, O. J., Sohn, K. H., and Jones, J. D. G. (2015), Two linked pairs of Arabidopsis TNL resistance genes independently confer recognition of bacterial effector AvrRps4. Nat Commun. 6.

Saur, I. M.-L., Conlan, B. F., and Rathjen, J. P. (2015), The N-Terminal Domain of the Tomato Immune Protein Prf Contains Multiple Homotypic and Pto Kinase Interaction Sites. Journal of Biological Chemistry. 290(18): p. 11258-11267.

Scacchi, E., Osmont, K. S., Beuchat, J., Salinas, P., Navarrete-Gómez, M., Trigueros, M., Ferrándiz, C., and Hardtke, C. S. (2009), Dynamic, auxin-responsive plasma membrane-to-nucleus movement of Arabidopsis BRX. Development. 136(12): p. 2059-2067.

Sela, H., Spiridon, L. N., Ashkenazi, H., Bhullar, N. K., Brunner, S., Petrescu, A. J., Fahima, T., Keller, B., and Jordan, T. (2014), Three-dimensional modeling and diversity analysis reveals distinct AVR recognition sites and evolutionary pathways in wild and domesticated wheat Pm3 R genes. Mol Plant Microbe Interact. 27(8): p. 835-45.

Sela, H., Spiridon, L. N., Petrescu, A. J., Akerman, M., Mandel-Gutfreund, Y., Nevo, E., Loutre, C., Keller, B., Schulman, A. H., and Fahima, T. (2012), Ancient diversity of splicing motifs and protein surfaces in the wild emmer wheat (Triticum dicoccoides) LR10 coiled coil (CC) and leucine-rich repeat (LRR) domains. Mol Plant Pathol. 13(3): p. $276-87$.

Shapiro, A. D. and Zhang, C. (2001), The role of NDR1 in avirulence gene-directed signaling and control of programmed cell death in arabidopsis. Plant Physiology. 127(3): p. 1089-1101.

Shen, Q.-H., Saijo, Y., Mauch, S., Biskup, C., Bieri, S., Keller, B., Seki, H., Ülker, B., Somssich, I. E., and Schulze-Lefert, P. (2007), Nuclear Activity of MLA Immune Receptors Links Isolate-Specific and Basal Disease-Resistance Responses. Science. 315(5815): p. 1098.

Sinapidou, E., Williams, K., Nott, L., Bahkt, S., Tor, M., Crute, I., Bittner-Eddy, P., and Beynon, J. (2004), Two TIR : NB : LRR genes are required to specify resistance to Peronospora parasitica isolate Cala2 in Arabidopsis. Plant Journal. 38(6): p. 898-909.

Slootweg, E., Roosien, J., Spiridon, L. N., Petrescu, A. J., Tameling, W., Joosten, M., Pomp, R., van Schaik, C., Dees, R., Borst, J. W., Smant, G., Schots, A., Bakker, J., and Goverse, A. (2010), Nucleocytoplasmic distribution is required for activation of resistance by the potato NB-LRR receptor $\mathrm{Rx} 1$ and is balanced by its functional domains. Plant Cell. 22(12): p. 4195-215.

Slootweg, E. J., Spiridon, L. N., Roosien, J., Butterbach, P., Pomp, R., Westerhof, L., Wilbers, R., Bakker, E., Bakker, J., Petrescu, A.-J., Smant, G., and Goverse, A. (2013), Structural Determinants at the Interface of the ARC2 and Leucine-Rich Repeat Domains Control the Activation of the Plant Immune Receptors Rx1 and Gpa2. Plant Physiology. 162(3): p. 1510-1528.

Smith, S. M. and Hulbert, S. H. (2005), Recombination events generating a novel Rp1 race specificity. Mol Plant Microbe Interact. 18(3): p. 220-8.

Smith, S. M., Steinau, M., Trick, H. N., and Hulbert, S. H. (2010), Recombinant Rp1 genes confer necrotic or nonspecific resistance phenotypes. Molecular genetics and genomics : MGG. 283(6): p. 591-602. ppublish. 
Snider, J. and Houry, W. A. (2008), AAA+ proteins: diversity in function, similarity in structure. Biochem Soc Trans. 36(Pt 1): p. $72-7$.

Sohn, K. H., Segonzac, C., Rallapalli, G., Sarris, P. F., Woo, J. Y., Williams, S. J., Newman, T. E., Paek, K. H., Kobe, B., and Jones, J. D. (2014), The Nuclear Immune Receptor RPS4 Is Required for RRS1SLH1-Dependent Constitutive Defense Activation in Arabidopsis thaliana. PLoS Genet. 10(10): e1004655.

Staal, J., Kaliff, M., Dewaele, E., Persson, M., and Dixelius, C. (2008), RLM3, a TIR domain encoding gene involved in broad-range immunity of Arabidopsis to necrotrophic fungal pathogens. Plant J. 2: p. 2.

Steinbrenner, A. D., Goritschnig, S., and Staskawicz, B. J. (2015), Recognition and Activation Domains Contribute to Allele-Specific Responses of an Arabidopsis NLR Receptor to an Oomycete Effector Protein. PLoS Pathog. 11(2): e1004665.

Stirnweis, D., Milani, S. D., Brunner, S., Herren, G., Buchmann, G., Peditto, D., Jordan, T., and Keller, B. (2014a), Suppression among alleles encoding nucleotide-binding-leucine-rich repeat resistance proteins interferes with resistance in F1 hybrid and allele-pyramided wheat plants. Plant J. 79(6): p. 893-903.

Stirnweis, D., Milani, S. D., Jordan, T., Keller, B., and Brunner, S. (2014b), Substitutions of Two Amino Acids in the Nucleotide-Binding Site Domain of a Resistance Protein Enhance the Hypersensitive Response and Enlarge the PM3F Resistance Spectrum in Wheat. Mol Plant Microbe Interact. 27(3): p. 265-76.

Sueldo, D. J., Shimels, M., Spiridon, L. N., Caldararu, O., Petrescu, A. J., Joosten, M. H., and Tameling, W. I. (2015), Random mutagenesis of the nucleotide-binding domain of NRC1 (NB-LRR Required for Hypersensitive Response-Associated Cell Death-1), a downstream signalling nucleotide-binding, leucine-rich repeat (NB-LRR) protein, identifies gain-of-function mutations in the nucleotide-binding pocket. New Phytol. 208(1): p. 210-23.

Takken, F. L. and Goverse, A. (2012), How to build a pathogen detector: structural basis of NB-LRR function. Curr Opin Plant Biol.

Takken, F. L. W., Albrecht, M., and Tameling, W. I. L. (2006), Resistance proteins: molecular switches of plant defence. Curr Opin Plant Biol. 9(4): p. 383-90.

Tameling, W. I. L. and Baulcombe, D. C. (2007), Physical association of the NB-LRR resistance protein Rx with a Ran GTPase-activating protein is required for extreme resistance to Potato virus X. Plant Cell. 19(5): p. 1682-94.

Tameling, W. I. L., Elzinga, S. D. J., Darmin, P. S., Vossen, J. H., Takken, F. L. W., Haring, M. A., and Cornelissen, B. J. C. (2002), The tomato R gene products I-2 and Mi-1 are functional ATP binding proteins with ATPase activity. Plant Cell. 14(11): p. 2929-2939.

Tameling, W. I. L., Vossen, J. H., Albrecht, M., Lengauer, T., Berden, J. A., Haring, M. A., Cornelissen, B. J. C., and Takken, F. L. W. (2006), Mutations in the NB-ARC Domain of I-2 That Impair ATP Hydrolysis Cause Autoactivation. Plant Physiol. 140(4): p. 1233-1245.

Tanigaki, Y., Ito, K., Obuchi, Y., Kosaka, A., Yamato, K. T., Okanami, M., Lehtonen, M. T., Valkonen, J. P., and Akita, M. (2014), Physcomitrella patens has kinase-LRR R gene homologs and interacting proteins. PLoS ONE. 9(4).

Ve, T., Williams, S. J., and Kobe, B. (2015), Structure and function of Toll/interleukin-1 receptor/resistance protein (TIR) domains. Apoptosis. 20(2): p. 250-61.

Wagner, S., Stuttmann, J., Rietz, S., Guerois, R., Brunstein, E., Bautor, J., Niefind, K., and Parker, J. E. (2013), Structural Basis for Signaling by Exclusive EDS1 Heteromeric Complexes with SAG101 or PAD4 in Plant Innate Immunity. Cell Host \& Microbe. 14(6): p. 619-630.

Wang, C.-I. A., Gun $\square$ ar, G., Forwood, J. K., Teh, T., Catanzariti, A.-M., Lawrence, G. J., Loughlin, F. E., Mackay, J. P., Schirra, H. J., Anderson, P. A., Ellis, J. G., Dodds, P. N., and Kobe, B. (2007a), Crystal Structures of Flax Rust 
Avirulence Proteins AvrL567-A and -D Reveal Details of the Structural Basis for Flax Disease Resistance Specificity. The Plant Cell. 19(9): p. 2898-2912.

Wang, G. F., Ji, J., El-Kasmi, F., Dangl, J. L., Johal, G., and Balint-Kurti, P. J. (2015a), Molecular and functional analyses of a maize autoactive NB-LRR protein identify precise structural requirements for activity. PLoS Pathog. 11(2).

Wang, G., Roux, B., Feng, F., Guy, E., Li, L., Li, N., Zhang, X., Lautier, M., Jardinaud, M.-F., Chabannes, M., Arlat, M., Chen, S., He, C., Noël, L. D., and Zhou, J.-M. (2015b), The Decoy Substrate of a Pathogen Effector and a Pseudokinase Specify Pathogen-Induced Modified-Self Recognition and Immunity in Plants. Cell Host \& Microbe. 18(3): p. 285-295

Wang, W., Devoto, A., Turner, J. G., and Xiao, S. (2007b), Expression of the Membrane-Associated Resistance Protein RPW8 Enhances Basal Defense Against Biotrophic Pathogens. Molecular Plant-Microbe Interactions. 20(8): p. 966-976.

Wang, X., Richards, J., Gross, T., Druka, A., Kleinhofs, A., Steffenson, B., Acevedo, M., and Brueggeman, R. (2013), The rpg4-mediated resistance to wheat stem rust (Puccinia graminis) in barley (Hordeum vulgare) requires Rpg5, a second NBS-LRR gene, and an actin depolymerization factor. Mol Plant Microbe Interact. 26(4): p. 407-18.

Wendler, P., Ciniawsky, S., Kock, M., and Kube, S. (2012), Structure and function of the AAA+ nucleotide binding pocket. Biochimica et Biophysica Acta (BBA) - Molecular Cell Research. 1823(1): p. 2-14.

Williams, S. J., Sornaraj, P., deCourcy-Ireland, E., Menz, R. I., Kobe, B., Ellis, J. G., Dodds, P. N., and Anderson, P. A. (2011), An autoactive mutant of the $M$ flax rust resistance protein has a preference for binding ATP, whereas wild-type M protein binds ADP. Mol Plant Microbe Interact. 24(8): p. 897-906.

Williams, S. J., Sohn, K. H., Wan, L., Bernoux, M., Sarris, P. F., Segonzac, C., Ve, T., Ma, Y., Saucet, S. B., Ericsson, D. J., Casey, L. W., Lonhienne, T., Winzor, D. J., Zhang, X., Coerdt, A., Parker, J. E., Dodds, P. N., Kobe, B., and Jones, J. D. G. (2014), Structural Basis for Assembly and Function of a Heterodimeric Plant Immune Receptor. Science. 344(6181): p. 299-303.

Wu, C.-H., Belhaj, K., Bozkurt, T. O., Birk, M. S., and Kamoun, S. (2015a), Helper NLR proteins NRC2a/b and NRC3 but not NRC1 are required for Pto-mediated cell death and resistance in Nicotiana benthamiana. New Phytologist. 209(4): n/a-n/a.

Wu, C.-H., Krasileva, K. V., Banfield, M. J., Terauchi, R., and Kamoun, S. (2015b), The "sensor domains" of plant NLR proteins: more than decoys? Frontiers in Plant Science. 6: p. 134.

Xiao, S., Ellwood, S., Calis, O., Patrick, E., Li, T., Coleman, M., and Turner, J. G. (2001), Broad-spectrum mildew resistance in Arabidopsis thaliana mediated by RPW8. Science. 291(5501): p. 118-20.

Xing, W., Zou, Y., Liu, Q., Liu, J., Luo, X., Huang, Q., Chen, S., Zhu, L., Bi, R., Hao, Q., Wu, J. W., Zhou, J. M., and Chai, J. (2007), The structural basis for activation of plant immunity by bacterial effector protein AvrPto. Nature. 449(7159): p. 243-7.

Xu, F., Kapos, P., Cheng, Y. T., Li, M., Zhang, Y., and Li, X. (2014), NLR-associating transcription factor bHLH84 and its paralogs function redundantly in plant immunity. PLoS Pathog. 10(8).

Xue, J.-Y., Wang, Y., Wu, P., Wang, Q., Yang, L.-T., Pan, X.-H., Wang, B., and Chen, J.-Q. (2012), A Primary Survey on Bryophyte Species Reveals Two Novel Classes of Nucleotide-Binding Site (NBS) Genes. PLoS ONE. 7(5): e36700.

Yan, N., Chai, J. J., Lee, E. S., Gu, L. C., Liu, Q., He, J. Q., Wu, J. W., Kokel, D., Li, H. L., Hao, Q., Xue, D., and Shi, Y. G. (2005), Structure of the CED-4-CED-9 complex provides insights into programmed cell death in Caenorhabditis elegans. Nature. 437(7060): p. 831-837. 
Yang, H., Shi, Y., Liu, J., Guo, L., Zhang, X., and Yang, S. (2010), A mutant CHS3 protein with TIR-NB-LRR-LIM domains modulates growth, cell death and freezing tolerance in a temperature-dependent manner in Arabidopsis. Plant J. 63(2): p. 283-96.

Yoon, S. I., Kurnasov, O., Natarajan, V., Hong, M., Gudkov, A. V., Osterman, A. L., and Wilson, I. A. (2012), Structural basis of TLR5-flagellin recognition and signaling. Science. 335(6070): p. 859-64.

Yue, J. X., Meyers, B. C., Chen, J. Q., Tian, D., and Yang, S. (2012), Tracing the origin and evolutionary history of plant nucleotide-binding site-leucine-rich repeat (NBS-LRR) genes. New Phytol. 193(4): p. 1049-63.

Zhao, M., He, L., Gu, Y., Wang, Y., Chen, Q., and He, C. (2014), Genome-Wide Analyses of a Plant-Specific LIM-Domain Gene Family Implicate Its Evolutionary Role in Plant Diversification. Genome Biology and Evolution. 6(4): p. 1000-1012.

Zhao, T., Rui, L., Li, J., Nishimura, M. T., Vogel, J. P., Liu, N., Liu, S., Zhao, Y., Dangl, J. L., and Tang, D. (2015), A truncated NLR protein, TIR-NBS2, is required for activated defense responses in the exo70B1 mutant. PLoS Genet. 11(1).

Zhou, M., Li, Y., Hu, Q., Bai, X. C., Huang, W., Yan, C., Scheres, S. H., and Shi, Y. (2015), Atomic structure of the apoptosome: mechanism of cytochrome c- and dATP-mediated activation of Apaf-1. Genes Dev. 29(22): p. $2349-61$.

Zhu, Z., Xu, F., Zhang, Y., Cheng, Y. T., Wiermer, M., Li, X., and Zhang, Y. (2010), Arabidopsis resistance protein SNC1 activates immune responses through association with a transcriptional corepressor. Proceedings of the National Academy of Sciences. 107(31): p. 13960-13965. 


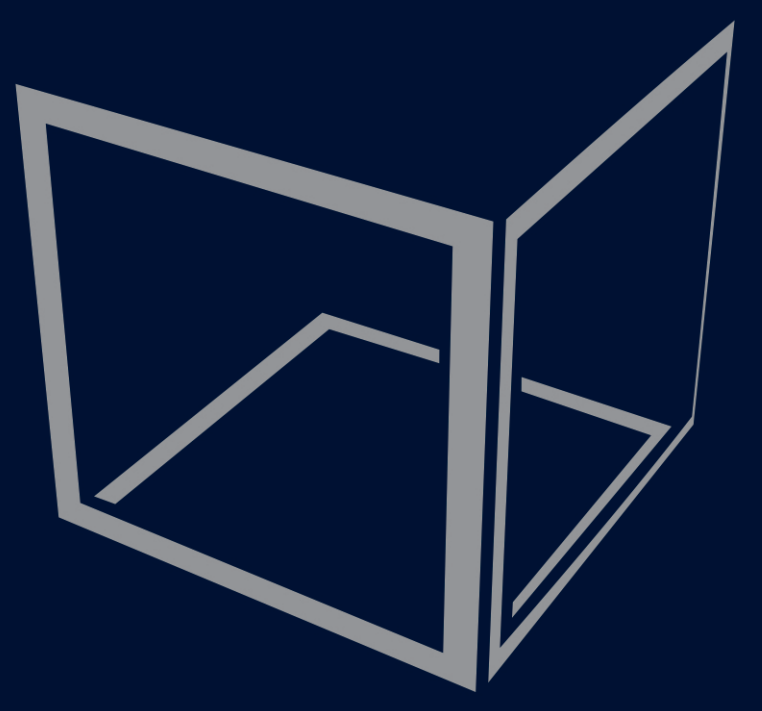

Octavina C. A Sukarta ${ }^{1}$

Eliza C. Martin ${ }^{2}$

Erik J. Slootweg ${ }^{1}$

Erin Bakker ${ }^{1}$

Ruben Schoen ${ }^{1}$

Vera Putker ${ }^{1}$

Marian E. P. Oortwijn ${ }^{3}$

Geert Smant ${ }^{1}$

Andrei J. Petrescu ${ }^{2}$

Aska Goverse ${ }^{1}$

${ }^{1}$ Laboratory of Nematology, Wageningen University \& Research, Wageningen, the Netherlands.

${ }^{2}$ Institute of Biochemistry of the Romanian Academy, Bucharest, Romania 


\section{Chapter}

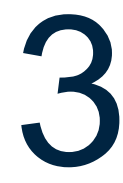

A Tomato Homolog of the Potato CC-NB-LRR Immune Receptor Rx1 Differs in Domain Cooperativity and Complex Formation Despite Sharing High Sequence Similarity 


\section{ABSTRACT}

Plant Nucleotide-Binding Leucine Rich Repeat (NB-LRR) immune receptors are pivotal components of innate immunity by mediating a specific, inducible branch of defence. These receptors comprise a flexible architecture, which can tolerate alterations such as sequence and domain exchange events. In the plant genome, this typically occurs between paralogs originating from a single gene-cluster, allowing plants to generate receptors with novel recognition specificities. Here, we expand further on the concept of domain exchangeability by investigating NB-LRRs derived from syntenic loci of related crop species. This will elucidate the extent by which mechanisms regulating receptor activity is conserved between homologous NB-LRRs. To that end, we performed sequence exchanges between homologs of the Rx1 immune receptor. Rx1 is a CC-NB-LRR originating from potato that confers resistance against Potato Virus X (PVX). We identified a full-length homolog of $R \times 1$ (designated Rslyc) from the tomato cultivar Katinka Cherry, which shares 85\% identity at the peptide level but has a yet undefined function. Despite being highly homologous, we show that a majority of the Rx1 and Rslyc domains cannot be exchanged without negatively impacting functionality of the receptor in trans. We infer that this is directly linked to differences in in the ability of the domains to form complexes in planta between the two systems. Notably, co-immunoprecipitation studies indicate that unlike Rx1, the NB-ARC domain of Rslyc cannot bind to its own or heterogenous LRRs in planta. This is supported by homology modelling indicating that the proximal NB-ARC and LRR regions are more basic in nature in Rslyc when compared to $\mathrm{Rx} 1$, which may affect receptor assembly and overall switch function. Furthermore, in contrast to Rx1, the distal CC and LRR domains of Rslyc can associate independently in trans. We speculate the CC/LRR interaction observed in Rslyc compensates for the weak binding at the NB-ARC/LRR interface, thereby preventing constitutive activation of defence by keeping the receptor in a closed state. Collectively, our data illustrate that despite their broad similarities, the function of homologous NB-LRRs is fine-tuned by distinct intradomain requirements.

\section{INTRODUCTION}

The heterogeneity and omnipresence of pathogens pose a serious risk to plants. As such, plants have developed a sophisticated, cell-autonomous innate immune system to recognize and neutralize 
microbial invasion (Bentham et al. 2017, Kapos et al. 2019). The plant's surveillance system relies on a consortium of diverse receptor proteins acting as molecular sensors. Referred to as Resistance proteins ( $\mathrm{R}$ proteins), a majority of these receptors belongs to the family of Nucleotide-Binding Leucine-Rich Repeat (NB-LRR) proteins. Plant NB-LRRs function intracellularly where they detect directly or indirectly specific, pathogen-derived molecules termed effectors. Successful effector recognition jump-starts a suite of potent defence responses, often manifesting in a form of localized cell death aimed at limiting further spreading of the pathogen.

In plants, classical NB-LRR prototypes have a conserved nucleotide binding module at their core referred to as the Nucleotide Binding adaptor shared by APAF-1, Resistance Proteins and CED4 (NB-ARC) domain (Bentham et al. 2017). Flanking the NB-ARC are two adjacent regions, including a hypervariable Leucine-Rich Repeat (LRR) domain at the C-terminus. The LRR tends to undergo diversifying selection and is therefore, generally associated with non-self-recognition events. On the other hand, the receptor's N-terminal end typically harbours a coiled-coil (CC) or Toll-Interleukin 1 Receptor (TIR) domain (van der Biezen et al. 1998). The CC/TIR region acts as a scaffold for interaction with other cellular factors involved in downstream signalling and/or indirect recognition. The concerted interplay between the various NB-LRR subdomains allows the receptor to undergo a coordinated switch between ADP-bound 'inactive' versus ATP-bound 'active' states, and initiate defence signalling upon pathogen perception (Tameling et al. 2006, Williams et al. 2011, Bernoux et al. 2016).

As spurious activation can lead to unwanted cell death, NB-LRRs are subject to strict regulation in the cell (van Wersch et al. 2020). Hereby, co-operation between the receptor subdomains constitutes an important form of control. Characterizing the physical and functional interactions between and within NB-LRRs domains is, therefore, pertinent to advance our understanding on the functioning of these receptors. In the past decade, studies across different NB-LRR systems have shown diverse structural requirements for proper activation and functioning (Moffett et al. 2002, Rairdan et al. 2008, Rairdan et al. 2006, Slootweg et al. 2013, Qi et al. 2012, Wang et al. 2015). While the specifics may differ, a body of evidence indicates that disturbing the underlying intradomain interactions can directly impair receptor activity (reviewed in Bentham et al. 2017, Jones et al. 2016, Kapos et al. 2019). This can, for instance, lead to autoactive phenotypes resulting in constitutive cell death activation. A majority of the autoactivating mutations described, thus far, map within close 
proximity to the bound nucleotide in the central NB-switch (Tornero et al. 2002, Tameling et al. 2006, Bendahmane et al. 2002). Notably, mutations in the conserved RNBS-A, RNBS-D, or MHD motifs have been shown to result in more sensitive or activable versions of the receptor. Aside from the NB, exchanging fragments of the LRR can also lead to aberrant NB-LRR functioning. For instance, systematically shuffling the LRR regions of the Mi-1.2 resistance protein with the corresponding fragments of its homolog Mi-1.1 (that has unknown function) leads to a chimera with autonomous activity (Hwang et al. 2000). Studies such as these exemplify how minor structural mismatches in an NB-LRR receptor can yield auto-activating or loss-of-function phenotypes. This leads to a model wherein NB-LRRs are kept in a primed state granted by proper folding of the receptor subdomains (Rairdan et al. 2006). To counterbalance malfunctioning phenotypes in one domain, mutations are often needed elsewhere in the receptor module. For example, while targeting residues in the LRR of the $\mathrm{R} \times 1$ receptor can broaden its recognition specificity, it also resulted in a dampened immune response (Farnham et al. 2006). Additional sensitizing mutations in the NB-ARC were needed to reinstate full-immunity (Harris et al. 2013). These studies collectively suggest interdependency and co-evolution existing between the various NB-LRR subdomains to allow proper functioning.

The potato Rx1 and Gpa2 belong to the group of CC-NB-LRR-type immune receptors and locate to the same gene cluster on chromosome XII of potato (Solanum tuberosum spp. andigena). They share high sequence similarity of $88 \%$ at the amino acid level, yet mediate resistance against taxonomically unrelated pathogens (van der Vossen et al. 2000, Bendahmane et al. 1997 ). Rx1 recognizes the coat proteins $(\mathrm{CP})$ of potexviruses including Potato Virus $X(P V X)$ to instigate rapid arrest of viral spreading in a process coined as extreme resistance (Bendahmane et al. 1999, Bendahmane et al. 1995). However, heterologous gene expression assays have also shown that $\mathrm{Rx} 1$ is capable of inducing classical cell death phenotypes. In contrast to Rx1, Gpa2 acts belowground in roots infected by the potato cyst nematode Globodera pallida by conferring a delayed resistance response that blocks nematode feeding cell development (Rouppe van der Voort et al. 1997, van der Vossen et al. 2000). However, transient experiments in Nicotiana benthamiana demonstrate that Gpa2 can trigger cell death upon recognition of GpRBP-1 effectors produced in the dorsal gland of the nematode (Sacco et al. 2009). Together, this makes Rx1 and Gpa2 a favourable model system to address questions related to NB-LRR immune receptor functioning, specificity and evolution at $R$ gene clusters. Previously, for example, $\mathrm{R} \times 1$ and $\mathrm{Gpa} 2$ were used to elucidate 
the role of intradomain interactions in modulating the function of plant CC-NB-LRRs (Moffett et al. 2002, Rairdan et al. 2008, Rairdan et al. 2006, Slootweg et al. 2013). One remarkable finding is that domain co-operativity limits the degree by which coding sequences can be exchanged between homologous receptors. For instance, swapping the C-terminal regions of the Rx1/Gpa2 LRR can switch disease resistance specificity between the two homologs (Slootweg et al. 2017). However, this is based on the premise that compatibility between the ARC2 and LRR regions is maintained, which is crucial for the assembly of the receptor complex. This finding also highlights that the CC-NB-ARC modules of Rx1/Gpa2 are functionally interchangeable and able to confer resistance via (partially) overlapping downstream immune responses. This convergence of defence responses might be the result of preserving interactions with common host-factors such as the RanGTPase Activating Protein 2 (RanGAP2). RanGAP2 interacts via surface-exposed residues on the Rx1 CC-domain and is required for full-functionality of the receptor (Sacco et al. 2007, Tameling et al. 2007, Hao et al. 2013). More detailed molecular studies have shown that RanGAP2 regulates the subcellular distribution of Rx1 and may have a role in mediating Gp-RBP-1 recognition by Gpa2.

Based on scenarios as sketched above, NB-LRR receptors, therefore, comprise of structures with remarkable plasticity that can tolerate some degree of alterations imposed on the complex (Slootweg et al. 2013, Sacco et al. 2009). The presence of NB-LRR clusters in the plant genome provides a genetic framework for the diversification of novel resistance genes based on sequence exchange events between paralogs at a single R-gene locus. However, it is also clear that sequence exchange has functional constraints that can result in autoactive or loss of function mutants. This is thought to have contributed to the shaping of NB-LRR immune receptors during $R$ gene evolution, including the development of novel recognition specificities. For example, homologs of Rx1 have been identified in other potato species like Rx2 in Solanum acaule or syntenic regions on Chromosome XII of other Solanaceous crops like tomato and pepper (Ritter et al. 1991, Mazourek et al. 2009, Shi et al. 2011). Whereas Rx2 still confers specific resistance to PVX, the most distant example Bs2 from pepper (Capsicum annuum) does not. Bs2 activates defence against the bacteria Xanthomonas campestris via classical cell death responses (Hibberd et al. 1987). Hence, during evolution of Solanaceous crop species, sequence variation at the Rx1 locus adds to the diversification of the recognition repertoire to a wide range of taxonomically unrelated species like a virus, bacterium, and nematode. However, 
the mechanistic/structural basis as well as the extent by which sequence variation contributes to receptor function and the generation of novel recognition spectra are not fully elucidated.

Here, we aim to elucidate whether mechanisms regulating complex formation and receptor function are conserved between NB-LRR homologs originating from related crop species. To address this, we performed sequence-exchange experiments between homologs of $\mathrm{R} \times 1$ from potato and tomato. The tomato homolog (designated Rslyc) is $85 \%$ similar at the amino acid level but its recognition specificity is unknown. We used Rslyc as a proxy to decipher novel molecular determinants underlying differences in PVX recognition and cell death activation. We present evidence that the Rslyc receptor complex is regulated by multiple domain associations, but these interactions do not fully overlap with those reported previously for $\mathrm{R} \times 1 / \mathrm{Gpa} 2$. Particularly, we demonstrate that unlike $\mathrm{R} \times 1$, the LRR domain of Rslyc can bind to the CC independently in trans without the need of a central NB-ARC domain. In a further characterization of Rslyc, we discovered that sequence and structural variations close to the nucleotide binding pocket may underly differences observed in the folding, localization, and intradomain co-operativity of the Rslyc NB-ARC. This includes compatibility at the ARC2/LRR interface, which was shown to differ between $\mathrm{R} \times 1$ and Rslyc in immunoprecipitation assays. Collectively, our data suggest that highly homologous receptors originating from closely related crop species can diversify in their domain interactions and complex formation in trans, and possibly in cis. Our main findings are further discussed in the framework of current activation and structural models as well as their implications for NB-LRR engineering.

\section{RESULTS}

\section{Identification and isolation of an $\mathrm{Rx} 1$ homolog from tomato}

Based on in silico screening of the genome of the tomato cultivar Katinka Cherry, a single gene (3136 bp) was found to encode a full-length homolog of the potato Rx1 receptor (henceforth, denoted as Rslyc). We subsequently amplified and sequenced Rslyc cDNA, thereby, confirming that the cultivar expresses a transcript corresponding to a full-length NB-LRR immune receptor. By comparing the genomic and cDNA sequences, the gene structure of Rslyc was elucidated (represented schematically in Fig. 1A). Similar to Rx1, Rslyc has two introns positioned at 3'end of 
the gene in a region encoding for the LRR domain. The exact borders of these introns, however, differ slightly. While the location of the first intron in Rslyc corresponds to that reported for Rx1, the boundary of the second intron begins at an earlier site closer to the 5'end (Bendahmane et al. 1999).

The translated polypeptide of Rslyc comprises 906 amino acids with a predicted molecular weight of $\sim 104.7 \mathrm{kDa}$. To gain better insight into the degree of conservation, we performed protein sequence alignment of Rslyc and Rx1 (Supplementals Fig. S1). Rx1 and Rslyc share 85\% identity at the amino acid level, with a majority of the hypervariable region concentrating to the LRR and ARC domains (Fig. 1B). To predict the putative number of LRR repeats in Rslyc, we used the recently published full-length structure of the Arabidopsis HOPZ-ACTIVATED-RESISTANCE 1 ZAR1 receptor as template (Wang et al. 2019). Our structural prediction suggests that the LRR domain of Rslyc is comparable in length to its $R \times 1$ counterpart, consisting of 15 LRR repeats. The latter includes a previously undefined 'linker' region (472-496) between the ARC2 and LRR (Slootweg et al. 2013). For both $\mathrm{Rx} 1$ and Rslyc, we also identified an additional insertion in repeat 9 of the LRR, which is absent in ZAR1. This insertion is enriched in aromatic residues and may, therefore, play a prominent role in mediating interactions with accessory proteins derived from the host (Supplementals Fig. S1). When examining polymorphisms identified within the LRRs of $\mathrm{Rx} 1$ and Rslyc, a majority is surface-exposed and occurs throughout the entire LRR region, with a slightly higher density at the $\mathrm{N}$-terminal half of the LRR. The core NB-ARC switch constitutes the most conserved module between the two receptors, with the Rslyc NB-ARC retaining hallmark functional motifs characterized in prior studies such as the P-loop, RNSB-A, RNSB-D and GXP. At the N-terminus, the CC domain of Rslyc bears residues for RanGAP2 binding and the EDMVD motif involved in intradomain associations with the NB-ARC-LRR (Rairdan et al. 2008). Further comparative analysis indicates that the $\mathrm{Rx} 1$ and Rslyc CC domains differ at 21 positions in the first 110 amino acids. From the four alpha helices that form the CC, the first helix is fully conserved while a majority of the polymorphisms locate in the second helix, which does not contain any previously characterized motifs.

Based on the presence of typical features of a CC-NB-LRR receptor as described above, we anticipate Rslyc to encode a functional immune receptor. However, its recognition specificity is unknown. To confirm that Rslyc does not mediate defence against PVX despite its high sequence similarity to Rx1, we inoculated the tomato cultivar Katinka Cherry with avirulent (UK3) and virulent 
(HB) PVX strains. PVX-levels in systemic leaves were quantified by DAS-ELISA using antibodies against the viral coat protein. The results indicate that Katinka Cherry is fully susceptible to PVX as evidenced by the high viral accumulation of both strains in these plants compared to the resistant potato SH line, which possesses Rx1 (Supplementals Fig. S2). Despite the high degree of sequence homology and conservation of functional motifs, our data show that Rslyc does not confer resistance against PVX.

A.
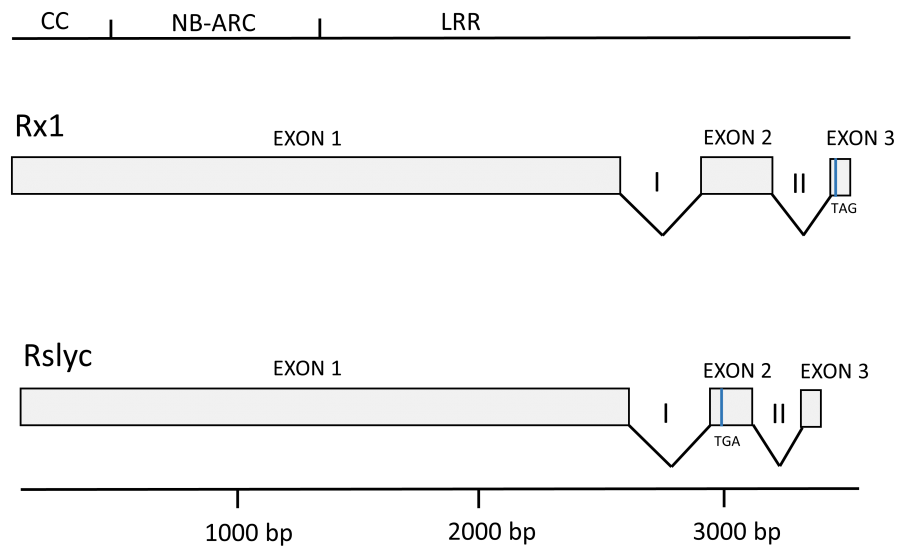

B.

Domain organization

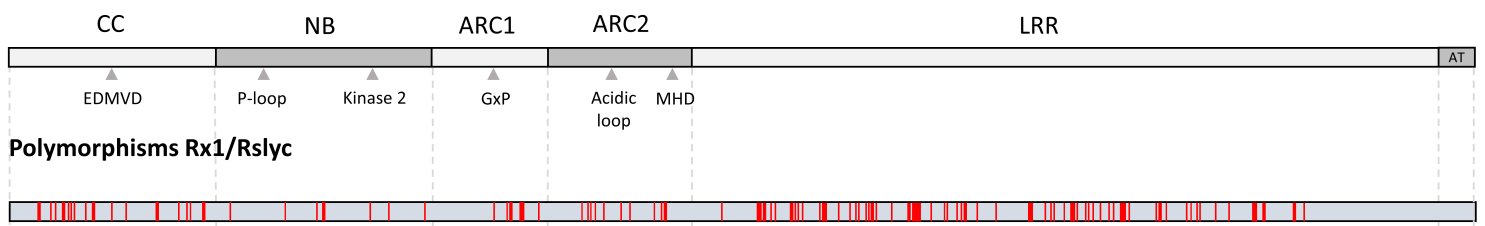

AA\% Rx1 versus Rslyc

$85 \%$

Polymorphisms mapped on available crystal structures or 3D models
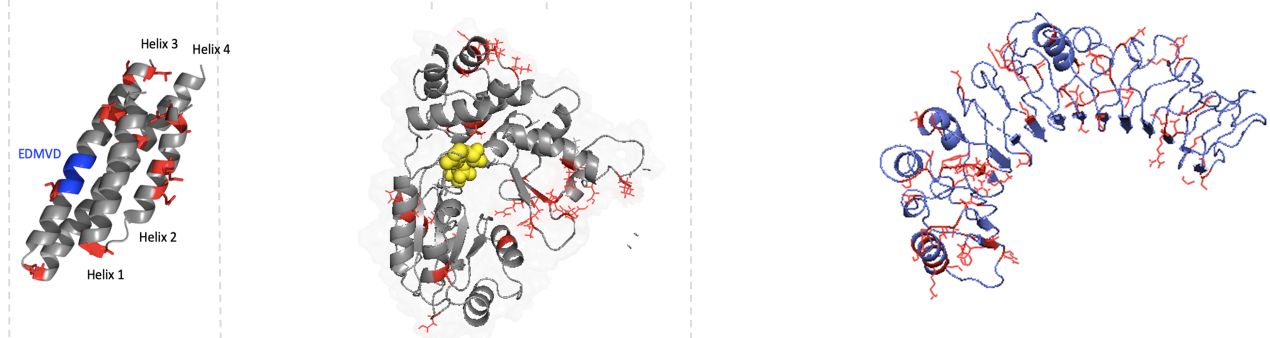

Fig. 1: The Rslyc gene has similar architecture and encodes a CC-NB-LRR protein homologous to $\mathrm{R} \times 1$. A) Diagram illustrating the gene-structures of Rslyc and $\mathrm{R} \times 1$. Open grey rectangles 
(Figure legend continued) represent exons whereas introns are shown by the inverted lines. Blue lines indicate the presence of a stop codon. B) Schematic representation of protein domains and variations therein between Rx1 and Rslyc. Positions of polymorphic amino acid residues are indicated by the red lines and mapped on the available crystal structure of the Rx1-CC or reported 3D remote homology models of the Gpa2-NB-ARC and LRR domains (Hao et al. 2013, Slootweg et al. 2013). The overall percentage identity (based on amino acid residues) of each domain is given in the next row. Key of the CC domain: $\mathrm{H} 1=$ Helix 1, H2 = Helix 2, H3 = Helix 3, H4 = Helix 4.

\section{The CC domains of Rx1 and Rslyc are functionally interchangeable}

Based on similarity of the primary sequence and conservation of functional motifs, we identified Rslyc as a suitable proxy to resolve critical determinants in NB-LRR activity using Rx1 as a functional template. We then performed a trans-complementation-based study in the model plant Nicotiana benthamiana to investigate the functionality of the Rslyc domains. Such approaches are based on the observation that the $\mathrm{R} \times 1$ subdomains can be expressed as separate constructs (in trans) but still reconstitute a functional receptor in planta (Moffett et al. 2002). We took advantage of this system to determine the capacity of various $\mathrm{Rx} 1$ and Rslyc domain combinations to form a functional receptor complex and confer resistance.

The $\mathrm{CC}$ domain is a modulator of $\mathrm{R} \times 1$ function and indispensable for complete resistance against PVX (Slootweg et al. 2018, Rairdan et al. 2008). Hence, we first tested whether the Rx1 and Rslyc CCs are functionally interchangeable despite differences observed at the sequence level. To that

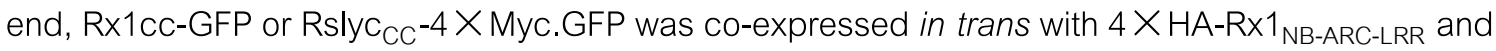
assessed for the ability to induce cell death upon elicitation by the avirulent PVX-CP UK3 (Fig. 2A). Similar domain combinations expressed with the coat protein of the PVX breaker strain (PVX-CP HB) were included as a specificity control. The degree of cell death in infiltrated $N$. benthamiana leaves was assessed by visual scoring and a chlorophyll assay. Our data show that trans-complementing

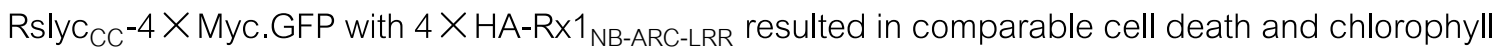
loss when co-expressed with PVX-CP UK3 compared to the Rx1 $1_{C C}-G F P / 4 \times H A-R \times 1_{\text {NB-ARC-LRR }}$

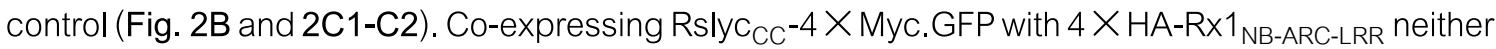
triggered autoactivity nor expanded recognition against PVX-CP HB. 
As Rx1 confers an extreme resistance response, we investigated whether the trans-complementation of Rslyc $\mathrm{CC}_{\mathrm{C}}$-GFP with $4 \times \mathrm{HA}-\mathrm{R} \times 1_{\mathrm{NB}-\mathrm{ARC}-\mathrm{LRR}}$ could elicit effective defence against an amplicon of PVX-UK3 in $N$. benthamiana. Quantification of viral levels by DAS-ELISA reveals that trans-complementing Rslyc $_{\mathrm{CC}}-4 \times$ Myc.GFP with $4 \times \mathrm{HA}-\mathrm{R} \times 1_{\mathrm{NB}-\mathrm{ARC}-\mathrm{LRR}}$ yielded comparable reduction of the virus at 5 days post infiltration (dpi) relative to the $\mathrm{Rx} 1$ control combinations (Fig. 2D). Together with the cell death data, this suggests that the CC domains of Rslyc and Rx1 can be exchanged in spite of the sequence variation observed.

We then determined whether the functional phenotypes observed link to the ability of the receptor domains to form complexes in planta. As sequence analysis shows that Rslyc CC retains the canonical EDVID motif, we anticipated that it would interact with the NB-ARC-LRR of Rx1. A Co-IP assay was, therefore, carried out using protein extracts of $N$. benthamiana leaves transiently co-expressing Rslyc $\mathrm{CC}$-GFP and $4 \times \mathrm{HA}-\mathrm{Rx} 1_{\mathrm{NB}-\mathrm{ARC}-\mathrm{LRR}}$. A combination of Rslyc $\mathrm{CC}_{\mathrm{CC}}-4 \times \mathrm{MyC}_{\mathrm{G}}$.GFP with the NB-ARC-LRR of Gpa2 ( 4 XHA-Gpa2 ${ }_{\text {NB-ARC-LRR }}$ ) was included as an additional specificity

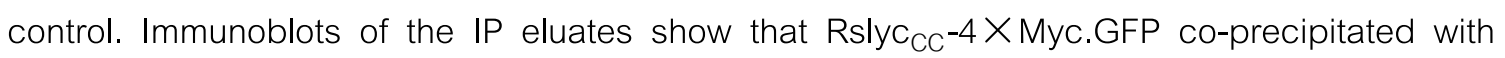

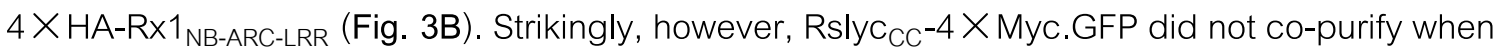
$4 \times$ HA-Gpa2 ${ }_{\text {NB-ARC-LRR }}$ was used as bait. Our data demonstrate that expressing Rslyc ${ }_{C C}$ with $\mathrm{R} \times 1_{\text {NB-ARC-LRR }}$ can result in the reconstitution of a functional $\mathrm{R} \times 1$ chimera, which is capable of triggering immunity upon elicitation by PVX.

Aside from mediating intramolecular-interactions, the $\mathrm{CC}$ domain constitutes the minimal unit for the association of Rx1 to a conserved WPP motif in RanGAP2 (Hao et al. 2013, Sacco et al. 2007, Tameling et al. 2007). Our sequence analysis indicates that hydrophobic residues required for RanGAP2 binding in Rx1 are conserved in helix 4 of the Rslyc CC (Hao et al. 2013). As such, we hypothesized that Rslyc CC could also bind with RanGAP2. Indeed, pulling down Rslyc $\mathrm{CC}^{-4} \times \mathrm{Myc}_{\mathrm{C}}$.GFP and Rx1cc-GFP in a Co-IP with $\alpha$-GFP beads co-purified RanGAP2-mCherry, indicative of a physical association in planta (Fig. 3C). It is worth noting that compared to full-length RanGAP2, immunoblots of the tagged proteins show that WPP-mCherry did not pull down with Rslyc $_{\mathrm{CC}}-4 \times \mathrm{Myc}_{\mathrm{C}}$.GFP or to a considerably lesser extent. However, we attribute this to the WPP protein being present less abundantly in the input for this infiltration combination. Based on our findings, we therefore conclude that similar to Rx1, Rslyc can form a heteromeric complex with the host-factor RanGAP2. 
The CC domain of Rx1 also dictates the nuclear localization of the receptor in the cell, which is crucial for activation of a complete defence response (Slootweg et al. 2010). We therefore investigated the subcellular-localization of Rslyc-4 X Myc.GFP by confocal laser scanning microscopy. The relative intensity ratios of GFP signal in the nucleus to the cytoplasm $\left(I_{N} / I_{C}\right)$ in each cell imaged was determined to quantify the cellular distribution of the expressed constructs (Slootweg et al. 2010). In line with existing studies, $\mathrm{Rx}{ }_{\mathrm{CC}}$-GFP was present in both the nucleus and cytoplasm with a higher signal in the nucleus (mean $I_{N} / I_{C}$ ratio of $\sim 2.0$ ). Our results show that Rslyc $\mathrm{CC}^{-4} \times$ Myc.GFP exhibits similar subcellular distribution patterns (mean $I_{N} / I_{C}$ ratio of $\sim 1.7$ ) as its Rx1 ${ }_{\text {CC }}$-GFP counterpart (Fig. 3A1 and 3A2). Taken together, we provide evidence that the CC domains of Rslyc and Rx1 are functionally similar, which is a reflection of the conservation of known motifs, intra/interdomain interactions, and subcellular localization. 
A.

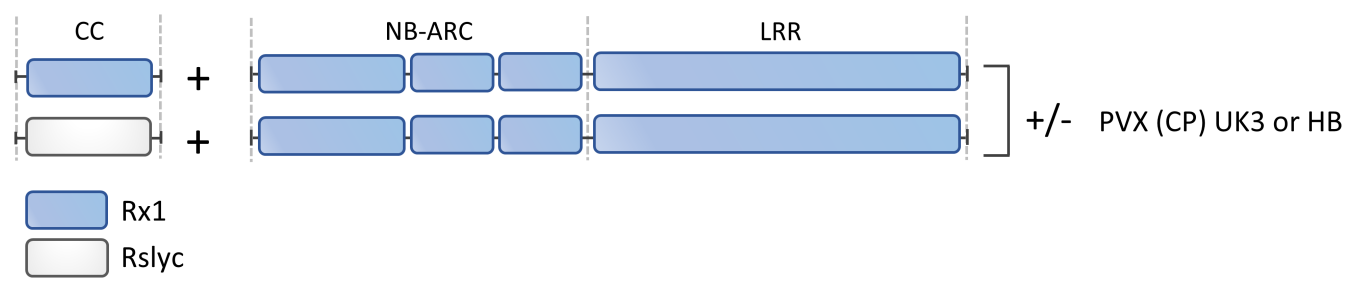

B.

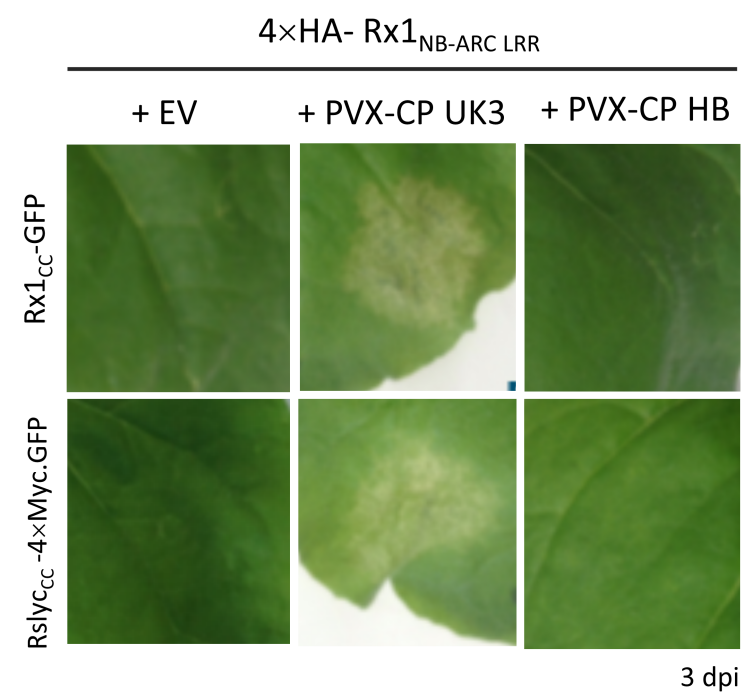

C1.

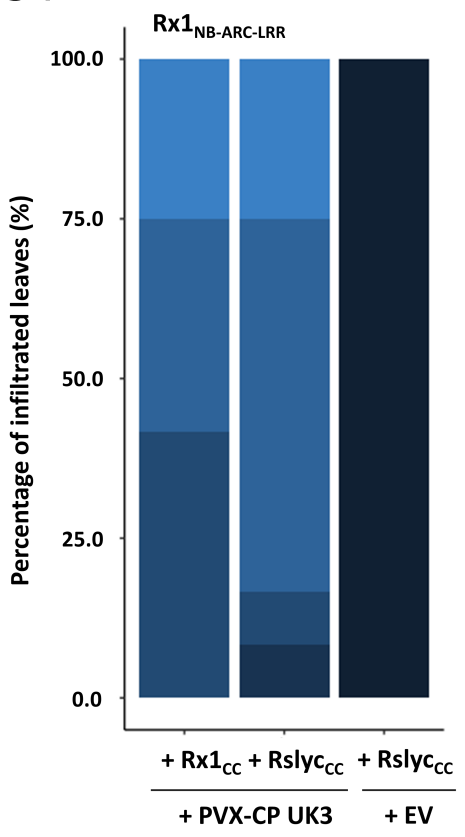

C2.

D.
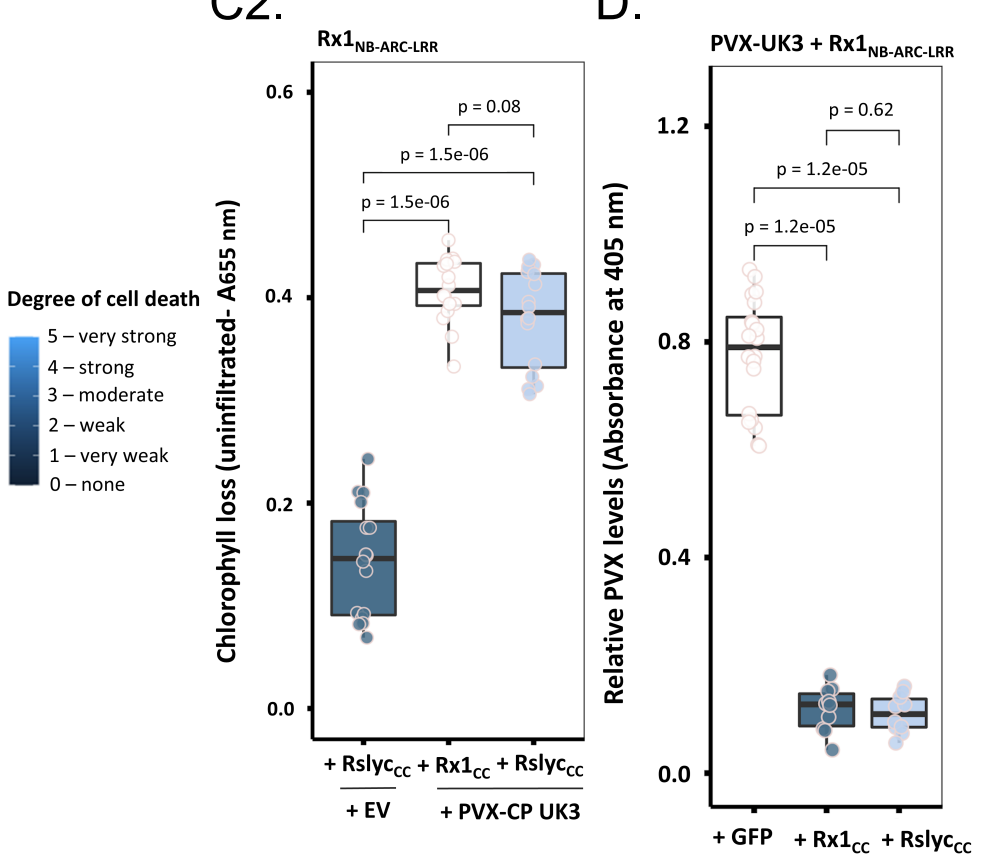

Fig. 2: The Rslyc CC domain can functionally complement the NB-ARC-LRR of Rx1 in trans.

A) Schematic diagram depicting the combination of constructs expressed in trans for the bioassays. 
(Figure legend continued) B) Representative photographs of $N$. benthamiana leaves infiltrated by $4 \times \mathrm{HA}^{-R \times 1_{\text {NB-ARC-LRR }}}$ and its matching CC domain or that of Rslyc in combination with empty vector (EV), PVX-CP UK3 or PVX-CP HB. Images were taken at 3 dpi. C1) Stacked bar chart summarizing the degree of cell death on infiltrated zones of $n=12$ samples as determined by visual scoring. Leaf samples were scored at 3 dpi. C2 and D) Boxplots of chlorophyll assay (C2) or PVX assay by DAS-ELISA (D) from a single representative experiment of three independent repeats. Whiskers represent interquartile range whereas the crossbar signifies the median. Each dot is representative of a biological replicate corresponding to leaf discs harvested from a single infiltrated leaf of $n \geq 10$ samples. Statistical significance was determined by ANOVA followed by a Tukey post-hoc analysis with $\alpha=0.05$.

Fig. 3 A1.

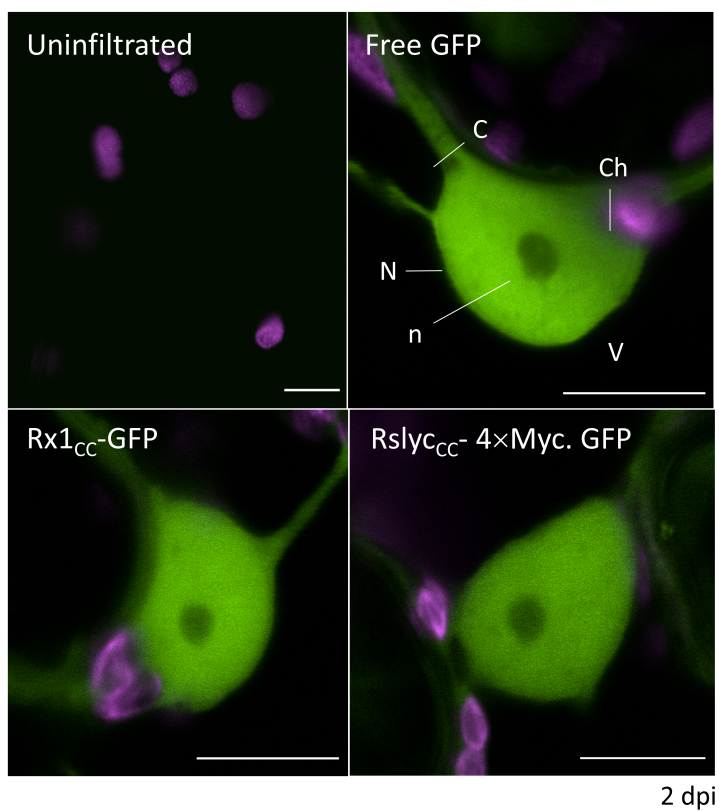

A2.

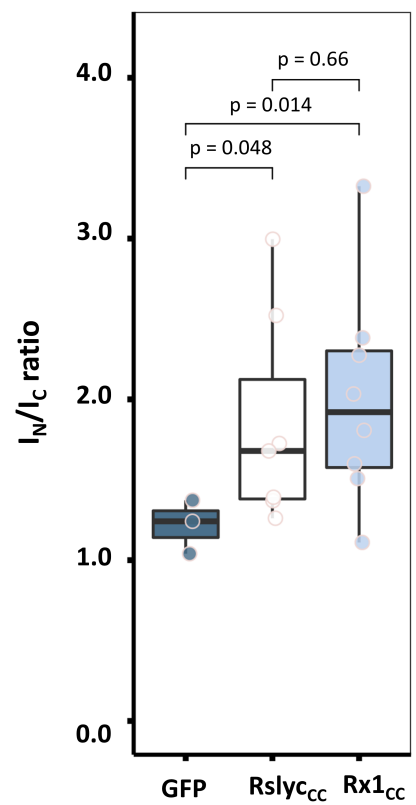




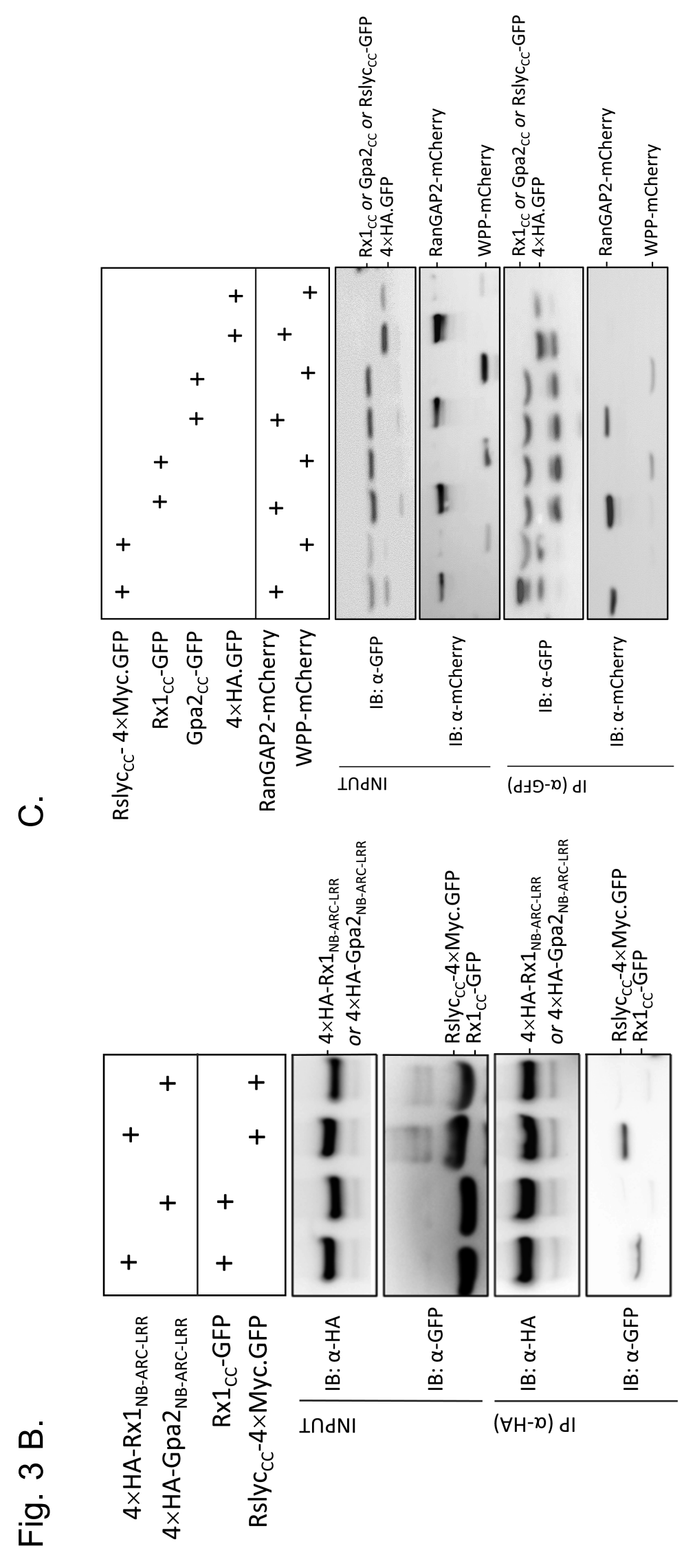


Fig. 3: The CC domain of Rslyc shares similar subcellular localization and inter/intradomain interactions with Rx1-CC. A1) Representative images of the nuclei of $N$. benthamiana cells uninfiltrated or transiently expressing free GFP, Rslyc $_{\mathrm{CC}}-4 \times$ Myc.GFP or Rx1 ${ }_{\mathrm{CC}}-\mathrm{GFP}$. Images were taken at 2 dpi by confocal laser scanning microscopy. Scale bar represents a width of $10 \mu \mathrm{m}$. Key: $\mathrm{N}$ = nucleus; $\mathrm{n}=$ nucleolus; $\mathrm{C}=$ cytoplasm; $\mathrm{V}=$ vacuole; $\mathrm{Ch}=$ chloroplast. Data shown is representative of three biological repeats. A2) Boxplot representing intensity ratios of GFP signal in the nucleus relative to the cytoplasm $\left(I_{N} / I_{C}\right)$ of $N$. benthamiana epidermal cells transiently expressing free GFP, Rslyc $\mathrm{CC}_{\mathrm{C}}-4 \times$ Myc.GFP or Rx1 ${ }_{\mathrm{CC}}$-GFP. Intensity was determined by the ImageJ software. Each dot is representative of a biological replicate corresponding to a single imaged cell. Statistical significance was determined by ANOVA followed by a Tukey post-hoc analysis with $\alpha=0.05$. B). Immunoblots of pull-downs of Rslyc $\mathrm{CC}_{\mathrm{C}}-4 \times$ Myc.GFP or Rx1 ${ }_{\mathrm{CC}}-\mathrm{GFP}$ with $4 \times \mathrm{HA}$-tagged versions of the Rx1 or Gpa2 NB-ARC-LRR fragments. Infiltrated leaves harvested at 3 dpi were used for Co-IP. Immunoprecipitation was performed using $\alpha$-HA magnetic beads and $\alpha$-GFP blots indicate the success of the pull-down. C). Immunoblots of the Co-IP of RanGAP2-mCherry or its WPP domain thereof with the CC domains of Rx1, Gpa2 or Rslyc. Bait proteins were pulled-down using $\alpha$-GFP beads. In the annotated immunoblots, "+" indicates the presence of a construct in the infiltration combination.

\section{Rx1 and Rslyc have different compatibilities at the NB-ARC and LRR interface}

Systemically shuffling the domains of $\mathrm{R} \times 1$ and $\mathrm{Gpa} 2$ demonstrates that co-operativity between the LRR and ARC interface constitutes an important regulatory control for receptor function (Slootweg et al. 2013, Rairdan et al. 2006). This is mediated by complementary acidic and basic patches in the $A R C 2$ and $\mathrm{N}$-terminal half of the LRR regions, which allow the receptor parts to assemble as a unit. Such studies illustrate the dual role of the LRR in regulating recognition specificity and the switch module. Given the significance of the compatibility between the ARC2 and LRR domains, we next investigated whether the ARC2-LRR interface of Rx1 and Rslyc is matching. To investigate this, we generated a chimeric LRR construct ( $4 \times H A-L R R_{\text {chimera }}$ ) containing the $\mathrm{N}$-terminal half of the Rslyc LRR and the minimal region required for PVX-recognition (Fig. 4A). The resultant LRR construct was used in trans-complementation assays with $4 \times \mathrm{MyC}_{\mathrm{C}} \mathrm{R} \times 1_{\mathrm{CC}-\mathrm{NB}-\mathrm{ARC}}$ to determine whether this combination can still confer a cell death and/or extreme resistance response. 
Our bioassays show that $4 \times$ MyC-R $1_{C C-N B-A R C}$ and $4 \times H A-L R R_{\text {chimera }}$ did not complement in trans to give a cell death or extreme resistance response (Fig. 4B1-B2 and 4C). This is in line with our interaction data demonstrating that the chimeric LRR construct did not co-precipitate with $4 \times$ MyC-R $1_{\text {CC-NB-ARC }} 2$ in a Co-IP (Fig. 5A). Based on these results, we infer that the combination of $4 \times$ Myc- $R \times 1$ CC-NB-ARC $_{\text {with }} 4 \times H A-L R R_{\text {chimera }}$ is non-functional as the complex cannot assemble efficiently in planta. The polymorphic residues present in the ARC2/LRR interface may compromise complex formation in trans due to mismatch as the domains are derived from two different homologs. This is further supported in a pull-down assay indicating that the receptor indeed interacts with the LRR chimera when Rslyc ${ }_{C C-N B-A R C}-4 \times M$ Mc.GFP was used as prey, thereby restoring compatibility

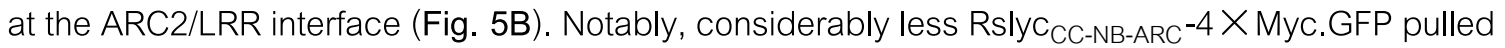
down with $4 \times \mathrm{HA}-\mathrm{LRR}_{\text {chimera }}$ compared to the $\mathrm{Rx} 1$ control combinations. This indicates that while the full receptor can still reconstitute in planta, the physical associations may be less efficient. In line with this, trans-complementing Rslyc ${ }_{\mathrm{CC}-\mathrm{NB}-\mathrm{ARC}}-4 \times \mathrm{Myc}$.GFP and $4 \times \mathrm{HA}-\mathrm{LRR}_{\text {chimera }}$ did not result in a cell death response compared to the wild-type Rx1 control (Fig. 4D and 4E1-E2). The addition of an autoactive mutation in the MHD motif of the ARC2 (D460V) in this truncated construct also did not potentiate the resistance response. A likely interpretation is that the ARC2/LRR association in Rslyc can occur to re-establish a receptor complex albeit with low affinity and thus, may be insufficient to trigger an effective defence response.

We then questioned whether these experimental results correlate to structural differences at the predicted LRR and ARC2 interface. To do so, we generated full-length models of Rx1 and Rslyc in their monomeric, ADP-bound states (Supplementals Fig. S4). The full-length homology models were built piecewise starting from the ZAR1 Cryo-EM structure in the auto-inhibited state (PDB: 6j5w - (Wang et al. 2019)) and from the potato Rx1 crystal structure of the CC domain (PDB: 4m70 - Hao et al. 2013). Molecular dynamics simulations were performed in parallel to assess the modeled domain stability and identify potential structural aspects responsible for the observed differences in intra and inter domain interactions between Rx1 and Rslyc. Our analysis reveals that point mutations in the NB-ARC and LRR regions induce significant differences in the electrostatic properties of the surface of these domains. Most strikingly, there appears to be two charged patches that are unique to the LRR domain of Rslyc (Supplementals Fig. S5), which render it more basic than the Rx1 LRR. The first of these patches (denoted as 'Patch P1+') resides on the concave side of the LRR 
within close vicinity to the ARC2. Hereby, there are five mutations, namely G525R, G526R, E528R, T548R, W549R, unique to Rslyc, which convert the otherwise neutral residues in Rx1 to positively charged ones. Consequently, these substitutions collectively create a largely positive patch specific to Rslyc (Fig. 5C). Imaginably, this may cause the proximal acidic loop (DEPDD in regions 220 -224) in the NB-ARC more prone to a structural drift towards 'LRR-Patch P1+'. Given the fact that NB-ARC-LRR folding plays a prominent role, such a slight deformation could possibly inflict activation problems. Together, our results suggest that experimentally observed incompatibilities between the ARC2/LRR-interface of Rslyc within itself and between Rx1 may be attributed to structural variation at these interfaces.

Fig. 4 A.

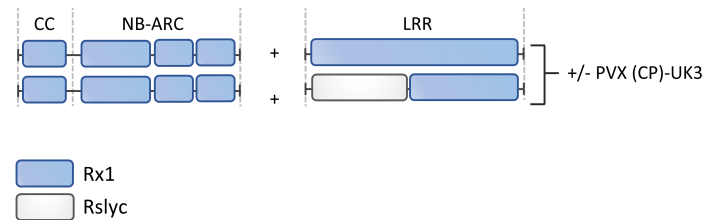

B1.

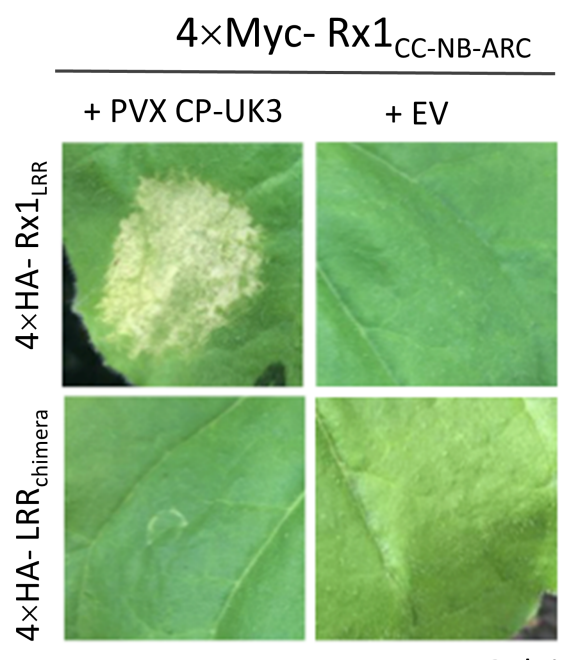

$3 \mathrm{dpi}$
D.

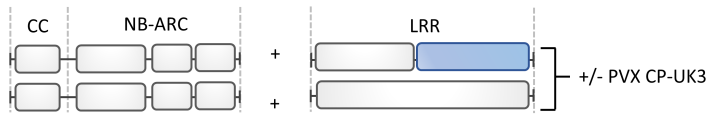

E1.

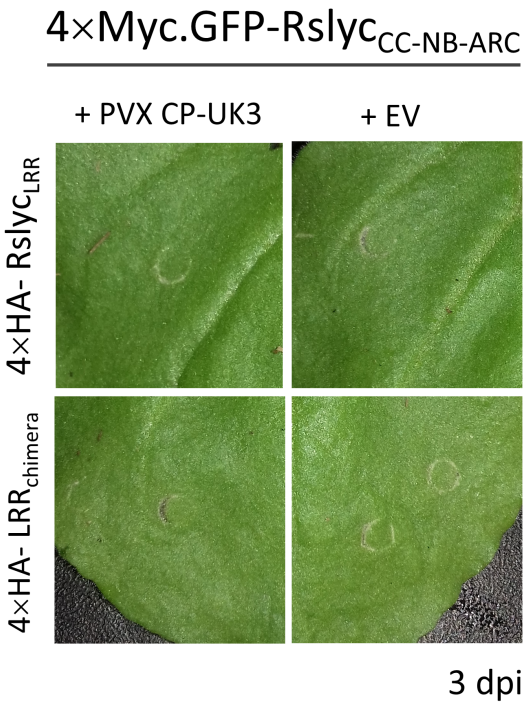




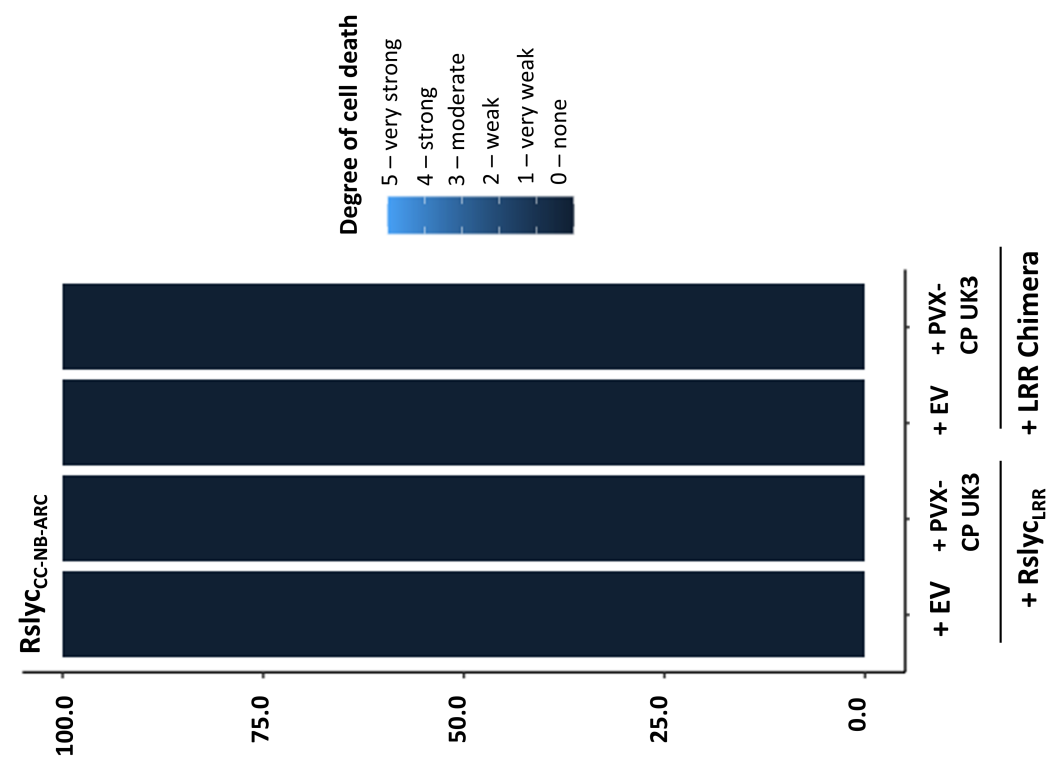

พ่

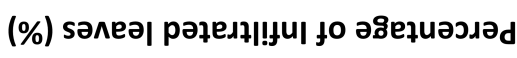

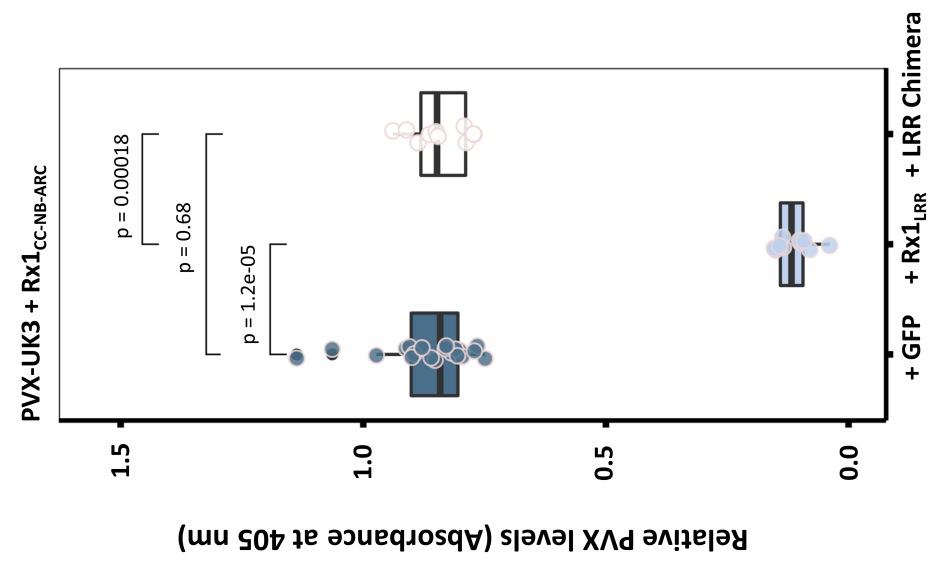

$\dot{0}$
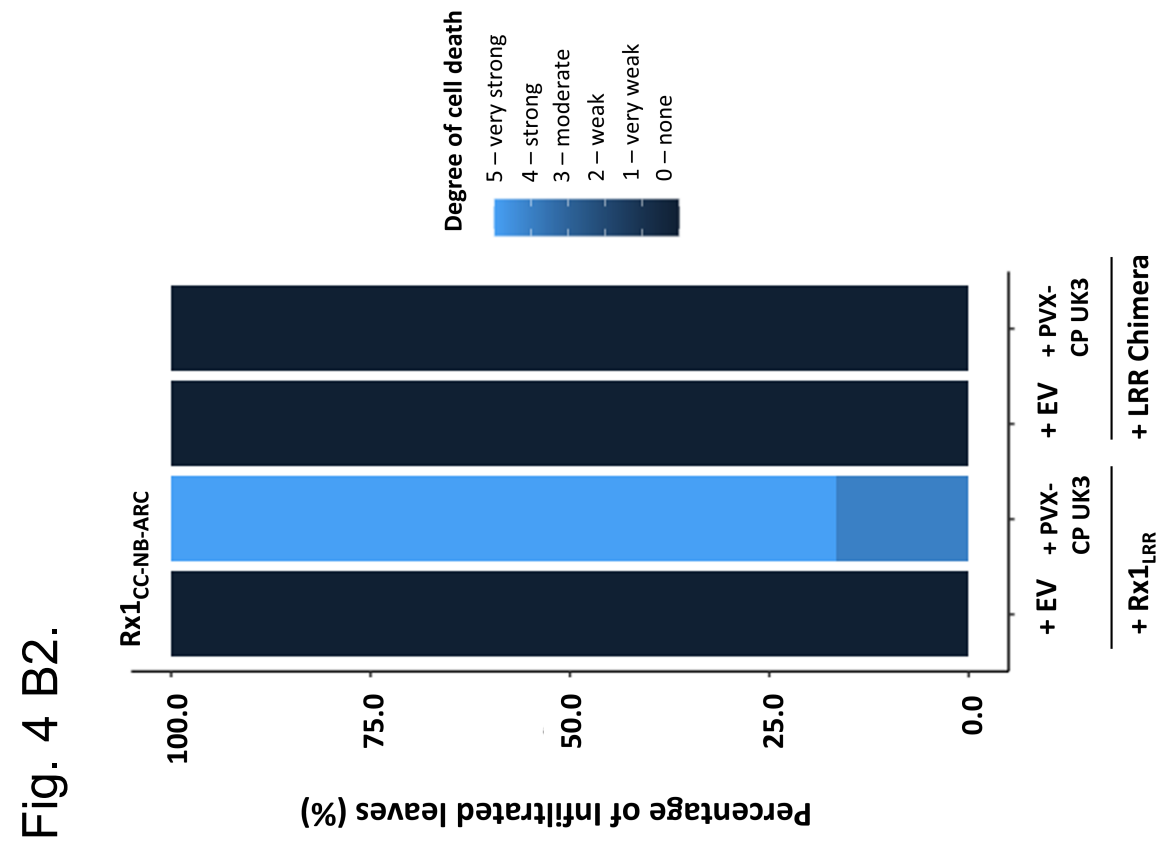
Fig. 4: The CC-NB-ARC domain of Rslyc does not functionally complement the Rx1-LRR in trans. A and D) Graphical summaries of infiltration combinations used in the trans-complementation assays. B1 and E1) Representative photographs of leaf phenotypes of $N$. benthamiana infiltrated by the CC-NB-ARC of Rslyc/Rx1 in combination with Rx1/Rslyc-LRR or a chimeric LRR construct. Images were taken at 3 dpi. B2 and E2) Stacked bar charts derived from the visual scoring of the degree of cell death in infiltrated zones of $n=12$ samples $C$ ) Boxplot of PVX assay quantifying viral levels as determined by DAS-ELISA involving infiltration combinations shown in A. Whiskers represent interquartile range whereas the crossbar signifies the median. Each dot is representative of a biological replicate corresponding to leaf discs harvested from a single infiltrated leaf. Statistical significance was determined by ANOVA followed by a Tukey post-hoc analysis with $\alpha=0.05$. 
A.

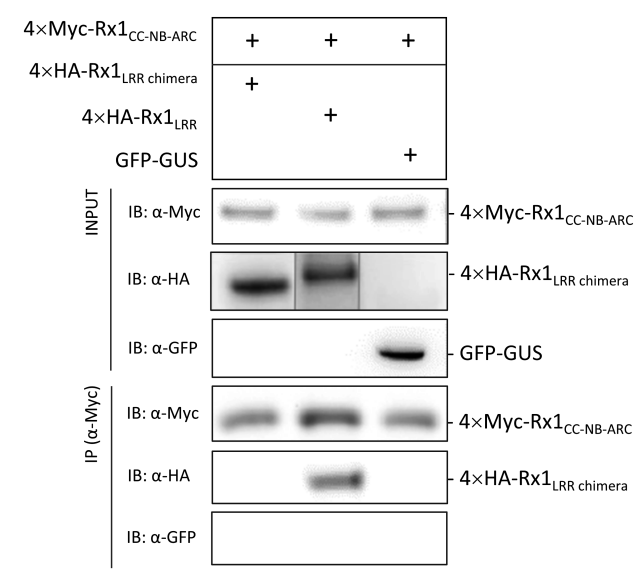

C.

$\mathrm{Rx} 1$
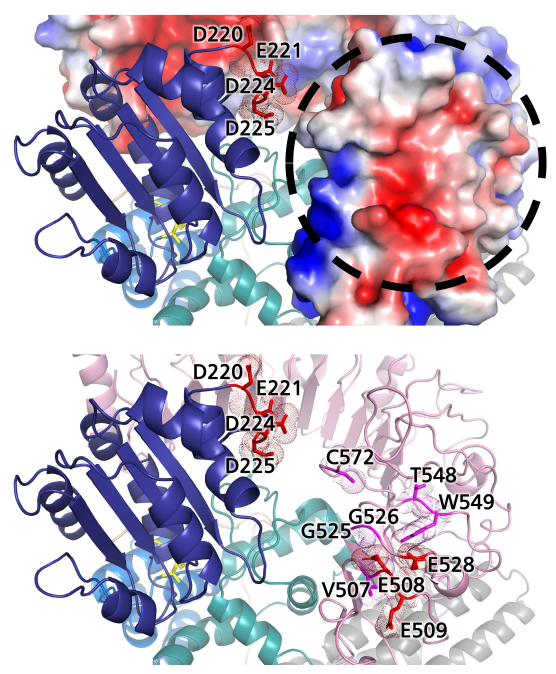

B.

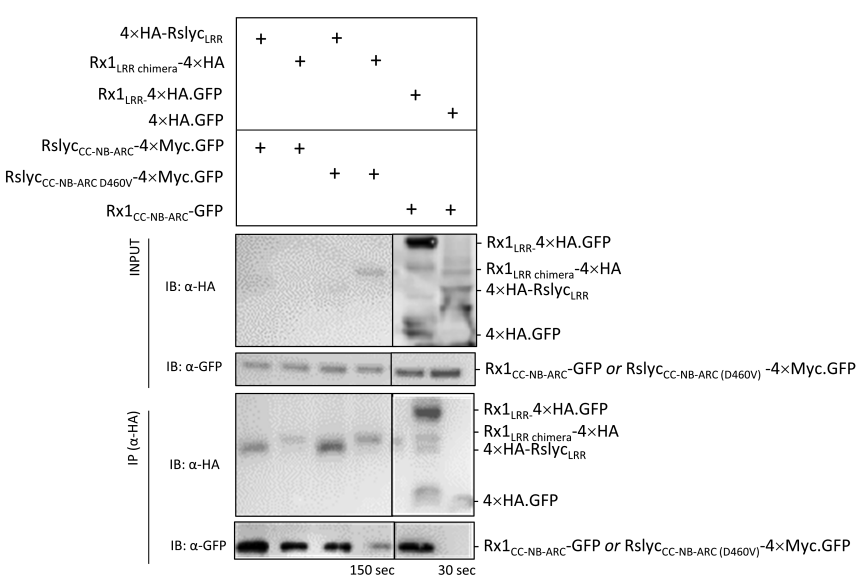

Rslyc
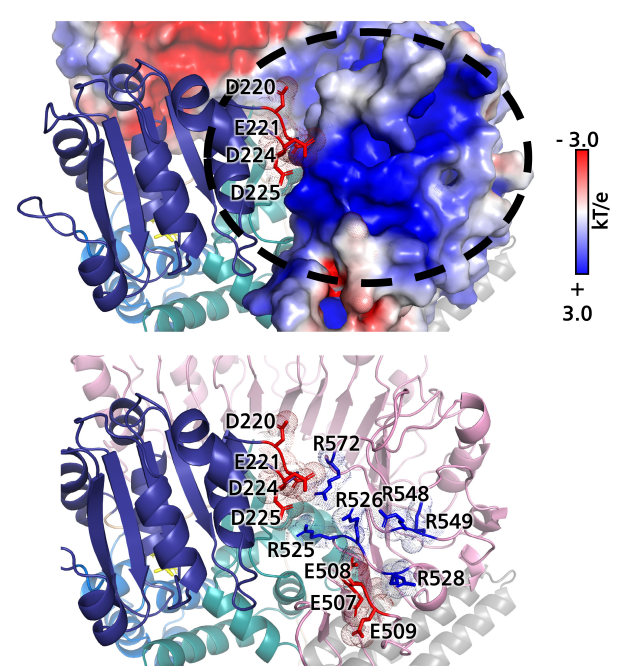

Fig. 5: A and B) Immunoblots of Co-IP investigating the interaction of Myc-tagged CC-NB-ARC domains of $\mathrm{R} \times 1$ (A) or Rslyc (B) with HA-tagged versions of the Rx1-LRR or chimeric LRR construct. Pull-down experiments were performed with infiltrated leaf extracts and using either $\alpha$-Myc or $\alpha$-HA magnetic beads. "+" indicates the presence of a construct in the infiltration combination. Exposure time used for the visualization of the blots are provided in B. C) Differences in surface electropotentials between Rx1- and Rslyc-LRRs in close proximity to the ARC2 as determined by APBS solver. The upper panels show the charge distribution in LRR-Patch P1+ (circled). Basic residues in Rslyc render this region to be more positively 
(Figure legend continued) charged. The polymorphisms mapped on the skeletal 3D-models of the LRR (pink) are provided in the lower panel. The figure additionally includes residues on the ARC2 domain (blue) in close proximity to the LRR. Color scheme depicted for the residues and surface potentials are according to their charge properties. Red = negative; Blue = positive; White $=$ neutral.

\section{The CC domain of Rslyc can associate independently with its LRR while the NB-ARC does not}

Our findings suggest that Rx1 and Rslyc differ in their how their subdomains may interact. To further probe at these differences, we systematically investigated complex formation between individual receptor subdomains. Prior studies have shown that the CC-NB-ARC of Rx1 can bind to its LRR in trans, although experimental evidence demonstrating that the CC or NB-ARC can interact independently with the LRR is lacking (Rairdan et al. 2008, Moffett et al. 2002). Therefore, we first assessed whether the NB-ARC and LRR fragments of Rslyc can physically associate in planta. To explore this, a Co-IP was performed using HA-tagged versions of Rx1, Rslyc, Gpa2 LRRs or the LRR

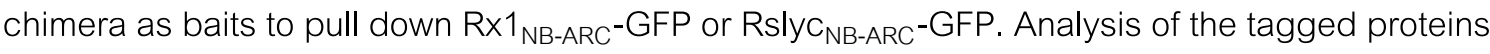
indicate that the NB-ARC domain of Rx1 co-precipitated with the various LRR domains but not the 4 XHA -GFP negative control (Fig. 6A1). Interestingly, however, the immunoblots show that the NB-ARC domain of Rslyc did not co-purify with any of the LRR-baits. These findings indicate that the NB-ARC regions of Rslyc and Rx1 differ in their capacities to bind LRRs. To ascertain whether these differences can be linked to intrinsic variation at the sequence/protein level, we mapped polymorphisms in these regions onto the Rslyc and Rx1 3D models in their ADP bound states. Our data show that the Rslyc-NB-ARC differs profoundly in its surface charges when compared to Rx1 (Supplementals Fig. S6). A remarkable difference lies in the ARC subdomain of Rslyc, which harbours more positively charged residues that render these regions to be more basic (circled region in Fig. 6A2). Imaginably, this may repulse the equally basic interface of the Rslyc-LRR and in turn, interfere with efficient complex formation as observed in our Co-IP.

We next investigated a potential CC/LRR interaction in Rslyc by carrying out an additional Co-IP assay using the $4 \times$ HA-LRR constructs as baits. Following the pull-down, the $\alpha$-GFP blots indicate that both the CCs of Rslyc and Rx1 were retained by the different LRR constructs (Fig. 6B1). Hereby, 
considerably greater amount of Rslyc CC co-purified with the LRRs compared to Rx1-CC, whose band fragment shows very weak band intensities following immunoprecipitation. Thus, in contrast to the NB-ARC domain, the CC domain of Rslyc can form a complex with the LRR domain irrespective of whether the LRR is derived from Rslyc or not. Structural modelling reveals that the CC domains of Rx1 and Rslyc are highly alike, both in terms of sequence homology and charge distributions. Though sequence polymorphisms do exist, very few have markedly different properties and occur within vicinity to the LRR. Notably, however, a Leucine residue on the CC that is unique to Rslyc (Serine in Rx1) lies nearby to the N-terminal end of the LRR. This substitution of Ser to Leu in Rslyc may increase the interface available for complex formation between the $\mathrm{CC}$ and hydrophobic residues in the LRR due to the larger side chain of the Leu residue (circles in Fig. 6B2). Assuming that the other mutations do not contribute, this would in turn be in line with the ability of the Rslyc-CC to interact with both its own and heterogenous LRRs, while the Rx1-CC does not.

Subsequently, we examined the impact of simultaneously expressing the three fragments on the separate domain interactions described above. In a similar vein, 4 XHA -tagged LRRs were used as baits to pull down the GFP tagged NB-ARC and CC domains. Combinations involving only the domains of Rx1 were expressed as control. Our data show that the addition of the NB-ARC of Rslyc does not disrupt the interaction between the CC and LRR (Supplementals Fig. S7). This observation, together with the absence of the Rx1 NB-ARC in the IP of the negative control, demonstrates that the pulldown was specific. Our data combined suggest that unlike Rx1, the CC and LRR domains of Rslyc can bind strongly, which is not dependent nor impacted by the NB-ARC domain.

Sequence and structural variations in the NB-ARC domains of Rx1 and Rslyc pinpoint to potential differences in the switch function

Aside from apparent differences in domain interactions, the variation in electrostatic charge and sequence polymorphisms described previously for the NB-ARC suggest that other functions of this domain may differ. This may include, for instance, discrepancies in the capacity of the Rx1 and Rslyc NB-ARCs to function as a switch. For example, a plausible scenario is that Rslyc may still be able to form a receptor complex and perceive a pathogen, but the NB-ARC core may be less competent to mount an effective defence response. This prompted us to investigate the structural 
fold of the Rx1 and Rslyc NB-ARC domains by remote homology modeling. From the 29 polymorphic residues in the NB-ARC, a number was observed to reside in functional motifs, namely: L153M in the hhGRExE, S191Y and G213S in the RNBS-A, and T336S in the GxP motif. Notably, the T336S substitution map within direct vicinity of the bound nucleotide (Fig. 7A1). Additional structural analysis indicates that a flexible linker connecting the CC to the NB-ARC also resides within proximity to the nucleotide-binding pocket. For both Rx1 and Rslyc, this linker is predicted to follow the groove formed by the charged surface of the NB-ARC domain (Fig. 7A2). The C-terminal end of the linker consists of a conserved region among Rx1, Rslyc and other plant $\mathrm{R}$ proteins that is composed of a "VG" (Valine, Glycine) motif and 1 or 2 hydrophobic residues upstream (Wróblewski et al. 2018). In the published ZAR1 cryo-EM structure, this region is located at the ADP binding site, and may therefore play an important role in occluding the release of ADP by acting as a barrier. The rest of the CC/NB-ARC linker in both Rx1 and Rslyc is rich in acidic amino acids, while the ZAR1 linker is rather neutral. These contrasting properties may affect the behaviour and fitting of the linker in the aforementioned groove, which may in turn impact the activity of the NB-ARC switch. 
A1.

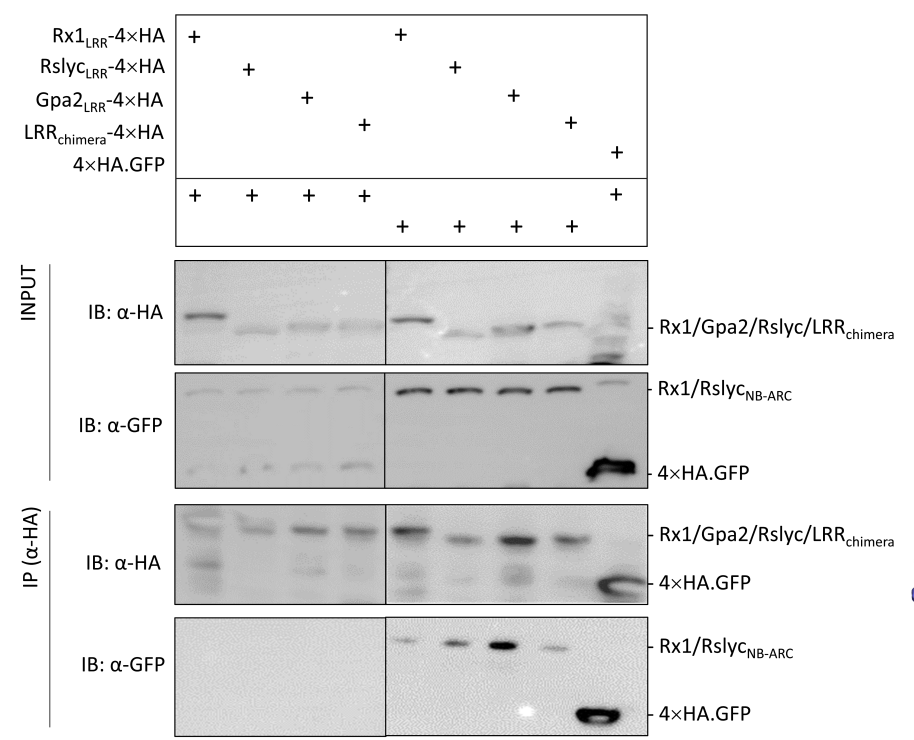

B1.

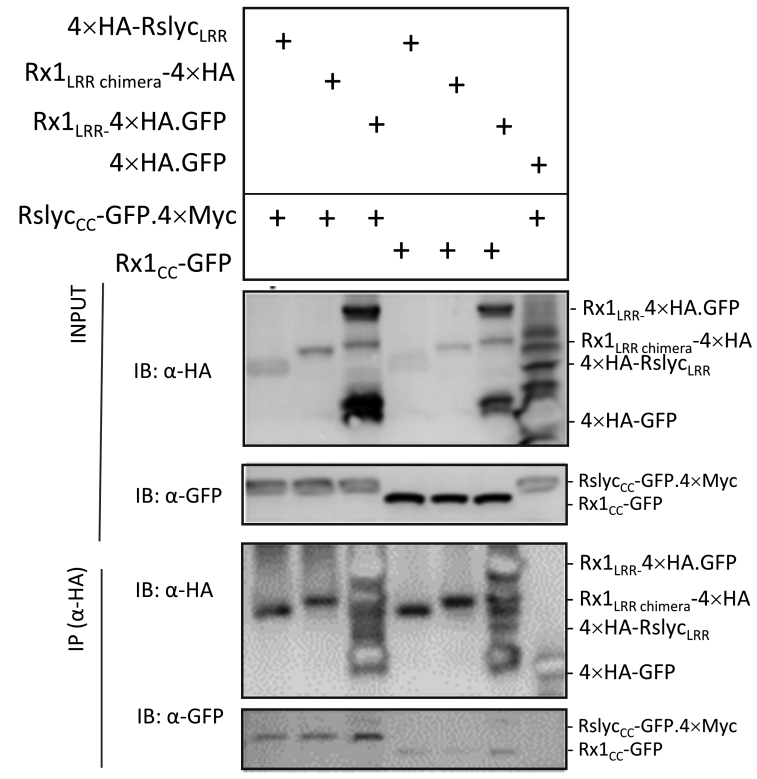

A2.

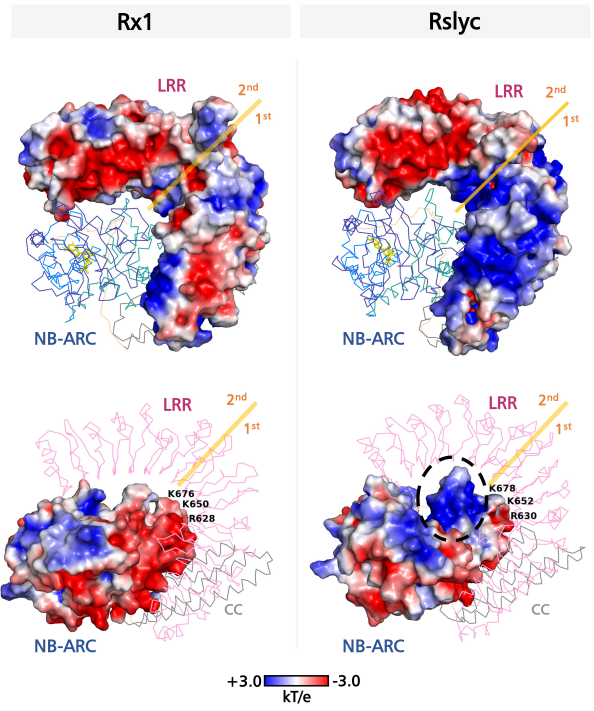

B2.

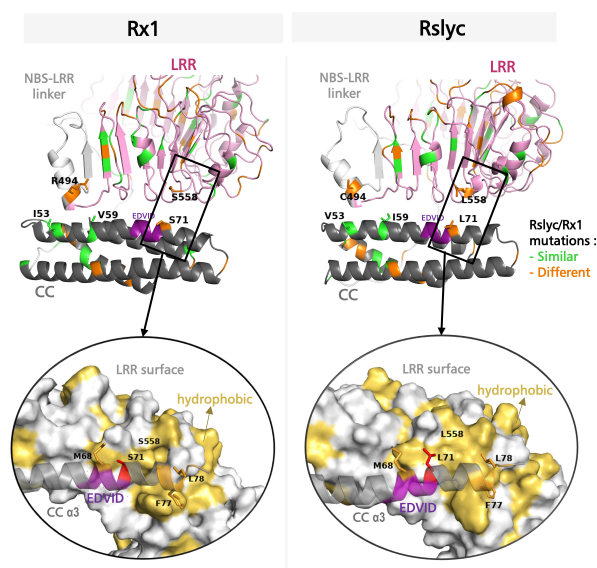

Fig. 6: Physical interactions of the Rslyc receptor subdomains. Immunoblots from pull-down experiments of various $4 \times$ HA-LRR variants of Rslyc, $R \times 1$ or a chimera thereof in combination with the NB-ARC (A1) or CC domain (B1) derived from Rslyc or Rx1. Immunoblots were performed using proteins extracted from agroinfiltrated leaf materials. "+" indicates the presence of a construct in the infiltration combination. A2) Estimation of electrostatic surface charge determined on homology models of the LRR and NB-ARC domains of Rx1 and Rslyc. The region discriminating the "two-halves" of the LRR as described in Slootweg et al. 2013 is indicated by the orange 
(Figure legend continued) line. Encircled region in the lower panel emphasizes the more positively charged patch unique to the Rslyc NB-ARC compared to Rx1. B2) Folding of the CC and LRR domains of Rx1 and Rslyc based on the ZAR1 template. Highlighted by the black boxes is the S71L substitution that is predicted to mediate the interaction between the CC and LRR domains. In circles are detailed projections of this interface, including an estimation of the hydrophobicity (yellow) of residues on the LRR surface.

Previously, the subcellular localization of Rx1 was shown to be affected by the status of its bound nucleotide (Tameling et al. 2010). Thus, we then determined whether polymorphisms observed at the sequence level can be reflected in differences on a cellular level. Confocal imaging indeed shows that RslyC $_{\text {NB-ARC }}-4 \times$ Myc.GFP has a different distribution pattern in the cell whereby its expression exhibits a greater signal in the cytoplasm compared to that of Rx1 or Gpa2 (Fig. 7B). In prior studies, it was shown that including the CC domain with the NB-ARC as a single construct affects the localization of the NB-ARC (Slootweg et al. 2010). It is, therefore, plausible that expressing Rslyc-NB-ARC with its CC in cis may allow the domain to acquire a similar localization pattern to that of Rx1. Interestingly, however, Rslyc $_{\mathrm{CC}-\mathrm{NB}-\mathrm{ARC}}{ }^{-4} \times \mathrm{MyC}$.GFP shows the same distribution as the NB-ARC domain alone. Apparently, the presence of the CC does not result in a shift in the subcellular distribution pattern of the NB-ARC. Such apparent differences in spatial distribution of $R \times 1$ and Rslyc (CC)-NB-ARCs hint towards functional differences of the NB-ARC module that affect its cellular localization and potentially, capacity of the core switch.

For $\mathrm{Rx} 1$, it has been reported that overexpressing the NB-ARC domain alone is sufficient to trigger a cell death response (Rairdan et al. 2008). We used this finding to determine whether the Rslyc-NB-ARC domain is signaling competent. Transiently overexpressing Rslyc NB-ARC $_{\text {n }}$ (Rslyc NB-ARC $-4 \times$ Myc.GFP) in $N$. benthamiana results in a weak cell death response that is significantly milder than observed for $\mathrm{Rx} 1_{\mathrm{NB}-\mathrm{ARC}}-\mathrm{GFP}$ (Fig. $7 \mathrm{C}$ ). Similar overexpressions of the Rslyc LRR or CC domains alone do not trigger cell death. Notably, immunoblotting assays show that RslyC $_{\mathrm{NB}-A R C}-4 \times$ Myc.GFP accumulates at a comparatively lower level to $\mathrm{R} \times 1_{\mathrm{NB}-\mathrm{ARC}}-\mathrm{GFP}$ (e.g. Fig. 6A1), which may, therefore, account for the weaker cell death output. However, as we have previously observed that the $\mathrm{CC}$ domain of Rslyc is also expressed at lower levels than $\mathrm{Rx} 1 \mathrm{CC}$, this suggests that expression levels may not be rate-limiting in our functional assays. 
Fig. 7 A1.

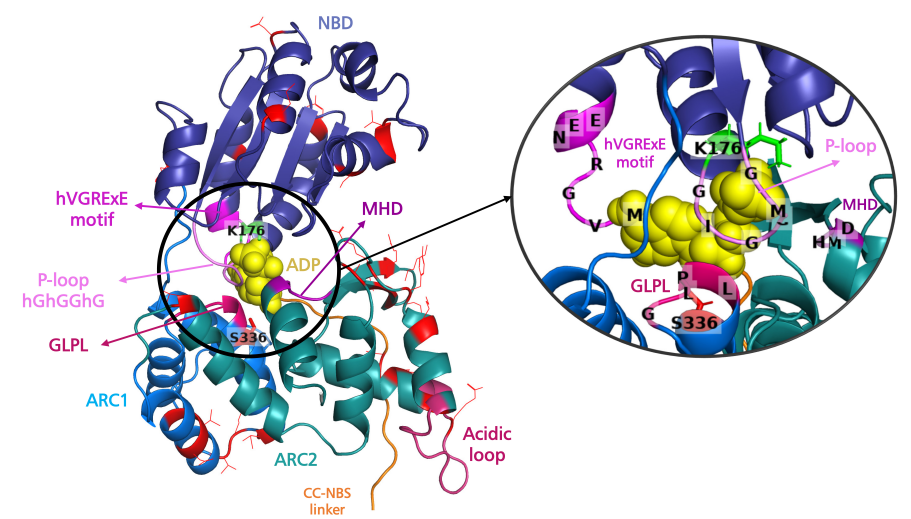

A2.
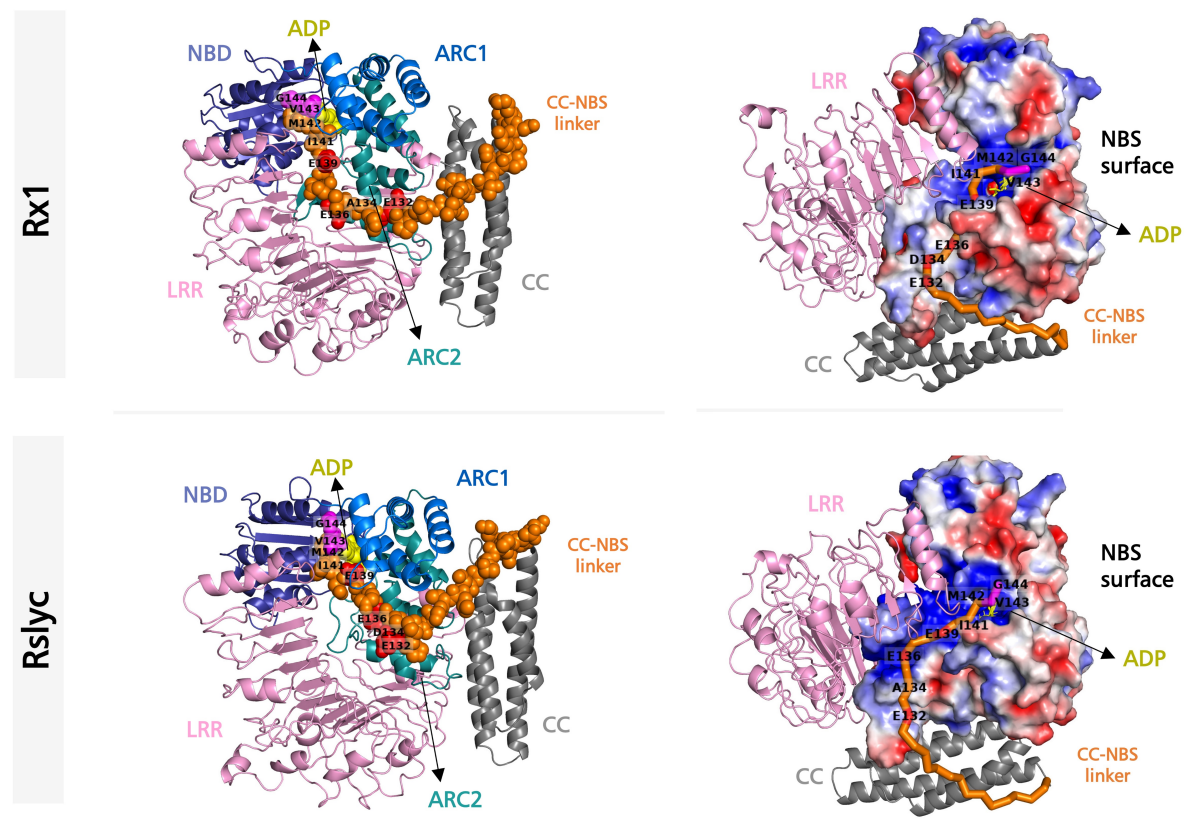
B.
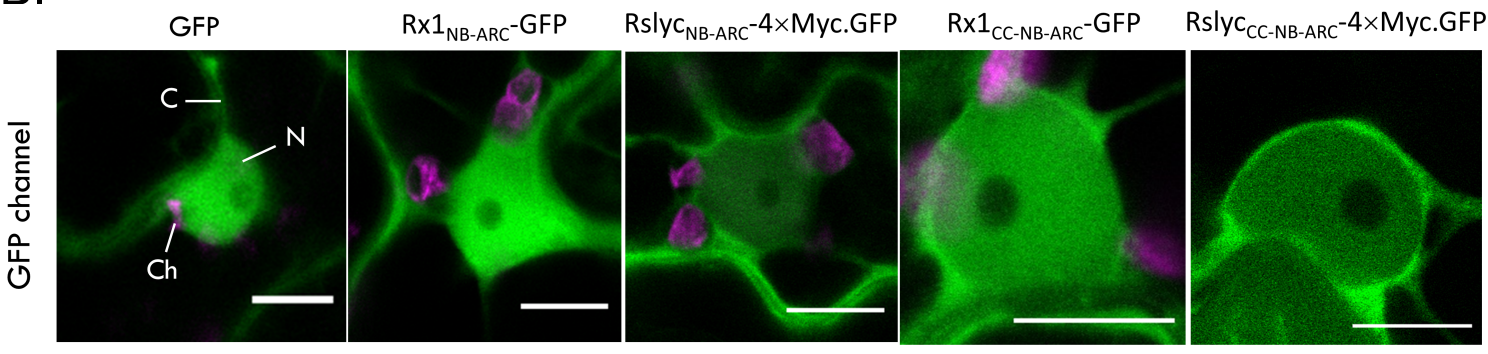

$2 \mathrm{dpi}$

C.

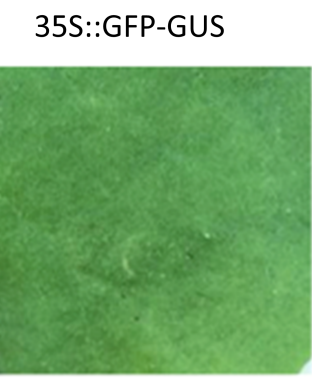

$35 \mathrm{~S}:: \mathrm{R} \times 1_{\mathrm{NB}-\mathrm{ARC}}$

35S::Rslyc NB-ARC $_{\text {AR }}$
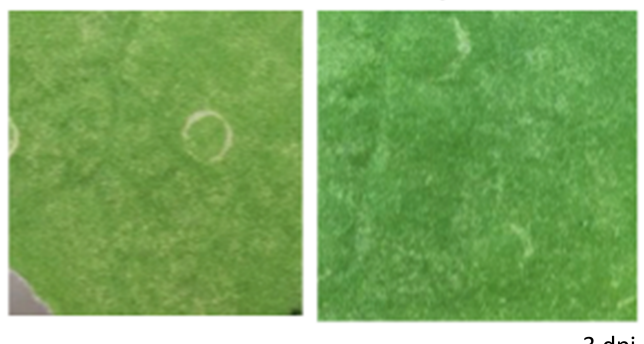

3 dpi

Fig. 7: Structural and functional differences in the NB-ARC domains of Rx1 and Rslyc. A1) 3D homology model of the Rslyc-NB-ARC domain (NB: dark blue; ARC1: blue; ARC2: cyan) with polymorphic residues (red) compared to Rx1. Conserved functional motifs are highlighted in magenta and the bound nucleotide is shown in yellow. A magnified image of the nucleotide binding pocket is shown in the circled region emphasizing the S336T residue (red) and kinase-dead mutant K176R (green) within close proximity to ADP. A2) A linker region (highlighted in orange) connecting the CC to the NB-ARC domain in Rx1 and Rslyc is predicted to form a hinge that contributes to preventing involuntary release of nucleotides. The linker is predicted to behave in accordance to its amino acid composition and enclosed environment formed by the groove and surface charges (shown in the second column) of the proximal NB-ARC domain. B) Detailed confocal images of nuclei and surrounding cytoplasm of $N$. benthamiana epidermal cells co-expressing mCherry with NB-ARC or CC-NB-ARC of Rx1, Gpa2 or Rslyc. Data shown is representative of three independent experimental repeats $\mathrm{C}$ ) Leaf images of $N$. benthamiana infiltrated by $A$. tumefaciens transiently expressing NB-ARC domain of Rx1, Rslyc or a GFP-GUS control indicating cell death lesions arising from the overexpression of the associated NB-ARCs. 


\section{DISCUSSION}

In the current research, we aimed to elucidate whether mechanisms regulating complex formation and receptor functions are conserved between homologous NB-LRR immune receptors originating from related crop species. To that end, we performed sequence-exchange studies between homologs of the $\mathrm{R} \times 1$ receptor from potato and tomato. First, we identified the tomato $\mathrm{Rx} 1$ homolog Rslyc, which has high sequence identity of $85 \%$ at the amino acid level with potato $\mathrm{R} \times 1$. We then used Rslyc as a proxy to elucidate molecular determinants defining the activity and function of plant CC-NB-LRRs. Data from structural modelling as well as trans-complementation assays in N. benthamiana suggest that co-operations between domains in Rslyc are inherently different from those characterized in $\mathrm{R} \times 1$ despite their overall sequence similarity. Whereas the $\mathrm{CC}$ domains can be interchanged freely between the two systems, this is not the case for the NB-ARC and LRR regions without losing functionality in trans. Though highly homologous, we therefore infer that functional Rx1 and Rslyc receptors have distinct domain requirements in trans and most likely, in cis to maintain the regulatory potential of the receptor in balance. This is further supported by the identification of polymorphic residues in the NB-ARC close to the nucleotide binding pocket, a structure that is essential for NB-LRR functioning as a molecular switch unit. Moreover, this central domain can affect the protein fold in the closed state, which could account for the observed differences in the subcellular distribution, interaction with the LRR as well as its sensitivity to trigger a cell death response. Based on these findings, we propose a working model highlighting differences in the modes of activation of $\mathrm{Rx} 1$ and Rslyc.

\section{Independent interaction of the Rslyc CC and LRR domains}

Compared to the highly variable C-terminal end of an NB-LRR, the N-terminus of this class of receptors constitutes a more conserved region. Yet, few studies have explored functional exchangeability of the CCs between homologous receptors. In our bioassays, we show that the CC domain of Rslyc confers both cell death and extreme resistance when co-expressed in trans with the NB-ARC-LRR of Rx1 (Fig. 2). We surmise that Rslyc and Rx1 CCs are, therefore, functionally similar despite differing in amino acids at 21 positions. This is consistent with the observation that Rslyc $\mathrm{CC}_{\mathrm{C}}$ has a similar distribution pattern in the cell as $\mathrm{R} \times 1 \mathrm{CC}$ and preserves the main functional motifs such 
as the EDVID and RanGAP2 binding sites. These findings suggest that Rslyc1 may form similar heterocomplexes for downstream signalling via the CC (Fig. 3B and 3C). Although the polymorphisms do not impede reconstitution in trans, they are solvent-exposed and as such, may still contribute to subtle differences in domain cooperativity. Remarkably, for example, we demonstrate that Rslyc $\mathrm{Cc}_{\mathrm{C}}$ can associate independently with its own and heterologous LRR domains in planta (Fig. 6B1). This contrasts to the CC domain of Rx1, which is thought to bind the NB-ARC and LRR simultaneously (Moffett et al. 2002). In line with previous reports, we only detected negligible interaction between $\mathrm{Rx} 1 \mathrm{cc}$ and the LRRs in the absence of the NB-ARC. Notably, differences in affinity between the interaction of Rslyc $\mathrm{CC}_{\mathrm{C}}$ and the various LRR domains may exist, although this cannot be distinguished accurately in our Co-IP settings. As the experiments were performed in vivo, it also raises the question if the complex formed could be mediated by another protein in the endogenous $N$. benthamiana background. These endogenous proteins may complement the absence of the NB-ARC domain in our study or the presence of other factors like RanGAP2, which contributes to the assembly of the receptor complex in planta. However, co-expression of the NB-ARC domain does not affect the CC/LRR interaction in Co-IP, thereby indicating that the interaction can occur regardless of its absence/presence (Supplementals Fig. S7). Furthermore, the CC and LRR constructs were overexpressed in our study, which most likely outcompetes such endogenous host proteins in complex formation.

Physical binding of the distal ends of an NB-LRR protein has been reported recently for the Arabidopsis ZAR1 in yeast and in planta (Baudin et al. 2020, Wang et al. 2019). This is supported by the three-dimensional structure of the receptor indicating that the CC and LRR domains are within close proximity in the closed ADP-bound state (Wang et al. 2019). In the presence of the cognate elicitor, ZAR1 reaches an intermediary state before being fully activated. In these states, the receptor undergoes structural re-arrangements, which may lead to unfolding of the CC from the LRR. The release of the CC/LRR interaction would be consistent with its postulated regulatory role in keeping the receptor inactive Baudin et al. 2020, Wang et al. 2019). The interaction site(s)/folds on the ZAR1-CC required for binding to the LRR and vice versa are not fully defined. However, mutations in the EDVID motif and helical turns compromise this association (Baudin et al. 2020, Wang et al. 2019). In the latter case, the primary sequences are not fully conserved in neither Rx1 nor Rslyc. The ZAR1-CC has a similar configuration of a four-helix bundle to that of Rx1-CC with the exception 
that it can dimerize upon activation and when overexpressed with an epitope tag (Baudin et al. 2017). Despite sharing the same 4 helical bundle architecture, the overall homology level between Rx1/Rslyc and ZAR1 CC domains is very low (17-19\% sequence identity) and unevenly distributed. We provide further insight from our homology modeling indicating that in the three receptor systems, a complementary acidic surface on the CC likely mediates the interaction with a basic patch on the LRR (Supplementals Fig. S8). However, in Rx1 and Rslyc, the basic LRR patch is larger, which may pinpoint to subtle differences in the folding of the CC relative to the LRR compared to ZAR1. This may in turn suggests that these receptors differ in the conformational changes and structural foldings they undergo during the resting, primed and activated states.

For Rslyc, it remains unknown whether the CC/LRR interaction observed has any genuine biological implications. This is relevant when considering that the combination of $\mathrm{Rs}_{\mathrm{lyc}} \mathrm{CC}_{\mathrm{C}}$ and $\mathrm{R \times 1}{ }_{\mathrm{NB}-\mathrm{ARC}-\mathrm{LRR}}$ is as functional as the Rx1 control (Fig. 2). In this context, although the CC and LRR may interact, it does not appear to influence functionality of the receptor in trans. Remarkably, we also demonstrate that Rslyc ${ }_{\mathrm{CC}}$ does not bind to the NB-ARC-LRR of Gpa2 in a Co-IP assay (Fig. 3B). One interpretation is that folding of the Gpa2 $2_{\mathrm{NB}-\mathrm{ARC}}$ can mask regions required for binding of the CC to the Gpa2-LRR. The specificity of the Rslyc $\mathrm{C}_{\mathrm{C}}$ for binding to the NB-ARC-LRR of Rx1 but not Gpa2 indicates that a degree of co-evolution exists between the $\mathrm{CC}$ domain and the distal ends of the receptor module in these systems.

Intrinsic differences in the NB-ARC domains of Rx1 and Rslyc

Although the NB-ARC domain of Rslyc constitutes the most conserved region when compared to $\mathrm{Rx} 1$, we demonstrate that it does not fully resemble its potato counterpart at a functional level.

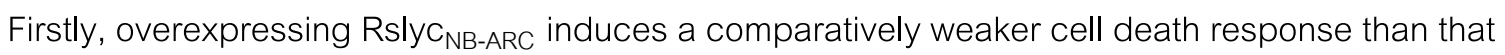
by $R \times 1_{\text {NB-ARC }}($ Fig. $7 C$ ). While we cannot fully exclude that this may be attributed to variation in expression levels, homology modelling and imaging studies hint towards intrinsic differences in the switch functions. Though it warrants more empirical proof, structural analysis suggests that this may refer to the nucleotide binding and/or DNA-binding properties of the NB-ARC. The former may be linked to variation in a CC-NB linker, which we postulate to block inappropriate exchange of ADP to ATP (Fig. 7A2). A prominent role of this intermediate linker region for CC-NB-LRR activity 
has been reported recently in a genome-wide functional study by (Wróblewski et al. 2018). The authors detailed that the CC-NB-ARC linker is required for 'partner selection' during multimerization and cell-death induction. An emerging, varied and significant role of the disordered linker should, therefore, be explored in future works.

We also demonstrate that unlike the CC, the NB-ARC domain of Rslyc does not associate independently with its LRR domain (Fig. 6A1). Consistent with this, Rslyc ${ }_{\mathrm{CC}-\mathrm{NB}-\mathrm{ARC}}$ also binds weakly to its own LRR and the chimeric version (Fig. 5B). These interactions are not sufficient in trans to give a cell death response (Fig. 4). Moreover, we identified polymorphisms and differences in charge distributions at the ARC2/LRR interface of $R \times 1$ and Rslyc that may account for differences in receptor assembly (Fig. 5C and 6A2). This coincides with a majority of the polymorphisms concentrating to the $\mathrm{N}$-terminal half of the LRR, pointing towards variation in the switch complex as opposed to recognition at the C-terminal end. As the ARC2/LRR compatibility is an important regulatory control, it is imaginable that weak interactions between the NB-ARC and LRR of Rslyc must be compensated elsewhere in the receptor module to prevent spontaneous activation. Given that the CC can associate independently with the LRR, we postulate that the CC/LRR interaction in Rslyc counterbalances the relatively weak binding between the NB-ARC and LRR.

To our knowledge, we provide first indications that the NB-ARC domain of $\mathrm{Rx} 1$ can associate with its LRR domain independently in planta. This has not been tested in previous studies, which made use of NB-ARC constructs in cis with the CC (Moffett et al. 2002, Rairdan et al. 2008). An independent NB-ARC/LRR interaction has also been documented for ZAR1, RPS5, and RPP1 where they are postulated to keep the receptor in an inactive state (Ade et al. 2007, Baudin et al. 2020, Qi et al. 2012). Unlike in Rslyc, efficient binding between the NB-ARC and $L R R$ in $R \times 1$ may also account for a better co-operation between the domains and nucleotide exchange. Interestingly, we show that binding of the Rx1 NB-ARC extends to heterologous LRRs including Gpa2, Rslyc and the chimeric LRR construct (Fig. 6A1). How do we then reconcile ubiquitous binding of the Rx1-NB with specificity of the CC-NB-ARC/LRR interaction reported in literature (Slootweg et al. 2013)? We speculate that the interdependency we hypothesized for the CC and LRR presents an additional layer of control for the successful reconstitution of a functional receptor molecule. On the one hand, electrostatic interaction between the NB-ARC and LRR intrinsically drives the initial physical assembly of receptor parts provided that complementary charges exist in these regions. Ultimately, however, a fully functional 
receptor unit is obtained when matching requirements between the CC and LRR are met. For $R \times 1$, the CC/LRR interaction is very weak but may be sufficient to avert aberrant complex formation arising from a mismatching CC-NB-ARC module. The model proposed is not entirely unlikely as Rairdan et al. 2008 has likewise postulated that activity of the NB core switch is fine-tuned by the combined actions of the CC and LRRs.

\section{A working model for the autoinhibition and activation of $\mathrm{Rx} 1$ and Rslyc}

From our data, we derive at a conceptual model (Fig. 8) whereby Rx1 and Rslyc may adopt an overall similar protein assembly but harbour subtle differences in the fitting and/or affinities of how the domains may interact. This may in turn impact the capacity of the receptor to adopt an open configuration upon activation and initiate downstream signalling (indicated by differently coloured nucleotides and thickness of arrows towards defence in Fig. 8C). Specifically, the relatively weak affinity of the NB-ARC/LRR interaction in Rslyc may allow sliding/inefficient fitting of the domains that impacts the overall fold of the receptor. In trans, this is reflected by the improper assembly of the receptor observed in our Co-IP assays. To compensate for this, we postulate that the CC-LRR interaction in Rslyc may contribute to allow the formation of an inactive receptor unit. Such a model highlights that some degree of interdependency between the CC/LRR ends may be required for regulating complex formation and switch functions. In $\mathrm{R} \times 1$, the relatively weak CC/LRR interaction is permissible as we demonstrate that the NB-ARC alone can associate with the LRR. From our data, we also attribute that differences in functionality between $\mathrm{R} \times 1$ and Rslyc may arise due to variation in the activities of the NB-ARC as a molecular switch. In the latter case, the NB-ARC of Rslyc may function less optimally due to a less favourable 'structural environment' to enable proper folding, associations and/or triggering of defence signalling. These factors combined may underpin differences in the capacity of Rslyc to recognize PVX and/or elicit an immune response.

Here, we illustrate that highly similar NB-LRRs from related crop species can have markedly different regulatory contact points between the receptor domains. As domain mismatches can yield a malfunctioning receptor, these findings also reflect a layer of functional constraint that shapes the evolution of new recognition specificities at R-gene clusters. In the case of Rslyc and Rx1, this constraint extends to multiple levels as discussed above. The strict functional constraints observed 
here contrast flexibility reported in earlier studies when exchanging the domains of the CC-NB-LRRs R3a and I2 (Ori et al. 1997, el-Kharbotly et al. 1994, Huang et al. 2005). These receptors localize to a syntenic region in chromosome 11 of tomato and potato, and mediate resistances to the fungus Fusarium oxysporum and oomycete Phytophthora infestans respectively. Targeting residues in the CC and NB-ARC of 12 can broaden recognition to P. infestans (Giannakopoulou et al. 2015). It is worth noting, however, that 12 has an existing capacity to mildly recognize the cognate effector of R3a, namely Avr3a. Intriguingly, some of the autoactivating mutations in the CC and NB-ARC of R3a are not directly translatable to expand the detection profile of I2. This pinpoints that although these receptors are more alike in how they need to be activated and elicit defence (when compared to Rx1 and Rslyc), subtle differences still exist to fine-tune their functions.

The identification of NB-LRRs homologs like Rslyc provides an impetus for synthetic NB-LRR engineering by serving as a template for the reconstitution of a functional $R$ gene in target crop species. Our data lays foundation for more in-depth molecular studies aiming to resolve specific residues that can be targeted for instance, by genome editing. Hereby, we anticipate that the modification of Rslyc for broadened recognition specificity should occur stepwise as reported by Harris et al. 2013. The first aims at adjusting intradomain interactions while the second focuses on the NB-ARC core to allow for a more sensitive version of the receptor. Notably, our studies do not address whether Rslyc has retained the recognition specificity for PVX. This could be investigated using a chimeric $R \times 1$ construct in which the C-terminal LRR fragment of $R \times 1$ is replaced by the complementary region of Rslyc. Furthermore, as the interaction assays are done in vivo, the current research also cannot formally rule out that other host factors specific to tomato are needed to mediate the functionality of Rslyc. 
A.

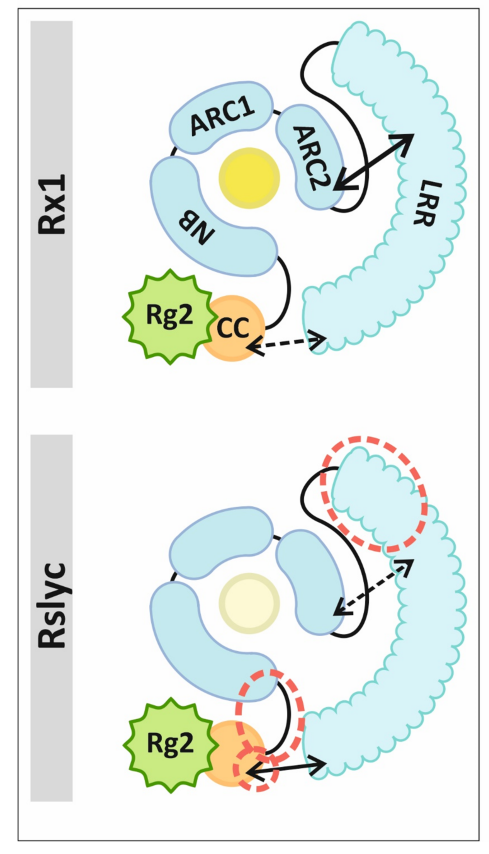

B.

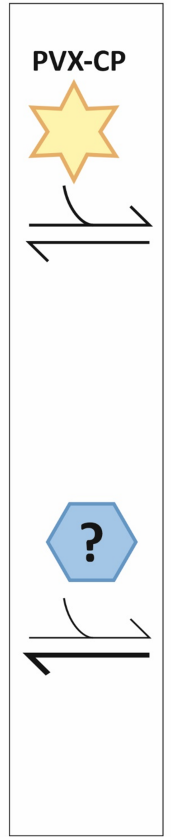

C.

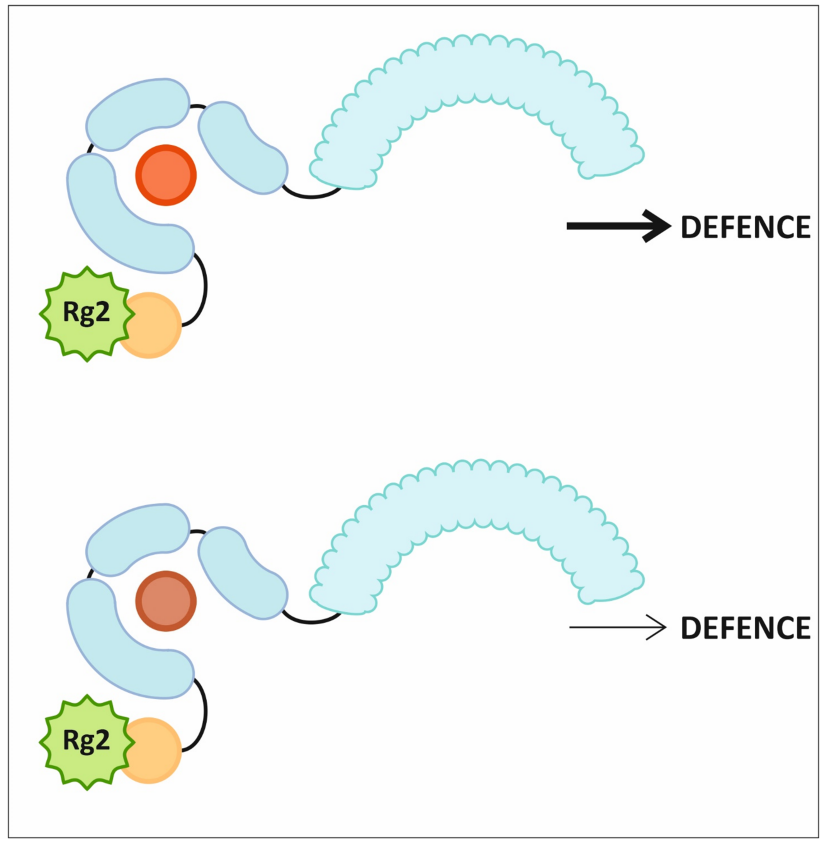

Fig. 8: Schematic diagram summarizing main functional differences between Rx1 and Rslyc based on studies performed in this research. Regions that exhibit different intradomain interactions and harbour polymorphisms that may be important on a functional level as pinpointed in our structural analysis are highlighted by dashed orange circles. A) In the OFF state, both receptors are subject to inter and intramolecular regulations that suppress immune activation until appropriate. With Rslyc, association between the NB-ARC/LRR domains is less efficient due to incompatibilities arising from the basic nature of the interface in both regions. We hypothesize this weak binding is in turn compensated by a CC/LRR interaction. The opposite is observed for Rx1 and these discrepancies may contribute to differences in how sensitive or easily the receptors can adopt an open configuration and prompt a defence response. Presence of the appropriate effector triggers a conformational change towards activation (B). In the case of Rslyc, the cognate effector is unknown. However, recognition may nonetheless be sufficient to relieve inhibitory forces allowing for receptor activation and downstream responses (C). Based on polymorphisms in the NB-ARC, we also speculate intrinsic differences in the switch capacity of this domain, further affecting interactions with adjacent regions and efficacy of triggering downstream defence reactions. These variations are depicted in the different colour intensities of the bound nucleotide. 


\section{MATERIALS AND METHODS}

Identification of Rslyc as a homolog of Rx1 from tomato

To identify the closest homolog of Rx1 in Katinka, we performed in silico screening using BLAST (Basic Local Alignment Search Tool) analysis in the program Geneious 7.1.7. A selection of candidate matches was made based on degree of homology including the number of BLAST hits and query coverage (length).

\section{Cloning of bacterial constructs}

Individual Rslyc CC, NB-ARC, LRR and CC-NB-ARC domain fragments were synthesized (Geneart) with flanking restriction sites as indicated in Supplementals Fig. S3. For generating the chimeric $L R R$ construct, the $\mathrm{N}$-terminal fragment of Rslyc LRR was fused with the C-terminal Rx1-LRR region by ligation using the complementary restriction site Xhol. All fragments were subcloned initially into the vector pRAP+ for addition of the appropriate epiptope tag (either a $4 \times$ Myc4-GFP or $4 \times \mathrm{HA}$ tag) (Schouten et al. 1997). Finally, subcloning was performed by Ascl/Pacl digestion into the binary plant expression vector pBIN+ between the Cauliflower Mosaic Virus (CamV35S) promotor and Tnos terminator (van Engelen et al. 1995). The generated pBIN+ constructs were transformed into Agrobacterium tumefaciens strain MOG101 to enable transient overexpression analysis in N. benthamiana by agroinfiltration.

\section{Transient expression by Agrobacterium tumefaciens transient assay}

Agrobacteria harbouring the appropriate constructs for agroinfiltration assays were prepared as reported in Slootweg et al. 2013. Briefly, A. tumefaciens was grown overnight in Lysogeny Broth (LB) medium supplemented by the relevant antibiotics $(25 \mu \mathrm{g} / \mathrm{ml}$ Rifampicyn and $5 \mu \mathrm{g} / \mathrm{ml}$ Kanamycin) at $28^{\circ} \mathrm{C}, 250$ rotation per minute (rpm). For agroninfiltration assays, the grown bacteria were spun down and resuspended in MMAi infiltration medium (2 g/L sucrose, $3.90 \mathrm{~g} / \mathrm{L} 2-(\mathrm{N}$ morpholino)-ethanesulfonic acid, $5 \mathrm{~g} / \mathrm{L}$ Murashige and Skoog salts and $200 \mathrm{mM}$ acetosyringone, Sigma Aldrich). $\mathrm{OD}_{600}$ measurements were taken and the bacteria was diluted to an $\mathrm{OD}_{600}$ value 
of 0.5 for all assays. The final suspensions were introduced on the underside side of the leaf by infiltration using a needleless syringe. Infiltrated plants were kept under standard greenhouse conditions ( $23^{\circ} \mathrm{C}$ with 18 hours of light and 6 hours of dark cycle). Cell death measurements were scored between 1-3 dpi whereas infiltrated leaves were harvested at 2-3 dpi for immunobloting and imaging studies. Infiltrated leaves for viral quantification by DAS-ELISA were harvested at $5 \mathrm{dpi}$.

\section{Cell death assay}

The degree of cell death was assessed at two levels, namely by visual scoring and chlorophyll assay. Visual assessment was based on a scale as described in Slootweg et al. 2013 ranging from 0 (no cell death) to 5 (extreme cell death response). Objective quantification by the chlorophyll assay was carried out in parallel as reported previously (Slootweg et al. 2018). Briefly, $12 \mathrm{~mm}$ leaf discs of infiltrated spots were harvested and incubated overnight at $37^{\circ} \mathrm{C}$ with $500 \mu \mathrm{l}$ chloroform at constant rotation (200 rpm). On the following day, the supernatant from each sample was measured at 655 $\mathrm{nm}$ and $450 \mathrm{~nm}$ using a microplate reader (BioRad model 680). The resultant values were subtracted from those of an uninfiltrated control to indicate the level of chlorophyll loss.

\section{Viral quantification by PVX-DAS ELISA}

The level of viral accumulation on infected leaves was determined by a Double Antibody Sandwich-Enzyme Linked Immunosorbent Assay (DAS-ELISA) as described in prior studies (Slootweg et al. 2018). Briefly, four $3 \mathrm{~mm}$ leaf discs were harvested from each infiltrated spot and lyzed in extraction buffer (50 mm phosphate buffer, $\mathrm{pH}$ 7) using the Tissue lyzer (Qiagen) for 30 seconds at $30 \mathrm{~Hz}$. The resultant supernatant was incubated in a plate coated with the primary antibody that is subjected against the PVX-CP (Prime Diagnostics) for 2 hours at $37^{\circ} \mathrm{C}$. The samples were subsequently incubated with a secondary conjugate antibody with alkaline phosphatase for 1 hour at $37^{\circ} \mathrm{C}$ before being exposed to a substrate solution containing 4-Nitrophenylphospphate. The absorption was measured at $405 \mathrm{~nm}$ with a reference filter of $655 \mathrm{~nm}$. 


\section{RNA isolation and cDNA synthesis}

RNA was isolated from $50 \mathrm{mg}$ of leaf materials using the The Maxwell囚16 LEV simplyRNA plant purification kit (Promega, 2014) according to the standard protocol provided by Maxwell囚. Following this, single-stranded cDNA was synthesied using the GoScriptTM Reverse transcription system (Promega) according to the manufacturer's protocol.

\section{Protein isolation and immunodetection}

Proteins were extracted from 100 -110mg of infiltrated leaf material as described previously (Slootweg et al. 2018). Briefly, leaf discs were resuspended in extraction buffer (1 mM EDTA, $150 \mathrm{mM} \mathrm{NaCl}, 50$ $\mathrm{mM}$ Tris- $\mathrm{HCl}, \mathrm{pH} 7.5,10 \%$ glycerol, $2 \%$ polyvinylpolpyrrolidone, and $0.5 \mathrm{mg} / \mathrm{mL}$ pefabloc SC protease inhibitor [Roche], 10 mM DTT) and lysed using the Qiagen Tissuelyzer II (15 Hz for 1 minute). The ground material was pelleted by centrifugation at $16 \times 000 \mathrm{~g}$ for $10 \mathrm{~min}$ and the supernatant was then run through a G-25 Sephadex column. The resultant eluate was subsequently collected, to which $4 x$ NuPage LDS sample buffer with 1M DTT (Invitrogen) was added. SDS-PAGE was then performed to separate the proteins using a NuPage 12\% Bis-tris gel (Invitrogen) run in 1XMOPS buffer. The BioRad pre-stained All Blue Protein Standard was run in parallel as marker. The separated proteins were then transferred to a PVDF membrane by Western blotting using the Turbo blot apparatus (Invitrogen) and recommended transfer buffer (supplemented with 10\% methanol) for a duration of $15 \mathrm{~min}$ at a constant volt of $25 \mathrm{~V}$. Immunodetection was performed by incubating with the membranes overnight in blocking buffer (5\% milk powder in PBST) supplemented with the appropriate antibodies depending upon the tagged-construct (GFP tags with $\alpha$-GFP/HRP (Abcam AB 290); Myc tags by Goat/c-myc (Abcam 9132) and Donkey anti goat Jackson (705-035-147) and HA tags with Rat/HA (Roche 12013819001)). Visualization of protein signal was performed using the G:Box imaging system (Syngene) following addition of the substrates Supersignal West Femto and Dura (Techno Pierce). Following visualization, the blots were stained with Coomassie brilliant blue (CBB) for indication of loading control by the RuBisCO proteins. 


\section{Co-Immunoprecipitation assays}

For protein-protein interaction studies by Co-IP, samples were harvested and extracted as detailed above. Prior to pull-downs, supernatant of the protein extracts was pre-cleared using a polyclonal Mouse IgG agarose (Sigma A2909) for 30 minutes at $4^{\circ} \mathrm{C}$ with constant, gentle rotation. Immunoprecipitation experiments were done by incubating the pre-cleared samples with magnetic $\mu$ MACS $\alpha$-HA or $\alpha$-Myc microbeads overnight at $4^{\circ} \mathrm{C}$ with gentle rotation. The following day, the samples were eluted through a seperation magnetic column ( $\mu$ MACS), separated on SDS-PAGE, immunoblotted and detected as described above.

\section{Localization studies by confocal microscopy}

Subcellular localization studies by confocal laser scanning microscopy was performed using the Zeiss LSM 510 confocal microscope (Carl Zeiss) under conditions and settings as described by Slootweg et al. 2010. For GFP measurements, leaf samples were excited using a $488 \mathrm{~nm}$ Argon laser and the emitted light was detected using a 505-550 nm band-pass filter. Autofluorescence from the chloroplasts was excited with the same laser source and detected through $650 \mathrm{~nm}$ long-pass filter. Images acquired were analysed using the Fiji (ImageJ) software for measurements of intensity ratios (Schindelin et al. 2012).

\section{Structural modelling}

Both Rslyc and Rx1 sequences were first profiled for a variety of structural and functional features including secondary structure, relative solvent accessibility and intrinsically disorder, with predictions performed with RaptorX-property (Wang et al. 2016), Psipred (Buchan et al. 2019), Jpred4 (Drozdetskiy et al. 2015), Spot-1D (Long et al. 2019) and Scratch (Cheng et al. 2005) software collections. Potential LRR motifs were in a first step identified with LRRpredictor while final repeat delineation was manually refined based of the above sequence profiling when motif probabilities were low or multiple motifs were detected within a given repeat range (Martin et al. 2020). Full length homology models were build for Rslyc and Rx1 starting from ZAR1 cryo-EM structure in the auto-inhibited ADP-binding monomeric state (PDB: 6j5w ) and from the potato Rx1 
crystal structure of the CC domain (PDB: 4m70), using Modeller v9.17 suite (Webb et al. 2016). The loop between helices $\alpha 2$ and $\alpha 3$ was missing in crystal structure - that was modeled to fit the CC structure constaints and minimise all sterical conflicts. As the CC/NB-ARC linker of Rslyc/Rx1 is 10aa shorter than in ZAR1 this region was modelled according to template over its C-ter region (aa 127 - 145) while the first 10 aa stretch (aa 117 - 126 ) was generated ab-initio and further optimized to adapt to the local structural environment. The NB-ARC and LRR domains were further modelled according to ZAR1. Finally, the acidic tail present in Rx1 was not included in the proposed homology model due to high uncertainties of its folding and orientation with respect to the rest of the protein. The initial models were refined thought iterative steps of energy minimization, simulated annealing and short molecular dynamics (MD) simulation using Modeller v9.17 (Webb et al. 2016) and Gromacs v2019.5 (Abraham et al. 2015). The stereochemical, geometrical and structural features quality assessment of the models was performed using Molprobity (Williams et al. 2018) and SAVES v5. The structural stability of the overall models was further investigated by longer MD simulations (50 ns) in explicit solvent consisting in water TIP3 and compensating ions using Gromacs (Abraham et al. 2015) and Charmm36-2019 forcefield (Huang et al. 2017, Huang et al. 2013) .

\section{Statistical analysis}

Data from cell death assays and confocal imaging were checked initially for normal distribution using the Shapiro-Wilk test before testing for statistical significance using ANOVA and a Tukey post-hoc analysis using the statistical program R (Team 2008). In the text, asterisks indicate statistical significance $\left({ }^{*} \alpha \leq 0.05\right.$ and $\left.{ }^{* *} \alpha \leq 0.01\right)$ whereas ns represents the absence thereof.

\section{ACKNOWLEDGEMENTS}

The following project was funded by the support of the Dutch Top Technology Institute Green Genetics (5CFD051RP), Dutch Technology Hotel grant and the Dutch Technology Foundation STW and Earth and Life Sciences ALW (STW-GG 14529), which are part of the Netherlands Organization for Scientific Research (NWO). We are also grateful for the technical assistance of Irene van Grinsven and Roy Toevank during the identification and isolation of Rslyc gene from the tomato cultivar Katinka cherry as well as viral disease assays performed in tomato and potato. 


\section{SUPPLEMENTALS}

Supplemental Fig. S1. Tomato cultivar Katinka Cherry encodes a full-length homolog of Rx1. Multiple sequence alignment and secondary structure prediction of Rslyc compared to ZAR1 and Rx1-like proteins. Separate subdomains are highlighted in colours according to the following scheme: blue $=$ CC, grey $=$ NB-ARC, LRR $=$ green. Highlighted in boxes are predicted LRR motifs that were deduced according to the approach described in materials and methods.

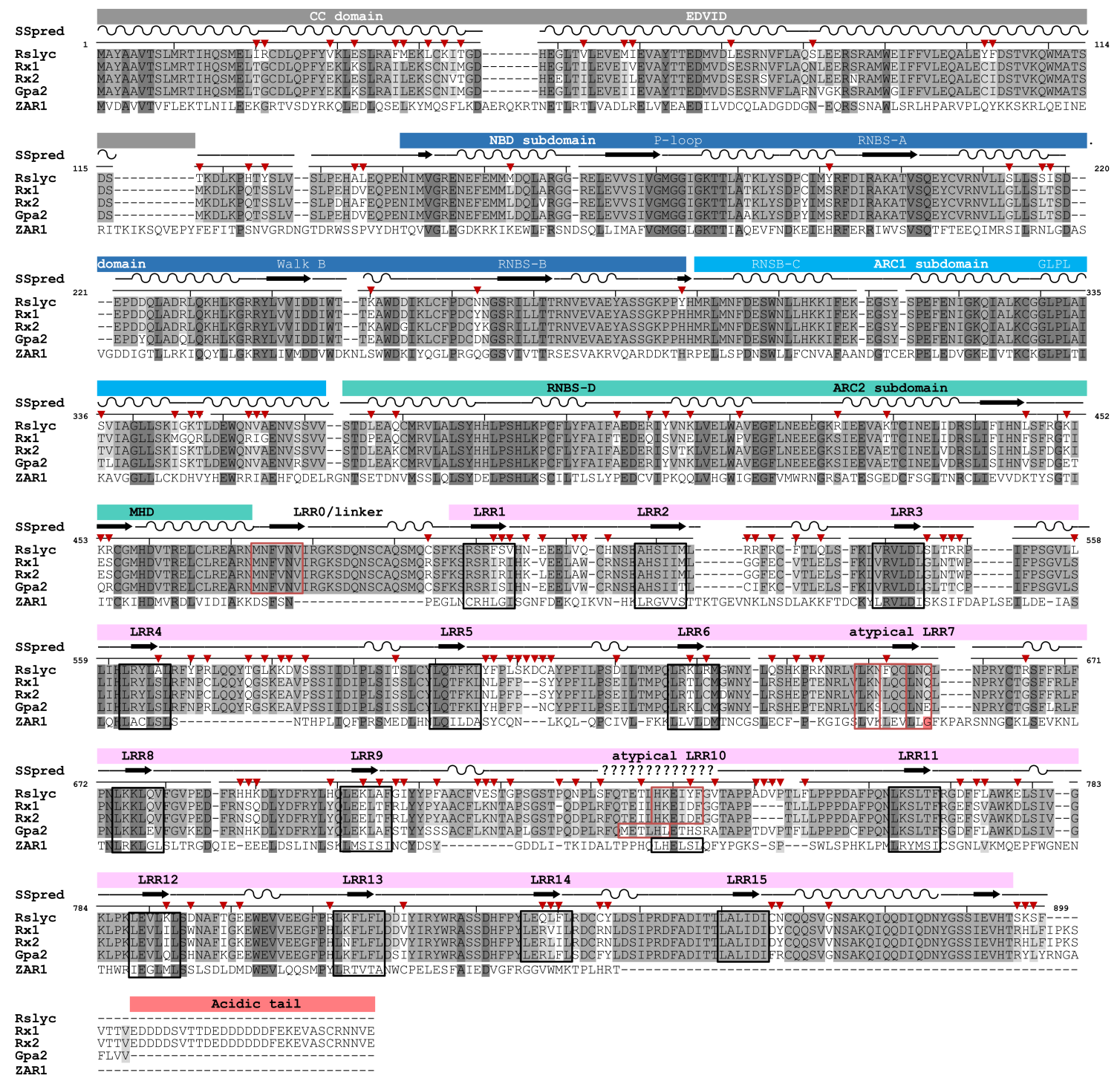


Supplemental Fig. S2. Tomato cultivar Katinka Cherry is susceptible to PVX-UK3 and PVX-HB. Barchart representing relative PVX levels in tomato or potato plants at $3 \mathrm{dpi}$. Viral load in infected leaf tissue material was quantified by DAS-ELISA. SH refers to the resistant cultivar background (harbouring Rx1 in the background) whereas Alisa Craig and LA2695 are two additional susceptible tomato controls. Error bars represent standard error of the mean.

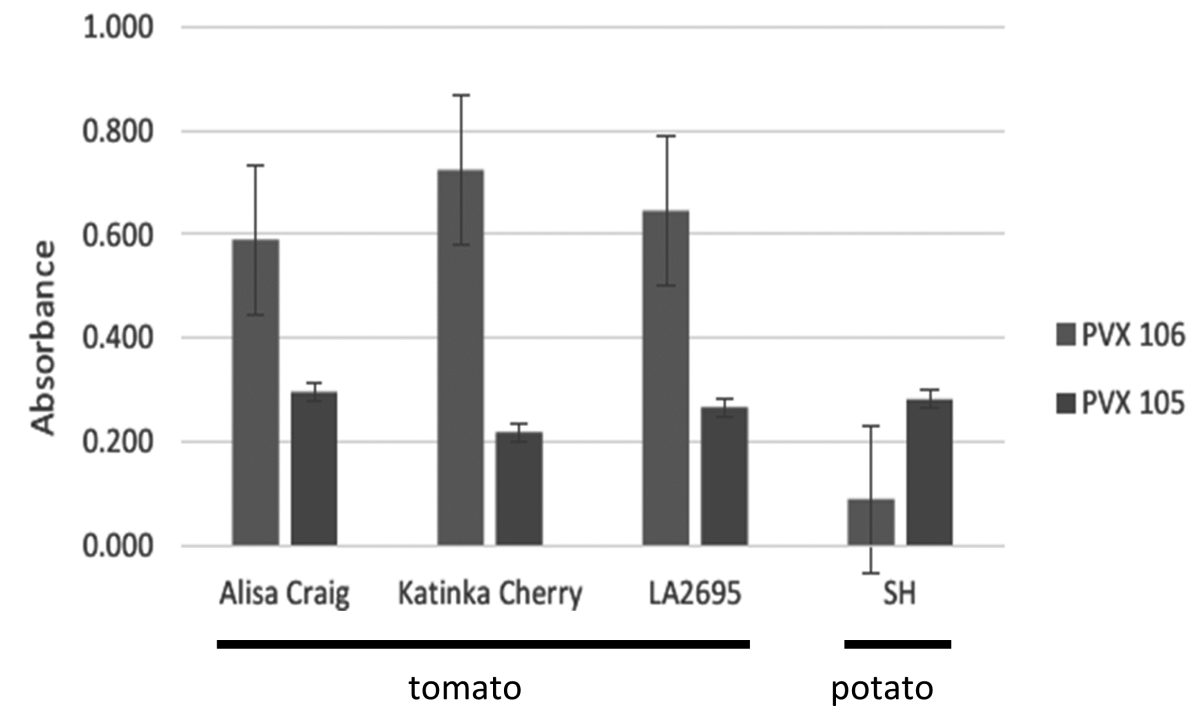




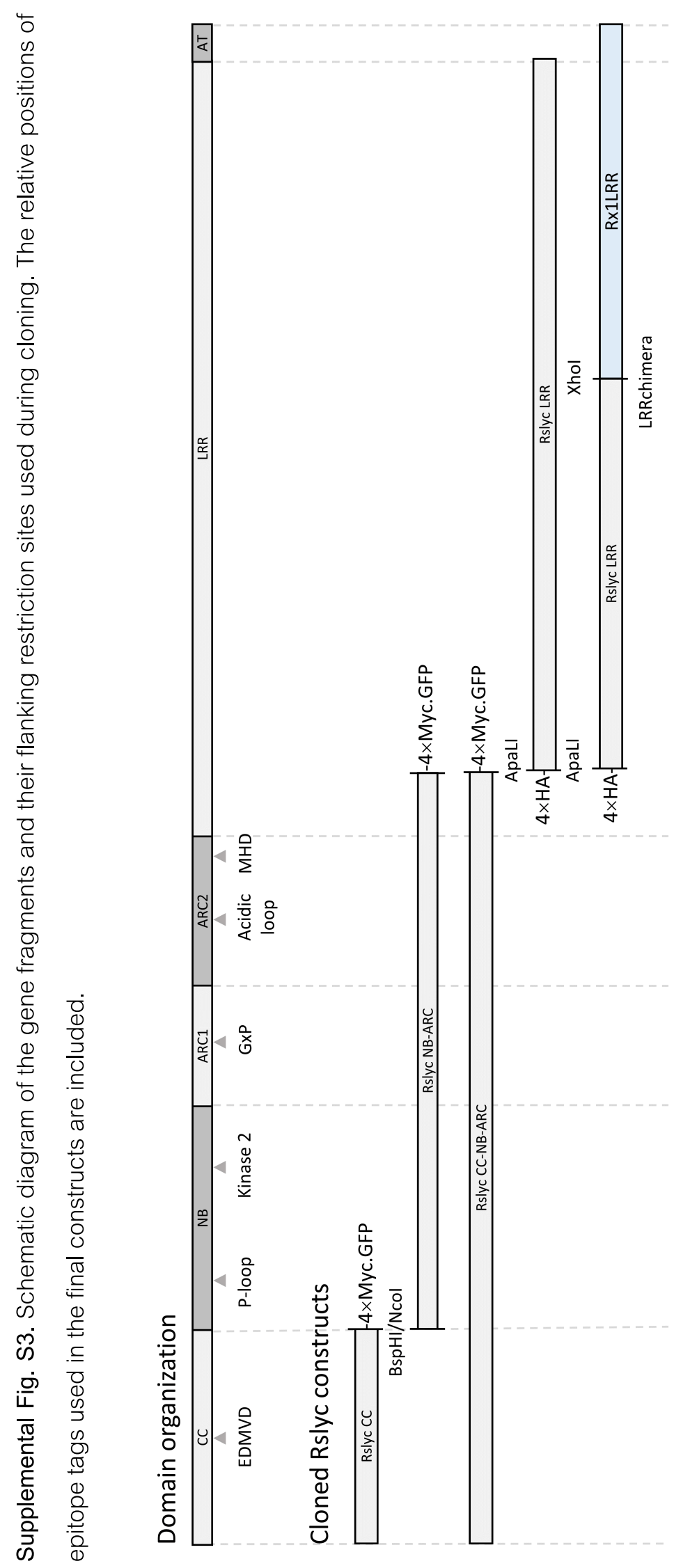


Supplemental Fig. S4. Full-length homology models of Rx1 (A) and Rslyc (B) in their ADP bound states based on the ZAR1 template and available crystal structure of the Rx1 CC domains. Domains are indicated as follows: LRR (pink), ARC2 (light blue), ARC1 (blue), NB (dark blue), CC (grey), and CC-NB linker (orange). The bound nucleotide is depicted in yellow.

\section{$\mathrm{R} \times 1$}

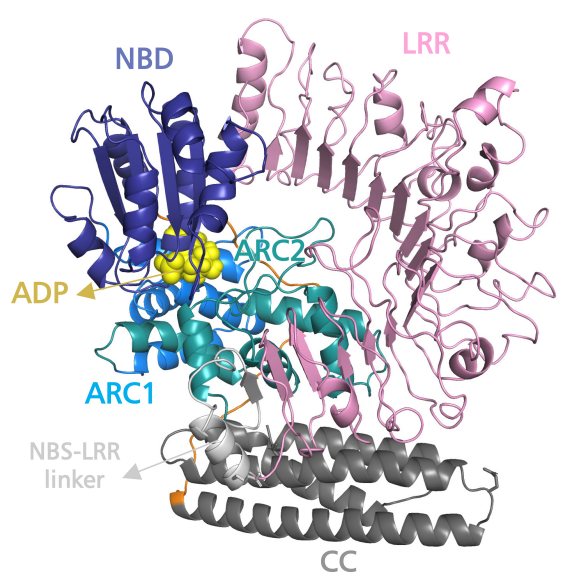

Rslyc

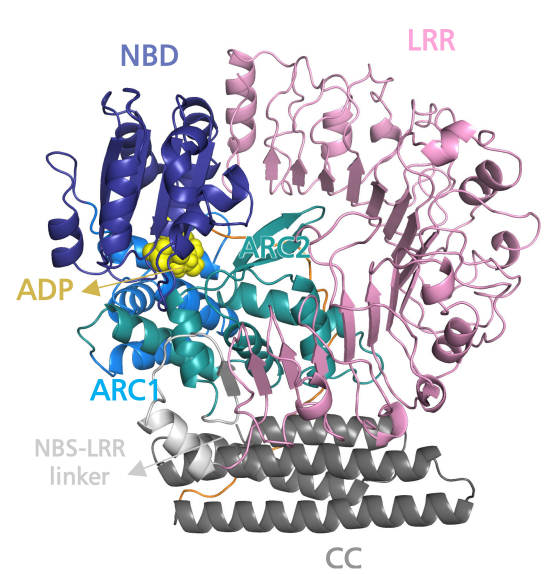


Supplemental Fig. S5. Differences in charge patches of the Rx1 and Rslyc LRR domains. A portion of the first patch $(\mathrm{P} 1+)$ is in contact with the ARC2 region. The second patch (P2+) of Rslyc is located far from the predicted CC-LRR interaction site but encompasses striking differences when compared to the other Rx1-like homologs. While in Rx1 and Gpa2 there are also several positively charged residues within this area, they are mixed with acidic and neutral counterparts. In Rslyc, however, some counterpart residues are mutated to Arganine or Lysine, generating an additive effect on the overall electrostatics of this region. No considerable differences were observed in Patch 3 (P3-) of Rx1 and Rslyc.
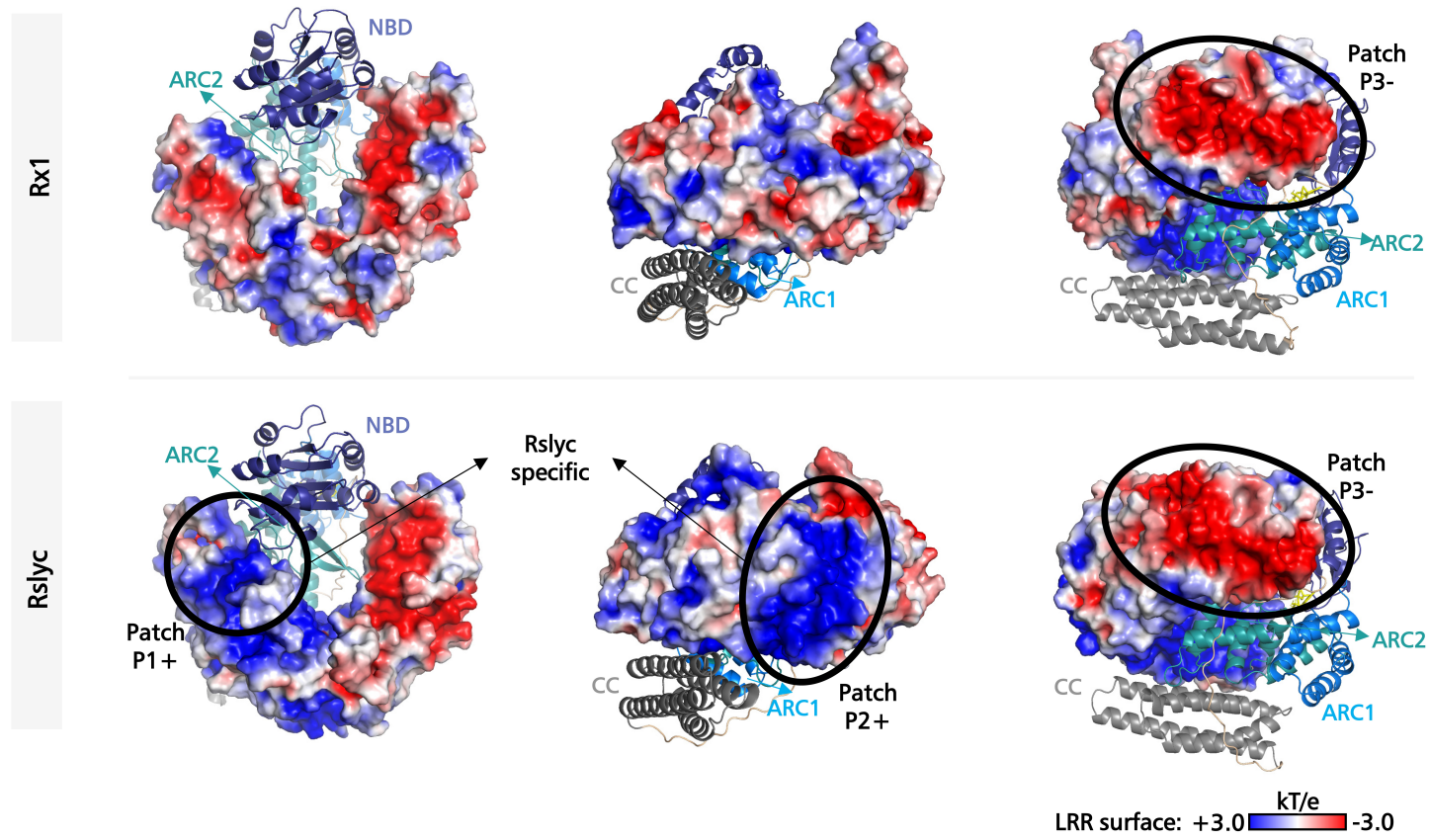
Supplemental Fig. S6. Variation in surface charges of the Rx1 and Rslyc NB-ARC domains. The Rslyc NB-ARC lacks charged patches otherwise present in Rx1 on the interfaces close to the CC and exterior side of the domain (first two columns). On the other hand, the region of the NB-ARC domain facing towards the LRR interface is prominently more basic in Rslyc (third column). The exact regions encompassing these main differences between the two receptor systems are highlighted in black circles in the figure.
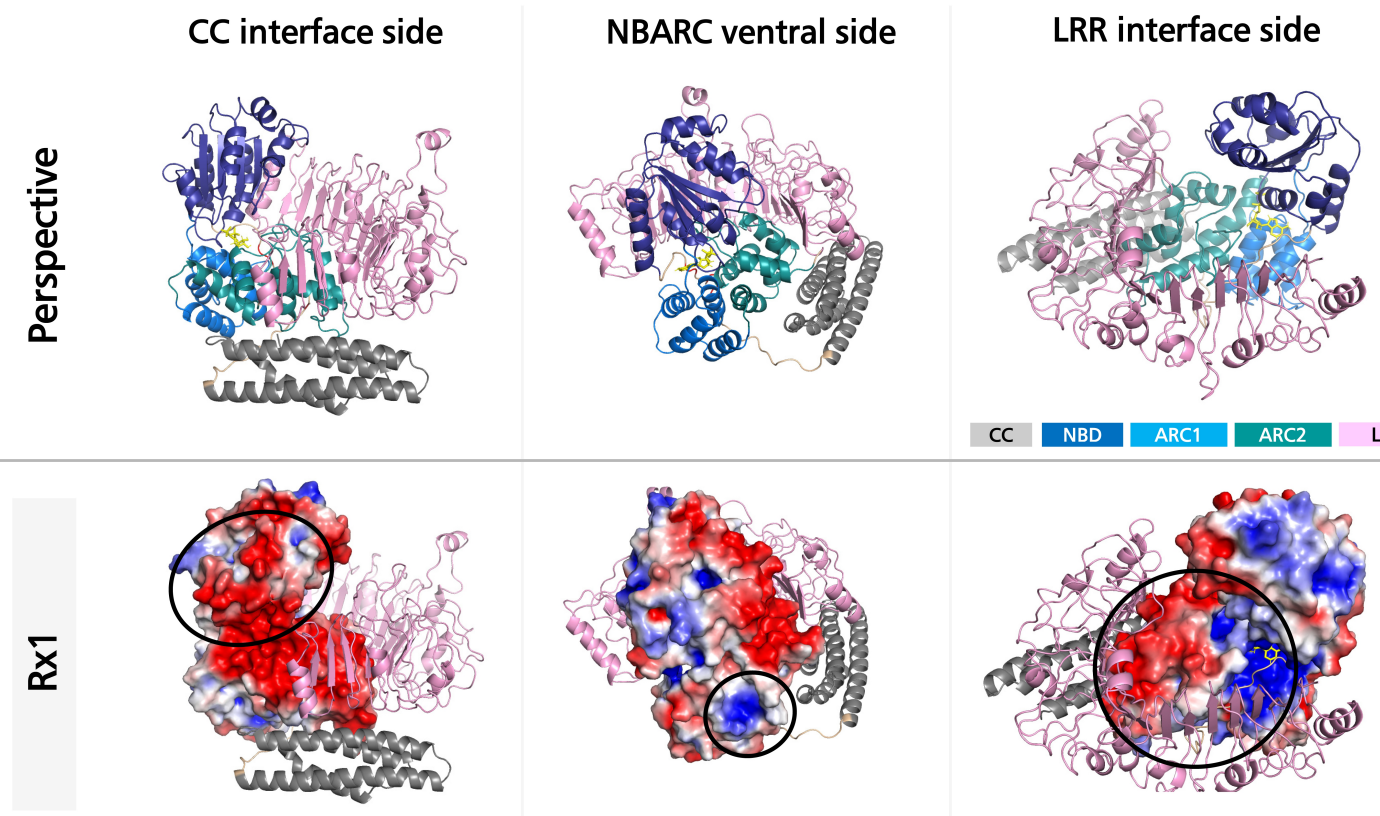

CC
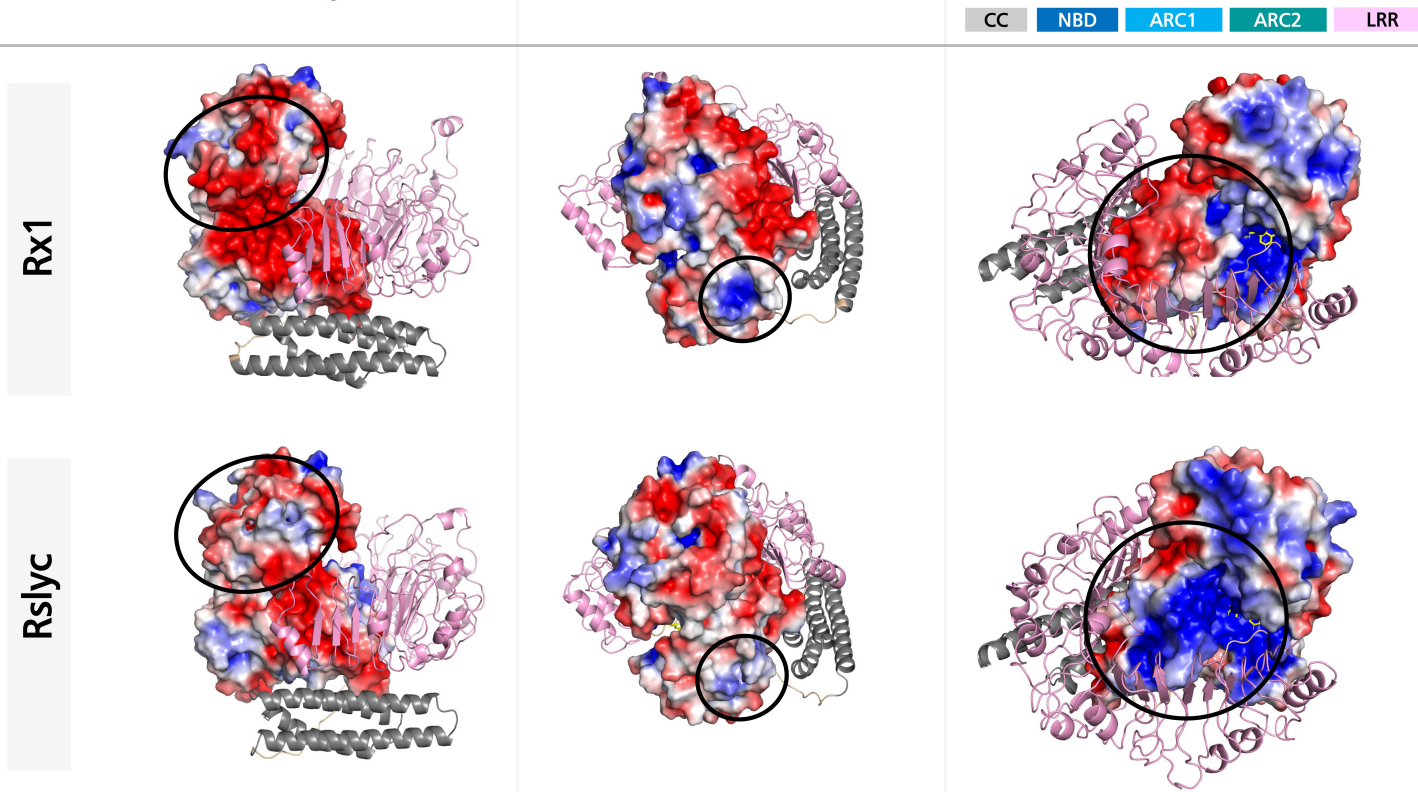

NB-ARC surface: $+3.0 \stackrel{\mathrm{kT} / \mathrm{e}}{\longrightarrow}-3.0$ 
Supplemental Fig. S7. Immunoblots from pull-down experiments of various $4 \times H A-L R R$ variants of Rslyc, Rx1 or a chimera thereof in combination with the NB-ARC and CC domains derived from Rslyc or Rx1. Immunoblots were performed using proteins extracted from agroinfiltrated leaf materials harvested at 3 dpi. $\alpha$-HA magnetic beads were used to pull down the LRR fragments as baits. "+" indicates the presence of a construct in the infiltration combination. The result shown is representative of three independent repeats.

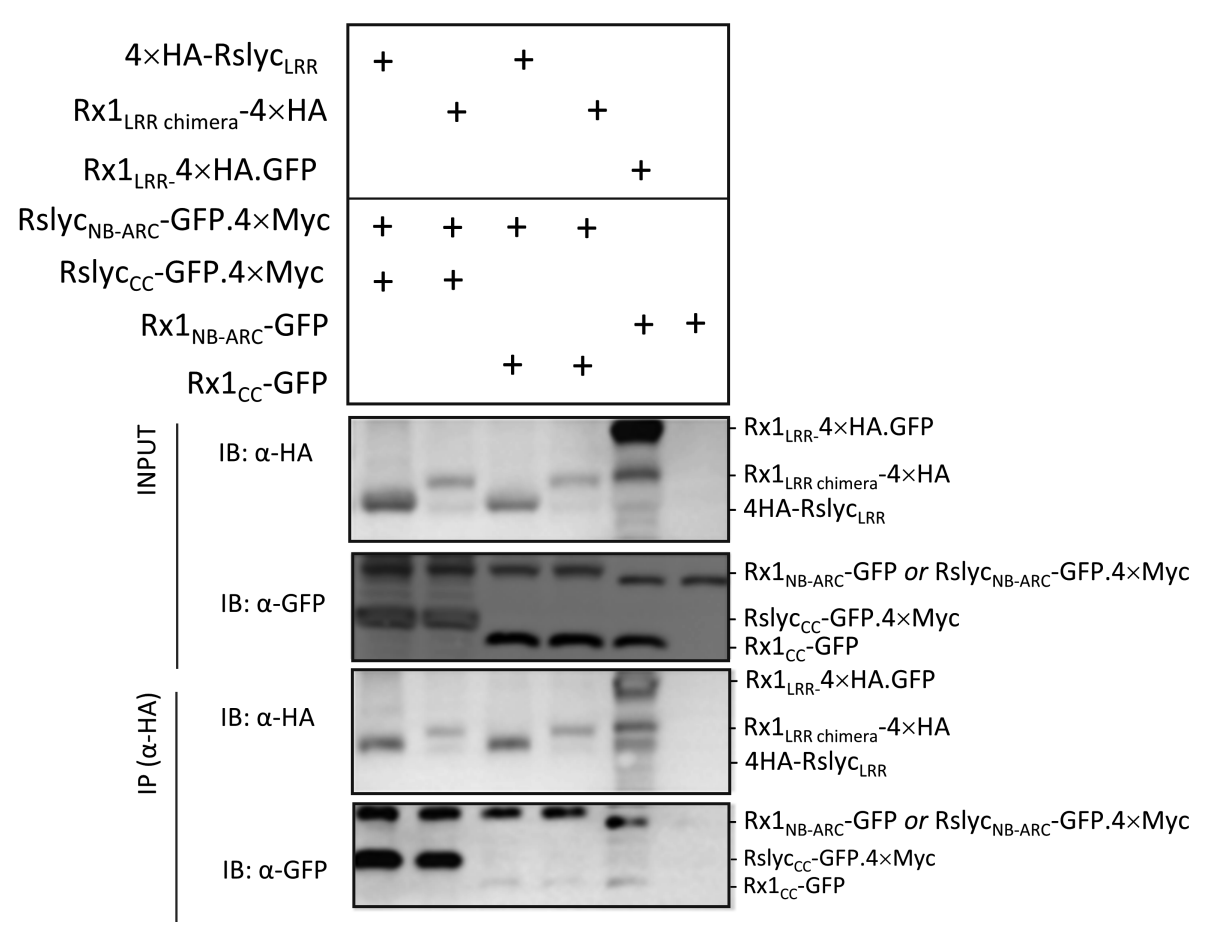


Supplemental Fig. S8. Differences in electorstatic surface potentials on the CC and LRR domains of ZAR1, Rx1 and Rslyc. For all three receptors, interaction between the CC and LRR is supported by complementary negative patch in the CC domain and positive patch in LRR (circle regions linked by arrows). Unlike ZAR1, however, the positively-charged patch at the distal end of the CC is absent in $\mathrm{R} \times 1$ and Rslyc. The latter group also has a more extended basic region in the first half of the LRR. Taken together, this may suggest differences in the manner by which the CC and LRR of ZAR1 versus Rx1 and Rslyc may form a complex.

\section{ZAR1}

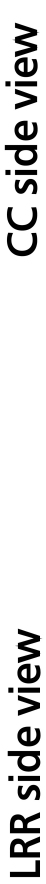

Rx1

LRR

ribbon

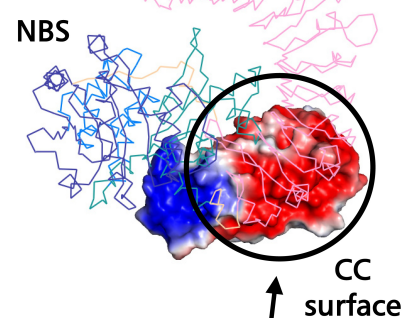

ace
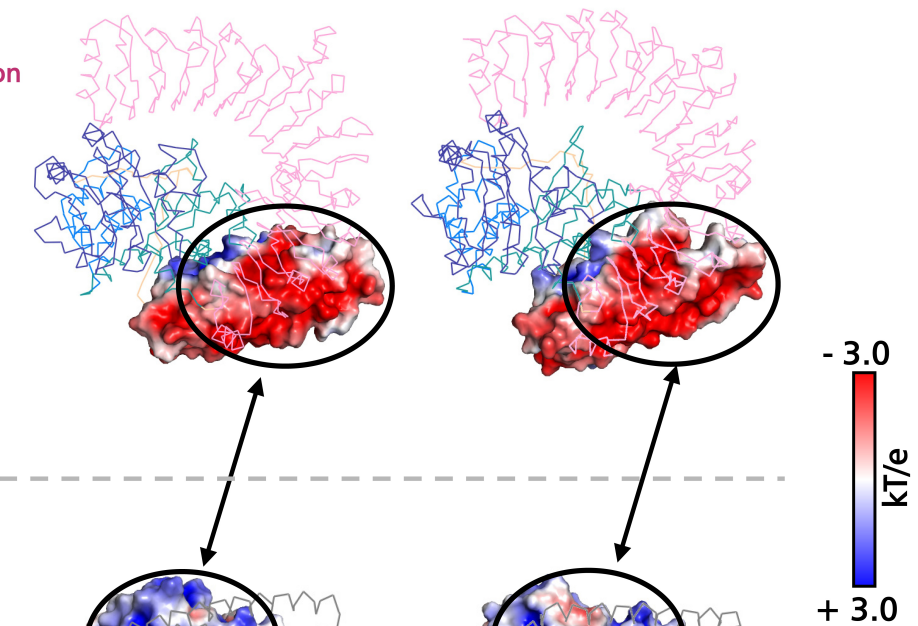

Rslyc

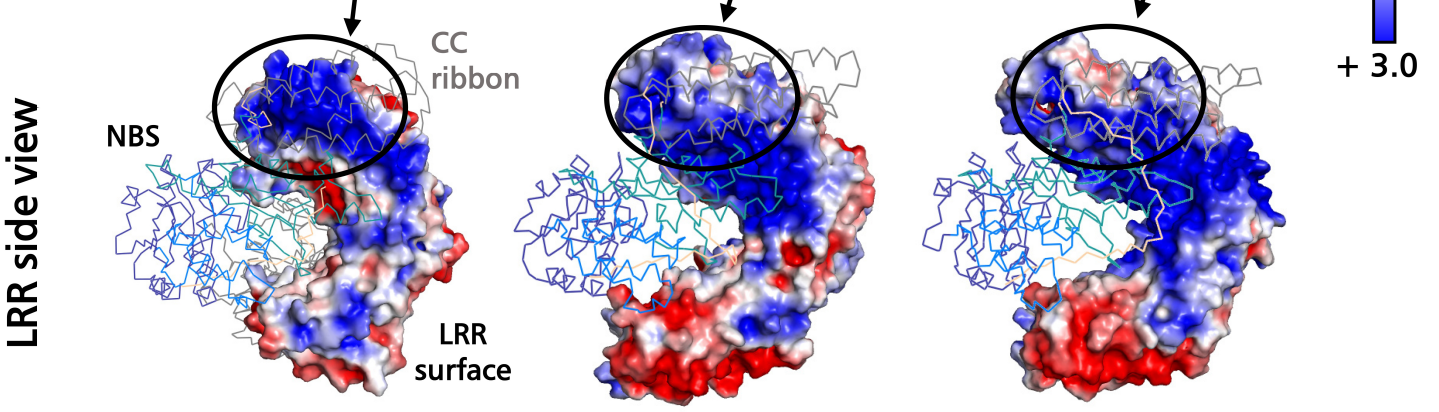




\section{References}

Abraham, M. J., Murtola, T., Schulz, R., Páll, S., Smith, J. C., Hess, B., and Lindahl, E. (2015), GROMACS: High performance molecular simulations through multi-level parallelism from laptops to supercomputers. SoftwareX. 1-2: p. 19-25.

Ade, J., DeYoung, B. J., Golstein, C., and Innes, R. W. (2007), Indirect activation of a plant nucleotide binding site-leucine-rich repeat protein by a bacterial protease. Proceedings of the National Academy of Sciences of the United States of America. 104(7): p. 2531-2536. ppublish.

Baudin, M., Hassan, J. A., Schreiber, K. J., and Lewis, J. D. (2017), Analysis of the ZAR1 Immune Complex Reveals Determinants for Immunity and Molecular Interactions. Plant Physiol. 174(4): p. 2038-2053.

Baudin, M., Schreiber, K. J., Martin, E. C., Petrescu, A. J., and Lewis, J. D. (2020), Structure-function analysis of ZAR1 immune receptor reveals key molecular interactions for activity. The Plant journal : for cell and molecular biology. 101(2): p. 352-370. ppublish.

Bendahmane, A., Farnham, G., Moffett, P., and Baulcombe, D. C. (2002), Constitutive gain-of-function mutants in a nucleotide binding site-leucine rich repeat protein encoded at the Rx locus of potato. Plant Journal. 32(2): p. 195-204.

Bendahmane, A., Kanyuka, K., and Baulcombe, D. (1997), High-resolution genetical and physical mapping of the Rx gene for extreme resistance to potato virus X in tetraploid potato. Theoretical and Applied Genetics. 95: p. 153-162.

Bendahmane, A., Kanyuka, K., and Baulcombe, D. C. (1999), The Rx gene from potato controls separate virus resistance and cell death responses. Plant Cell. 11.

Bendahmane, A., Köhm, B. A., Dedi, C., and Baulcombe, D. C. (1995), The coat protein of potato virus X is a strain-specific elicitor of $\mathrm{R} \times 1$-mediated virus resistance in potato. The Plant Journal. 8(6): p. 933-941.

Bentham, A., Burdett, H., Anderson, P. A., Williams, S. J., and Kobe, B. (2017), Animal NLRs provide structural insights into plant NLR function. Annals of Botany. 119(5): p. 698-702.

Bernoux, M., Burdett, H., Williams, S. J., Zhang, X., Chen, C., Newell, K., Lawrence, G. J., Kobe, B., Ellis, J. G., Anderson, P. A., and Dodds, P. N. (2016), Comparative Analysis of the Flax Immune Receptors L6 and L7 Suggests an Equilibrium-Based Switch Activation Model. Plant Cell. 28(1): p. 146-59.

Van der Biezen, E. A. and Jones, J. D. (1998), Plant disease-resistance proteins and the gene-for-gene concept. Trends Biochem Sci. 23.

Buchan, D. W. A. and Jones, D. T. (2019), The PSIPRED Protein Analysis Workbench: 20 years on. Nucleic Acids Research. 47(W1): W402-W407.

Cheng, J., Randall, A. Z., Sweredoski, M. J., and Baldi, P. (2005), SCRATCH: a protein structure and structural feature prediction server. Nucleic acids research. 33(Web Server issue): W72-W76.

Drozdetskiy, A., Cole, C., Procter, J., and Barton, G. J. (2015), JPred4: a protein secondary structure prediction server. Nucleic Acids Research. 43(W1): W389-W394.

Van Engelen, F. A., Molthoff, J. W., Conner, A. J., Nap, J. P., Pereira, A., and Stiekema, W. J. (1995), pBINPLUS: an improved plant transformation vector based on pBIN19. Transgenic research. 4(4): p. 288-290. ppublish.

Farnham, G. and Baulcombe, D. C. (2006), Artificial evolution extends the spectrum of viruses that are targeted by a disease-resistance gene from potato. Proc Natl Acad Sci U S A. 103(49): p. 18828-33.

Giannakopoulou, A., Steele, J. F. C., Segretin, M. E., Bozkurt, T. O., Zhou, J., Robatzek, S., Banfield, M. J., Pais, M., and Kamoun, S. (2015), Tomato 12 Immune Receptor Can Be Engineered to Confer Partial Resistance to the Oomycete Phytophthora infestans in Addition to the Fungus Fusarium oxysporum. Molecular Plant-Microbe Interactions: MPMI-07-15-0147-R. 
Hao, W., Collier, S. M., Moffett, P., and Chai, J. (2013), Structural basis for the interaction between the potato virus $\mathrm{X}$ resistance protein $(\mathrm{Rx})$ and its cofactor Ran GTPase-activating protein 2 (RanGAP2). The Journal of biological chemistry. 288(50): p. 35868-35876.

Harris, C. J., Slootweg, E. J., Goverse, A., and Baulcombe, D. C. (2013), Stepwise artificial evolution of a plant disease resistance gene. Proceedings of the National Academy of Sciences. 110(52): p. 21189-21194.

Hibberd, A. M., Bassett, M. J., and Stall, R. E. (1987), Allelism tests of three dominant genes for hypersensitive resistance to bacterial spot of pepper. Phytopathology. 77(9): p. 1304-1307.

Huang, J. and MacKerell Jr, A. D. (2013), CHARMM36 all-atom additive protein force field: Validation based on comparison to NMR data. Journal of Computational Chemistry. 34(25): p. 2135-2145.

Huang, J., Rauscher, S., Nawrocki, G., Ran, T., Feig, M., de Groot, B. L., Grubmüller, H., and MacKerell, A. D. (2017), CHARMM36m: an improved force field for folded and intrinsically disordered proteins. Nature Methods. 14(1): p. $71-73$.

Huang, S., van der Vossen, E. A. G., Kuang, H., Vleeshouwers, V. G. A. A., Zhang, N., Borm, T. J. A., van Eck, H. J., Baker, B., Jacobsen, E., and Visser, R. G. F. (2005), Comparative genomics enabled the isolation of the R3a late blight resistance gene in potato. The Plant journal : for cell and molecular biology. 42(2): p. 251-261. ppublish.

Hwang, C. F., Bhakta, A. V., Truesdell, G. M., Pudlo, W. M., and Williamson, V. M. (2000), Evidence for a role of the N terminus and leucine-rich repeat region of the Mi gene product in regulation of localized cell death. Plant Cell. 12(8): p. 1319-1329.

Jones, J. D. G., Vance, R. E., and Dangl, J. L. (2016), Intracellular innate immune surveillance devices in plants and animals. Science. 354(6316): aaf6395.

Kapos, P., Devendrakumar, K. T., and Li, X. (2019), Plant NLRs: From discovery to application. Plant Science. 279: p. 3-18.

el-Kharbotly, A., Leonards-Schippers, C., Huigen, D. J., Jacobsen, E., Pereira, A., Stiekema, W. J., Salamini, F., and Gebhardt, C. (1994), Segregation analysis and RFLP mapping of the R1 and R3 alleles conferring race-specific resistance to Phytophthora infestans in progeny of dihaploid potato parents. Molecular \& general genetics : MGG. 242(6): p. 749-754. ppublish.

Long, S. and Tian, P. (2019), Protein secondary structure prediction with context convolutional neural network. RSC Advances. 9(66): p. 38391-38396.

Martin, E. C., Sukarta, O. C. A., Spiridon, L., Grigore, L. G., Constantinescu, V., Tacutu, R., Goverse, A., and Petrescu, A. J. (2020), LRRpredictor-A New LRR Motif Detection Method for Irregular Motifs of Plant NLR Proteins Using an Ensemble of Classifiers. Genes (Basel). 11(3).

Mazourek, M., Cirulli, E. T., Collier, S. M., Landry, L. G., Kang, B. C., Quirin, E. A., Bradeen, J. M., Moffett, P., and Jahn, M. M. (2009), The fractionated orthology of Bs2 and Rx/Gpa2 supports shared synteny of disease resistance in the Solanaceae. Genetics. 182(4): p. 1351-64.

Moffett, P., Farnham, G., Peart, J., and Baulcombe, D. C. (2002), Interaction between domains of a plant NBS-LRR protein in disease resistance-related cell death. The EMBO Journal. 21(17): p. 4511-4519.

Ori, N., Eshed, Y., Paran, I., Presting, G., Aviv, D., Tanksley, S., Zamir, D., and Fluhr, R. (1997), The I2C family from the wilt disease resistance locus 12 belongs to the nucleotide binding, leucine-rich repeat superfamily of plant resistance genes. The Plant cell. 9(4): p. 521-532. ppublish.

Qi, D., DeYoung, B. J., and Innes, R. W. (2012), Structure-Function Analysis of the Coiled-Coil and Leucine-Rich Repeat Domains of the RPS5 Disease Resistance Protein. Plant Physiol. 158(4): p. 1819-32. 
Rairdan, G. J., Collier, S. M., Sacco, M. A., Baldwin, T. T., Boettrich, T., and Moffett, P. (2008), The Coiled-Coil and Nucleotide Binding Domains of the Potato Rx Disease Resistance Protein Function in Pathogen Recognition and Signaling. Plant Cell. 20(3): p. 739-751.

Rairdan, G. J. and Moffett, P. (2006), Distinct Domains in the ARC Region of the Potato Resistance Protein Rx Mediate LRR Binding and Inhibition of Activation. Plant Cell. 18(8): p. 2082-2093.

Ritter, E., Debener, T., Barone, A., Salamini, F., and Gebhardt, C. (1991), RFLP mapping on potato chromosomes of two genes controlling extreme resistance to potato virus X (PVX). Molecular \& general genetics : MGG. 227(1): p. 81-85. ppublish.

Rouppe van der Voort, J., Wolters, P., Folkertsma, R. T., Hutten, R., Zandvoort, P., Vinke, H., Kanyuka, K., Bendahmane, A., Jacobsen, E., and Janssen, R. (1997), Mapping of the cyst nematode resistance locus Gpa2 in potato using a strategy based on comigrating AFLP markers. Theor App/ Genet. 95.

Sacco, M. A., Koropacka, K., Grenier, E., Jaubert, M. J., Blanchard, A., Goverse, A., Smant, G., and Moffett, P. (2009), The cyst nematode SPRYSEC protein RBP-1 elicits Gpa2- and RanGAP2-dependent plant cell death. PLoS Pathog. 5.

Sacco, M. A., Mansoor, S., and Moffett, P. (2007), A RanGAP protein physically interacts with the NB-LRR protein Rx, and is required for Rx-mediated viral resistance. Plant J. 52(1): p. 82-93.

Schindelin, J., Arganda-Carreras, I., Frise, E., Kaynig, V., Longair, M., Pietzsch, T., Preibisch, S., Rueden, C., Saalfeld, S., Schmid, B., Tinevez, J. Y., White, D. J., Hartenstein, V., Eliceiri, K., Tomancak, P., and Cardona, A. (2012), Fiji: an open-source platform for biological-image analysis. Nat Methods. 9(7): p. 676-82.

Schouten, A., Roosien, J., de Boer, J. M., Wilmink, A., Rosso, M.-N., Bosch, D., Stiekema, W. J., Gommers, F. J., Bakker, J., and Schots, A. (1997), Improving scFv antibody expression levels in the plant cytosol 1. FEBS Letters. 415(2): p. 235-241.

Shi, J., Yeom, S.-I., Kang, W.-H., Park, M.-K., Choi, D., Kwon, J.-K., Han, J.-H., Lee, H.-R., Kim, B.-D., and Kang, B.-C. (2011), Isolation of an Rx homolog from C. annuum and the evolution of Rx genes in the Solanaceae family. Plant Biotechnology Reports. 5(4): p. 331-344.

Slootweg, E., Roosien, J., Spiridon, L. N., Petrescu, A. J., Tameling, W., Joosten, M., Pomp, R., van Schaik, C., Dees, R., Borst, J. W., Smant, G., Schots, A., Bakker, J., and Goverse, A. (2010), Nucleocytoplasmic distribution is required for activation of resistance by the potato NB-LRR receptor $R \times 1$ and is balanced by its functional domains. Plant Cell. 22(12): p. 4195-215.

Slootweg, E., Koropacka, K., Roosien, J., Dees, R., Overmars, H., Lankhorst, R. K., van Schaik, C., Pomp, R., Bouwman, L., Helder, J., Schots, A., Bakker, J., Smant, G., and Goverse, A. (2017), Sequence Exchange between Homologous NB-LRR Genes Converts Virus Resistance into Nematode Resistance, and Vice Versa. Plant physiology. 175(1): p. 498-510.

Slootweg, E. J., Spiridon, L. N., Martin, E. C., Tameling, W. I. L., Townsend, P. D., Pomp, R., Roosien, J., Drawska, O., Sukarta, O. C. A., Schots, A., Borst, J. W., Joosten, M. H., Bakker, J., Smant, G., Cann, M. J., Petrescu, A.-J., and Goverse, A. (2018), Distinct Roles of Non-Overlapping Surface Regions of the Coiled-Coil Domain in the Potato Immune Receptor Rx1. Plant Physiology. 178(3): p. 1310-1331.

Slootweg, E. J., Spiridon, L. N., Roosien, J., Butterbach, P., Pomp, R., Westerhof, L., Wilbers, R., Bakker, E., Bakker, J., Petrescu, A.-J., Smant, G., and Goverse, A. (2013), Structural Determinants at the Interface of the ARC2 and Leucine-Rich Repeat Domains Control the Activation of the Plant Immune Receptors Rx1 and Gpa2. Plant Physiology. 162(3): p. 1510-1528. 
Tameling, W. I. L. and Baulcombe, D. C. (2007), Physical association of the NB-LRR resistance protein Rx with a Ran GTPase-activating protein is required for extreme resistance to Potato virus X. Plant Cell. 19(5): p. 1682-94.

Tameling, W. I. L., Nooijen, C., Ludwig, N., Boter, M., Slootweg, E., Goverse, A., Shirasu, K., and Joosten, M. (2010), RanGAP2 Mediates nucleocytoplasmic partitioning of the NB-LRR immune receptor Rx in the solanaceae. Thereby dictating Rx function. Plant Cell. 22.

Tameling, W. I. L., Vossen, J. H., Albrecht, M., Lengauer, T., Berden, J. A., Haring, M. A., Cornelissen, B. J. C., and Takken, F. L. W. (2006), Mutations in the NB-ARC Domain of I-2 That Impair ATP Hydrolysis Cause Autoactivation. Plant Physiol. 140(4): p. 1233-1245.

Team, R. C. (2008), R: A language and environment for statistical computing. R Foundation for Statistical Computing. [Online].

Tornero, P., Chao, R. A., Luthin, W. N., Goff, S. A., and Dangl, J. L. (2002), Large-scale structure-function analysis of the Arabidopsis RPM1 disease resistance protein. The Plant cell. 14(2): p. 435-450.

Van der Vossen, E. A. G., van der Voort, J., Kanyuka, K., Bendahmane, A., Sandbrink, H., Baulcombe, D. C., Bakker, J., Stiekema, W. J., and Klein-Lankhorst, R. M. (2000), Homologues of a single resistance-gene cluster in potato confer resistance to distinct pathogens: a virus and a nematode. Plant J. 23.

Wang, G. F., Ji, J., El-Kasmi, F., Dangl, J. L., Johal, G., and Balint-Kurti, P. J. (2015), Molecular and functional analyses of a maize autoactive NB-LRR protein identify precise structural requirements for activity. PLoS Pathog. 11(2).

Wang, J., Hu, M., Wang, J., Qi, J., Han, Z., Wang, G., Qi, Y., Wang, H. W., Zhou, J. M., and Chai, J. (2019), Reconstitution and structure of a plant NLR resistosome conferring immunity. Science. 364(6435).

Wang, S., Li, W., Liu, S., and Xu, J. (2016), RaptorX-Property: a web server for protein structure property prediction. Nucleic Acids Res. 44(W1): W430-5.

Webb, B. and Sali, A. (2016), Comparative Protein Structure Modeling Using MODELLER. Curr Protoc Bioinformatics. 54: p. 5.6.1-5.6.37.

Van Wersch, S., Tian, L., Hoy, R., and Li, X. (2020), Plant NLRs: The Whistleblowers of Plant Immunity. Plant Communications. 1(1): p. 100016.

Williams, C. J., Headd, J. J., Moriarty, N. W., Prisant, M. G., Videau, L. L., Deis, L. N., Verma, V., Keedy, D. A., Hintze, B. J., Chen, V. B., Jain, S., Lewis, S. M., Arendall W. B., 3., Snoeyink, J., Adams, P. D., Lovell, S. C., Richardson, J. S., and Richardson, D. C. (2018), MolProbity: More and better reference data for improved all-atom structure validation. Protein Sci. 27(1): p. 293-315.

Williams, S. J., Sornaraj, P., deCourcy-Ireland, E., Menz, R. I., Kobe, B., Ellis, J. G., Dodds, P. N., and Anderson, P. A (2011), An autoactive mutant of the $\mathrm{M}$ flax rust resistance protein has a preference for binding ATP, whereas wild-type M protein binds ADP. Mol Plant Microbe Interact. 24(8): p. 897-906.

Wróblewski, T., Spiridon, L., Martin, E. C., Petrescu, A.-J., Cavanaugh, K., Truco, M. J., Xu, H., Gozdowski, D., Pawlowski, K., Michelmore, R. W., and Takken, F. L.W. (2018), Genome-wide functional analyses of plant coiled-coil NLR-type pathogen receptors reveal essential roles of their N-terminal domain in oligomerization, networking, and immunity. PLOS Biology. 16(12): e2005821. 


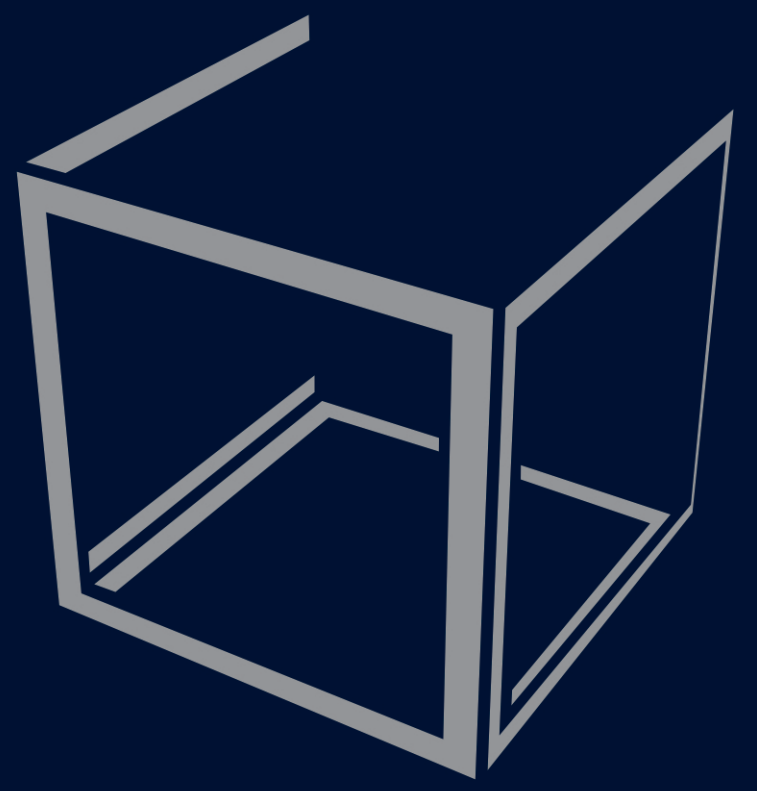

Octavina C. A Sukarta ${ }^{1}$

Erik J. Slootweg ${ }^{1}$

Amalia D. G Munoz ${ }^{1}$,

Somnath Pokare ${ }^{2}$,

Jan Roosien ${ }^{1}$,

Rikus Pomp ${ }^{1}$,

Abdenaser Elashry²,

Florian Grundler ${ }^{2}$,

Geert Smant ${ }^{1}$,

Aska Goverse $^{1}$

${ }^{1}$ Laboratory of Nematology, Wageningen University \& Research, Wageningen, the Netherlands.

${ }^{2}$ Department of Molecular Phytomedicine, INRES, University of Bonn, Bonn, Germany 


\section{Chapter}

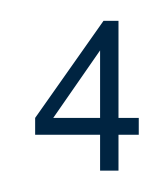

The RanGTPase Activating Protein 2

(RanGAP2) is a Common Target of the

Cognate Effectors of its Bound Receptors,

Gpa2 and $R \times 1$ 


\section{ABSTRACT}

The Gpa2 and Rx1 immune receptors encode for canonical CC-NB-LRR proteins belonging to the same gene cluster in potato. Despite sharing high sequence homology, they have evolved to provide defence against unrelated pathogens. Gpa2 detects Gp-RBP1 effectors secreted by the potato cyst nematode Globodera pallida whereas Rx1 recognizes the viral coat protein (CP) of Potato Virus X (PVX). How Gpa2 and Rx1 perceive their cognate effectors remains unknown. However, artificial tethering studies of Gpa2 to Gp-RBP1 suggest that this may occur indirectly through the shared co-factor, RanGTPase Activating Protein 2 (RanGAP2) (Sacco et al. 2009). Using a combination of Co-Immunoprecipitation and cellular imaging studies, we show that both the virulent and avirulent variants of Gp-RBP1 and PVX-CP effectors can interact with the RanGAP1 and RanGAP2 homologs in planta. Additionally, knocking-down both RanGAP homologs impair infection of cyst nematodes in Arabidopsis thaliana and PVX in Nicotiana benthamiana. These findings suggest that plant RanGAPs are common virulence targets of different classes of plant pathogens with distinct lifestyles. In resistant backgrounds, RanGAP1 and RanGAP2 form a heteromeric complex with Gpa2 and Rx1. We, therefore, investigated the impact of RanGAP2 effector targeting on the association with its bound receptors. Immunoprecipitation experiments show that the RanGAP2-receptor complex is not disrupted by the presence of Gp-RBP1 and PVX-CP upon overexpression. Combined with earlier studies, we propose a model of indirect recognition by Rx1 and Gpa2 in which RanGAP2 contributes to but is insufficient for effector recognition and immune activation. 


\section{INTRODUCTION}

Effective immunity against invading pathogens hinges on the successful recognition of non-self. In plants, this process is mediated by a group of cell-autonomous receptor proteins, most of which belong to the family of Nucleotide-Binding Leucine-Rich Repeats (NB-LRR) receptors (van Wersch et al. 2020). Stereotypical NB-LRR modules are composed of three domains: a central Nucleotide Binding adaptor shared by APAF-1, Resistance Protein and CED4 (NB-ARC) core, a hypervariable C-terminal Leucine-Rich Repeat (LRR) domain and an N-terminal coiled-coil (CC) or Toll Interleukin-1 like receptor (TIR) domain. The NB-ARC region constitutes an ATP/ADP nucleotide-binding pocket, enabling NB-LRRs to act as a molecular switch that can toggle readily between inactive and active states (Takken et al. 2006, Bernoux et al. 2016). The switch function is activated upon the detection of specific pathogen-derived molecules termed effectors, which are translocated into the host to exploit the cellular machinery and promote virulence. Upon recognition, NB-LRRs trigger a suit of defence response, often manifesting in the form of localized cell death, termed the Hypersensitive Response (HR).

Over the years, several studies have detailed how plant NB-LRRs can perceive pathogens, allowing us to come to an understanding of the mechanistic basis of effector recognition (reviewed in van Wersch et al. 2020). The most simplistic is a receptor-ligand model, which involves direct interaction between the NB-LRR and its cognate effector. This was first documented in the rice CC-NB-LRR (CNL) Pita, whose functionality is compromised when substituting a single amino acid in its Leucine-Rich Repeat (LRR) domain that abolished interaction with the Magnaporthe grisea effector Avr-Pita (Jia et al. 2000). It appears, however, that the direct recognition model applies only to a few exceptional cases (Dodds et al. 2010, Ravensdale et al. 2012). Instead, a majority of NB-LRRs indirectly senses pathogen-induced modifications of effector targets or their mimics (Dodds et al. 2010). In this manner, it is believed that the plant can circumvent rapidly evolving pathogens by enabling a single NB-LRR to detect multiple effectors that act on a single host target. Today, a wide variety of models for pathogen detection have been described that reconcile the detection of a plethora of invading pathogens with only a limited set of NB-LRR immune receptors (van Wersch et al. 2020). 
The CC-NB-LRRs Gpa2 and Rx1 from potato (Solanum tuberosum spp. andigena) are highly homologous immune receptors that mediate distinct defence responses against evolutionarily unrelated pathogens (van der Vossen et al. 2000, Bendahmane et al. 1995, Bendahmane et al. 1999). Gpa2 confers defence against the cyst nematode Globodera pallida by detecting the nematode-secreted effector Gp-RBP1. This induces a hypersensitive type of cell death of cells surrounding the nematode's feeding site, which it requires for further development of the cyst. Heterologous studies have shown, however, that Gpa2 can also trigger HR in the leaves of Nicotiana benthamiana (Sacco et al. 2009). Rx1 gives immunity to Potato Virus $X(P V X)$, a filamentous positive-sense RNA virus that infects aerial parts of Solanaceous plants. Upon recognition of the viral coat protein (PVX-CP), Rx1 activates a symptomless defence response referred to as extreme resistance that effectively limits infection to initially affected cells (Bendahmane et al. 1995). However, $\mathrm{R} \times 1$ also has the capacity to induce a classical HR response, such as when overexpressed or when there is an overaccumulation of PVX-CP. PVX-CP and GpRBP-1 effectors share no sequence nor structural similarities, and thus, $\mathrm{Rx} 1$ and Gpa2 offer an excellent platform to resolve the basis of diversification of recognition in homologous NB-LRRs.

The mechanistic basis of effector recognition by Gpa2 and Rx 1 is mostly unknown. For both receptors, direct perception of neither Gp-RBP1 nor PVX-CP has been shown. It is clear, however, that recognition specificity of $\mathrm{R} \times 1$ and Gpa2 is confined to the C- terminal end of the LRR domain (Farnham et al. 2006, Slootweg et al. 2017, Rairdan et al. 2006). Sequence exchange within this region allows $\mathrm{R} \times 1$ to confer resistance against $G$. pallida in the roots without eliciting autoactivity or compromising the functionality of the immune receptor (Slootweg et al. 2017). Such findings pinpoint to a functional bifurcation of the receptor in which recognition specificity is determined by the hypervariable LRR domain, whereas defence activation is confined to the CC-NB-ARC moiety. As the CC-NB-ARC is interchangeable, this also suggests that Gpa2 and Rx1 are likely to share similar host-factors and downstream components. Indeed, the CC domains of both receptors form complexes with the RanGTPase activating protein 2 (RanGAP2) protein (Tameling et al. 2007, Sacco et al. 2007). RanGAP2 plays a vital role in the cell by regulating mitosis and nucleocytoplasmic transport during plant development (Sacco et al. 2009, Pay et al. 2002, Rose et al. 2001). Earlier works also demonstrate that RanGAP2 modulates the stability and partitioning of Rx1 in the nucleus and cytoplasm (Tameling et al. 2010). More importantly, however, RanGAP2 contributes to Rx1 
and Gpa2-mediated defence responses, although the underpinning mechanisms remain unclear (Tameling et al. 2007, Sacco et al. 2007).

Previously, YFP-complementation experiments showed that HR by Gpa2 in N. benthamiana is enhanced when Gp-RBP1 is artificially tethered to RanGAP2 (Sacco et al. 2009). This hints that RanGAP2 may contribute to immunity by facilitating Gp-RBP1 recognition. Such findings prompted us to test whether Gp-RBP1 effectors could associate with RanGAP2 in the plant cell. Given that RanGAP2 is also a co-factor of Rx1, we also determined whether PVX-CPs could target RanGAP2. Using a combination of Co-Immunoprecipitation (Co-IP) and cellular imaging studies, we report that RanGAP2 can form protein complexes with Gp-RBP1 and PVX-CPs in planta via a conserved WPP domain. We further demonstrate that these effectors commonly target the RanGAP1 homolog, shown previously to also interact with $\mathrm{Rx} 1$ in a yeast-two-hybrid assay (Tameling et al. 2010). Interestingly, both eliciting and non-eliciting variants of PVX-CP and Gp-RBP1 can associate with RanGAP1 and RanGAP2, suggesting a broader role for this effector target in promoting nematode and viral pathogenicities. Indeed, knocking down either or both RanGAP homologs reduced infection by PVX in N. benthamiana and the cyst nematode Heterodera schachtii in Arabidopsis thaliana. These data support a model of plant RanGAPs as a common virulence target of a virus and nematode. As RanGAP2 also associates with Rx1 and Gpa2, we examined whether effector targeting can influence the preformed immune complex, which may elucidate mechanisms of pathogen detection and receptor activation. Co-IP experiments show that in the presence of the cell-death inducing effectors, RanGAP2 remains bound to $R \times 1$ and Gpa2. Based on these data, we conclude that RanGAP binding in planta is insufficient for recognition. From this, a tripartite model emerges in which RanGAP2 acts as a bait to facilitate effector perception by Gpa2 and Rx1. This model also sheds light on the bifurcation of recognition specificities of these receptors. We further discuss our findings in the context of effector recognition and activation models of NB-LRRs in plants. 


\section{RESULTS}

\section{Virulent and avirulent effectors of G. pallida and PVX interact with full-length RanGAP1 and RanGAP2 in planta}

To discern whether RanGAP2 and Gp-RBP1 can form a complex in planta, we performed a Co-IP assay. To that end, full-length RanGAP2-GFP was co-expressed transiently in leaves of $N$. benthamiana by agroinfiltration with $8 \times$ HA-tagged versions of the Gpa2-activating or non-activating Gp-RBP1 variants, namely D383-1 and Rook4. In plants, RanGAP2 is homologous to RanGAP1, sharing $66.2 \%$ identity at the amino acid level in N. benthamiana. Both proteins are functionally redundant with a function as activators of RanGTPase as part of the nucleocytoplasmic transport cycle (Rodrigo-Peiris et al. 2011, Xu et al. 2008). We, therefore, sought to investigate whether RanGAP1 could also associate with Gp-RBP1. For pull-downs, RanGAP2-GFP or RanGAP1-GFP was captured with $\alpha$-GFP conjugated paramagnetic beads as bait, and the bound proteins were analysed by immunoblotting (Fig. 1A). Our data show that there were no changes in protein stability of RanGAP1-GFP, RanGAP2-GFP or the Gp-RBP1 effectors when co-expressed. Both D383-1-8XHA and Rook4-8 X HA specifically co-immunoprecipitated with both RanGAP1-GFP and RanGAP2-GFP. Interestingly, these G. pallida effectors co-purified more intensely with the RanGAP2 homolog compared to RanGAP1. Moreover, stronger band intensity for the non-eliciting Rook4 variant was consistently observed after IP when RanGAP1 and RanGAP2 was used as bait. This suggests that various Gp-RBP1 effectors may differ in their binding affinities for RanGAP2 and RanGAP1. Combined, our results demonstrate that both virulent and avirulent Gp-RBP1 effectors can form complexes with both RanGAP homologs in planta.

As RanGAP2 was initially found to be a co-factor of Rx1, we expanded our Co-IP studies to explore whether RanGAP1 and/or RanGAP2 can also form a complex with PVX-CP (Tameling et al. 2007, Sacco et al. 2007). Full-length RanGAP1-GFP and RanGAP2-GFP were used as bait to pull-down $4 \times$ HA-tagged versions of the coat proteins from the avirulent UK3 (CP106) and virulent HB (CP105) PVX strains. In line with the interaction data for Gp-RBP1, we did not observe changes in protein stabilities of RanGAP1-GFP, RanGAP2-GFP, CP105-4 XHA, or CP106-4XHA upon co-expression (Fig. 1B). Both CP106-4 XHA and CP105-4 XHA variants co-immunoprecipitated with RanGAP2-GFP, whereas no detectable amounts purified with the $\alpha$-GFP beads alone. Notably, 
both viral coat proteins consistently co-purified in lower quantities when RanGAP1-GFP was used as bait. Our findings, therefore, reveal that activating and non-activating variants of PVX-CP can associate with both RanGAP homologs in planta as has been observed for Gp-RBP1. RanGAP1 and RanGAP2 are, therefore, common targets of structurally divergent effector types from pathogens with distinct lifestyles.

A.

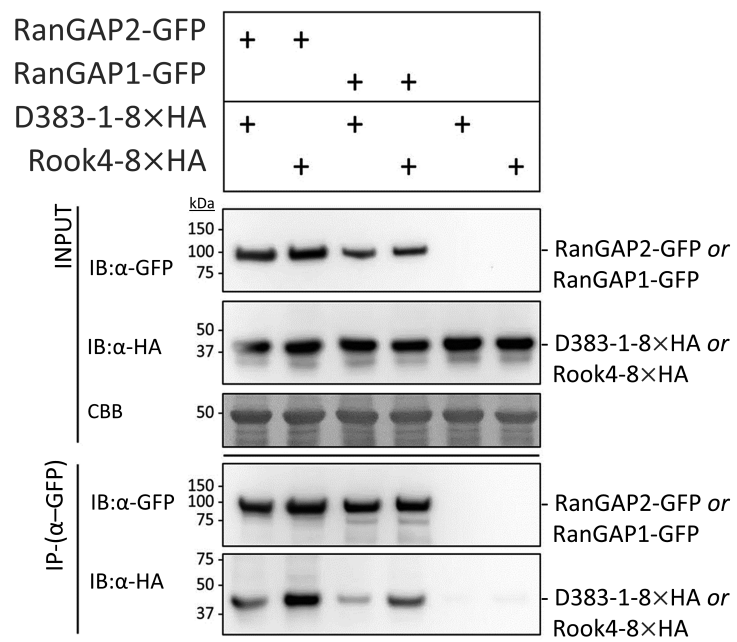

B.

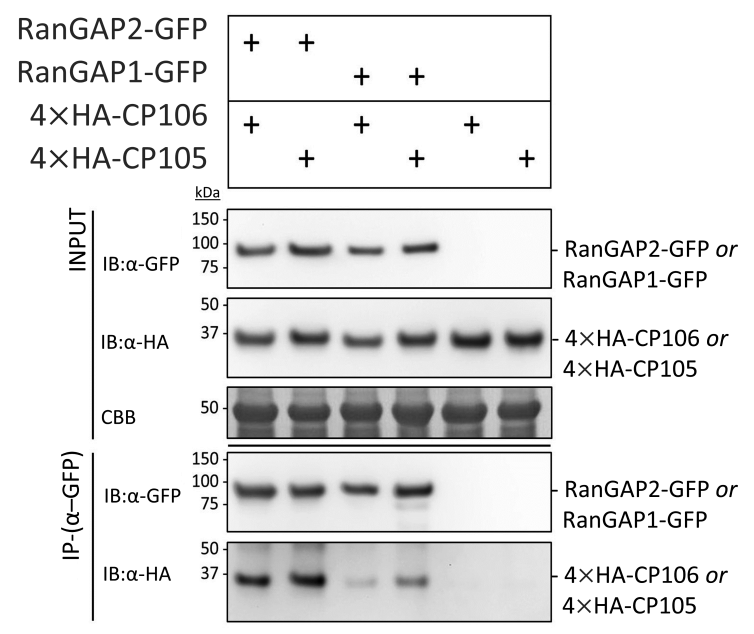

Fig. 1: Gp-RBP1 of G. pallida and CP of PVX can associate with RanGAP1 and RanGAP2 in planta. Co-immunoprecipitation of full-length RanGAP1-GFP or RanGAP2-GFP as bait and HA-tagged Gp-RBP1 (A) or PVX-CP (B) effector proteins. A. tumefaciens harbouring constructs for the pull-downs were co-expressed in N. benthamiana leaves and harvested at 2 dpi. "+" indicates the presence of a construct in the infiltration combination. The soluble extract was used for Co-IP studies using $\alpha$-GFP conjugated beads to precipitate the bait. The immunoblots (IB) with $\alpha$-GFP and $\alpha$-HA antibodies of the input material are shown in the top half of the image and the results of Co-IP in the two bottom panels of the figure. Coomassie brilliant blue (CBB) stained blots serve as a loading control for the input material. Data shown are representative of three independent repeats.

\section{RanGAP1 and RanGAP2 contribute to nematode and viral infections}

Given that RanGAP1 and RanGAP2 can interact with both the eliciting and non-eliciting variants of PVX-CP and Gp-RBP1, we hypothesized that plant RanGAPs could fulfill a broader role beyond 
functioning as a co-factor in pathogen recognition. For instance, the effector targeting of host proteins is known to be directly used by pathogens to promote virulence (Carella et al. 2018). Thus, we investigated whether RanGAP1 and/or RanGAP2 can contribute to nematode and/or viral infection. Due to the lack of more efficient molecular genetic tools in tomato and potato, we took an alternative plant system to test the contribution of RanGAP2 and RanGAP1 to nematode parasitism by challenging the Arabidopsis thaliana rangap1 and rangap2 mutant plants (rg1-1 and rg2-2) with the beet cyst nematode Heterodera schachtii, which has a similar mode of parasitism as $G$. pallida on potato. Our data indicate that the total number of nematodes infecting the roots of $r$ 1-1 was significantly lower as compared to the wild-type control (Col-0), after 2 weeks of infection (Fig. 2A1). Although this is less significant in rg2-2, a consistently lower trend was observed between different experimental repeats. In cyst nematodes, sex determination is dependent on environmental conditions. Auspicious conditions favour the development of female over male nematodes. Therefore, we also investigated the proportion of male and female nematodes at 2 weeks post-infection. Interestingly, both rg1-1 and rg2-2 plants harbour significantly fewer females than wild-type plants (Fig. 2A2). The reduction in the total number of nematodes and proportions of females infecting the roots of mutant plants collectively pinpoint that both RanGAP homologs contribute to the susceptibility of the roots of $A$. thaliana and thus, to cyst nematode virulence.

To investigate the function of RanGAP1 and RanGAP2 in PVX infection, we performed Tobacco Rattle Virus-Based Virus-Induced Gene Silencing (TRV-VIGS) in N. benthamiana using constructs described in Tameling et al. 2007 and Tameling et al. 2010. After 21 days post TRV-VIGS, leaves of TRV-silenced $N$. benthamiana plants were infiltrated with agrobacteria harboring amplicons of PVX-106 (avirulent) or PVX-105 (virulent). Viral levels were quantified in the infiltrated zones within 1-5 dpi by DAS-ELISA. Our data show that when RanGAP2 or RanGAP1 is knocked-down transiently, there is significantly less viral accumulation compared to the TRV:GFP control irrespective of the viral strain at $3 \mathrm{dpi}$ but not at 4-5 dpi (data at these later time-points not shown) (Fig. 2B). Simultaneously silencing RanGAP1 and RanGAP2 by VIGS results in greater suppression of either virus. Our TRV-VIGS data, therefore, illustrate that both RanGAP2 and RanGAP1 contribute to PVX virulence in $N$. benthamiana, particularly during the early stages of viral infection. Combined with the disease assay performed in Arabidopsis, we demonstrate that plant RanGAPs contribute to the infections of unrelated pathogens. 
A1.

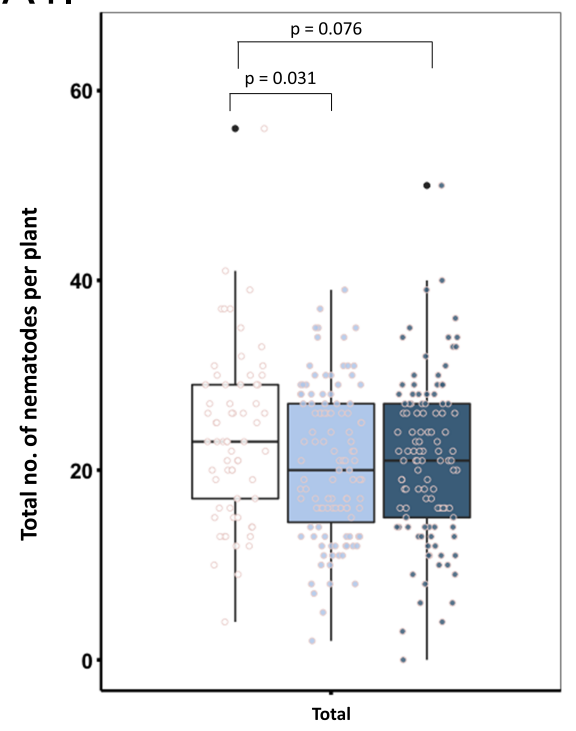

B.

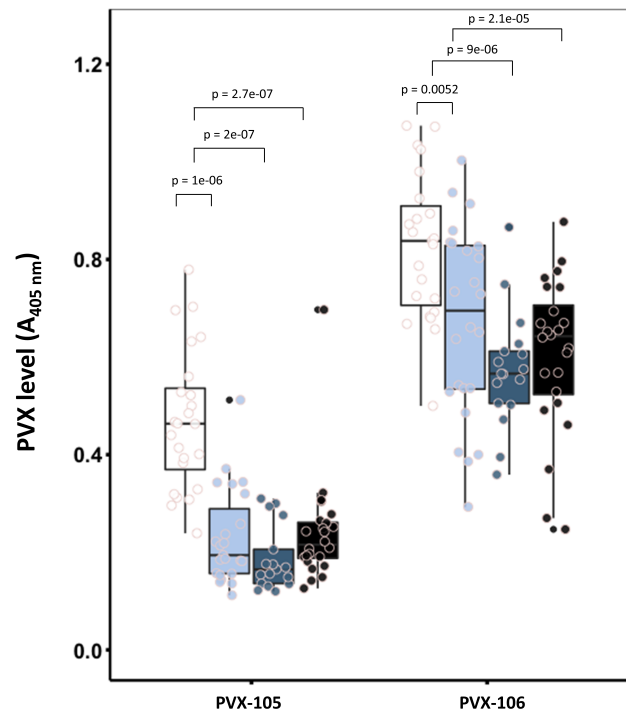

A2.

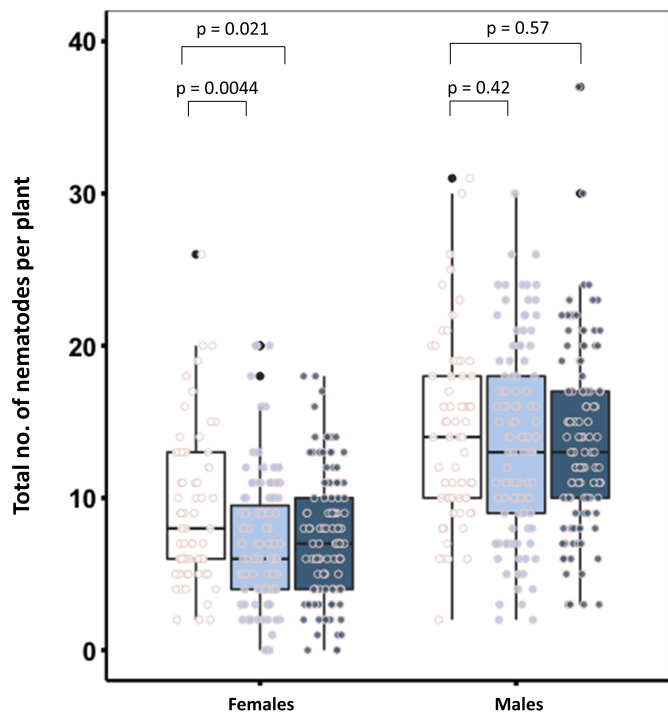

plant

宊 Col-0

审 rg1-1

审 rg2-2

Fig. 2: RanGAP1 and RanGAP2 contribute to pathogenicity of cyst nematodes in $A$. thaliana and PVX in N. benthamiana. The total (A1) and average number of female and male (A2) nematodes per plant in A. thaliana roots after 2 weeks of infection. Boxes indicate the 75 th and 25th percentile, and whiskers show the 95th and 5th percentile. Data shown is from 4 independent repeats where statistical significance difference was calculated using T-test with $\alpha=0.05$. B). PVX virulence assay on TRV-VIGS N. benthamiana plants silenced for RanGAP2, RanGAP1 or combinations thereof in $N$. benthamiana. Silenced plants were infiltrated at 21 days post TRV-VIGS treatment with Agrobacteria for expression of the amplicon of either PVX105 or PVX106. Infiltrated leaf samples were harvested at 3 dpi for viral quantification by DAS-ELISA. Statistically significant difference was compared to 
(Figure legend continued) TRV:GFP samples using the Wilcoxon-Signed Rank test ( $\alpha=0.05$ ). Data shown is the combination of two independent experimental repeats consisting of 24 biological replicates.

\section{Gp-RBP1 and PVX-CP associate with the RanGAP2-WPP domain in planta}

To gain further insight into the effector targeting of RanGAP2, we next resolved the RanGAP domains involved in the association with Gp-RBP1 and PVX-CP. Plant RanGAPs are characterized by a unique, N-terminal WPP domain (so-called for a conserved Tryp-Pro-Pro motif), which anchors the protein to the nuclear envelope (Pay et al. 2002). To test if the WPP domain is sufficient for the interaction with G. pallida and PVX effectors, we co-expressed GFP/CFP versions of the effectors with a RanGAP2-WPP variant tagged with the red fluorescent protein mCherry and a nuclear localization signal (NLS). The NLS-tagged RanGAP2-WPP was targeted to localize exclusively in the nucleus. On the other hand, both Gp-RBP1 and PVX-CP have a more or less equal nucleocytoplasmic distribution (Slootweg et al. 2010, Cruz et al. 1996). It was anticipated that co-expressing WPP-NLS-mCh would shift the subcellular localization of these effectors towards the nucleus when these proteins exist in the same complex. This shift in nucleocytoplasmic distribution can be quantified by determining the fluorescence intensity ratio between the GFP-tagged protein in the nucleus and cytoplasm $\left({ }^{\prime}{ }^{/ /} C\right)$, as described previously in Slootweg et al. 2010. Confocal imaging was performed at 2 days post infiltration (2 dpi). Free CFP, which does not form a complex with the RanGAP2-WPP construct, was used as a negative control. Remarkably, our imaging data show that relatively higher nuclear intensities for Rook4-GFP-4 XHA, D383-1-GFP-4 XHA, CFP-CP106, and CFP-CP105 were observed during co-expression with WPP-NLS-mCh in support of an interaction (Fig. 3). This apparent shift in cellular distribution supports a model in which the WPP domain of RanGAP2 is sufficient for complex formation with Gp-RBP1 and PVX-CP. The nucleocytoplasmic distribution of the CFP negative control was not altered when co-expressed with the WPP-NLS-mCh construct. Overall, our findings demonstrate that the physical association of Gp-RBP1 and PVX-CP locates to a functional motif in RanGAP2. 
A1.

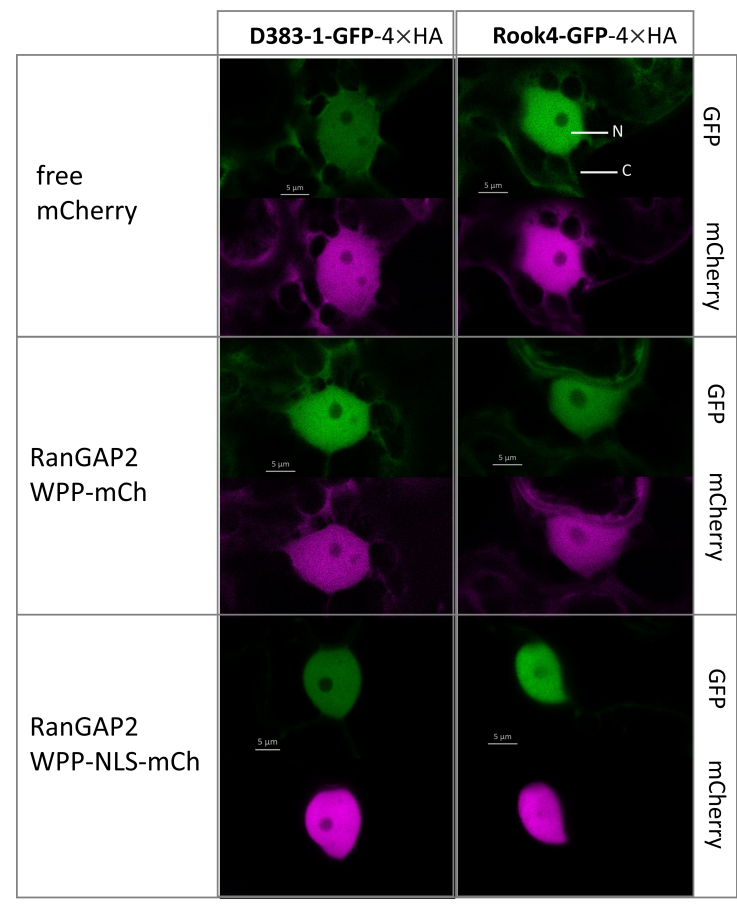

B1.

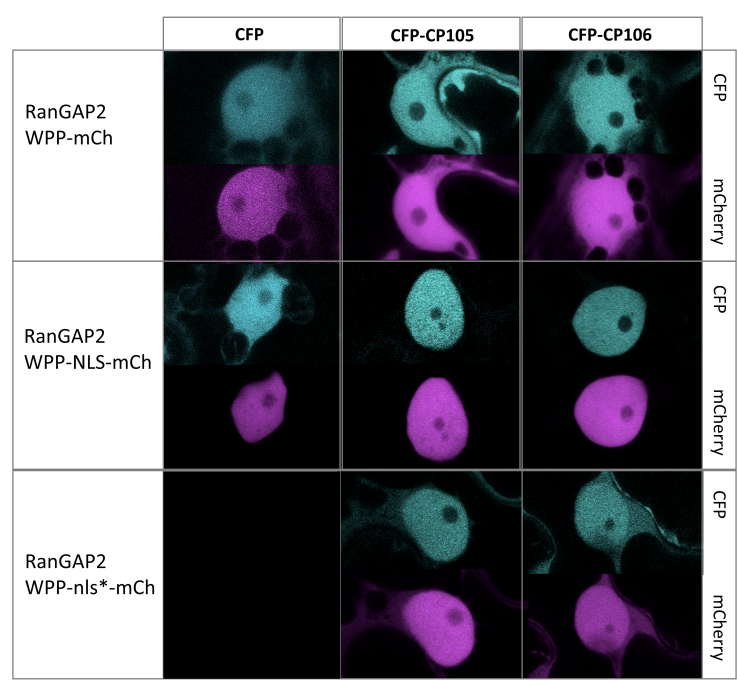

A2.

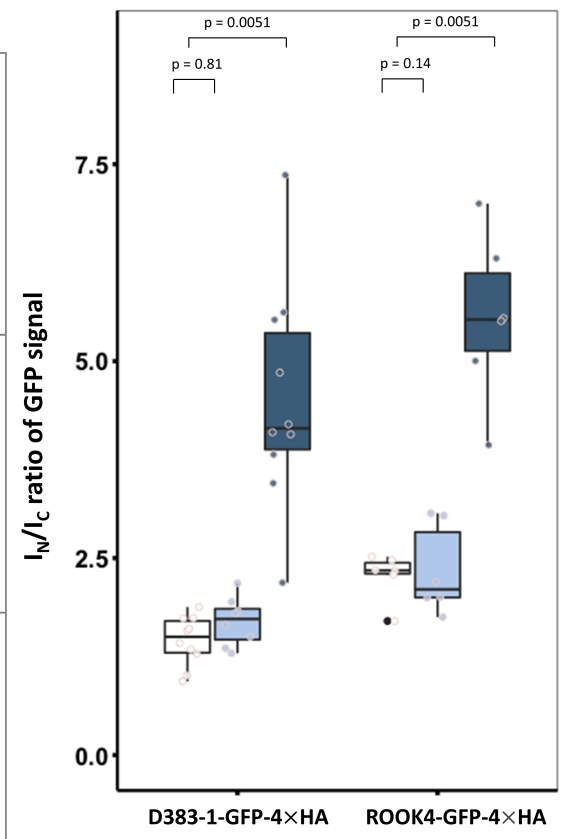

Sample

审 $\mathrm{mch}$

臿 WPP-mCh

睧 WPP-NLS-mCh

B2.

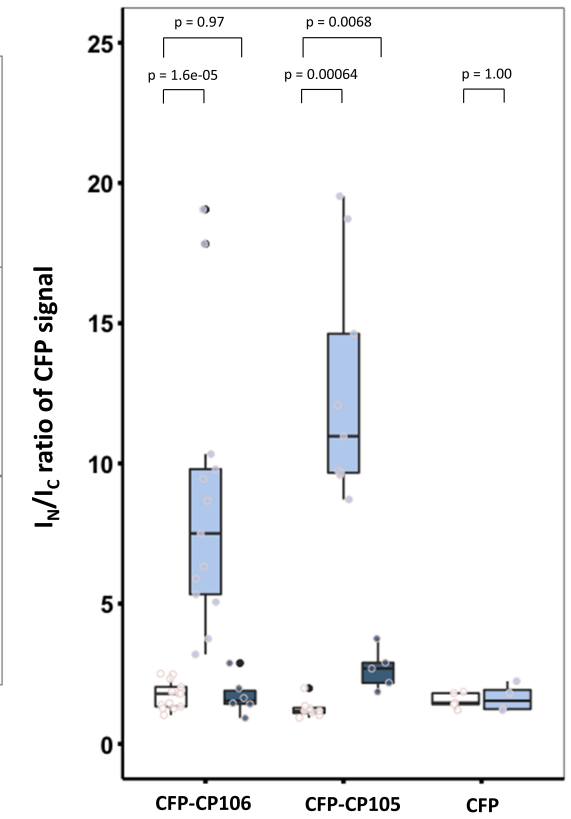

Sample

演 WPP-mCh 후 WPP-nls*-mCh
舅 WPP-NLS-mCh

Fig. 3: The WPP domain of RanGAP2 is sufficient for the interaction with Gp-RBP1 (A) and PVX-CP (B). Representative confocal images of nuclei $(\mathrm{N})$ and surrounding cytoplasm $(\mathrm{C})$ of cells expressing mCherry-tagged RanGAP2-WPP and GFP-tagged Gp-RBP1 (Rook4 or D383-1) (A1) or CFP-tagged PVX-CP constructs (PVX-CP 106 or 105) (B1). The CFP/GFP and mCherry channels are shown 
(Figure legend continued) side by side for each combination. Quantification of the fluorescence intensity ratios $\left(I_{N} / I_{C}\right.$ ratio) is represented in the accompanying boxplots (A2 and B2). Boxes indicate the interquartile range with whiskers indicating the maximum and minimum values. Statistical significance difference was calculated using the Wilcoxon-Signed Ranked test with $\alpha=0.05$. For both PVX-CP and Gp-RBP1, data shown is the combination of at least two independent repeats.

\section{Effector targeting of RanGAP2 does not affect the RanGAP2-receptor complex}

RanGAP2 forms a heteromeric complex with Gpa2 and Rx1 in planta, which relies on an interaction between the receptor CC domain and the RanGAP2-WPP region (Hao et al. 2013, Tameling et al. 2007, Sacco et al. 2007). Our data indicate that the association of Gp-RBP1 and PVX-CP with RanGAP2 overlaps at the WPP domain. It is, therefore, conceivable that effector targeting could affect the RanGAP2-receptor complex. To explore this, full-length Gpa2 N-terminally tagged with $4 \times$ Myc was co-expressed with RanGAP2-GFP and either D383-1-8 $\times$ HA or Rook4-8 $\times$ HA. The coat proteins $4 \times$ HA-CP106 and $4 \times$ HA-CP105 were also included as controls as they do not activate Gpa2 but bind RanGAP2. Agroinfiltrated leaves were harvested at $2 \mathrm{dpi}$, before a visible cell death response to harvest sufficient protein for detection by Western blot. Indeed, immunoblotting showed that co-expressing these effectors did not strongly affect the protein levels of $4 \times$ Myc-Gpa2 and RanGAP2-GFP at this time point (Fig. 4A). To study the interaction between Gpa2 and RanGAP2 under influence of the co-expressed effectors, we performed a Co-IP with $4 \times$ Myc-Gpa2 as bait and RanGAP2-GFP as prey. RanGAP2-GFP was pulled down with $4 \times$ Myc-Gpa2, but not in the absence of $4 \times$ Myc-Gpa2 as bait. Co-expressing with either Gp-RBP1 or PVX-CPs did not alter the amount of RanGAP2-GFP pulled down along with 4×Myc-Gpa2 (Fig. 4A). Therefore, the interaction between Gpa2 and RanGAP2 is not affected by the cell death eliciting variant of Gp-RBP1 at 2 dpi.

We likewise investigated whether the complex of Rx1/RanGAP2 would be affected by its interaction with PVX-CP. To test this, $4 \times$ Myc-Rx1 was co-expressed with RanGAP2 and the PVX-CPs for 24 hours before the leaves were harvested. At this time point, no cell death was visible, and the protein levels were adequate for detection on Western blot. As described for Gpa2, Rx1 and RanGAP2-GFP protein levels were not affected by the co-expressed effectors. Following IP, RanGAP2-GFP specifically co-immunoprecipitated with $4 \times$ Myc-Rx1 using $\alpha$-Myc beads, but not with $\alpha$-Myc beads alone (Fig. 
4B). Similar to Gpa2, the presence of the effectors does not affect the Rx1/RanGap2 complex. Based on these findings, we, therefore, conclude that the association between Gpa2 and Rx1 with RanGAP2 appears unchanged by the cell death eliciting effectors at this time-point. We corroborated these findings by examining the impact of effector targeting on the Rx1-CC and RanGAP2 interaction alone, which does not elicit cell death. Our immunoprecipitation data shows that PVX-CP and Gp-RBP1 also do not affect complex formation between Rx1-CC with the RanGAP2 (Supplementals Fig. S2). Together, these data suggest that neither the virulent nor avirulent effector variants induced the dissociation of the CC for complex formation with RanGAP2. 
A.

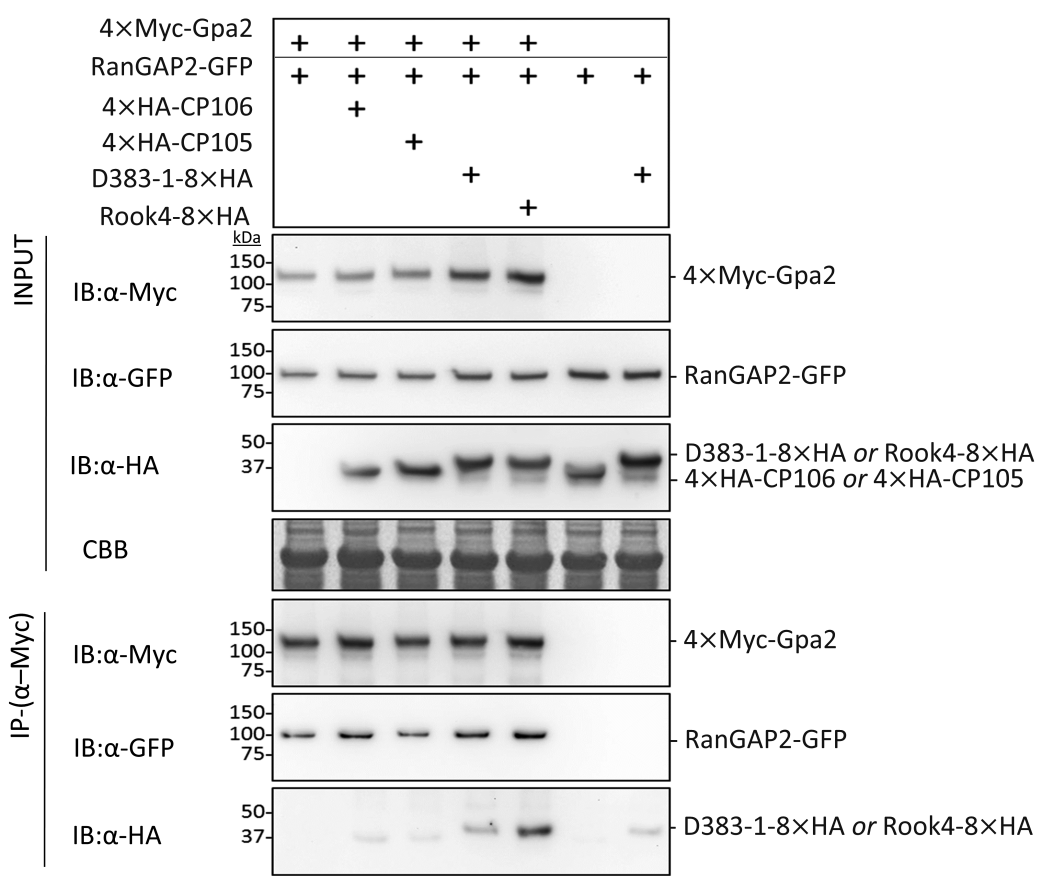

B.

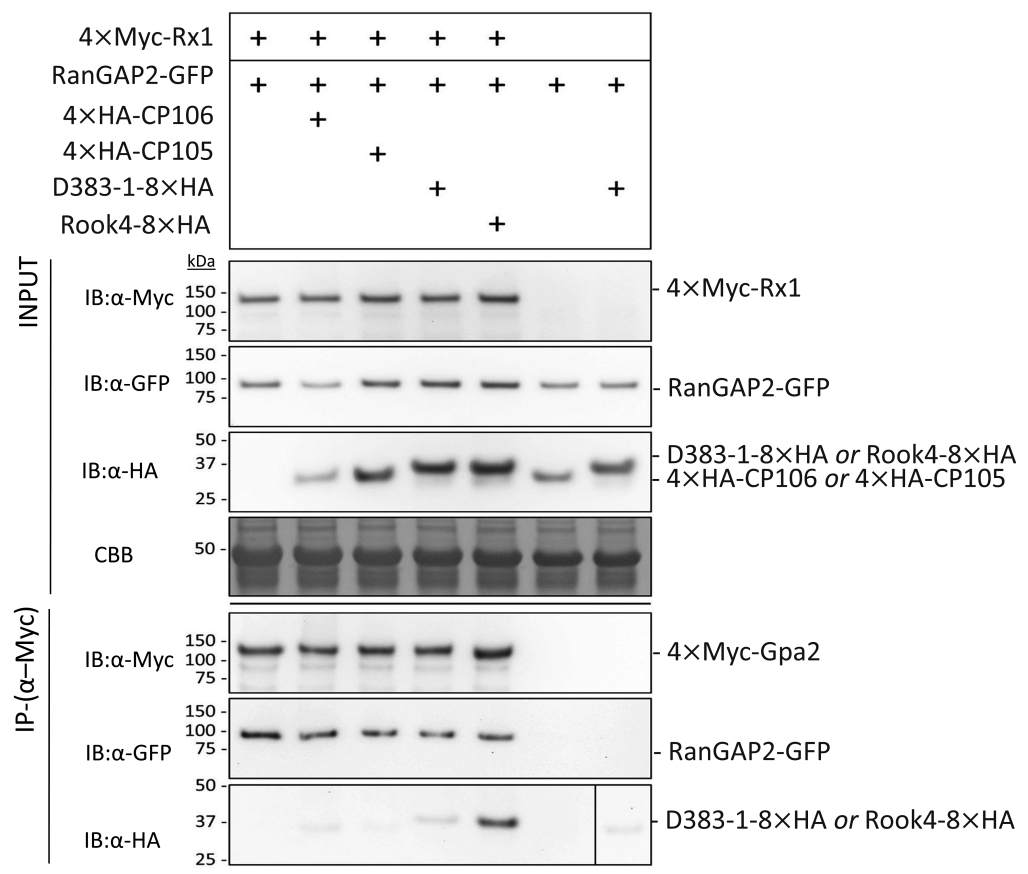

Fig. 4: The targeting of RanGAP2 by Gp-RBP1 or PVX-CP does not hamper its association with Gpa2 and Rx1. Shown in the figure are immunoblots from Co-IP experiments where $4 \times$ Myc-tagged Gpa2 and Rx1 constructs were used as bait and RanGAP2-GFP as prey. HA-tagged versions of 
(Figure legend continued) CP106, CP105, or the Gp-RBP1 D383-1 and Rook4 were additionally co-expressed. The top three immunoblot (IB) panels represent the input material. Coomassie brilliant blue (CBB) stained blots on which RuBisCO is visible are used as a control for equal loading for the input material. The lower three panels show the protein pulled down in the $\alpha$-Myc immunoprecipitation. A) Co-immunoprecipitation to test if the interaction between full-length Gpa2 and RanGAP2 is affected by the virulent and avirulent PVX-CPs or by the Gp-RBPs D383-1 or Rook4. The samples were harvested at 48 hours post agroinfiltration before cell death would occur in the combination of Gpa2 and D383-1. B) Co-immunoprecipitation to test if the interaction between full-length Rx1 and RanGAP2 is affected by the same sets of effectors as in (A). The proteins were co-expressed for 24 hours and harvested before cell death would occur in the combination of Rx1 and CP106. Data shown is representative of three independent repeats. "+" indicates the presence of a construct in the infiltration combination. Coomassie brilliant blue (CBB) stained blots serve as a loading control for the input material.

\section{DISCUSSION}

The RanGAP2 protein has long been established as a co-factor of the closely-related intracellular immune receptors Gpa2 and Rx1 (Sacco et al. 2007, Tameling et al. 2007). Nevertheless, how RanGAP2 functions in immunity provided by Gpa2 and Rx1 remains vague. In this study, we expand further on this function by providing evidence for the physical association of RanGAP1 and RanGAP2 with the cognate effectors of Gpa2 and Rx1, namely Gp-RBP1 and PVX-CP. We discovered that the interaction with RanGAP2 and RanGAP1 is not restricted to the cell-death inducing effector variants. Moreover, both RanGAP homologs contribute to infection by these pathogens. These data combined suggest that RanGAP2 and RanGAP1 are common targets of effectors from two unrelated pathogens. Interestingly, we further demonstrate that effector targeting does not interfere with the complex of RanGAP2 with Gpa2 and Rx1. This implies that receptor activation is a multistep event, which may initiate with the docking of effector molecules to RanGAP2. A model for the role of RanGAP2 as a bait in the stepwise detection of Gp-RBP1 and PVX-CP is proposed. 


\section{RanGAP1 and RanGAP2 interact with structurally divergent effectors}

The PVX-CP and Gp-RBP1 effectors bear no sequence or structural resemblance. Nevertheless, we show that both effectors can form a heteromeric complex with RanGAP2 in planta (Fig. 1A and 1B). This is intriguing when considering that the physical association observed may occur directly, as demonstrated in a FRET-FLIM (Förster Resonance Energy Transfer (FRET) by Fluorescence Lifetime Imaging) analysis of Gp-RBP1 and RanGAP2 (Supplementals Fig. S4). Unlike interactions shown by Co-IPs, which can be mediated by other factors in the complex, those demonstrated in a FRET-FLIM assay are most likely to be direct as the phenomenon of energy transfer requires two proteins to be within a distance of $\leq 10 \mathrm{~nm}$ (Bücherl et al. 2010). Though we have yet to demonstrate that PVX-CP can also directly associate with RanGAP2, it will be fascinating to unravel whether these structurally dissimilar effectors can overlap (compete) in the regions of RanGAP2 where they bind to. More detailed mutational and interaction studies are, thus, needed to further resolve underlying interacting surfaces on the effectors and RanGAPs.

It is worth noting that previous studies could not establish the complex formation of RanGAP2 with PVX-CP by Co-IP (Tameling et al. 2007). Given that the associations described here are seen at both the biochemical and cellular levels, however, our findings are unlikely to be the outcome of experimental artifacts, for example, due to overexpression. We attribute these apparent disparities in results to variation in platforms and setups used. Most notably, earlier approaches made use of C-terminally tagged PVX-CP constructs (Tameling et al. 2010). Here, PVX-CP tagged at the $\mathrm{N}$-terminus was employed instead as the C-terminal variant has been proven to compromise viral infection (Cruz et al. 1996). Whether the loss of CP function is directly linked to impaired RanGAP2 binding also warrants further investigation. Taken together, however, this hints that the PVX-CP C-terminus may be necessary for mediating the association to RanGAP2. This contrasts with that reported previously for the $N$. benthamiana PCIP1 protein, whose interaction relies on residues in a conserved alpha-helix at the $\mathrm{N}$-terminal end of the viral coat protein (Park et al. 2013). These findings suggest that despite its relatively small size, PVX-CP is a flexible module that can accommodate multiple contact points for interdomain associations.

In addition to RanGAP2, we demonstrate that both PVX-CP and Gp-RBP1 can associate with the RanGAP1 homolog in planta, albeit less strongly (Fig. 1A and Fig. 1B). Immunoblotting assays 
indicate that both RanGAP homologs are expressed at comparable levels, minimizing the likelihood that the observed quantitative differences in interaction are due to RanGAP1 being present in lower abundance. Interestingly, this is reminiscent of earlier works reporting that Rx1 interacts less strongly and is less efficiently relocated to the cytoplasm by RanGAP1 than by RanGAP2 (Tameling et al. 2010). It is, therefore, tempting to correlate the weaker interaction of RanGAP1 with the effectors to that with its bound receptors. Alternatively, a more likely possibility is that the observed findings are due to intrinsic differences between the RanGAP2 and RanGAP1 proteins, with the N. benthamiana homologs sharing an overall sequence identity of $66.29 \%$ (65.26\% at the WPP domain and $67.61 \%$ at the LRR domain).

\section{Potential roles of RanGAP1 and RanGAP2 in virulence by cyst nematodes and PVX}

Our interaction data suggest that complex formation of effector variants of Gp-RBP1 and PVX-CP with RanGAP1 and RanGAP2 is independent of the recognition specificity of Rx1 and Gpa2. Additionally, we reveal that both RanGAP2 and RanGAP1 contribute to infection by cyst nematodes and PVX (Fig. 2A and 2B). This indicates that plant RanGAPs are functional host targets of two taxonomically unrelated pathogens. As per our current understanding, this is the first report for the role of RanGAP1 and RanGAP2 in pathogenicity. It is worth noting that the disease assays performed in this study were not done in with mutant potato plants. However, as we observed an effect in virulence when RanGAP1 and RanGAP2 were down-regulated in diverse genetic backgrounds ( $A$. thaliana and $N$. benthamiana respectively) supports a broader role of RanGAPs in the infectivity of cyst nematodes and viruses. Remarkably, a more substantial influence of the depletion of RanGAP1 was consistently found during infection by cyst nematodes in A. thaliana as compared to RanGAP2. This suggests that the RanGAP2 and RanGAP1 homologs may have a yet undefined, differing role in processes that cyst nematodes exploit for their fitness. Additionally, it remains to be determined whether these differences are linked to variation in binding affinities of RanGAP1 and RanGAP2 with Gp-RPB1, as observed in our interaction studies (Fig. 1A).

Our finding that RanGAP2 and RanGAP1 are common host targets of unrelated pathogens is in line with an emerging picture that diverse pathogens utilize a small and overlapping set of host proteins to benefit their fitness (Weßling et al. 2014). The convergence of multiple, unrelated effector 
molecules on a single host protein is proposed to grant pathogens with leverage by providing the flexibility to shift to new hosts and/or effectively suppress 'defence hubs' (Carella et al. 2018). A number of excellent reviews have inventoried the growing catalogue of common effector targets, with prominent examples being the molecular chaperone EDS1 and protease Rcr3 (Song et al. 2009, Bhattacharjee et al. 2011). These common targets are proposed to be central for a limited range of cellular processes that pathogens require for survival (Weßling et al. 2014). This pinpoints that a fundamental function of RanGAP1 and RanGAP2 is targeted to facilitate disease progression. This, in turn, aligns with our findings demonstrating that the virulence functions of RanGAPs are highly conserved in diverse genetic backgrounds.

During interphase, RanGAP is involved in the maintenance of a RanGTP/RanGDP gradient required for macromolecule transport between the nucleus and the cytoplasm (Pay et al. 2002). Interestingly, both nucleocytoplasmic trafficking, as well as mitotic activity, are crucial host cell processes involved in viral pathogenicity and cyst nematode syncytium formation (Quentin et al. 2013, Liu et al. 2008). At least one virus is known to disrupt this gradient by targeting Ran to interfere with nuclear efflux of antiviral factors (Porter et al. 2006). In this light, it is fascinating to note that preliminary immunoprecipitation data show that the association of PVX-CP106 to RanGAP2 is impaired when mutating a conserved Aspartate residue to Alanine in the RanGAP2-LRR (Supplementals Fig. S3). This mutation has been shown previously to impair the Ran-binding and GAP activity of RanGAP2, suggesting that PVX-CP may physically target an interface in the RanGAP2-LRR domain required for RAN association and/or activation (Tameling et al. 2010). However, whether targeting by PVX-CP directly impacts these RanGAP2-related functions and the precise implications thereafter require more concrete molecular and biochemical studies. We further demonstrate that the interaction of both Gp-RBP1 and PVX-CP locate to the WPP domain, which is required to anchor RanGAP2 to the nuclear envelope (Fig. 3 and Supplementals Fig. S4). Simultaneous targeting of the WPP and LRR domains may thus collectively disturb the cellular distribution and GAP activity of RanGAP2, affecting the overall biological functions of the protein (e.g. in nuclear trafficking). It would, therefore, be worthwhile to further investigate in what way PVX and cyst nematodes benefit from interacting with RanGAP. 


\section{Potential roles of RanGAP2 in effector recognition by Rx1/Gpa2}

Earlier studies have implicated RanGAP2 in effector recognition by Rx1 and Gpa2, mainly due to the lack of an apparent function of RanGAP2 in defence signalling (Tameling et al. 2007, Sacco et al. 2007). Moreover, the role of RanGAP2 as a cytoplasmic retention factor also coincides with previous findings that Rx1 needs to be localized in the cytoplasm for recognition (Slootweg et al. 2010). The physical association of RanGAP2 with the cognate effectors of its immune regulators reported here further reinforces this model. We, therefore, questioned: how can one reconcile the targeting of a conserved host-factor (RanGAP2) with specific recognition by Rx1/Gpa2?

One could envision several ways in which RanGAP2 functions as a recognition co-factor. The most classical view involves its role as a guarded host protein modified by the effector. This is in line with the critical cellular function of RanGAP2 that may be targeted to promote pathogen virulence, which is further reinforced by the binding of Gp-RBP1 and PVX-CP to a functional motif in RanGAP2 (Fig. 2, Fig.

3, Supplementals Fig. S3 and Fig. S4). An experimental system based on co-immunoprecipitations and Western blotting allowed us to evaluate the hypothesis that the interaction of PVX-CP or Gp-RBP1 could de-stabilize the complex of RanGAP2 with Rx1 or Gpa2. However, we were unable to detect any apparent changes in the stability, size, or banding pattern of RanGAP1, RanGAP2 or its bound receptors in our assays. Notably, we do not rule out the possibility that the effectors may impose other or more subtle modifications leading to the perturbations of RanGAP1/2. We, therefore, cannot formally exclude that RanGAP2 acts as a virulence target guarded by two NB-LRRs with distinct recognition specificities. The role of RanGAP2 as a guardee, however, contradicts earlier works detailing the lack of positively-selected residues on the RanGAP2 surface (Carpentier et al. 2013). This is expected from a guarded host protein as it would need to co-evolve with the pathogen. Additionally, for sensing perturbations of RanGAP2 (as proposed in the guard hypothesis), one would anticipate that a single NB-LRR receptor would be sufficient. This presumption holds unless the targeting of RanGAP2 by PVX-CP and Gp-RBP1 results in different modifications of the protein.

Our interaction data also suggest that RanGAP2 could serve as a bait that promotes direct recognition of the effectors. Here, the term 'bait' is generally ascribed to accessory host-factors that facilitate effector detection (Collier et al. 2009). The question then arises, how does RanGAP2 function as a bait that enhances the detection of PVX-CP and Gp-RBP1? Earlier works have shown that the surface 
for RanGAP2-binding in Rx1 lies within proximity to the EDVID motif that is required for the interaction of the Rx1-CC with the rest of the receptor protein (Hao et al. 2013). It is, therefore, imaginable that molecular events affecting the RanGAP2/CC complex are translated to the rest of the receptor module. For example, effector targeting of RanGAP2 may disturb the association of RanGAP2 with Rx1/Gpa2 causing a conformational change that leads to receptor activation as reported for EDS1 and RPS4, RPS6 (Bhattacharjee et al. 2011). Based on co-immunoprecipitation experiments, however, this was not the case (Fig. 4). This suggests that different surface regions of the RanGAP2-WPP domain may be required for binding to Gpa2/Rx1 and their cognate effectors, possibly allowing for the concurrent formation of a tripartite complex. We, therefore, propose that recognition of PVX-CP/Gp-RBP1 is a two-step event according to the bait-and-switch model (Collier et al. 2009). This model involves the initial 'docking' of the effectors to the bait, in this case, RanGAP2 via the WPP domain (Fig. 5). However, the landing of effectors to RanGAP2 is insufficient for recognition and subsequent receptor activation, given that the non-eliciting effectors also bind. Instead, we hypothesize that this docking brings the effector in closer proximity to the LRR, which is then able to directly sense structural determinants on an accessible/exposed side of the effector. In the case of $\mathrm{R} \times 1$ and Gpa2, this refers to sensing by the C-terminal half of the LRR, which has been shown to confer recognition specificity (Rairdan et al. 2006). Subsequently, specific effector recognition lifts autoinhibitory forces imposed by the LRR-ARC2 interface leading to activation (Slootweg et al. 2013, Collier et al. 2009). Such a model is in accordance with the divergence of the Rx1 and Gpa2 LRRs to sense structurally unrelated effectors. It is also consistent with our findings that different interfaces of Gp-RBP1 may be involved in RanGAP2 binding and detection by the receptor. Specifically, tagging Gp-RBP1 at the $\mathrm{N}$-terminus with a fluorophore prevents energy transfer in FRET-FLIM assay and co-localization with a WPP-NLS construct (Supplementals Fig. S4). This suggests that the $\mathrm{N}$-terminus may be involved for RanGAP2 binding. On the other hand, a proline to serine substitution at position 187 in the C-terminus determines recognition specificity but is not required for the binding of RanGAP (Sacco et al. 2009). This polarization in regions of Gp-RBP-1 required for RanGAP2 binding and recognition further reinforces the function of RanGAP2 as a molecular bait in the switch model.

In the model proposed, we therefore view RanGAP2 as fulfilling a modulatory role rather than being an absolute necessity for Gp-RBP1/PVX-CP recognition. This coincides with recent findings that Rx1 mutants impaired in RanGAP2 binding are still functional (Slootweg et al. 2018). We hypothesize that 
the facilitative role of RanGAP2 lies in bringing the effector in closer proximity to the cognate LRR domain and/or enhancing the effector/LRR binding affinity. In this scenario, binding to RanGAP2 would, thus, be critical when there is a low abundance of effectors in the cell to enhance sensitivity for effective detection of the effector. The function we ascribe is also reminiscent of that described for the extra Solanaceous Domain (SD) in the Sw-5b receptor protein, which is likewise postulated to enhance effector detection by the LRR (Li et al. 2019). The attributed role of RanGAP2 could be further linked to the finding that the N-terminus of Gp-RBP1 mediates the binding to RanGAP2 with variation in this region having been described to contribute to the strength in inducing Gpa2-mediated HR (Sacco et al. 2009). More detailed mechanistic studies are needed to resolve whether this directly correlates with the capacity to bind RanGAP2. The future challenge lies in uncovering the precise basis of RanGAP2-mediated activation of Rx1/Gpa2-like immune receptors by specific effector variants. Our finding that GpRBP-1 and PVX-CP interact with RanGAP homologs provides an important stepping-stone towards this goal.
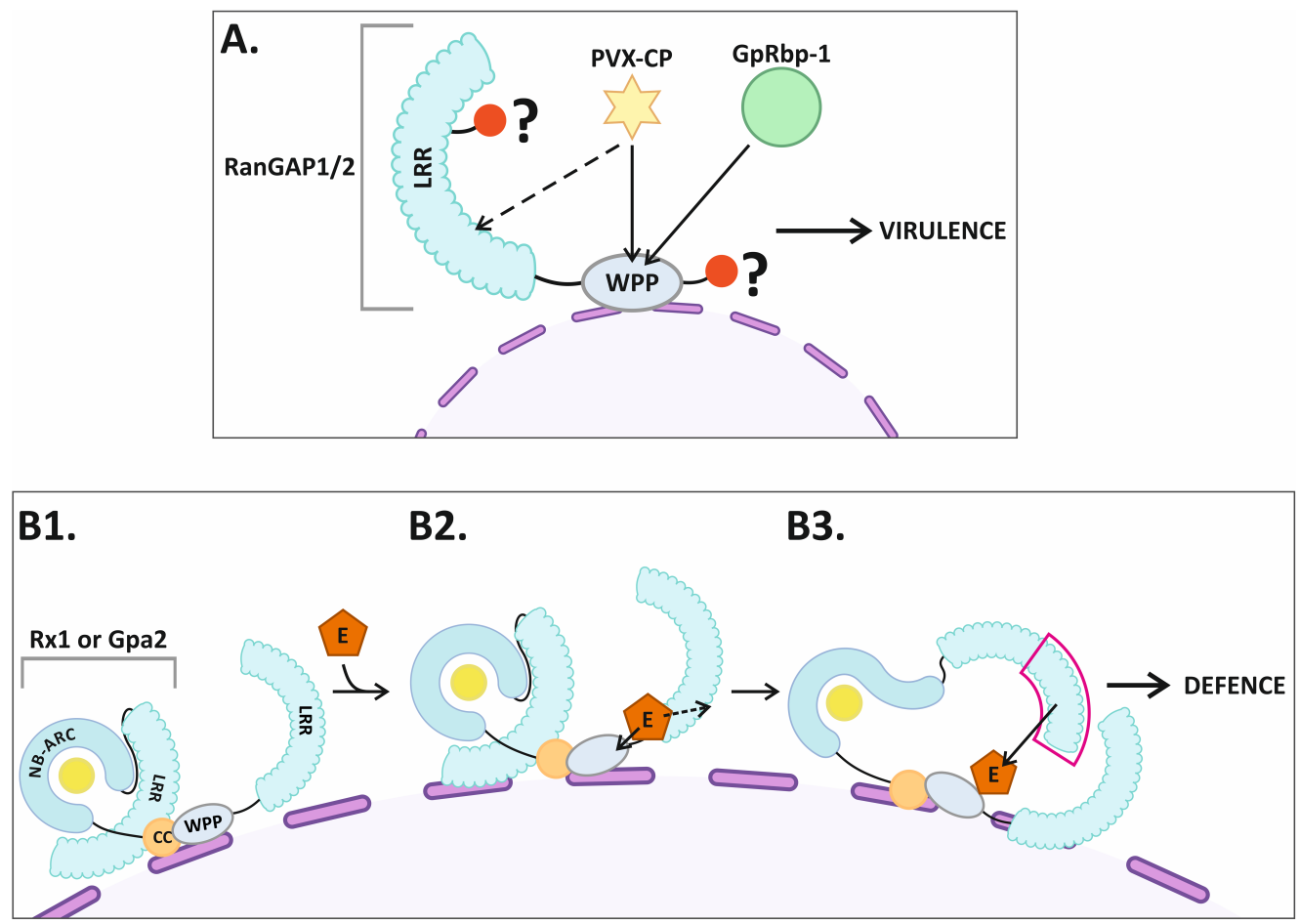

Fig. 5: Working model for the role of RanGAP1 and RanGAP2 in virulence (A) and recognition events by Gpa2/Rx1 (B). In the latter case, a model for only RanGAP2 is shown, for which a clearer role in resistance of Gpa2 and Rx1 has been established. A). In the absence of a matching NB-LRR 
(Figure legend continued) receptor, Gp-RBP1 and PVX-CP effectors target both RanGAP1 and RanGAP2 via the WPP and potentially, LRR domains, which contributes to viral disease and nematode feeding cell formation in a yet undisclosed manner (red circle). B) When Rx1/Gpa2 is present, the receptors tether to RanGAP1 and RanGAP2 at the nuclear envelope via its WPP domain (B1). During invasion, both virulent and avirulent forms of Gp-RBP1 and PVX-CP (orange pentagon labelled 'E') initially dock to RanGAP2, bringing the effector in close proximity to the immune receptor (B2). The C-terminal half of the receptor LRR (highlighted in red) then directly recognizes the presence of its cognate elicitor (B3) via an exposed surface of the effector that is not bound to RanGAP2. In the case of Gp-RBP1, this includes recognition of the C-terminal regions containing the S/P substitution which determines Gpa2 recognition, while the N-terminal Gp-RBP1 end associates with RanGAP2. Successful recognition leads to downstream events towards defence.

\section{MATERIALS AND METHODS}

\section{Plasmid constructs}

Constructs for expression of RanGAP2-mCherry, WPP-NLS-mCherry and WPP-nls*-mCherry were as described previously (Tameling et al. 2010). To obtain Gp-RBP1 variants D383-1 and Rook4 with $\mathrm{N}$ or C-terminally tagged GFP or HA, the target genes were initially subcloned into the pRAP vector by Ncol/Kpnl digestion (Schouten et al. 1997). A similar strategy was followed for cloning of CFP-tagged PVX-CPs. For transient expression experiments in N. benthamiana, the tagged-effector constructs were finally subcloned into the $\mathrm{pBIN}+$ binary vector and transformed into Agrobacterium tumefaciens strain MOG10 (van der Vossen et al. 2000).

\section{Heterologous expression by A. tumefaciens transient assay in N. benthamiana}

Heterologous protein expression was carried out by $A$. tumefaciens transient assay (ATTA) in plants, as described previously (Slootweg et al. 2010). Briefly, Agrobacteria strains carrying the expression vectors were grown in Yeast Extract Broth (YEB) medium ( $5 \mathrm{~g} / \mathrm{L}$ peptone, $1 \mathrm{~g} / \mathrm{L}$ yeast extract, $5 \mathrm{~g} / \mathrm{L}$ beef extract, $5 \mathrm{~g} / \mathrm{L}$ sucrose and $2.5 \mathrm{~g} / \mathrm{L} \mathrm{NaCl}$ and $2 \mathrm{ml}_{1} \mathrm{M} \mathrm{MgSO}_{4}$ ) overnight. Grown bacterial cells were spun down and re-suspended in infiltration medium and optical densities at wavelength $600 \mathrm{~nm}$ 
$\left(\mathrm{OD}_{600}\right)$ were adjusted to final $\mathrm{OD}_{600}$ values of 0.2-0.4 for all constructs in co-immunoprecipitation and imaging assays unless otherwise stated. A. tumefaciens suspensions were then infiltrated on the abaxial surface of the leaves of $N$. benthamiana plants using needleless syringes. Infiltrated spots were harvested for protein extraction or examined by microscopy at 2 days post infiltration (dpi).

\section{Transient silencing by TRV-VIGS and PVX resistance assay in N. benthamiana}

Constructs used for RanGAP1, RanGAP2 and RanGAP1 + 2 TRV-VIGS silencing in N. bethamiana are as described previously (Tameling et al. 2007, Tameling et al. 2010). Agroinfiltration was performed in a similar way as for the $N$. benthamiana agroinfiltrations (see above). Briefly, bacteria are grown overnight in YEB medium and re-suspended in MMAi containing $200 \mu \mathrm{M}$ acetosyringone. Final $\mathrm{OD}_{600}$ of TRV1 and TRV:Rg1, TRV:RG1+2 or TRV:RG2 mix were adjusted to 0.5 for infiltration. Infiltrated plants were grown for 21 days to allow for systematic silencing before use in viral infection assays as described previously in Slootweg et al. 2018. Briefly, Agrobacteria carrying amplicons for PVX105 or PVX106 were infiltrated on TRV-silenced plants at $\mathrm{OD}_{600}$ values of 0.002. Between 1-5 dpi, 13 $\mathrm{mm}$ leaf discs were harvested from infiltrated spots, extracted in phosphate buffer $(\mathrm{pH}=7)$ and finely ground using Tissuelyzer II (Qiagen) with settings of 30 seconds at $30 \mathrm{~Hz}$. Ground materials were incubated in a 96-well plate coated with polyclonal antibody targeted against the PVX-CP (Prime Diagnostics) at $37^{\circ} \mathrm{C}$ for 2 hours, before a second round of incubation with a conjugate antibody carrying alkaline phosphatase. Viral levels were quantified by absorbance measurements at $405 \mathrm{~nm}$ with the BioRad microplate reader (model 680) following a reaction with the substrate p-Nitrophenol.

\section{In planta co-immunoprecipitation and detection of recombinant proteins}

Total protein extracts were prepared by grinding leaf material in protein extraction buffer $(20 \%(\mathrm{v} / \mathrm{v})$ glycerol, 50 mM Tris-HCl pH 7.5, 2 mM EDTA, 300 mM NaCl, 0.6 mg/ml Pefabloc SC plus (Roche, Basel, Switzerland), 2\% (w/v) polyclar-AT polyvinylpolypyrrolidone (Serva, Heidelberg, Germany), 10 $\mathrm{mM}$ dithiothreitol and $0.1 \%(\mathrm{v} / \mathrm{v})$ Tween20) on ice. For co-immunoprecipitation, protein extracts were passed through a Sephadex G-25 column (GE Healthcare, Chicago, Illinois) and pre-cleared by 
treatment with rabbit-IgG agarose (Sigma, $50 \mu \mathrm{L}$ slurry per $60 \mu \mathrm{L}$ protein extract). The cleared protein extract was incubated with $\mu$ MACS $\alpha$-GFP paramagnetic (Miltenyi, Bergisch Gladbach, Germany) for $1 \mathrm{~h}$ at $4^{\circ} \mathrm{C}$. Columns were washed with washing buffer $(20 \%(\mathrm{v} / \mathrm{v})$ glycerol, $50 \mathrm{mM}$ Tris-HCl pH 7.5 , $2 \mathrm{mM}$ EDTA, $300 \mathrm{mM} \mathrm{NaCl}, 0.10 \%(\mathrm{v} / \mathrm{v})$ Nonidet 40 and $5 \mathrm{mM}$ dithiothreitol) five times and eluted by removing the column from the $\mu \mathrm{MACS}$ collector and adding $45 \mu \mathrm{L}$ of the washing with the washing solution. The input samples were mixed with 1X NuPage LDS sample buffer with $0.25 \mathrm{M}$ dithiothreitol and incubated at $95^{\circ} \mathrm{C}$ for 5 minutes.

For western blotting, proteins were separated by SDS-PAGE on NuPage $12 \%$ Bis-Tris gels (Invitrogen) and blotted to $0.45 \mu \mathrm{m}$ polyvinylidene difluoride membrane (Thermo Scientific). Before immunodetection we blocked the membranes for $1 \mathrm{~h}$ at room temperature in $5 \%(\mathrm{w} / \mathrm{v})$ powder milk in PBS with $0.1 \%$ Tween20. For immunodetection rabbit $\alpha$-GFP (Abcam, Cambridge, United Kingdom) with horseradish peroxidase-conjugated donkey $\alpha$-rabbit (Jackson ImmunoResearch, Ely, United Kingdom) or horseradish peroxidase-conjugated rat $\alpha$ - HA (Roche) were used. Peroxidase activity was visualized using SuperSignal West Femto or Dura substrate (Thermo Scientific) and imaging of the luminescence with G:BOX gel documentation system (Syngene, United Kingdom).

\section{Confocal laser scanning and FRET-FLIM microscopy}

Confocal microscopy was performed on N. benthamiana epidermal cells using a Zeiss LSM 510 confocal microscope (Carl-Zeiss) with a 40X 1.2 numerical aperture water-corrected objective. For co-localization studies the argon laser was used to excite at $488 \mathrm{~nm}$ for GFP and chlorophyll, and the HeNe laser at 543nm to excite mCherry. GFP and chlorophyll emission were detected through a band-pass filter of 505 to $530 \mathrm{~nm}$ and through a $650 \mathrm{~nm}$ long-pass filter, respectively. mCherry emission was detected through a band-pass filter of 600 to $650 \mathrm{~nm}$. Nuclear and cytoplasmic fluorescence intensities were quantified using ImageJ (Schindelin et al. 2012). For FRET-FLIM analysis, the FRET between GFP and mCherry was detected via Fluorescent Lifetime Imaging Microscopy. The HYD SMD detector of a Leica SP5 CLSM (Leica, Wetzlar, Germany) was used to measure the emission and fluorescent lifetime of GFP $(495-545 \mathrm{~nm})$ and the red fluorescent $\mathrm{mCh}$ emission (570-625 nm). The excitation of the GFP chromophore was measured using a white light laser (488 nm). The Time-correlated single-photon counting (TCSPC) was performed using a Becker 
\& Hickl FLIM system FLIM analysis of TCSPC was performed with the B\&H SPCImage software (Becker \& Hickl GmbH, Berlin, Germany).

\section{Nematode infection assays in $A$. thaliana}

rg1-1;rg1-1/rg2-2;rg2-2 mutant plants were obtained from the group of I. Meier. All A. thaliana genotypes used in the experiments are in the Columbia 0 genetic background. The presence of T-DNA inserts in the lines was confirmed by PCR using specific primers designed with the iSect Primers tool of the SIGNAL SALK database (Supplementals Table S1), in combination with the universal LB primer (Alonso et al. 2003). For nematode assays, seeds were vapour sterilized and vernalized at $4^{\circ} \mathrm{C}$ in the dark for 4 days to break seed dormancy. After vernalisation the seeds were plated in pairs in $9 \mathrm{~cm}$ petri dishes containing Knop's modified medium. Plants were grown at $25^{\circ} \mathrm{C}$ under a 16h/8h light-dark cycle. 10 day-old seedlings were inoculated with 60-70 surface-sterilized H. schachtii infective juveniles. After 2 weeks of infection, the number of males and females present in the roots of Arabidopsis plants were counted visually and the size of females and syncytia were calculated with Leica M165C Binocular (Leica Microsystems, Wetzlar, Germany) and the Leica Application Suite software (Leica Microsystems). To combine results from 4 biological replicates, we weighted the measures of association from each replicate by the inverses of their variances. The variance of such weighted average is simply the inverse of the sum of the inverses of the variances which allow standard methods to be used to test for the overall significance at the $5 \%$ level of the genotype and the number of nematodes per plant. Such approach corresponds to methods to combine studies under a fixed effects model.

\section{Statistical test}

Statistical analyses were performed in R studio Version 1.1.456. Data from assays performed in this study were checked for normality using Shapiro-Wilk Test. Depending upon the outcome, statistical level was determined either by T-test or Wilcoxon-Signed Rank Test with $\alpha=0.05$ as specified in the text. 


\section{ACKNOWLEDGEMENTS}

The works described in this thesis benefits from the financial support of the Dutch Top Technology Institute Green Genetics (5CFD051RP), Dutch Technology Hotel grant and the Dutch Technology Foundation STW and Earth and Life Sciences ALW (STW-GG 14529), which are part of the Netherlands Organization for Scientific Research (NWO). We also thank Matthieu Joosten for providing TRV:VIGS, RanGAP1 and RanGAP2-GFP and RanGAP2-mCherry mutant constructs. 


\section{SUPPLEMENTALS}

Supplemental Fig. S1: The Gp-RBP1s D383-1 and Rook4 interact with the RanGAP2-WPP domain in an immunoprecipitation assay. $\alpha$-HA magnetic beads were used for the pull-down of Gp-RBP1 variants as baits in combination with the RanGAP2-WPP domain. 4 XHA-GFP was included as negative control. Protein extracts of leaves harvested at 2 dpi were used for the immunoprecipitation assay. "+" indicates the presence of a construct in the co-expressed combination.

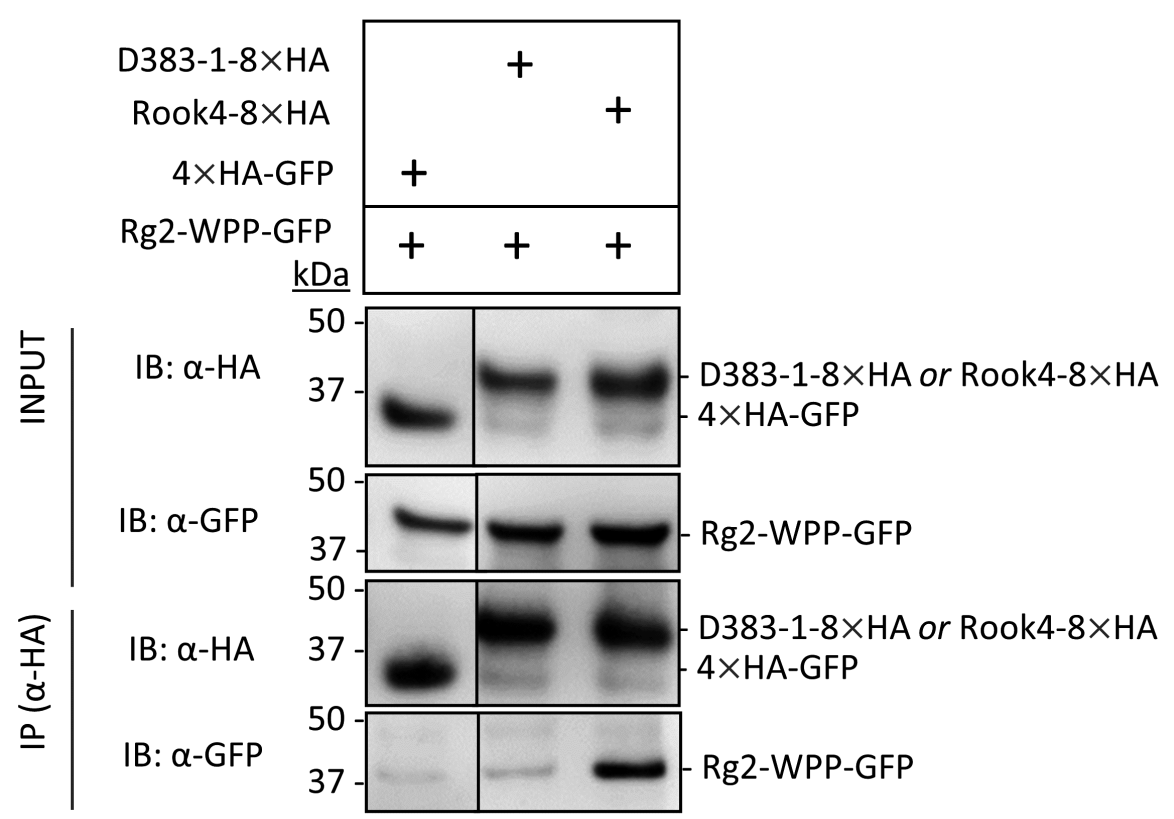


Supplemental Fig. S2: The binding of RanGAP2 to the CC domain of Rx1 is not disrupted in the presence of the effectors studied. Co-immunoprecipitation investigating whether the interaction between the CC domain of Rx1 and RanGAP2 is affected by the coat proteins of avirulent and virulent PVX-CP strains or by the Gp-RBPs D383-1 or Rook4. The samples were harvested at 48 hours post agroinfiltration. As a control for aspecific binding, 4 X Myc-GFP was used as bait. "+" indicates the presence of a construct in the co-expressed combination.

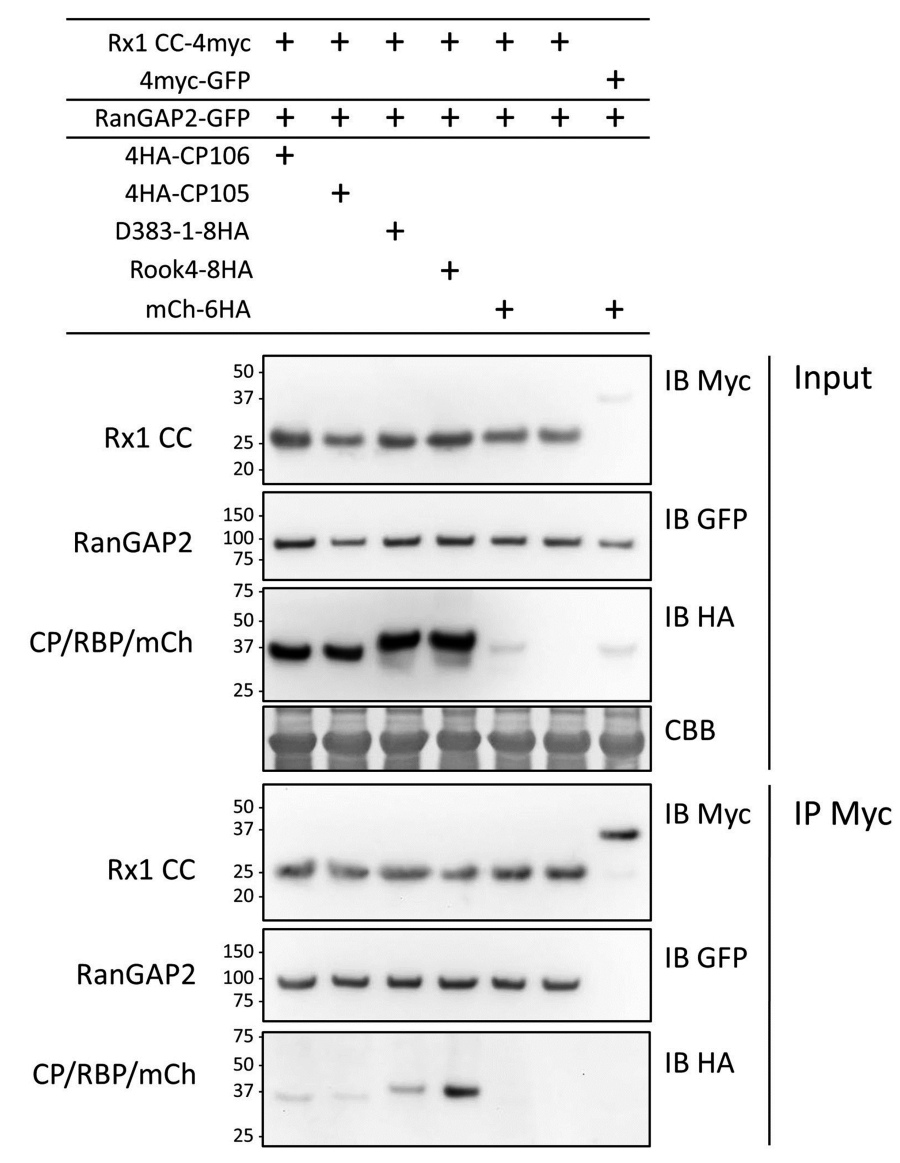


Supplemental Fig. S3: Mutating a conserved Aspartic acid residue in the LRR domain of RanGAP2 compromises its interaction with CP106. $\alpha$-HA magnetic beads were used precipitate $4 \times$ HA-CP106 as baits in an immunoprecipitation assay to test the physical association with RanGAP2-mCherry or mutant variants (Rg2 D335A-mCherry or Rg2 D335N-mCherry). Rx1-4 XHA.GFP was included as positive control. Infiltrated $N$. benthamiana leaves harvested at $2 \mathrm{dpi}$ were used in the immunoprecipitation assay. "+" indicates the presence of a construct in the co-expressed combination.

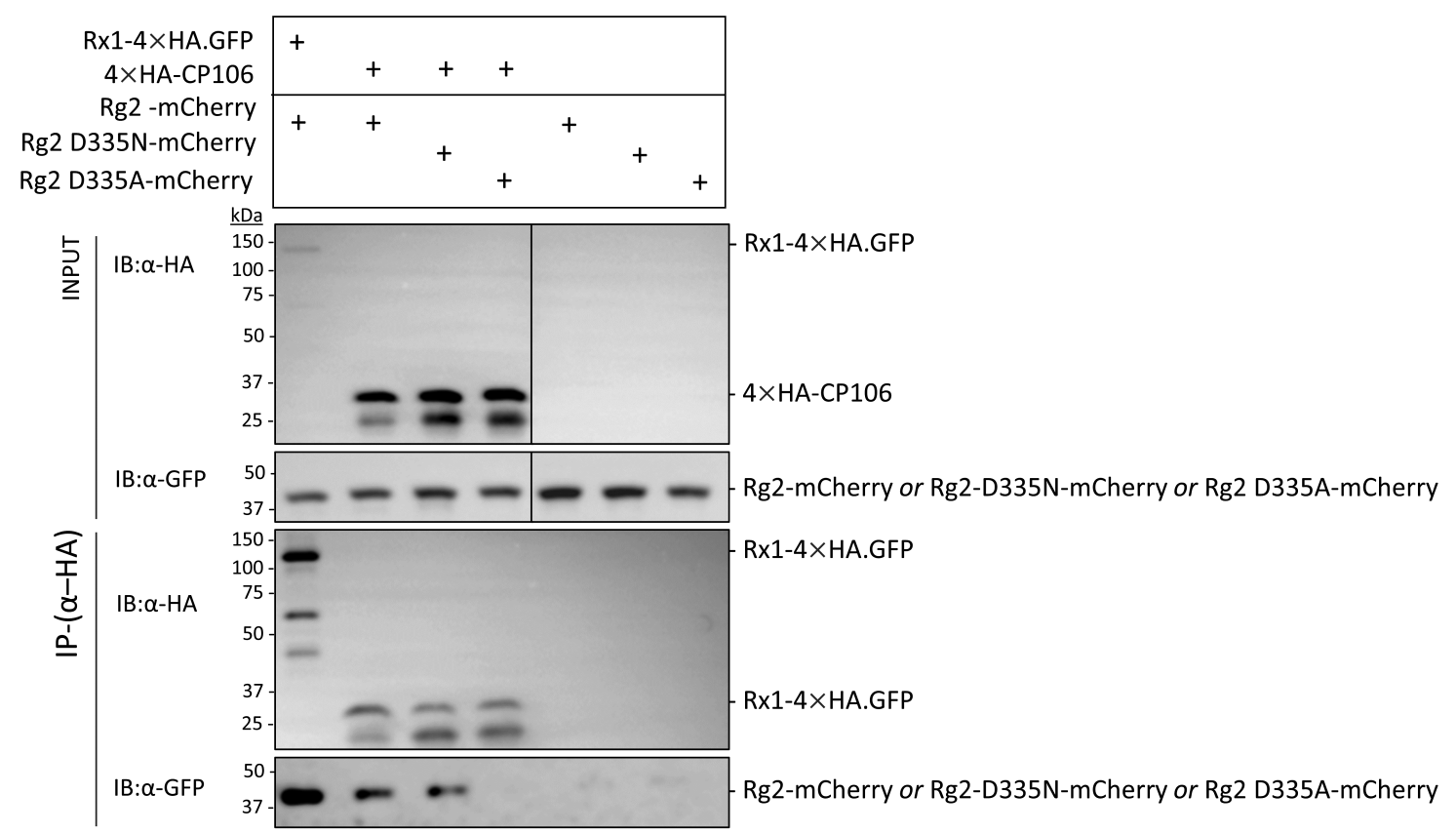


Supplemental Fig. S4: Tagging Gp-RBP1 with a fluorescence protein at the N-terminus impairs its interaction with RanGAP2 (WPP domain). A). Confocal imaging of RanGAP2-WPP-NLS constructs co-expressed with Rook4 or D383-1 tagged with GFP at the N or C termini. Representative images of nuclei in infiltrated $N$. benthamiana epidermal cells for combinations involving $\mathrm{N}$-terminally tagged Gp-RBP1s are given in A1. Key: N = nucleus; C = cytoplasm. Quantification of cellular distribution by ${ }^{N} / / C$ measurements is summarized in boxplot of $\mathrm{A} 2$ with boxes representing the interquartile range. Data shown is the combination of two experimental repeats. B) Boxplot indicating lifetime (picoseconds) from a FRET-FLIM experiment whereby full length RanGAP2-mCh is co-expressed with the same set of effectors as described in A2. Data shown is pooled from three experimental repeats.

A1.

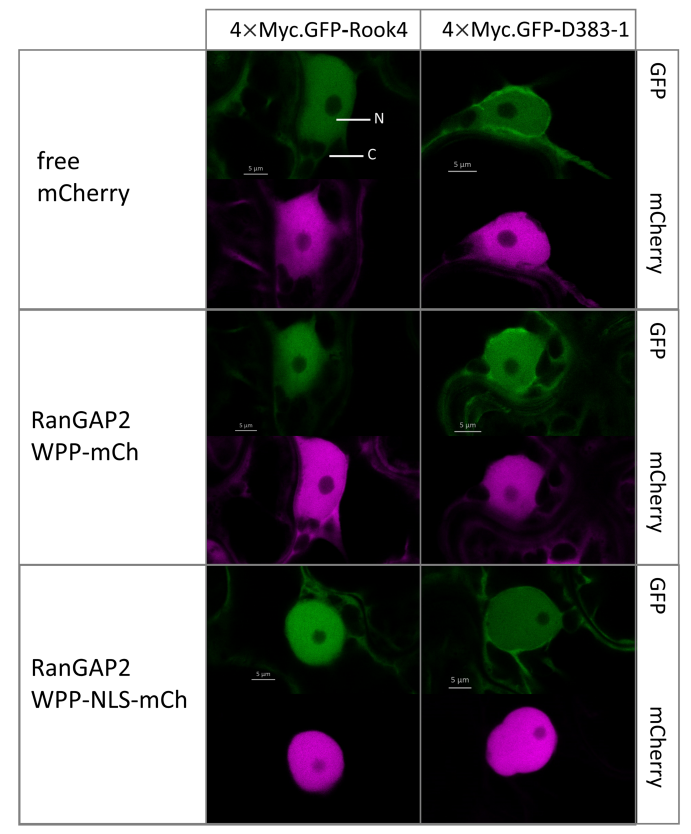

A2.

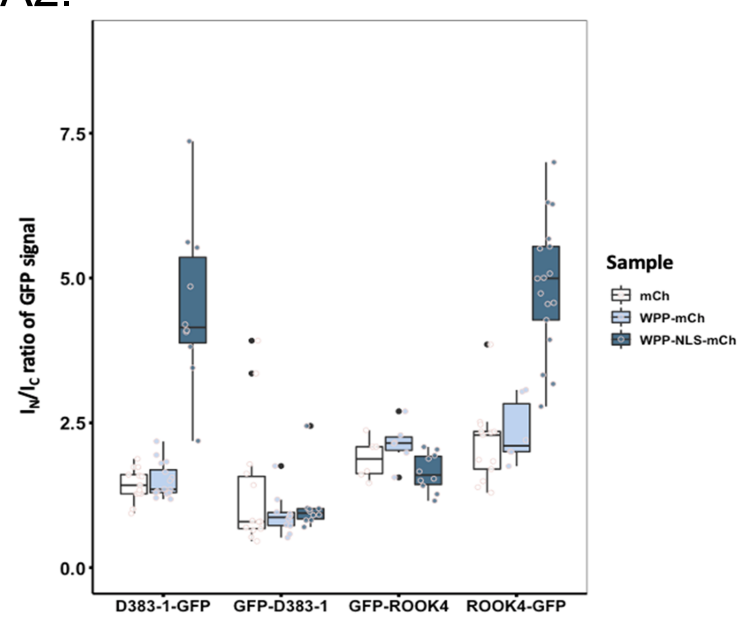

B.

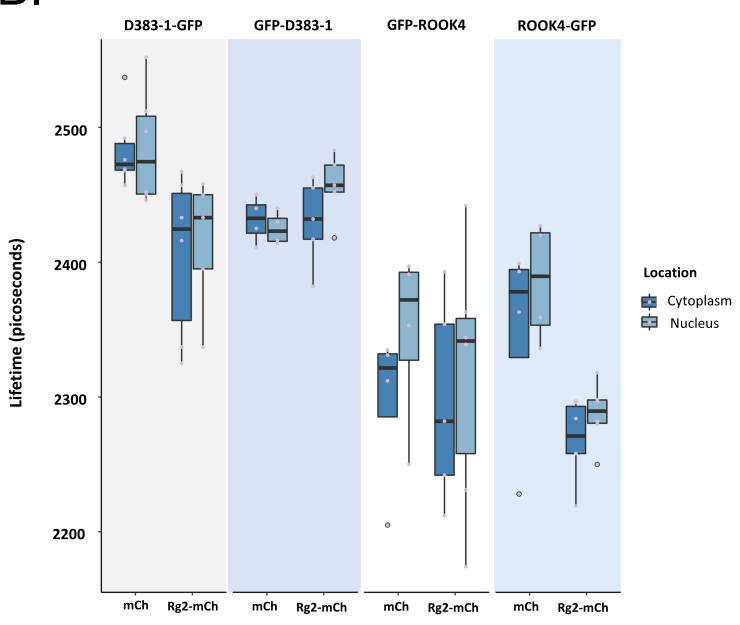


Supplemental Table S1: List of primers used in the study for the genotyping of $A$. thaliana RanGAP mutants.

\begin{tabular}{cc}
\hline Primer name & Sequence \\
\hline SALK_058630_LP $(r g 1-1)$ & 5' - CAAAGGAACAAGCTTGTCCAG - 3' \\
SALK_058630_RP $(r g 1-1)$ & 5' - TCCATTTCTCCAACGAATCTG - 3' \\
SALK_006398_LP $($ rg2-2 $)$ & 5' - CTCCTCTGATCACAATCGGTC - 3' \\
SALK_006398_RP $(r g 2-2)$ & 5' - TGGTCTTTGGTTAAGCTACCG - 3' \\
\hline
\end{tabular}




\section{References}

Alonso, J. M., Stepanova, A. N., Leisse, T. J., Kim, C. J., Chen, H., Shinn, P., Stevenson, D. K., Zimmerman, J., Barajas, P., Cheuk, R., Gadrinab, C., Heller, C., Jeske, A., Koesema, E., Meyers, C. C., Parker, H., Prednis, L., Ansari, Y., Choy, N., Deen, H., Geralt, M., Hazari, N., Hom, E., Karnes, M., Mulholland, C., Ndubaku, R., Schmidt, I., Guzman, P., Aguilar-Henonin, L., Schmid, M., Weigel, D., Carter, D. E., Marchand, T., Risseeuw, E., Brogden, D., Zeko, A., Crosby, W. L., Berry, C. C., and Ecker, J. R. (2003), Genome-Wide Insertional Mutagenesis of Arabidopsis thaliana. Science. 301(5633): p. 653.

Bendahmane, A., Kanyuka, K., and Baulcombe, D. C. (1999), The Rx gene from potato controls separate virus resistance and cell death responses. Plant Cell. 11.

Bendahmane, A., Köhm, B. A., Dedi, C., and Baulcombe, D. C. (1995), The coat protein of potato virus X is a strain-specific elicitor of Rx1-mediated virus resistance in potato. The Plant Journal. 8(6): p. 933-941.

Bernoux, M., Burdett, H., Williams, S. J., Zhang, X., Chen, C., Newell, K., Lawrence, G. J., Kobe, B., Ellis, J. G., Anderson, P. A., and Dodds, P. N. (2016), Comparative Analysis of the Flax Immune Receptors L6 and L7 Suggests an Equilibrium-Based Switch Activation Model. Plant Cell. 28(1): p. 146-59.

Bhattacharjee, S., Halane, M. K., Kim, S. H., and Gassmann, W. (2011), Pathogen Effectors Target Arabidopsis EDS1 and Alter Its Interactions with Immune Regulators. Science. 334(6061): p. 1405-1408.

Bücherl, C., Aker, J., de Vries, S., and Borst, J. W. (2010), Probing Protein-Protein Interactions with FRET-FLIM. In: Plant Developmental Biology: Methods and Protocols. Ed. by L. Hennig and C. Köhler. Totowa, NJ: Humana Press, p. 389-399.

Carella, P., Evangelisti, E., and Schornack, S. (2018), Sticking to it: phytopathogen effector molecules may converge on evolutionarily conserved host targets in green plants. Current opinion in plant biology. 44: p. 175-180.

Carpentier, J., Grenier, E., Esquibet, M., Hamel, L.-P., Moffett, P., Manzanares-Dauleux, M. J., and Kerlan, M.-C. (2013), Evolution and variability of Solanum RanGAP2, a cofactor in the incompatible interaction between the resistance protein GPA2 and the Globodera pallida effector Gp-RBP-1. BMC Evolutionary Biology. 13(1): p. 1-14.

Collier, S. M. and Moffett, P. (2009), NB-LRRs work a "bait and switch" on pathogens. Trends in Plant Science. 14(10): p. $521-529$.

Cruz, S. S., Chapman, S., Roberts, A. G., Roberts, I. M., Prior, D. A., and Oparka, K. J. (1996), Assembly and movement of a plant virus carrying a green fluorescent protein overcoat. Proc Natl Acad Sci U S A. 93(13): p. 6286-90.

Dodds, P. N. and Rathjen, J. P. (2010), Plant immunity: towards an integrated view of plant-pathogen interactions. Nat Rev Genet. 11(8): p. 539-48.

Farnham, G. and Baulcombe, D. C. (2006), Artificial evolution extends the spectrum of viruses that are targeted by a disease-resistance gene from potato. Proc Natl Acad Sci U S A. 103(49): p. 18828-33.

Hao, W., Collier, S. M., Moffett, P., and Chai, J. (2013), Structural basis for the interaction between the potato virus $\mathrm{X}$ resistance protein $(\mathrm{Rx})$ and its cofactor Ran GTPase-activating protein 2 (RanGAP2). The Journal of biological chemistry. 288(50): p. 35868-35876.

Jia, Y., McAdams, S. A., Bryan, G. T., Hershey, H. P., and Valent, B. (2000), Direct interaction of resistance gene and avirulence gene products confers rice blast resistance. The EMBO journal. 19(15): p. 4004-4014.

Li, J., Huang, H., Zhu, M., Huang, S., Zhang, W., Dinesh-Kumar, S. P., and Tao, X. (2019), A Plant Immune Receptor Adopts a Two-Step Recognition Mechanism to Enhance Viral Effector Perception. Molecular Plant. 12(2): p. $248-262$.

Liu, J. and Coaker, G. (2008), Nuclear Trafficking During Plant Innate Immunity. Molecular Plant. 1(3): p. 411-422. 
Park, M.-R. and Kim, K.-H. (2013), Molecular characterization of the interaction between the N-terminal region of Potato virus X (PVX) coat protein (CP) and Nicotiana benthamiana PVX CP-interacting protein, NbPCIP1. Virus Genes. 46(3): p. 517-523.

Pay, A., Resch, K., Frohnmeyer, H., Fejes, E., Nagy, F., and Nick, P. (2002), Plant RanGAPs are localized at the nuclear envelope in interphase and associated with microtubules in mitotic cells. Plant J. 30.

Porter, F. W., Bochkov, Y. A., Albee, A. J., Wiese, C., and Palmenberg, A. C. (2006), A picornavirus protein interacts with Ran-GTPase and disrupts nucleocytoplasmic transport. Proceedings of the National Academy of Sciences of the United States of America. 103(33): p. 12417-12422.

Quentin, M., Abad, P., and Favery, B. (2013), Plant parasitic nematode effectors target host defense and nuclear functions to establish feeding cells. Frontiers in Plant Science. 4(53).

Rairdan, G. J. and Moffett, P. (2006), Distinct Domains in the ARC Region of the Potato Resistance Protein Rx Mediate LRR Binding and Inhibition of Activation. Plant Cell. 18(8): p. 2082-2093.

Ravensdale, M., Bernoux, M., Ve, T., Kobe, B., Thrall, P. H., Ellis, J. G., and Dodds, P. N. (2012), Intramolecular Interaction Influences Binding of the Flax L5 and L6 Resistance Proteins to their AvrL567 Ligands. PLoS Pathog. 8(11): e1003004.

Rodrigo-Peiris, T., Xu, X. M., Zhao, Q., Wang, H.-J., and Meier, I. (2011), RanGAP is required for post-meiotic mitosis in female gametophyte development in Arabidopsis thaliana. Journal of Experimental Botany. 62(8): p. 2705-2714.

Rose, A. and Meier, I. (2001), A domain unique to plant RanGAP is responsible for its targeting to the plant nuclear rim. Proc Natl Acad Sci USA. 98.

Sacco, M. A., Koropacka, K., Grenier, E., Jaubert, M. J., Blanchard, A., Goverse, A., Smant, G., and Moffett, P. (2009), The cyst nematode SPRYSEC protein RBP-1 elicits Gpa2- and RanGAP2-dependent plant cell death. PLoS Pathog. 5.

Sacco, M. A., Mansoor, S., and Moffett, P. (2007), A RanGAP protein physically interacts with the NB-LRR protein Rx, and is required for Rx-mediated viral resistance. Plant J. 52(1): p. 82-93.

Schindelin, J., Arganda-Carreras, I., Frise, E., Kaynig, V., Longair, M., Pietzsch, T., Preibisch, S., Rueden, C., Saalfeld, S., Schmid, B., Tinevez, J. Y., White, D. J., Hartenstein, V., Eliceiri, K., Tomancak, P., and Cardona, A. (2012), Fiji: an open-source platform for biological-image analysis. Nat Methods. 9(7): p. 676-82.

Schouten, A., Roosien, J., de Boer, J. M., Wilmink, A., Rosso, M.-N., Bosch, D., Stiekema, W. J., Gommers, F. J., Bakker, J., and Schots, A. (1997), Improving scFv antibody expression levels in the plant cytosol 1. FEBS Letters. 415(2): p. 235-241.

Slootweg, E., Roosien, J., Spiridon, L. N., Petrescu, A. J., Tameling, W., Joosten, M., Pomp, R., van Schaik, C., Dees, R., Borst, J. W., Smant, G., Schots, A., Bakker, J., and Goverse, A. (2010), Nucleocytoplasmic distribution is required for activation of resistance by the potato NB-LRR receptor $\mathrm{Rx} 1$ and is balanced by its functional domains. Plant Cell. 22(12): p. 4195-215.

Slootweg, E., Koropacka, K., Roosien, J., Dees, R., Overmars, H., Lankhorst, R. K., van Schaik, C., Pomp, R., Bouwman, L., Helder, J., Schots, A., Bakker, J., Smant, G., and Goverse, A. (2017), Sequence Exchange between Homologous NB-LRR Genes Converts Virus Resistance into Nematode Resistance, and Vice Versa. Plant physiology. 175(1): p. 498-510.

Slootweg, E. J., Spiridon, L. N., Martin, E. C., Tameling, W. I. L., Townsend, P. D., Pomp, R., Roosien, J., Drawska, O., Sukarta, O. C. A., Schots, A., Borst, J. W., Joosten, M. H., Bakker, J., Smant, G., Cann, M. J., Petrescu, A.-J., and Goverse, A. (2018), Distinct Roles of Non-Overlapping Surface Regions of the Coiled-Coil Domain in the Potato Immune Receptor Rx1. Plant Physiology. 178(3): p. 1310-1331. 
Slootweg, E. J., Spiridon, L. N., Roosien, J., Butterbach, P., Pomp, R., Westerhof, L., Wilbers, R., Bakker, E., Bakker, J., Petrescu, A.-J., Smant, G., and Goverse, A. (2013), Structural Determinants at the Interface of the ARC2 and Leucine-Rich Repeat Domains Control the Activation of the Plant Immune Receptors Rx1 and Gpa2. Plant Physiology. 162(3): p. 1510-1528.

Song, J., Win, J., Tian, M., Schornack, S., Kaschani, F., llyas, M., van der Hoorn, R. A. L., and Kamoun, S. (2009), Apoplastic effectors secreted by two unrelated eukaryotic plant pathogens target the tomato defense protease Rcr3. Proceedings of the National Academy of Sciences. 106(5): p. 1654.

Takken, F. L. W., Albrecht, M., and Tameling, W. I. L. (2006), Resistance proteins: molecular switches of plant defence. Curr Opin Plant Biol. 9(4): p. 383-90.

Tameling, W. I. L. and Baulcombe, D. C. (2007), Physical association of the NB-LRR resistance protein Rx with a Ran GTPase-activating protein is required for extreme resistance to Potato virus X. Plant Cell. 19(5): p. 1682-94.

Tameling, W. I. L., Nooijen, C., Ludwig, N., Boter, M., Slootweg, E., Goverse, A., Shirasu, K., and Joosten, M. (2010), RanGAP2 Mediates nucleocytoplasmic partitioning of the NB-LRR immune receptor Rx in the solanaceae. Thereby dictating Rx function. Plant Cell. 22.

Van der Vossen, E. A. G., van der Voort, J., Kanyuka, K., Bendahmane, A., Sandbrink, H., Baulcombe, D. C., Bakker, J., Stiekema, W. J., and Klein-Lankhorst, R. M. (2000), Homologues of a single resistance-gene cluster in potato confer resistance to distinct pathogens: a virus and a nematode. Plant J. 23.

Van Wersch, S., Tian, L., Hoy, R., and Li, X. (2020), Plant NLRs: The Whistleblowers of Plant Immunity. Plant Communications. 1(1): p. 100016.

Weßling, R., Epple, P., Altmann, S., He, Y., Yang, L., Henz, S. R., McDonald, N., Wiley, K., Bader, K. C., Gläßer, C., Mukhtar, M. S., Haigis, S., Ghamsari, L., Stephens, A. E., Ecker, J. R., Vidal, M., Jones, J. D. G., Mayer, K. F. X., Ver Loren van Themaat, E., Weigel, D., Schulze-Lefert, P., Dangl, J. L., Panstruga, R., and Braun, P. (2014), Convergent Targeting of a Common Host Protein-Network by Pathogen Effectors from Three Kingdoms of Life. Cell Host \& Microbe. 16(3): p. 364-375.

Xu, X. M., Zhao, Q., Rodrigo-Peiris, T., Brkljacic, J., He, C. S., Müller, S., and Meier, I. (2008), RanGAP1 is a continuous marker of the Arabidopsis cell division plane. Proceedings of the National Academy of Sciences. 105(47): p. 18637-18642. 
Octavina C. A Sukarta ${ }^{1,6}$

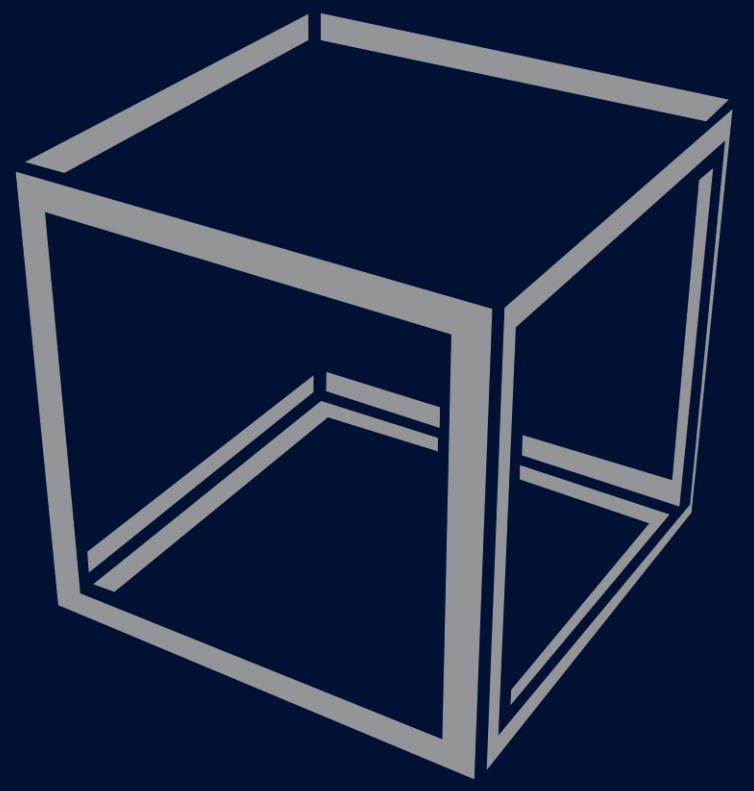

Philip D. Townsend ${ }^{2,3,6}$

Alexander Llewelyn ${ }^{2,3}$

Christopher H. Dixon ${ }^{2,3}$

Erik J. Slootweg ${ }^{1}$

Lars-Olof Pålsson ${ }^{3,4}$

Frank L.W. Takken ${ }^{5}$

Aska Goverse ${ }^{1}$

Martin J Cann ${ }^{2,3}$

${ }^{1}$ Laboratory of Nematology, Department of Plant Sciences, Wageningen University \& Research, 6708 PB, Wageningen, The Netherlands.

${ }^{2}$ Department of Biosciences, ${ }^{3}$ Biophysical Sciences Institute, ${ }^{4}$ Department of Chemistry, Durham University, South Road, Durham, DH1 3LE, United Kingdom.

${ }^{5}$ Molecular Plant Pathology, Swammerdam Institute for Life Sciences, University of Amsterdam, Science Park 904, 1098 XH, Amsterdam, The Netherlands.

${ }^{6}$ These authors contributed equally to this work 


\section{Chapter}

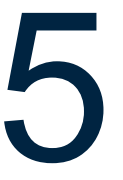

A DNA-Binding Bromodomain-Containing

Protein Interacts with and Reduces

Rx1-Mediated Immune Response to Potato

Virus $X$

"A slightly modified version of this chapter has been published in Plant Communications, 2020,

Volume 1, Issue 4, 100086 


\section{ABSTRACT}

Plant NB-LRR proteins enable the immune system to recognise and respond to pathogen attack. An early consequence of immune activation is transcriptional reprogramming. Some NB-LRRs have been shown to act in the nucleus and interact with transcription factors. The Rx1 NB-LRR protein of potato has been shown previously to bind and distort double-stranded DNA. However, the components of the chromatin localized Rx1-complex are largely unknown. Here we report a physical and functional interaction between $\mathrm{R} \times 1$ and $\mathrm{NbDBCP}$, a bromodomain-containing chromatin-interacting protein. NbDBCP accumulates in the nucleolus, interacts with chromatin and redistributes $\mathrm{R} \times 1$ to the nucleolus in a subpopulation of imaged cells. Rx1 over-expression reduces NbDBCP interactions with chromatin. $\mathrm{NbDBCP}$ is a negative regulator of $\mathrm{R} \times 1$-mediated immune responses to potato virus $\mathrm{X}(\mathrm{PVX})$ and this activity requires an intact bromodomain. Previously, $\mathrm{R} \times 1$ has been shown to regulate the DNA-binding activity of a Golden2-like transcription factor, NbGlk1. Rx1 and NbDBCP act synergistically to reduce NbGIk1 DNA-binding suggesting a mode of action for NbDBCP's inhibitory effect on immunity. This study provides new mechanistic insight into how a chromatin localised NB-LRR complex co-ordinates immune signalling following pathogen perception. 


\section{INTRODUCTION}

The plant innate immune system enables cell-autonomous defence responses upon pathogen perception (Jones et al. 2016, Maekawa et al. 2011a). Plant nucleotide-binding leucine-rich repeat (NB-LRR)-type immune receptors directly or indirectly detect pathogen-produced effector protein and mediate the immune responses to the invading pathogen (Eitas et al. 2010, Maekawa et al. 2011a, Staskawicz et al. 2001). NB-LRR proteins are members of the signal-transduction ATPases with numerous domains (STAND) and have a multidomain structure that allows them to function as a sensor, switch and response factor (Leipe et al. 2004, Takken et al. 2009). Plant NB-LRR immune receptors can be broadly separated into three distinct domains. The $\mathrm{N}$-terminus typically consists of either a coiled-coil (CC) or Toll-interleukin 1 receptor domain involved in inter- and intramolecular interactions (Maekawa et al. 2011b). The nucleotide-binding (NB) domain, sometimes

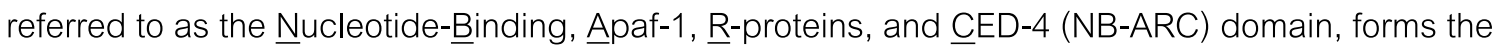
central domain and is proposed to function as a nucleotide-dependent molecular switch in NB-LRR activation (Takken et al. 2006, Takken et al. 2009, Tranier et al. 2000, van der Biezen et al. 1998). Finally, the C-terminal leucine rich repeat (LRR) domain determines pathogen recognition specificity and maintains the NB-LRR protein in an auto-inhibited state in the absence of a pathogen-derived signal. The NB-ARC domains of NB-LRRs are ADP-bound in the off state (Maekawa et al. 2011b, Tameling et al. 2006, Williams et al. 2011). Pathogen recognition by the NB-LRR was hypothesised to activate nucleotide exchange of ADP for ATP allowing the NB-ARC domain to adopt an activated state triggering immune signalling, while hydrolysis of ATP to ADP is proposed to re-establish the inactivated state (Tameling et al. 2006). ATP binding in potato $\mathrm{R} \times 1$ has been linked to its in vitro activity (Fenyk et al. 2015). Recent elucidation of the cryoEM structure of Arabidopsis ZAR1 in its autoinhibited and activated state provide structural support for this ADP/ATP exchange model (Wang et al. 2019a, Wang et al. 2019b). The NB-ARC domain, however, might not be a strict ATPase for all plant NB-LRRs as for example the NB subdomains of rice Os2g_25900, maize PSiP and Arabidopsis Rpm1 can sequentially cleavage phosphates from the nucleotide to the nucleoside in vitro, although the role of this activity in in vivo remains to be determined (Fenyk et al. 2012).

Proper NB-LRR function often requires a nucleocytoplasmic distribution in the cell. A subset of NB-LRR proteins, including N, MLA10, and Rx1 have dynamic nuclear-cytoplasmic distribution, 
while RRS1-R becomes localised to the nucleus, dependent upon the PopP2 effector (Burch-Smith et al. 2007, Caplan et al. 2008, Deslandes et al. 2003, Shen et al. 2007, Slootweg et al. 2010). Several other NB-LRRs, including barley MLA1 and MLA10, Arabidopsis RPS4 and SNC1 and the tobacco N protein also show nuclear localization (Bai et al. 2012, Shen et al. 2007, Wirthmueller et al. 2007, Zhu et al. 2010). Nuclear expulsion of Rx1, MLA10, N, RPS4, and SNC1 compromises immune activation suggestive of NB-LRR-dependent signalling components residing in the nucleus (Burch-Smith et al. 2007, Cheng et al. 2009, Shen et al. 2007, Wirthmueller et al. 2007). The identity of these signalling components is, therefore, of considerable interest.

In vitro biochemistry demonstrates that at least a subset of plant NB-LRRs are directly active at DNA. A significant structural homology was proposed between the NB-LRR NB-ARC domain and the DNA replication origin-binding Cdc6/Orc1 proteins. In line with this observation, a direct interaction of Rx1 of potato, I-2 of tomato and the orphan NB-LRR PSiP of maize with DNA has been observed in vitro (Fenyk et al. 2016, Fenyk et al. 2015). The $R \times 1$ gene, introgressed in potato from the wild species $\mathrm{mm}$ tuberosum ssp. andigena, confers resistance to potato virus $\mathrm{X}(\mathrm{PVX})$ upon recognition of its coat protein (Bendahmane et al. 1999, Bendahmane et al. 1995). The Rx1 protein binds to genomic DNA in situ on immune activation (Fenyk et al. 2015). In addition, Rx1 induces ATP-dependent bendingand melting of DNA in vitro. Analysis of Rx1-binding to a variety of DNA structures demonstrated a preference for topologies resembling transcription bubbles. Rx1 therefore binds, bends and distorts DNA in a manner reminiscent of the formation of the transcription initiation complex (Finzi et al. 2010, Kim et al. 2012, Liu et al. 2010, Tang et al. 2011). A further interesting parallel between Rx1 and the eukaryotic Cdc6/Orc1 proteins is that eukaryotic ORCs lack DNA sequence specificity in vitro, but show higher affinity for specific DNA topologies (MacAlpine et al. 2010, Remus et al. 2004). Consistent with this, $\mathrm{R} \times 1$ showed no observed sequence specificity but an increased affinity for branched and melted DNA topologies over linear dsDNA.

One of the most important and earliest consequences of immune activation is transcriptional reprogramming (Navarro et al. 2004, Tsuda et al. 2008, Garner et al. 2016). The association of MLA10 with Myb and WRKY transcription factors and N with the SPL6 transcription factor suggests that plant NB-LRRs themselves might directly regulate transcription in the immune response (Chang et al. 2013, Roberts et al. 2013, Padmanabhan et al. 2013). The specificity of an immune-dependent transcription response is difficult to reconcile with the observation that Rx1 DNA-binding specificity 
might be mediated by local DNA topology rather than sequence specificity, implying the involvement of other factors conferring specificity. Indeed, the $\mathrm{CC}$ of $\mathrm{R} \times 1$ has been shown to interact with a Golden2-like (GLK) Transcription factor (TF), NbGlk1 (Townsend et al. 2018). NbGlk1 binds distinct consensus DNA sequences and this binding affinity in vitro is reduced upon its interaction with $\mathrm{R} \times 1$. Moreover, overexpression of NbGlk1 activates immune responses to PVX. Such a direct involvement of GLK-like TFs in defence signalling has been reported also in Arabidopsis toward cucumber mosaic virus (Han et al. 2016) and to the fungal pathogens Fusarium graminearum (Savitch et al. 2007) and Hyaloperonospora arabidopsidis (Murmu et al. 2014).

Transcriptional reprogramming initiated as part of an immune response must be under tight control and is likely exercised at multiple levels (Garner et al. 2016). Rx1 associates with NbGIk1 and prevents its assembly on DNA unless $R \times 1$ is activated by PVX, representing one level of transcriptional control (Townsend et al. 2018). Among other potential mechanisms, histone modifications are of particular interest since they represent an important mechanism to control transcription of defence-related genes (Espinas et al. 2016). For example, histone modification through the removal of acetyl groups from modified lysine residues by histone deacetylases can suppress immunity (Wang et al. 2017, Ding et al. 2012). We here set out to identify nuclear regulators of $R \times 1$ function and investigate their role in immunity. We report that $\mathrm{R} \times 1$ interacts directly with a DNA-binding bromodomain-containing protein (NbDBCP) thereby identifying a new member in nuclear DNA bound NB-LRR complexes controlling plant immunity. The finding that NbDBCP acts as a negative regulator of Rx1-mediated immune responses to PVX provides a direct link between chromatin and immunity.

\section{RESULTS}

\section{The Rx1 CC domain interacts with a bromodomain containing protein}

To provide further insight into Rx1's mechanism of action, and its control of the NbGIk1 TF, we screened for additional Rx1 interactors. NbGlk1 was previously identified in a yeast Two-Hybrid (Y2H) screen as an interactor of the CC domain of Rx1 (amino acids 1-144). Here we used the same CC domain to perform an additional $\mathrm{Y} 2 \mathrm{H}$ screen of a random-primed Nicotiana benthamiana mixed tissue cDNA library. Niben101Scf17137g00006.1 (https://solgenomics.net) was identified in seven clones 
corresponding to four individual cDNAs of which two were isolated twice each. Individual clones were presumably obtained multiple times due to amplification of the random-primed cDNA library. The full-length cDNA for Niben101Scf17137g00006.1 encodes a 664-amino acid protein with a predicted molecular weight of $74814 \mathrm{Da}$. The protein carries a single Swi3, Ada2, N-Cor, and TFIIIB (SANT)-type helix-turn-helix domain and a single bromodomain of 111 amino acids (BD; Fig. 1A), hence we named it N. bethamiana DNA-binding BD-containing Protein (NbDBCP). We used the Simple Modular Architecture Research Tool (Letunic et al. 2018) to identify all proteins having a similar domain structure of a SANT-type domain and a BD. Proteins with a similar domain structure to NbDBCP were identified as uncharacterised proteins found in both dicots (e.g. At2g44430 of Arabidopsis thaliana) and monocots (e.g. LOC4346003 of Oryza sativa Japonica Group). A maximum likelihood phylogenetic analysis demonstrated that the proteins formed two distinct phylogenetic clades with branches for each clade having strong support with Bootstrap values of 0.98 (Supplemental Fig. S1). Of the 57 plant proteins having a similar domain structure of NbDBCP, 43 (including NbDBCP) also carried a coiled-coil domain between the SANT-type domain and the BD. NbDBCP is therefore representative for a larger protein family with a conserved domain structure.

\section{$\mathrm{Rx} 1$ interacts directly with $\mathrm{NbDBCP}$ in vitro and in vivo}

A BD typically recognises acetylated lysine residues. Since all NbDBCP clones identified from the $\mathrm{Y} 2 \mathrm{H}$ screen encompass the BD, $\mathrm{R} \times 1$ likely interacts with this domain. To test this, we assessed Rx1-binding to the NbDBCP BD in vitro. The NbDBCP BD is encoded by amino acids 293-414 of the NbDBCP ORF (Fig. 1A). We expressed amino acids 293-414 of NbDBCP (NbDBCP-BD) as a recombinant protein and examined its interaction with a glutathione-S-transferase (GST)-tagged Rx1-CC domain (Rx1(GST-1-144)) by size exclusion chromatography. The extreme C-terminus of Rx1(GST-1-144) was susceptible to some proteolytic cleavage during purification resulting in the purified protein running as a doublet in SDS-PAGE. We noted shifts in the peak bands corresponding to Rx1(GST-1-144) (Fig. 1B, SDS-PAGE panel 1, capped green bar) and NbDBCP-BD (Fig. 1B, SDS-PAGE panel 2 , capped red bar) when co-incubated (Fig. 1B, panel 3). GST alone (Fig. 1B, SDS-PAGE panel 4) showed no peak shift when co-incubated with NbDBCP-BD (26306038, SDS-PAGE panel 5) and hence from these results we conclude that $\mathrm{NbDBCP} B D$ and $\mathrm{R} \times 1-\mathrm{CC}$ interact in vitro. 
We next examined whether full-length $\mathrm{NbDBCP}$ interacts with full length $\mathrm{R} \times 1$ in planta. Co-immunoprecipitation experiments were performed using full-length $\mathrm{NbDBCP}$ fused to a $\mathrm{N}$ - or C-terminal $4 \times \mathrm{HA}$ epitope tag (Fig. 1C; $4 \times \mathrm{HA}-\mathrm{NbDBCP}$ and NbDBCP-4$\times \mathrm{HA}$ ) with full length $\mathrm{R} \times 1$ fused to a $4 \times$ Myc epitope tag (Fig. 1C; Rx1-4 X Myc). Experiments were also performed with the Rx1 CC domain fused to a $4 \times$ Myc epitope tag (Fig. 1C; Rx1-CC-4 XMyc). GFP fused to a $4 \times$ Myc epitope tag (Fig. 1C; 4 XMyc-GFP) was included as a control for non-specific interactions with NbDBCP. Two different elution protocols were used to optimise recovery of potential complexes formed between NbDBCP and Rx1-4 XMyc (Fig. 1C; elution 1) and Rx1-CC-4 XMyc (Fig. 1C; elution 2).

Both the $N$-terminal and C-terminal $4 \times$ HA NbDBCP fusions could be detected with an $\alpha$-HA tag antibody after immunoprecipitation with an $\alpha$-myc antibody when co-expressed with Rx1-CC-4 $\times$ Myc (Fig. 1C; elution 2) and Rx1-4 XMyc (Fig. 1C; elution 1). The amount of $\mathrm{N}$ - and C-terminally tagged NbDBCP that co-immunoprecipitated with the full-length $\mathrm{R} \times 1-4 \times \mathrm{Myc}$ was less then with Rx1-CC-4 XMyc. Nevertheless, the amount of $N$ - and C-terminally tagged NbDBCP that co-immunoprecipitated with the full-length $\mathrm{R} \times 1$ construct was consistently and repeatedly above background levels. Elution 1 did not liberate Rx1-CC-4 XMyc (Fig. 1C; elution 1; 4 XMyc panel), while elution 2 did release the Rx1-4XMyc (Fig. 1C; elution 2; $4 \times$ Myc panel). However, the $\alpha$-HAWestern Blot is optimised to observe NbDBCP interactions with Rx1-CC-4XMyc and was not exposed sufficiently long to observe NbDBCP interactions with Rx1-4 $\times$ Myc. Why less NbDBCP was immunoprecipitated with full-length $\mathrm{R} \times 1$ molecule compared to the CC-domain is unknown. Nonetheless, the interaction between full-length $\mathrm{NbDBCP}$ and $\mathrm{R} \times 1$ in planta was observed across independent experiments and thus represents a genuine interaction.

\section{$\mathrm{Rx} 1$ and $\mathrm{NbDBCP}$ localise to the nucleus in situ}

Having established that $\mathrm{R} \times 1$ and NbDBCP interact in vitro and in planta, we set out to identify the intracellular localisation of this event. We used confocal laser scanning microscopy to examine the cellular localisation of a C-terminal fusion of NbDBCP with GFP (NbDBCP-GFP) in N. bethamiana epidermal cells in the absence and presence of $\mathrm{R} \times 1$ ( $\mathrm{R} \times 1$-mCherry). The subcellular distribution of Rx1-mCherry in the cytoplasm and nucleus was within the range reported in existing studies 
(Supplemental Fig. S2) (Slootweg et al. 2010, Tameling et al. 2010). NbDBCP-GFP in combination with free mCherry (Fig. 2A; mCh) is localised in the nucleoplasm and nucleolus (Fig. 2A; left and middle panels; Supplemental Fig. S3). These localization patterns do not vary largely between 2 and $3 \mathrm{dpi}$ as well as in the presence or absence of the P19 silencing suppressor, minimizing the likelihood that the nucleolar distribution of NbDBCP-GFP is an artefact of overexpression (Supplemental Fig. S3). The amount of nucleoplasmic signal varied between cells (compare Fig. 2A to GFP channel of Fig. 2B panels 4-9) and in a few cells NbDBCP-GFP aggregated in subnuclear bodies (Fig. 2A; image at lower right panel taken at $3 \mathrm{dpi}$ ). No signal was observed in uninfiltrated cells. The distribution of NbDBCP-GFP differed from that of free GFP confirming that the localization for NbDBCP-GFP is genuine (Fig. 2A; upper and lower right panels). 
A.

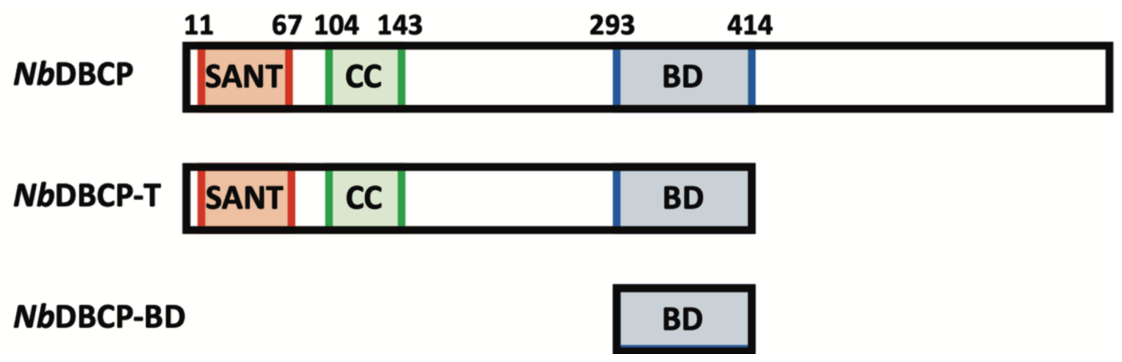

B.

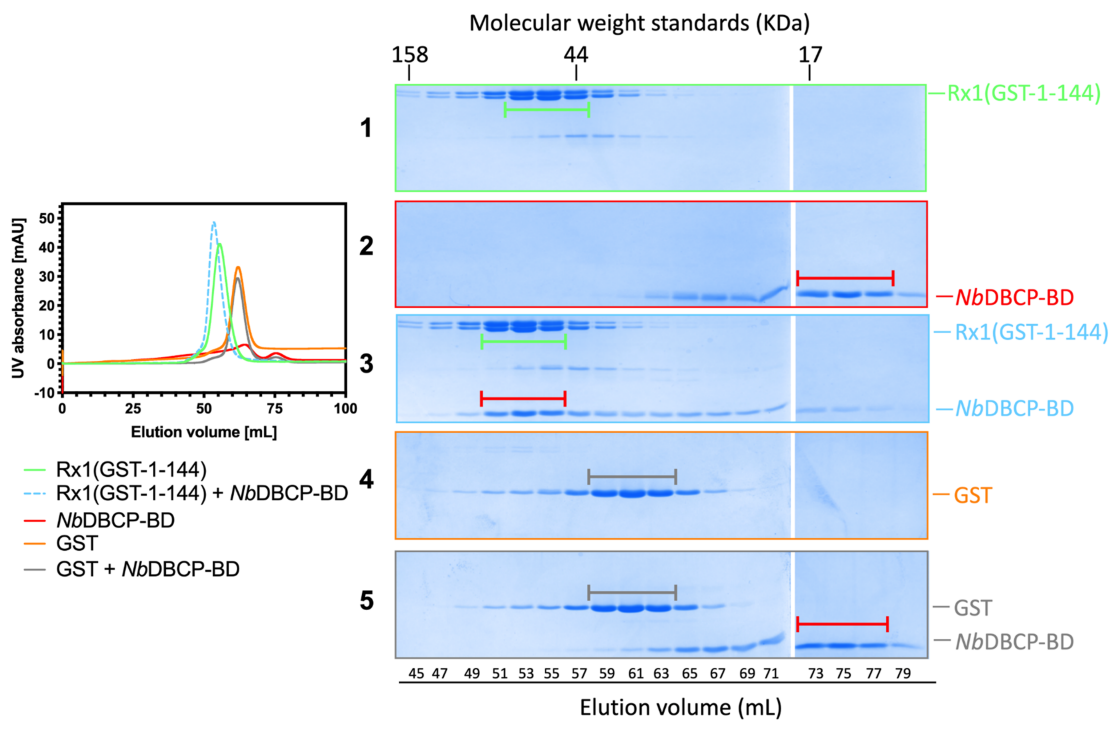

C.

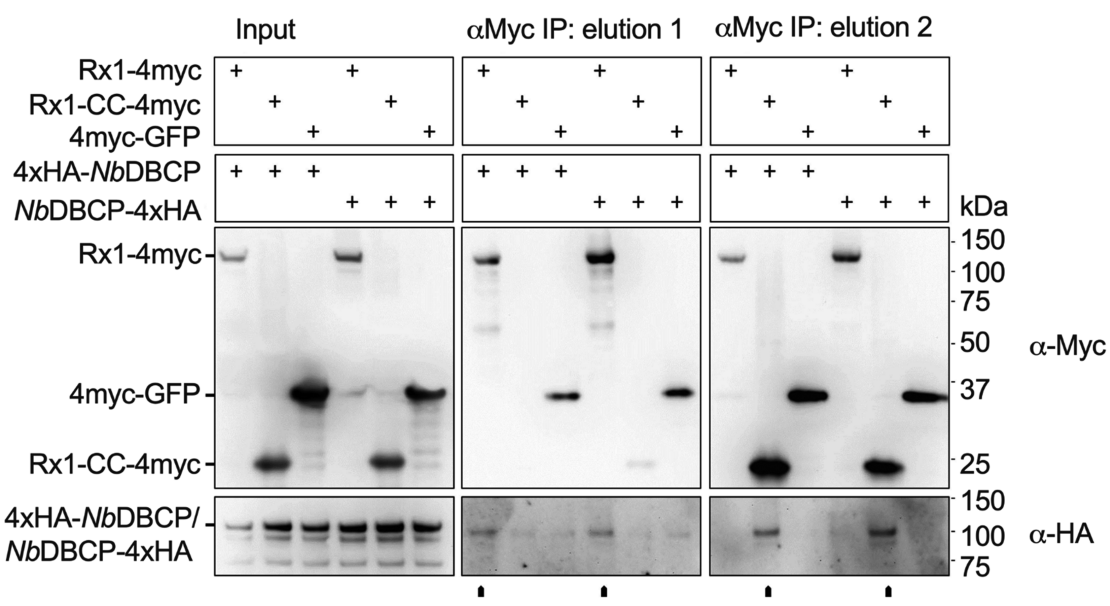

Fig. 1: Rx1 binds NbDBCP in vitro and in planta. A) Top-cartoon of the NbDBCP domain structure. Middle and Bottom-NbDBCP-T and NbDBCP-BD represents proteins used in this study. Numbers represent amino acid residue. SANT-DNA-binding SANT-type helix-turn-helix 
(Figure legend continued) domain. CC-coiled-coil domain. BD-bromodomain. B) Interaction of Rx1(GST-1-144) with NbDBCP-BD. On the left are representative gel-filtration chromatograms of Rx1(GST-1-144), NbDBCP-BD, GST, Rx1(GST-1-144) incubated with NbDBCP-BD and GST incubated with NbDBCP-BD. Peak fractions were visualized by SDS-PAGE and are represented by capped bars. C. Co-immunoprecipitation of 4xmyc-tagged full-length $R \times 1$ ( $R \times 1-4 \times M y c)$ or $R \times 1-C C$ (Rx1-CC-4 $\times$ Myc) when co-expressed in planta with $\mathrm{N}$ - and $\mathrm{C}$-terminally $4 \times \mathrm{HA}$-tagged $\mathrm{NbDBCP}$ ( $4 \times$ HA-NbDBCP and NbDBCP-4 $\times$ HA). The labels on the figure are as follows; Input-denotes the constructs agroinfiltrated into $N$. bethamiana leaves; $\alpha$-Myc-an immunoblot performed using an $\alpha$-Myc epitope tag antibody; $\alpha$-an immunoblot performed using an $\alpha$-HA epitope tag antibody; $\alpha$-Myc IP-immunoprecipitation of the denoted input samples with an $\alpha$-Myc epitope tag antibody; elution indicates the method used to release immunoprecipitated protein (see Experimental Procedures). Immunoblot bands corresponding to Rx1-4 X Myc, Rx1-CC-4 X Myc, 4 × Myc-GFP and NbDBCP from the $\alpha-M y c$ and $\alpha-H A$ immunoblots are indicated.

The nuclear localization pattern of NbDBCP-GFP was unaltered when co-expressed with Rx1-mCherry (Fig. 2B; GFP channel; compare panel 1 to 4-9). In addition, the cellular distribution of Rx1-mCherry in the nucleus relative to cytoplasm was mostly unaffected and similar as upon co-expression with GFP-GUS (Fig. 2B; mCherry channel; compare panel 3 to 4-6). We confirmed this by determining the relative intensity ratios of mCherry signal in the nucleus relative to cytoplasm $\left(I_{N} / I_{C}\right)$ as described (Slootweg et al. 2010). The $I_{N} / I_{C}$ ratios of Rx1-mCherry do not differ significantly in the presence or absence of NbDBCP-GFP (Supplemental Fig. S2). However, in some cells we observed that Rx1-mCherry relocalized to the nucleolus and to subnuclear bodies when co-expressed with NbDBCP-GFP (23 $\pm 7 \%$ of imaged cells (S.D.; 51 cells imaged in four biologically independent experiments; Fig. 2B; mCherry channel; compare panels 4-6 to 7-9). Rx1-mCherry is present in the cytoplasm and nuclear envelope when not relocalized to the nucleolus (Fig. 2B panels 4-6). The weaker signal of mCherry is due to a lower quantum yield compared to GFP and the apparent brighter signal for $\mathrm{R} \times 1$ in the nucleolus (Fig. 2B 7-9) is therefore likely a reflection of a local high concentration. Additionally, the Rx1-mCherry construct used in this study is intact (as demonstrated by immunoblotting in Supplemental Fig. S4) but expressed at significantly lower levels than free mCherry. 
Given that only a low percentage of cells shows the re-distribution of Rx1-mCherry into the nucleolus, we quantified this shift in all cells imaged. To do so, the relative intensity ratios of Rx1-mCherry in the nucleolus relative to the nucleoplasm $\left(I_{\text {Nucleolus }} / I_{\text {Nucleoplasm }}\right)$ was determined. Data from independent experiments indicate that there is a statistically significant increase in nucleolar signal of Rx1-mCherry when co-expressed with NbDBCP-GFP but not NbDBCP-HA (Supplemental Fig. S5). Thus, a possibility still remains of an overexpression artefact. To explore this, we demonstrated that NbDBCP-GFP exhibited very low levels of bleed-through in the mCherry channel when expressed alone (Fig. 2B panel 1; note the lack of signal in the mCherry channel compared to the GFP channel). The observed redistribution of Rx1-mCherry upon co-expression with NbDBCP-GFP far exceeds this background level (Fig. 2B; mCherry channel; panels 2 to 9). Additionally, this redistribution was not observed when Rx1-mCherry was expressed with GFP-GUS (Fig. 2B; mCherry channel; panel 3; Supplemental Fig. S5). These factors combined minimize the likelihood that the accumulation of $R \times 1$ into subnuclear bodies is due to experimental artefacts. In summary, the observed shared subcellular distribution of $\mathrm{Rx} 1$ and $\mathrm{NbDBCP}$ in the nucleus (and nucleolus in a small subset of cells) is consistent with their ability to interact. 
A.

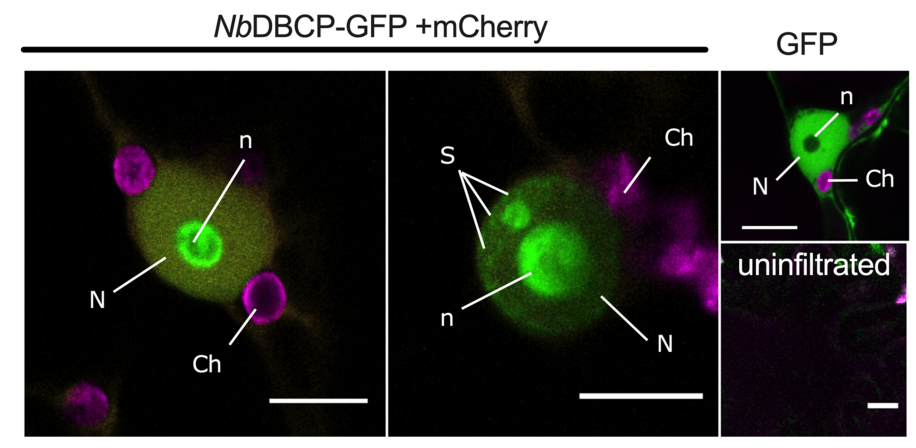

B.

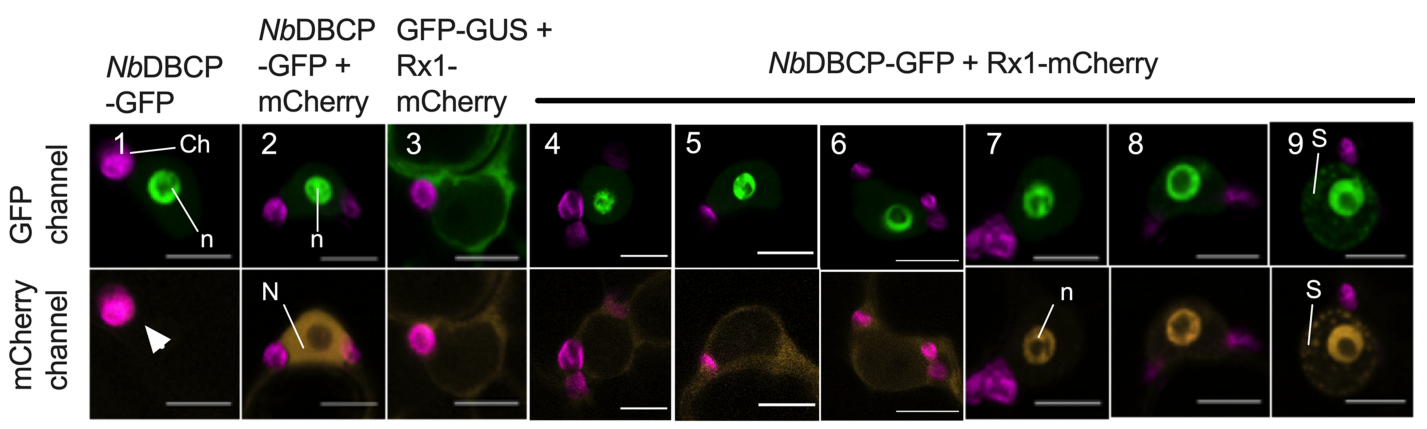

Fig. 2: Subcellular distribution of NbDBCP-GFP in planta. A) Representative overlay confocal images of $N$. bethamiana leaf epidermal cells transiently expressing NbDBCP-GFP + P19 (left and middle panels), free GFP (upper right panel) or an uninfiltrated leaf (lower right panel). Images were taken at 2 dpi. Scale bar $=10 \mu \mathrm{m}$. $\mathrm{N}=$ nucleus, $\mathrm{n}=$ nucleolus, $\mathrm{S}=$ subnuclear bodies, $\mathrm{Ch}=$ chloroplasts. $\mathrm{B})$ Subcellular distribution of NbDBCP-GFP with or without mCherry or Rx1-mCherry. The P19 silencing suppressor was included for all combinations. Images were taken at 2 dpi. Scale bar $=10 \mu \mathrm{m}$. White arrow indicates bleed through of GFP in mCherry channel as observed in the nucleus. Similar settings were used for all images shown including $488 \mathrm{~nm}$ and $543 \mathrm{~nm}$ laser intensities (3\% and 55-56.5\% respectively). 1. NbDBCP-GFP expressed alone. 2. NbDBCP-GFP expressed with mCherry. 3. GFP-GUS expressed with Rx1-mCherry. 4-9. NbDBCP-GFP expressed with Rx1-mCherry. $\mathrm{N}=$ nucleus, $\mathrm{n}=$ nucleolus, $\mathrm{S}=$ subnuclear bodies, $\mathrm{Ch}=$ chloroplasts.

Rx1 and NbDBCP interact with DNA in situ

The co-localisation of Rx1 and NbDBCP to the nucleus prompted us to investigate whether Rx1 and NbDBCP interact at DNA in planta. We studied Rx1-NbDBCP-DNA interactions using Förster 
resonance energy transfer-fluorescence lifetime imaging microscopy (FRET-FLIM). GFP (negative control), histone H2B fused to GFP (GFP-H2B; positive control), full-length Rx1 with or without an $N$-terminal GFP tag, or full-length NbDBCP with or without an N-terminal GFP tag were transiently expressed in N. bethamiana. The constituent fluorescence lifetimes for the GFP tag were examined in leaves counterstained with the nucleic acid stain LDS 751 (Fig. 3). GFP showed two distinct fluorescence lifetimes at $\sim 0.5$ and $\sim 1.5 \mathrm{~ns}$. The fluorescence lifetime at $\sim 0.5 \mathrm{~ns}$ can be explained by energy transfer from GFP to an acceptor fluorophore (Fenyk et al. 2015). Increased energy transfer from GFP to acceptor LDS 751 (indicative for physical proximity to nucleic acids) increases the relative contribution of the $\sim 0.5 \mathrm{~ns}$ fluorescent lifetime. A shift in the ratio of the $\sim 1.5$ (long) to $\sim 0.5$ (short) ns GFP lifetimes is indicative for interactions with DNA (Fenyk et al. 2015) at distances within the Förster radius (likely $<50 \AA$ ). We monitored the interaction of a NbDBCP-GFP fusion with DNA with or without $\mathrm{R} \times 1$ (untagged) in the presence or absence of the Rx1-activating PVX coat protein CP106. This experiment would reveal whether NbDBCP interacts with chromatin in situ and whether this interaction is altered by co-expression of $\mathrm{R} \times 1$ and CP106. Consistent with the localisation of NbDBCP to the nucleus (Fig. 2), NbDBCP-GFP bound DNA when expressed alone (Fig. 3A) However, NbDBCP was not observed to bind DNA when co-expressed with either Rx1 or CP106 despite an equivalent fluorescent signal indicating that $\mathrm{NbDBCP}$ is still expressed. The ability of CP106 alone to displace NbDBCP from DNA prompted us to ask whether CP106 and NbDBCP are able to physically interact. CP106 did not interact with NbDBCP in a Y2H assay (Supplemental Fig. S6) suggesting that the effect observed on NbDBCP DNA-binding is due to an indirect interaction in planta. While we cannot formally exclude such an interaction, there is currently no data to support it.

We next monitored the interaction of a Rx1-GFP fusion with chromatin with or without $\mathrm{NbDBCP}$ (untagged) in the presence or absence of the Rx1-activating (avirulent) PVX coat protein (CP106) (Fig. 3B). In line with our previous studies, Rx1-GFP expressed without NbDBCP only bound DNA in the presence of CP106 (Fenyk et al. 2012). The Rx1-GFP fusion was not observed to interact with chromatin when co-expressed with NbDBCP (untagged) (Fig. 3B). This result is consistent with the observation that NbDBCP-GFP was not observed to interact with chromatin when co-expressed with Rx1 (untagged) (Fig. 3A). Interestingly, when Rx1-GFP was co-expressed with NbDBCP and CP106 it was observed to interact with chromatin (Fig. 3B). 

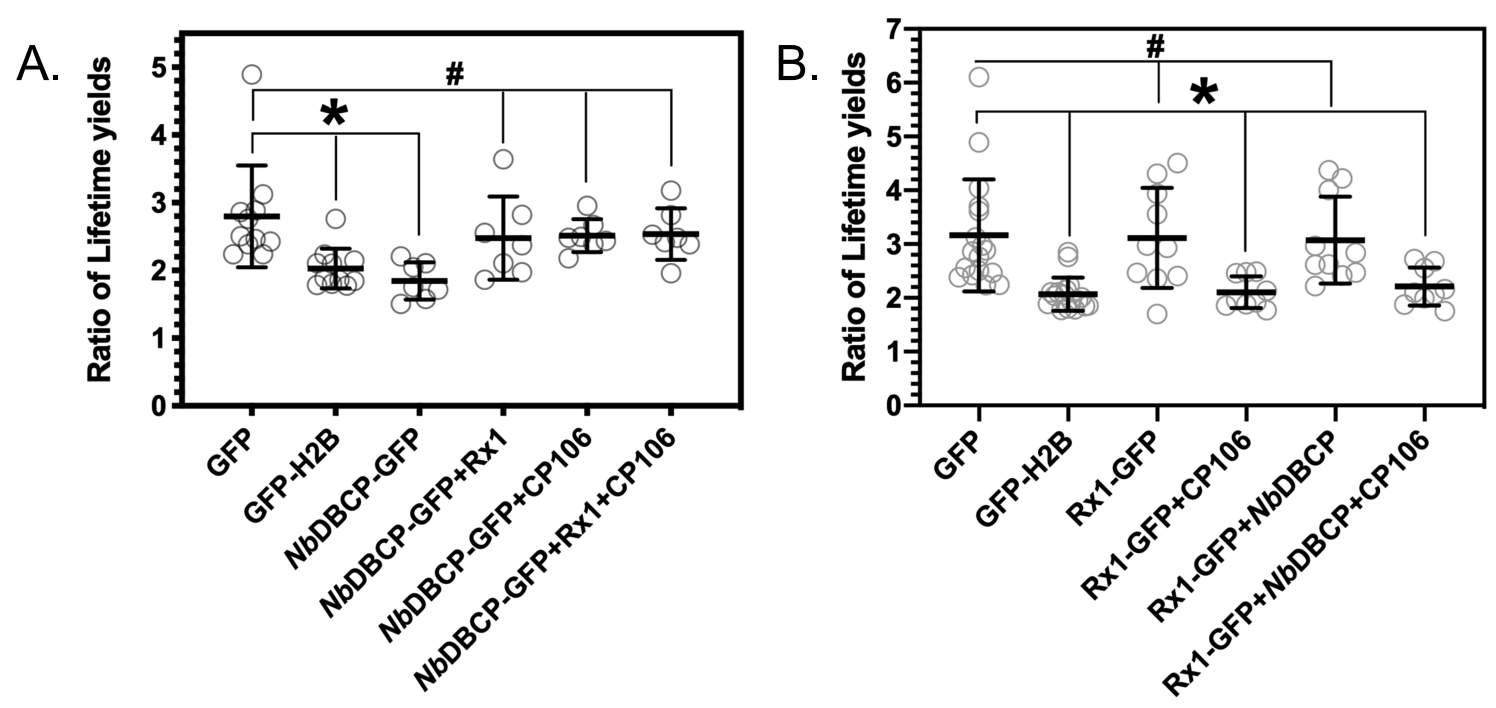

Fig. 3: Binding of $R \times 1$ and NbDBCP proteins to chromatin in situ. A) Ratio of the long to short GFP lifetimes for NbDBCP-GFP full-length construct alone and upon co-expression with $\mathrm{R} \times 1$ and the avirulent CP106 allele of the PVX CP (scatter plot $\pm S . D ;{ }^{*} p<0.05$; \# $p>0.05$; one-way ANOVA with post hoc Dunnett's multiple comparison). B. Ratio of the long to short GFP lifetimes for Rx1-GFP full-length construct alone and upon co-expression with NbDBCP and the avirulent CP106 allele of the PVX CP (scatter plot $\pm S$.D; * $p<0.05$; \# $p>0.05$; one-way ANOVA with post hoc Dunnett's multiple comparison).

\section{NbDBCP reduces $\mathrm{Rx1}$-mediated immune responses}

The observed interaction between NbDBCP and Rx1 suggests a functional relation between these proteins. Rx1 can trigger two distinct types of immune outputs; 1) a cell death response that can be induced by overexpression of the PVX coat protein and 2) symptomless extreme-resistance response that results in inhibition of viral replication (Bendahmane et al. 1999). Virus-induced gene silencing (VIGS) employs a recombinant TRV virus carrying part of a designated plant gene sequence targeted for silencing (Lange et al. 2013). We used VIGS to silence the endogenous NbDBCP-encoding gene of $\mathrm{N}$. bethamiana to investigate its requirement for $\mathrm{R} \times 1$-mediated immune responses. Two independent VIGS constructs directed against different regions of the NbDBCP transcript were used to ensure that the results are not due to off-target effects. Down-regulation of NbDBCP mRNA by VIGS was examined by real-time quantitative PCR (Fig. 4A). Rx1-mediated immunity was assessed 
by measuring PVX coat protein accumulation following agroinfiltration using an infectious PVX clone. A VIGS construct targeting GFP serves as negative control on Rx1-mediated immune responses. No change in PVX coat protein accumulation (in the absence of $\mathrm{R} \times 1$ ) was observed in NbDBCP silenced plants (pTRV2-NbDBCP-1 and -2) when compared to GFP silenced plants (pTRV2-GFP; Fig. 4B). NbDBCP itself therefore does not influence PVX-accumulation.

As expected PVX coat protein accumulation was reduced when Rx1 was co-expressed with PVX in either non-silenced or GFP silenced plants (pTRV2-GFP; Fig. 4B). As a positive control for a compromised Rx1 function a TRV::SGT1 silencing construct was used. SGT1 is a homologue of a yeast ubiquitin ligase-associated protein and has a general role in NB-LRR-mediated immunity and is required for Rx1 function (Lu et al. 2003, Peart et al. 2002, Hoser et al. 2014, Slootweg et al. 2010). PVX coat protein accumulation was unaffected in SGT1 silenced plants (pTRV2-SGT1) in the absence of $R \times 1$, however, in the presence of $R \times 1$ silencing of SGT1 eliminated immunity resulting in increased PVX coat protein accumulation (Fig. 4B). PVX coat protein accumulation was also significantly reduced when Rx1 and PVX were co-expressed in NbDBCP silenced plants ( $\mathrm{PTRV} 2-\mathrm{NbDBCP}-1$ and -2) when compared to GFP silenced plants (pTRV2-GFP) (Fig. 4B). These findings suggest that NbDBCP has a suppressive role in the Rx1-mediated resistance response. Our qRT-PCR analysis demonstrated a trend of decreased relative NbDBCP expression with pTRV2:NbDBCP-1 and -2 compared to pTRV2:GFP plants. Despite the minor effect and variability in gene silencing (see Fig. 4A, pTRV2:NbDBCP-1), the findings from our disease assays were robust and replicable across experiments (Fig. 4). This might reflect the fact that $\mathrm{R} \times 1$-mediated immunity is sensitive to a relatively small drop in NbDBCP expression. Overall, these data suggest that NbDBCP is a negative regulator of Rx1-mediated immunity. 
A. พู

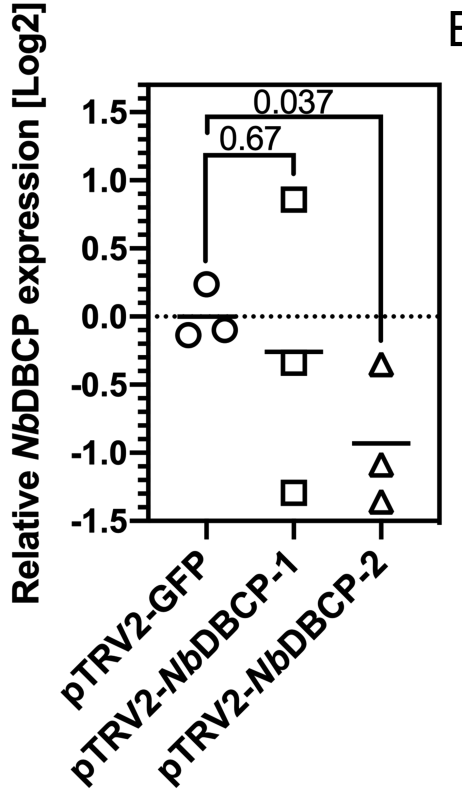

B.

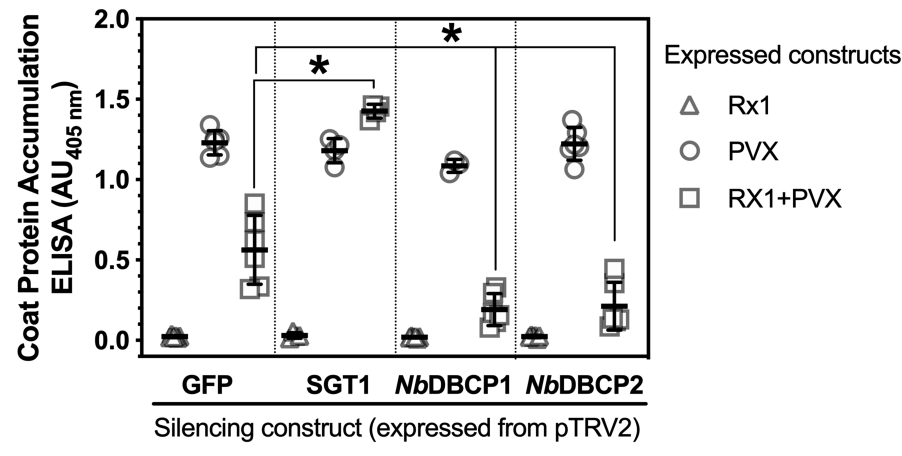

Fig. 4: The influence of NbDBCP gene-silencing on susceptibility to PVX. A) Relative expression level of endogenous NbDBCP in N. bethamiana TRV-VIGS plants as determined by qRT-PCR analysis. Each data point represents a biological replicate consisting of pooled leaf materials from at least 3 different plants and 3 technical replicates. Significance level is calculated based on Log2 transformation of $2^{-\Delta \Delta C t}$ using a paired student T-test with $\alpha=0.05$. Crossbar designates the mean relative $\mathrm{NbDBCP}$ expression. B) Absorbance at $405 \mathrm{~nm}$, indicative of PVX coat protein accumulation, in four VIGS strain (pTRV2-GFP (negative control), pTRV2-SGT1 (positive control), pTRV2-NbDBCP-1, and pTRV2-NbDBCP-2) when co-infiltrated with Rx1 and PVX (scatter plot \pm S.D; $n=3-6$; * $p<0.05$ compared to pTRV2-GFP RX1 + PVX; one-way ANOVA with Dunnett's multiple comparison). The data derives from six independent experiments for pTRV2-GFP, pTRV2-NbDBCP-1, and PTRV2-NbDBCP-2 and four independent experiments for PTRV2-SGT1.

The NbDBCP bromodomain affects immune responses to PVX

The NbDBCP BD is sufficient for the interaction between Rx1 and NbDBCP (Fig. 1B). We therefore investigated whether the BD was crucial to the NbDBCP-mediated immunity to PVX. The NbDBCP BD was analysed using the Phyre2 protein fold recognition engine and modelled using the BPTF $\mathrm{BD}$ in complex with histone $\mathrm{H} 4$ acetylated at Lys16 (PDB accession number 3QZT). NbDBCP Y336 and E386 were identified as residues likely to interact with acetyl-lysine and therefore 
required for BD-dependent function (Fig. 5A). NbDBCP-Y336F and NbDBCP-E386L mutants were generated, conserving amino acid side-chain bulk while ablating intermolecular interactions at the site, to study their effect on immune responses to PVX. The NbDBCP-Y336F variant could not be expressed in planta and was not studied further. The cellular localisation of a C-terminal fusion of NbDBCP-E386L with GFP (NbDBCP-E386L-GFP) in N. bethamiana epidermal cells was determined using confocal laser scanning microscopy. NbDBCP-E386L-GFP was co-expressed with the P19 silencing suppressor to enhance expression and NbDBCP-E386L-GFP was observed to localise to the nucleoplasm and nucleolus (Fig. 5B; GFP channel) similar to NbDBCP-GFP wild type protein. NbDBCP-E386L expression in planta was compared to NbDBCP wild type by Western Blot. Expression levels for NbDBCP-E386L were consistently lower than wild type NbDBCP but protein is detectable (Fig. 5C). It was therefore not possible to assess the influence of the E386L mutation on NbDBCP suppression of Rx1-mediated immune responses through assessment of a loss-of-function phenotype, as the data could be explained by reduced NbDBCP-E386L protein levels.

We therefore investigated whether NbDBCP-E386L confers a possible gain-of-function phenotype in planta, as such a phenotype is less likely to arise through decreased protein expression. We investigated the effect of $\mathrm{NbDBCP}$ wild type and E386L overexpression on phenotypes induced by PVX. NbDBCP and PVX were transiently expressed in N. bethamiana upon A. tumefaciens infiltration. As expected, no immune response was observed when PVX was co-expressed with GFP (Fig. 5D and S7; A1), NbDBCP-GFP-WT (Fig. 5D and S7; A2), or NbDBCP-GFP-E386L (Fig. 5D and S7; A3) in the absence of Rx1. However, a hypersensitive response was observed when PVX and GFP were co-expressed with Rx1 (Fig. 5D and S7; B1) and when PVX and NbDBCP-GFP-WT were co-expressed with Rx1 (Fig. 5D and S7; B2). Interestingly, levels of cell death were qualitatively reduced when PVX and NbDBCP-GFP-E386L were co-expressed with Rx1 (Fig. 5D and S7; B3). The gain-of-function phenotype for NbDBCP-E386L is not explained by a failure to interact with Rx1 as both NbDBCP wild type and NbDBCP-E386L interact with the $\mathrm{Rx} 1 \mathrm{CC}$ domain in a $\mathrm{Y} 2 \mathrm{H}$ assay (Supplemental Fig. S8).

To support the interpretation of the data above, we directly measured virus coat protein accumulation in infiltrated N. bethamiana leaves to provide quantitative support for the qualitative analysis of Fig. 5D. Virus coat protein accumulation in leaf infiltrates were measured by double antibody sandwich enzyme-linked immunosorbent assay (DAS-ELISA) using an antibody that recognises the PVX coat 
protein. In the absence of $\mathrm{R} \times 1$, both $\mathrm{NbDBCP}$ wild type and E386L proteins increased the basal immune response of $\mathrm{N}$. bethamiana cells (Fig. 5E; compare second and third data sets to the first), which is evident through a decrease in the accumulation of PVX coat protein. $\mathrm{R} \times 1$ co-expression significantly reduced PVX virus expression (Fig. 5E; compare the first and fourth data sets) as expected. Notably, more virus accumulated when Rx1 was co-expressed with NbDBCP-WT-GFP than when Rx1 was co-expressed with GFP (Fig. 5E; compare the fourth and sixth data sets). This supports the observation from VIGS that NbDBCP-WT suppresses Rx1 activity. Significantly, less viral accumulation was observed when Rx1 was co-expressed with NbDBCP-E386L-GFP (Fig. 5E; compare fourth and fifth data sets). The significant increase in $\mathrm{R} \times 1$-mediated immunity can therefore be attributed to a gain-of-function phenotype conferred by the NbDBCP-E386L-GFP variant, showing that $\mathrm{NbDBCP}$ is a negative regulator of $\mathrm{R} \times 1$-function. 
A.

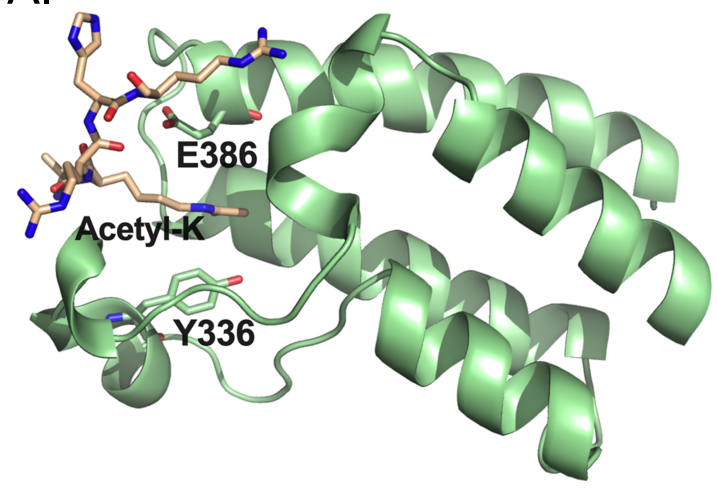

C.

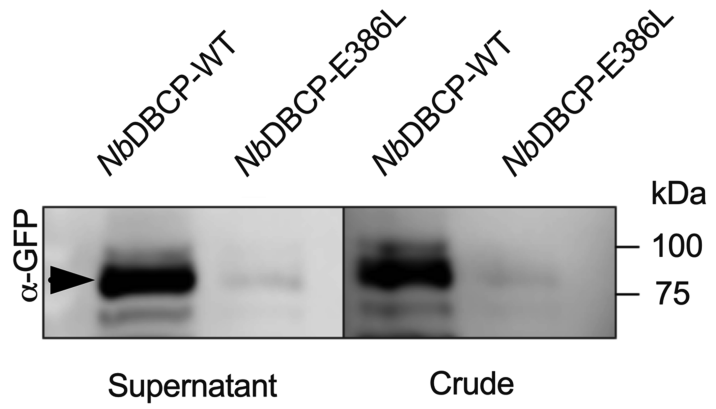

D.

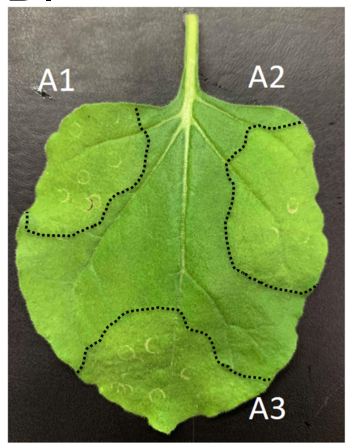

B.

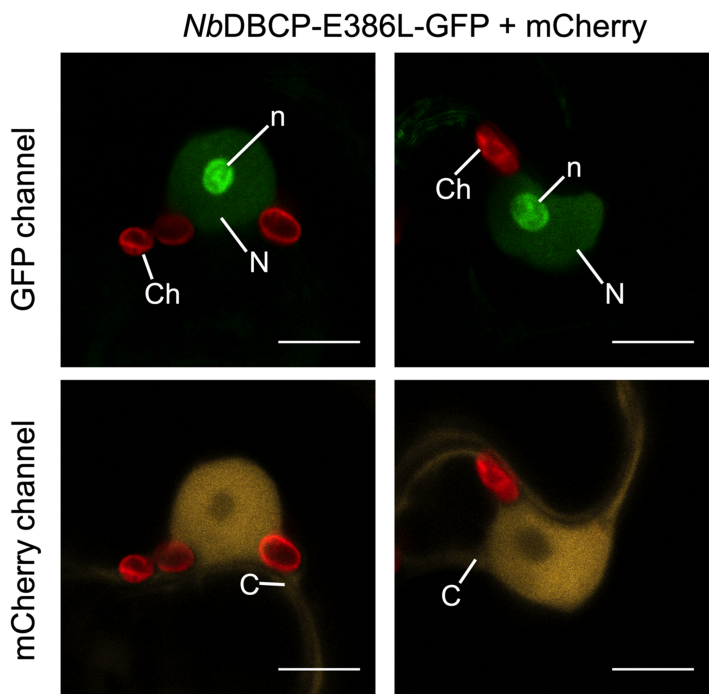

E.

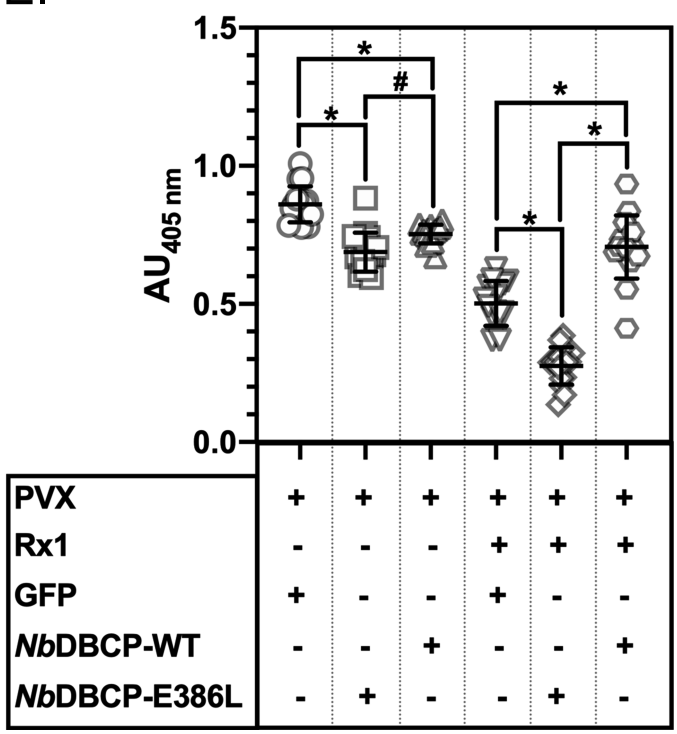

Fig. 5: The influence of $N b D B C P$ BD mutation on Rx1-mediated reductions in susceptibility to PVX. A) Structural homology model for the NbDBCP bromodomain. BD residues Y336 and E386 are shown as is the acetyl-lysine of a modelled target protein. B) Representative images of the nuclei of $N$. bethamiana leaf epidermal cells co-expressing NbDBCP-E386L-GFP and free mCherry. Images were taken at 2 dpi by confocal microscopy with consistent results in all imaged cells. Scale bar represents width of $10 \mu \mathrm{m}$. Key: $\mathrm{N}=$ nucleus; $\mathrm{n}=$ nucleolus; $\mathrm{C}=$ cytoplasm; $\mathrm{Ch}=$ chloroplast. C) Western blot of NbDBCP-GFP wild type and E386L protein expression + P19 in planta. $\alpha$-GFP immunoblot performed using an $\alpha$-GFP antibody. Equal protein loading was assessed by a Coomassie Blue protein loading control. Leaf samples harvested at $3 \mathrm{dpi}$ were 
(Figure legend continued) used for protein extraction. kDa-molecular weight markers. D) Representative photographs of N. bethamiana leaves infiltrated with pGR208 (that drives expression of a PVX amplicon) and/or full-length $\mathrm{R} \times 1$ in the presence/absence of NbDBCP-WT or E386L variant. Images were taken at 5 dpi with consistent results between leaf samples. Key: A1 = pGR208 + GFP; $\mathrm{A} 2=\mathrm{pGR} 208+$ NbDBCP $-\mathrm{WT} ; \quad \mathrm{A} 3=\mathrm{pGR} 208+$ NbDBCP $-\mathrm{E} 386 \mathrm{~L} ; \quad \mathrm{B} 1=\mathrm{pGR} 208+\mathrm{R} \times 1+\mathrm{GFP} ;$ $\mathrm{B} 2=\mathrm{pGR208}+\mathrm{R} \times 1+\mathrm{NbDBCP}-\mathrm{WT} ; \quad \mathrm{B} 3=\mathrm{pGR} 208+\mathrm{R} \times 1+\mathrm{NbDBCP}-\mathrm{E} 386 \mathrm{~L} . \quad \mathrm{E})$ Chart representing relative PVX levels measured by DAS-ELISA in N. bethamiana leaves infiltrated with pGR208 and/or Rx1 in the presence or absence of NbDBCP-WT or E386L. Leaves were harvested at 5 dpi. Error bars represent the standard deviation (means \pm S.D..; $n=16 ;{ }^{*} p<0.05 ; \# p>0.05$; one-way ANOVA with post hoc Tukey multiple comparison test).

\section{NbDBCP reduces NbGlk1-mediated DNA binding}

We hypothesised that $\mathrm{N} b \mathrm{DBCP}$ might inhibit the pro-immune activity of $\mathrm{Rx} 1$ as part of a larger complex. NbGIk1 binds to consensus GLK DNA-binding sites and its binding affinity for these sites is reduced by $\mathrm{Rx} 1$ (Townsend et al. 2018). As Rx1 is a negative regulator of NbGIk1 DNA-binding we investigated whether NbDBCP might reduce Rx1-mediated immunity through an influence on NbGIk1 DNA-binding. We were unable to express the full-length NbDBCP molecule as a recombinant protein in vitro. We were, however, able to express a $C$-terminally truncated variant of NbDBCP consisting of amino acids 1-414 (NbDBCP-T). We measured the Kd value of NbGlk1 for a dsDNA substrate containing a concatenated GGATATCC NbGlk1 binding site (Townsend et al. 2018) in the presence or absence of Rx1(GST-1-144) and/or NbDBCP-T by fluorescence anisotropy (Fig. 6A). $\mathrm{R} \times 1$ (GST-1-144) reduced the binding affinity of NbGIk1 for its dsDNA substrate as expected from $0.11 \pm 0.00 \mu \mathrm{M}$ (S.D.) to $0.15 \pm 0.01 \mu \mathrm{M}$ (S.D.). Interestingly, NbDBCP-T also reduced the binding affinity of NbGIk1 for its dsDNA substrate to $0.16 \mu 0.01 \mu \mathrm{M}$ (S.D.). Rx1(GST-1-144) and NbDBCP-T gave a synergistic effect with a much further reduction in the binding affinity of NbGlk1 for its dsDNA substrate to $0.26 \pm 0.03 \mu \mathrm{M}$ (S.D.).

As the $\mathrm{Rx} 1$ interaction with $\mathrm{NbDBCP}$ is dependent on the $\mathrm{BD}$ we investigated whether the NbDBCP-BD was sufficient to reduce the binding affinity of NbGlk1 for its dsDNA substrate. NbDBCP-BD did not alter NbGlk1 binding to dsDNA in the presence or absence of $R \times 1$ indicating that the site of 
the Rx1-NbDBCP interaction is not required for the influence of NbDBCP on NbGlk1 DNA-binding (Fig. 6B). The experiment of Fig. 6B was performed using NbDBCP-T in place of NbDBCP-BD (Fig. 6C). NbDBCP-T reduced NbGlk1 binding to dsDNA in the presence of Rx1 supporting the findings of Fig. $6 \mathrm{~A}$ and confirming that the failure of NbDBCP-BD to reduce NbGlk1 DNA binding was not specific to the assay conditions. The ability of $\mathrm{Rx} 1$ and $\mathrm{NbDBCP}$ to synergistically occlude NbGlk1 DNA-binding suggests that they are able to form a larger protein complex. We therefore investigated evidence for a complex between NbGlk1 and NbDBCP in vitro. We therefore expressed amino acids 1-414 of NbDBCP (NbDBCP-T) as a recombinant protein and examined its interaction with amino acids 83-402 of NbGlk1 (NbGlk1(83-402)) by size exclusion chromatography. Both NbDBCP-T and NbGlk1 (83-402) represent the largest recombinant protein variants we have been able to produce as soluble proteins in E. coli. We noted a shift in the peak band corresponding to NbGIk1(83-402) (Fig. 6D, SDS-PAGE panel 1, capped green bar) when co-incubated with NbDBCP-T (Fig. 6D, panel 3, capped green bar) indicative of complex formation. No shift in the peak band corresponding to NbDBCP-T (Fig. 6D, SDS-PAGE panel 2, capped red bar) was obvious when incubated with NbGlk1(83-402) (Fig. 6D, panel 3, capped red bar). However, a more sensitive quantitative examination of the distribution of NbDBCP protein to the left (higher molecular weight) and right-hand side of the protein peak at an elution volume of $12 \mathrm{~mL}$ by densitometry revealed an enhanced distribution of NbDBCP-T towards a higher molecular weight. The left:right ratio of NbDBCP around the $12 \mathrm{~mL}$ elution volume was 1.37 for NbDBCP-T alone but 1.60 when NbDBCP-T was incubated with NbGlk1(83-402), indicative for a shift due to complex formation.

The interaction between NbGIk1 and NbDBCP observed through fluorescence anisotropy is therefore evidenced through gel filtration analysis. However, gel filtration analysis indicates that the interaction is relatively transient and only weakly detectable on transit of the complex through a gel filtration column. Accordingly, we were unable to observe a three-way interaction between $\mathrm{Rx} 1, \mathrm{NbDBCP}$ and NbGlk1 by gel filtration chromatography.

We performed additional confocal imaging to investigate the influence of NbDBCP overexpression on the cellular localization of NbGLK1-GFP. Experiments were performed using an HA-tagged version of NbDBCP as an NbDBCP-mCherry construct was not available. Immunoblotting demonstrated that all proteins used for imaging were expressed and intact (Fig. S9A). The data demonstrates that NbDBCP-HA overexpression does not alter the subcellular distribution of NbGLK1-GFP (Fig. S9B). 
This does not preclude an interaction may exist between the two, but that it may occur transiently. In conclusion, NbDBCP is an immune regulator that acts at a Rx1 and NbGlk1 complex at chromatin and whose ability to modulate Rx1 activity is dependent upon an intact BD.

A.

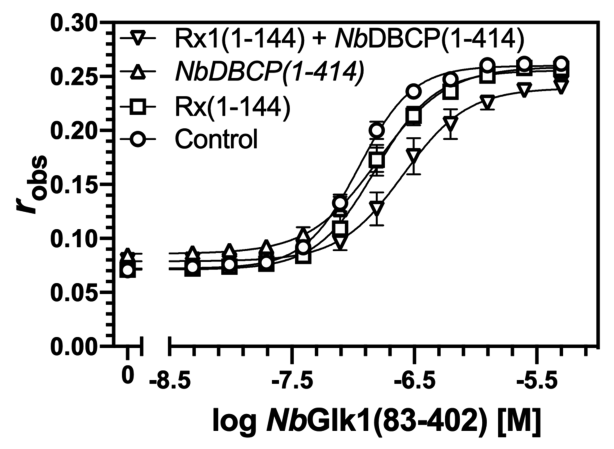

B.

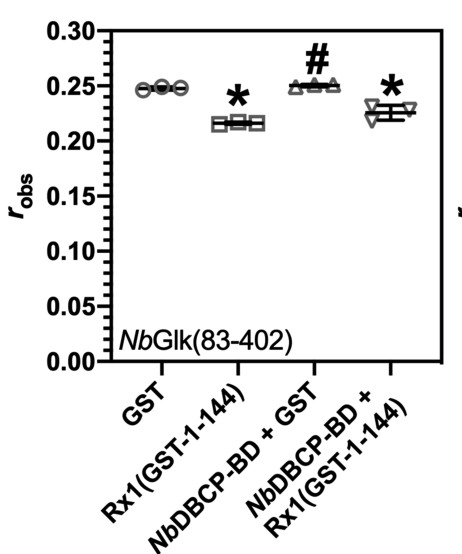

C.

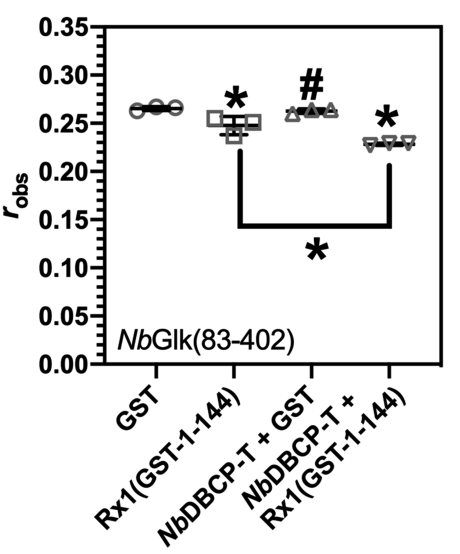

D.

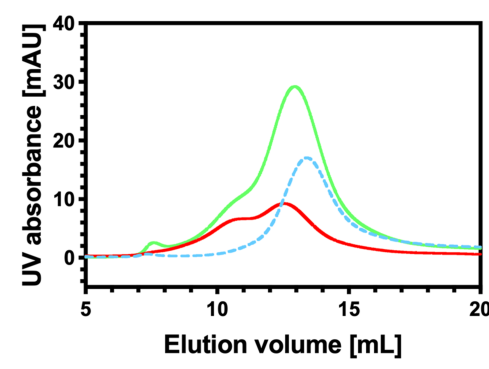

-- NbDBCP-T + NbGlk1(83-402)

- NbGlk1(83-402)

- NbDBCP-T

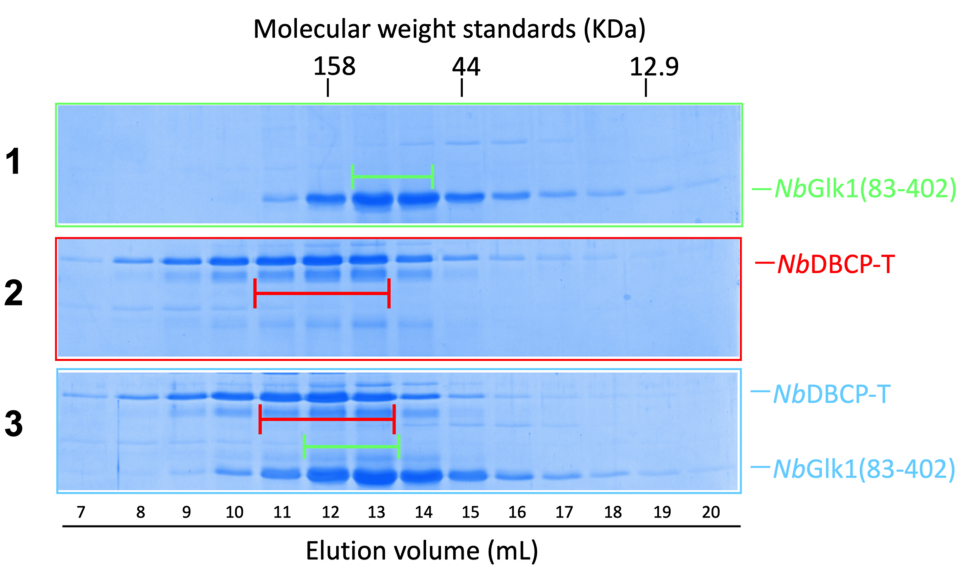

Fig. 6: The influence of Rx1 and NbDBCP on NbGlk1 DNA-binding. A) Fluorescence anisotropy values plotted against log protein concentration for NbGlk1(83-402) in the presence or absence of Rx1(1-144) or NbDBCP-T (n=3). B) DNA binding for NbGlk1 (83-402) was measured by fluorescence anisotropy in the presence or absence of GST, Rx1(GST-1-144) and NbDBCP-BD (means \pm S.E.M.; $n=3$; * $p<0.05$; \# $p>0.05$; one-way ANOVA with post hoc Tukey multiple comparison test). C) DNA binding for NbGlk1 (83-402) was measured by fluorescence anisotropy in the presence or absence of GST, Rx1(GST-1-144) and NbDBCP-T (means \pm S.E.M.; $n=3$; * $p<0.05$; \# $p>0.05$; one-way ANOVA with post hoc Tukey multiple comparison test). D) Interaction of NbGlk1(83-402) with NbDBCP-T. On the left are representative gel-filtration chromatograms of NbGlk1(83-402), NbDBCP-T and 
(Figure legend continued) NbGlk1(83-402) incubated with NbDBCP-T. Peak fractions were visualized by SDS-PAGE and are represented by capped bars.

\section{DISCUSSION}

Here we identify the BD-containing protein NbDBCP as a Rx1-interacting protein (Fig. 1). At least one NbDBCP-like protein is predicted within the genome sequences of a range of dicot and monocot species (Supplemental Fig. S1). NbDBCP-like protein are therefore widespread in higher plants but of unknown function. NbDBCP localisation to the nucleolus suggests broadly conserved function in ribosome biogenesis in plants that awaits further investigation. The identification of NbDBCP provides a potential direct link between histone modification and NB-LRR activity. Antibodies for detection of endogenous NbDBCP were not available. However, based on qPCR analysis (Fig. 4A), we can infer relative expression levels by comparison to actin in TRV:GFP plants. Data from independent repeats demonstrates that the average Ct values between actin and NbDCBP are within one Ct difference. This indicates that expression levels of endogenous NbDBCP is within a comparable range to actin.

NbDBCP was shown to localise to the nucleolus (Fig. 2) and co-expression with Rx1 resulted in approximately a quarter of the cells showing a redistribution of $\mathrm{Rx} 1$ to the nucleolus (Fig. 2). The reason for this localisation is unknown. We note that the nucleolus is a key target for plant viruses (Kalinina et al. 2018, Ding et al. 2020). Nucleolar chromatin is also subject to histone modification (Sáez-Vásquez et al. 2019). It is therefore tempting to speculate that localisation of Rx1 and NbDBCP to the nucleolus functions as part of a sub-organellar specific defence response, but this awaits future investigation. The question of whether $\mathrm{Rx} 1$ and $\mathrm{NbDBCP}$ is further redistributed on $\mathrm{PVX}$ infection is an open question requiring further investigation. When expressed alone NbDBCP-GFP interacts with plant chromatin in situ (Fig. 3A) and its DNA-binding in situ appeared to be inhibited by either $\mathrm{R} \times 1$ or CP106 overexpression. It is interesting to note that Rx1-GFP does interact with chromatin when co-expressed with NbDBCP and CP106 (Fig. 3B). This suggests that CP106 rearranges a complex at chromatin, that results in the loss of a NbDBCP interaction with chromatin while $\mathrm{R} \times 1$ is able to interact with chromatin. The FRET-FLIM experiment measures a ratio and so this observation is not an artefact of protein expression. This surprising finding suggests that CP106 can affect NbDBCP 
activity in a Rx1 independent manner. The PVX coat protein has been proposed to interact with multiple host proteins in N. bethamiana (Park et al. 2009), the identity of which remains a question for future research. Our interpretation of these confocal microscopy and FRET-FLIM data is that NbDBCP localises in the nucleolus where it interacts - or is closely associated - with chromatin. Such an interaction is consistent with the known role of BDs to bind acetyl-lysine that is typically found in histones (Marmorstein et al. 2001, Dhalluin et al. 1999). These latter interactions can be disturbed upon overexpression of $\mathrm{R} \times 1$ or the $\mathrm{CP}$, possibly via a third protein that interacts with all partners. However, a caveat with the interpretation of the FRET-FLIM data is the possibility of a false negative result. If the expressed NbDBCP-GFP fusion protein has saturated all available DNA-binding sites, the accumulation of an increased pool of non-DNA-bound protein will shift the ratio of the long to short lifetimes to the GFP negative control. In the absence of an available alternative method not susceptible to the same issue of false negatives, however, the interpretation of a negative result should be viewed with some caution. A conservative interpretation of the data therefore is that NbDBCP is able to interact with DNA in situ with some evidence that either $\mathrm{Rx} 1$ or $\mathrm{CP} 106$ redistributes $\mathrm{NbDBCP}$ from this site. It is interesting to note that a similar observation was previously made for the interaction of NbGlk1 with DNA in situ (Townsend et al. 2018). Rx1 redistributed NbGlk1 from DNA in situ and reduced the binding affinity for NbGlk1 for DNA in vitro.

We were unable to demonstrate an interaction between NbDBCP-BD and the small molecules $\mathrm{N}$-acetyl-lysine and $\omega$-acetyl-histamine (which mimics the $\mathrm{N}$-acetyl-lysine side chain) by isothermal calorimetry. However, BD substrate specificity for acetylated proteins is also dependent upon binding interactions with the amino acids surrounding the acetylated lysine (Mujtaba et al. 2002). The binding affinity for the acetylated lysine is therefore possibly too low to observe in the absence of an appropriate peptide sequence. An alternative possibility is that the BD shows specificity for a different chromatin modification. For example, the typical $Y N$ motif of the $B D L_{B C}$ loop in NbDBCP is replaced by YF. The bulky phenylalanine may therefore clash with $\mathrm{N}$-acetyl-lysine as observed previously for BDs with alternative residues at this site (Wen et al. 2014).

We took an indirect approach to identify whether histone modification binding might be required for NbDBCP function. Using structural modelling of NbDBCP-BD we identified two residues (Y336 and E386) possibly involved in NbDBCP-BD interactions with a modified target protein (Fig. 5A). Only the NbDBCP E386L variant could be expressed in planta but it accumulated at levels below 
that of the wild type protein. However, upon its accumulation the NbDBCP-E386L variant showed a gain-of-function phenotype resulting in a potentiated Rx1-mediated immune response to PVX as compared to the wild type protein (Fig. 5D-E). Note that as a gain-of-function phenotype, the finding is genuine despite the reduced level of expression of the mutant compared to the wild type protein. This finding is consistent with the VIGS data of Fig. 4B that demonstrates that NbDBCP is a negative regulator of Rx1-mediated immunity. Extreme resistance and cell death are thought to constitute distinct pathways of $\mathrm{Rx} 1$ immunity (Bendahmane et al. 1999). The common view is that cell death occurs as a secondary resistance response in case extreme resistance proves insufficient. As cell death is qualitatively more prominent, these data suggest that NbDBCP wild type expression reduces extreme resistance (consistent with the gene silencing data of Fig. 4B) while NbDBCP E386L expression enhances extreme resistance (due to less cell death). The precise mechanism for the gain-of-function phenotype for NbDBCP-E386L is not known. It might, for example, form non-functional complexes with the wild type protein resulting in a net reduction in the negative effect on $\mathrm{R} \times 1$ responses. This mechanism awaits future investigation.

$\mathrm{R} \times 1$ and NbDBCP acted synergistically to reduce the affinity of NbGlk1 for dsDNA (Fig. 6). The DNA-binding assay used has no histones present and so the observed effect on NbGlk1 is unlikely to involve binding to a chromatin modification in this assay. This is further evidenced by the observation that the isolated NbDBCP BD does not influence NbGlk1 DNA-binding (Fig. 6). It is most likely, therefore, that $\mathrm{R} \times 1$ and $\mathrm{NbDBCP}$ act in a complex to physically occlude NbGlk1 from DNA. We have some evidence that NbDBCP and NbGlk1 are able to form a complex (Fig. 6). It is interesting to note that the NbDBCP-T protein had an approximate molecular weight, as assessed by gel filtration chromatography, consistent with dimer formation (Fig. 6D). The functional relevance of a higher order structure for NbDBCP-T is unknown. Unfortunately, NbDBCP-T-E386L could not be produced as a recombinant protein in $E$. coli to investigate whether altered formation of such a higher order structure can explain the phenotype of this mutant and to investigate the relevance of such a structure. A crucial direction for future work, therefore, will be to fully define this larger Rx1-NbGIk1-NBDBCP complex (and potential homo-oligomerization) in planta and the requirements for its formation and complete role in immunity.

A possible interpretation of the data presented here and elsewhere (Townsend et al. 2018) centers on chromatin. NbDBCP interacts with chromatin, presumably at the nucleolus, and suppresses 
immune responses associated with $\mathrm{R} \times 1$ through an unknown mechanism but which likely depends on a functional BD (Fig. 7A). Co-expressed Rx1 and NbGlk1 form an inactive complex at DNA (Townsend et al. 2018) (Fig. 7B). NbDBCP forms a complex with co-expressed Rx1 and is released from chromatin. How and when endogenous $\mathrm{R} \times 1$ releases $\mathrm{NbDBCP}$ from chromatin awaits future investigation. Immune activation of Rx1 and/or NbDBCP via PVX's CP permits an uncharacterized change in the Rx1-NbGIk1 complex permitting Rx1 binding to chromatin and NbGlk1 to interact with its consensus DNA-binding sequence (Townsend et al. 2018) (Fig. 7C). Immune signaling is activated once NbGIk1 is stably bound to its consensus sequences and $\mathrm{NbDBCP}$ is removed from its inhibitory site. In conclusion, we identify NbDBCP as an immune supressing protein acting at chromatin and regulated by $\mathrm{R} \times 1$. Rx1 provides a direct link between the perception of PVX and transcriptional processes at DNA. Furthermore, the CC domain appears to function as a complex scaffold for nuclear proteins involved in transcriptional reprogramming. This immune regulated complex at chromatin is regulated by nuclear-localised $R \times 1$ to suppress immune activation until the perception of an appropriate pathogen signal. 
A
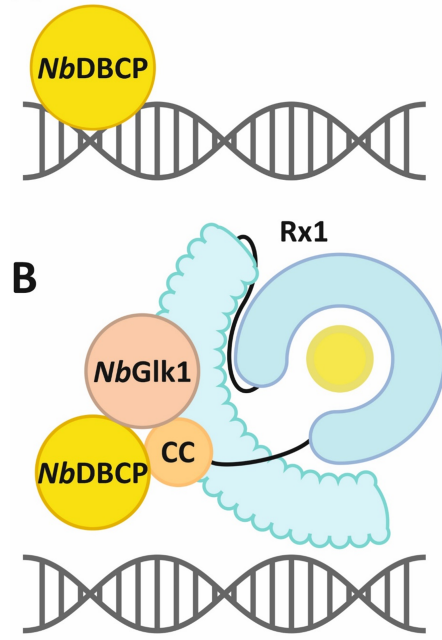

C PVX

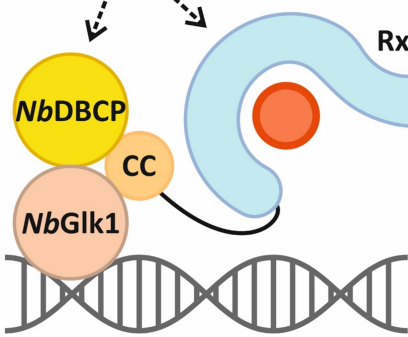

Rx1

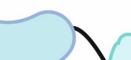

Fig. 7: Model for protein interactions of NbDBCP and NbGLK1 with Rx1. A) NbDBCP interacts with DNA. B) In the absence of PVX Rx1 interacts with NbDBCP and NbGlk1 at DNA and reduces the affinity of NbGlk1 for DNA (Townsend et al. 2018). NbDBCP suppresses immunity downstream of the $\mathrm{R} \times 1 / \mathrm{NbGIk1-DNA}$ interaction. C) PVX acts via $\mathrm{R} \times 1$ and/or NbDBCP resulting in a complex where $\mathrm{R} \times 1$ but not NbDBCP interacts with chromatin. Instead, NbGlk1 interacts with chromatin (Townsend et al. 2018).

\section{MATERIALS AND METHODS}

\section{Plasmids}

The NbDBCP (Niben101Scf17137g00006.1 (https://solgenomics.net) open-reading frame was amplified by PCR from cDNA synthesized from $N$. bethamiana whole leaf material and the DNA was 
sequenced on both strands using Sanger DNA sequencing. Several differences with the computed open-reading frame for Niben101Scf17137g00006.1 were noted. The cloned open-reading frame is deposited at NCBI with Accession number MN594539. A PCR product spanning NbDBCP residues 293-414 was cloned into the Xhol site of pET14b (pET14b- NbDBCP-BD) and fitted with a hexahistidine tag for affinity purification of recombinant protein. The oligonucleotides used to construct pET14b- NbDBCP-BD were NbDBCP-1 and NbDBCP-2. A PCR product spanning NbDBCP residues 1-414 was cloned into the Xhol site of pET14b (pET14b- NbDBCP-T) and fitted with a hexahistidine tag for affinity purification of recombinant protein The oligonucleotides used to construct pET14b- NbDBCP-BD were NbDBCP-7 and NbDBCP-8.

PCR products spanning nucleotides 1-300 (NbDBCP-1) and 645-945 (NbDBCP-2) of the NbDBCP coding sequence were cloned into the $\mathrm{Ncol}$ and Xhol sites of pTRV2 (pTRV2-NbDBCP-1 and pTRV2-NbDBCP-2). pTRV2-SGT1 is as previously described (Gabriëls et al. 2006, Gabriëls et al. 2007). A PCR product encompassing the full-length NbDBCP cDNA was cloned into the Ncol and Notl sites of pRAP35S-YFP-4HA to make pRAP35S-NbDBCP-4HA. The oligonucleotides used to construct pRAP35S-NbDBCP-4HA were NbDBCP-3 and NbDBCP-4. An Ascl/Pacl fragment from pRAP35S-NbDBCP-4HA encompassing the 35S promoter and NbDBCP-4HA fusion was cloned into the corresponding sites of the binary vector pBIN+ to make pBIN35S-NbDBCP-4HA. A PCR product encompassing the full-length native NbDBCP cDNA was introduced into Gateway donor vector pDONR207 (Invitrogen) to make pDONR207-NbDBCP. The NbDBCP gene was then recombined into the Gateway destination binary vector pK7WGF2 (Karimi et al. 2002) to make GFP-NbDBCP. The oligonucleotides used to make pDONR207-NbDBCP were NbDBCP-5 and NbDBCP-6. Mutant constructs were generated by site directed mutagenesis. The oligonucleotides used to make NbDBCP E386L variants were NbDBCP-9 and NbDBCP-10. The oligonucleotides used to make NbDBCP Y336F variants were NbDBCP-11 and NbDBCP-12.

Plasmids pET14b-NbGIk1(83-402), pET22b-Rx1-CCNBARC, pGEX-6P-1-Rx1(GST-1-144) and pBIN35S-NbGIk1-4HA are as described (Townsend et al. 2018). pBIN-35S-based plasmids corresponding to Rx1, GFP-H2B, Rx1-GFP, Rx1-mCherry, Rx1-4myc and CP106 are as described (Townsend et al. 2018). The PVX:GFP construct used were pGr106 (Townsend et al. 2018) and pGr208 which is based on pGr106 and modified to express GFP5 (Haseloff et al. 1997) driven by a 
duplicated PVX coat protein promoter. For TRV-VIGS silencing, the pTRV1 construct was used as described (Liu et al. 2002).

\section{Protein Expression and Purification}

Protein corresponding to $\mathrm{R} \times 1-C C N B A R C$ was expressed from $p E T 22 b-R \times 1-C C N B A R C$ as described

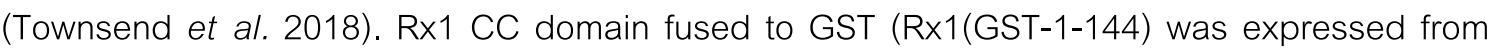
pGEX-6P-1-Rx1(GST-1-144) as described (Townsend et al. 2018). Protein corresponding to NbGlk1(83-402) was expressed from pET14b-NbGlk1(83-402) as described (Townsend et al. 2018).

Protein corresponding to NbDBCP-BD wild type and NbDBCP-BD-Y336F was expressed from pET14b-NbDBCP-BD and pET14b-NbDBCP-BD-Y336F plasmids in E. coli BL21(DE3) pLysS. A starter culture was grown overnight at $37^{\circ} \mathrm{C}$ in Luria broth supplemented with $50 \mu \mathrm{gL}-1$ kanamycin and $34 \mu \mathrm{g} \mathrm{mL}-1$ chloramphenicol. The overnight culture was diluted 1:50 into fresh Luria broth with antibiotics and grown with shaking at $37^{\circ} \mathrm{C}$ to $\mathrm{A} 600 \mathrm{~nm}=0.8$. Protein production was induced at $37^{\circ} \mathrm{C}$ with $1 \mathrm{mM}$ isopropyl- $\beta$-D-thiogalactoside for 2 hours. Cells were centrifuged $(4,000 \mathrm{~g}, 20$ minutes, $\left.4^{\circ} \mathrm{C}\right)$. Pelleted cells were washed with $50 \mathrm{mM}$ Tris- $\mathrm{HCl}$ pH 8.5, $1 \mathrm{mM}$ EDTA and centrifuged $(5,500 \mathrm{~g}$, $20 \mathrm{~min}, 4^{\circ} \mathrm{C}$ ). Cells were resuspended in twice their volume of lysis buffer (50 mM Tris-HCl pH 8.0, $200 \mathrm{mM} \mathrm{NaCl}, 40 \mathrm{mM}$ imidazole, 5 mM $\beta$-mercaptoethanol, and SIGMAFAST TM Protease Inhibitor Cocktail Tablets). Cells were lysed by sonication (150 s) and the lysate cleared by centrifugation at $\left(42,000 \mathrm{~g}, 60\right.$ minutes, $\left.4^{\circ} \mathrm{C}\right)$. The supernatant was loaded onto a $5 \mathrm{~mL}$ HisPrep HP Ni-NTA column (GE Healthcare) on an AKTA Pure chromatography system at $2 \mathrm{~mL}$ min-1 (GE Healthcare). The column was washed with 5 bed volumes of lysis buffer, 20 bed volumes of wash buffer (lysis buffer +1 $\mathrm{M} \mathrm{NaCl}$ ), 5 bed volumes of lysis buffer, and eluted with lysis buffer supplemented with $500 \mathrm{mM}$ imidazole. Peak fractions were assessed by SDS-PAGE, pooled, concentrated, exchanged into storage buffer (50 mM Tris-HCl pH 7.5, 500 mM NaCl, 1 mM EDTA, 1 mM DTT, 20 \% (v/v) glycerol), and stored at $-80^{\circ} \mathrm{C}$.

Protein corresponding to NbDBCP-T was expressed from pET14b-NbDBCP-T plasmid in E. coli BL21(DE3). A starter culture was grown overnight at $37^{\circ} \mathrm{C}$ in Luria broth supplemented with 50 $\mu \mathrm{g} \mathrm{mL-1} \mathrm{kanamycin.} \mathrm{The} \mathrm{overnight} \mathrm{culture} \mathrm{was} \mathrm{diluted} \mathrm{1:50} \mathrm{into} \mathrm{fresh} \mathrm{Luria} \mathrm{broth} \mathrm{with} \mathrm{antibiotics}$ 
and grown with shaking at $37^{\circ} \mathrm{C}$ to $A_{600 \mathrm{~nm}}=0.6$. Protein production was induced at $22^{\circ} \mathrm{C}$ with 0.5 $\mathrm{mM}$ isopropyl- $\beta$-D-thiogalactoside for 18 hours. Cells were centrifuged $\left(4,000 \mathrm{~g}, 20\right.$ minutes, $\left.4^{\circ} \mathrm{C}\right)$. Pelleted cells were washed with $50 \mathrm{mM}$ Tris-HCl pH 8.5, $1 \mathrm{mM}$ EDTA and centrifuged $(5,500 \mathrm{~g}$, $20 \mathrm{~min}, 4^{\circ} \mathrm{C}$ ). Cells were resuspended in twice their volume of lysis buffer (50 $\mathrm{mM} \mathrm{Na}_{2} \mathrm{PO}_{4} \mathrm{pH} 7.5$, $200 \mathrm{mM} \mathrm{NaCl}, 1 \mathrm{mM}$ DTT and SIGMAFAST TM Protease Inhibitor Cocktail Tablets). Cells were lysed by sonication $(150 \mathrm{~s})$ and the lysate cleared by centrifugation at $\left(42,000 \mathrm{~g}, 60\right.$ minutes, $\left.4^{\circ} \mathrm{C}\right)$. The supernatant was loaded onto a $5 \mathrm{~mL}$ HisPrep HP Ni-NTA column (GE Healthcare) on an AKTA Pure chromatography system at $2 \mathrm{~mL}$ min-1 (GE Healthcare). The column was washed with 5 bed volumes of lysis buffer, 20 bed volumes of wash buffer (lysis buffer $+500 \mathrm{mM} \mathrm{NaCl}$ ), 5 bed volumes of lysis buffer, and eluted with lysis buffer supplemented with $200 \mathrm{mM}$ imidazole. Peak fractions from the Ni-NTA column were pooled, concentrated and loaded onto a Superdex 200 16/600 and eluted with $50 \mathrm{mM} \mathrm{Na}_{2} \mathrm{PO}_{4} \mathrm{pH}$ 7.5, $200 \mathrm{mM} \mathrm{NaCl}, 1 \mathrm{mM}$ DTT, $1 \mathrm{mM}$ EDTA). Peak fractions were assessed by SDS-PAGE, pooled, concentrated, exchanged into storage buffer (50 mM Tris-HCl pH 7.5, 500 $\mathrm{mM} \mathrm{NaCl}, 1 \mathrm{mM}$ EDTA, $1 \mathrm{mM}$ DTT, $20 \%$ (v/v) glycerol), and stored at $-80^{\circ} \mathrm{C}$.

\section{Yeast Two-Hybrid Analyses}

Hybrigenics Services SAS (Paris, France) performed the yeast two-hybrid screen using Rx1 (amino acids 1-144) cloned into pB27 bait plasmid as a C-terminal fusion to LexA (N-LexA-Rx1-C). The screen was performed against a random-primed $N$. bethamiana mixed tissue cDNA library constructed into pP6 prey plasmid. A total of 96.6 million clones (approximately 9-fold library coverage) were

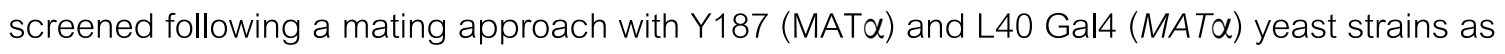
described (Fromont-Racine et al. 1997). To confirm protein-protein interactions, freshly transformed yeast colonies were resuspended in $1 \mathrm{~mL}$ sterile deionized water, and $10 \mu \mathrm{L}$ aliquots were spotted onto medium lacking leucine and tryptophan $(-\mathrm{L} / \mathrm{-W})$ and medium lacking leucine, tryptophan, histidine (-L/-W/-H), supplemented with 10 or $50 \mathrm{mM}$ 3-Amino-1,2,4-triazole (3-AT). Growth was scored after 5 to $7 \mathrm{~d}$ of incubation at $28^{\circ} \mathrm{C}$. CP106 (amino acids 1-237) was cloned into pB66 bait plasmid as a C-terminal fusion to Gal4 (N-Gal4-CP106-C). 


\section{Gel Filtration Analysis}

Gel filtration analysis of protein was performed at $4^{\circ} \mathrm{C}$ using an Sephacryl HiPrep 16/60 S200 HR column (GE Healthcare) on an AKTA Pure chromatography system (GE Healthcare). Protein was dialysed overnight against running buffer (20 mM Tris-HCl pH 7.4, $140 \mathrm{mM} \mathrm{NaCl}, 1 \mathrm{mM}$ DTT). Proteins were incubated on ice for $30 \mathrm{~min}$ individually or together and then centrifuged at 12,000 $\mathrm{g}$ for 30 min. Protein loading concentration was approximately $75 \mu \mathrm{M}$. Columns were run at a flow rate of 0.5 $\mathrm{mL} / \mathrm{min}$ in running buffer. Thirty $\mu \mathrm{L}$ of each eluted fraction was subjected to SDS-polyacrylamide gel electrophoresis (SDS-PAGE) and visualization with Quick Coomassie stain (Generon). Molecular weights were calibrated with gel filtration standards (BioRad): bovine thyroglobulin (670 kDa), bovine $\gamma$-globulin (158 kDa), chicken ovalbumin (44 kDa) and equine myoglobin (17 kDa).

\section{Co-immunoprecipitation}

N. bethamiana leaves were infiltrated with Agrobacterium tumefaciens strains (GV3101, MOG101) transformed with combinations of pBIN35S-NbDBCP-4HA, pBIN35S-Rx-CC-4myc and pBIN35S-Rx1-4myc binary vectors and leaf material was harvested 2 days after infiltration. $100 \mathrm{mg}$ of leaf material was ground in liquid nitrogen and resuspended in $1.5 \mathrm{~mL}$ extraction buffer $(10 \%$ (v/v) glycerol, 25 mM Tris-HCl pH 7,5, 1 mM Na2EDTA, 150 mM NaCl, 0.6 mg/mL Pefabloc SC, 20 $\mathrm{mg} / \mathrm{mL}$ polyvinylpolypyrrolidone, $0.1 \%(\mathrm{v} / \mathrm{v})$ Tween 20, $5 \mathrm{mM}$ DTT). The supernatant was passed through a $5 \mathrm{~mL}$ G25 Sephadex column after pelleting the cell debris. The resulting sample was incubated at $4^{\circ} \mathrm{C}$ with $50 \mu \mathrm{L}$ magnetic beads (Miltenyi $\mu \mathrm{MACs}$ ) for 2 hours. Unbound proteins were removed by washing 5 times with washing buffer (10\% (v/v) glycerol, $25 \mathrm{mM}$ Tris-HCl pH 7.5, 1 mM Na2EDTA, $150 \mathrm{mM} \mathrm{NaCl}$, 0.15\% (v/v) Nonidet P-40, 5 mM DTT). For Elution 1 the column was removed from the magnetic holder and moved to an Eppendorf tube. $45 \mu \mathrm{L}$ of washing buffer was added to remove the magnetic beads from the column. Subsequently $15 \mu \mathrm{L}$ of $4 \times$ NuPAGE LDS sample buffer ( $60 \mathrm{mM} \mathrm{DTT}$ ) was added and the samples were incubated at $95^{\circ} \mathrm{C}$ for 5 minutes. For Elution 2, $20 \mu \mathrm{L}$ of $1 \times$ NuPAGE LDS sample buffer $\left(95^{\circ} \mathrm{C}\right)$ was added directly to the column and incubated for 5 minutes. After 5 minutes the column was removed from the magnetic holder and moved to an Eppendorf tube. An additional $50 \mu \mathrm{L}$ 1x NuPAGE LDS sample buffer $\left(95^{\circ} \mathrm{C}\right)$ was added to the column to remove the beads and protein from the column. Start material (before incubation 
with beads), the unbound fraction and the captured proteins were separated on NuPAGE novex $12 \%$ bis-tris gels in MES buffer (50 mM MES, $50 \mathrm{mM}$ Tris base, $0.1 \%$ SDS, $1 \mathrm{mM}$ EDTA pH 7.3) and blotted on polyvinylidene difluoride (PVDF) membranes for immunoblot analysis. Affinity-tagged proteins were detected using peroxidase conjugated antibodies (c-Myc: goat $\alpha$-c-Myc (Abcam 9132) and donkey $\alpha$-goat peroxidase conjugated (Jackson 705-035-147), HA: rat $\alpha$-HA HRP conjugated (Roche 12013819001)). Peroxidase activity was visualized with the SuperSignal ${ }^{\text {TM }}$ West Dura and Femto Substrates (Thermo Scientific) and imaged in a Syngene G:BOX Chemi HR-16 Gel documentation system.

\section{Fluorescence Anisotropy}

Double-stranded DNA substrates with a concatenated GGATATCC site NbGlk1-binding site (GGATATCC) was made by annealing synthetic oligonucleotides FA-5 and FA-6. FA-5 was end-labelled with FAM. Double-stranded DNA was annealed by mixing $10 \mu \mathrm{M}$ concentrations of complementary oligonucleotides in $150 \mathrm{mM} \mathrm{NaCl}, 15 \mathrm{mM} \mathrm{NaCitrate}$, heating to $95^{\circ} \mathrm{C}$, and cooling to room temperature over 5 hours. Changes in anisotropy were measured using a Synergy ${ }^{\mathrm{TM}} \mathrm{H} 4$ Fluorescence Spectrophotometer (BioTek) fitted with polarizing filters $\left(\lambda_{\mathrm{em}}=528 \mathrm{~nm}, \lambda_{\mathrm{ex}}=485 \mathrm{~nm}\right.$, bandwidth $=20 \mathrm{~nm}$, averaging time $=10 \mathrm{~s}$ ). Anisotropy was determined using $10 \mathrm{nM}$ fluorescein end-labelled oligonucleotides (Eurofins MWG) with variable protein in $20 \mathrm{mM}$ Tris- $\mathrm{HCl}$ pH 7.4, 140 $\mathrm{mM} \mathrm{NaCl}, 1$ mM DTT. Anisotropy was calculated using Gen5 software (BioTek).

\section{Laser Scanning Confocal Microscopy}

Subcellular distribution studies were performed using a Zeiss LSM 510 confocal microscope (Carl Zeiss) and a $\times 401.2$ numerical aperture water-corrected objective as previously described (Slootweg et al. 2010). A. tumefaciens strain GV3101 (pMP90) transformed with GFP-fusion constructs of $\mathrm{NbDBCP}$ wild type or E386L mutant variant were infiltrated on $\mathrm{N}$. bethamiana leaves at final $\mathrm{OD}_{600}$ values of 0.5 . Leaf epidermal cells transiently expressing these constructs were harvested between 48-72 dpi and imaged by confocal microscopy. For GFP and chlorophyll imaging, N. bethamiana epidermal cells were excited using an Argon laser at $488 \mathrm{~nm}$ and emissions were detected through 505-550 nm band-pass and $650 \mathrm{~nm}$ long-pass filters, respectively. A HeNe $543 \mathrm{~nm}$ laser-line was 
used to excite leaf samples for mCherry imaging and a 600-650 nm band-pass filter was used for detection. Confocal images were analysed using the Java application ImageJ (Abramoff et al. 2004).

\section{Time Resolved FRET In Situ}

A. tumefaciens strain GV3101 (pMP90) was transformed with constructs pK7WGF2 (GFP negative control), pK7WGF2-H2B (GFP-H2B positive control), pBIN35S-CP106, pBIN35S-Rx1, pK7WGF2-GFP-NbDBCP (GFP-NbDBCP) and experiments were performed as described (Fenyk et al. 2015).

\section{Virus Induced Gene Silencing (VIGS)}

Three-week old N. bethamiana plants were co-infiltrated with Agrobacterium GV3101 strains for co-expression of TRV1 and TRV2 vectors. Silencing vectors used were pTRV2-GFP, pTRV2-SGT1, pTRV2- $\Delta$ NbDBCP-1 and pTRV2- $\Delta$ NbDBCP-2. Three or four weeks after inoculation, the upper leaves of the plant were used for transient expression and qPCR experiments to check for silencing levels using oligonucleotides F-q1 and R-q1.

\section{PVX Resistance Assay}

The resistance mediated by Rx1 against PVX was assessed by co-expressing $R \times 1$ with Agrobacterium GV3101 harbouring an amplicon of the PVX genome in N. bethamiana leaves. To observe either an increase or decrease in PVX resistance the $\mathrm{R} \times 1$ constructs were expressed at low levels using a leaky scan promoter (Slootweg et al. 2010) and relatively low densities of the Agrobacterium strain for agroinfiltration $(A 600 \mathrm{~nm}=0.1-0.05)$. Under these conditions $\mathrm{Rx} 1$ does not exhibit complete resistance and factors affecting $\mathrm{Rx} 1$ function are reflected in changes in PVX accumulation. Agrobacterium GV3101for expression of PVX (pPVX:GFP) was agroinfiltrated at $A_{600 \mathrm{~nm}}=0.002$. At five days post-infiltration $3 \times 6 \mathrm{~mm}$ diameter leaf discs were collected per infiltration spot and ground in $250 \mu \mathrm{L} 50 \mathrm{mM}$ phosphate buffer (pH 7) using a Tissuelyser II (Qiagen; $3 \mathrm{~mm}$ ball bearing, $2 \times 1 \mathrm{~min}, 30 \mathrm{~Hz}$ ). Cell debris was centrifuged for $10 \mathrm{~min} 5,000 \mathrm{~g} .50 \mu \mathrm{L}$ of the 
supernatant was used in a DAS-ELISA (Mäki-Valkama et al. 2000) for PVX as described (Slootweg et al. 2010).

\section{Expression Analysis by Quantitative Real-Time PCR}

Total RNA was extracted from $50 \mathrm{mg}$ leaf tissue using the Maxwell 16 simpleRNA extraction kit (Promega). cDNA synthesis was performed using the SuperScript III First-Strand Synthesis System (Invitrogen). Gene expression levels were analysed by qPCR in a reaction mix of $25 \mu$ l consisting of: $0.2 \mu \mathrm{M}$ forward and reverse primers (each), $10 \mathrm{ng}$ cDNA, $12.5 \mu \mathrm{l}$ Absolute qPCR Sybergreen Fluorescein mix (ThermoFisher), and $12.5 \mu \mathrm{MQ}$ water. qPCR data was normalized against the actin reference gene. Relative expression levels were analysed by the comparative method $\left(2^{-\Delta \Delta C t}\right.$ ) using average threshold (Ct) values (Schmittgen et al. 2008).

\section{Structural Modelling}

Protein fold searches using the Phyre ${ }^{2}$ protein homology/analogy recognition engine version 2.0 (Kelley et al. 2009) were undertaken using amino acids 304-405 of NbDBCP, using both normal and intensive modelling modes. The model was based on the crystal structure of the BPTF BD in complex with histone $\mathrm{H} 4$ acetylated at Lys16 (PDB accession number 3QZT) made using Chainsaw within the CCP4 package (Winn et al. 2011) and sequence alignments were generated by the Phyre2 server. Side chain packing and energy minimization was performed using GalaxyRefine (Heo et al. 2013). Figures were generated using the PyMOL molecular graphics system (LLC 2010).

\section{Statistical Analysis}

Error bars represent the standard error of the mean with the number of biologically independent replicates as indicated in the legend. Statistical comparisons ( $p$ values) were obtained from one-way ANOVA with the indicated post-hoc test unless otherwise indicated. $p$ values in statistical comparisons are indicated in figures and indicate compared data sets as described in the figure legends. 


\section{ACKNOWLEDGEMENTS}

This work was supported by Biotechnology and Biological Sciences Research Council Grant BB/M007405/1 (to M.J.C. and L.O.P.), the Dutch Technology Foundation STW and Earth and Life Sciences ALW (to E.J.S., O.C.A.S. and A.G.) and VIVO project No. 865.14.003 (to F.L.W.T.) (Netherlands Organization for Scientific Research). The authors declare that they have no conflicts of interest with the contents of this article. 


\section{SUPPLEMENTALS}

Supplemental Table (Related to Supplemental Methods). The oligonucleotides used for this study.

\begin{tabular}{|c|c|c|}
\hline Primer name & Orientation & Sequence \\
\hline NbDBCP-1 & Sense & 5' - GGC CTC GAG GCC GTC GGA CAT ATT AAA G - 3' \\
\hline NbDBCP-2 & Antisense & 5' - GGC CTC GAG TTA GGG CCC AGG TGA TGA ATG - 3' \\
\hline NbDBCP-3 & Sense & 5' - GGC TCA TGA TCG AAA AAG AGT ACG GCA CAA C - 3' \\
\hline $\mathrm{NbDBCP}-4$ & Antisense & 5' - GGC GCG GCC GCC CTC CTT GAC CGT TTC TTT G - 3' \\
\hline \multirow[t]{2}{*}{ NbDBCP-5 } & Sense & 5' - GGG GAC AAG TTT GTA CAA AAA AGC AGG CTA CAT GGA AAA AGA \\
\hline & & GTA CGG C - 3' \\
\hline \multirow[t]{2}{*}{ NbDBCP-6 } & Antisense & 5' - GGG GAC CAC TTT GTA CAA GAA AGC TGG GTC ACC TCC TTG ACC \\
\hline & & GTT TCT TTG - 3' \\
\hline NbDBCP-7 & Sense & 5' - GGC CTC GAG ATG ATC GAA AAA GAG TAC - 3' \\
\hline NbDBCP-8 & Antisense & 5' - GGC CTC GAG TTA GGG CCC AGG TGA TGA ATG - 3' \\
\hline NbDBCP-9 & Sense & 5' - CCC TAA GTC ATC CCC ACT GTC AAC TGC AGC TAA TG - 3' \\
\hline NbDBCP-10 & Antisense & 5' - CAT TAG CTG CAG TTG ACA GTG GGG ATG ACT TAG GG - 3' \\
\hline NbDBCP-11 & Sense & 5' - CAG AAA ACG GAC AAG TTC AAG AAT ATG ATC CGA C - 3' \\
\hline NbDBCP-12 & Antisense & 5' - GTC GGA TCA TAT TCT TGA ACT TGT CCG TTT TCT G - 3' \\
\hline \multirow[t]{2}{*}{ FA-5 } & Sense & 5' - CGG ATA TCC ACG GAT ATC CAC GGA TAT CCA CGG ATA TCC ACG \\
\hline & & GAT ATC CC - 3' \\
\hline \multirow[t]{2}{*}{ FA-6 } & Antisense & 5' - GGG ATA TCC GTG GAT ATC CGT GGA TAT CCG TGG ATA TCC GTG \\
\hline & & GAT ATC CG - 3' \\
\hline $\mathrm{F}-\mathrm{q} 1$ & Sense & 5' - CCG TCG AAG AGG AGT GTA GG - 3' \\
\hline R-q1 & Antisense & 5' - CCG TCG AAG AGG AGT GTA GG - 3' \\
\hline
\end{tabular}


Fig. S1 (Related to Fig. 1). Maximum-likelihood phylogenetic tree showing the calculated relationship between higher plant species with a conserved SANT-type domain and BD structure. Numbers represent bootstrap values. Branch terminal labelling is in the format UniProt ID_Species name. $\mathrm{NbDBCP}$ is boxed in red.

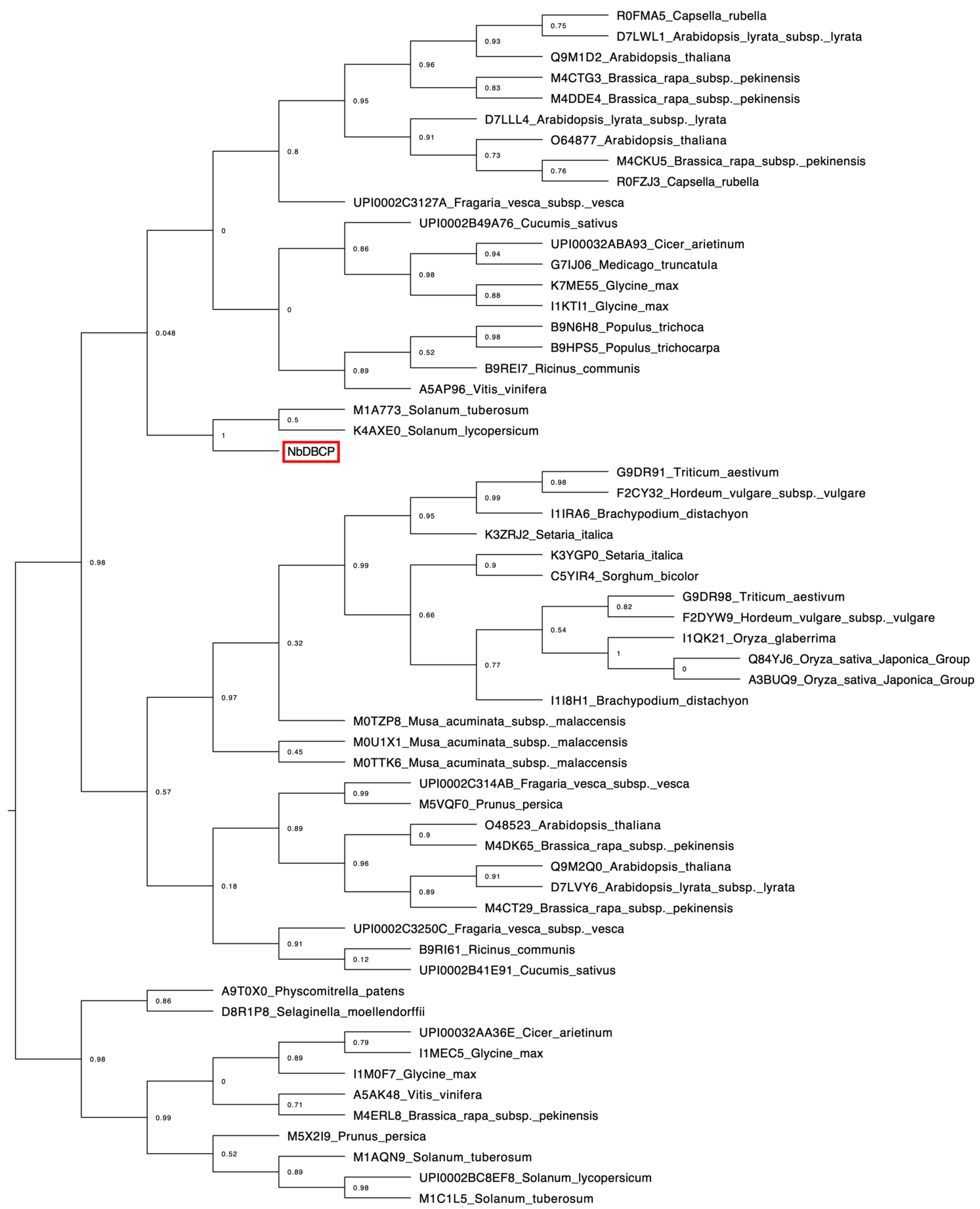


Fig. S2 (Related to Fig. 2). $I_{N} / I_{C}$ ratio for Rx1-mCherry expressed with either GFP or NbDBCP-GFP (mean $\pm 95 \%$ C.I) as determined using the ImageJ software.

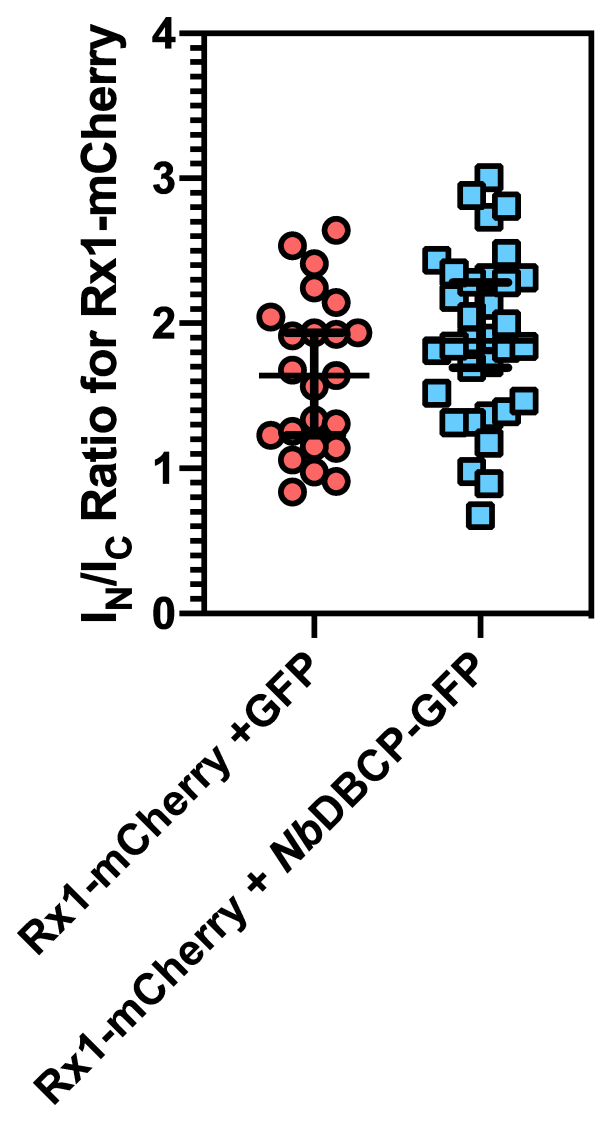


Fig. S3 (Related to Fig. 2). Subcellular distribution of NbDBCP-GFP in planta. Representative overlay confocal images of $N$. benthamiana leaf epidermal cells transiently expressing NbDBCP-GFP with or without P19, free GFP or an uninfiltrated leaf. Images were taken at 2 or $3 \mathrm{dpi}$. Scale bar $=10 \mathrm{~mm}$. $\mathrm{N}=$ nucleus, $\mathrm{n}=$ nucleolus, $\mathrm{SB}=$ subnuclear bodies, $\mathrm{C}=$ cytoplasm, $\mathrm{Ch}=$ chloroplasts .
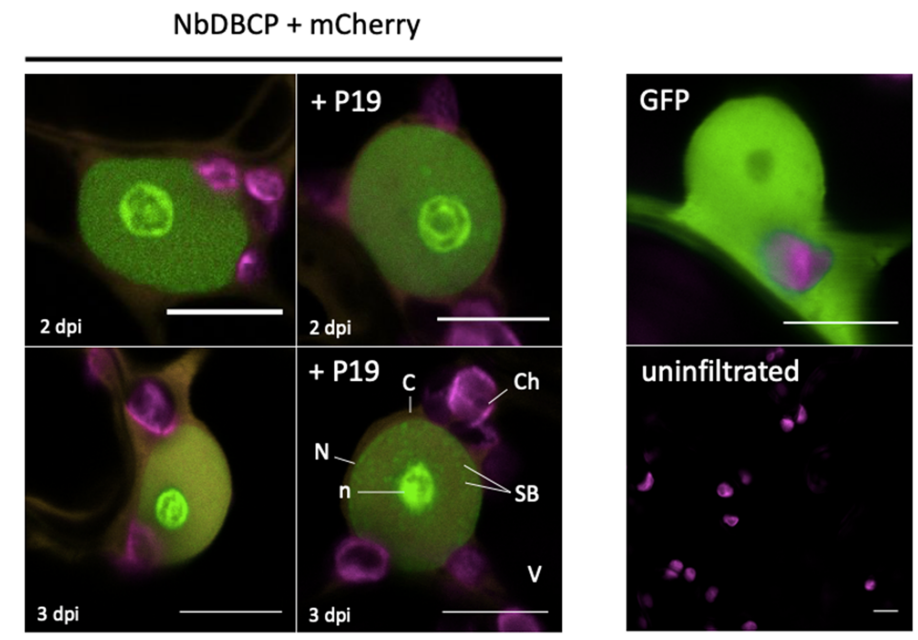

Fig. S4 (Related to Fig. 2). Immunoblot of mCherry constructs used for the confocal microscopy experiments. Infiltrated $N$. benthamiana leaf samples were harvested at $3 \mathrm{dpi}$ and protein from either total extract or the soluble fractions were used for used for Western blotting with an $\alpha$-mCherry antibody. The black triangle indicates the presence of a protein band with the expected size of Rx1-mCherry. CBB denotes Coomassie Blue stain loading control for the denoted samples.

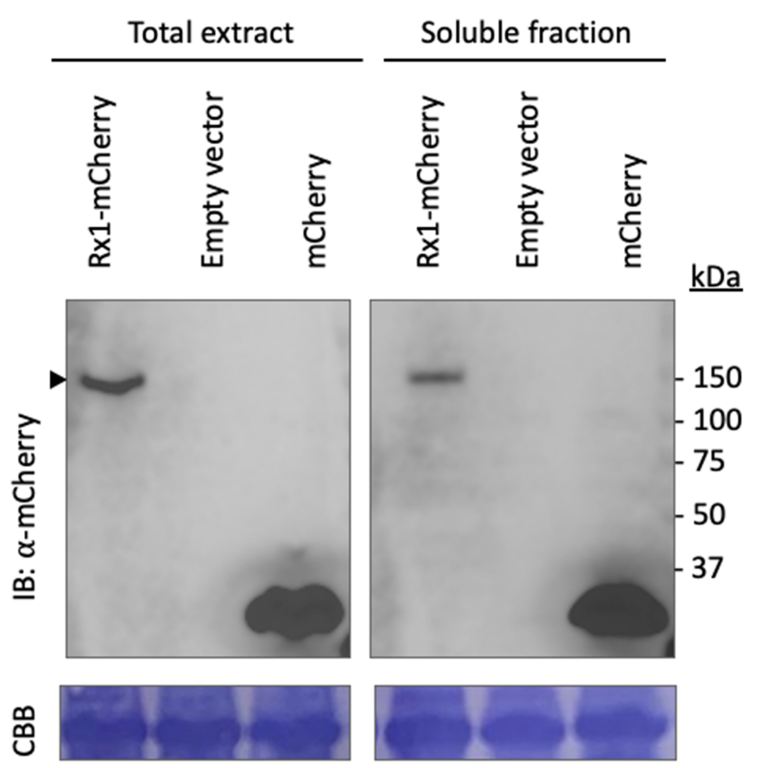


Fig. S5 (Related to Fig. 2). Co-expressing Rx1-mCherry with NbDBCP-GFP but not NbDBCP-HA results in a significant increase in signal of the receptor in the nucleolus. Relative intensity ratios of $\mathrm{R} \times 1$-mCherry or free-mCherry in the nucleolus relative to the nucleoplasm $\left(I_{\text {Nucleolus }} / I_{\text {Nucleoplasm }}\right)$ in the presence or absence of NbDBCP-GFP. Crossbar represents the mean values. Data was taken from four independent experimental repeats and tested for normality using the Shapiro-Wilk test. Subsequently, statistical significance was calculated using the Wilcoxon Signed-Rank Test with $\alpha=0.05$. Intensity of Rx1-mCherry in each compartment was determined using the ImageJ software. Signal from the vacuole was considered as background and subtracted from the signal of each compartment before taking a ratio.

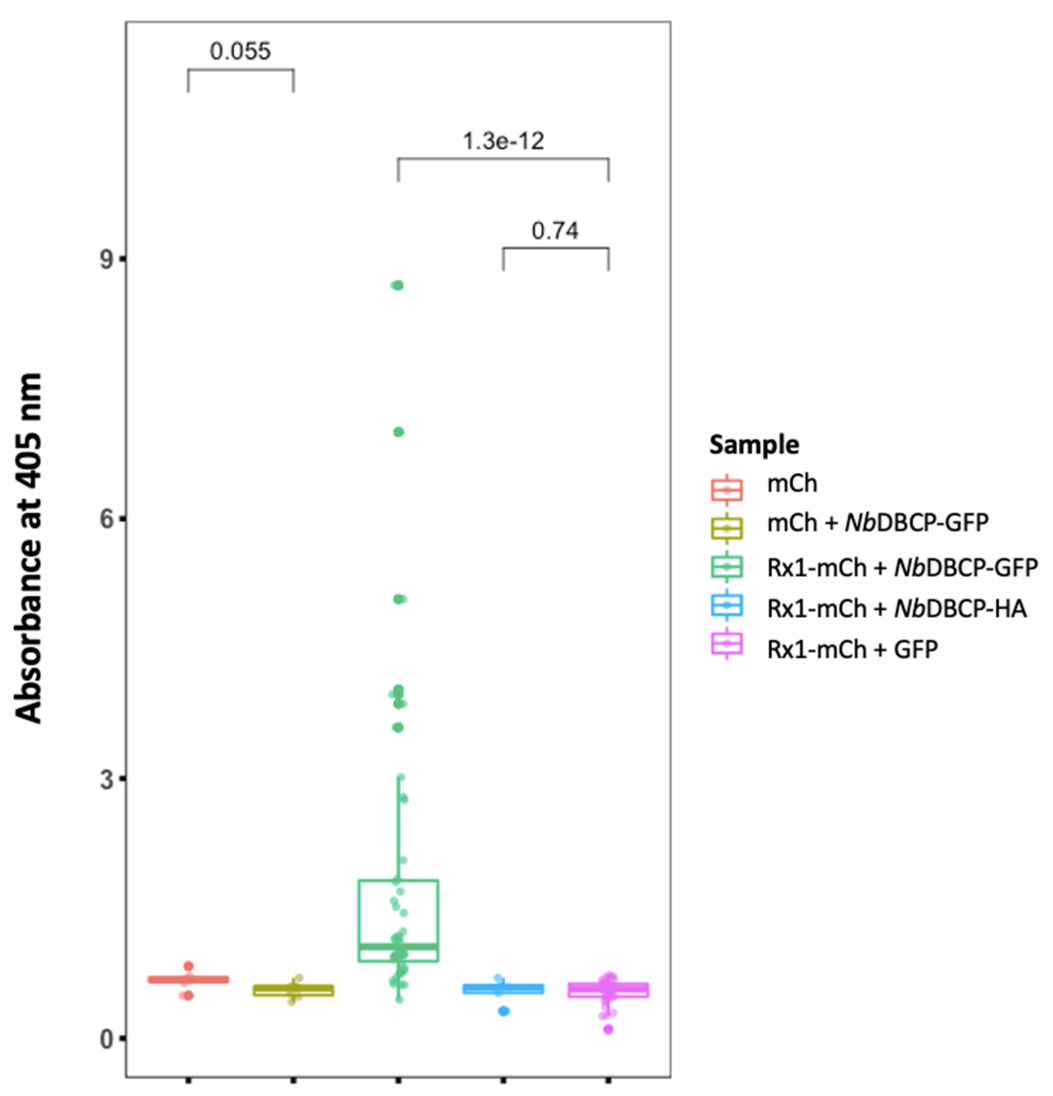


Fig. S6 (Related to Fig. 3). CP106 does not interact with NbDBCP by Y2H. 1x1 Yeast Two-Hybrid screen of CP106(1-237) against amino acids 265-493 of NbDBCP. CP106(1-237) was fused to the Gal4 DNA-binding domain and NbDBCP(265-493) wild type and E386L was fused to the Gal4 activation domain. Plates were grown on medium lacking leucine and tryptophan $(-\mathrm{L} / \mathrm{-W})$ and medium lacking leucine, tryptophan, histidine (-L/-W/-H). A) Smad vs Smurf positive control B) Empty pB66 bait vs NbDBCP(265-493) wild type in prey negative control. C) CP106(1-237) in pB66 bait plasmid vs empty pP7 prey negative control. D) CP106(1-237) in pB66 bait plasmid with NbDBCP(265-493) wild type in pP7 prey plasmid.

A.

\section{pB27-Smad vs pP7-Smurf (positive control)}

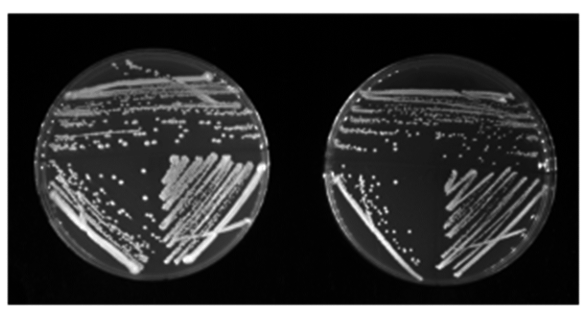

C.

\section{pB27-CP106(1-237) vs pP7 (negative control)}

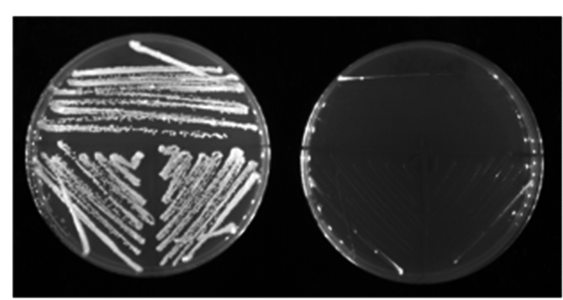

$-L /-W \quad-L /-W /-H$
B. pB66 vs pP7-NbDBCP(265-493) (negative control)

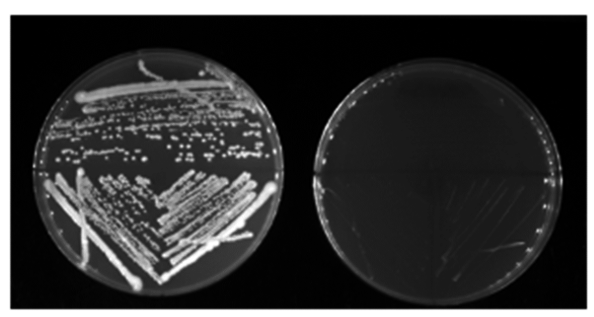

$$
-L /-W \quad-L /-W /-H
$$

D.

\section{pB27-NbDBCP(265-493) vs pP7-CP106(1-237) (test)}

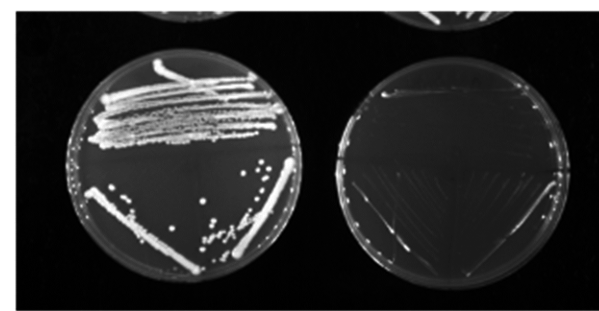

$-L /-W /-T \quad-L /-W /-H$ 
Fig. S7 (Related to Fig. 5). Representative photographs of N. benthamiana leaves infiltrated with pGR208 (that drives expression of a PVX amplicon) and/or full-length Rx1 in the presence/absence of NbDBCP-WT or E386L variant. Images were taken at 5 dpi. Independent biological repeats are shown to demonstrate consistent results between leaf samples. Key: A1 = pGR208 + GFP; $\mathrm{A} 2=\mathrm{pGR208}+\mathrm{NbDBCP}-\mathrm{WT} ; \quad \mathrm{A} 3=\mathrm{pGR} 208+$ NbDBCP $-\mathrm{E} 386 \mathrm{~L} ; \quad \mathrm{B} 1=\mathrm{pGR} 208+\mathrm{R} \times 1+\mathrm{GFP} ;$ $\mathrm{B} 2=\mathrm{pGR} 208+\mathrm{Rx} 1+\mathrm{NbDBCP}-\mathrm{WT} ; \mathrm{B} 3=\mathrm{pGR} 208+\mathrm{R} \times 1+\mathrm{NbDBCP}-\mathrm{E} 386 \mathrm{~L}$.
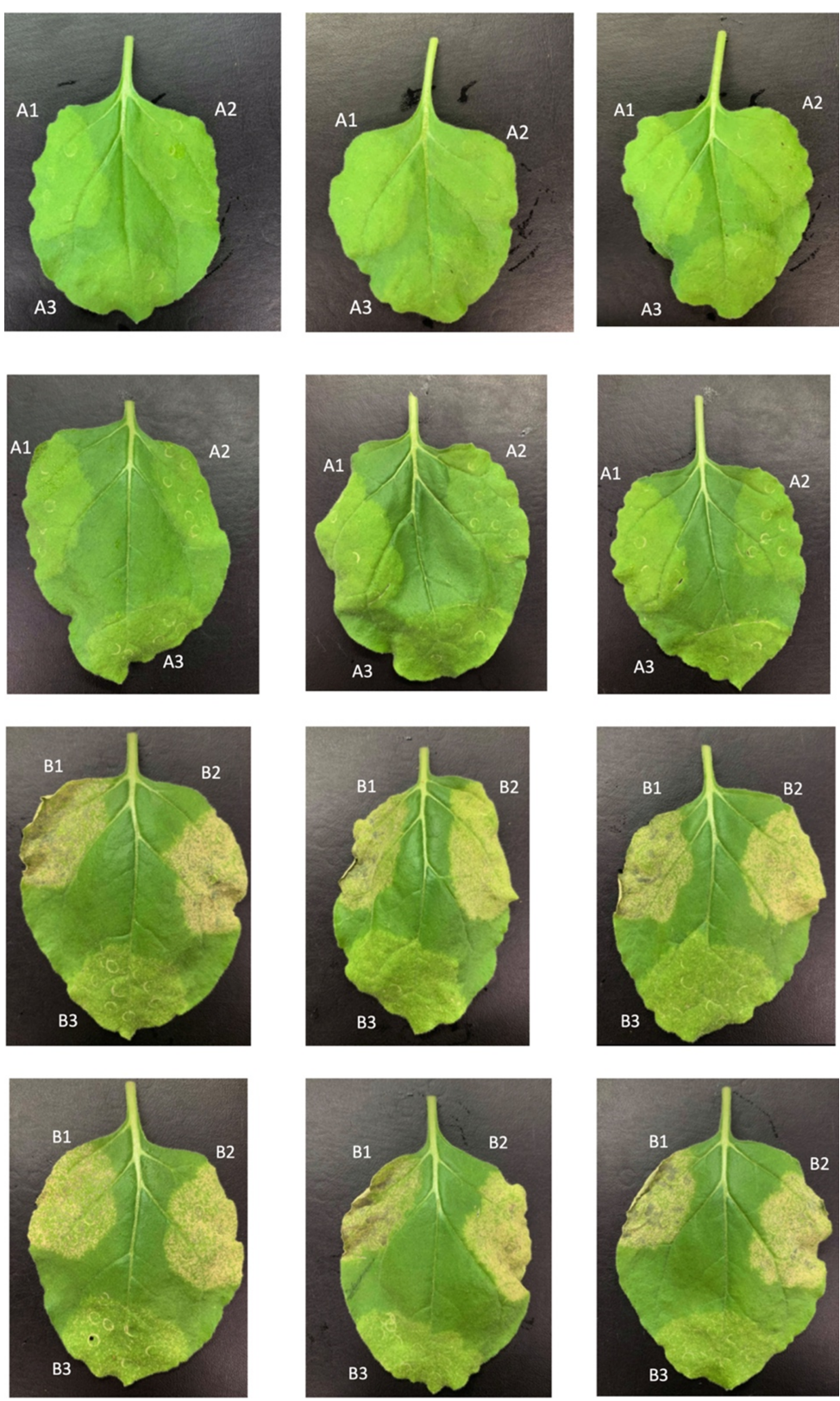
Fig. S8 (Related to Fig. 5). The N-terminus of the Rx1 NB-LRR protein interacts with NbDBCP wild type and E386L. 1x1 Yeast Two-Hybrid screen of Rx1(1-144) against amino acids 265-493 of NbDBCP. Rx1(1-144) was fused to the Gal4 DNA-binding domain and NbDBCP(265-493) wild type and E386L was fused to the Gal4 activation domain. Plates were grown on medium lacking leucine and tryptophan $(-\mathrm{L} /-\mathrm{W})$ and medium lacking leucine, tryptophan, histidine $(-\mathrm{L} /-\mathrm{W} /-\mathrm{H})$, supplemented with 5, 10 or 20 mM 3-Amino-1,2,4-triazole (3AT). A) Smad vs Smurf positive control B) Empty pB27 bait vs NbDBCP(265-493) wild type in prey negative control. C) Empty pB27 bait vs NbDBCP(265-493) E386L in prey negative control. D) Rx1(1-144) containing bait vs empty pP7 prey negative control. E) Rx1(1-144) in pB27 bait plasmid with NbDBCP(265-493) wild type in pP7 prey plasmid. F) Rx1(1-144) in pB27 bait plasmid with NbDBCP(265-493) E386L in pP7 prey plasmid.
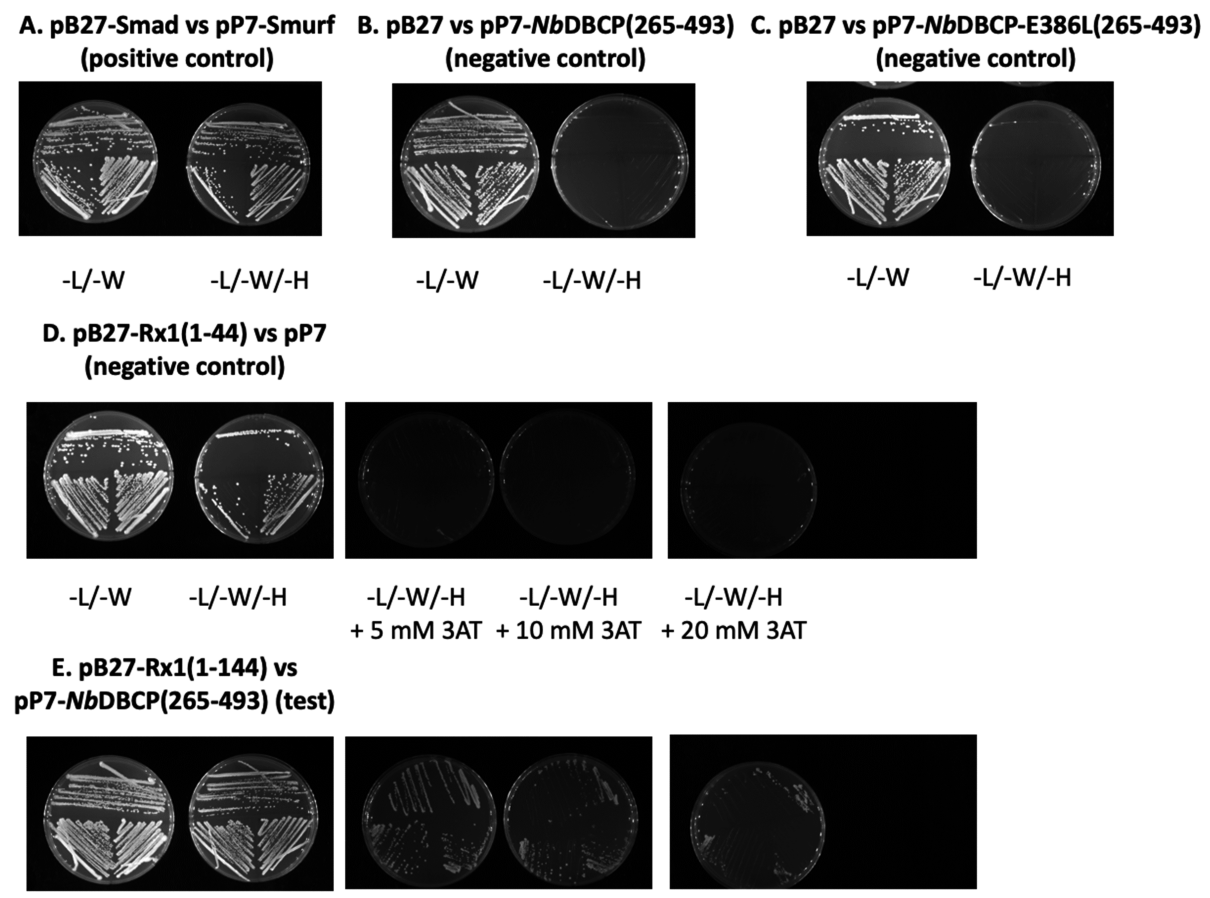

$-L /-W /-H \quad-L /-W /-H$

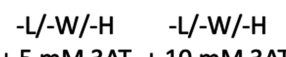

$-\mathrm{L} / \mathrm{-W} / \mathrm{-H}$

$+5 \mathrm{mM} 3 \mathrm{AT}+10 \mathrm{mM} 3 \mathrm{AT}+20 \mathrm{mM} 3 \mathrm{AT}$

F. pB27-Rx1(1-144) vs PP7-NbDBCP-E386L(265-493) (test)
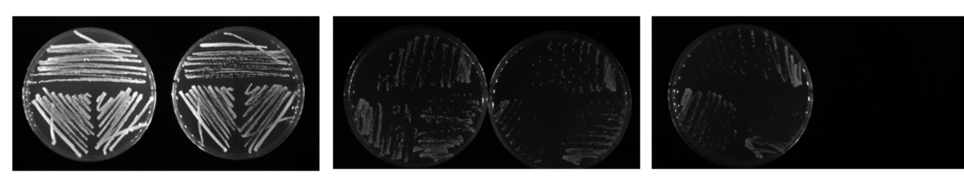

$$
\begin{aligned}
& -\mathrm{L} /-\mathrm{W} /-\mathrm{H} \quad-\mathrm{L} / \mathrm{W} /-\mathrm{H} \quad-\mathrm{L} /-\mathrm{W} /-\mathrm{H} \quad-\mathrm{L} / \mathrm{-W} /-\mathrm{H} \quad-\mathrm{L} / \mathrm{W} / \mathrm{-H} \\
& +5 \mathrm{mM} 3 \mathrm{AT}+10 \mathrm{mM} \text { 3AT }+20 \mathrm{mM} 3 \mathrm{AT}
\end{aligned}
$$


Fig. S9 (Related to Fig. 6). A) Immunoblotting of constructs used for the confocal imaging of NbGLK1-GFP and NbDBCP-HA. Total and soluble protein extracts from N. benthamiana leaves harvested at $2 \mathrm{dpi}$ were used for Western blotting. Staining of membrane by Coomassie-Brilliant Blue (CBB) was performed as loading control. B) Subcellular localization patterns of NbGLK1-GFP in the presence or absence of NbDBCP-HA. Imaging was performed at 2 dpi by confocal microscopy of $N$. benthamiana epidermal cells expressing the appropriate construct combinations. White bar represents a scale of $10 \mu \mathrm{m} . \mathrm{N}=$ nucleus; $\mathrm{n}=$ nucleolus; $\mathrm{V}=$ vacuole; $\mathrm{Ch}=$ chloroplast.

A.

Total extract Soluble fraction
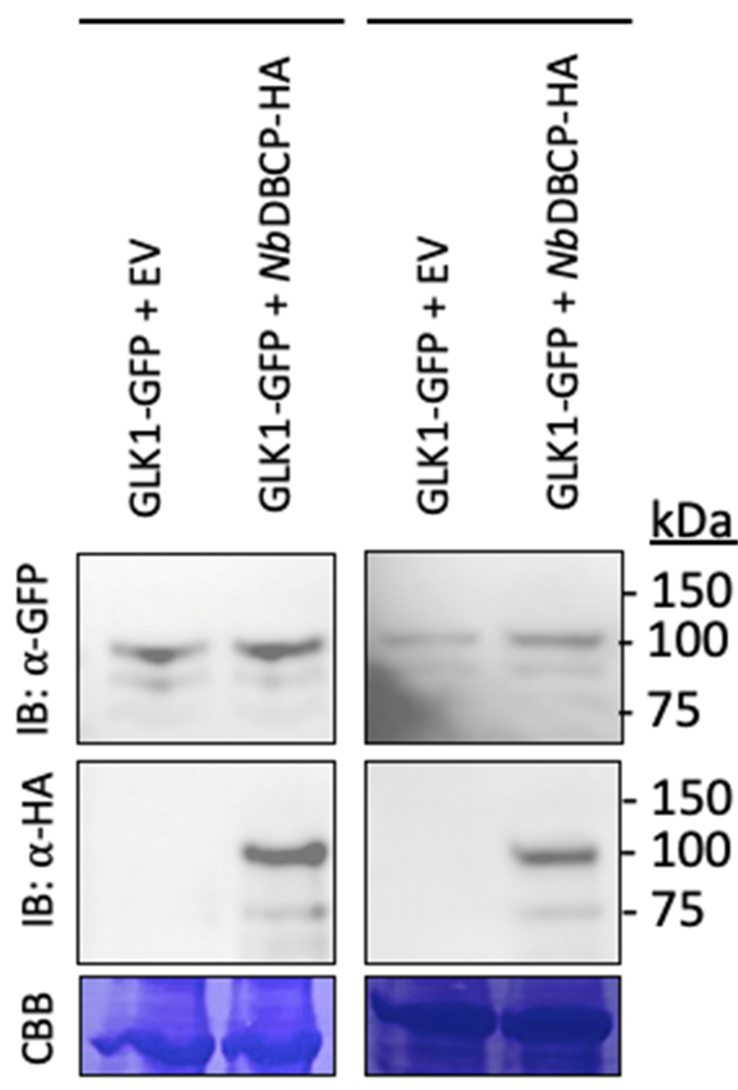

B.
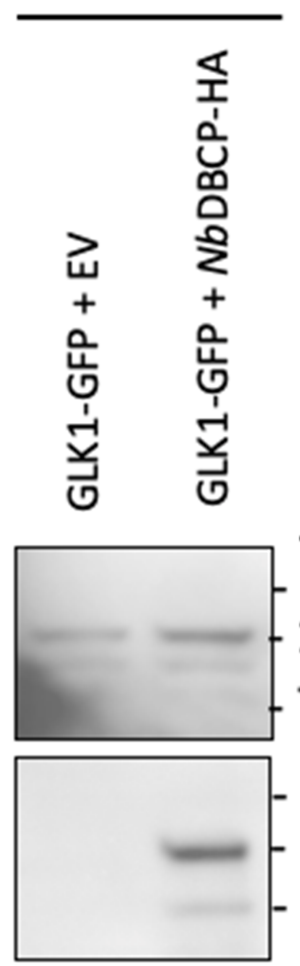

$\mathrm{kDa}$

150

100

75

150

100

75
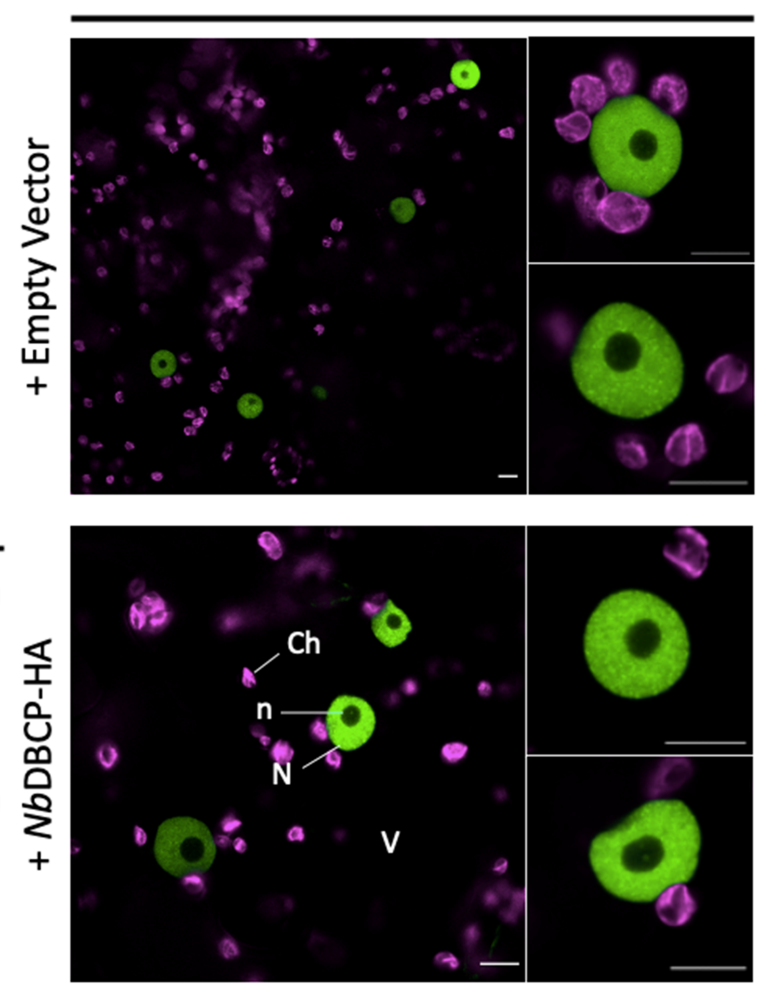


\section{References}

Abramoff, M., Magalhães, P. J., and Ram, S. J. (2004), Image processing with ImageJ. Biophotonics international. 11(7): p. 36-42.

Bai, S., Liu, J., Chang, C., Zhang, L., Maekawa, T., Wang, Q., Xiao, W., Liu, Y., Chai, J., Takken, F. L., Schulze-Lefert, P., and Shen, Q. H. (2012), Structure-function analysis of barley NLR immune receptor MLA10 reveals its cell compartment specific activity in cell death and disease resistance. PLoS Pathog. 8(6): e1002752.

Bendahmane, A., Kanyuka, K., and Baulcombe, D. C. (1999), The Rx gene from potato controls separate virus resistance and cell death responses. Plant Cell. 11.

Bendahmane, A., Köhm, B.A., Dedi, C., and Baulcombe, D. C. (1995), The coat protein of potato virus $X$ is a strain-specific elicitor of Rx1-mediated virus resistance in potato. The Plant Journal. 8(6): p. 933-941.

Van der Biezen, E. A. and Jones, J. D. G. (1998), The NB-ARC domain: A novel signalling motif shared by plant resistance gene products and regulators of cell death in animals. Current Biology. 8(7): R226-R227.

Burch-Smith, T. M., Schiff, M., Caplan, J. L., Tsao, J., Czymmek, K., and Dinesh-Kumar, S. P. (2007), A novel role for the TIR domain in association with pathogen-derived elicitors. PLoS Biol. 5(3): e68.

Caplan, J. L., Mamillapalli, P., Burch-Smith, T. M., Czymmek, K., and Dinesh-Kumar, S. P. (2008), Chloroplastic protein NRIP1 mediates innate immune receptor recognition of a viral effector. Cell. 132(3): p. 449-462.

Chang, C., Yu, D., Jiao, J., Jing, S., Schulze-Lefert, P., and Shen, Q.-H. (2013), Barley MLA Immune Receptors Directly Interfere with Antagonistically Acting Transcription Factors to Initiate Disease Resistance Signaling. The Plant Cell Online. 25(3): p. 1158-1173.

Cheng, Y. T., Germain, H., Wiermer, M., Bi, D., Xu, F., García, A. V., Wirthmueller, L., Després, C., Parker, J. E., Zhang, Y., and Li, X. (2009), Nuclear Pore Complex Component MOS7/Nup88 Is Required for Innate Immunity and Nuclear Accumulation of Defense Regulators in <em>Arabidopsis</em>. The Plant Cell. 21(8): p. 2503-2516.

Deslandes, L., Olivier, J., Peeters, N., Feng, D. X., Khounlotham, M., Boucher, C., Somssich, I., Genin, S., and Marco, Y. (2003), Physical interaction between RRS1-R, a protein conferring resistance to bacterial wilt, and PopP2, a type III effector targeted to the plant nucleus. Proceedings of the National Academy of Sciences. 100(13): p. 8024-8029.

Dhalluin, C., Carlson, J. E., Zeng, L., He, C., Aggarwal, A. K., Zhou, M.-M., and Zhou, M.-M. (1999), Structure and ligand of a histone acetyltransferase bromodomain. Nature. 399(6735): p. 491-496.

Ding, B., Bellizzi, M. d. R., Ning, Y., Meyers, B. C., and Wang, G.-L. (2012), HDT701, a histone H4 deacetylase, negatively regulates plant innate immunity by modulating histone $\mathrm{H} 4$ acetylation of defense-related genes in rice. The Plant cell. 24(9): p. 3783-3794.

Ding, Y. and Lozano-Durán, R. (2020), The Cajal Body in Plant-Virus Interactions. Viruses. 12(2): p. 250.

Eitas, T. K. and Dangl, J. L. (2010), NB-LRR proteins: pairs, pieces, perception, partners, and pathways. Curr Opin Plant Biol. 13(4): p. 472-7.

Espinas, N. A., Saze, H., and Saijo, Y. (2016), Epigenetic Control of Defense Signaling and Priming in Plants. Frontiers in plant science. 7: p. 1201-1201.

Fenyk, S., Campillo, A. d. S. E., Pohl, E., Hussey, P. J., and Cann, M. J. (2012), A nucleotide phosphatase activity in the nucleotide binding domain of an orphan resistance protein from rice. The Journal of biological chemistry. 287(6): p. 4023-4032.

Fenyk, S., Dixon, C. H., Gittens, W. H., Townsend, P. D., Sharples, G. J., Pålsson, L.-O., Takken, F. L. W., and Cann, M. J. (2016), The Tomato Nucleotide-Binding Leucine-Rich Repeat (NLR) Immune Receptor I-2 Couples DNA-Binding to Nucleotide-Binding Domain Nucleotide Exchange. Journal of Biological Chemistry. 291(3): p. 1137-1147. 
Fenyk, S., Townsend, P. D., Dixon, C. H., Spies, G. B., de San Eustaquio Campillo, A., Slootweg, E. J., Westerhof, L. B., Gawehns, F. K. K., Knight, M. R., Sharples, G. J., Goverse, A., Pålsson, L.-O., Takken, F. L. W., and Cann, M. J. (2015), The Potato Nucleotide-binding Leucine-rich Repeat (NLR) Immune Receptor Rx1 Is a Pathogen-dependent DNA-deforming Protein. The Journal of biological chemistry. 290(41): p. 24945-24960. ppublish.

Finzi, L. and Dunlap, D. D. (2010), Single-molecule approaches to probe the structure, kinetics, and thermodynamics of nucleoprotein complexes that regulate transcription. The Journal of biological chemistry. 285(25): p. 18973-18978.

Fromont-Racine, M., Rain, J. C., and Legrain, P. (1997), Toward a functional analysis of the yeast genome through exhaustive two-hybrid screens. Nat Genet. 16(3): p. 277-82.

Gabriëls, S. H., Takken, F. L., Vossen, J. H., de Jong, C. F., Liu, Q., Turk, S. C., Wachowski, L. K., Peters, J., Witsenboer, H. M., de Wit, P. J., and Joosten, M. H. (2006), CDNA-AFLP combined with functional analysis reveals novel genes involved in the hypersensitive response. Mol Plant Microbe Interact. 19(6): p. 567-76.

Gabriëls, S. H., Vossen, J. H., Ekengren, S. K., van Ooijen, G., Abd-El-Haliem, A. M., van den Berg, G. C., Rainey, D. Y., Martin, G. B., Takken, F. L., de Wit, P. J., and Joosten, M. H. (2007), An NB-LRR protein required for HR signalling mediated by both extra- and intracellular resistance proteins. Plant J. 50(1): p. 14-28.

Garner, C. M., Kim, S. H., Spears, B. J., and Gassmann, W. (2016), Express yourself: Transcriptional regulation of plant innate immunity. Semin Cell Dev Biol. 56: p. 150-162.

Han, X.-Y., Li, P.-X., Zou, L.-J., Tan, W.-r., Zheng, T., Zhang, D.-W., and Lin, H.-H. (2016), GOLDEN2-LIKE transcription factors coordinate the tolerance to Cucumber mosaic virus in Arabidopsis. Biochemical and Biophysical Research Communications. 477(4): p. 626-632.

Haseloff, J., Siemering, K. R., Prasher, D. C., and Hodge, S. (1997), Removal of a cryptic intron and subcellular localization of green fluorescent protein are required to mark transgenic Arabidopsis plants brightly. Proc Natl Acad Sci U S A. 94(6): p. 2122-7.

Heo, L., Park, H., and Seok, C. (2013), GalaxyRefine: Protein structure refinement driven by side-chain repacking. Nucleic acids research. 41: W384-W388. ppublish.

Hoser, R., Lichocka, M., Zurczak, M., Hennig, J., and Krzymowska, M. (2014), Emerging role of SGT1 as a regulator of NB-LRR-receptor nucleocytoplasmic partitioning. Plant Signal Behav. 9(4).

Jones, J. D. G., Vance, R. E., and Dangl, J. L. (2016), Intracellular innate immune surveillance devices in plants and animals. Science. 354(6316): aaf6395.

Kalinina, N. O., Makarova, S., Makhotenko, A., Love, A. J., and Taliansky, M. (2018), The Multiple Functions of the Nucleolus in Plant Development, Disease and Stress Responses. Frontiers in plant science. 9: p. 132-132.

Karimi, M., Inzé, D., and Depicker, A. (2002), GATEWAY vectors for Agrobacterium-mediated plant transformation. Trends Plant Sci. 7(5): p. 193-5.

Kelley, L. A. and Sternberg, M. J. (2009), Protein structure prediction on the Web: a case study using the Phyre server. Nat Protoc. 4(3): p. 363-71.

Kim, H., Tang, G. Q., Patel, S. S., and Ha, T. (2012), Opening-closing dynamics of the mitochondrial transcription pre-initiation complex. Nucleic Acids Res. 40(1): p. 371-80.

Lange, M., Yellina, A. L., Orashakova, S., and Becker, A. (2013), Virus-induced gene silencing (VIGS) in plants: an overview of target species and the virus-derived vector systems. Methods Mol Biol. 975: p. 1-14.

Leipe, D. D., Koonin, E. V., and Aravind, L. (2004), STAND, a class of P-loop NTPases including animal and plant regulators of programmed cell death: Multiple, complex domain architectures, unusual phyletic patterns, and evolution by horizontal gene transfer. Journal Of Molecular Biology. 343(1): p. 1-28. 
Letunic, I. and Bork, P. (2018), 20 years of the SMART protein domain annotation resource. Nucleic Acids Res. 46(D1): p. D493-d496.

Liu, X., Bushnell, D. A., Wang, D., Calero, G., and Kornberg, R. D. (2010), Structure of an RNA Polymerase II-TFIIB Complex and the Transcription Initiation Mechanism. Science. 327(5962): p. 206.

Liu, Y., Schiff, M., and Dinesh-Kumar, S. P. (2002), Virus-induced gene silencing in tomato. The Plant journal : for cell and molecular biology. 31(6): p. 777-786. ppublish.

LLC, S. (2010), The PyMOL Molecular Graphics System, Version 1.3r1.

Lu, R., Malcuit, I., Moffett, P., Ruiz, M. T., Peart, J., Wu, A.-J., Rathjen, J. P., Bendahmane, A., Day, L., and Baulcombe, D. C. (2003), High throughput virus-induced gene silencing implicates heat shock protein 90 in plant disease resistance. The EMBO journal. 22(21): p. 5690-5699.

MacAlpine, H. K., Gordân, R., Powell, S. K., Hartemink, A. J., and MacAlpine, D. M. (2010), Drosophila ORC localizes to open chromatin and marks sites of cohesin complex loading. Genome research. 20(2): p. 201-211.

Maekawa, T., Kufer, T. A., and Schulze-Lefert, P. (2011a), NLR functions in plant and animal immune systems: So far and yet so close. Nature Immunology. 12(9): p. 818-826.

Maekawa, T., Cheng, W., Spiridon, L. N., Töller, A., Lukasik, E., Saijo, Y., Liu, P., Shen, Q.-H., Micluta, M. A., Somssich, I. E., Takken, F. L. W., Petrescu, A.-J., Chai, J., and Schulze-Lefert, P. (2011b), Coiled-Coil Domain-Dependent Homodimerization of Intracellular Barley Immune Receptors Defines a Minimal Functional Module for Triggering Cell Death. Cell Host \& Microbe. 9(3): p. 187-199.

Mäki-Valkama, T., Valkonen, J. P., Kreuze, J. F., and Pehu, E. (2000), Transgenic resistance to PVY(O) associated with post-transcriptional silencing of $\mathrm{P} 1$ transgene is overcome by $\mathrm{PVY}(\mathrm{N})$ strains that carry highly homologous $\mathrm{P} 1$ sequences and recover transgene expression at infection. Mol Plant Microbe Interact. 13(4): p. 366-73.

Marmorstein, R. and Berger, S. L. (2001), Structure and function of bromodomains in chromatin-regulating complexes. Gene. 272(1): p. 1-9.

Mujtaba, S., He, Y., Zeng, L., Farooq, A., Carlson, J. E., Ott, M., Verdin, E., and Zhou, M. M. (2002), Structural basis of lysine-acetylated HIV-1 Tat recognition by PCAF bromodomain. Mol Cell. 9(3): p. 575-86.

Murmu, J., Wilton, M., Allard, G., Pandeya, R., Desveaux, D., Singh, J., and Subramaniam, R. (2014), Arabidopsis GOLDEN2-LIKE (GLK) transcription factors activate jasmonic acid (JA)-dependent disease susceptibility to the biotrophic pathogen Hyaloperonospora arabidopsidis, as well as JA-independent plant immunity against the necrotrophic pathogen Botrytis cinerea. Mol Plant Pathol. 15(2): p. 174-84.

Navarro, L., Zipfel, C., Rowland, O., Keller, I., Robatzek, S., Boller, T., and Jones, J. D. (2004), The transcriptional innate immune response to flg22. Interplay and overlap with Avr gene-dependent defense responses and bacterial pathogenesis. Plant Physiol. 135(2): p. 1113-28.

Padmanabhan, M. S., Ma, S., Burch-Smith, T. M., Czymmek, K., Huijser, P., and Dinesh-Kumar, S. P. (2013), Novel positive regulatory role for the SPL6 transcription factor in the N TIR-NB-LRR receptor-mediated plant innate immunity. PLoS pathogens. 9(3): e1003235. ppublish.

Park, M. R., Park, S. H., Cho, S. Y., and Kim, K. H. (2009), Nicotiana benthamiana protein, NbPCIP1, interacting with Potato virus $X$ coat protein plays a role as susceptible factor for viral infection. Virology. 386(2): p. 257-69.

Peart, J. R., Lu, R., Sadanandom, A., Malcuit, I., Moffett, P., Brice, D. C., Schauser, L., Jaggard, D. A. W., Xiao, S., Coleman, M. J., Dow, M., Jones, J. D. G., Shirasu, K., and Baulcombe, D. C. (2002), Ubiquitin ligase-associated protein SGT1 is required for host and nonhost disease resistance in plants. Proceedings of the National Academy of Sciences of the United States of America. 99(16): p. 10865-10869. 
Remus, D., Beall, E. L., and Botchan, M. R. (2004), DNA topology, not DNA sequence, is a critical determinant for Drosophila ORC-DNA binding. The EMBO journal. 23(4): p. 897-907.

Roberts, M., Tang, S., Stallmann, A., Dangl, J. L., and Bonardi, V. (2013), Genetic requirements for signaling from an autoactive plant NB-LRR intracellular innate immune receptor. PLoS genetics. 9(4): e1003465-e1003465.

Sáez-Vásquez, J. and Delseny, M. (2019), Ribosome Biogenesis in Plants: From Functional 45S Ribosomal DNA Organization to Ribosome Assembly Factors. The Plant cell. 31(9): p. 1945-1967.

Savitch, L. V., Subramaniam, R., Allard, G. C., and Singh, J. (2007), The GLK1 'regulon' encodes disease defense related proteins and confers resistance to Fusarium graminearum in Arabidopsis. Biochemical and Biophysical Research Communications. 359(2): p. 234-238.

Schmittgen, T. D. and Livak, K. J. (2008), Analyzing real-time PCR data by the comparative C(T) method. Nat Protoc. 3(6): p. 1101-8.

Shen, Q.-H., Saijo, Y., Mauch, S., Biskup, C., Bieri, S., Keller, B., Seki, H., Ülker, B., Somssich, I. E., and Schulze-Lefert, P. (2007), Nuclear Activity of MLA Immune Receptors Links Isolate-Specific and Basal Disease-Resistance Responses. Science. 315(5815): p. 1098.

Slootweg, E., Roosien, J., Spiridon, L. N., Petrescu, A. J., Tameling, W., Joosten, M., Pomp, R., van Schaik, C., Dees, R., Borst, J. W., Smant, G., Schots, A., Bakker, J., and Goverse, A. (2010), Nucleocytoplasmic distribution is required for activation of resistance by the potato NB-LRR receptor $R \times 1$ and is balanced by its functional domains. Plant Cell. 22(12): p. 4195-215.

Staskawicz, B. J., Mudgett, M. B., Dangl, J. L., and Galan, J. E. (2001), Common and contrasting themes of plant and animal diseases. Science. 292(5525): p. 2285-9.

Takken, F. L. W., Albrecht, M., and Tameling, W. I. L. (2006), Resistance proteins: molecular switches of plant defence. Curr Opin Plant Biol. 9(4): p. 383-90.

Takken, F. L. W. and Tameling, W. I. L. (2009), To nibble at plant resistance proteins. Science (New York, N.Y.) 324(5928): p. 744-746. ppublish.

Tameling, W. I. L., Nooijen, C., Ludwig, N., Boter, M., Slootweg, E., Goverse, A., Shirasu, K., and Joosten, M. (2010), RanGAP2 Mediates nucleocytoplasmic partitioning of the NB-LRR immune receptor Rx in the solanaceae. Thereby dictating Rx function. Plant Cell. 22.

Tameling, W. I. L., Vossen, J. H., Albrecht, M., Lengauer, T., Berden, J. A., Haring, M. A., Cornelissen, B. J. C., and Takken, F. L. W. (2006), Mutations in the NB-ARC Domain of I-2 That Impair ATP Hydrolysis Cause Autoactivation. Plant Physiol. 140(4): p. 1233-1245.

Tang, G.-Q., Deshpande, A. P., and Patel, S. S. (2011), Transcription factor-dependent DNA bending governs promoter recognition by the mitochondrial RNA polymerase. The Journal of biological chemistry. 286(44): p. 38805-38813.

Townsend, P. D., Dixon, C. H., Slootweg, E. J., Sukarta, O. C. A., Yang, A. W. H., Hughes, T. R., Sharples, G. J., Pålsson, L. O., Takken, F. L. W., Goverse, A., and Cann, M. J. (2018), The intracellular immune receptor Rx1 regulates the DNA-binding activity of a Golden2-like transcription factor. J Biol Chem. 293(9): p. 3218-3233.

Tranier, S., Bouthors, A. T., Maveyraud, L., Guillet, V., Sougakoff, W., and Samama, J. P. (2000), The high resolution crystal structure for class A beta-lactamase PER-1 reveals the bases for its increase in breadth of activity. J Biol Chem. 275(36): p. 28075-82.

Tsuda, K., Sato, M., Glazebrook, J., Cohen, J. D., and Katagiri, F. (2008), Interplay between MAMP-triggered and SA-mediated defense responses. Plant J. 53(5): p. 763-75.

Wang, J., Hu, M., Wang, J., Qi, J., Han, Z., Wang, G., Qi, Y., Wang, H. W., Zhou, J. M., and Chai, J. (2019a), Reconstitution and structure of a plant NLR resistosome conferring immunity. Science. 364(6435). 
Wang, J., Wang, J., Hu, M., Wu, S., Qi, J., Wang, G., Han, Z., Qi, Y., Gao, N., Wang, H. W., Zhou, J. M., and Chai, J. (2019b), Ligand-triggered allosteric ADP release primes a plant NLR complex. Science. 364(6435).

Wang, Y., Hu, Q., Wu, Z., Wang, H., Han, S., Jin, Y., Zhou, J., Zhang, Z., Jiang, J., Shen, Y., Shi, H., and Yang, W. (2017), HISTONE DEACETYLASE 6 represses pathogen defence responses in Arabidopsis thaliana. Plant Cell Environ. 40(12): p. 2972-2986.

Wen, H., Li, Y., Xi, Y., Jiang, S., Stratton, S., Peng, D., Tanaka, K., Ren, Y., Xia, Z., Wu, J., Li, B., Barton, M. C., Li, W., Li, H., and Shi, X. (2014), ZMYND11 links histone H3.3K36me3 to transcription elongation and tumour suppression. Nature. 508(7495): p. 263-8.

Williams, S. J., Sornaraj, P., deCourcy-Ireland, E., Menz, R. I., Kobe, B., Ellis, J. G., Dodds, P. N., and Anderson, P. A. (2011), An autoactive mutant of the $M$ flax rust resistance protein has a preference for binding ATP, whereas wild-type M protein binds ADP. Mol Plant Microbe Interact. 24(8): p. 897-906.

Winn, M. D., Ballard, C. C., Cowtan, K. D., Dodson, E. J., Emsley, P., Evans, P. R., Keegan, R. M., Krissinel, E. B., Leslie, A. G. W., McCoy, A., McNicholas, S. J., Murshudov, G. N., Pannu, N. S., Potterton, E. A., Powell, H. R., Read, R. J., Vagin, A., and Wilson, K. S. (2011), Overview of the CCP4 suite and current developments. Acta crystallographica. Section D, Biological crystallography. 67(Pt 4): p. 235-242.

Wirthmueller, L., Zhang, Y., Jones, J. D. G., and Parker, J. E. (2007), Nuclear Accumulation of the Arabidopsis Immune Receptor RPS4 Is Necessary for Triggering EDS1-Dependent Defense. Current Biology. 17(23): p. 2023-2029.

Zhu, Z., Xu, F., Zhang, Y., Cheng, Y. T., Wiermer, M., Li, X., and Zhang, Y. (2010), Arabidopsis resistance protein SNC1 activates immune responses through association with a transcriptional corepressor. Proceedings of the National Academy of Sciences. 107(31): p. 13960-13965. 


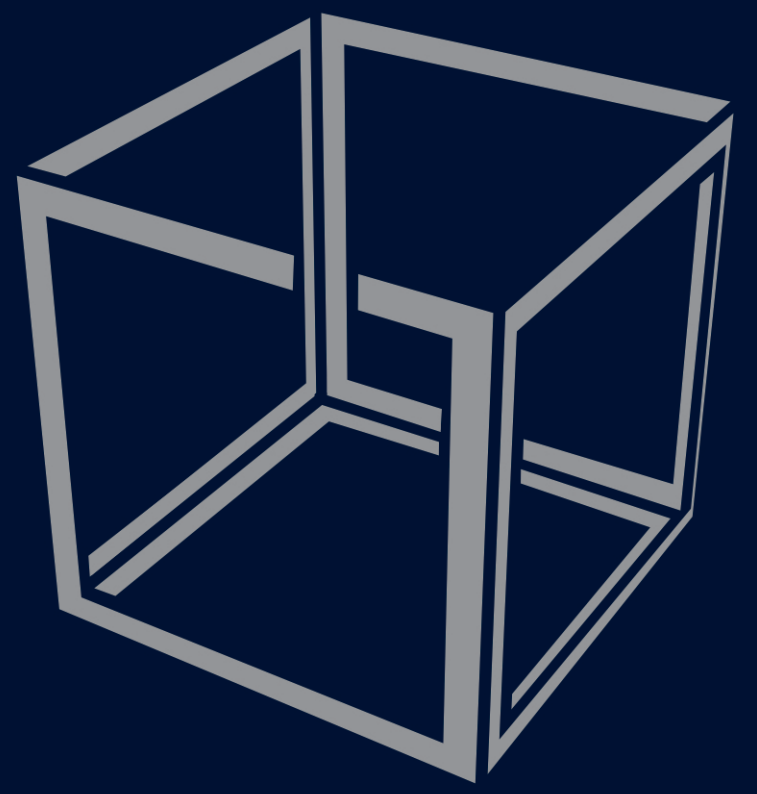

Octavina C. A Sukarta ${ }^{1}$

Qi Zheng ${ }^{1}$

Erik J. Slootweg ${ }^{1}$

Melanie Mendel ${ }^{1}$

Mark Mekken ${ }^{1}$

Vera Putker ${ }^{1}$

Rikus Pomp ${ }^{1}$, Jan Roosien ${ }^{1}$, Sjef Boeren ${ }^{2}$

Martin J Cann ${ }^{3}$

Geert Smant $^{1}$, Aska Goverse ${ }^{1}$

${ }^{1}$ Laboratory of Nematology, Wageningen University \& Research, Wageningen, the Netherlands.

${ }^{2}$ Laboratory of Biochemistry, Wageningen University \& Research, Wageningen, The Netherlands

${ }^{3}$ Department of Biosciences, Biophysical Sciences Institute, Durham University, Durham, United Kingdom 


\section{Chapter}

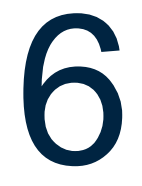

The Glycine-Rich RNA-Binding Protein 7

(GRP7) is a Novel Component belonging to the

Resistance Complexes of the Potato

CC-NB-LRRs Rx1 and Gpa2 


\section{ABSTRACT}

The activity of intracellular plant Nucleotide-Binding Leucine-Rich Repeat (NB-LRR) immune receptors is fine-tuned by an intricate interplay between the receptors and their interacting partners. Identifying the pool of host-proteins associated with NB-LRRs is, therefore, crucial to advance our understanding of how these receptors function. In a Co-Immunoprecipitation/Mass-Spectrometry screening, we discovered the Nicotiana benthamiana homolog of the Glycine-Rich RNA-Binding Protein 7 (NbGRP7) as a novel interactor of the potato CC-NB-LRR Gpa2, which confers resistance against the potato cyst nematode Globodera pallida. We show that NbGRP7 can also form a complex with the homologous $\mathrm{Rx} 1$ receptor, which recognizes the coat protein of Potato Virus $\mathrm{X}$ (PVX). In additional immunoprecipitation studies, we demonstrate that NbGRP7 binds to Rx1 via the Rx1-CC domain at an interface distinct from the cytoplasmic co-factor RanGTPase Activating Protein 2 (RanGAP2). Using a combination of heterologous overexpression and silencing assays in $N$. benthamiana, we further show that NbGRP7 acts as a positive regulator of extreme resistance by Rx1 and elicitor-induced cell death by Gpa2. Moreover, we specify the role of NbGRP7 in Rx1 immunity by mutating a conserved Arginine residue in the RNA-binding domain. Contrary to the wildtype protein, these mutant derivatives are compromised in their ability to potentiate the extreme resistance response of Rx1. Based on subsequent expression analyses, we speculate that NbGRP7 regulates the steady-state levels of Rx1 transcripts pre- and post-activation. Our research implicates novel NbGRP7-related cellular activities, including its RNA-chaperone functions, in defence by intracellular plant NB-LRRs

\section{INTRODUCTION}

Innate immunity in plants is orchestrated by a consortium of receptor proteins. These receptors occupy various compartments of the cell where they act as molecular sentinels, detecting and responding to a pathogenic intrusion (Bezerra-Neto et al. 2020). At the plant membrane, Pattern Recognition Receptors (PRRs) survey the extracellular space for conserved microbial features (so called Pathogen Associated Molecular Patterns or PAMPs) or collateral damage inflicted on the host (termed Danger Associated Molecular Pattern or DAMPs) (Zipfel 2014, Jones et al. 2006). Detection of PAMPs/DAMPs triggers the plant basal defence response that is effective against a broad range 
of assailants in a process coined as PAMP-triggered immunity (PTI). Pathogens, however, can adapt by evolving an arsenal of virulence-promoting effector molecules that are introduced into the host environment to interfere with cellular processes and/or disarm PTI (Jones et al. 2006). In this evolutionary interplay, plants have developed Resistance proteins ( $R$ proteins) that mostly reside intracellularly and recognize specific effectors or their activity. The suit of responses that follows can effectively limit further ingress by the pathogen. Referred to as Effector Triggered Immunity (ETI), this process is often hallmarked by a visible sign of programmed cell death called the Hypersensitive Response (HR) (Balint-Kurti 2019).

The majority of R proteins annotated to date belongs to the family of Nucleotide-Binding Leucine-Rich Repeat (NB-LRR) receptors (Bezerra-Neto et al. 2020). Classical NB-LRRs modules have a tri-domain architecture consisting of a central Nucleotide-Binding APAF-1, R-Protein and CED4 NB-ARC) region framed by a C-terminal Leucine Rich Repeat (LRR) and N-terminal domains. In plants, the $N$-terminus is typically a coiled-coil (CC) or Toll/Interleukin Receptor-like (TIR) domain (van der Biezen et al. 1998, Jones et al. 2016). The concerted activities and functions of these subdomains enable NB-LRRs to act as a molecular switch. The NB-ARC core forms a deep groove with the capacity to bind ADP/ATP and depending on the nature of bound nucleotide, the pocket can adopt different conformations. This in turn impacts the folding of the adjacent LRR and CC/TIR domains. These distal $\mathrm{C}$ and $\mathrm{N}$-terminal regions partake in intramolecular regulation, pathogen perception and/or downstream signalling (Jones et al. 2016, Bezerra-Neto et al. 2020). The switch function of an NB-LRR receptor is activated by recognition of strain-specific effectors, prompting an exchange of ADP for ATP. This triggers a series of conformational changes and initiates downstream signalling. However, the sequence of events, thereafter, leading to immunity remains largely unresolved. Thus, there is an increasing interest in the field to uncover components driving NB-LRR mediated immunity and how they are fine-tuned in the cell.

The modular architecture of NB-LRR receptors suggests that they are likely to be part of larger multimeric complexes. Indeed, plant NB-LRRs have been shown to engage in various interactions within the host proteome (Sacco et al. 2007). The common view is that these interactions modulate immunity by regulating recognition, defence signalling, and/or directly affecting the stability, localization or activity of the receptor (Sukarta et al. 2016). Here, we refer to the co-operative pool of NB-LRR-associated factors as the interactome, which may be composed of multiple host proteins. 
In a vast majority of cases, binding to these co-factors is mediated by the receptor's $N$-terminal end (Sun et al. 2020). This is consistent with reports showing that the CC/TIR domains of a few NB-LRR systems can multimerize, which is thought to increase the surface area available for scaffolding interacting partners (Bentham et al. 2018). The nature of proteins known to bind to an NB-LRR receptor varies, ranging from well-established molecular-chaperones (e.g. SGT1 and RAR1) to transcription factors (Tameling et al. 2007, de la Fuente van Bentem et al. 2005, Leister et al. 2005, Bieri et al. 2004). These proteins may exist as pre-formed complexes with the receptor or are recruited de novo during an active defence response, suggesting that the conformation of an NB-LRR domain may be vital for effective docking of co-factors (Chang et al. 2013). For example, the TIR-NB-LRR receptor $N$ associates with the transcription factor Squoamosa Promoter Binding Protein-Like 6 (SPL6) in the nucleus only upon activation by its cognate effector, namely the helicase domain (p50) of the Tobacco Mosaic Virus (TMV) replicase protein (Padmanabhan et al. 2013). Conversely, the CC domain of barley MLA10 recruits Myb and WRKY1/2 transcription factors for defence (Sarris et al. 2016, Shen et al. 2007, Chang et al. 2013). For a more extensive catalogue of NB-LRR interacting partners, we direct our readers to a recent review in (Sun et al. 2020). Thus, it has become increasingly apparent that aside from a few exceptions, the identity and functions of $\mathrm{N}$-terminal interacting partners remain elusive.

The potato (Solanum tuberosum) CC-NB-LRR RX1 confers defence against Potato Virus X (PVX) upon recognition of the viral coat protein (CP) (Bendahmane et al. 1995). This triggers an Extreme Resistance (ER) response, which is robust and can effectively restrict viral spread without the need to elicit cell death (Bendahmane et al. 1995, Bhattacharjee et al. 2009). In the genome of potato, the $\mathrm{R} \times 1$ locus locates to a small gene cluster on chromosome XII together with at least three other homologs. The best characterized of these is Gpa2, which encodes a protein with high sequence similarity to $\mathrm{Rx} 1$ (88\% identity at the protein level) (van der Vossen et al. 2000). Unlike Rx1, however, Gpa2 detects specific effector variants produced in the dorsal oesophageal gland of the potato cyst nematode Globodera pallida termed GpRBP-1 (Arntzen et al. 1993, van der Vossen et al. 2000). Previously, structure-functional studies have shown that exchanging the CC-NB-ARC of Gpa2 with the complementary portion from $\mathrm{Rx} 1$ can still reconstitute a functional receptor unit, assuming that compatibility with essential co-factors at the ARC-LRR interface is maintained (Slootweg et al. 2017). This finding suggests that $R \times 1$ and Gpa2 may converge in their use of co-factors and signalling 
requirements despite the bifurcation of their recognition specificity. In line with this view, the CC domains of Rx1 and Gpa2 are highly alike (differing in only 6 amino acid positions) and can both interact with the RanGTPase Activating Protein 2 (RanGAP2) (Sacco et al. 2007, Tameling et al. 2007). The cellular function of RanGAP2 is to mediate nucleocytplasmic trafficking of macromolecules (Pay et al. 2002). In Rx1, the interaction with RanGAP2 retains a subpopulation of the receptor to the cytoplasm, which is required to recognize PVX and prompt a complete defence response (Slootweg et al. 2010, Tameling et al. 2010). The role of RanGAP2 in Gpa2-mediated defence is less clear. However, artificially tethering RanGAP2 to GpRBP-1 enhances the cell death response, hinting that RanGAP2 may contribute to effector recognition (Sacco et al. 2009). The ability of Rx1 and Gpa2-CCs to recruit RanGAP2 is consistent with a role of this domain as a molecular scaffold. This is further supported by the finding that $\mathrm{R} \times 1$ can co-opt and modulate the DNA-binding activity of the Golden2-like transcription factor (GLK1) via its CC domain (Townsend et al. 2018). However, these receptors likely associate with a wider pool of host proteins at various stages of their activity. Thus, the components regulating Gpa2 and Rx1 immunities remain poorly defined, which hampers our understanding of how they function.

Here, we performed a Co-Immunoprecipitation/Mass-Spectrometry (Co-IP/MS) study in Nicotiana benthamiana to identify novel proteins associated with Gpa2. Given the role of the CC as a molecular scaffold, the CC domain of Gpa2 was used as bait. From our screening, Glycine-Rich RNA-Binding Protein 7 (NbGRP7) emerged as a candidate interactor. Similar to other RNA-Binding Proteins (RBPS), NbGRP7 harbours a canonical RNA-binding domain but is further characterized by the presence of an additional glycine-rich region at the C-terminus (Ciuzan et al. 2015). Plant GRP7s are most extensively studied for their role in responses to abiotic stresses, particularly in cold tolerance (Miura et al. 2013). However, existing works pinpoint that they may also function in plant-biotic interactions (Fu et al. 2007, Lee et al. 2012). For instance, the RNA- binding function of Arabidopsis thaliana GRP7 (AtGRP7) is ADP-ribosylated by the Pseudomonas syringae effector HopU1 to promote virulence of the bacteria (Fu et al. 2007). A more recent study implicates that AtGRP7 contributes to basal defence against a broader group of pathogens, TMV (Lee et al. 2012). As per our current understanding, however, the function of a plant GRPs in ETI has not been described. Here, we present genetic and molecular evidence that NbGRP7 can complex with Gpa2 and its close homolog Rx1 via the receptor CC domain. Based on functional analyses, we show that NbGRP7 can 
potentiate the defence responses of these immune receptors. Impairing the RNA-binding capacity of NbGRP7 by substituting a conserved Arginine residue compromises its pro-immune activity in $\mathrm{R} \times 1$ defence, suggesting that this may be an important feature underlying the function of NbGRP7 in NB-LRR-mediated immunity. In addition, we show that NbGRP7 regulates the steady-state transcript and protein levels of $\mathrm{R} \times 1$ before and during an active defence response. This most likely occurs indirectly, as preliminary data suggests NbGRP7 does not associate with $\mathrm{R} \times 1$ transcripts in vitro. Our findings are further discussed in the context of other interactors involved in NB-LRR functioning, as well as their role in the post-transcriptional regulation of NB-LRRs.

\section{RESULTS}

\section{Identification and isolation of NbGRP7 as a Gpa2-interacting protein}

To screen for putative proteins associated with the Gpa2-CC domain, we adopted a proteomics approach using Co-IP/MS analysis as described in previous studies identifying RanGAP2 as a bona fide interactor of the Rx1-CC domain (Tameling et al. 2007, Sacco et al. 2007). To that end, constructs for the expression of a C-terminally GFP-tagged Gpa2-CC domain or free GFP (negative control) were generated under the control of the Cauliflower Mosaic Virus 35S (CaMV35S) promoter for use as baits. Transient expression of the fusion proteins in planta was achieved by Agrobacterium-mediated infiltration in N. benthamiana. To prevent artefacts associated with the overexpression of recombinant proteins, infiltrated leaf samples were harvested at 2 days post infiltration (dpi). Given the significance of localization to receptor function, we performed an additional cellular fractionation step prior to purification, which allowed us to isolate candidate interactors specific to either the cytoplasm or nucleus (Slootweg et al. 2010). As anticipated, MS analysis of the eluted fractions showed an overrepresentation of the Gpa2-CC bait in both cellular extracts. We also co-purified RanGAP2 exclusively in the cytoplasmic fraction of the pull-down. These findings collectively support that the technical approach and stringency used for the data analysis were sound. Interestingly, we found a protein that co-precipitated consistently with the Gpa2-CC nuclear fraction and has significant peptide hits matching to a GRP7 homolog in the N. benthamiana EST database (Supplementals Fig. S1). Given the specificity and reproducibility of the interaction observed, GRP7 was prioritized for further studies. 
To facilitate the functional analysis of GRP7- Gpa2 interaction, we isolated the predicted cDNA of the N. benthamiana GRP7 homolog (henceforth, denoted as NbGRP7) based on the N. benthamiana draft genome (University of Sydney and Solgenomics) and existing sequence of the Arabidopsis thaliana GRP7 orthologoue. Sequence analysis of the isolated NbGRP7 transcript shows the fragment to be $501 \mathrm{bp}$ long, encoding a protein of 167 amino acids with an approximated weight of $\operatorname{sim} 16.9$ $\mathrm{kDa}$. By performing a protein alignmen with additional homologs, we found that NbGRP7 exhibits strong similarity with other plant-derived GRPs (Supplementals Fig. S2). NbGRP7 shares the highest sequence identity $(73-75 \%$ at the amino acid level) to the Solanum tuberosum GRP7 variants (XP_006365106.1 and XP_006365107.1). The N-terminus of NbGRP7 constitutes the most conserved region, wherein the canonical RNA Recognition Motif (RRM) resides. Positioned within this region are also the Ribonucleoprotein motifs (RNP1 and RNP2) and Arginine residues required for RNA binding (summarized schematically in Fig. 1A). The variable and highly disordered glycine-rich region accounts for the remaining $\mathrm{C}$-terminal half of the protein, which is further interspersed with aromatic amino acids.

Consistent with the high degree of homology observed in the protein alignment, we demonstrated that a $4 \times$ Myc.GFP construct of NbGRP7 has a similar distribution pattern in the cell as reported for AtGRP7 (Kim et al. 2008). More specifically, confocal imaging shows $4 \times$ Myc.GFP-NbGRP7 to locate in both the cytoplasm and nucleus (Fig. 1B). In the latter case, a non-homogenous distribution of the nucleoplasm was observed with GFP signals accumulating in subnuclear bodies. These cellular structures are typically associated with RNA processing, which coincides with the expected function of a GRP7 homolog (Spector et al. 2011). The nuclear localization of $4 \times$ Myc.GFP-NbGRP7 is also in agreement with our Co-IP/MS data. Notably, however, while NbGRP7 was detected exclusively in the nuclear fraction of Gpa2-CC, confocal microscopy shows that $4 \times$ Myc.GFP-NbGRP7 also localizes in the cytoplasm. On the one hand, the observed cytoplasmic distribution may indicate a subpopulation of cleaved-off GFP, as is visible in several immunoblots performed in this study. Alternatively, interaction between Gpa2-CC and endogenous NbGRP7 in the cytoplasm may not meet the threshold for detection by Co-IP/MS due to lower abundance of NbGRP7 in this compartment. This may be overcome in the setting of an overexpression assay, which allows a higher accumulation of NbGRP7 in the cytoplasm. 


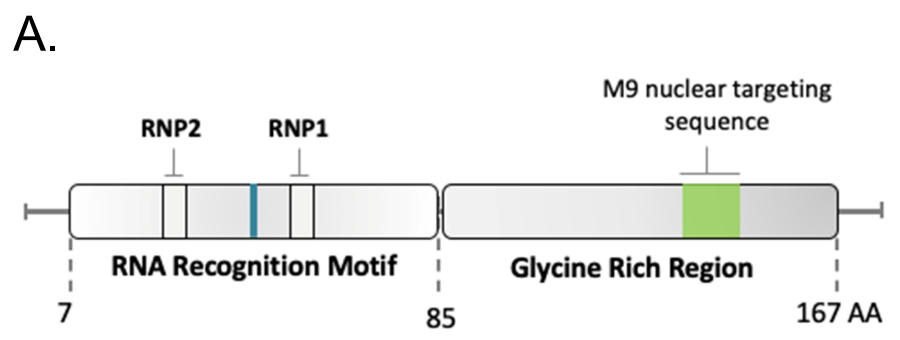

B.

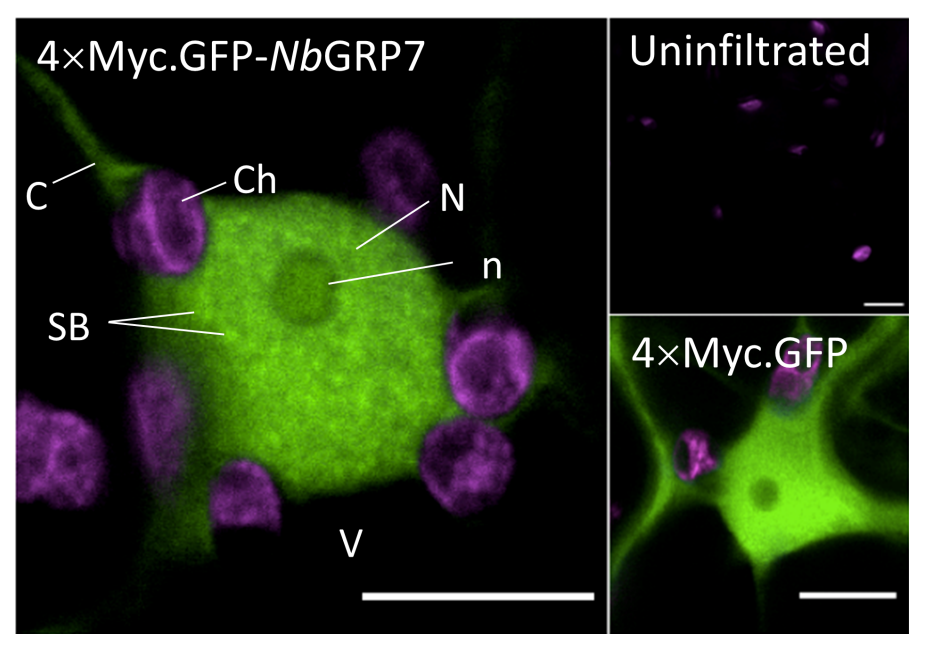

Fig. 1: Identification of a NbGRP7 homolog in N. benthamiana.

A) Schematic diagram representing the full-length NbGRP7 homolog isolated from N. benthamiana cDNA. The conserved Arginine residue required for RNA binding is highlighted in blue. RNP1 = ribonucleoprotein motif 1; RNP2 = ribonucleoprotein motif 2. B) Representative confocal images of detailed nuclei of uninfiltrated $N$. benthamiana epidermal cells or those expressing $4 \times$ Myc.GFP-NbGRP7 or 4 X Myc.GFP. Images were taken at $2 \mathrm{dpi}$ with the same magnification and settings. Scale bar represents width of $10 \mu \mathrm{m}$. Key: $\mathrm{N}=$ nucleus; $\mathrm{n}=$ nucleolus; $\mathrm{C}=$ cytoplasm; $\mathrm{Ch}=$ chloroplast; $\mathrm{v}=$ vacuole; $\mathrm{SB}=$ subnuclear bodies.

NbGRP7 interacts with full-length Gpa2 and Rx1 in planta

We next sought to investigate whether NbGRP7 can bind to the full-length Gpa2 immune receptor in planta. A Co-IP assay was therefore performed to assess a potential interaction with the complete Gpa2 protein. Although NbGRP7 was originally found as an interactor of the Gpa2-CC, we 
expanded our Co-IP study to include Rx1 given its close homology with Gpa2, particularly in the CC domain (95.7\% identity at the protein level). To that end, 4 X Myc.GFP-NbGRP7 was co-expressed transiently in combination with Haemagglutinin (HA)-tagged version of the full-length receptors as bait ( $4 \times$ HA-Gpa2 and Rx1-4XHA.GFP). Combinations involving the CC domain of Gpa2 (4XHA-Gpa2-CC) or 4 XHA-GFP were included as positive and negative controls, respectively. Infiltrated leaf materials were harvested at $2 \mathrm{dpi}$ and the proteins extracted were subjected to a Co-IP using the $\alpha$-HA magnetic beads system ( $\mu$ MACS). Immunoblotting with $\alpha$-Myc antibodies was used to determine whether 4 XMyc.GFP-NbGRP7 could pull down successfully. Western blotting of the eluates further demonstrated that $4 \times$ Myc.GFP-NbGRP7 can co-precipitate with both $4 \times$ HA-Gpa2 and Rx1-4XHA.GFP (Fig. 2A). Consistent with the Co-IP/MS screening, we also observed 4 X Myc.GFP-NbGRP7 to co-purify with 4 XHA-Gpa2-CC but not 4 XHA.GFP. Notably, $4 \times$ Myc.GFP-NbGRP7 was pulled-down with the Rx1 CC fragment. Taken together, our data show that a N. benthamiana homolog of the GRP7 protein can form a complex with the highly homologous Gpa2 and Rx1 receptors in planta.

\section{NbGRP7 interacts with $\mathrm{R} \times 1$ via the CC domain at a surface region distinct from RanGAP2}

As NbGRP7 interacts with full-length Gpa2 and Rx1, we next determined whether other receptor domains may contribute to complex formation in addition to the CC. To address this, we performed a pull-down assay to delineate the physical association of 4 XMyc.GFP-NbGRP7 with HA or GFP-tagged fusions of the Rx1 NB-ARC and LRR domains (GFP-R $\times 1$ NB-ARC and $4 \times H A-R \times 1$ LRR respectively). Interestingly, precipitating NbGRP7 with $\alpha-M y c$ beads exclusively co-purifies $4 \times \mathrm{HA}-\mathrm{R} \times 1 \mathrm{CC}$ but not the other receptor domains nor 4 X HA.GFP control (Fig. 2B). This suggests that the $\mathrm{CC}$ is required for the interaction with NbGRP7.

The Rx1-CC crystal structure reveals a relatively compact fold composed of a four-helix bundle (Hao et al. 2013). Targeted mutations at the protein surface show that different regions of the CC partake in the modulation of the immune response of Rx1 (Slootweg et al. 2018). Thus, discerning interacting regions on the CC can help pinpoint a specific role NbGRP7 in Rx1-mediated defence. To further localize the structural determinants involved in NbGRP7 binding, we used available surface mutants of the Rx1-CC domain as described in (Slootweg et al. 2018). Particularly, mutants aimed at 
locally disrupting hydrophobic surface residues in helixes 1 and 4 of the CC were tested (referred to as S1 and S4 mutations accordingly). The latter disrupts the hydrophobic patch essential for RanGAP2-binding while S1 mutations have reduced intramolecular binding to the NB-ARC and LRR domains. Constructs of the surface mutants in the context of the full-length immune receptor were included in the assay. When using the full-length and CC fragments as baits, we show that $4 \times$ Myc.GFP-NbGRP7 can co-precipitate with the mutant variants (CC and full-length) similar to the wildtype control (Fig. 2C1 and C2). While the immunoblot shows that Rx1 S1-4 XHA.GFP pulled down at a greater extent compared to the wild-type and $\mathrm{S} 4$ derivates (Fig. 2C2), this was not consistent between experimental repeats. These findings suggest that S1 and S4 surface regions of the CC are most likely not involved in complex formation with NbGRP7. Overall, we show that the $\mathrm{CC}$ domain constitutes the minimal binding platform needed for the interaction of Rx1 with NbGRP7, which involves distinct surface regions than those reported previously for intramolecular interactions with the NB-ARC-LRR domain or intermolecular interactions with RanGAP2. 


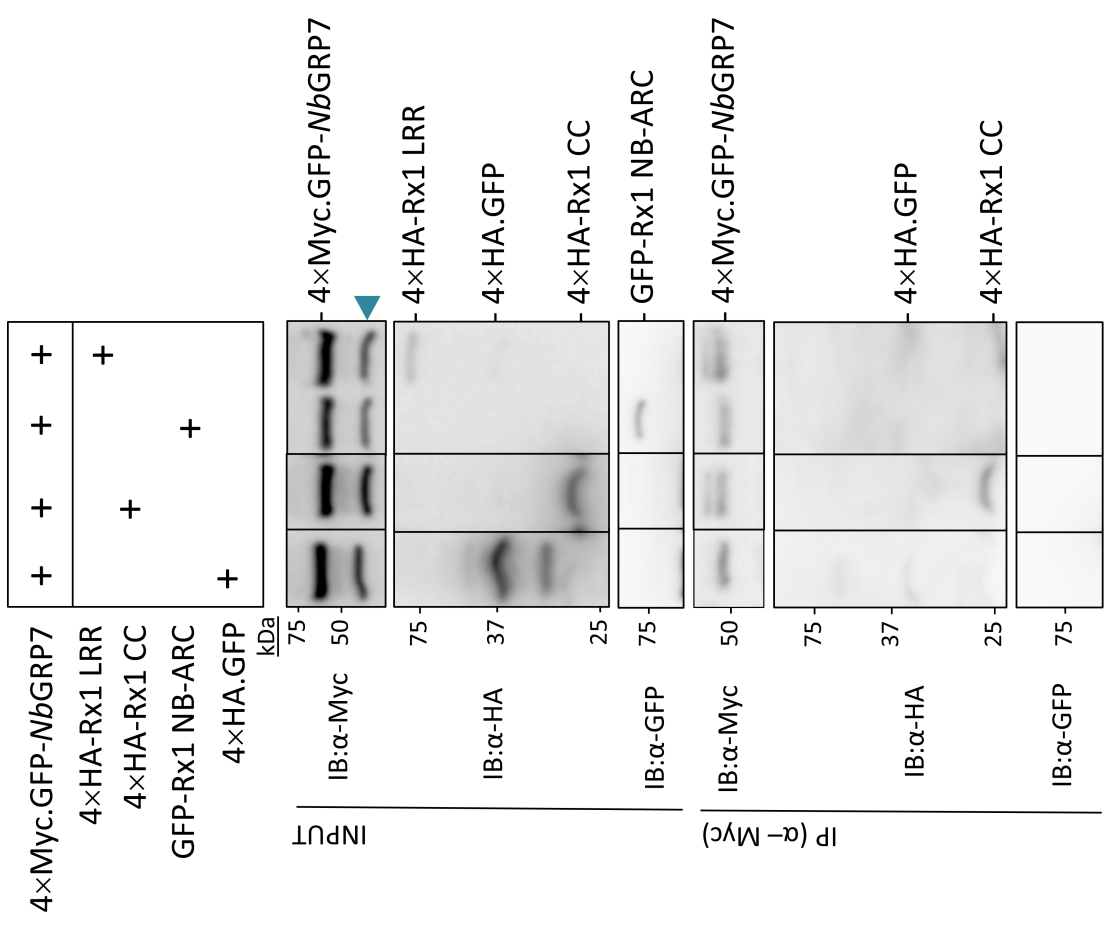

6

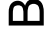

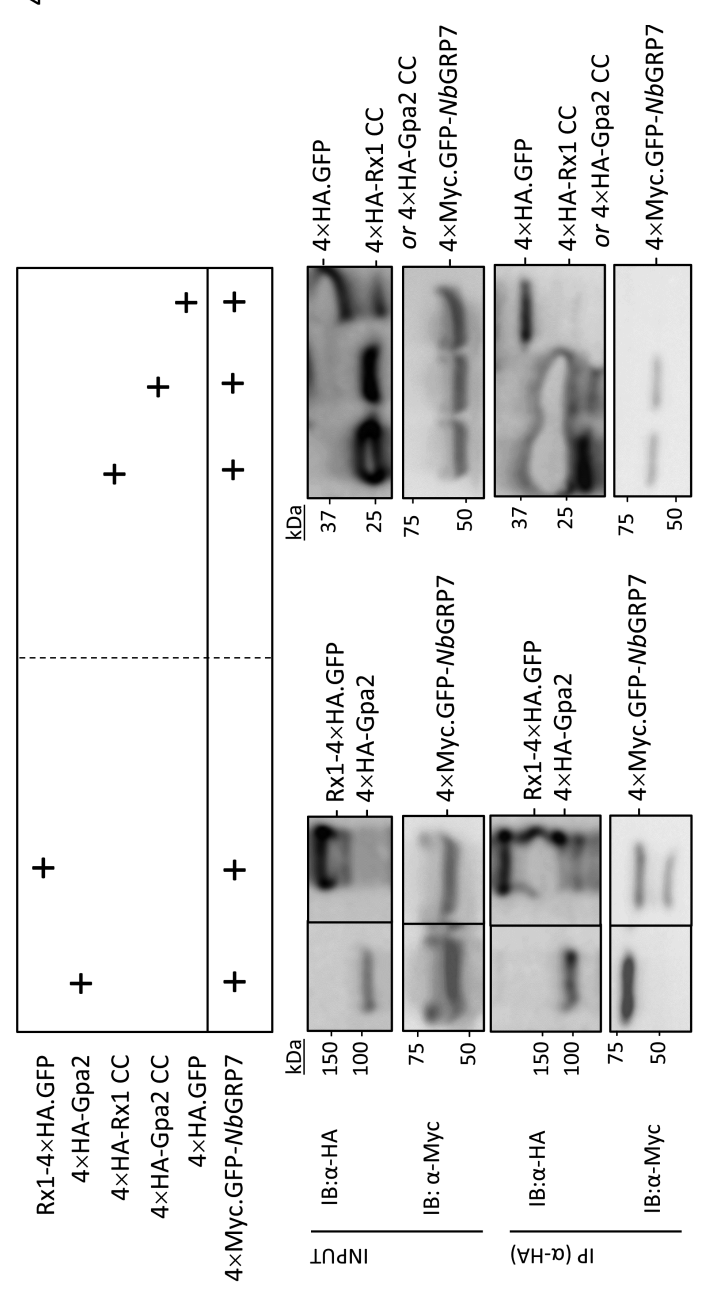




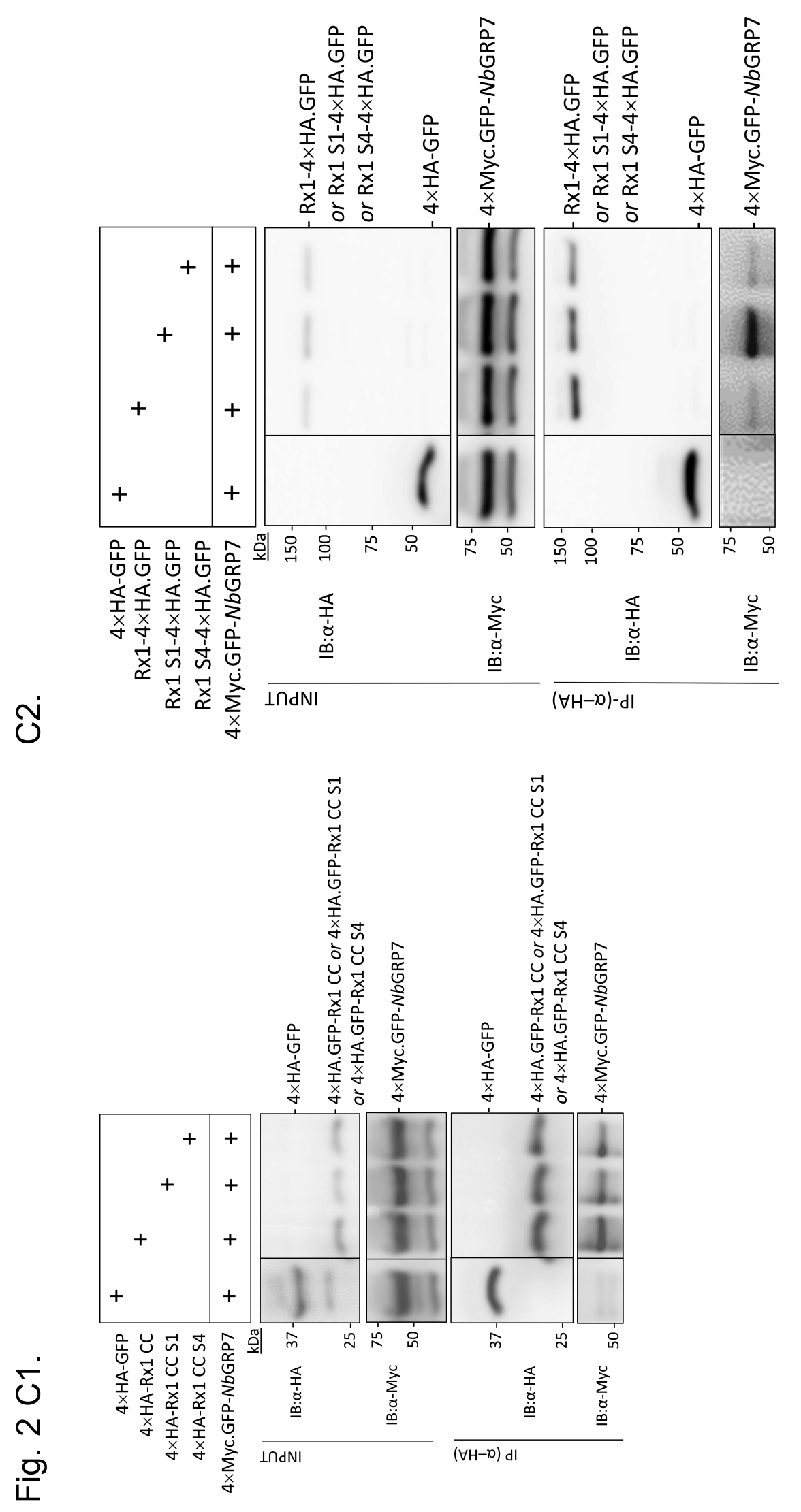


Fig. 2: NbGRP7 forms complexes with $\mathrm{Gpa} 2$ and $\mathrm{Rx} 1$ in planta via the CC domain. A) Immunoblots from Co-IP of NbGRP7 with full-length Gpa2/Rx1 or their CC domains. For the pull-downs, crude extracts of $\mathrm{N}$. benthamiana co-expressing the appropriate protein combinations were incubated with $\alpha$-HA magnetic beads ( $\mu$ MACS). Gpa2/Rx1 constructs or the negative $4 \times$ HA.GFP control were used as baits to co-purify $4 \times$ Myc. GFP-NbGRP7. Conversely, in B, NbGRP7 was used as bait for the Co-IP with the various Rx1 subdomains (LRR, NB-ARC and CC). C) Pull-down investigating the potential interaction of NbGRP7 as bait with Rx1 (CC) surface mutants (S1 or S4). In C1, interaction with the mutated CC domains were investigated whereas in $\mathrm{C} 2$, full-length $\mathrm{Rx} 1$ containing these mutations were used. Data shown for each IP is representative of at least two independent repeats. "+" indicates the presence of a construct in the infiltration combination used for immunoprecipitation. In these assays, $4 \times$ Myc.GFP of the NbGRP7 fusion construct tends to be cleaved-off under the extraction conditions used. This is indicated by the triangle as exemplified in B.

\section{NbGRP7 contributes to defences by Gpa2 and Rx1 in N. benthamiana}

Host proteins interacting with the $\mathrm{CC}$ domains of $\mathrm{Rx} 1$ and $\mathrm{Gpa} 2$ directly regulate immune responses by these receptors (Townsend et al. 2018, Sacco et al. 2007, Tameling et al. 2007). We thus questioned whether the physical interaction observed between NbGRP7 and Rx1/Gpa2 could likewise translate to a biological effect in defence. To directly assess the function of NbGRP7 in Rx1 and/or Gpa2 immunity, transient overexpression assays were done in N. benthamiana. Agrobacteria harbouring $4 \times$ Myc.GFP-NbGRP7 was co-infiltrated with those for the expression of Rx1/Gpa2 and their cognate elicitors, namely PVX-CP UK3 or GpRBP-1 respectively. Infiltrated spots were examined for the progression of cell death within 3-5 dpi by measurements of chlorophyll loss.

Remarkably, we observed that overexpressing NbGRP7 potentiates elicitor-induced cell HR by Gpa2 at 5 dpi (Fig. 3A). We observed that macroscopic signs of Gpa2-dependent cell death in agroinfiltrated lead areas were more pronounced in the presence of NbGRP7, which was further confirmed by the greater chlorophyll loss. To determine whether the pro-immune functions of NbGRP7 was elicitor-induced, the same experimental setup was use with an autoactive Gpa2 construct (p35S:Gpa2 D460V). In contrast to HR triggered by GpRBP-1, overexpressing NbGRP7 does not influence the cell-death induced by autoactive Gpa2. Collectively, these results show that NbGRP7 specifically contributes to elicitor-induced HR by Gpa2. For Rx1, cell death in N. benthamiana is 
typically a quick response. We, therefore, compared cell death induced by Rx1 constructs cloned under the control of the endegonous ( $\mathrm{pR \times 1}$ ), CaMV35S (p35S), or leaky scan promotor ( $\mathrm{p} 35_{\mathrm{LS}}$ as described in (Slootweg et al. 2010)). Contrary to Gpa2, our data show negligible effects of NbGRP7 transient overexpression on Rx1-dependent cell death (Fig. 3B). No significant differences in chlorophyll loss relative to the control could be observed reproducibly when overexpressing $\mathrm{R} \times 1$, the viral elicitor/effector PVX-CP UK3 and NbGRP7 irrespective the promotor under which the immune receptor was cloned. Likewise, co-expressing NbGRP7 with an autoactive Rx1 construct (D460V) did not impact the cell death output.

As cell death is not the sole resistance response characterized for $\mathrm{R} \times 1$, we also tested the effect of NbGRP7 overexpression on extreme resistance to PVX. N. benthamiana leaves were infiltrated with Agrobacteria harbouring an amplicon of the avirulent PVX-UK3 strain and viral levels were quantified by DAS-ELISA within 1-5 dpi. Our overexpression data further demonstrate that NbGRP7 potentiates Rx1-mediated extreme resistance against PVX-UK3 between 3-5 dpi, as shown by a significantly greater reduction in virus levels compared to the control (Fig. 3C). This effect is lost at later time points, which may correspond to a decrease in level of overexpressed NbGRP7 and thereby, possibly promoting viral escape. Additionally, overexpressing NbGRP7 in the absence of Rx1 reduced susceptibility for PVX-UK3 (Supplementals Fig. S5), consistent with existing studies implicating the role of GRP7 in basal defence (Lee et al. 2012). To complement our overexpression studies, TRV-VIGS silencing of NbGRP7 was performed. However, TRV-VIGS silenced plants showed severe developmental phenotypes at 3 weeks post-silencing (data not shown), most likely due to pleiotropic effects of NbGRP7 silencing on accumulation of TRV. We, therefore, abandoned this approach and alternatively, performed local transient hairpin silencing of NbGRP7 (Shin et al. 2017). Hairpin constructs (denoted as HpNbGRP7) were designed to knock-down transcript levels of endogenous NbGRP7 specifically (Supplementals Fig. S3). Similar Rx1 and PVX-UK3 combinations as described above were co-infiltrated with hpNbGRP7, and virus levels were quantified at $3 \mathrm{dpi}$. The results demonstrated that transient silencing of NbGRP7 leads to significantly higher accumulation of PVX coat protein, indicating that $R \times 1$ resistance was hampered (Fig. 3D). These findings complement our overexpression analysis and collectively, support the role of NbGRP7 in extreme resistance by $\mathrm{R} \times 1$. Taken together, our data demonstrate that NbGRP7 acts a pro-immune component in Gpa2 and Rx1-meditated defences. 
A.

Sample

自 GPRBP-1+GFP

审 GpRBP-1 + NbGRP7
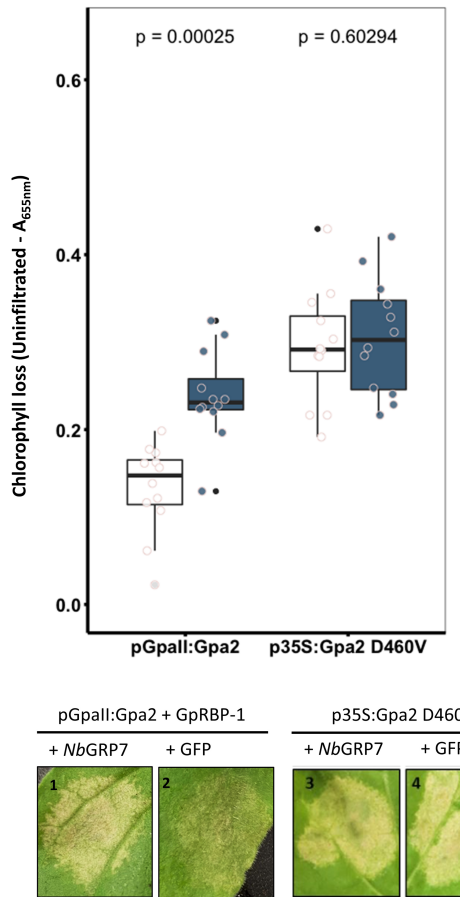

C.

Sample

穴 PVX-UK3 + GFP + GFP

定 $P V X-U K 3+R \times 1_{L S}+G F P$

审 PVX-UK3 + RX1 $1_{L S}+N b G R P 7$

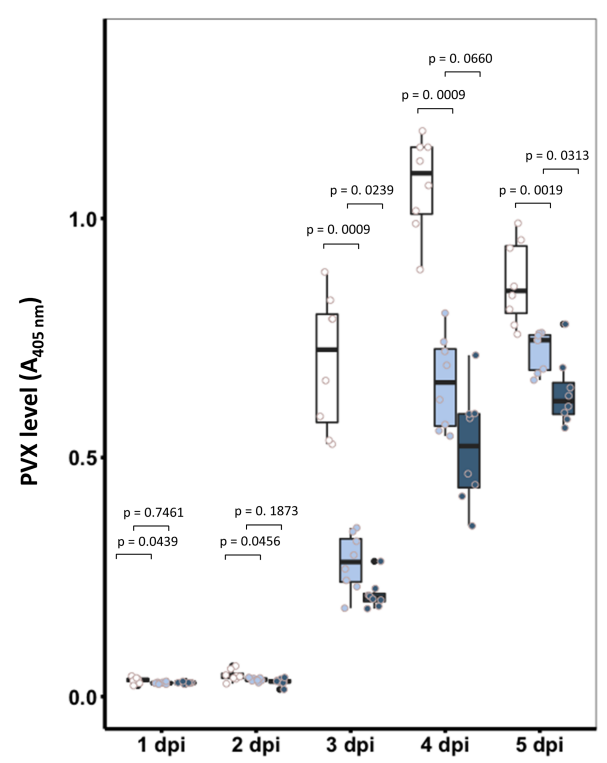

B.

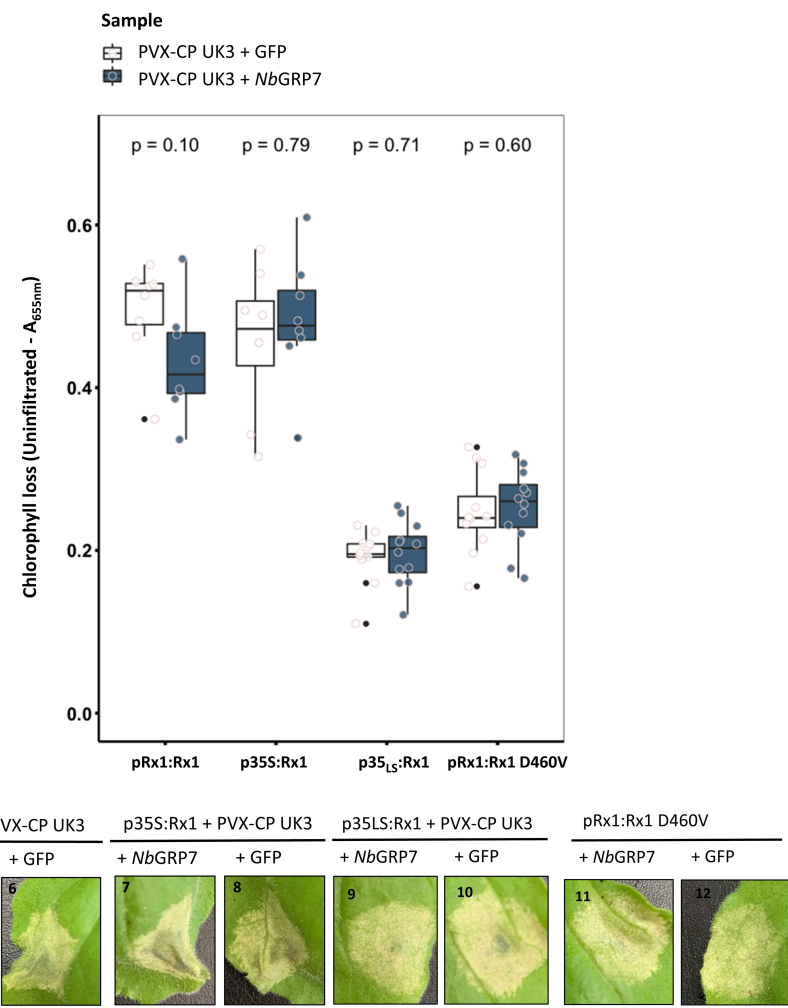

D.

Sample

宇 HpNbGRP7

审 HpGUS

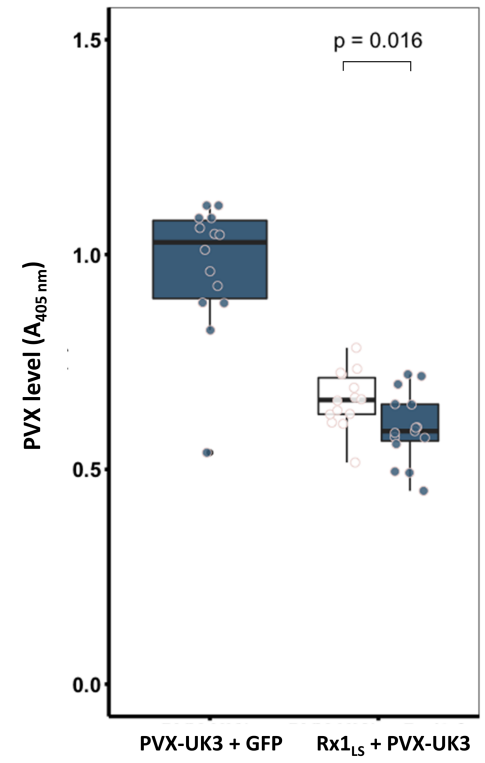

Fig. 3: NbGRP7 contributes to defences by Gpa2 (A) and Rx1 (B-D). Boxplots representing chlorophyll loss of elicitor-induced or independent cell death upon overexpression of NbGRP7 
(Figure legend continued) at 3-5 dpi (A and B). Bars represent the interquartile range while the cross indicates the median. The whiskers mark the minimal and maximal data points. Significance was calculated using Wilcoxon-Signed Rank test with $\alpha=0.05$ from $n \geq 12$ samples. Data shown is representative of at least three independent repeats. For the cell death assay, constructs of Rx1 under the control of either a 35S, endogenous or leaky scan promotor was used whereas Gpa2 was cloned under the control of its endogenous promotor ( $p$ Gpall). Representative photographs of infiltrated leaf zones are provided in the next row. Boxplots of absorbance at $405 \mathrm{~nm}$, indicating levels of PVX-UK3 upon transient overexpression (C) or silencing (D) of NbGRP7 in the context of $\mathrm{R} \times 1$-mediated responses. Data shown is representative of at least three independent repeats with similar results. Significance was calculated using Wilcoxon-Signed Rank test with $\alpha=0.05$ from $n \geq$ 8 samples.

Targeting a conserved Arginine residue in the RNA binding domain of NbGRP7 compromises its Rx1-related functions

Nicaise et al. 2013 demonstrated that the RNA binding activity of AtGRP7 is crucial for its role in regulating the function of the surface-localized immune receptor FLS2 (Nicaise et al. 2013). To address whether similar mechanisms underly the function of NbGRP7 in immunities by Rx1 and Gpa2, we targeted a conserved Arginine residue at position 49 of NbGRP7 to generate mutant variants (NbGRP7 R49K and NbGRP7 R49Q) that are impaired in their RNA binding capacities as described in earlier works (Nicaise et al. 2013). These mutants were cloned in the same p35S:4×Myc.GFP backbone as the wild type NbGRP7 variant. Western blot analysis indicates that $4 \times$ Myc.GFP-NbGRP7 R49K and 4×Myc.GFP-NbGRP7 R49Q are expressed in N. benthamiana as stably as wild type NbGRP7 (Supplementals Fig. S4A). Surprisingly, however, they show markedly different distribution patterns in the cell. The subnuclear bodies characteristic of NbGRP7 are considerably less present upon expression of the mutants, as would be expected with reduced RNA chaperone activities (Supplementals Fig. S4B). In addition to these targeted mutations, we created larger deletion fragments of NbGRP7 such as those without the RRM. However, the resultant proteins showed the propensity to multimerize in immunoblotting experiments and these constructs were, therefore, excluded in further studies. 
To illustrate the significance of RNA binding for NbGRP7 function in defence by Rx1/Gpa2, we tested the effect of overexpressing these mutants on the extreme resistance response. Thus, we transiently co-expressed 4×Myc.GFP-NbGRP7, 4×Myc.GFP-NbGRP7 R49Q, 4×Myc.GFP-NbGRP7 R49K, or 4×Myc.GFP alone with $\mathrm{p} 35_{\mathrm{LS}}: \mathrm{Rx} 1$ and PVX-UK3 in N. benthamiana. Quantification of virus levels shows that both mutant constructs do not suppress PVX accumulation as much as wild-type NbGRP7 at 3 dpi as determined by DAS-ELISA (Fig. 4A). To corroborate these findings, an additional qPCR analysis was performed, which indicates that levels of virus transcripts increased in tissues where $\mathrm{p} 35_{\mathrm{LS}}: \mathrm{Rx} 1$ was co-expressed with the mutants compared to those with wild-type NbGRP7 (Fig. 4B1-B2). These findings overall suggest that the potentiating effects of Rx1-mediated defence by NbGRP7 depends on an intact RNA binding domain.

Alternatively, differences in complex binding and/or affinities between wild-type NbGRP7 and its mutant derivatives with $\mathrm{Rx} 1$ may account for the phenotypes seen in our bioassays. To explore this possibility, we conducted a Co-IP using similar experimental set-ups as described beforehand. As expected, wild type $4 \times$ Myc.GFP-NbGRP7 was enriched following purification when either $4 \times H A-R \times 1-C C$ or $4 \times H A-R \times 1$ was used as baits (Fig. 4C1 and Fig. 4C2). Interestingly, we observed that $4 \times \mathrm{HA}-\mathrm{R} \times 1-\mathrm{CC}$ or $4 \times \mathrm{HA}-\mathrm{R} \times 1$ pulled-down the NbGRP7 R49Q variant consistently to a greater extent than wild-type NbGRP7. These results suggest that mutating the conserved Arginine residue in NbGRP7 to an amino acid with markedly different properties can also impact its interaction with Rx1. Conversely, western blotting further showed that the $4 \times$ Myc.GFP-NbGRP7 R49K co-immunoprecipitated at comparable levels with $4 \times \mathrm{HA}-\mathrm{R} \times 1-\mathrm{CC}$ and $4 \times \mathrm{HA}-\mathrm{R} \times 1$ as the wild-type NbGRP7. We, therefore, conclude that the loss of pro-immune activity of NbGRP7 R49K is not due to a lack of complex formation with the Rx1-CC, but most likely caused by the loss of its RNA binding capacity. 

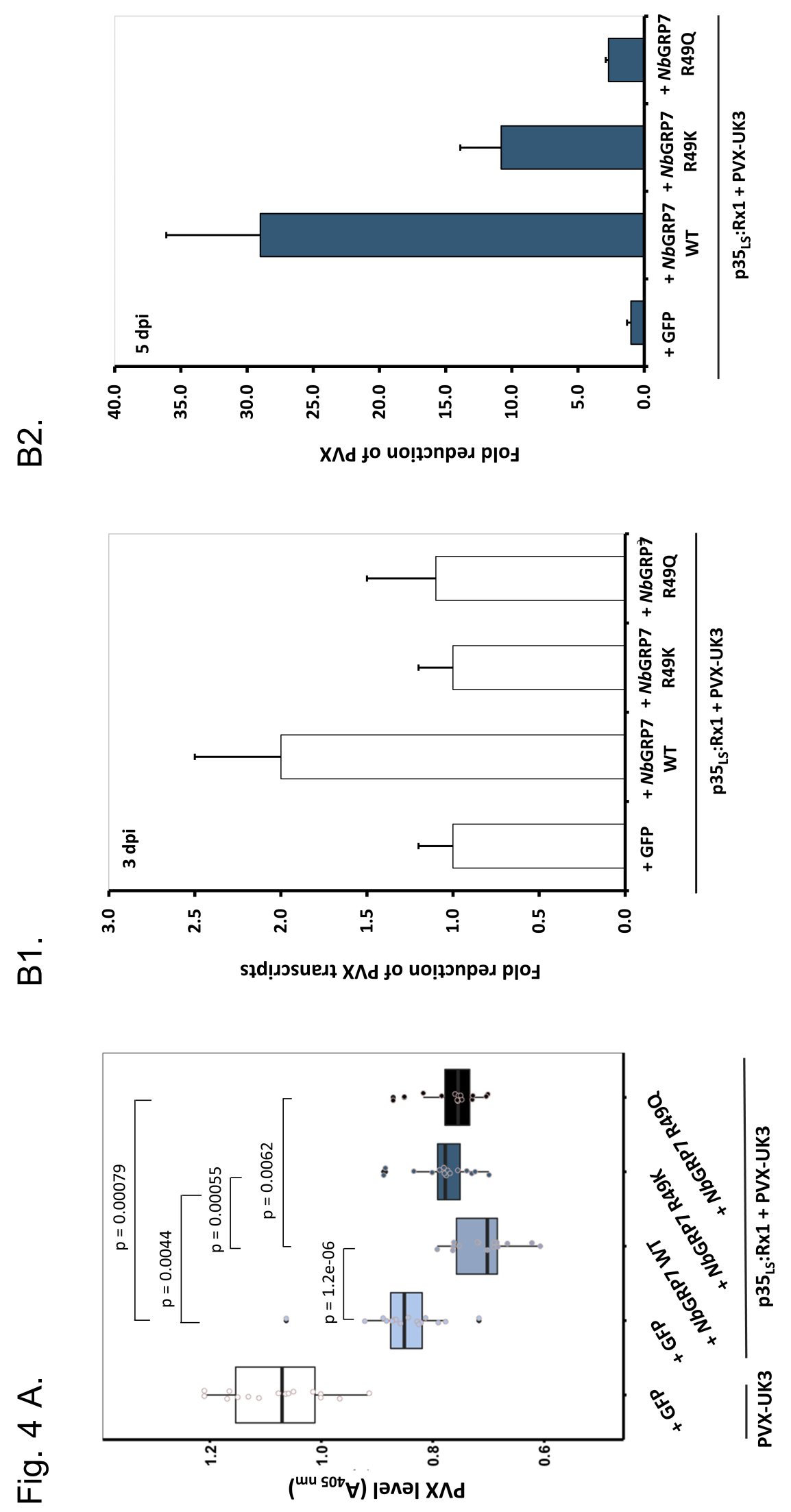
Fig. 4 C1.

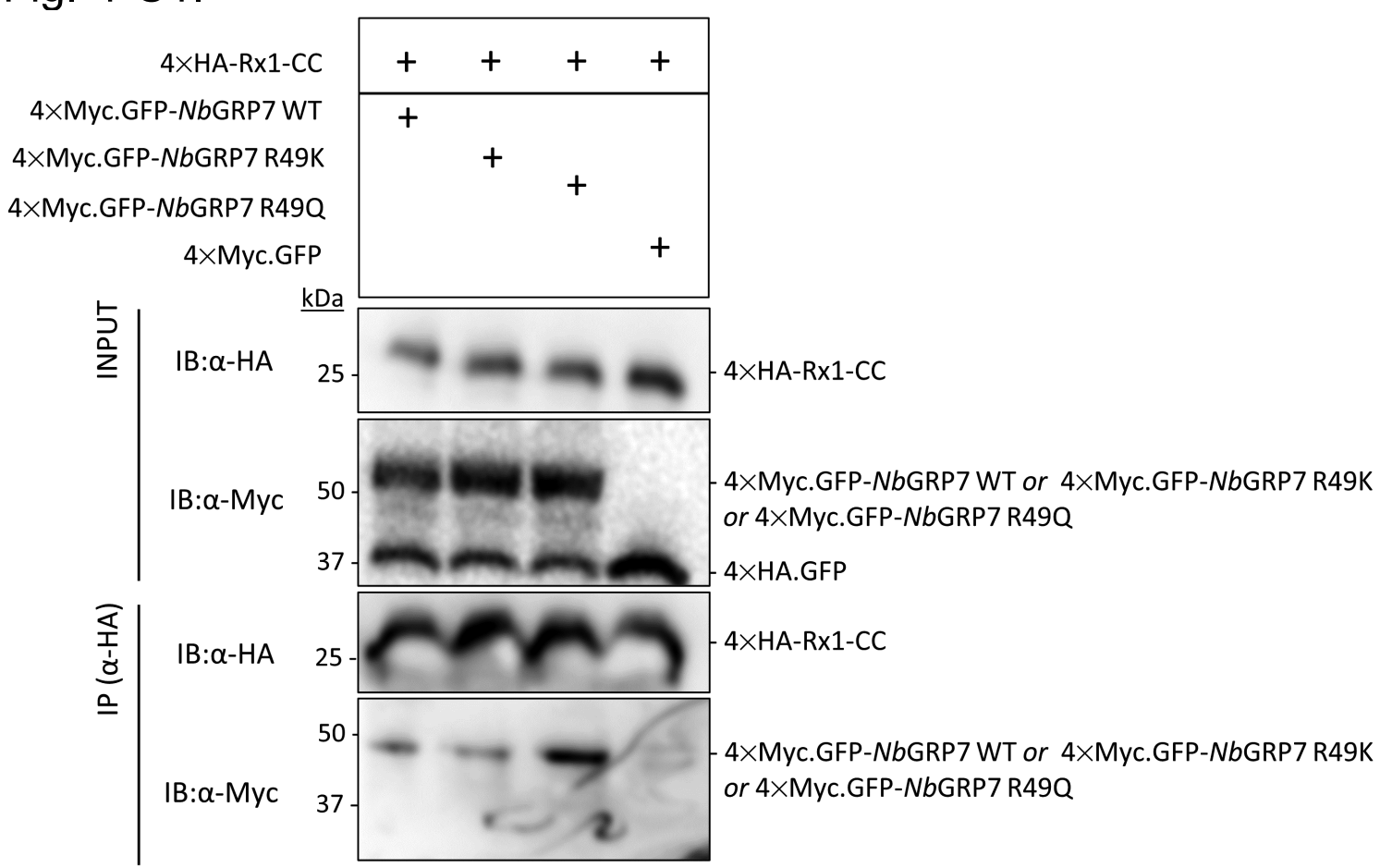

C2.

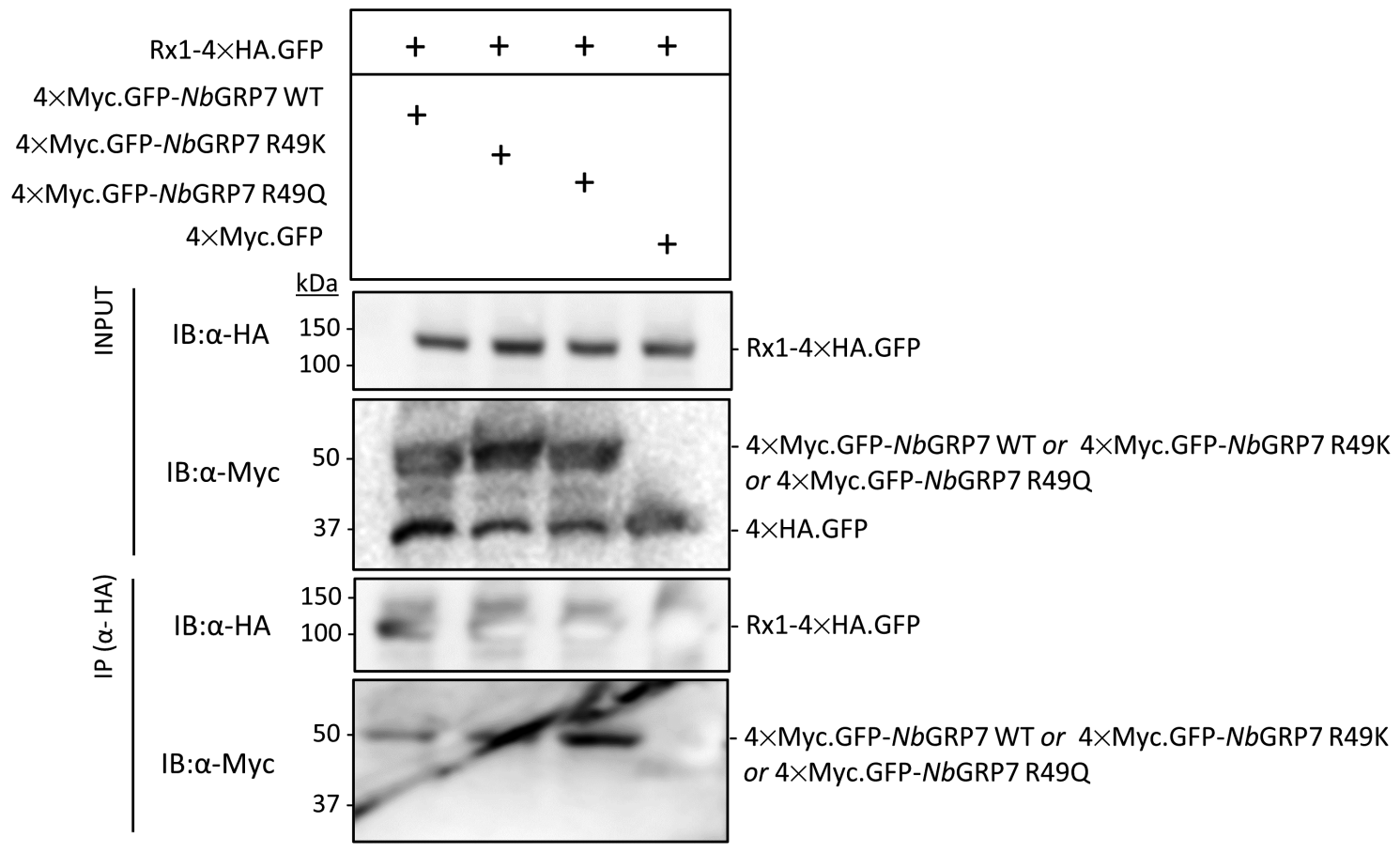

Fig. 4: The RNA binding activity of NbGRP7 contributes to Rx1-mediated extreme resistance against PVX-UK3 in N. A) Boxplots of a DAS-ELISA assay from transient overexpression of 
(Figure legend continued) p35 LS:RX1-GFP and PVX-UK3 in combination with $4 \times$ Myc.GFP-NbGRP7 WT, NbGRP7 R49K or NbGRP7 R49Q. Bars represent the interquartile range and the cross indicates the median. The whiskers mark the minimal and maximal data points. Statistical significance was calculated using Wilcoxon-Signed Rank test with $\alpha=0.05$ from $n \geq 12$ samples. B) Bar graphs of qRT-PCR analysis of viral transcript levels as determined using primers specific for the PVX coat protein. RNA from infected $N$. benthamiana leaves harvested at $3 \mathrm{dpi}$ (B1) and $5 \mathrm{dpi}$ (B2) were used for the analysis. Data shown is representative of two different experiments, with each sample consisting of a pool of at least 5 different plants. To obtain the relative fold change, samples were first normalized to the actin reference gene and then compared to the combination of Rx1 ${ }_{L S}+P V X-U K 3+G F P$. Error bars represent standard error. C1 and C2). Co-IP of HA-tagged Rx1-CC domain or the full-length immune receptor in combination with WT or mutated variants of 4 XMyc.GFP-NbGRP7. $\alpha$-HA beads were used to pull-down the receptor fragments. The success of the Co-IP is detected in the $\alpha$-Myc immunoblot. "+"indicates the presence of a particular construct in the infiltration combination.

\section{NbGRP7 regulates the transcripts of $\mathrm{R} \times 1$ pre- and post-activation}

Our data suggest that the RNA binding functions of NbGRP7 may contribute to its immune-related activities in $\mathrm{R} \times 1$ defence. We then questioned the nature of RNA that could be the target of this binding? AtGRP7 is well-documented for its role in the processing and metabolism of its target transcripts, including the PRRs FLS2 and EFR (Nicaise et al. 2013). In a similar vein, we investigated whether NbGRP7 can also regulate Rx1 mRNA in the cell. qPCR analysis was performed to provide initial evidence for potential effects on Rx1 transcripts under conditions when NbGRP7 is expressed ectopically in $N$. benthamiana. We found an increase in relative transcript abundance of $R \times 1$ upon NbGRP7 overexpression that peaks at 5 dpi in the absence of PVX (Fig. 5A1). Complementarily, silencing NbGRP7 in N. benthamiana resulted in decreased $R \times 1$ transcript levels. Similar patterns in transcript profiles were observed during immune activation by the avirulent PVX-UK3 strain when NbGRP7 was overexpressed and silenced (Fig. 5A2). In parallel, we performed an immunoblotting assay to determine whether the effects observed upon overexpression or silencing of NbGRP7 translate to different $\mathrm{R} \times 1$ protein levels in planta. Overexpressing $4 \times$ Myc.GFP-NbGRP7 led to higher protein accumulation of $\mathrm{p} 35_{\mathrm{LS}}$ :GFP-Rx1 while silencing reduced this amount (Supplementals Fig. S6). Repeating these assays with overexpression constructs of the NbGRP7 RNA binding 
mutants NbGRP7 R49K and NbGRP7 R49Q show reduced transcript levels of R×1 when compared to wild-type NbGRP7 both in the presence and absence of PVX-UK3 (Fig. 5B1 and 5B2). Collectively, our findings indicate that modulating the levels of NbGRP7 impacts the steady-state level of Rx1 pre- and post-activation, which may explain its pro-immune activity in $\mathrm{R} \times 1$ mediated plant defence. This is in turn directly or indirectly dependent on the RNA binding capacity of NbGRP7. 
A1.

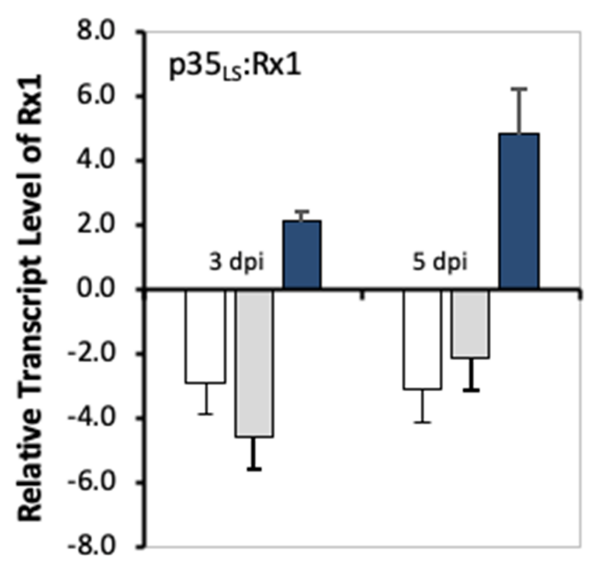

B1.

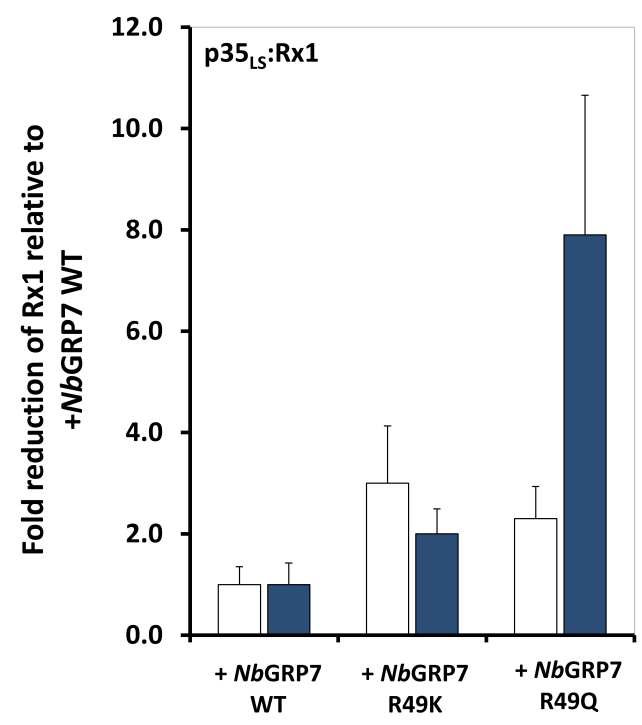

A2.

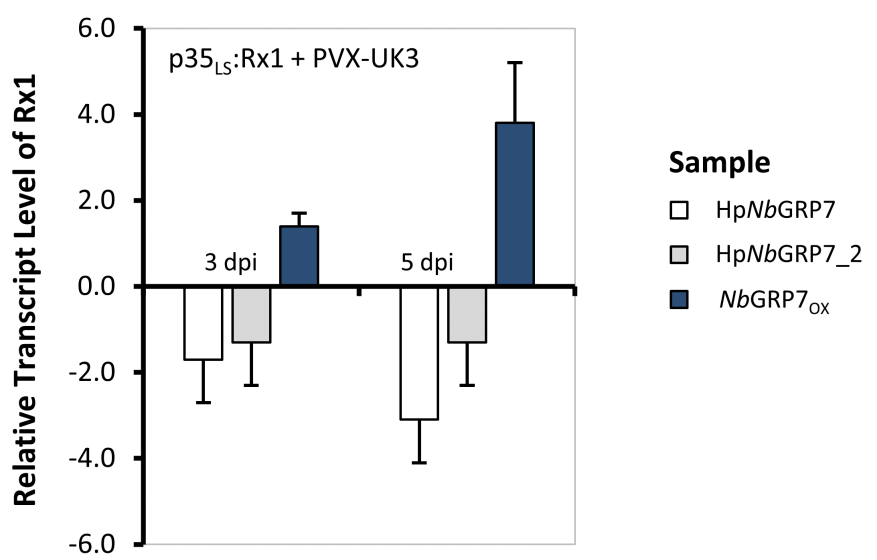

B2.

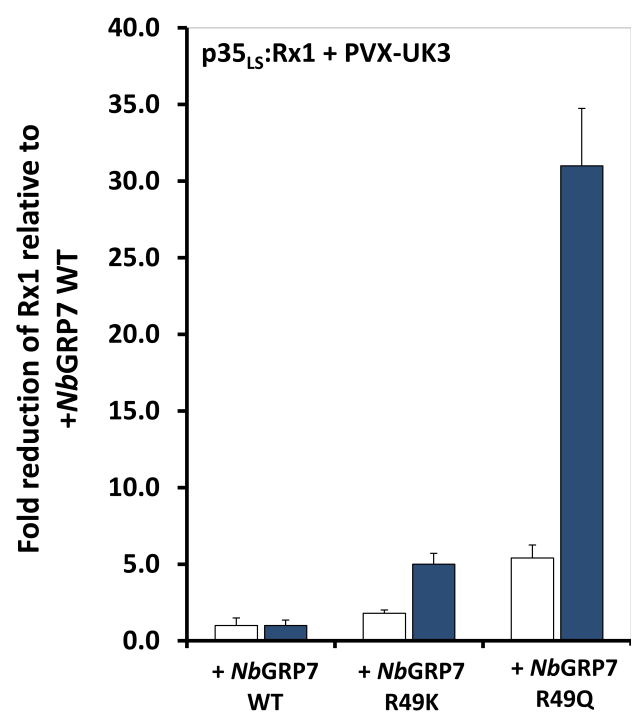

Fig. 5: NbGRP7 regulates Rx1 transcripts pre- and post-activation by PVX-UK3. A1 and A2) qRT-PCR showing expression profile of Rx1 transcript co-expressed with a construct whereby NbGRP7 is either overexpressed or silenced in N. benthamiana upon activation by PVX-UK3 or in the absence of the pathogen. Leaf samples were harvested at either 3 or $5 \mathrm{dpi}$. For each combination shown, data was obtained from a pool of at least 5 different plants. $R \times 1$ transcript levels were normalized to the actin reference gene and the fold change was calculated relative to HpGUS (for hairpin silencing experiments) or GFP-GUS (for NbGRP7 overexpression experiments). Error bars represent the standard error. B1 and B2) qRT-PCR quantifying the relative transcript abundance of $\mathrm{R} \times 1$ pre and post-activation in the presence of wild-type or mutant NbGRP7 constructs at 3 or $5 \mathrm{dpi}$. 
(Figure legend continued) Fold change was derived following normalization to the actin reference gene and compared to the combination containing wild-type NbGRP7. Error bars represent the standard error.

\section{DISCUSSION}

Although the role of GRP7 in PTI has been established, its involvement in ETI remains unknown. From a Co-IP/MS screening, we discovered a N. benthamiana homolog of the GRP7 protein (NbGRP7) as a novel interactor of the intracellular Gpa2 and Rx1-CC-NB-LRR immune receptors. Transient overexpression and silencing experiments demonstrated that NbGRP7 positively regulates the extreme resistance of RX1 and elicitor dependent cell death responses of Rx1 and Gpa2, respectively. Interestingly, we further showed that ectopic expression of NbGRP7 impacts the transcript and protein abundance of $\mathrm{R} \times 1$ pre- and post-activation. Both the pro-immune activity and transcript regulation of RX1 by NbGRP7 rely on an intact RNA-binding domain. However, we were unable to demonstrate that these phenomena are linked with direct binding of NbGRP7 to the receptor transcripts. Taken together, we conclude that NbGRP7 acts as a co-factor regulating the stability of its bound receptors prior to and during an active immune response.

\section{The function of the CC domain as scaffold for interacting proteins including NbGRP7}

We demonstrate that the Rx1 and Gpa2-CC-domains mediate the heteromeric complex with NbGRP7 (Fig. 2). Current views of NB-LRR functioning place the receptor $N$-terminus at the nexus of downstream interactions (Sukarta et al. 2016, Jones et al. 2016, Kapos et al. 2019). This function as a molecular scaffold is in agreement with previous reports that the Rx1 CC domain is also the minimal-interacting module required for the association with RanGAP2 and GLK1 (Sacco et al. 2007, Townsend et al. 2018). The CC domain of Rx1 has a nucleocytoplasmic distribution in the cell. The dual compartmentalization of the CC may expand the repertoire of co-factors that can be recruited via the same or overlapping surface regions. Specifically, the CC accounts for the localization of the immune receptor to the nucleus (Slootweg et al. 2010). This is thought to occur by virtue of binding with nuclear components, which is consistent with the identification of the GLK1 transcription factor as an Rx1-interacting protein. In our Co-IP/MS screening, NbGRP7 co-purifies with the nuclear fraction 
of Gpa2-CC. However, whether NbGRP7 exclusively interacts with Rx1 in the nucleus warrants further investigation. Confocal imaging further shows that a prominent feature of NbGRP7 localization are the speckle-like structures in the nucleoplasm (Fig. 1B). These subnuclear bodies have been linked to splicing factors and active regions of transcription (Spector et al. 2011). Thus, it is tempting to speculate that NbGRP7 belongs to the complex of $\mathrm{Rx} 1$ at the DNA to regulate its function in the nucleus. Given the mRNA chaperone activity of plant GRPs, one hypothesis is that NbGRP7 may co-ordinate the processing of nascent RNA transcribed by Rx1-associated transcription factors e.g. GLK1. It would, therefore, be interesting to determine how NbGRP7 would co-operate with existing nuclear interactors of $R \times 1$. This would require us to first discern whether the nuclear pool of NbGRP7 is in fact important for its functions in effector-triggered immunity by NB-LRRs.

Our results suggest that distinct surface regions of the $\mathrm{CC}$ domain are required for intra and intermolecular regulations. Our analysis revealed that NbGRP7 can still form a complex in planta with a mutant derivative of the Rx1-CC (S1) that no longer interacts with the NB-ARC-LRR (Fig. $2 \mathrm{C} 1$ and $2 \mathrm{C} 2$ ). This is reminiscent of the finding that the interface required for RanGAP2-binding on the Rx1-CC does not coincide with those for associating with adjacent receptor domains (e.g. the EDVID motif) (Hao et al. 2013). We further show that NbGRP7 does not share an interacting surface with RanGAP2 in helix four of the CC (Fig. $2 \mathrm{C} 1$ and 2C2). Our data, therefore, do not exclude simultaneous binding of RanGAP2 and NbGRP7 as a tripartite complex with Rx1/Gpa2, consistent with their shared subcellular distribution in the cytoplasm. In this regards, it will be interesting to uncover the structural basis underlying the complex of Rx1-CC/NbGRP7 and how this deviates from the more established interaction of Rx1 with RanGAP2. Given the wide spectre of targets and overall protein fold of plant GRPs, we predict that binding of NbGRP7 occurs via its disordered glycine-rich region to surface-exposed residues in the Rx1/Gpa2-CC. An overall interpretation of our data is that a dedicated interface is unlikely to be present at the $\mathrm{CC}$ for heterocomplex formation, which in turn reflects flexibility of the CC as a protein domain. Indeed, functional and biochemical studies have shown that the $\mathrm{CC}$ is a versatile moiety, which can accommodate various inter/intradomain interactions and tolerate drastic structural re-arrangements upon activation (Wang et al. 2019, Slootweg et al. 2018). It would, therefore, be fascinating to resolve the spatio-temporal dynamics of the CC with their bound interactors pre- and post-activation to better understand the mechanistic basis of how the $\mathrm{N}$-termini of plant NB-LRRs fine-tune immunity. 
The dual role of NbGRP7 in basal and effector-triggered immunity to pathogens

Strikingly, we show that modulating the levels of NbGRP7 in N. benthamiana either by transient overexpression or silencing affects extreme resistance by $\mathrm{R} \times 1$ and elicitor-induced cell death responses by Gpa2 (Fig. 3A-3D). Despite several optimization attempts, however, we were unable to demonstrate similar effects of NbGRP7 ectopic expression on HR induced by PVX-CP (Fig. 3B). One possibility is that the cell death response by Rx1 has reached its maximum level. Thus, residual NbGRP7 from overexpression cannot further boost this response in the current experimental settings. Remarkably, compared to the negligible effect on cell death mediated by $R \times 1$, overexpression of NbGRP7 potentiates GpRBP-1 induced cell death response by Gpa2 (Fig. 3A). This is intriguing considering the high similarity of the Rx1 and Gpa2-CC domains. We attribute this difference owing to the comparatively mild nature of the Gpa2 cell death response, which offers more room to observe subtle differences. A general paradigm is that cell death is prerequisite for disease resistance. However, accumulating evidence show that it is dispensable and can be genetically uncoupled from resistance (extensively reviewed in Künstler et al. 2016). In a similar vein, extreme resistance by Rx1 to PVX was postulated to be epistatic to cell death (Bendahmane et al. 1999). The latter is viewed as a secondary latent response, reserved by the host till immunity proves insufficient e.g. when there is an over-abundance of the viral coat protein such as during heterologous expression assays. This is further reinforced by structure-functional studies of the Rx1-CC, indicating that different surface regions of the domain can be linked to cell death and extreme resistance (Slootweg et al. 2018). Thus, it is imaginable that NbGRP7 may function in regulating extreme resistance while having a limited role in the cell death pathway. Similar outcomes were noted for GLK1, whose overexpression only impacts extreme resistance (Townsend et al. 2018). In the future, it will be interesting to investigate a wider pool of NB-LRRs and determine whether the diverse defence phenotypes of NbGRP7 can be linked to structural and/or interaction differences with the receptors associated.

In addition, we demonstrate that NbGRP7 can enhance basal resistance against PVX, consistent with the role of AtGRP7 in general susceptibility (Supplementals Fig. S5) (Lee et al. 2012). RNA silencing has long been viewed as the primary defence mechanism against single-stranded RNA viruses like PVX (Huh et al. 2013). RNA binding proteins can play a role by directly binding to viral transcripts and/or regulate the activity of components involved in this process. For PVX, translational control also represents an important strategy, which coincides with the association of AtGRP7 with translational 
machinery (Jaubert et al. 2011, Nicaise et al. 2013). NbGRP7 may thus represent an essential component in antiviral defence. Conversely, whether NbGRP7 can play a role in antinematode defence requires further studies. However, as AtGRP7 has been show to confer resistance against a diverse spectrum of pathogens, this may not be unlikely (Saqlan Naqvi et al. 1998, Lee et al. 2012). To be effective against a wide pool of pathogens, it is, therefore, imaginable that the mechanisms underpinning NbGRP7 function should be broad-acting and extend at multitude levels. This is in agreement with the role of AtGRP7 in regulating salicylic acid-responses that are induced by multiple pathogen types including plant-pathogen nematodes (Hackmann et al. 2014, Zinovieva et al. 2011).

The broad function of plant GRPs is intriguing in light of our finding that NbGRP7 can mediate ETI, a specific branch of plant innate immunity. RNA-binding proteins have recently been linked to NB-LRR-mediated resistance in a study of the unconventional rice transcription factors harbouring an RRM, which contributes to blast resistance by PigmR and other structurally similar NB-LRRs (Zhai et al. 2019). To the best of our knowledge, however, ours is the first study reporting the role of a homolog in the GRP class of RNA binding proteins in NB-LRR mediated immunity. Our findings therefore illustrate that NbGRP7 is a core component linking PTI and ETI. A parallel can also be drawn with GLK1, which potentiates both extreme resistance and basal resistance against PVX (Townsend et al. 2018). In hindsight, this shows that a single NB-LRR protein can tap into hubs of defence signalling, coinciding with a general view that ETI and PTI responses overlap. This is also exemplified in recent studies demonstrating that a range of NB-LRRs (including Rx1, Gpa2, Bs2, R1 and Rpi-blb2) rely on the activity of NRC 'helper' NB-LRRs, which are also apparently required for PAMP perception (Leibman-Markus et al. 2018). As a whole, our data fit within a general theme of convergent cell surface-localised and intracellular immune signalling in plant defence.

\section{A model for the post-transcriptional regulation of plant immune responses by NbGRP7}

Earlier works involving AtGRP7 and FLS2 offers a mechanistic basis for the function of GRP7 in PTI (Nicaise et al. 2013, Fu et al. 2007). AtGRP7 was shown to bind directly to the PRR-encoding transcripts and translational machinery in vivo, although the consequence of such bindings remains unclear. Here, we demonstrate that overexpressing NbGRP7 directly enhances the transcript levels of 
Rx1 pre- and post-activation (Fig. 5). It is worth noting that heterologous overexpression of NbGRP7 may impose artefacts affecting the cellular transcriptome. However, our expression analysis did not indicate any aspecific impacts on the housekeeping gene actin. Additionally, as complementary phenotypes were observed when silencing NbGRP7, we believe these to be true biological effects. We, therefore, infer that the qualitative rise in receptor abundance may be directly linked to a concomitant increase in immune output as observed in our bioassays (Fig. 3A and 3C). Following this rationale, we also anticipate that the elicitor-independent responses of $R \times 1$ and $G$ pa2 would be enhanced, which was not the case. The autoactive mutant D460V used in this study is impaired in its binding between the NB-ARC and LRR domains, facilitating the exchange of ADP to ATP. We predict that this structural relaxation may override the effect of increased transcript levels needed to surpass the activation threshold. Additionally, the autoactive response may rely on other host components, which may be rate-limiting for the process but is not regulated by NbGRP7.

Co-ordinated control of plant NB-LRRs transcripts is key for appropriate defence activation. This has led to an extensive evolution of various molecular checkpoints to fine-tune the dosage of NB-LRRs in the cell. As a corollary, there is ample evidence for splicing, lifetime, and export of mRNAs as a differential response to biotic stress (extensively reviewed in Lai et al. 2018). Studies performed in potato protoplasts have shown that the extreme resistance response of $\mathrm{R} \times 1$ to $\mathrm{PVX}$ does not require de novo synthesis of defence transcripts (Gilbert et al. 1998). In this model, it is, therefore, imperative that a sufficient pool of pre-existing components is available for defence. This puts post-transcriptional regulation at the forefront for regulating $R \times 1$ function. This is in accordance with reports demonstrating that $\mathrm{Rx} 1$ transcripts are subject to regulation by 22 -nt microRNAs ( $\mathrm{Li}$ et al. 2012). The biological role of plant GRPs also fits perfectly within this framework. Although we currently do not have a clear picture of the mechanistic basis of how NbGRP7 contributes to $\mathrm{R} \times 1$ and Gpa2 defences, targeting a conserved Arginine residue (R49K/R49Q) indicates that the RNA binding capacity of NbGRP7 is involved (Fig. 4). It will be of interest to determine whether NbGRP7 has a similar direct role in the turnover of Rx1/Gpa2 transcripts as described for AtGRP7 and FLS2 (Nicaise et al. 2013). However, our preliminary data does not favour physical binding of NbGRP7 with the Rx1 transcript. Studies using RNA-immunoprecipitation (RIP) indicate that Rx1 transcripts are only slightly enriched when purifying $4 \times$ Myc.GFP-NbGRP7 with $\alpha$-GFP beads compared to the FLS2/GRP7 control (Supplementals Fig. S7). We corroborated these in planta 
assays by performing fluorescence anisotropy in vitro. Prior studies have shown that AtGRP7 has a preference for binding to the 3'UTR regions of its target genes (Nicaise et al. 2013). Following this rationale, we designed RNA probes to cover the 3'UTR of Rx1. As full-length NbGRP7 could not be expressed stably in E. coli, its RRM domain alone was produced and incubated with the fluorescently labelled RNA probes. Independent experimental repeats demonstrated that NbGRP7 does not alter the fluorescence anisotropy of any of the probes tested (Supplementals Table S1). These findings, therefore, showed that NbGRP7-RRM does not bind to the 3'UTR region of Rx1 in vitro. From these data, we cannot conclude that NbGRP7 directly associates with $\mathrm{R} \times 1$ transcripts in vivo and in vitro. This is reminiscent of the regulation of PR-1 by AtGRP7, which also does not involve direct binding to the PR-1 transcript (Hackmann et al. 2014). Future studies should, therefore, aim at elucidating the nature of the cargo bound to NbGRP7. Additionally, our interaction data shows that $4 \times$ Myc.GFP-NbGRP7 R49Q pulls-down more efficiently with $4 \times H A-R \times 1$, indicative of stronger physical association (Fig. 4C1 and 4C2). Thus, an influence of NbGRP7 on Rx1 -defence response is likely due to the combined effects of changes in the molecular function of NbGRP7 as well its interaction with the immune receptor.

Amassing the results of the current study, we propose a model for the role of NbGRP7 in Rx1 and Gpa2-mediated immunities (Fig. 6). We envision that specific activation of the immune receptor may alter interaction surfaces of the receptor-NbGRP7 complex, which impacts the cellular function of NbGRP7. More detailed molecular and biochemical studies are needed to fully elucidate how and which biological function(s) of NbGRP7 are affected. Given our qPCR data, however, we postulate that the activated forms of Rx1/Gpa2 directly or indirectly affects the RNA chaperone activity of NbGRP7 to ensure an effector-free pool of the immune receptor is sufficiently present to mount and/or sustain a defence response. Such feedback-loops to replenish receptor abundance are reminiscent of ligand induced-translation and polysome recruitment by membrane bound receptors at the cell-surface in both animal and plant cells (Tcherkezian et al. 2010, Carvalho et al. 2008). In hindsight, the model we propose is also appealing in light of recent reports that plant NB-LRRs may oligomerize as inflammasomes, which is deemed as an irreversible process (Wang et al. 2019). Previous works in potato have shown that modulating Rx1 and Gpa2 transcript/protein abundance directly impacts defence output, indicating that exerting control at a post-transcriptional level is important in fine-tuning immunity. The 
functional data presented in this research, therefore, adds to the framework of (post)-transcriptional regulation guided by NB-LRRs. The future challenge lies in resolving the precise role and structural basis of NbGRP7 in NB-LRR immunity and how this activity is in turn regulated by the bound receptors.

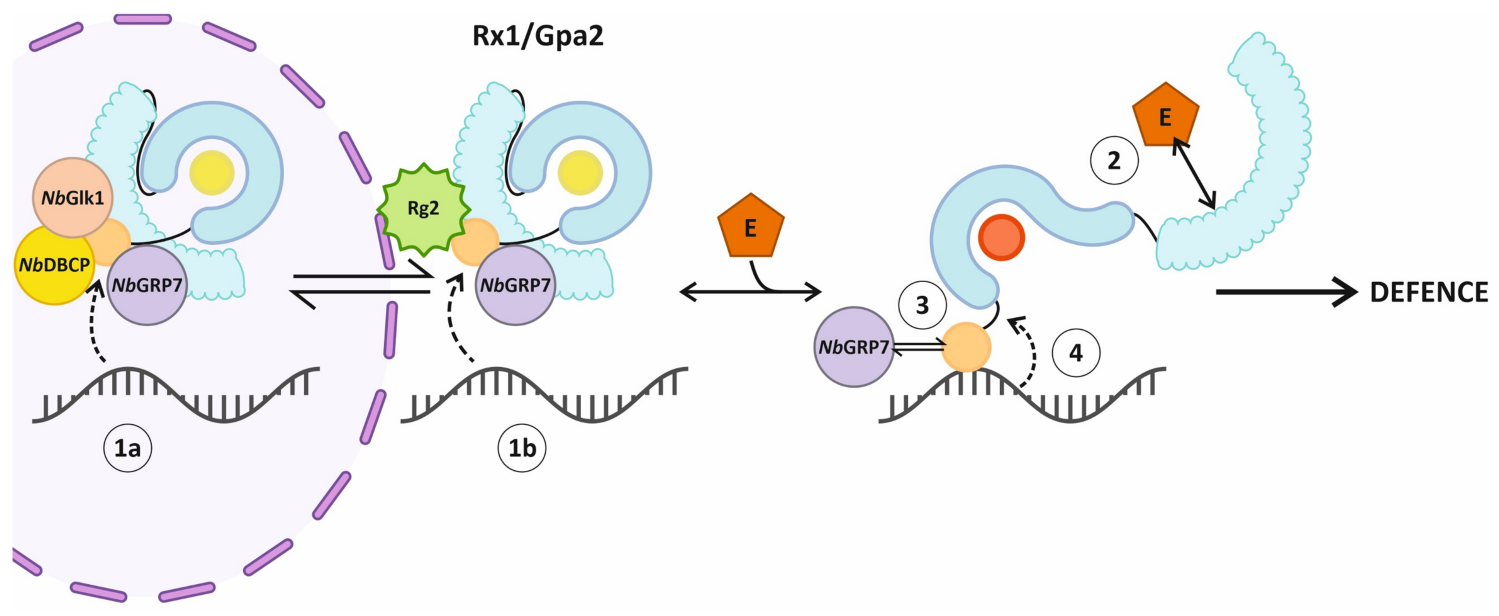

Fig. 6: Schematic representation of a working model proposed for the role of $N b G R P 7$ in immunity by Rx1/Gpa2. NbGRP7 exists as pre-formed complexes with the receptor proteins in either the nucleus (1a) and/or cytoplasm (1b). NbGRP7 is presumed to regulate the receptor transcript and protein levels in the cell through its RNA chaperone activity via a yet undefined mechanism (curved dashed line). Presence of the appropriate elicitor (E) is recognized in the cytoplasm and triggers a conformational switch in Rx1/Gpa2 (2). Structural rearrangements of the receptor architecture are predicted to affect downstream complexes such as those with NbGRP7, in turn impacting the cellular activity of NbGRP7 (3). Collectively, these changes ensure that a balanced and steady abundance of $\mathrm{R} \times 1 / \mathrm{Gpa} 2$ is present to sustain an immune response (4; curved dashed line).

\section{MATERIALS AND METHODS}

\section{Plasmid construction}

To isolate full-length genes of NbGRP7, similarity searches by BLAST were performed (BLOSUM62 matrix) against the $N$. benthamiana genome database. The search was done against both the University of Sydney (http://benth-web-pro-1.ucc.usyd.edu.au/blast/blast.php) and Solgenomics 
databases (http://solgenomics.net/tools/blast/index.pl?db_id=196). Hereby, Express Sequenced Tags (ESTs) sequences retrieved from the MS analysis were used as input queries. The best hit(s) retrieved was used as reference to design gene-specific primers for isolation of the full-length genes (listed in Supplementals Table S2). The genes were isolated from N. benthamiana cDNA as Ncol-Kpnl or Ncol-Bglll fragments and subcloned into the pRAP and pBinPLUS expression cassettes with an $N$-terminal $4 \times$ Myc.GFP tag. To do this, High Fidelity PCR (Promega) was performed according to the manufacturer's protocol. Purified fragments were initially ligated into pGEMT-easy high copy number vector and the products transformed into E. coli TOP 10 (Thermofischer Scientific) by heat shock. Positive clones were grown overnight at $37^{\circ} \mathrm{C}$, plasmid-prepped (Promega) and checked by sequencing. Sequence-verified clones were then cloned into the pRAP vector (Schouten et al. 1997) in frame with the $N$-terminal 4 X Myc.GFP tag by 3-way ligation (1:1:1 ratio) following additional digestion reactions with the BspHI enzyme. Positive clones were checked by colony PCR, mini-prepped and cloned into pBINPLUS (van der Vossen et al. 2000) as Ascl-Pacl fragments in A. tumefaciens MOG101. Correct fusion protein expression was confirmed by Western blotting (using $\alpha$-Myc antibodies) and localization studies.

For targeted substitution of NbGRP7 R49Q and R49K, nested PCR was performed using primers listed in Supplementals Table S2 and Ready-ToGo beads (illustra PuReTaq PCR Beads, GE Healthcare). In the first round of PCR, primers were used to amplify regions encompassing the mutation in the RRM. The resultant fragment was used as template in a second round of PCR with overlapping extensions to obtain the full-length NbGRP7 fragment with the expected substitution of arginine residue. The same cloning steps for addition of $4 \times$ Myc.GFP tag and into the binary pBIN vector was performed as listed above.

For the hairpin constructs, potential silencing regions in NbGRP7 were screened using the Solgenomics VIGS tool (http://solgenomics.net/tools/vigs) against the $N$. benthamiana gene models database v.04.4. Selection of optimal regions included least probability of off-target effects. To validate this, prioritized regions were further BLASTed for aspecificity against available $N$. benthamiana draft genome as provided in the University of Sydney database (http://benth-web-pro-1.ucc.usyd.edu.au/blast/blast.php). Target sequences were ordered synthetically (Genescript) in antisense orientation with a spacer in between as specified in 
Supplementals Table S3. These were subcloned into the destination vector pPT2 (Shin et al. 2017) by BamHI/Xbal digestion first in E. coli TOP10 and finally, A.tumefaciens strain MOG101.

\section{Transient protein expression by Agrobacterium tumefaciens transient assay}

Agrobacterium tumefaciens transient assay (ATTA) was used as a system for heterologous protein expression in plants. Briefly, Agrobacteria strains carrying the appropriate expression vectors were grown in Yeast Extract Broth medium ( $5 \mathrm{~g} / \mathrm{L}$ peptone, $1 \mathrm{~g} / \mathrm{L}$ yeast extract, $5 \mathrm{~g} / \mathrm{L}$ beef extract, 5

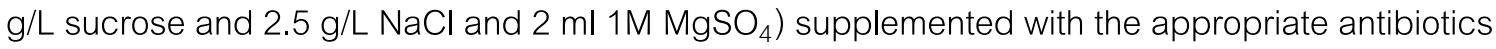
overnight at $28^{\circ} \mathrm{C}$ with constant shaking (250 rpm). Grown bacterial cells were spun down at 4,000 $\mathrm{g}$ for 10 minutes and re-suspended in MMAi infiltration medium ( $5 \mathrm{~g} / \mathrm{L}$ Murashige and Skoog salts, $3.90 \mathrm{~g} / \mathrm{L}$ 2-(N morpholino)ethanesulfonic acid, $2 \mathrm{~g} / \mathrm{L}$ sucrose and $1 \mathrm{ml} 200 \mathrm{mM}$ acetosyringone; Sigma Aldrich). Measurements of the optical density at wavelength $600 \mathrm{~nm}\left(\mathrm{OD}_{600}\right)$ were then taken. Agrobacterial suspensions were diluted to final $\mathrm{OD}_{600}$ values according to each assay. After 2 hours incubation at room temperature, agroinfiltration was performed on the underside of the leaves of 2-3 weeks old $N$. benthamiana plants using needleless syringes. Plants were grown before and after agroinfiltration under standard greenhouse conditions at a constant temperature of $23^{\circ} \mathrm{C}$ with light and dark cycle of L18:D6. Infiltrated spots were screened for the development of HR, harvested for protein extraction or examined by microscopy at $2-5$ days post infiltration (dpi) depending on the assay and construct.

\section{Protein extraction and immunodetection}

Protein extraction was performed as described in (Slootweg et al. 2010). Briefly, 50-100 mg of leaf material was grounded in extraction buffer (10 mM DTT, $150 \mathrm{mM} \mathrm{NaCl}, 50 \mathrm{mM}$ Tris-HCl, pH 7.5, 1 mM EDTA, $10 \%$ glycerol, $2 \%$ polyvinylpolpyrrolidone, and $0.5 \mathrm{mg} / \mathrm{mL}$ pefabloc SC protease inhibitor [Roche]), and spun down at $16,000 \mathrm{rpm}$ for 5 minutes at $4^{\circ} \mathrm{C}$. The supernatant was run through a G25-sephadex column and the eluate was used for subsequent pull-down assays or mixed directly with 4X Nupage LDS sample buffer with 1M DTT (Invitrogen). Proteins extracted were then separated by loading onto $12 \%$ Sodium dodecyle sulfate- Polyacrylamide gel electrophoresis (SDS-PAGE) run in 1X MOPS buffer and visualized by Commassie Brilliant Blue staining or wet blotting. Myc-tagged 
candidate interactors were detected using Goat $\alpha$-Myc polyclonal antibodies (Abcam) in subsequent western blot analysis as described by Tian et al. 2014. However, hereby immunodetection was achieved using a second polyclonal antibody conjugated with Horse-Radish Peroxidase (Abcam). Conversely, HA and GFP-tagged fusion proteins were detected using a Peroxidase-conjugated $\alpha$-HA (Roche) or $\alpha$-GFP (Abcam) antibodies respectively. Finally, peroxidase activity was detected by reacting with the Dura luminescenet and SuperSignal West Femto substrates (1:1 ratio; Thermo Scientific, Pierce) using the G:Box gel documentation system (Syngene).

\section{In planta Co-Immunoprecipitation assays (Co-IP)}

N-terminally tagged constructs for expression of p35S:Rx1-4 XHA.GFP, p35S:Rx1 S1-4XHA.GFP,

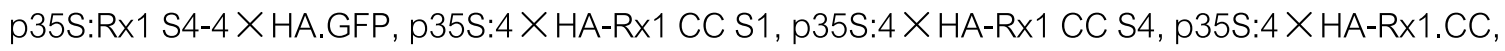
p35S:4 XHA-Gpa2.CC, p35S:4 X HA.Gpa2 and p35SLS:4 XHA.GFP were as described in (Slootweg et al. 2010) and (Slootweg et al. 2018). N. benthamiana leaves infiltrated by the appropriate protein combinations (at $\mathrm{OD}_{600}$ of 0.3-0.5) were harvested at 2-3 dpi. For Co-IP, proteins were extracted as described previously. Prior to the pull-down, pre-clearing of the protein extract was performed by incubation with mouse IgG1 agarose beads. After mixing with $\alpha$-GFP, $\alpha$-Myc or $\alpha$-HA magnetic beads ( $\mu$ MACS) and washing, eluted proteins were run in an SDS-PAGE system (Bis-Tris gel, 12\%, Invitrogen) with 1 XMOPS buffer and blotted onto PVDF membrane. Immunodetection was then performed as described beforehand using the appropriate antibodies.

\section{In planta Co-IP/MS analysis}

For proteomics analysis, p35S:Gpa2.CC-GFP or p35S:GFP was expressed transiently in $N$. benthamiana between 22-28 hours. Proteins were extracted from leaf samples according to the descriptions detailed above with a few exceptions. Particularly, cell fractionation was performed prior to affinity purification as described in (Slootweg et al. 2010). Bait proteins were precipitated using micromacs $\alpha$-GFP beads (Miltenyi) as described above. Peptides were generated by on-beads trypsin digestion of the pull-down samples, which were subsequently sent for MS analysis at the Proteomics Centre at WUR Biochemistry (Wageningen). For identification of proteins, the spectra of 
each run was matched using a MaxQuant software via a database consisting of translated ESTs and UniProt data referring to $\mathrm{N}$. benthamiana and N. tabacum.

\section{Cellular localization studies by confocal laser scanning microscopy}

Cellular localization patterns of the individual candidate interactors were examined by the Zeiss LSM 510 confocal microscope (Carl Zeiss) as described previously in (Slootweg et al. 2010). Agrobacteria harboring p35S:4 X Myc. GFP constructs of NbGRP7 (WT or mutant variants) were infiltrated on $N$. benthamiana leaves at final $\mathrm{OD}_{600}$ values of 0.3 . Leaf epidermal cells transiently expressing the candidates were harvested at $48 \mathrm{hpi}$ and imaged by confocal microscopy unless otherwise stated. Quantitative analysis of fluorescence intensities was performed using the ImageJ application software.

\section{PVX resistance assay and DAS-ELISA}

The effect of NbGRP7 silencing and overexpression was tested on extreme resistance against PVX by Rx1 using similar approaches as described in (Slootweg et al. 2010). Co-agroinfiltration experiments were performed using p35 $\mathrm{LS}: \mathrm{R} \times 1$ and PVX-GFP constructs. At 3-5 dpi, the level of viral accumulation was quantified by DAS-ELISA. ELISA plates were coated with polyclonal antibodies $(1: 1000)$ raised against the viral CP (Prime Diagnostics). A second polyclonal antibody conjugated with alkaline phosphatase was used for immunodetection (1:1000) at wavelength $405 \mathrm{~nm}$ (BioRad Microplate Reader model 680) via the substrate p-nitrophenyl- phosphate. Absorbance Measurements were taken with a reference filter of $655 \mathrm{~nm}$.

\section{Expression analysis by qRT-PCR}

Total RNA extraction was performed using 50 mg leaf tissues using the Promega Maxwell 16 simpleRNA extraction kit according to the manufacturer's protocol. First-strand cDNA synthesis was directly performed using the SuperScript III First-Strand Synthesis System (Invitrogen). To analyze expression levels, qRT-PCR was done (BioRad System) in a total reaction mix of $25 \mu$ l consisting of: $1 \mu \mathrm{l}$ forward and reverse primers ( $5 \mathrm{mM}$ each), $8.5 \mu$ Taq ready mix and $12.5 \mu \mathrm{MQ}$ water. qPCR 
was run using the following program: initial denaturation at $95^{\circ} \mathrm{C}$ for 15 min followed by 40 cycles of amplification at $95^{\circ} \mathrm{C}$ for $30 \mathrm{~s}, 60^{\circ} \mathrm{C}$ for $30 \mathrm{~s}, 72^{\circ} \mathrm{C}$ for $30 \mathrm{sec}$ and final elongation at $72^{\circ} \mathrm{C}$ for $60 \mathrm{~s}$ with a $90 \mathrm{X}$ melting curve at $50^{\circ} \mathrm{C}$ for $10 \mathrm{~s}$. To promote reproducibility, each sample was analyzed in duplo. In addition, a standard no template control was included to indicate the presence of contaminating DNA. GPCR data was normalized against the actin housekeeping gene. Finally, relative expression levels were analyzed by the comparative method $\left(2^{-\Delta \Delta C t}\right)$ using the average threshold values as described in (Schmittgen et al. 2008).

\section{In vivo RNA binding assay by RNA Immunoprecipitation}

RNA immunoprecipitation was performed as described in (Marmisolle et al. 2018). Briefly, GFP-TRAP ®beads (Chromotek) were washed in RIP-lysis buffer (20 mM Tris- $\mathrm{HCl}([\mathrm{pH}=7.5], 150 \mathrm{mM} \mathrm{NaCl}, 1$ $\mathrm{mM} \mathrm{MgCl}_{2}, 1 \mathrm{mM} \mathrm{CaCl} 2,0.1 \%$ SDS, $1 \%$ Sodium Deoxycholate, 1\% Triton X-100, $5 \mathrm{mM} \mathrm{PMSF}$ and $5 \mathrm{mM}$ DTT; $50 \mathrm{U}$ RNase inhibitor) three times and incubated overnight at $4^{\circ} \mathrm{C}$ with gentle rotation. Infiltrated leaf materials were harvested at $3 \mathrm{dpi}$, cross-linked in 1\% Formaldehyde solution (1XPBS) for 15 minutes by vacuum infiltration and reverse-linked by addition of $125 \mathrm{mM}$ glycine. $500 \mathrm{mg}$ of leaf materials were subsequently lyzed in RIP-lysis buffer using the TissueLyser ( $15 \mathrm{~Hz}$ for 1 minute). The samples were separated by centrifugation at 16,000 rpm, and the resultant supernatant was mixed in RIP-dilution buffer (10 mM Tris- $\mathrm{HCl}[\mathrm{pH}=7,5], 150 \mathrm{mM} \mathrm{NaCl}, 0,5 \mathrm{mM}$ EDTA) and incubated overnight with the pre-washed GFP-TRAP $®$ beads (Chromotek) at $4^{\circ} \mathrm{C}$. On the following day, IP samples were washed three times with RIP-washing buffer (50 mM Tris-HCl $[\mathrm{pH}=7,5], 500 \mathrm{mM}$ $\mathrm{NaCl}, 4 \mathrm{mM} \mathrm{MgCl} 2$, 0,5\% Sodium Deoxycholate, 0.1\% SDS, 2 M Urea, 2 mM DTT). RNA was isolated from the beads using the TRIzol extraction (Invitrogen) and treated to remove DNAse using the RQ1 DNAse solution (Promega) according to the manufacturer's protocol. cDNA synthesis and qRT-PCR analyses were performed as described previously.

\section{In vitro RNA binding assay by fluorescence anisotropy}

For in vitro production of NbGRP7, fragments corresponding to the RNA Binding Domain and full-length NbGRP7 were ordered from Genescript in pET-vectors with sequences optimized for production in E. coli and transformed into BL21 expression cells. E. coli cells harbouring the 
appropriate constructs were grown overnight in Terrific Broth medium (23.6g/L Yeast Extract, 11.8g/L Tryptone, 9.4g/L K $\mathrm{HPO}_{4}, 2.2 \mathrm{~g} / \mathrm{L} \mathrm{KH}_{2} \mathrm{PO}_{4} 4 \mathrm{ml} / \mathrm{L}$ Glycerol) supplemented with Ampicillin at $18^{\circ} \mathrm{C}$ and induced with 400 um IPTG. On the following day, samples were spun down and lysed as described in (Fenyk et al. 2015).

To generate fluorescently-labbelled RNA probes, in vitro transcription was performed using the TranscriptAid T7 High Yield Transcription Kit (ThermoFischer Scientific) according to the manufacter's protocol. Hereby, gene fragments of the NbGRP7 3'UTR were PCR amplified using primers listed in Supplementals Table S2 and the resultant products were directly used as template for the run-off reactions. Following in vitro transcription, probes were purified by native polyacrylamide gel electrophoresis (12\% gel), extracted in anisotropy buffer (20 mM Tris (pH 7.4), $140 \mathrm{mM} \mathrm{NaCl}, 1 \mathrm{mM}$ DTT, DEPC treated) and incubated at $37^{\circ} \mathrm{C}$ for 3 hours. The supernatant consisting of purified probes were used in anisotropy measurements, which was performed using the Synergy $\mathrm{H} 4$ fluorescence plate reader. Briefly, $10 \mathrm{nM}$ of RNA probes were mixed with $1 \mu$ protein solutions of NbGRP7 or the Ubiquitin negative control. Fluorescence was measured at 485/20 nm and 528/20 nm respectively.

\section{Statistical test}

Statistical analyses was performed in R studio Version 1.1.456. Data from assays performed in this study were checked for normality using Shapiro-Wilk Test. Depending upon the outcome, statistical level was determined either by T-test or Wilcoxon-signed rank test with $\alpha=0.05$ as specified in the text.

\section{ACKNOWLEDGEMENTS}

The current work benefits from funding by the Dutch Top Technology Institute Green Genetics (5CFD051RP), Dutch Technology Hotel grant and the Dutch Technology Foundation STW and Earth and Life Sciences ALW (STW-GG 14529), which are part of the Netherlands Organization for Scientific Research (NWO). Additionally, we thank Jan-Wilem Borst and Arjen Badder from Wageningen Light and Microspectroscopy Centre for providing imaging facilities and their technical expertise, and 
Alexander Llewlyn for performing and analyzing data of the fluorescence anisotropy. We are also grateful for the technical assistance and support of Charlotte Hijmans. 


\section{SUPPLEMENTALS}

Supplemental Fig. S1. Peptide hits and their locations in the full-length primary sequence of the NbGRP7 homolog identified in the Co-IP/MS screening (shown as bold, underlined sequences). * indicates a ratio referring to peptide abundance in the test samples relative to negative GFP control as determined by the label-free MaxQuant algorithm.

\begin{tabular}{cc}
\hline NbGRP7 (Gpa2-CC-GFP nuclear extract) \\
\hline Peptide hits & 2 \\
\hline Unique peptide & 1 \\
Ratio Int LFQ* & 1.729 \\
\hline \multicolumn{2}{c}{ Peptide Hits } \\
CFVGGLAWATTDR \\
NITVNEAQSR \\
\hline
\end{tabular}

MAAEVEYRCFVGGLAWATTDRTLGDAFAHYGEVVDSKIINDRETGRSRGFGFVTFSDEKAMRDAIEGM NGQNLDGRNITVNEAQSRGSGGGGGGFGGGRRREGGYSGGGGYGGGSGGYGGGRREGGYSGG GGGYGGGYGGGRNRGYGGGYGGGGGDGGSRYSRGGGASEGSWRN 
Supplemental Fig. S2. Multiple protein alignment of NbGRP7 with characterized paralogs from Arabidopsis, potato and pepper generated using the Geneious software ver. 2020.1. Detailed alignment of specific functional motifs and conserved residues are provided in the next row. Orange highlight in the consensus sequence indicates boundaries of the RNA Recognition Motif.

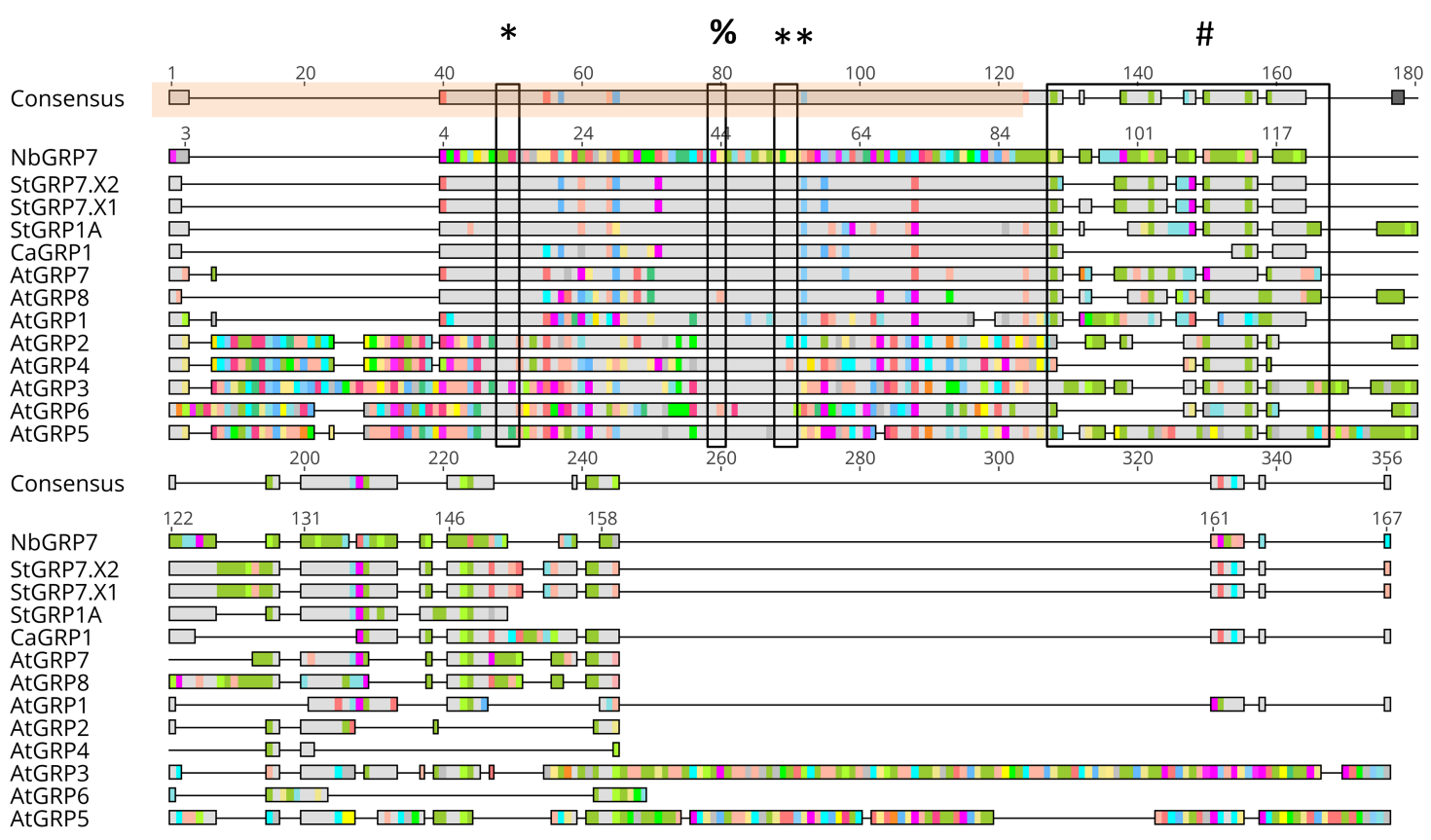

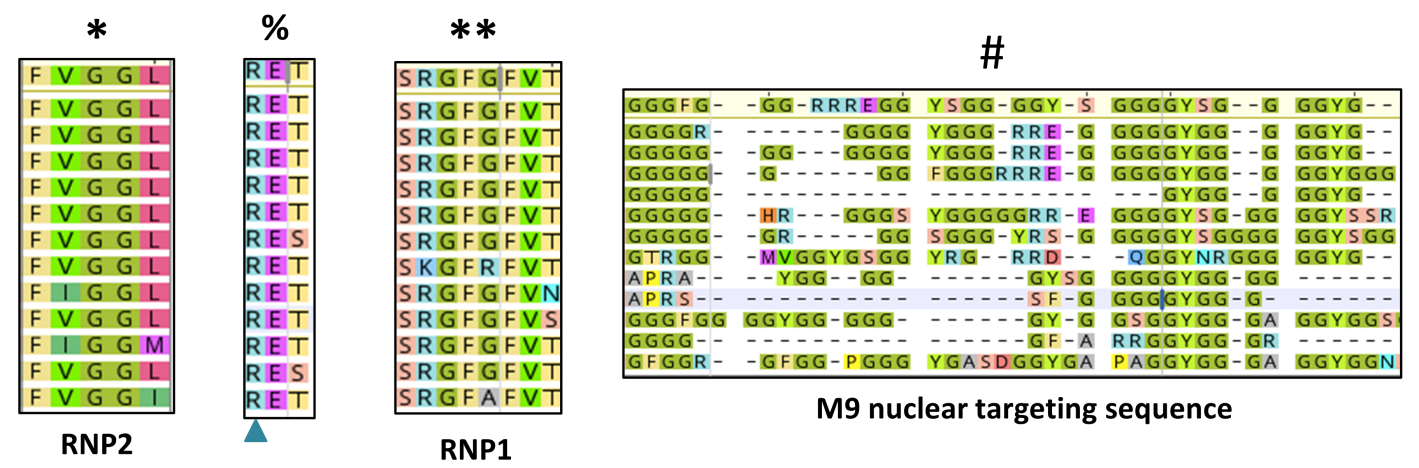


Supplemental Fig. S3. A) Schematic representation of the construct design for hairpin silencing of NbGRP7. Regions corresponding to characteristic domains are indicated above the gene-structure. RRM = RNA Recognition Motif; GRR: Glycine Rich Region. B) Efficiency of silencing on endogenous NbGRP7 transcript is represented as bargraph using RNA extracted from leaf materials harvested at 3 dpi. C) Efficiency of silencing was also tested by immunoblotting experiments of an overexpressed $4 \times$ Myc.GFP-NbGRP7 construct in combination with HpGUS control or the two silencing constructs HpNbGRP7 and HpNbGRP7_2. CBB-stained membrane of the RuBisCO protein is provided as loading control.

A.

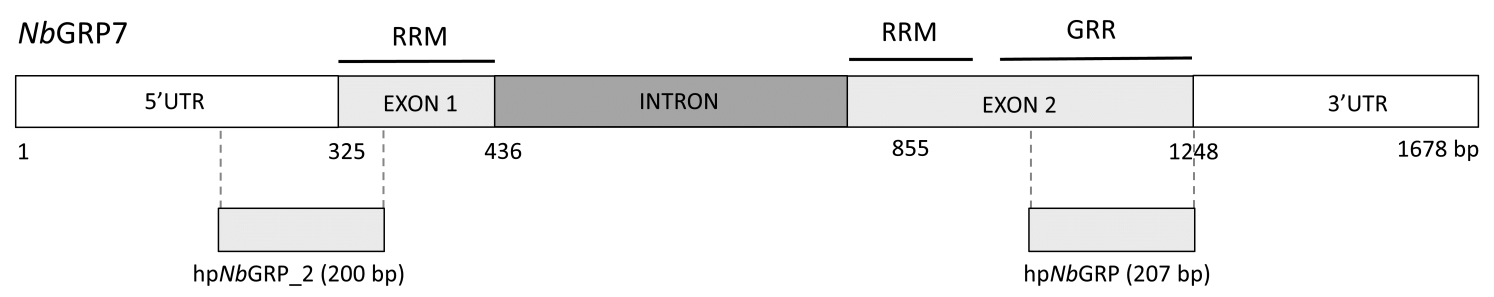

B.

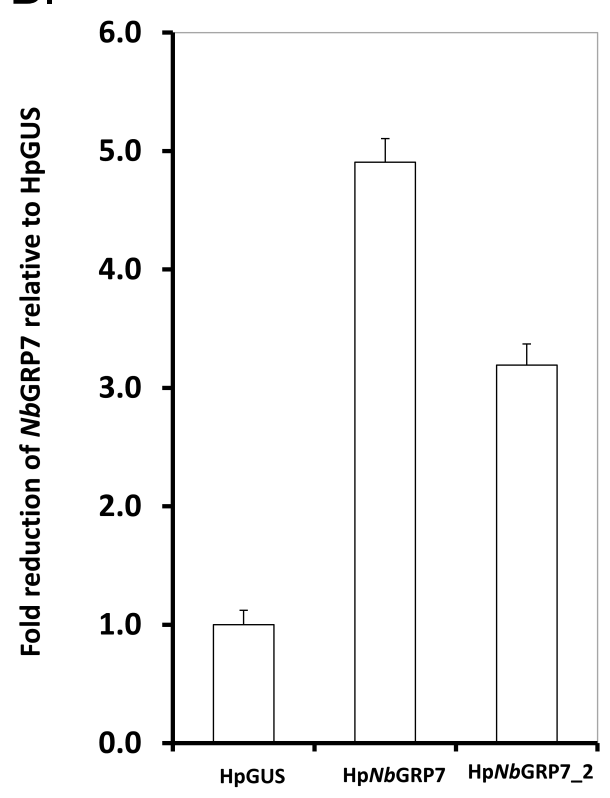

C.

\section{4×Myc.GFP-NbGRP7}

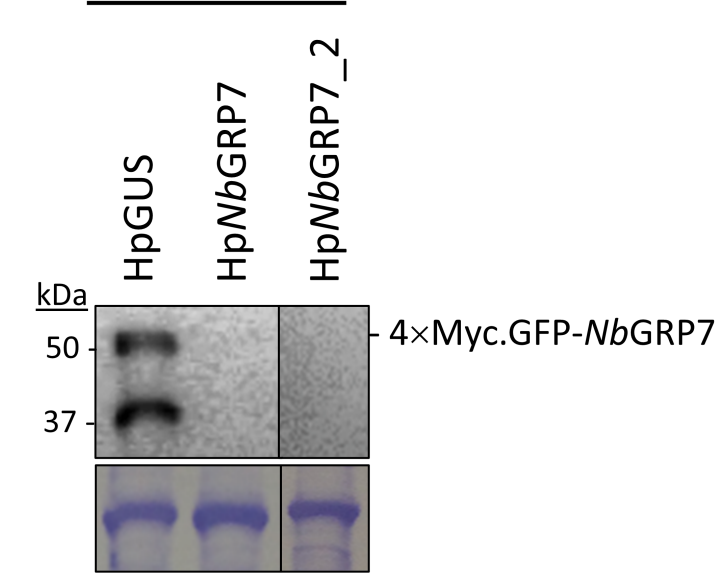

IB: $\alpha-G F P$

CBB 
Supplemental Fig. S4. Western blot $(A)$ and confocal imaging (B) of RNA binding mutants of 4 XMyc.GFP-NbGRP7 indicating that they are stably expressed in planta but localize to different regions in the nucleoplasm. Imaging in B was performed at 3 dpi. CBB-stained membrane in A serves as loading control as indicated by levels of the RuBisCO protein.

A.

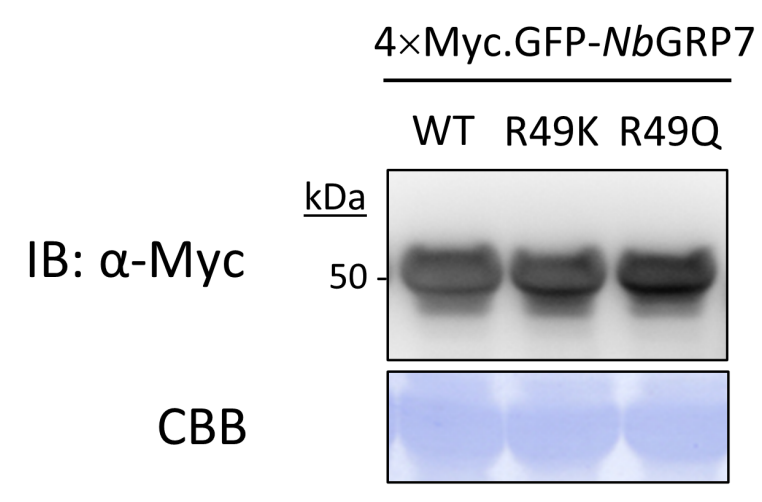

B.

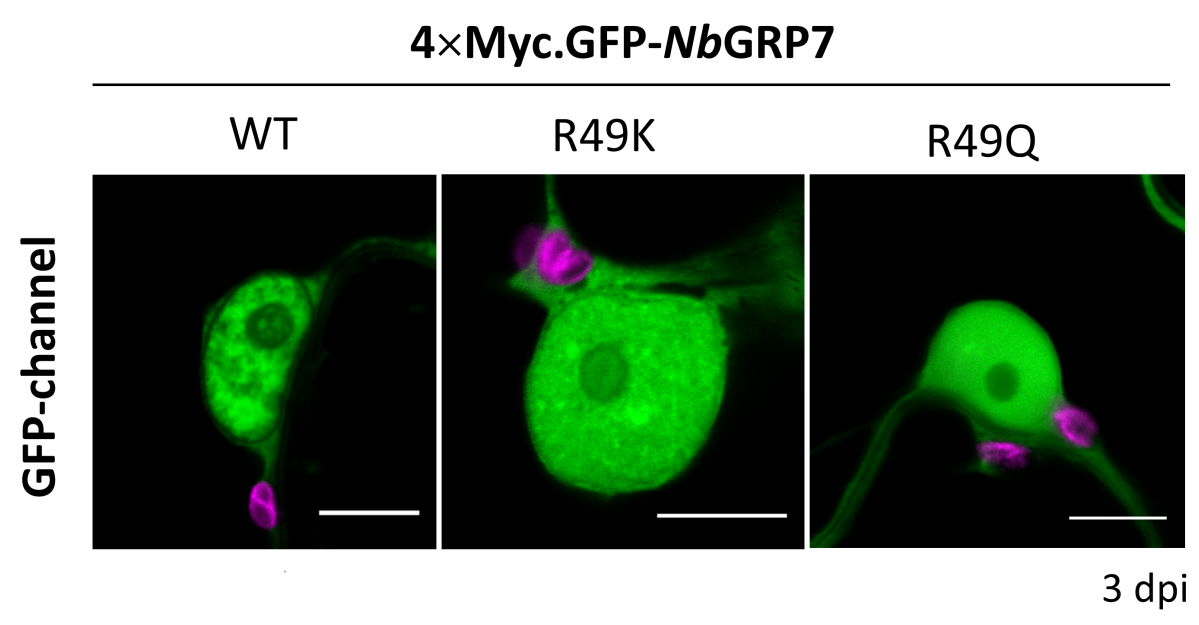


Supplemental Fig. S5. NbGRP7 potentiates immunity against PVX-UK3 independent of Rx1. Boxplots representing absorbance at $405 \mathrm{~nm}$, indicating levels of PVX-UK3 upon transient overexpression of NbGRP7. Bars represent the interquartile range, and the median is indicated by the crossbar. Whiskers show the maximum and minim data points respectively. Data shown is from a single representative experiment ( $n=8$ samples) with similar results from at least three independent repeats. Significance difference was calculated using Wilcoxon-Signed Rank test with $\alpha=0.05$.

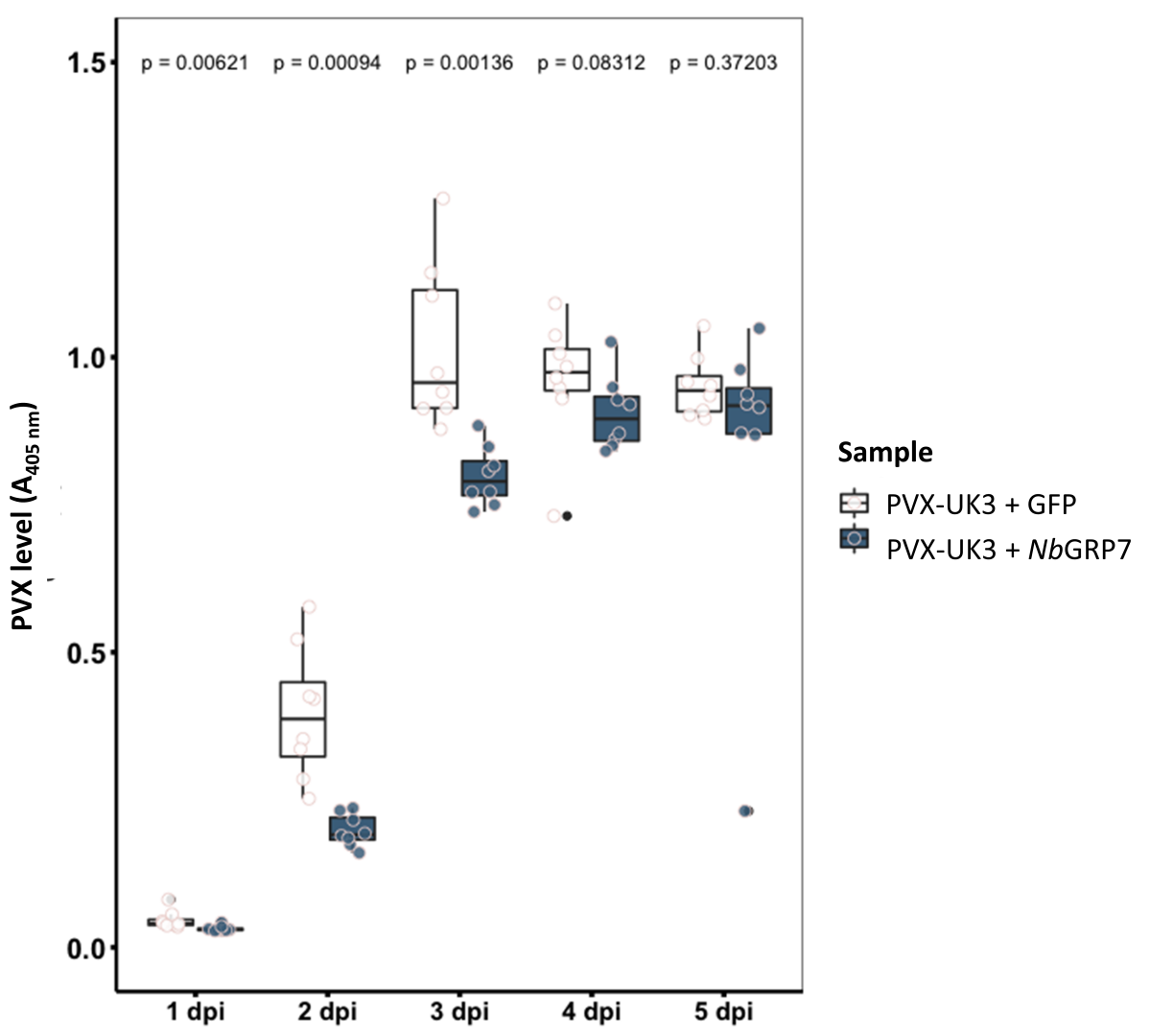


Supplemental Fig. S6. Ectopic expression of NbGRP7 affects the protein abundance of $\mathrm{R} \times 1$. Immunoblotting of protein extracts from $N$. benthamiana leaves co-expressing full-length $\mathrm{Rx} 1$ in combination with $4 \times$ Myc.GFP, $4 \times$ Myc.GFP-NbGRP7 or the hairpin silencing constructs. Leaf samples were harvested at $3 \mathrm{dpi}$. Data shown is from a single representative experiment. CBB-stained membrane of the RuBisCO protein served as loading control.

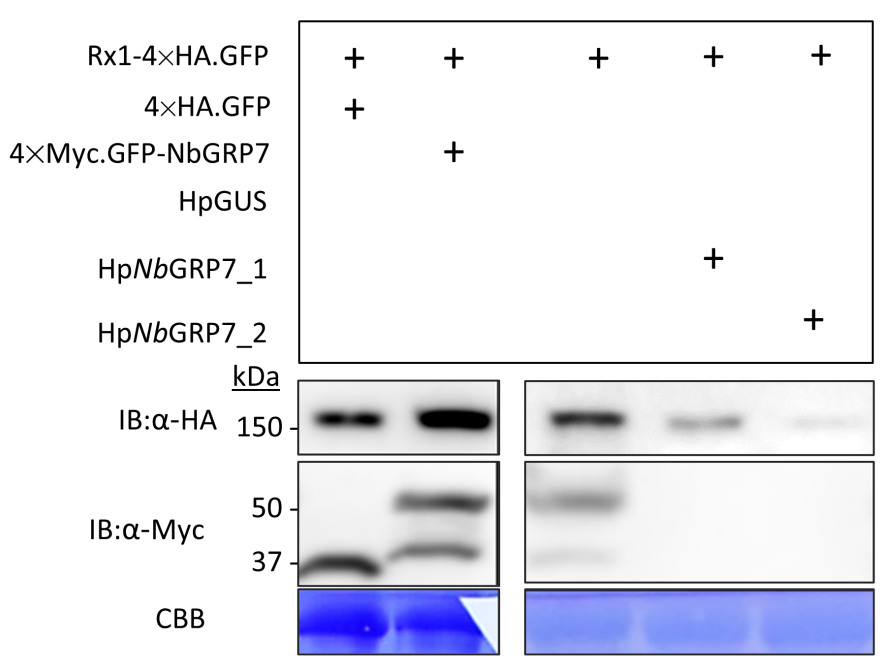


Supplemental Fig. S7. NbGRP7 does not bind to Rx1 in vivo as demonstrated by RIP. For RIP, total proteins and RNA were extracted from infiltrated leaves for co-expression of $4 \times$ Myc.GFP-NbGRP7 with FLS2-mCherry or Rx1-4XHA.GFP. Extracted materials were crosslinked and used for immunoprecipitation using GFP-trap agarose beads (Chromotek) to pull-down 4 X Myc.GFP-NbGRP7 as bait. The amount of bound RNA enriched following purification was quantified by qRT-PCR using primers to specifically amplify the receptors. Fold enrichment of Rx1 or FLS2 transcripts was normalized against input samples and calculated relative to the background $4 \times$ Myc.GFP control as described in (Marmisolle et al. 2018).

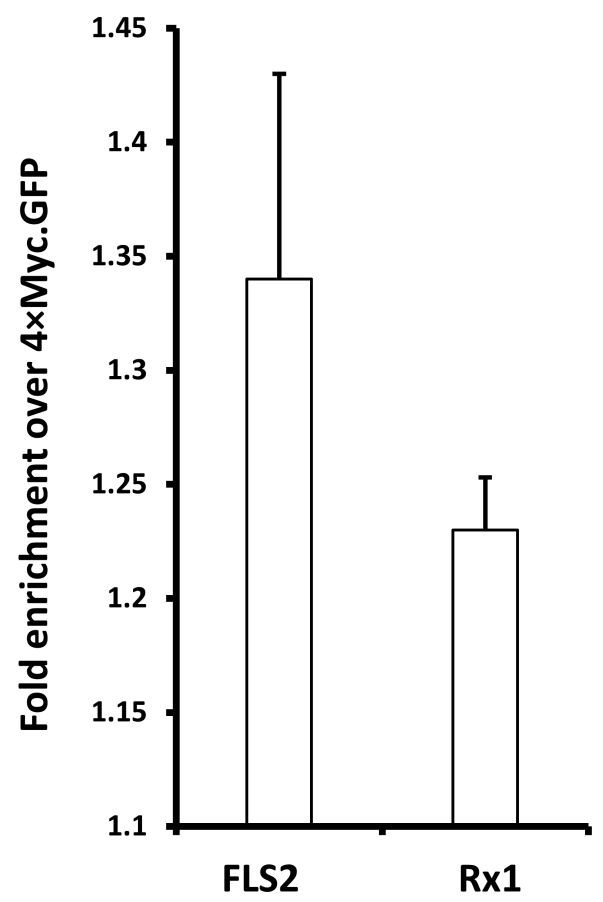


Supplemental Table S1. NbGRP7 does not bind to the 3'UTR regions of Rx1 in vitro. Fluorescence anisotropy measurements (Robs) of 5'Fam-labelled RNA probes corresponding to the 3'UTR regions of the Rx1 transcript when incubated with E. coli produced NbGRP7 or the Haemoglobin specificity control. Robs values for the buffer of each probe are included as indications of background. The results shown are representative of three independent repeats.

\begin{tabular}{llll}
\hline Probe & $75-175 \mathrm{bp}$ & $150-250 \mathrm{bp}$ & $225-280 \mathrm{bp}$ \\
\hline \multicolumn{3}{c}{ Robs } \\
\hline Buffer & 0.213 & 0.150 & 0.058 \\
Haemoglobin & 0.286 & 0.189 & 0.094 \\
NbGRP7 & 0.200 & 0.162 & 0.092 \\
\hline
\end{tabular}


Supplemental Table S2. Primers used in this study as listed according to the assays performed.

\begin{tabular}{|c|c|}
\hline \multicolumn{2}{|l|}{ Cloning } \\
\hline Primer Name & Sequence \\
\hline NbGRP7 & $\begin{array}{l}\text { F: 5' - TGGGCTACCACCGATAGAAC - 3' } \\
\text { R: 5' - TGCGTCCCTCATAGCTTTCT - 3' }\end{array}$ \\
\hline NbGRP7 R49K & $\begin{array}{l}\text { F: 5' - GAGACTGGAAGATCAAAAGGATTTGGCTTTGTT - 3' } \\
\text { R: 5' - AACAAAGCCAAATCCTTTTGATCTTCCAGTCTC - 3' }\end{array}$ \\
\hline NbGRP7 R49Q & $\begin{array}{l}\text { F: 5' - GAGACTGGAAGATCACAGGGATTTGGCTTTGTT - 3' } \\
\text { R: 5' - AACAAAGCCAAATCCCTGTGATCTTCCAGTCTC - 3' }\end{array}$ \\
\hline In vitro Transcription & \\
\hline 75-175 bp Rx1 3’UTR & $\begin{array}{l}\text { F: 5' - TAATACGACTCACTATAGGGATTATAATGTTTTGTTCGTTTTCA - 3' } \\
\text { R: 5' - CCACTGACAAATGGCTATACGAAAGATCA - 3' }\end{array}$ \\
\hline 150-250 bp Rx1 3’UTR & $\begin{array}{l}\text { F: 5' - TAATACGACTCACTATAGGGATTATAATTCTTTCGTATAGCCA - 3' } \\
\text { R: 5' - AACACACGTTACTGGAGTCTGTTCTG - 3' }\end{array}$ \\
\hline 225-280 bp Rx1 3’UTR & $\begin{array}{l}\text { F: 5' - TAATACGACTCACTATAGGGATTATAATCAGAACAGACTCCA - 3' } \\
\text { R: 5' - CCCGGGAGATTGAGGACTCC - 3' }\end{array}$ \\
\hline qPCR analysis & \\
\hline NbGRP7 qPCR & $\begin{array}{l}\text { F: 5' - GAGGATACAGTGGTGGTGGA - 3' } \\
\text { R: 5' - CTCTTGAGTAGCGGGAACCA - 3' }\end{array}$ \\
\hline $\mathrm{Rx} 1 \mathrm{qPCR}$ & $\begin{array}{l}\text { F: 5' - TTGAGGGAAGCTCGAAACAT - 3' } \\
\text { R: 5' - ACGACACCAAGCCAATTCTT - 3' }\end{array}$ \\
\hline Actin qPCR & $\begin{array}{l}\text { F: 5' - CCGAGCGGGAAATTGTTAGG - 3' } \\
\text { R: 5' - CACGGATGAGCTGGTCTTTG - 3' }\end{array}$ \\
\hline
\end{tabular}


Supplemental Table S3. Sequences of hairpin constructs used in this study. Spacer sequence used in the construct for the inverted repeat is highlight in blue. Sites for the restriction enzymes Xbal and BamHI used in sub-cloning are underlined.

\begin{tabular}{|c|c|c|}
\hline \multirow[t]{12}{*}{ HpNbGRP7 } & $207 \mathrm{bp}$ & TCTAGAAGGCGGATACAGTGGTGGTGGAGGATACAGTGGTGGTGGAGGATACAGTG \\
\hline & & GCGGCGGCGGCTATGGAGGTGGAAGACGTGAGGGTGGCTACGGTGGTGGTTATGG \\
\hline & & AGGTGGCCGTGACCGTGGATATGGTGGCGGTTATGGCGGTGGTGATGGTGGTTCCC \\
\hline & & GCTACTCAAGAGGTGGTGGTGCATCCGAGGGAAGCTGGAGGAATTAACACTGCACG \\
\hline & & GTATGCTCCTCTTCTTGTTCATGGTCATGATCCTTATATGAGCAGGGAAAGTCCAGTTT \\
\hline & & AGACTTGTAGTTAGTTACTCTTCGTTATAGGATTTGGATTTCTTGCGTGTTTATGGTTTTA \\
\hline & & GTTTCCCTCCTTTGATGAATAAAATTGAATCTTGTATGAGTTTCATATCCATGTTGTGAA \\
\hline & & TCTTTTTGGAGACGCAGCTAGTAATTCCTCCAGCTTCCCTCGGATGCACCACCACCTC \\
\hline & & TTGAGTAGCGGGAACCACCATCACCACCGCCATAACCGCCACCATATCCACGGTCA \\
\hline & & CGGCCACCTCCATAACCACCACCGTAGCCACCCTCACGTCTTCCACCTCCATAGCC \\
\hline & & GCCGCCGCCACTGTATCCTCCACCACCACTGTATCCTCCACCACCACTGTATCCGCC \\
\hline & & TGGATCC \\
\hline \multirow[t]{11}{*}{ HpNbGRP7_2 } & $200 \mathrm{bp}$ & TCTAGAATTCACATCAGCTCTTCTCTTAATTAATTAACTCTCTCTTGTTTGTTTACATTTAT \\
\hline & & AATCTCTTTCAAGAAAAAAGAGAAAGAAAAACAATGGCAGCTGAGGTTGAGTACAGG \\
\hline & & TGCTTCGTAGGTGGGCTGGCATGGGCTACCACTGATAGAACGTTAGGAGATGCTTTT \\
\hline & & GCTCACTACGGCGAAGTTGTCGACTCGAAGACACTGCACGGTATGCTCCTCTTCTTG \\
\hline & & TTCATGGTCATGATCCTTATATGAGCAGGGAAAGTCCAGTTTAGACTTGTAGTTAGTTA \\
\hline & & CTCTTCGTTATAGGATTTGGATTTCTTGCGTGTTTATGGTTTTAGTTTCCCTCCTTTGATG \\
\hline & & AATAAAATTGAATCTTGTATGAGTTTCATATCCATGTTGTGAATCTTTTTGGAGACGCAG \\
\hline & & CTAGCTTCGAGTCGACAACTTCGCCGTAGTGAGCAAAAGCATCTCCTAACGTTCTATC \\
\hline & & AGTGGTAGCCCATGCCAGCCCACCTACGAAGCACCTGTACTCAACCTCAGCTGCCAT \\
\hline & & TGTTTTTCTTTCTCTTTTTTCTTGAAAGAGATTATAAATGTAAACAAACAAGAGAGAGTTA \\
\hline & & ATTAATTAAGAGAAGAGCTGATGTGAATGGATCC \\
\hline \multirow[t]{9}{*}{ HpGUS } & $145 \mathrm{bp}$ & TCTAGACCGCGTCTTTGATCGCGTCAGCGCCGTCGTCGGTGAACAGGTATGGAATTT \\
\hline & & CGCCGATTTTGCGACCTCGCAAGGCATATTGCGCGTTGGCGGTAACAAGAAAGGGA \\
\hline & & TCTTCACTCGCGACCGCAAACCGAAGTCGGCGGCTTTTACACTGCACGGTATGCTCC \\
\hline & & TCTTCTTGTTCATGGTCATGATCCTTATATGAGCAGGGAAAGTCCAGTTTAGACTTGTA \\
\hline & & GTTAGTTACTCTTCGTTATAGGATTTGGATTTCTTGCGTGTTTATGGTTTTAGTTTCCCTC \\
\hline & & CTTTGATGAATAAAATTGAATCTTGTATGAGTTTCATATCCATGTTGTGAATCTTTTTGCA \\
\hline & & GACGCAGCTAGAAAAGCCGCCGACTTCGGTTTGCGGTCGCGAGTGAAGATCCCTTT \\
\hline & & CTTGTTACCGCCAACGCGCAATATGCCTTGCGAGGTCGCAAAATCGGCGAAATTCCA \\
\hline & & TACCTGTTCACCGACGACGGCGCTGACGCGATCAAAGACGCGGGGATCC \\
\hline
\end{tabular}




\section{References}

Arntzen, F. K., Vinke, J. H., and Hoogendoorn, J. (1993), Inheritance and Level of Resistance to Potato Cyst Nematodes (Globodera pallida), Derived from Solanum tuberosum spp. In: Durability of Disease Resistance. Ed. by T. Jacobs and J. E. Parlevliet. Springer Netherlands.

Balint-Kurti, P. (2019), The plant hypersensitive response: concepts, control and consequences. Molecular Plant Pathology. 20(8): p. 1163-1178.

Bendahmane, A., Kanyuka, K., and Baulcombe, D. C. (1999), The Rx gene from potato controls separate virus resistance and cell death responses. Plant Cell. 11.

Bendahmane, A., Köhm, B. A., Dedi, C., and Baulcombe, D. C. (1995), The coat protein of potato virus X is a strain-specific elicitor of Rx1-mediated virus resistance in potato. The Plant Journal. 8(6): p. 933-941.

Bentham, A. R., Zdrzalek, R., De la Concepcion, J. C., and Banfield, M. J. (2018), Uncoiling CNLs: Structure/Function Approaches to Understanding CC Domain Function in Plant NLRs. Plant \& cell physiology. 59(12): p. 2398-2408.

Bezerra-Neto, J. P., Araújo, F. C., Ferreira-Neto, J. R. C., Silva, R. L. O., Borges, A. N. C., Matos, M. K. S., Silva, J. B., Silva, M. D., Kido, E. A., and Benko-Iseppon, A. M. (2020), NBS-LRR genes - Plant health sentinels: Structure, roles, evolution and biotechnological applications. In: Applied Plant Biotechnology for Improving Resistance to Biotic Stress. Ed. by P. Poltronieri and Y. Hong. Academic Press, p. 63-120.

Bhattacharjee, S., Zamora, A., Azhar, M. T., Sacco, M. A., Lambert, L. H., and Moffett, P. (2009), Virus resistance induced by NB-LRR proteins involves Argonaute4-dependent translational control. The Plant Journal. 58(6): p. 940-951.

Bieri, S., Mauch, S., Shen, Q. H., Peart, J., Devoto, A., Casais, C., Ceron, F., Schulze, S., Steinbiss, H. H., Shirasu, K., and Schulze-Lefert, P. (2004), RAR1 positively controls steady state levels of barley MLA resistance proteins and enables sufficient MLA6 accumulation for effective resistance. Plant Cell. 16(12): p. 3480-3495.

Van der Biezen, E. A. and Jones, J. D. (1998), Plant disease-resistance proteins and the gene-for-gene concept. Trends Biochem Sci. 23.

Carvalho, C. M., Santos, A. A., Pires, S. R., Rocha, C. S., Saraiva, D. I., Machado, J. P. B., Mattos, E. C., Fietto, L. G., and Fontes, E. P. B. (2008), Regulated nuclear trafficking of rpL10A mediated by NIK1 represents a defense strategy of plant cells against virus. PLoS pathogens. 4(12): e1000247-e1000247.

Chang, C., Yu, D., Jiao, J., Jing, S., Schulze-Lefert, P., and Shen, Q.-H. (2013), Barley MLA Immune Receptors Directly Interfere with Antagonistically Acting Transcription Factors to Initiate Disease Resistance Signaling. The Plant Cell Online. 25(3): p. 1158-1173.

Ciuzan, O., Hancock, J., Pamfil, D., Wilson, I., and Ladomery, M. (2015), The evolutionarily conserved multifunctional glycine-rich RNA-binding proteins play key roles in development and stress adaptation. Physiologia Plantarum. 153(1): p. 1-11.

Fenyk, S., Townsend, P. D., Dixon, C. H., Spies, G. B., de San Eustaquio Campillo, A., Slootweg, E. J., Westerhof, L. B., Gawehns, F. K. K., Knight, M. R., Sharples, G. J., Goverse, A., Pålsson, L.-O., Takken, F. L. W., and Cann, M. J. (2015), The Potato Nucleotide-binding Leucine-rich Repeat (NLR) Immune Receptor Rx1 Is a Pathogen-dependent DNA-deforming Protein. The Journal of biological chemistry. 290(41): p. 24945-24960. ppublish.

Fu, Z. Q., Guo, M., Jeong, B.-r., Tian, F., Elthon, T. E., Cerny, R. L., Staiger, D., and Alfano, J. R. (2007), A type III effector ADP-ribosylates RNA-binding proteins and quells plant immunity. Nature. 447(7142): p. 284-288.

De la Fuente van Bentem, S., Vossen, J. H., de Vries, K. J., van Wees, S., Tameling, W. I. L., Dekker, H. L., de Koster, C. G., Haring, M. A., Takken, F. L. W., and Cornelissen, B. J. C. (2005), Heat shock protein 90 and its co-chaperone 
protein phosphatase 5 interact with distinct regions of the tomato I-2 disease resistance protein. The Plant journal : for cell and molecular biology. 43(2): p. 284-298. ppublish.

Gilbert, J., Spillane, C., Kavanagh, T. A., and Baulcombe, D. C. (1998), Elicitation of Rx-Mediated Resistance to PVX in Potato Does Not Require New RNA Synthesis and May Involve a Latent Hypersensitive Response. Molecular Plant-Microbe Interactions ${ }^{\circledR} .11(8)$ : p. 833-835.

Hackmann, C., Korneli, C., Kutyniok, M., Köster, T., Wiedenlübbert, M., Müller, C., and Staiger, D. (2014), Salicylic acid-dependent and -independent impact of an RNA-binding protein on plant immunity. Plant, Cell \& Environment. 37(3): p. 696-706.

Hao, W., Collier, S. M., Moffett, P., and Chai, J. (2013), Structural basis for the interaction between the potato virus $X$ resistance protein $(\mathrm{Rx})$ and its cofactor Ran GTPase-activating protein 2 (RanGAP2). The Journal of biological chemistry. 288(50): p. 35868-35876.

Huh, S. U. and Paek, K.-H. (2013), Plant RNA binding proteins for control of RNA virus infection. Frontiers in Physiology. 4(397).

Jaubert, M., Bhattacharjee, S., Mello, A. F., Perry, K. L., and Moffett, P. (2011), ARGONAUTE2 mediates RNA-silencing antiviral defenses against Potato virus $X$ in Arabidopsis. Plant Physiol. 156(3): p. 1556-64.

Jones, J. D. and Dangl, J. L. (2006), The plant immune system. Nature. 444(7117): p. 323-9.

Jones, J. D. G., Vance, R. E., and Dangl, J. L. (2016), Intracellular innate immune surveillance devices in plants and animals. Science. 354(6316): aaf6395.

Kapos, P., Devendrakumar, K. T., and Li, X. (2019), Plant NLRs: From discovery to application. Plant Science. 279: p. 3-18.

Kim, J. S., Jung, H. J., Lee, H. J., Kim, K. A., Goh, C.-H., Woo, Y., Oh, S. H., Han, Y. S., and Kang, H. (2008), Glycine-rich RNA-binding protein 7 affects abiotic stress responses by regulating stomata opening and closing in Arabidopsis thaliana. The Plant journal : for cell and molecular biology. 55(3): p. 455-466. ppublish.

Künstler, A., Bacsó, R., Gullner, G., Hafez, Y. M., and Király, L. (2016), Staying alive - is cell death dispensable for plant disease resistance during the hypersensitive response? Physiological and Molecular Plant Pathology. 93: p. $75-84$.

Lai, Y. and Eulgem, T. (2018), Transcript-level expression control of plant NLR genes. Molecular Plant Pathology. 19(5): p. $1267-1281$.

Lee, H. J., Kim, J. S., Yoo, S. J., Kang, E. Y., Han, S. H., Yang, K.-Y., Kim, Y. C., McSpadden Gardener, B., and Kang, H. (2012), Different roles of glycine-rich RNA-binding protein7 in plant defense against Pectobacterium carotovorum, Botrytis cinerea, and tobacco mosaic viruses. Plant Physiology and Biochemistry. 60: p. 46-52.

Leibman-Markus, M., Pizarro, L., Bar, M., Coaker, G., and Avni, A. (2018), NRC proteins - a critical node for pattern and effector mediated signaling. Plant signaling \& behavior. 13(8): e1507404-e1507404.

Leister, R. T., Dahlbeck, D., Day, B., Li, Y., Chesnokova, O., and Staskawicz, B. J. (2005), Molecular genetic evidence for the role of SGT1 in the intramolecular complementation of Bs2 protein activity in Nicotiana benthamiana. Plant Cell. 17(4): p. 1268-1278.

Li, F., Pignatta, D., Bendix, C., Brunkard, J. O., Cohn, M. M., Tung, J., Sun, H., Kumar, P., and Baker, B. (2012), MicroRNA regulation of plant innate immune receptors. Proc Natl Acad Sci U S A. 109(5): p. 1790-5.

Marmisolle, F. E., García, M. L., and Reyes, C.A. (2018), RNA-binding protein immunoprecipitation as a tool to investigate plant miRNA processing interference by regulatory proteins of diverse origin. Plant methods. 14: p. 9-9.

Miura, K. and Furumoto, T. (2013), Cold signaling and cold response in plants. International journal of molecular sciences. 14(3): p. 5312-5337. epublish. 
Nicaise, V., Joe, A., Jeong, B.-r., Korneli, C., Boutrot, F., Westedt, I., Staiger, D., Alfano, J. R., and Zipfel, C. (2013), Pseudomonas HopU1 modulates plant immune receptor levels by blocking the interaction of their mRNAs with GRP7. The EMBO Journal. 32(5): p. 701-712.

Padmanabhan, M. S., Ma, S., Burch-Smith, T. M., Czymmek, K., Huijser, P., and Dinesh-Kumar, S. P. (2013), Novel positive regulatory role for the SPL6 transcription factor in the N TIR-NB-LRR receptor-mediated plant innate immunity. PLoS pathogens. 9(3): e1003235. ppublish.

Pay, A., Resch, K., Frohnmeyer, H., Fejes, E., Nagy, F., and Nick, P. (2002), Plant RanGAPs are localized at the nuclear envelope in interphase and associated with microtubules in mitotic cells. Plant J. 30.

Sacco, M. A., Koropacka, K., Grenier, E., Jaubert, M. J., Blanchard, A., Goverse, A., Smant, G., and Moffett, P. (2009), The cyst nematode SPRYSEC protein RBP-1 elicits Gpa2- and RanGAP2-dependent plant cell death. PLoS Pathog. 5

Sacco, M. A., Mansoor, S., and Moffett, P. (2007), A RanGAP protein physically interacts with the NB-LRR protein Rx, and is required for Rx-mediated viral resistance. Plant J. 52(1): p. 82-93.

Saqlan Naqvi, S. M., Park, K.-S., Yi, S.-Y., Lee, H.-W., Hae Bok, S., and Choi, D. (1998), A glycine-rich RNA-binding protein gene is differentially expressed during acute hypersensitive response following Tobacco Mosaic Virus infection in tobacco. Plant Molecular Biology. 37(3): p. 571-576.

Sarris, P. F., Cevik, V., Dagdas, G., Jones, J. D., and Krasileva, K. V. (2016), Comparative analysis of plant immune receptor architectures uncovers host proteins likely targeted by pathogens. BMC Biol. 14(1): p. 016-0228.

Schmittgen, T. D. and Livak, K. J. (2008), Analyzing real-time PCR data by the comparative C(T) method. Nat Protoc. 3(6): p. 1101-8

Schouten, A., Roosien, J., de Boer, J. M., Wilmink, A., Rosso, M.-N., Bosch, D., Stiekema, W. J., Gommers, F. J., Bakker, J., and Schots, A. (1997), Improving scFv antibody expression levels in the plant cytosol 1. FEBS Letters. 415(2): p. 235-241.

Shen, Q.-H., Saijo, Y., Mauch, S., Biskup, C., Bieri, S., Keller, B., Seki, H., Ülker, B., Somssich, I. E., and Schulze-Lefert, P. (2007), Nuclear Activity of MLA Immune Receptors Links Isolate-Specific and Basal Disease-Resistance Responses. Science. 315(5815): p. 1098.

Shin, Y.-J., Castilho, A., Dicker, M., Sádio, F., Vavra, U., Grünwald-Gruber, C., Kwon, T.-H., Altmann, F., Steinkellner, H., and Strasser, R. (2017), Reduced paucimannosidic N-glycan formation by suppression of a specific $\beta$-hexosaminidase from Nicotiana benthamiana. Plant biotechnology journal. 15(2): p. 197-206. ppublish.

Slootweg, E., Roosien, J., Spiridon, L. N., Petrescu, A. J., Tameling, W., Joosten, M., Pomp, R., van Schaik, C., Dees, R., Borst, J. W., Smant, G., Schots, A., Bakker, J., and Goverse, A. (2010), Nucleocytoplasmic distribution is required for activation of resistance by the potato NB-LRR receptor $\mathrm{Rx} 1$ and is balanced by its functional domains. Plant Cell. 22(12): p. 4195-215.

Slootweg, E., Koropacka, K., Roosien, J., Dees, R., Overmars, H., Lankhorst, R. K., van Schaik, C., Pomp, R., Bouwman, L., Helder, J., Schots, A., Bakker, J., Smant, G., and Goverse, A. (2017), Sequence Exchange between Homologous NB-LRR Genes Converts Virus Resistance into Nematode Resistance, and Vice Versa. Plant physiology. 175(1): p. 498-510.

Slootweg, E. J., Spiridon, L. N., Martin, E. C., Tameling, W. I. L., Townsend, P. D., Pomp, R., Roosien, J., Drawska, O., Sukarta, O. C. A., Schots, A., Borst, J. W., Joosten, M. H., Bakker, J., Smant, G., Cann, M. J., Petrescu, A.-J., and Goverse, A. (2018), Distinct Roles of Non-Overlapping Surface Regions of the Coiled-Coil Domain in the Potato Immune Receptor Rx1. Plant Physiology. 178(3): p. 1310-1331.

Spector, D. L. and Lamond, A. I. (2011), Nuclear speckles. Cold Spring Harb Perspect Biol. 3(2). 
Sukarta, O. C. A., Slootweg, E. J., and Goverse, A. (2016), Structure-informed insights for NLR functioning in plant immunity. Seminars in Cell \& Developmental Biology. 56: p. 134-149.

Sun, Y., Zhu, Y.-X., Balint-Kurti, P. J., and Wang, G.-F. (2020), Fine-Tuning Immunity: Players and Regulators for Plant NLRs. Trends in Plant Science. 25(7): p. 695-713.

Tameling, W. I. L. and Baulcombe, D. C. (2007), Physical association of the NB-LRR resistance protein Rx with a Ran GTPase-activating protein is required for extreme resistance to Potato virus X. Plant Cell. 19(5): p. 1682-94.

Tameling, W. I. L., Nooijen, C., Ludwig, N., Boter, M., Slootweg, E., Goverse, A., Shirasu, K., and Joosten, M. (2010), RanGAP2 Mediates nucleocytoplasmic partitioning of the NB-LRR immune receptor Rx in the solanaceae. Thereby dictating Rx function. Plant Cell. 22.

Tcherkezian, J., Brittis, P. A., Thomas, F., Roux, P. P., and Flanagan, J. G. (2010), Transmembrane receptor DCC associates with protein synthesis machinery and regulates translation. Cell. 141(4): p. 632-644.

Tian, D., Wang, J., Zeng, X., Gu, K., Qiu, C., Yang, X., Zhou, Z., Goh, M., Luo, Y., Murata-Hori, M., White, F. F., and Yin, Z. (2014), The rice TAL effector-dependent resistance protein XA10 triggers cell death and calcium depletion in the endoplasmic reticulum. The Plant cell. 26(1): p. 497-515.

Townsend, P. D., Dixon, C. H., Slootweg, E. J., Sukarta, O. C. A., Yang, A. W. H., Hughes, T. R., Sharples, G. J., Pålsson, L. O., Takken, F. L. W., Goverse, A., and Cann, M. J. (2018), The intracellular immune receptor Rx1 regulates the DNA-binding activity of a Golden2-like transcription factor. J Biol Chem. 293(9): p. 3218-3233.

Van der Vossen, E. A. G., van der Voort, J., Kanyuka, K., Bendahmane, A., Sandbrink, H., Baulcombe, D. C., Bakker, J., Stiekema, W. J., and Klein-Lankhorst, R. M. (2000), Homologues of a single resistance-gene cluster in potato confer resistance to distinct pathogens: a virus and a nematode. Plant J. 23.

Wang, J., Hu, M., Wang, J., Qi, J., Han, Z., Wang, G., Qi, Y., Wang, H. W., Zhou, J. M., and Chai, J. (2019), Reconstitution and structure of a plant NLR resistosome conferring immunity. Science. 364(6435).

Zhai, K., Deng, Y., Liang, D., Tang, J., Liu, J., Yan, B., Yin, X., Lin, H., Chen, F., Yang, D., Xie, Z., Liu, J.-Y., Li, Q., Zhang, L., and He, Z. (2019), RRM Transcription Factors Interact with NLRs and Regulate Broad-Spectrum Blast Resistance in Rice. Molecular Cell. 74(5): 996-1009.e7.

Zinovieva, S. V., Vasyukova, N. I., Udalova, Z. V., Gerasimova, N. G., and Ozeretskovskaya, O. L. (2011), Involvement of salicylic acid in induction of nematode resistance in plants. Biology Bulletin. 38(5): p. 453.

Zipfel, C. (2014), Plant pattern-recognition receptors. Trends in Immunology. 35(7): p. 345-351. 


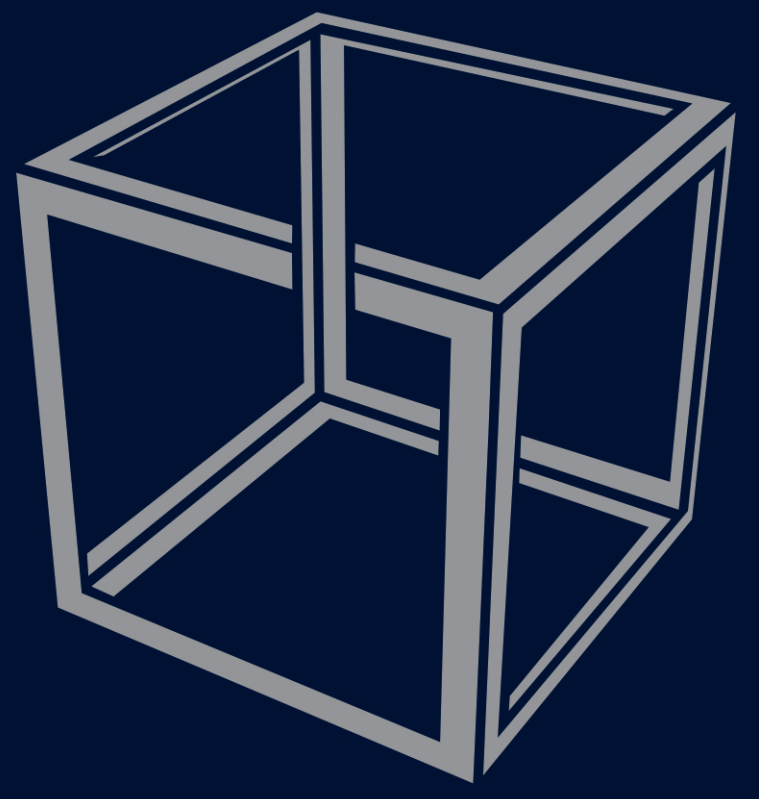

Octavina C. A Sukarta

Laboratory of Nematology, Wageningen University \& Research, Wageningen, The Netherlands. 
Chapter

7

General Discussion 


\section{PREFACE}

Plant NB-LRR immune receptors make a remarkable academic pursuit, owing to their high agronomic value but also at a fundamental level. They have deceivingly simple structures but are, in reality, complex in function. Both in plants and animals, NB-LRRs are wired to surveil alien invaders and trigger the appropriate defence response to curtail damage inflicted on the host (van Wersch et al. 2020). Since they were first described 20 years ago, we have progressed in our understanding of how this fascinating group of receptor proteins functions (Chapters $1 \& 2$ ). Extraordinarily, however, our knowledge of the series of events linking effector recognition to defence output remains mostly fragmentary. Thus, to shed light on mechanisms of plant NB-LRR function, in this dissertation, I investigated the roles of intra and interdomain interactions in regulating the activity of the potato CC-NB-LRR Rx1 immune receptor (Thesis Objectives). This involved exploring the co-operative assembly of the various building blocks that make up an NB-LRR protein. These intradomain associations help to keep $\mathrm{R} \times 1$ in an autoinhibited state in unchallenged cells while still allowing the receptor to swiftly relay defence signaling upon microbial detection (Chapter 3 ). In a second arm of the thesis, I stepped beyond the level of a single receptor protein by characterizing novel components belonging to the Rx1 complex, either in the cytoplasm or nucleus (Chapters 4,5 \& 6). By addressing both aims, I illustrate that $\mathrm{R} \times 1$ functions as an analog rather than a digital switch whereby the strength and specificity of its immune response can be fine-tuned to adapt to a rapidly changing environment. In the individual research chapters of this thesis (Chapters 3-6), I have provided a detailed discussion of the scientific context related to each main finding. Here, I intend to further link, integrate, and digest my results in the framework of current scientific discussions pertaining to Rx1 but also the broader NB-LRR field. In theme with the rest of the thesis, the chapter will center on the mechanistic and molecular aspects. I finally propose a refined, working model of $\mathrm{Rx} 1$ function in relation to its localization in the cell by reflecting on my main findings and drawing parallels from a few systems. However, as is usually the case in research, the works presented here incite further questions. I, therefore, close the thesis by highlighting several knowledge gaps that may shape the direction of future research concerning Rx1 but also plant NB-LRRs in general. 


\section{Beauty in the Design: No Two Soldiers are Alike}

\section{Different strengths on the battlefield}

In plant immunity, the dynamic interplay that comes with the interaction of a plant and its pathogen is often described in the setting of a molecular warfare. The host is akin to a country, which deploys immune receptors acting as soldiers in defence against an army of invading pathogens. As many more commonalities can be drawn in this regard, I will maintain the analogy of a warfare as a conceptual supplement in this chapter. To begin with, the strength of a soldier on the battlefield lies not necessarily at the weapon he carries, but rather his innate built. I, therefore, start my discussion by revisiting the most rudimentary portion of a battleplan: the makings of a soldier. This is analogous to the modular parts of an NB-LRR receptor. Even in much earlier studies, elucidating the function and co-operation of independent receptor (sub)domains was recognized as an essential aspect of plant NB-LRR research (Moffett et al. 2002, Rairdan et al. 2006, Bezerra-Neto et al. 2020, van Wersch et al. 2020). Due to the similar architectures of these subdomains, it was initially proposed that they may have a conserved molecular function across various receptor systems. By extension, it was therefore thought that NB-LRRs sharing some degree of structural homology might exhibit comparable mechanisms of pre and post-activation controls (McHale et al. 2006). However, differences in the functioning of NB-LRRs can be determined by subtle changes in their sequence, even at the level of single amino acid residues. This is exemplified in a study of the rice Necrotic Leaf Sheath 1 (NLS1) mutant, whereby a single substitution in the receptor can result in constitutive defence activation (Tang et al. 2011). However, the mechanistic basis by which sequence variation contributes to NB-LRR function is largely unexplored. Thus, in Chapter 3 , we probed further at this prospect by investigating domain exchangeability between the potato Rx1 and its tomato homolog (denoted as Rslyc). A striking and unexpected finding is that although these receptors bear considerable sequence similarity ( $85 \%$ at the amino acid level), the few polymorphisms that exist can strictly dictate the switch function as well as the capacity of the domains to interact. Consequently, we could not freely exchange the subdomains of Rx1 and Rslyc according to the framework established for Rx1 and Gpa2 without compromising the functionality of the receptor in trans (Slootweg et al. 2017). This finding is the more intriguing when considering that based on overall sequence identity, Rslyc and Gpa2 are equally as homologous to Rx1. 
The question therefore arises: what are the constraints limiting the functional exchangeability of $R \times 1$ and Rslyc? Based on our structural analysis, we predict that the collective nature of the polymorphisms observed in Rslyc renders it to be more biochemically divergent to $R \times 1$ than Gpa2 is. Our findings thus illustrate that even highly homologous NB-LRRs can vary in the protein configurations during their dormant and activated states and hence, modes of activation. This has important implications on resistance engineering efforts where the focus is to use a well-characterized R protein as a proxy to guide the modification of a non-functional allele (e.g. by targeted mutagenesis). Thus, it appears that we may not always be able to directly translate knowledge from a single R-gene to another, even if it is a close homolog. This is further reinforced in the study of R3a and I2, whereby the direct transfer of autoactivating mutations identified in R3a does not result in an expanded response spectrum of $\mathrm{I} 2$ but instead requires the introduction of an additional sensitizing mutation in the same region (Giannakopoulou et al. 2015). Information retrieved from the primary sequence must, therefore, be coupled with structural knowledge of the protein at hand and the complexes formed during the off, primed, and on states. This would also rely on an iterative exchange of insights between in silico modeling and experimental work.

One naturally has to wonder, why is there a lack of uniformity in how NB-LRRs need to be activated? The answer likely stems from a combination of factors. One plausible scenario is that this relates to the stoichiometry of the receptor relative to its microbial signal in the cell, which may vary across different NB-LRR systems. For instance, in situations where the eliciting factors are present less abundantly, receptors that are more trigger-happy or sensitive with less stringent intradomain controls might be favorable. This is, therefore, also linked to differences in the various modes of pathogen invasion and effector delivery. Likewise, diversity in the structural and functional features of the effector (including post-translational modifications) may also be a contributing factor. These traits can directly affect binding affinity to the NB-LRR or (perturbation of) recognition co-factor and hence, the capacity of the effector to act as an eliciting-stimulus. Lastly, we cannot rule out that variation in the genetic background of the host plant may present unique cellular constraints/requirements (e.g. co-factors) that favor certain modes of receptor activation.

Soldiers of diverse specters build an army

Surprisingly, the few polymorphisms identified in Chapter 3 have also allowed us to use Rslyc as an excellent platform to resolve novel regions/interfaces needed for fine-tuning Rx1 activity. Based 
on earlier works, compatibility at the NB-ARC/LRR regions was identified as a critical structural determinant for the reconstitution of a functional Rx1 receptor (Rairdan et al. 2006, Slootweg et al. 2013). Using mainly trans-complementation and homology modeling approaches, we discovered two additional components that may be required for modulating the receptor complex and/or the central nucleotide switch. The first refers to compatibility between the CC and LRR domains (Chapter 3 Fig. 6). An interaction between the distal ends of the receptor has not been observed previously for Rx1 but is reported to help maintain autoinhibition in the Arabidopsis HOPZ-ACTIVATED RESISTANCE1 (ZAR1) (Baudin et al. 2017). Furthermore, homology modeling predicts that a linker between the CC and NB domains may act as a barricading hinge that prevents the inadvertent release of the nucleotide in the dormant state of the receptor (Chapter 3 Fig. 7).

The identification of the aforementioned features as novel structural determinants means that intradomain requirements of $\mathrm{R} \times 1$ are multi-layered and spread over the entire receptor unit. In $\mathrm{R} \times 1$, the NB-ARC domain acts as the principal signaling moiety, and this function is modulated by its interactions with adjacent subdomains such as the LRR (Rairdan et al. 2008). Given the proximity of both the linker and CC/LRR interface to the central NB-ARC domain, it is imaginable that they may impact its signaling capacity by exerting an influence on the 'priming' of the NB-ARC for activation. This process involves the receptor domains to form a perfect fit, thereby permitting the nucleotide-binding pocket to adopt a conformation that is precariously repressed such that it is readily activated when needed (Rairdan et al. 2008). However, we are not fully aware of the relative significance of both the linker and CC/LRR interface for regulating the function of $R \times 1$ in this state. In this respect, the homology models and surface characteristics identified in Chapter 3 provide a testable basis for more detailed mutagenesis studies to determine their functional contributions. Notably, these domain interactions alone are also unlikely to be sufficient for modulating the function of Rx1. For instance, the CC of Rslyc was reported to associate with heterogenous LRRs in trans (Chapter 3 Fig. 6). Likewise, $\mathrm{Rx} 1$ has been shown to interact with domains of homologous NB-LRRs including Bs2 and HRT (Rairdan et al. 2006). This degree of promiscuity suggests that physical interaction in the receptor unit does not always equate functional communication. Other factors (e.g. host proteins) are thus needed to successfully reconstitute the functional signaling competent conformation/state of the receptor. 
Taken together, we can extrapolate several important paradigms based on the works described in Chapter 3. Primarily, each domain of an NB-LRR receptor appears to possess the capacity to regulate the folding and activity of the receptor i.e. there is no clear division in tasks between the subdomains. The observation that several intradomain controls exist also puts into question their individual weight for regulating receptor function. Imaginably, some structural constraints may exert more prominent control than others, which may also alter depending upon the stage of the receptor activation/deactivation cycle. Overall, however, it is likely that their concerted activity rather than these individual structural controls matters. For most NB-LRR systems, it is unclear how the various intramolecular contact points interact and influence one another in time and space. One can envision that some may act synergistically while others are antagonistic to keep the overall regulatory potential of the receptor in balance. Given that NB-LRRs are relatively compact modules, it is also conceivable that a single domain could share multiple contact points with other regions in the protein such that any change is mutually adjusted. For example, a destabilization in one interface would be accommodated by another in close proximity. This leads to the well-known concept of domain co-evolution. Sound knowledge of the dynamic interplay between the various regulatory contact points in an NB-LRR receptor is thus crucial, which will require more detailed studies by domain exchange approaches and the introduction of epistatic mutations. The resultant findings of these studies should also be complemented with structural data whereby the emergence of powerful techniques such as Cryo-Electron Microscopy is pivotal to capture three-dimensional snapshots of stage-specific conformational changes.

A final emphasis should also be made on the observation that intradomain requirements can vary profoundly even between highly similar receptor systems. As one tends to look for the general pattern in science, such findings prompt the need to study beyond individual NB-LRRs (Chapter 2). This will become particularly relevant with the development of improved methods for NB-LRR discovery that have spurred a growing catalog of novel receptor-types, thereby adding to the heterogeneity of receptor domain functions (Bezerra-Neto et al. 2020, Lolle et al. 2020). In conclusion, although all soldiers are trained for the same purpose, their potential can differ immensely from one soldier to the next. As an army, however, they can together provide plant cells with immunity to a wide range of intruders. Appreciating this diversity will allow us to conjure more creative solutions for breeding and engineering resistance. 


\section{Equipped for Battle: Weapons Galore}

\section{Collecting Ammunition}

When a soldier steps into the battlefield, he is well-equipped with the necessary provision and ammunition. In a similar vein, NB-LRR receptors are not autonomous beings as accessory proteins are needed to regulate their folding, localization, and/or signaling (Bezerra-Neto et al. 2020, van Wersch et al. 2020, Sun et al. 2020). Given that plant NB-LRRs are composed of only a few modular units, it is understandable that they recruit other co-factors for their function. However, aside from RanGAP2, GLK1, and the molecular chaperones SGT1 and RAR1, no other proteins are known to be definitively required for Rx1 function (Sacco et al. 2007, Tameling et al. 2007, Townsend et al. 2018, Slootweg et al. 2010). This may indicate that a shorter signaling route is needed by $R \times 1$ to initiate defence than thought originally. Such hypotheses are in line with the finding that DNA is a direct molecular target of the Rx1 NB-ARC domain upon specific activation by PVX-CP (Fenyk et al. 2015). Alternatively, the scarcity of Rx1-interacting proteins reported to date may be the outcome of difficulties in their identification. Efforts to resolve the Rx1 heterocomplex have spanned for almost over a decade and continued in the present study. These screening efforts include a combination of genetic and biochemical/proteomics-based assays (Tameling et al. 2007, Sacco et al. 2007, Townsend et al. 2018). Similar to other R-genes, fishing for proteins associated to $R \times 1$ is notoriously challenging due to the low abundance, instability, and difficulty of expressing the full-length receptor (Sun et al. 2020). Furthermore, the lack of an efficient molecular toolkit impedes downstream functional studies in the native potato background. In this thesis, we have, therefore, adopted a heterologous screening approach via either yeast-two hybrid or overexpression-based affinity purification in Nicotiana benthamiana (Chapters 5 and 6). Given the artificial nature of both assays, an important caveat is the ability to distinguish bona fide interactors from promiscuous plant proteins. As illustrated in our research chapters, it has, therefore, become a standard to enforce additional in vitro and in planta assays to validate the interactions reported in a pairwise fashion.

To circumvent the low expression levels associated with full-length $R \times 1$, we opted to employ the $\mathrm{R} \times 1-\mathrm{CC}$ alone as bait in our screening efforts (Chapters 5 and 6 ). In hindsight, the use of a truncated domain may not fully reflect the activation status of $\mathrm{R} \times 1$. This is relevant as the nucleotide-dependent conformation and folding of an NB-LRR may affect the ability of the receptor to form heterocomplexes, for instance, by exposing and/or occluding (new) surfaces for interactions (Lukasik-Shreepaathy et al. 
2012 ). In the natural setting, one also does not expect infection nor defence to be a monotonous process but instead exhibits phases that may oscillate over time. In a recent study, for instance, the activation of Rx1 was shown to trigger phased and discreet immune responses (Knip et al. 2019). Depending upon the functional stage of the receptor, distinct host-factors may, therefore, come into play at different time points and are further spatially separated. A focus of future studies should, therefore, be to capture the differential signaling dynamics post-activation. This would require more elaborate and sensitive time-course experiments to resolve interactors associated with activated NB-LRR proteins, which could also potentially form complexes more transiently. For Rx1, the controlled, inducible system and response timeline established by Knip et al. 2019 would serve as an important starting point for such experiments. This can be coupled with exciting technological developments such as proximity-based labeling methods by Biotinylation-ID to meet the demands of a better resolution in demonstrating (transient) protein-protein interactions (Khan et al. 2018). In such approaches, the bait is engineered as a fusion with the biotin ligase BirA, which has been mutated to biotinylate any proteins within a $10 \mathrm{~nm}$ radius. When expressed in planta, any endogenous protein within this vicinity could later be efficiently purified with a streptavidin-based column. The utility of this approach has been demonstrated in $N$. benthamiana during the successful identification of the putative E3 Ubiquitin ligase UBR7 as an interactor of the TIR domain of the $\mathrm{N}$ receptor, thereby serving as a convenient basis for future screening efforts (Zhang et al. 2019).

\section{The Arsenal}

In Chapters 5 and 6, we demonstrate that a single R protein, Rx1, can associate with diverse host components and in various compartments of the cell. A logical question would, therefore, be as to why Rx1 would co-op multiple interacting partners? The recruitment of various host proteins may relate to distinct, compartment-specific functions of $\mathrm{R} \times 1$ during different stages of its activation/deactivation cycle. It has been established that the cytoplasmic pool of Rx1 surveils for the presence and/or activity of PVX-CP (discussed more extensively in Section 3 of this chapter) (Slootweg et al. 2010). In contrast, the nuclear function of Rx1 was unclear until recently, with reports of its activity at the chromatin (Fenyk et al. 2015). The subsequent discovery that Rx1 exists as a heterocomplex with factors that possess particular DNA-binding motifs (e.g. GLK1) offers a link for the DNA binding of Rx1 to specific immune activation (Townsend et al. 2018). In Chapter 5, we further resolved this complex by identifying the histone-associated NbDBCP protein as a novel component. Based on 
functional data, we demonstrate that ectopic expression of $R \times 1$ influences the capacity of $\mathrm{NbDBCP}$ to associate with DNA, similarly to how it has been shown for GLK1 (Chapter 5 Fig. 3). A picture, therefore, emerges in which $\mathrm{R} \times 1$ acts as a master control unit, coordinating the function of proteins with specific nuclear activities towards immunity. The model proposed echoes those deduced previously for the barley Mla receptor and mammalian NLR CIITA (Chang et al. 2013, Devaiah et al. 2013). It is fascinating to note that CIITA was also shown to recruit and modulate multiple transcription factors and histone-associated enzymes (Devaiah et al. 2013). Unlike Mla and CIITA, however, Rx1 may represent a unique and isolated example as these receptors do not require DNA binding to exert their regulatory functions. What purpose does the association to DNA have for Rx1, and is there a selective advantage to do so? A rather straightforward hypothesis is that the tethering of $\mathrm{R} \times 1$ to nucleic acids allows it to be in constant proximity to its associated partners. It may also be a manner by which $\mathrm{R} \times 1$ imposes negative regulation by outcompeting specific DNA binding sites. Finally, and not mutually exclusive, an exciting theory is that since PVX can induce DNA damage, Rx1 may also sense the integrity of endogenous DNA to initiate a downstream response (discussed further in Section 4 of this chapter) (Cerovska et al. 2014).

The finding that Rx1 also forms complexes with NbGRP7 (Chapter 6) potentially links (epigenetic) processes at the DNA with post-transcriptional events. Notably, we have yet to establish physical association and functional dependency of NbGRP7 with neither NbDBCP nor GLK1. However, the local co-existence of various molecular (transcriptional/post-transcriptional) checkpoints in a single Rx1 complex would provide an efficient means to modulate the target transcript, especially during situations where a rapid response is needed such as in the event of a pathogen infection. In mammals, for instance, there is evidence that post-transcriptional processes (e.g. alternative splicing) act in tandem with and are determined by epigenetic processes (Naftelberg et al. 2015). It will, therefore, be intriguing to determine the spatio-temporal dynamics and co-operativity of the $\mathrm{R} \times 1$ nuclear complex. An important question then becomes: what targets do the regulators modulate? Histone modification has been reported to be involved in the chromatin remodeling and expression of a subset of NB-LRRs transcripts (Lee et al. 2015). For example, methylation of histones by SDG8 is important for the transcriptional control of several NB-LRRs including RPM1 and RPS5 (Palma et al. 2010). Control of $R$ protein transcript also links to the observation that NbGRP7 can stabilize 
Rx1 transcript (Chapter 6 Fig. 5). This, therefore, represents an obvious target for future research, which should link the nuclear activity of Rx1 with more in-depths transcriptomics studies.

\section{Brothers in Arms}

A soldier does not go into battle alone and is a part of a team where he fulfills a specific role. In the same manner, many plant NB-LRRs do not act as 'singletons' but band together with other NB-LRRs to confer defence (Adachi et al. 2019b). In Chapter 2, we discussed how the function of plant NB-LRRs could extend beyond the textbook definition and split into sensor and helper roles. In this respect, defence by $\mathrm{Rx} 1$ is (functionally) dependent on the paralogs of the helper NLR-REQUIRED FOR CELL DEATH (NRC) (Wu et al. 2017). Transiently silencing NRC2, NRC3, and NRC4 compromised resistance to PVX by $\mathrm{Rx} 1$ in $N$. benthamiana. By this definition, $\mathrm{R} \times 1$, therefore, acts as a classical sensor NB-LRR that is specialized to recognize the pathogen and requires the activity of its executor helper NB-LRRs (i.e. NRCs) to translate recognition events into immune signaling. However, this strict definition does not fully accommodate the ability of $R \times 1$ to bind DNA for the induction of downstream defence responses. Additionally, how can we reciprocate this with the dependency and association of Rx1 to other host-factors? The mechanistic and molecular basis by which NRCs mediate downstream signaling of their sensors is still largely unknown. As some of the interactions we identified (e.g. NbGRP7) are via in planta immunoprecipitation approaches, the possibility that the complex formations observed with $\mathrm{R} \times 1$ are mediated by other intermediary proteins such as NRCs cannot be excluded. For this reason, binding studies should be performed in (plant) cell-free systems. However, whether NRCs share the same cellular distribution and physically interact with $\mathrm{R} \times 1$ and/or its co-factors pre- and post-activation should be investigated to support the model of an NRC-dependent helper/sensor complex for Rx1. In the future, it will, therefore, be increasingly important to establish a suitable platform for demonstrating concurrent and larger heterocomplexes in planta. Likewise, does Rx1 adopt a different conformation in these various compartments and how do the interactions with its host-proteins occur in real-time? To address this, obtaining structural data of the receptor as a (transient) complex with its host targets will be key in future works.

Taken together, we have sketched a scenario wherein NB-LRRs are capable of engaging in multiple associations with the host proteome, most likely in the context of an extensive, interconnected network unique to the receptor and its interactome (helpers and co-factors alike). For example, NRCs 
are reported to form a well-supported phylogenetic superclade with their related sensor NB-LRRs in Solanaceae (Wu et al. 2017). An exciting concept to explore is thus the evolution of interactors with the receptors depending on the composition of NB-LRRs and vice versa. Thus, diversification of NB-LRR proteins is also the complex outcome of changes in the interactions with their partners. The existence of an interactome network specific to each receptor may also underpin discrepancies in the activities of Rx1 and Rslyc, whereby Rslyc may require a specific tomato-helper/co-factor to confer full-immunity (Chapter 3). Indeed, the impact of genetic background on a functional phenotype is a well-observed phenomenon in biology (Gallois et al. 2018). This is supported by a number of mechanistic studies indicating that (natural) variation of a single molecular factor can modulate the immune output on a molecular level by affecting the stability, turnover, and/or signaling of the NB-LRR (reviewed extensively in Gallois et al. 2018). The activity of an NB-LRR is, therefore, also shaped by the make-up of its interactome. Interestingly, despite the difference in modes of activation, NB-LRRs signaling is known to converge onto similar downstream components (Sun et al. 2020). Rslyc and Rx1, for instance, seem to differ in their activation mechanism but both physically interact with RanGAP2 (as is also the case for Gpa2) (Chapter 3). Hence, although different NB-LRRs need to adapt to rapidly evolving pathogens, some degree of similarity is preserved to still allow them to associate and make use of a similar pool of co-factors and immune pathways (Mazourek et al. 2009).

\section{Out in the Field: Sounding the Battle Cry}

Equipped with weapons, a soldier goes out into the battlefield. Hereby, the most important task then becomes the ability to distinguish an enemy from the crowd. Likewise, understanding how exogenous signals are perceived specifically by the receptor is crucial. In hindsight, for Rx1, fundamental questions pertaining to effector recognition still linger. Though it is clear that PVX-CP is the virus-derived factor that triggers Rx1 resistance, it is unknown whether it also constitutes the signal that is directly perceived by the receptor (Bendahmane et al. 1995). In Chapter 4, we proposed that recognition of PVX-CP occurs stepwise, which involves the initial binding of PVX-CP to RanGAP2. This was based on the finding that PVX-CP can physically associate with RanGAP2 in planta (Chapter 4 Fig. 1). However, RanGAP2 targeting also extends to the virulent effector variant and does not impose any obvious changes to its complex with Rx1 (Chapter 4 Fig. 4). We, therefore, 
hypothesized that upon binding to RanGAP2, PVX-CP is still directly perceived by Rx1 via the LRR domain. Apparently, however, the physical association of Rx1 (LRR)/PVX-CP is too transient or occurs with too low affinity to capture by immunoprecipitation approaches. Perhaps, this correlates to the overall sensitivity of the receptor, whereby more transient interactions are sufficient to release autoinhibition in receptors that are more trigger-happy. If $\mathrm{Rx} 1$ were, in fact, to recognize PVX-CP directly, this would mean that a structural feature of the coat-protein is perceived (Bendahmane et al. 1995). In this regard, it is worth noting that $\mathrm{Rx} 1$ can recognize the coat proteins of other (potex) viruses that have low homology ( $40 \%)$ to PVX-CP in transgenic N. benthamiana plants (Baurès et al. 2008). Rx1, therefore, most likely recognizes a conserved structural feature/fold of the CP to allow for multiple, direct recognition events (Duff-Farrier et al. 2016). This is reminiscent of Sw5-b that also recognizes a conserved 21 AA-peptide fragment of the NSm movement protein (Zhu et al. 2017). In this scenario, however, one has to wonder how recognition specificity is conferred. Apparently, mutations in the coat proteins of virulent breakers introduce drastic enough alterations in the CP to disrupt the recognizable portion of the conserved fold.

As we demonstrate that RanGAP2 is targeted by PVX-CP, an alternative possibility is that Rx1 directly detects modifications on RanGAP2 as postulated in the guard hypothesis (Chapter 4) (Bezerra-Neto et al. 2020). However, as discussed in Chapter 4, there are various underlying factors as to why RanGAP2 may not fit the definition of a classical guardee. As such, we have allocated a more neutral term of a 'bait' to represent its facilitative role as a recognition co-factor. In retrospect, RanGAP2 may still be 'guarded' by Rx1 from the effector targeting of its conserved function. This may underly why RanGAP2 would interact with $R \times 1$ and other Rx1-like homologs including Gpa2 and Rslyc in the first place (Chapter 3). At the moment, however, we have little empirical support that the biological function of RanGAP2 is in fact targeted by PVX-CP and whether its potential guarding is intrinsic to Rx1-type immune receptors. A better mechanistic understanding is thus required to elucidate how RanGAP2 co-operate with the LRR to allow specific effector recognition. Nonetheless, the case of RanGAP2 illustrates the need to relax our current definitions of co-factors for NB-LRR recognition. This is particularly relevant as compared to mammals that recognize PAMPs, the diversity of NB-LRR architectures in plants represents a more colorful scope of recognition mechanisms. Given that Rx1 can confer resistance to other potexvirsuses, we also do not exclude the possibility that a single NB-LRR protein, like $R \times 1$, can recognize diverse pathogens via various modes and/or baits. It is 
possible, for instance, depending upon the partners recruited, different recognition specificities can be acquired with distinct baits presenting a unique set of effector proteins. As described in Chapter 2, for example, the Arabidopsis ZAR1 recruits various Receptor-Like Cytoplasmic Kinases and pseudokinases for the recognition of distinct bacterial effectors (Jubic et al. 2019). In systems where bait proteins are required for recognition, they must, therefore, be taken into account when attempting to understand the mechanistic underpinnings of recognition specificity conferred by the LRR and vice versa.

Aside from unraveling the nature of the eliciting signal that is directly perceived by $\mathrm{R} \times 1$, a pending, decade-old question is: what is the exact (biochemical) force needed to relieve autoinhibition post-effector recognition? For $\mathrm{Rx} 1$, this cue should relieve structural restraints imposed by the NB-ARC/LRR, CC/LRR, and barricading linker to allow the receptor to progress towards an activated state. If sensing of the pathogen were to occur indirectly, disturbing the complex of the host protein with its co-receptor may suffice (Collier et al. 2009). This would then involve the transmission of signal/changes in the $\mathrm{CC}$ to the remainder of the receptor module. For $\mathrm{Rx} 1$, this is in agreement with the fact that the RanGAP2 binding sites in the CC domain lie in close vicinity to the EDVID motif required for binding of the CC to the NB-ARC-LRR (Hao et al. 2013). Effector targeting of RanGAP2 may, therefore, outcompete its interaction with $\mathrm{R} \times 1$ subdomains, thereby disrupting the receptor module towards activation. However, we found no evidence that this could be the case as the complex of RanGAP2 and Rx1 is not altered upon co-expression with PVX-CP (Chapter 4 Fig. 4). Alternatively, the biochemical cue here is likely to be perturbation and/or destabilization of the LRR as a direct consequence of binding to PVX-CP. Homology modeling indeed indicates that the LRR can, in theory, accommodate such complex formation as it harbors patches with the potential to accommodate protein-protein interaction (Chapter 3 Supplemental Fig. S5). In this case, whether effector perception translates to a loss of inhibition, therefore, still depends on the LRR. This upstream change/perturbation in the LRR is then transmitted to the rest of the receptor module by virtue of the compact folding of the receptor. However, similar activation mechanisms and/or biochemical stimuli are unlikely to be the case for all R-genes. For instance, in Bs2, the CC-NB-ARC remains bound to the LRR in the presence of the avirulence factor (Leister et al. 2005). Indeed, the autoactivity of most $R$-genes can be elicited at several points of the receptor, in support that receptor activation may occur via various forms of perturbations/alterations. 


\section{Landing the Finishing Blow: So Many Ways to Die?}

Once an enemy is spotted, the battle cry is set loose, and the true duty of a soldier is put in motion. His sole focus will then be fixated on ensuring the survival of his countrymen using the armaments he is equipped with. For Rx1, the nature of the bullet that is fired (i.e. its immune outputs), is known and will be elaborated more extensively in the later paragraphs of this section. However, how the firing of weaponry begins is much less clear. That is, what are the functional aspects leading to immunity post-activation? Our knowledge is presently limited to the identification of the $R \times 1$ (CC)-NB-ARC fragment as a signaling moiety, which has spurred massive efforts for elucidating how this region initiates downstream responses (Rairdan et al. 2008). As detailed previously, this led to the discovery that the Rx1-NB-ARC domain alone can associate with DNA, where it complexes with and modulates the activity of nuclear-associated factors (Fenyk et al. 2015, Townsend et al. 2018). This unconventional finding incites new questions as to how the nuclear heterocomplex of $\mathrm{R} \times 1$ co-operates to initiate conserved immune responses such as transcriptional activation and programmed cell death. In this regard, an additional, significant aspect of the DNA binding of $\mathrm{R} \times 1$ is that it can also distort the nucleic acid bound (Fenyk et al. 2015). It is, therefore, tempting to imagine that DNA damage could act as a cue that triggers the immune output. Likewise, studies have shown that the onset of immune responses in plants is often accompanied by an increased level of DNA damage, which is typically linked to an intrinsic increase in Salycylic Acid (SA) accumulation (Rodriguez et al. 2018). In mammals, however, UV-induced DNA damage is shown to initiate the oligomerization and activation of inflammatory responses by the NLR NLRP3 (Hasegawa et al. 2016). Thus, the sensing of DNA by Rx1 remains to be highly speculative as it is uncertain whether the damage inflicted is a consequence rather than a cause of immunity. Additionally, we did not identify any components belonging to the DNA repair machinery in our screening assays, as would otherwise be expected for a non-canonical pathway towards immunity.

\section{A Pentameric Death Wheel}

Recently, a ground-breaking structural study of the Arabidopsis ZAR1 offers an alternative route by which plant NB-LRRs can trigger immunity (Wang et al. 2019). Similar to mammals, the authors proposed that plant NB-LRRs can form toxic, funnel-like structures upon activation that will lead to perturbation of membrane integrity and the eventual collapse of the cell. The defence is therefore thought to occur without relying on an extensive suite of downstream signaling pathways (Adachi 
et al. 2019b). This hypothesis was built upon data from Cryo-EM imaging studies demonstrating that ZAR1 can oligomerize to form a pentameric supermolecular structure (so-called the wheel of death or 'resistosome') in the presence of its cognate effector (Wang et al. 2019). The formation of a resistosome involves proximity-induced chain-activation of an inactive ZAR1 driven by the NB-ARC domain of the initially activated receptor. Naturally, one musts wonder to what degree does this model of a resistosome applies to $R \times 1$ ? The propensity of $R \times 1$ to oligomerize has not been successfully shown despite various attempts until recently in the setting of an immunoprecipitation assay where it was demonstrated to self-associate when co-expressed with PVX-CP (Knip et al. 2019). Undoubtedly, the Rx1 homodimer is far from the pentameric arrangement described for ZAR1. It does, however, bring forth the potential of $\mathrm{R} \times 1$ to achieve a 1:1 stoichiometry if a resistosome were to ever arise. Given that $\mathrm{R} \times 1$ is dependent on NRC helpers for its function, an alternative scenario is that the $\mathrm{R} \times 1$ resistosome is composed of a hetero-oligomeric complex as observed for the mammalian inflammasome of the sensor family of apoptosis inhibitory proteins NAIP and CARD-domain containing 4 protein NLRC4 (Vance 2015). However, as mentioned previously, the physical interaction of Rx1 and NRCs needed to support this model has yet to be demonstrated.

In ZAR1, the formation of pore-like structures is by virtue of a terminal alpha helix ( $\alpha 1$ helix) projecting from the CC domain as a consequence of drastic conformational re-arrangement of the domain when ZAR1 is activated (Wang et al. 2019). When considering the resistosome model, the next question would then relate to whether the Rx1-CC can yield comparable pore-like structures and if so, where this would take place in the cell. Earlier works report that physical association between the Rx1-CC domain and NB-ARC-LRR is disrupted when PVX-CP is co-expressed (Moffett et al. 2002). This suggests that the Rx1-CC is released from the remainder of the receptor upon elicitation, which would imaginably, render the domain more accessible for pore formation. As we have shown that Rx1 remains tethered to RanGAP2 when defence is activated (Chapter 4 Fig. 4), the nuclear envelope constitutes the most probable site for the funneling of the Rx1-CC. Indeed, in mammals, the exact location for forming pores is not limited to the plasma membrane but has also been observed in the mitochondria (Tait et al. 2010). In this regard, it is fascinating to note studies reporting that homomeric interactions of nucleoporins channels are disrupted during an active Effector Triggered Immunity (ETI) response in plants to permeabilize the nuclear membrane and allow significant trafficking of protein cargoes (Gu et al. 2016). However, at present, it is still challenging to reconcile 
how the potential formation of an Rx1 resistosome and/or perturbation of the nuclear barrier is linked to the activities at the DNA.

\section{Do All Roads Lead to Rome?}

When discussing the potential model of an $\mathrm{R} \times 1$ resistosome, one must take into account that $\mathrm{R} \times 1$ is not fully analogous to ZAR1 at the functional level. Indeed, there are several arguments as to why it may not induce immunity by similar mechanisms. For example, it was recently shown that a signature motif composed of 29 AA (referred to as the MADA motif) in the $\alpha 1$ helix of the CC is critical for the cell death-inducing capacity of approximately $20 \%$ of plant CC-NB-LRRs (Adachi et al. 2019a). Though this was originally reported for NRC4, a matching consensus sequence was also found in ZAR1. The authors, therefore, proposed that the MADA motif may account for the funnel-like formation of the ZAR1 resistosome. The Rx1-CC, however, does not harbor this motif and is insufficient to induce cell death. Rx1 may, therefore, represent a different subclass of plant CC-NB-LRRs that triggers immunity in a manner dissimilar from ZAR1 or which requires NRC helper proteins (possessing the MADA motif) to achieve the same effect. In further support of this difference, distinct from most NB-LRRs, disease resistance by $\mathrm{R} \times 1$ is temperature-insensitive and does not require the induction of cell death (Richard et al. 2020). Therefore, there appears to be multiple ways to immunity. Whereas the ZAR1 model represents a 'dead-end' road to resistance, which culminates in the anchoring of the NB-LRR to a fixed structure, in Chapter 2, we have also indicated that alternative models of activation exist. This includes the 'equilibrium' model, which postulates that an NB-LRR continuously shifts between its ADP/ATP bound states even in unchallenged plants. Infection by the pathogen tilts the equilibrium towards the ATP bound state as a consequence of effector binding stabilizing this activated form (Bernoux et al. 2016). This model may, for instance, allow more flexibility in NB-LRRs systems to toggle and adjust the amplitude of responses triggered, depending upon a number of factors such as the severity of infection and efficiency of recognition. This would be relevant for Rx1 where elicitation by $\mathrm{PVX}-\mathrm{CP}$ can induce a range of responses. Hereby, Extreme Resistance (ER) represents one end of the spectrum, resulting in complete abrogation of the virus without the need to sacrifice host tissue. In scenarios where the virus can escape ER (e.g. under overexpression conditions of heterologous systems), a trailing cell death response is triggered that is insufficient to curb the rapid movement of the virus but will eventually lead to Hypersensitive Response $(H R)$. Though $E R, H R$, and trailing necrosis are described to be epistatic, experimental data at the molecular level in support of this 
model is currently lacking (Bendahmane et al. 1995, Gilbert et al. 1998, Kohm et al. 1993). However, it was shown that the antiviral response by $\mathrm{R} \times 1$ involves specific translational repression of PVX transcript, representing an additional framework by which the endpoint can be achieved (Meteignier et al. 2016). Multiple routes to immunity are also observed in mammals for instance, where the activation of an NLR receptor is translated through various, discreet pathways depending upon the type of ligand-bound and the cellular context (Bentham et al. 2017). How does this correlate with the fact that most NB-LRRs converge into common defence hubs or co-factors? One possibility is that although the responses of different NB-LRRs may qualitatively overlap, variation in the immune response elicited can instead arise from quantitative rather than qualitative difference, such as by gene dosage. In line with this, a likely interpretation is that although ER and HR may largely function as separate pathways, some degree of commonality may exist, which would enable Rx1 to swiftly shift to cell death in scenarios where ER proves insufficient.

\section{Conclusions and Outlook: Through Colored lenses}

When I first embarked on my dissertation, I wanted to contribute to the field of plant immunity by advancing our conceptual understanding of NB-LRR function. For this purpose, I adopted the well-studied potato Rx1 as a model system from which I may extend findings to other structurally similar CC-NB-LRRs. Combined with earlier studies in literature, results from the years of work performed in this thesis have allowed me to come at a refined model describing how the activity of $\mathrm{Rx} 1$ is driven by various molecular checkpoints in the cell (represented Fig. 1). In unchallenged plants, the concerted actions of a complex and dynamic suite of inter and intradomain interactions help to maintain a pool of primed/readily activated $R \times 1$ molecules in the nucleus and cytoplasm (Fig. 1-1A and 1B). The extent by which cellular context impacts the composition and interplay of these molecular determinants, as well as the configuration of $\mathrm{R} \times 1$, will be an exciting area to explore in the near future. Upon infection by PVX, the viral coat protein is sensed by cytoplasmic Rx1 via a process we hypothesize to be facilitated by effector binding to RanGAP2 (Fig. 1-2). Sensing of PVX-CP induces as a set of conformational changes in Rx1 that impacts pre-existing intra and heterocomplexes. The precise nature of this stepwise process is unknown but should ultimately permit the NB-ARC domain to adopt an open conformation, undergo nucleotide exchange, and initiate signaling (Fig. 1-3). Amongst other host-factors, downstream signaling of Rx1 is functionally 
dependent on the NRC helper NB-LRR (Fig. 1-4). The formation of higher-level, supermolecular structures and toxic pores may follow suit, most likely at the nuclear envelope (Fig. 1-5). By a yet undefined mechanism, the signal from the perception of effectors in the cytoplasm is transduced to the complex of Rx1 at the DNA. Consequently, the nuclear pool of Rx1 then co-ordinates the activities of DNA associated components (Fig. 1-6) (NbGLK1 and NbDBCP) towards defence, currently described to be in the form of either ER or HR (Fig. 1-7).

Needless to say, the scenario as sketched above is centered solely on Rx1. To expand on the broader horizon, we identify several key questions that would be important to address in future research lines as highlighted in the following paragraphs. Firstly, the works presented in this thesis are seen through the lens of the NB-LRR alone. As there are two sides in every battle, one can, therefore, envision that future work will move beyond the R-protein by investigating the receptor as a continuum with its cognate effector. We can further extend this concept when considering that plant NB-LRRs, their interactomes and potentially, effectors (co)-exist in a complex, intricate network architecture (Adachi et al. 2019b). In this setting, the function or activity of a component is then not completely independent of another in the same/co-existing network. In this respect, it will be fascinating to perform studies at the whole-proteome scale to understand the full-scope of residue co-evolution within and between components of these networks as a co-evolving system.

Secondly, NB-LRRs are not at the frontline of the battle. A further step is to go beyond the binary/tertiary interaction of an NB-LRR, its co-factor, and effector. As highlighted in Chapter 6, downstream components of ETI are often shared with PTI. For instance, NbGRP7 and GLK1 were found to potentiate both basal and Rx1-mediated immunities against PVX. Interestingly, studies are emerging demonstrating that PTI responses amplify ETI and vice versa (Ngou et al. 2020). The synergistic interplay between the two arms of plant immunity is thought to enable a more robust response than if the branches were to exist as separate entities. Thus, to what extent do PTI and ETI functionally co-operate and how does this relate to NB-LRRs on a molecular level? The concurrent study of ETI and PTI is a largely unchartered territory but one important enough to venture on.

Finally, in a time where global food security is directly linked to climate change, knowledge of how plant NB-LRRs adapt to their abiotic surroundings constitutes a critical area of research. This is a pressing matter as already, studies are detailing the adverse effects associated with elevated temperatures on the stability and activities of NB-LRRs and/or their associated co-factors (reviewed 
in Venkatesh et al. 2019). As a plant's habitat is in constant flux, the idea that NB-LRRs can respond to environmental cues is not far-fetched. This may, in turn, be mediated by their interacting partners that often have conserved, multipurpose functions in various plant physiological processes (Sun et al. 2020). Investigating the crosstalk between immunity and abiotic stimuli will, therefore, become increasingly important, which may demand complex systems biology approaches. In present times where considerable efforts are needed from resistance engineering, multidisciplinary approaches are pivotal in providing sound fundamental insight to support rationale breeding strategies. 


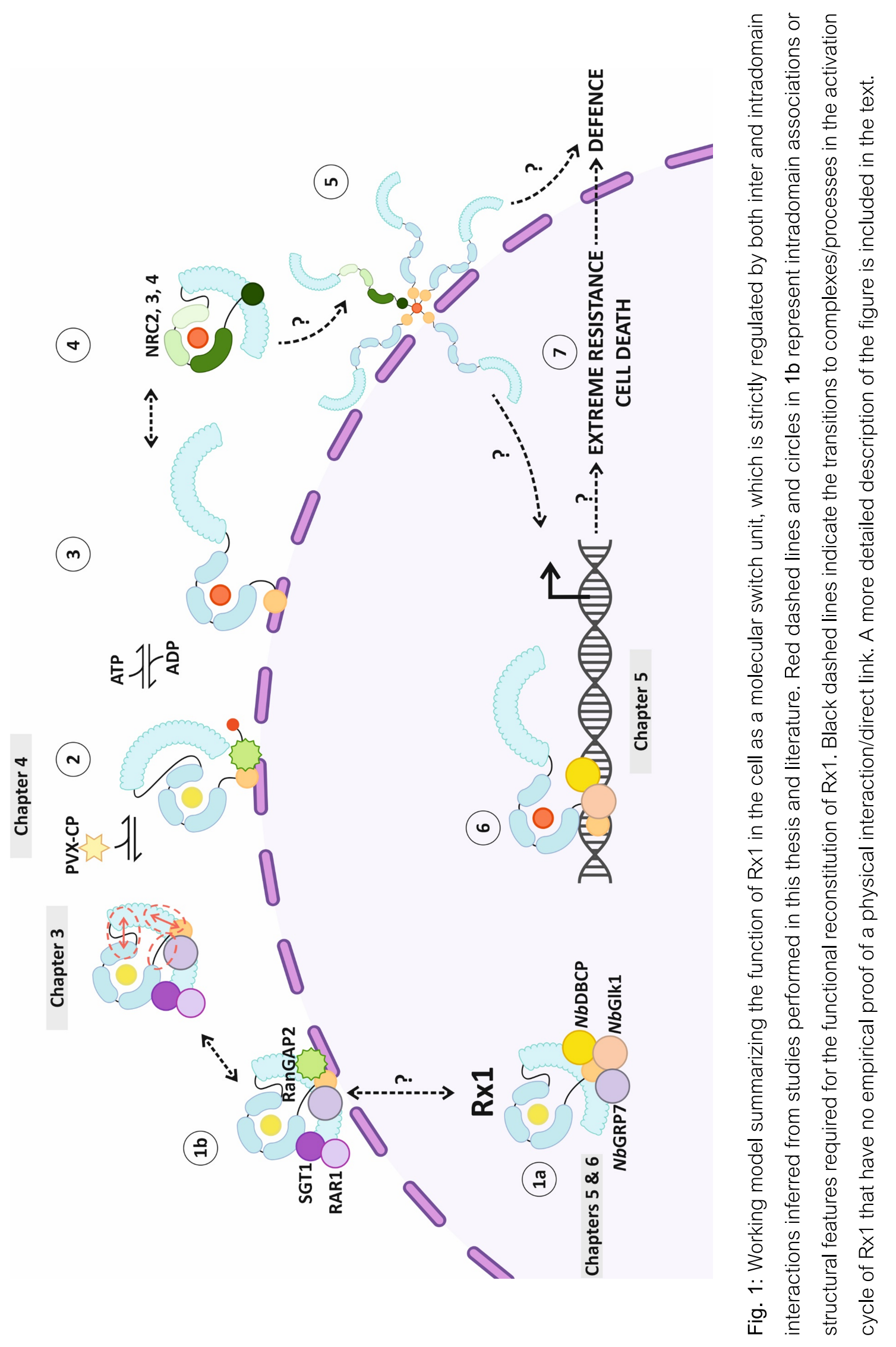




\section{References}

Adachi, H., Contreras, M. P., Harant, A., Wu, C.-h., Derevnina, L., Sakai, T., Duggan, C., Moratto, E., Bozkurt, T. O., Maqbool, A., Win, J., and Kamoun, S. (2019a), An N-terminal motif in NLR immune receptors is functionally conserved across distantly related plant species. eLife. 8: e49956.

Adachi, H., Derevnina, L., and Kamoun, S. (2019b), NLR singletons, pairs, and networks: evolution, assembly, and regulation of the intracellular immunoreceptor circuitry of plants. Current Opinion in Plant Biology. 50: p. 121-131.

Baudin, M., Hassan, J. A., Schreiber, K. J., and Lewis, J. D. (2017), Analysis of the ZAR1 Immune Complex Reveals Determinants for Immunity and Molecular Interactions. Plant Physiol. 174(4): p. 2038-2053.

Baurès, I., Candresse, T., Leveau, A., Bendahmane, A., and Sturbois, B. (2008), The Rx gene confers resistance to a range of potexviruses in transgenic Nicotiana plants. Mol Plant Microbe Interact. 21(9): p. 1154-64.

Bendahmane, A., Köhm, B. A., Dedi, C., and Baulcombe, D. C. (1995), The coat protein of potato virus X is a strain-specific elicitor of Rx1-mediated virus resistance in potato. The Plant Journal. 8(6): p. 933-941.

Bentham, A., Burdett, H., Anderson, P. A., Williams, S. J., and Kobe, B. (2017), Animal NLRs provide structural insights into plant NLR function. Annals of Botany. 119(5): p. 698-702.

Bernoux, M., Burdett, H., Williams, S. J., Zhang, X., Chen, C., Newell, K., Lawrence, G. J., Kobe, B., Ellis, J. G., Anderson, P. A., and Dodds, P. N. (2016), Comparative Analysis of the Flax Immune Receptors L6 and L7 Suggests an Equilibrium-Based Switch Activation Model. Plant Cell. 28(1): p. 146-59.

Bezerra-Neto, J. P., Araújo, F. C., Ferreira-Neto, J. R. C., Silva, R. L. O., Borges, A. N. C., Matos, M. K. S., Silva, J. B., Silva, M. D., Kido, E. A., and Benko-Iseppon, A. M. (2020), NBS-LRR genes - Plant health sentinels: Structure, roles, evolution and biotechnological applications. In: Applied Plant Biotechnology for Improving Resistance to Biotic Stress. Ed. by P. Poltronieri and Y. Hong. Academic Press, p. 63-120.

Cerovska, N., Plchova, H., Vaculik, P., Moravec, T., and Gichner, T. (2014), Potato virus X induces DNA damage in leaf nuclei of the host plant Nicotiana tabacum L. var. xanthi. Biologia Plantarum. 58(4): p. 783-787.

Chang, C., Yu, D., Jiao, J., Jing, S., Schulze-Lefert, P., and Shen, Q.-H. (2013), Barley MLA Immune Receptors Directly Interfere with Antagonistically Acting Transcription Factors to Initiate Disease Resistance Signaling. The Plant Cell Online. 25(3): p. 1158-1173.

Collier, S. M. and Moffett, P. (2009), NB-LRRs work a "bait and switch" on pathogens. Trends in Plant Science. 14(10): p. 521-529.

Devaiah, B. N. and Singer, D. S. (2013), CIITA and Its Dual Roles in MHC Gene Transcription. Front Immunol. 4: p. 476.

Duff-Farrier, C.R., Candresse, T., Bailey, A. M., Boonham, N., and Foster, G. D. (2016), Evidence for different, host-dependent functioning of Rx against both wild-type and recombinant Pepino mosaic virus. Mol Plant Pathol. 17(1): p. 120-6.

Fenyk, S., Townsend, P. D., Dixon, C. H., Spies, G. B., de San Eustaquio Campillo, A., Slootweg, E. J., Westerhof, L. B., Gawehns, F. K. K., Knight, M. R., Sharples, G. J., Goverse, A., Pålsson, L.-O., Takken, F. L. W., and Cann, M. J. (2015), The Potato Nucleotide-binding Leucine-rich Repeat (NLR) Immune Receptor Rx1 Is a Pathogen-dependent DNA-deforming Protein. The Journal of biological chemistry. 290(41): p. 24945-24960. ppublish. 
Gallois, J.-L., Moury, B., and German-Retana, S. (2018), Role of the Genetic Background in Resistance to Plant Viruses. International journal of molecular sciences. 19(10): p. 2856.

Giannakopoulou, A., Steele, J. F. C., Segretin, M. E., Bozkurt, T. O., Zhou, J., Robatzek, S., Banfield, M. J., Pais, M., and Kamoun, S. (2015), Tomato I2 Immune Receptor Can Be Engineered to Confer Partial Resistance to the Oomycete Phytophthora infestans in Addition to the Fungus Fusarium oxysporum. Molecular Plant-Microbe Interactions: MPMI-07-15-0147-R.

Gilbert, J., Spillane, C., Kavanagh, T. A., and Baulcombe, D. C. (1998), Elicitation of Rx-Mediated Resistance to PVX in Potato Does Not Require New RNA Synthesis and May Involve a Latent Hypersensitive Response. Molecular Plant-Microbe Interactions ${ }^{\circledR}$. 11(8): p. 833-835.

Gu, Y., Zebell, S. G., Liang, Z., Wang, S., Kang, B.-H., and Dong, X. (2016), Nuclear Pore Permeabilization Is a Convergent Signaling Event in Effector-Triggered Immunity. Cell. 166(6): 1526-1538.e11.

Hao, W., Collier, S. M., Moffett, P., and Chai, J. (2013), Structural basis for the interaction between the potato virus $X$ resistance protein $(\mathrm{Rx})$ and its cofactor Ran GTPase-activating protein 2 (RanGAP2). The Journal of biological chemistry. 288(50): p. 35868-35876.

Hasegawa, T., Nakashima, M., and Suzuki, Y. (2016), Nuclear DNA damage-triggered NLRP3 inflammasome activation promotes UVB-induced inflammatory responses in human keratinocytes. Biochem Biophys Res Commun. 477(3): p. 329-35.

Jubic, L. M., Saile, S., Furzer, O. J., El Kasmi, F., and Dangl, J. L. (2019), Help wanted: helper NLRs and plant immune responses. Current Opinion in Plant Biology. 50: p. 82-94.

Khan, M., Youn, J.-Y., Gingras, A.-C., Subramaniam, R., and Desveaux, D. (2018), In planta proximity dependent biotin identification (BiolD). Scientific Reports. 8(1): p. 9212.

King, S. R. F., McLellan, H., Boevink, P. C., Armstrong, M. R., Bukharova, T., Sukarta, O., Win, J., Kamoun, S., Birch, P. R. J., and Banfield, M. J. (2014), Phytopthora infestans RXLR effector PexRD2 interacts with host MAPKKK $\boldsymbol{\varepsilon}$ to suppress plant immune signaling. The Plant cell. 26(3): p. 1345-1359. ppublish.

Knip, M., Richard, M. M. S., Oskam, L., van Engelen, H. T. D., Aalders, T., and Takken, F. L. W. (2019), Activation of immune receptor Rx1 triggers distinct immune responses culminating in cell death after 4 hours. Molecular plant pathology. 20(4): p. 575-588.

Kohm, B. A., Goulden, M. G., Gilbert, J. E., Kavanagh, T. A., and Baulcombe, D. C. (1993), A Potato Virus X Resistance Gene Mediates an Induced, Nonspecific Resistance in Protoplasts. The Plant Cell. 5(8): p. 913-920.

Lee, H. A. and Yeom, S. I. (2015), Plant NB-LRR proteins: tightly regulated sensors in a complex manner. Brief Funct Genomics. 14(4): p. 233-42.

Leister, R. T., Dahlbeck, D., Day, B., Li, Y., Chesnokova, O., and Staskawicz, B. J. (2005), Molecular genetic evidence for the role of SGT1 in the intramolecular complementation of Bs2 protein activity in Nicotiana benthamiana. Plant Cell. 17(4): p. 1268-1278.

Lolle, S., Stevens, D., and Coaker, G. (2020), Plant NLR-triggered immunity: from receptor activation to downstream signaling. Current Opinion in Immunology. 62: p. 99-105. 
Lukasik-Shreepaathy, E., Vossen, J. H., Tameling, W. I. L., de Vroomen, M. J., Cornelissen, B. J. C., and Takken, F. L. W. (2012), Protein-protein interactions as a proxy to monitor conformational changes and activation states of the tomato resistance protein I-2. Journal of experimental botany. 63(8): p. 3047-3060.

Martin, E. C., Sukarta, O. C. A., Spiridon, L., Grigore, L. G., Constantinescu, V., Tacutu, R., Goverse, A., and Petrescu, A. J. (2020), LRRpredictor-A New LRR Motif Detection Method for Irregular Motifs of Plant NLR Proteins Using an Ensemble of Classifiers. Genes (Basel). 11(3).

Mazourek, M., Cirulli, E. T., Collier, S. M., Landry, L. G., Kang, B. C., Quirin, E. A., Bradeen, J. M., Moffett, P., and Jahn, M. M. (2009), The fractionated orthology of Bs2 and Rx/Gpa2 supports shared synteny of disease resistance in the Solanaceae. Genetics. 182(4): p. 1351-64.

McHale, L., Tan, X., Koehl, P., and Michelmore, R. W. (2006), Plant NBS-LRR proteins: adaptable guards. Genome Biology. 7(4): p. 212.

Meteignier, L.-V., Zhou, J., Cohen, M., Bhattacharjee, S., Brosseau, C., Caamal Chan, M. G., Robatzek, S., and Moffett, P. (2016), NB-LRR signaling induces translational repression of viral transcripts and the formation of RNA processing bodies through mechanisms differing from those activated by UV stress and RNAi. Journal of Experimental Botany. 67(8): p. 2353-2366.

Moffett, P., Farnham, G., Peart, J., and Baulcombe, D. C. (2002), Interaction between domains of a plant NBS-LRR protein in disease resistance-related cell death. The EMBO Journal. 21(17): p. 4511-4519.

Naftelberg, S., Schor, I. E., Ast, G., and Kornblihtt, A. R. (2015), Regulation of alternative splicing through coupling with transcription and chromatin structure. Annu Rev Biochem. 84: p. 165-98.

Ngou, B. P. M., Ahn, H.-K., Ding, P., and Jones, J. D. (2020), Mutual Potentiation of Plant Immunity by Cell-surface and Intracellular Receptors. bioRxiv: p. 2020.04.10.034173.

Palma, K., Thorgrimsen, S., Malinovsky, F. G., Fiil, B. K., Nielsen, H. B., Brodersen, P., Hofius, D., Petersen, M., and Mundy, J. (2010), Autoimmunity in Arabidopsis acd11 is mediated by epigenetic regulation of an immune receptor. PLoS pathogens. 6(10): e1001137-e1001137.

Rairdan, G. J., Collier, S. M., Sacco, M. A., Baldwin, T. T., Boettrich, T., and Moffett, P. (2008), The Coiled-Coil and Nucleotide Binding Domains of the Potato Rx Disease Resistance Protein Function in Pathogen Recognition and Signaling. Plant Cell. 20(3): p. 739-751.

Rairdan, G. J. and Moffett, P. (2006), Distinct Domains in the ARC Region of the Potato Resistance Protein Rx Mediate LRR Binding and Inhibition of Activation. Plant Cell. 18(8): p. 2082-2093.

Richard, M. M. S., Knip, M., Aalders, T., Beijaert, M. S., and Takken, F. L. W. (2020), Unlike Many Disease Resistances, Rx1-Mediated Immunity to Potato Virus X Is Not Compromised at Elevated Temperatures. Frontiers in genetics. 11: p. 417. epublish.

Rodriguez, E., Chevalier, J., El Ghoul, H., Voldum-Clausen, K., Mundy, J., and Petersen, M. (2018), DNA damage as a consequence of NLR activation. PLOS Genetics. 14(2): e1007235.

Sacco, M. A., Mansoor, S., and Moffett, P. (2007), A RanGAP protein physically interacts with the NB-LRR protein RX, and is required for Rx-mediated viral resistance. Plant J. 52(1): p. 82-93. 
Slootweg, E., Roosien, J., Spiridon, L. N., Petrescu, A. J., Tameling, W., Joosten, M., Pomp, R., van Schaik, C.,

Dees, R., Borst, J. W., Smant, G., Schots, A., Bakker, J., and Goverse, A. (2010), Nucleocytoplasmic distribution is required for activation of resistance by the potato NB-LRR receptor $\mathrm{Rx} 1$ and is balanced by its functional domains. Plant Cell. 22(12): p. 4195-215.

Slootweg, E., Koropacka, K., Roosien, J., Dees, R., Overmars, H., Lankhorst, R. K., van Schaik, C., Pomp, R., Bouwman, L., Helder, J., Schots, A., Bakker, J., Smant, G., and Goverse, A. (2017), Sequence Exchange between Homologous NB-LRR Genes Converts Virus Resistance into Nematode Resistance, and Vice Versa. Plant physiology. 175(1): p. 498-510.

Slootweg, E. J., Spiridon, L. N., Martin, E. C., Tameling, W. I. L., Townsend, P. D., Pomp, R., Roosien, J., Drawska, O., Sukarta, O. C. A., Schots, A., Borst, J. W., Joosten, M. H., Bakker, J., Smant, G., Cann, M. J., Petrescu, A.-J., and Goverse, A. (2018), Distinct Roles of Non-Overlapping Surface Regions of the Coiled-Coil Domain in the Potato Immune Receptor Rx1. Plant Physiology. 178(3): p. 1310-1331.

Slootweg, E. J., Spiridon, L. N., Roosien, J., Butterbach, P., Pomp, R., Westerhof, L., Wilbers, R., Bakker, E., Bakker, J., Petrescu, A.-J., Smant, G., and Goverse, A. (2013), Structural Determinants at the Interface of the ARC2 and Leucine-Rich Repeat Domains Control the Activation of the Plant Immune Receptors Rx1 and Gpa2. Plant Physiology. 162(3): p. 1510-1528.

Sukarta, O. C. A., Slootweg, E. J., and Goverse, A. (2016), Structure-informed insights for NLR functioning in plant immunity. Seminars in Cell \& Developmental Biology. 56: p. 134-149.

Sukarta, O. C. A., Townsend, P. D., Llewelyn, A., Dixon, C. H., Slootweg, E. J., Pålsson, L.-O., Takken, F. L. W., Goverse, A., and Cann, M. J. (2020), A DNA-Binding Bromodomain-Containing Protein Interacts with and Reduces Rx1-Mediated Immune Response to Potato Virus X. Plant communications. 1(4): p. 100086. epublish.

Sun, Y., Zhu, Y.-X., Balint-Kurti, P. J., and Wang, G.-F. (2020), Fine-Tuning Immunity: Players and Regulators for Plant NLRs. Trends in Plant Science. 25(7): p. 695-713.

Tait, S. W. G. and Green, D. R. (2010), Mitochondria and cell death: outer membrane permeabilization and beyond. Nature Reviews Molecular Cell Biology. 11(9): p. 621-632.

Tameling, W. I. L. and Baulcombe, D. C. (2007), Physical association of the NB-LRR resistance protein Rx with a Ran GTPase-activating protein is required for extreme resistance to Potato virus X. Plant Cell. 19(5): p. 1682-94.

Tang, J., Zhu, X., Wang, Y., Liu, L., Xu, B., Li, F., Fang, J., and Chu, C. (2011), Semi-dominant mutations in the CC-NB-LRR-type R gene, NLS1, lead to constitutive activation of defense responses in rice. Plant J. 66(6): p. 996-1007.

Townsend, P. D., Dixon, C. H., Slootweg, E. J., Sukarta, O. C. A., Yang, A. W. H., Hughes, T. R., Sharples, G. J., Pålsson, L. O., Takken, F. L. W., Goverse, A., and Cann, M. J. (2018), The intracellular immune receptor Rx1 regulates the DNA-binding activity of a Golden2-like transcription factor. J Biol Chem. 293(9): p. 3218-3233.

Vance, R. E. (2015), The NAIP/NLRC4 inflammasomes. Current opinion in immunology. 32: p. 84-89.

Venkatesh, J. and Kang, B.-C. (2019), Current views on temperature-modulated R gene-mediated plant defense responses and tradeoffs between plant growth and immunity. Current Opinion in Plant Biology. 50: p. 9-17. 
Wang, J., Hu, M., Wang, J., Qi, J., Han, Z., Wang, G., Qi, Y., Wang, H. W., Zhou, J. M., and Chai, J. (2019), Reconstitution and structure of a plant NLR resistosome conferring immunity. Science. 364(6435).

Warmerdam, S., Sterken, M. G., van Schaik, C., Oortwijn, M. E. P., Sukarta, O. C. A., Lozano-Torres, J. L., Dicke, M., Helder, J., Kammenga, J. E., Goverse, A., Bakker, J., and Smant, G. (2018), Genome-wide association mapping of the architecture of susceptibility to the root-knot nematode Meloidogyne incognita in Arabidopsis thaliana. The New phytologist. 218(2): p. 724-737. ppublish.

Warmerdam, S., Sterken, M. G., Sukarta, O. C. A., van Schaik, C. C., Oortwijn, M. E. P., Lozano-Torres, J. L., Bakker, J., Smant, G., and Goverse, A. (2020), The TIR-NB-LRR pair DSC1 and WRKY19 contributes to basal immunity of Arabidopsis to the root-knot nematode Meloidogyne incognita. BMC plant biology. 20(1): p. 73. epublish.

Van Wersch, S., Tian, L., Hoy, R., and Li, X. (2020), Plant NLRs: The Whistleblowers of Plant Immunity. Plant Communications. 1(1): p. 100016.

Wu, C. H., Abd-El-Haliem, A., Bozkurt, T. O., Belhaj, K., Terauchi, R., Vossen, J. H., and Kamoun, S. (2017), NLR network mediates immunity to diverse plant pathogens. Proc Natl Acad Sci U S A. 114(30): p. 8113-8118.

Zhang, Y., Song, G., Lal, N. K., Nagalakshmi, U., Li, Y., Zheng, W., Huang, P.-j., Branon, T. C., Ting, A. Y., Walley, J. W., and Dinesh-Kumar, S. P. (2019), TurboID-based proximity labeling reveals that UBR7 is a regulator of N NLR immune receptor-mediated immunity. Nature Communications. 10(1): p. 3252.

Zhu, M., Jiang, L., Bai, B., Zhao, W., Chen, X., Li, J., Liu, Y., Chen, Z., Wang, B., Wang, C., Wu, Q., Shen, Q., Dinesh-Kumar, S. P., and Tao, X. (2017), The Intracellular Immune Receptor Sw-5b Confers Broad-Spectrum Resistance to Tospoviruses through Recognition of a Conserved 21-Amino Acid Viral Effector Epitope. The Plant cell. 29(9): p. 2214-2232. 
Summary

Acknowledgments

About the author

List of Publications

Education statement 


\section{SUMMARY}

In plants, each cell is wired with the inherent ability to fend-off invading microbes by means of an innate immune system. An integral component of plant immunity is a repertoire of receptor proteins, which act as molecular sentinels surveying the intracellular or extracellular space for signs of microbial invasion. This thesis focused on elucidating the function of intracellular plant immune receptors belonging to the family of Nucleotide-Binding Leucine-Rich Repeats (NB-LRRs). NB-LRRs mediate an inducible form of defence upon recognition of specific pathogen-derived effector molecules. Over the past years, studies have shown that the plant genome harbors a vast variety of NB-LRR receptor types that are characterized by distinct structural features. This diversity is further reflected in the various manners by which NB-LRRs can undergo recognition, activation, and defence signaling (Chapter 2). However, though our catalog of plant NB-LRRs has rapidly expanded, the mechanistic basis by which these receptors function and are regulated in the cell remains fragmentary. Thus, to address this knowledge gap, the current thesis uses the potato $\mathrm{Rx} 1$ CC-NB-LRR as a model system, which confers immunity against Potato Virus X (PVX). The works presented collectively illustrate that the activity of $\mathrm{Rx} 1$ is modulated by a complex set of intra and intermolecular interactions to fine-tune its immune output.

NB-LRRs are composed of modular units, which provide the receptor with an intrinsic form of control. This is achieved by the folding of the subdomains in a manner that allows the receptor to remain autoinhibited in unchallenged plants, while also readily activated during a pathogenic invasion. One primary objective of the thesis was to elucidate the degree by which domain co-operation is conserved across different NB- LRRs system. As there are no apparent differences in their features, classical beliefs postulate that similar NB-LRR types may exhibit comparable intra-domain controls. The works presented in Chapter 3, however, illustrate that this does not always prove to be the case. By performing in silico screening, a tomato homolog (Rslyc) of the potato Rx1 was identified from the cultivar Katinka Cherry, which does not confer defence against PVX. Functional analysis performed in Nicotiana benthamiana indicates that Rx1 and Rslyc demand distinct structural requirements for their functions despite sharing high sequence similarity. Based on trans-complementation and immunoprecipitation approaches, it was demonstrated that the subdomains of Rx1 and Rslyc undergo different intradomain associations. Specifically, the distal ends of the receptor in Rslyc (i.e. the CC 
and LRR domains) can physically associate, whereas, in $\mathrm{R} \times 1$, this interaction occurs negligibly. Unlike Rx1, the NB-ARC and LRR domains of Rslyc were also found to weakly form complexes, which may hinder the efficient reconstitution of the receptor in the cell. Structural prediction analysis performed in parallel further indicates that sequence and structural variation in the central NB-ARC domain may provide an additional constraint accounting for differences in the capacity of $\mathrm{R} \times 1$ and Rslyc to recognize PVX. Particularly, this refers to the composition and behavior of a linker region connecting the $\mathrm{CC}$ and NB-ARC domains that is predicted to help prevent inadvertent nucleotide exchange events. Taken together, these results can have important implications for breeding strategies whereby the tinkering of an NB-LRR receptor would require a structure-informed, stepwise approach.

In addition to intra-domain interactions, the function of an NB-LRR receptor is regulated by heterocomplex formation with accessory host proteins. For Rx1, the RanGTPase-activating protein 2 (RanGAP2) is deemed to be a bona fide co-factor required to confer a full immune response. However, aside from regulating the subcellular localization of Rx1, the exact role of RanGAP2 in Rx1 immunity is vague. Based on findings of artificial tethering studies with the cognate effector of the homologous Gpa2 receptor (which confers defence against the potato cyst nematode Globodera pallida), it was hypothesized that RanGAP2 might mediate recognition processes. In support of this model, in Chapter 4, a combination of immunoprecipitation and cellular approaches demonstrate that RanGAP2 can interact with the cognate effectors of Rx1 and Gpa2 in planta. Remarkably, however, this interaction extends to the non-activating effector variants, suggesting a broader role for the effector targeting of RanGAP2. Indeed, the findings show that RanGAP2 contributes to the infection of PVX in N. benthamiana and cyst nematodes in Arabidopsis. To explore a potential manner by which RanGAP2 could contribute to the perception of effectors by $\mathrm{R} \times 1$, we determined the influence of effector targeting on the complex of RanGAP2 with Rx1/Gpa2. However, Co-IP experiments show that RanGAP2 remains bound to Rx1 and Gpa2 in the presence of the effectors. Based on these findings, we proposed a model for the potential role of RanGAP2 as a bait whereby initial docking to RanGAP2 is involved in but not required for the recognition by and activation of $\mathrm{R} \times 1$.

Aside from RanGAP2, few components belonging to the Rx1 resistance complex has been reported to date. In Chapters 5 and 6 , we therefore adopted two separate approaches in an effort to identify novel 
$\mathrm{R} \times 1$-associated proteins. In both screenings, the $\mathrm{N}$-terminal $\mathrm{CC}$ domain was used as bait, in light of its role as a scaffold for downstream interactions. Moreover, as the subcellular distribution of $R \times 1$ has been shown to dictate its function, we aimed to further resolve nuclear and/or cytoplasmic interactors. Based on the yeast-2-hybrid platform (Chapter 5), a DNA-Binding Bromodomain-Associated protein (denoted $\mathrm{NbDBCP}$ ) was found to interact with the Rx1-CC. Co-immunoprecipitation approaches further verified that the interaction is maintained with the full-length $\mathrm{Rx} 1$ in planta. NbDBCP was further shown to negatively regulate the extreme resistance response of $R \times 1$ in $N$. benthamiana, which is dependent on an intact bromodomain. In a further characterization of its function, the DNA binding capacity of NbDBCP is impaired in conditions where $\mathrm{Rx} 1$ is present abundantly. Additionally, NbDBCP and Rx1 were shown to synergistically prevent the DNA binding activity of the pro-immune component Golden-like Transcription Factor (GLK1), providing a basis for the suppressive function of $\mathrm{NbDBCP}$ in $\mathrm{Rx} 1$ defence. These findings suggest that $\mathrm{R} \times 1$ can recruit and regulate the activity of nuclear-associated host proteins with specific DNA binding activity towards defence.

In Chapter 6, a Glycine Rich RNA binding protein 7 (NbGRP7) was additionally discovered to interact with the $\mathrm{N}$-terminal CC domain of the Gpa2 receptor in a Co-IP/MS analysis performed in $N$. benthamiana. NbGRP7 was also shown to form complexes with full-length $R \times 1$ in an immunoprecipitation assay via the receptor CC domain. To elucidate the biological implications underlying the physical association of NbGRP7 with Gpa2 and Rx1, the impact of transiently overexpressing or silencing NbGRP7 was determined on defence responses. The results indicate that NbGRP7 is a pro-immune component, which contributes to cell death response by Gpa2 and extreme resistance (ER) by Rx1. In an effort to decipher the mechanistic underpinnings of NbGRP7 in NB-LRR mediated immunity, mutant variants of the protein were generated targeting a conserved Arginine residue. These mutants were found to have reduced capacities to potentiate ER by $R \times 1$, indicating that its RNA-binding capacity may be crucial for its activities in Rx1-defence. In relation, qPCR assays further demonstrate that the transcript abundance of $\mathrm{Rx} 1$ is affected during ectopic expression of NbGRP7. These findings suggest that NbGRP7 may regulate RNA levels of Rx1 before and during an active defence response. Thus, in addition to transcriptional components, $\mathrm{R} \times 1$ can co-opt proteins that are involved in post-transcriptional processes.

The main findings and experimental approaches adopted in this thesis were further discussed in light of recent, fundamental discoveries related to Rx1 and plant NB-LRRs in general (Chapter 7). 
The scope covered entails mechanisms pre- and post-activation, including a discussion on potential resistosome formation and the uncoupling of defence pathways. Based on these reflections, a working model describing the immune functioning of $\mathrm{Rx} 1$ was proposed, which may, to some degree, apply to other NB- LRRs sharing structural homology. Overall, it was concluded that a diverse scope of inter and intramolecular determinants are required to control the compartment-specific activities of Rx1 in the cell. More detailed studies, however, are needed to resolve the spatial and temporal dynamics in which these various regulatory (check) points act in concert. 


\section{DUTCH SUMMARY}

In planten bezit elke cel het inherente vermogen om binnendringende microben af te weren door middel van een aangeboren immuunsysteem. Een integraal onderdeel van de immuniteit van planten is een repertoire van receptoreiwitten, die fungeren als moleculaire schildwachten die de intracellulaire of extracellulaire ruimte onderzoeken op tekenen van microbiële invasie. Dit proefschrift richtte zich op het ophelderen van de functie van intracellulaire immuunreceptoren van planten die behoren tot de familie van Nucleotide-Binding Leucine Rich-Repeats (NB-LRRs). NB-LRRs mediëren een induceerbare vorm van verdediging bij herkenning van specifieke pathogeen-afgeleide effectormoleculen. Studies hebben de afgelopen jaren aangetoond dat het plantengenoom een grote verscheidenheid aan NB-LRR-receptortypen herbergt die worden gekenmerkt door verschillende structurele kenmerken. Deze diversiteit wordt verder weerspiegeld in de verschillende manieren waarop NB-LRRs herkenning, activering en afweer signalering kunnen ondergaan (Hoofdstuk 2). Hoewel onze catalogus van NB-LRRs van planten snel is uitgebreid, blijt de mechanistische basis waarmee deze receptoren functioneren en worden gereguleerd in de cel fragmentarisch. Om deze kenniskloof te dichten gebruikt dit proefschrift de aardappel Rx1 CC-NB-LRR als een modelsysteem, dat immuniteit tegen Potato Virus X (PVX) verleent. Het proefschrift als geheel illustreert dat de activiteit van Rx1 wordt gemoduleerd door een complexe reeks intra- en intermoleculaire interacties om de immuunrespons te verfijnen.

NB-LRRs zijn samengesteld uit modulaire eenheden die de receptor een intrinsieke vorm van controle geven. Dit wordt bereikt door het vouwen van de sub domeinen op een manier die het mogelijk maakt dat de receptor auto-geremd blijft in planten die niet geïnfecteerd zijn, terwijl hij ook gemakkelijk wordt geactiveerd tijdens een infectie met een pathogeen. Een van de belangrijkste doelstellingen van het proefschrift was om de mate waarin domeinsamenwerking behouden blijft in verschillende NB-LRR-systemen te verduidelijken. Aangezien er geen duidelijke verschillen zijn in hun kenmerken, veronderstellen klassieke opvattingen dat vergelijkbare NB-LRR-typen vergelijkbare intra-domeincontroles kunnen vertonen. De resultaten die in Hoofdstuk 3 worden gepresenteerd, illustreren echter dat dit niet altijd het geval blijkt te zijn. Door een in silico-screening uit te voeren, werd een homoloog in tomaat (Rslyc) van de aardappel Rx1 geïdentificeerd in de cultivar Katinka Cherry, die geen verdediging biedt tegen PVX. Functionele analyse uitgevoerd in Nicotiana benthamiana geeft aan dat Rx1 en Rslyc verschillende structurele vereisten voor hun 
functies hebben, ondanks gedeelde hoge sequentiegelijkenis. Op basis van transcomplementatieen immunoprecipitatiebenaderingen werd aangetoond dat de subdomeinen van $\mathrm{R} \times 1$ en Rslyc verschillende intra-domeinassociaties ondergaan. In het bijzonder kunnen de distale uiteinden van de receptor in Rslyc (d.w.z. de CC- en LRR-domeinen) fysiek associëren, terwijl in Rx1 deze interactie verwaarloosbaar plaatsvindt. In tegenstelling tot Rx1 bleken de NB-ARC- en LRR-domeinen van Rslyc ook zwak complexen te vormen, die de efficiënte reconstructie van de receptor in de cel kunnen belemmeren. Parallel uitgevoerde structurele voorspellingsanalyse geeft verder aan dat sequentie en structurele variatie in het centrale NB-ARC-domein een extra beperking kunnen bieden die rekening houdt met verschillen in het vermogen van Rx1 en Rslyc om PVX te herkennen. Dit verwijst in het bijzonder naar de samenstelling en het gedrag van een gelinkt gebied dat de CC- en NB-ARC-domeinen verbindt en waarvan wordt voorspeld dat het onbedoelde nucleotide-uitwisselingsgebeurtenissen helpt voorkomen. Alles bij elkaar genomen kunnen deze resultaten belangrijke implicaties hebben voor kweekstrategieën waarbij het sleutelen aan een NB-LRR-receptor een structuur-geïnformeerde, stapsgewijze aanpak vereist.

Naast intra-domeininteracties wordt de functie van een NB-LRR-receptor gereguleerd door heterocomplexvorming met bijkomende gastheereiwitten. Voor Rx1 wordt het RanGTPase-activating protein 2 (RanGAP2) beschouwd als een bona fide co-factor die nodig is om een volledige immuunrespons te verlenen. Afgezien van het reguleren van de intracellulaire lokalisatie van $R \times 1$, is de exacte rol van RanGAP2 in Rx1-immuniteit vaag. Op basis van bevindingen van kunstmatige tethering-studies met de verwante effector van de homologe Gpa2-receptor (die bescherming biedt tegen het aardappelcysteaaltje Globodera pallida), werd verondersteld dat RanGAP2 herkenningsprocessen zou kunnen mediëren. Ter ondersteuning van dit model laat in Hoofdstuk 4, een combinatie van immunoprecipitatie en cellulaire benaderingen zien dat RanGAP2 kan interageren met de verwante effectoren van Rx1 en Gpa2 in planta. Opmerkelijk is echter dat deze interactie zich uitstrekt tot de niet-activerende effectorvarianten, wat een bredere rol suggereert voor de effector-targeting van RanGAP2. De bevindingen tonen inderdaad aan dat RanGAP2 bijdraagt aan de infectie van PVX in N. benthamiana en cysteaaltjes in Arabidopsis. Om een mogelijke manier te onderzoeken waarop RanGAP2 zou kunnen bijdragen aan de perceptie van effectoren door Rx1, hebben we de invloed van effector-targeting op het complex van RanGAP2 bepaald met Rx1/Gpa2. Co-IP-experimenten tonen echter aan dat RanGAP2 gebonden blijft aan Rx1 en 
Gpa2 in aanwezigheid van de effectoren. Op basis van deze bevindingen hebben we een model voorgesteld voor de mogelijke rol van RanGAP2 als aas, waarbij aanvankelijke koppeling aan RanGAP2 betrokken is bij maar niet vereist is voor de herkenning door en activering van $\mathrm{R} \times 1$.

Afgezien van RanGAP2 zijn er tot op heden enkele componenten gerapporteerd die tot het Rx1-resistentiecomplex behoren. In de Hoofdstukken 5 en 6, hebben we daarom twee verschillende benaderingen aangenomen om nieuwe Rx1-geassocieerde eiwitten te identificeren. In beide screenings werd het $N$-terminale CC-domein als lokaas gebruikt, gezien zijn rol als platform voor stroomafwaartse interacties. Bovendien, aangezien is aangetoond dat de intracellulaire distributie van Rx1 zijn functie dicteert, wilden we de nucleaire en/of cytoplasmatische interactoren opsporen. Gebaseerd op de yeast-2-hybride platforms (Hoofdstuk 5), bleek een DNA-binding Bromodomain-Associated Protein (aangeduid als NbDBCP) interactie te hebben met de Rx1-CC. Co-immunoprecipitatiebenaderingen bevestigden verder dat de interactie wordt gehandhaafd met de Rx1 van volledige lengte in planta. Verder werd aangetoond dat NbDBCP de extreme weerstandsrespons van $\mathrm{Rx} 1$ in $N$. benthamiana, die afhankelijk is van een intact bromodomein, negatief reguleert. In een verdere karakterisering van zijn functie is het DNA-bindende vermogen van $\mathrm{NbDBCP}$ aangetast in omstandigheden waarin $\mathrm{Rx} 1$ overvloedig aanwezig is. Bovendien werd aangetoond dat NbDBCP en Rx1 synergetisch de DNA-bindingsactiviteit van de pro-immuuncomponent Golden-like Transcription Factor (GLK1) verhinderen, wat een basis vormt voor de onderdrukkende functie van NbDBCP bij Rx1-verdediging. Deze bevindingen suggereren dat $\mathrm{R} \times 1$ de activiteit van nucleair-geassocieerde gastheereiwitten kan rekruteren en reguleren met specifieke DNA-bindende activiteit voor verdediging.

In Hoofdstuk 6, werd in een Co-IP/MS-analyse uitgevoerd in N. benthamiana bovendien een Glycine Rich RNA-Binding Protein 7 (NbGRP7) ontdekt dat een interactie aangaat met het $N$-terminale CC-domein van de Gpa2-receptor. NbGRP7 bleek ook complexen te vormen met Rx1 van volledige lengte in een immunoprecipitatietest via het receptor CC-domein. Om de biologische implicaties die ten grondslag liggen aan de fysieke associatie van NbGRP7 met Gpa2 en Rx1 op te helderen, werd de impact van tijdelijk overexpressie of uitschakeling van NbGRP7 op afweerreacties bepaald. De resultaten geven aan dat NbGRP7 een pro-immuuncomponent is, die bijdraagt aan celdoodrespons door Gpa2 en extreme resistentie (ER) van Rx1. In een poging om de mechanistische onderbouwing van NbGRP7 in NB-LRR-gemedieerde immuniteit te ontciiferen, 
werden mutanten van het eiwit gegenereerd die gericht waren op een geconserveerd arginineresidu. Deze mutanten bleken verminderde capaciteiten te hebben om ER door Rx1 te versterken, wat aangeeft dat het RNA-bindende vermogen ervan cruciaal kan zijn voor zijn activiteiten in $\mathrm{R} \times 1$-verdediging. In verband daarmee tonen qPCR-assays verder aan dat de transcriptie van Rx1 wordt beïnvloed tijdens ectopische expressie van NbGRP7. Deze bevindingen suggereren dat NbGRP7 RNA-niveaus van Rx1 vóór en tijdens een actieve afweerreactie kan reguleren. Dus, naast transcriptionele componenten, kan Rx1 eiwitten coöpteren die betrokken zijn bij post-transcriptionele processen.

De belangrijkste bevindingen en experimentele benaderingen die in dit proefschrift zijn toegepast, werden verder besproken in het licht van recente, fundamentele ontdekkingen met betrekking tot $\mathrm{Rx} 1$ en NB-LRRs in planten in het algemeen (Hoofdstuk 7). De behandelde reikwijdte omvat mechanismen voor en na activering, inclusief een discussie over mogelijke resistosoomvorming en de ontkoppeling van verdedigingsroutes. Op basis van deze reflecties werd een werkmodel voorgesteld dat de immuunfunctie van $\mathrm{R} \times 1$ beschrijft, dat tot op zekere hoogte van toepassing kan zijn op andere NB-LRRs die structurele homologie delen. Over het algemeen werd geconcludeerd dat een diverse reikwijdte van inter- en intramoleculaire determinanten nodig zijn om de compartimentspecifieke activiteiten van $\mathrm{R} \times 1$ in de cel te beheersen. Meer gedetailleerde studies zijn echter nodig om de ruimtelijke en temporele dynamiek op te lossen waarin deze verschillende regelgevende (check) punten samenwerken. 


\section{ACKNOWLEDGMENTS}

Whenever I receive a PhD thesis from a friend or colleague, the acknowledgements is one of the chapters that I look most forward to reading. Simply, because it feels like the heart of the book. It is personal and above all, meaningful. As I am writing my very own, I cannot help but feel an immense sense of gratitude to those who have helped me along the way.

To those who know me personally, you are aware that I like to spend my free time (maybe a little too much) gaming to unwind, with one of my favourite genres being MMORPGs (Massively Multiplayer Online Role-Playing Game). So, it probably is not a surprise that I write my acknowledgements by drawing parallels in this regard. When you start an MMORPG game, your character is introduced to several NPCs (non-playable characters) who are meant to guide and mentor you in order to reach 'cap level'. During my PhD, I was extremely fortunate to have Aska and Geert as my direct supervisors. Aska, thank you for allowing me to be a part of your group since my MSc theses (so that is a whopping 6-7 years now!). I have enjoyed having scientific discussions with you as I always left feeling immensely inspired, recharged and equipped with a bag full of ideas. Thank you for entrusting me with the helm during my PhD, even though my ideas often did not go as planned. Above all, thank you for providing a nurturing environment where I felt safe to communicate openly. In my last year of $\mathrm{PhD}$, I had the opportunity to share an office with you and Liesbeth, which allowed me to get to know you both on a more personal level. Thank you for welcoming me so warmly to our little workspace these past months. Geert, I (and a few others) always joked that you may come across as a little intimidating and Amalia aptly described why in her acknowledgement. I share the same sentiment as she does in that we admire how you are able to effortlessly marry Science and human connection. I therefore feel incredibly privileged to have you complement Aska's supervision and as my promotor.

Jaap, thank you for welcoming me to Nematology! Frankly, it was quiet unfortunate that I did not get to know you as much as I would like to, but I have always enjoyed the exchanges we had in the corridor and coffee corner. Thank you for fostering Nematology as a close-knit research group, and for inviting us to your homes during the yearly BBQs. Arjen, Hans and Jan, thank you also for making Nematology a scientifically diverse, dynamic, and collaborative working environment. 
Rikus, Hein, Joost, Casper, and Sven: without you, we can all agree that the labs would not stay afloat. Rikus, it is not an exaggeration when I say that I owe all my knowledge of protein work to you. Thank you for supervising me during my first western blot and co-immunoprecipitation that have become rudimentary in my thesis! Hein, I have always admired your thoroughness in lab-work and the fact that you were still willing to go the extra mile to help others. Also, thank you for passing down your cat goodies to Rob and myself! Casper, you always kept Nema interesting! Thank you for giving me the best deals for hair dyes and allowing me to tag along with your nematode work so I can at least say that I have handled them during my PhD (to a certain extent). Joost, we share a common ground in Indonesia, but I also came to realize that you are one of the kindest people at Nema who always had a show-stopping dish up his sleeve for the Christmas-lunch! Last but certainly not least, Sven, my FERTILIZER. Whenever I felt down, it always helped when I bumped into you in the hallway and have our cheerful little exchanges. During Covid-19, I have missed this immensely along with stories of your travel, children, and furniture building.

Lisette, Manouk, and Crystel: you are the backbones of Nematology that keep it running behind the scene. I want to sincerely thank you for taking care of essential administrative details that I often overlooked and, of course, for motivating me to practice my Dutch!

A core part of any MMORPG is the dungeons, which are obstacles designed to help your character progress in level. The key to overcoming them is to go through the experience as a team. Likewise, I was blessed enough to have the opportunity to have several collaborations during my PhD. Jan-Willem, thank you for teaching me the ropes in confocal microscopy and your availability whenever I needed help! The biochemical work in Chapters 5 and 6 would not be possible without the expertise of Martin and Alex. Martin, thank you for the opportunity to be involved in the DBCP paper, and it was almost cathartic to finally have the story out. Andrei and Eliza, your structural modeling in Chapter 3 has shed new light on our biological data and inspired many new research lines. I am excited to see how we can shape this chapter into a manuscript in the coming months. Marian, you are a true expert in cloning and I was so incredibly fortunate to have you onboard to help get through that long list of constructs for the TTW project!

To my paranymphs, Sarlita and Mark. Sarlita, I cannot thank you enough for everything. More especially, for being there in the last few days (EVEN minutes) of the chaos that was my reading 
draft submission. It meant a lot to me that you made the trip from Germany despite your paranoia about Covid-19. For the sake of it: jijik, meni geleuh.

Mark and Anne. M, thank you for adopting Rob and me into Herenstraat and imparting us with the house. You are the Regina of Nema (in all the right ways), and together with Anne, I can always count on you both for fun dinners and advice. Anne, it was such a pleasure to work with you on my cover and layout, and I hope I was not too much of a nightmare!

The beauty of an MMO game is that you do not venture through the journey alone. There are others embarking on the same trip whom you may share joy, frustration and success with. This section is, therefore, dedicated to my fellow group members and PhDs. I would like to start with the NB-LRR team where I spent a few years directly working with. Erik, you were my MSc thesis supervisor and then post-doc during the first three years. At the start, it was quite difficult for me to approach you and I regret not having taken more opportunities to pick your brain because you were (are) quiet literally a walking NB-LRR-encyclopedia. Nonetheless, thank you for being open to my impromptu chats even after you have left. Qi, it was great having you as a student and now, a colleague. I really enjoyed sharing a room and spending my time with you in Glasgow during MPMI because I got to really know you! I am sure you will finish your thesis with flying colors, and if you ever need anything, you can always count on me $:-$ Vera, you are the newest addition to the NB-LRR team. I am honestly chuffed that someone with such enthusiasm for NB-LRRs has joined the group. Wishing you the best!

To my colleagues who started their PhD journey at more or less the same time as I did: Koen, thanks for all the laughs and allowing me to play DOTA with you that one time (and never again). I wish you success in completing the book and I promise not to spit in my future mini-preps. Kim, together with Koen, you were my LMA lab-mate. I really love the exchanges we had over our shared interest in confocal microscopy and life in general; I could always count on you for useful advice! Ava, I have always appreciated your positive outlook in pretty much everything and it was also fun spending a few days with you in Vienna when I tried my hardest to keep up with your pace of walking. Lisa, I always enjoyed hearing your stories during breaks, especially those involving high maintenance pets, which we share in common. Success with your final steps! Katharina, thank you for bringing the much-needed sensibility and balance to the crazy mix of PhDs at Nema. 
Matthijs, Jaap-Jan (JJ), Nina, Yuqing and Joris, you all started your PhD a few years after I did. Matthijs, during the second half of my PhD, I shared my lab bench with you, and though I seldom met you there, I have fond memories of popping by your (office) desk. These visits were unannounced for the most part, but you always welcomed me warmly for a lively discussion. I will really miss that! $\mathrm{JJ}$, in my last year of $\mathrm{PhD}$, we undertook the immense duty of organizing the yearly Christmas lunch. Thanks for all the dedication you put into shooting that pipette tip into the box (and that it is caught on camera). Also, when this is all over, it is a little sad to think that there will be no-one to call me cebol and say bau ketiak. Nina, you are one of the saner few in the bunch but I have always enjoyed my talks with you about life and science. Success with your PhD! Yuqing, we share a love for gaming, but we never had the chance to play online together. If you ever feel like playing League of Legends, let me know and we will do a classic or ARAM! Joris, so much kudos to anyone that is willing to dedicate 4 years to bioinformatics. Thanks for answering my questions if I ever had some.

Myrna, Marijke, Iqbal, and Sarah: sadly, most of you came by to Nematology as I was finishing up and for this reason, we had little time to get to know each other. Nonetheless, I wish you all the best in your exciting adventures!

Fortunately, in an MMO, you are also at the company of more experienced players whom you can learn from. Amalia, aside from our scientific collaboration, you were always ready to lend me your shoulder when I needed one, so I am immensely thankful for that. Paula, you have brought a lot of laughter during my time at Nema! Particularly, thank you for being inclusive of all topics during the breaks as well as offering your expertise on matters related to personal hygiene. Ruud, though our first exchange was not that ideal (recounting the instance of when I asked for spare restriction enzyme), thereafter, I am always grateful that I can turn to you for perspectives inside and outside academia. Casper Q, Yiru, Sonja, Jose, Jet, Martijn and Lotte: you are all experts in your respective fields and I appreciate how I could always turn to you for questions whenever I needed to.

At some point, you have gone through enough dungeons to be able to start teaching others a few tricks or two. Likewise, I had the opportunity to supervise MSc and BSc students during the course of my PhD. Erwin, Oscar, Ghotam, Dian, Beta, Ruben, Sergio, Steven, Jingyi, Henriette, Charlotte, Esmee, Mark, Melanie and Alex: thank you for the dedication you have put into the often experimental projects I have assigned you on. You all made me realize how rewarding and fun supervising could be! 
MMORPGs are not always about running dungeons as you often find yourself in a guild with others. I was lucky enough to spend a year at the Wageningen PhD council (WPC) and two years at the EPS PhD council. During this time, I have met others whom I shared many hard work and fond memories with. So, I would like to thank my council members: Vera, Tieme, Leena, Stuart, Daniel, Hao, Sietske, Ivo, Michelle, Valerie, Daan, Irene, and Mandy. A special thankyou to Susan and Heleen for always willing to lend a helping hand in organizing these events.

I am also grateful to those who remind me that there is more to life outside the game. Azim (Mr. Tea), Elaine (Lynne), Tammy, Sam, Eugene, Mas Donny, Mba Tita, (Mas) Ferdy, Beta and Mas Margi: in one way or another, you all help remind me of my roots- Indonesia! Though most of us no longer live in the same cities let alone continent anymore, it is always a blessing to catch up like old times when we meet. Richard, you are only a train ride away but honestly, we do not visit each other enough. It is absolute fun though when we do reunite and I cannot wait till we organize our next catch-up (in Fiji?)! Savio, thanks for carrying me to the bus stop that one time I hurt my ankle and pretty much for always being there. It is amazing how far our friendship has come, and I am sure it will continue to grow! Rian, I have known you since you were 2-3 years old back in Aberdeen and it is pretty awesome that you are here with us now in NL. Deetcha and I gotchu. My beloved Trash Queens: Muffy, Sina, Layal, Lucy, Odette and Sarah: though we are all worlds apart, our little friend group and time on AC have helped me immensely with getting through the last stretch of my $\mathrm{PhD}$ thesis these last few months. Love you all!

I am so incredibly grateful to my extended family. The Canadas (Cees, Margaret, Gerard, Bart, and Eric) thank you for always being so warm and giving the best hugs! Bets, Ed, Ernee, Nikkie and Pimmie: you are all some of my favourite people because of your wit, humour and strong family values! Thank you for being so welcoming to this little Asian. The Stokmans: Wout, Marleen, Wout (Jr.), Mona, Saar and Lola: I could never ask for a better, more supportive schoonfamilie as you all have made the Netherlands truly feel like home.

To my sister Mba Oppie, I remember in school, you helped me carry my heavy textbooks and said that if I do not enrol in a PhD program, you would make me regret it (out of love, I am sure). It is rather difficult to think that my biggest hype woman would not be able to make it in person on the day that I would be receiving that degree. But, I know that you would still be my no. 1 supporter even from across the globe. Love you, dude. To my brother Adit/Deetcha, 'you're my chick fillet' $\odot$. 
On a serious note, growing up we were raised almost like twins in our matching GAP sweaters. I am so proud to see how far you have come since the old days when I used to tell you (and your friends) off during prefect duty. Thank you for always being a voice of reason in my life and love you, bro. To my parents: Mamah dan Papah, terima makasih untuk semua doanya dan kepercayaan di Uchie. Untuk Papah khususnya, terima kasih atas dukungan dan telah memberi Uchie kebebasan untuk memilih studynya.

Rob (liefudie), thank you for being my rock and partner in crime. I am honestly so humbled everyday by your love and endless support. I am sure we will continue to make amazing memories in the years to come together with Jack, Dexter and maybe a doggo or two? $:-$

To all others who have helped me but whom I failed to mention: thank you, terima kasih, bedankt! 


\section{ABOUT THE AUTHOR}

Octavina Citra Ayudhany Sukarta was born on October 28, 1990 in Bandung, Indonesia. Along with her two siblings, she was raised as a third culture kid moving between countries. During her elementary and secondary education, she developed a deep curiosity for biology, which she later pursued in her bachelor studies.

Drawn by the applied approach to teaching, Octavina decided to do a BSc in the technical institute Hogeschool van Arnhem en Nijmegen

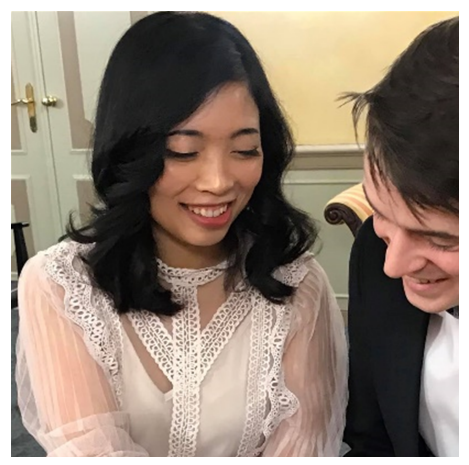
(HAN) in Nijmegen, the Netherlands, which she completed in 2012 with distinction. In the course of her BSc studies, she had the opportunity to fulfill her internships at the James Hutton Institute in Dundee, Scotland (under the supervision of Prof. Dr. Paul Birch) and Plant Breeding at Wageningen University in Wageningen, the Netherlands (in the group of Dr. ir. Vivianne Vleeshouwers). It was during this time that she became enthralled with the dynamics of Plant-Microbe Interactions. To further explore this interest, in 2013, she enrolled in a Plant Biotechnology Master of Science program at Wageningen University (specialization Molecular Plant Pathology), where she graduated with cum laude. In the Msc course 'Host-Parasite Interactions', she was introduced into the topic of plant NB-LRR immune receptors, which she quickly gravitated towards. This prompted her to perform her MSc theses in the same subject at the Laboratory of Nematology. Owing to the collaborative atmosphere (and the abundance of coffee-break cakes), she accepted an offer for a PhD position at Nematology following her MSc.

In 2015, she was appointed as a PhD candidate in the group of Dr. ir. Aska Goverse and Prof. Dr. Geert Smant. During her PhD, she focused more intensely on the molecular and mechanistic aspects of NB-LRR biology. At this time, she was fortunate enough to supervise BSc and MSc students as well as various (practical) courses. Aside from scientific research, she was also an internal secretary of the Wageningen PhD council (WPC) and member of the local EPS graduate school council. In 2016, Octavina met Rob Stokman, whom she later married and shares a profound love for cats and gaming. On December 4, 2020, she will be given the opportunity to defend her thesis. 


\section{LIST OF PUBLICATIONS}

1. Sukarta, O. C. A., Townsend, P. D., Llewelyn, A., Dixon, C. H., Slootweg, E. J., Pålsson, L.-O., Takken, F. L. W., Goverse, A., and Cann, M. J. (2020), A DNA-Binding Bromodomain-Containing Protein Interacts with and Reduces Rx1-Mediated Immune Response to Potato Virus X. Plant communications. 1(4): p. 100086. epublish

2. Martin, E. C., Sukarta, O. C. A., Spiridon, L., Grigore, L. G., Constantinescu, V., Tacutu, R., Goverse, A., and Petrescu, A. J. (2020), LRRpredictor-A New LRR Motif Detection Method for Irregular Motifs of Plant NLR Proteins Using an Ensemble of Classifiers. Genes (Basel). 11(3)

3. Warmerdam, S., Sterken, M. G., Sukarta, O. C. A., van Schaik, C. C., Oortwijn, M. E. P., Lozano-Torres, J. L., Bakker, J., Smant, G., and Goverse, A. (2020), The TIR-NB-LRR pair DSC1 and WRKY19 contributes to basal immunity of Arabidopsis to the root-knot nematode Meloidogyne incognita. BMC plant biology. 20(1): p. 73. epublish

4. Warmerdam, S., Sterken, M. G., van Schaik, C., Oortwijn, M. E. P., Sukarta, O. C. A., Lozano-Torres, J. L., Dicke, M., Helder, J., Kammenga, J. E., Goverse, A., Bakker, J., and Smant, G. (2018), Genome-wide association mapping of the architecture of susceptibility to the root-knot nematode Meloidogyne incognita in Arabidopsis thaliana. The New phytologist. 218(2): p. 724-737. ppublish

5. Townsend, P. D., Dixon, C. H., Slootweg, E. J., Sukarta, O. C. A., Yang, A. W. H., Hughes, T. R., Sharples, G. J., Pålsson, L. O., Takken, F. L. W., Goverse, A., and Cann, M. J. (2018), The intracellular immune receptor $\mathrm{R} \times 1$ regulates the DNA-binding activity of a Golden2-like transcription factor. J Biol Chem. 293(9): p. 3218-3233

6. Slootweg, E. J., Spiridon, L. N., Martin, E. C., Tameling, W. I. L., Townsend, P. D., Pomp, R., Roosien, J., Drawska, O., Sukarta, O.C.A., Schots, A., Borst, J.W., Joosten, M. H., Bakker, J., Smant, G., Cann, M. J., Petrescu, A.-J., and Goverse, A. (2018), Distinct Roles of Non-Overlapping Surface Regions of the Coiled-Coil Domain in the Potato Immune Receptor Rx1. Plant Physiology. 178(3): p. 1310-1331

7. Sukarta, O. C. A., Slootweg, E. J., and Goverse, A. (2016), Structure-informed insights for NLR functioning in plant immunity. Seminars in Cell \& Developmental Biology. 56: p. 134-149

8. King, S. R. F., McLellan, H., Boevink, P. C., Armstrong, M. R., Bukharova, T., Sukarta, O., Win, J., Kamoun, S., Birch, P. R. J., and Banfield, M. J. (2014), Phytopthora infestans RXLR effector PexRD2 interacts with host MAPKKK $\varepsilon$ to suppress plant immune signaling. The Plant cell. 26(3): p. 1345-1359. ppublish 


\section{Education Statement of the Graduate School Experimental Plant Sciences}

\section{Issued to: Octavina Citra Ayudhany Sukarta \\ Date: \\ 4 December 2020 \\ Group: Laboratory of Nematology \\ University: Wageningen University \& Research}

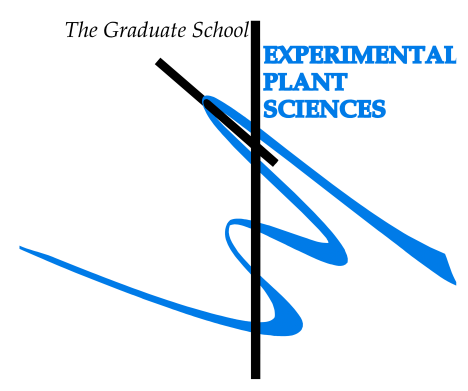

\section{1) Start-Up Phase}

- First presentation of your project

Title: Opening the molecular toybox: Functional characterization of host 14 Apr $2016 \quad 1.5$ interactors of the potato CC-NB-LRR immune receptors Rx1 and Gpa2

- Writing or rewriting a project proposal

- Writing a review or book chapter

Review: 'Structure-Informed Insights for NLR functioning in Plant Immunity', Seminars in Cell \& Developmental Biology; 2016,

Dec $2015-$

Feb 2016 56:134-149, DOI:10.1016/j.semcdb.2016.05.012

MSc courses

\section{2) Scientific Exposure}

- EPS PhD student days

Symposium \& Workshops: EPS PhD Get2Gether, 2016, Soest, the Netherlands

Symposium \& Workshops: EPS PhD Get2Gether, 2017, Soest, the Netherlands

Symposium \& Workshops: EPS PhD Get2Gether, 2018, Soest, the Netherlands

Symposium \& Workshops: EPS PhD Get2Gether, 2019, Soest, the Netherlands

Symposium \& Workshops: EPS PhD Get2Gether, 2020, Soest, the Netherlands

\section{- EPS theme symposia}

Symposium: EPS Theme 2 Symposium, Interactions between Plants and Biotic Agents, combined with Willie Commelin

$\underline{\text { date }}$

Scholten Day, Wageningen, The Netherlands

Symposium: EPS Theme 2 Symposium, Interactions between

Plants and Biotic Agents, combined with Willie Commelin

$\begin{array}{cc}\frac{c p}{\text { date }} & \underline{c p} \\ \text { 28-29 Jan 2016 } & 0.6 \\ \text { 9 Feb 2017 } & 0.3 \\ \text { 15-16 Feb 2018 } & 0.6 \\ \text { 11-12 Feb 2019 } & 0.6 \\ \text { 10-11 Feb 2020 } & 0.6 \\ & \end{array}$

23 Jan 2017

0.3

24 Jan 2018

0.3

Scholten Day, Amsterdam, The Netherlands

Symposium: EPS Theme 2 Symposium, Interactions between

Plants and Biotic Agents, combined with Willie Commelin

1 Feb 2019

0.3

Scholten Day, Wageningen, The Netherlands 


\section{- Lunteren Days and other national platforms}

Annual meeting: 'Experimental Plant Sciences', Lunteren, The Netherlands

Annual meeting: 'Experimental Plant Sciences', Lunteren, The Netherlands

Annual meeting: 'Experimental Plant Sciences', Lunteren, The Netherlands

Annual meeting: 'Experimental Plant Sciences', Lunteren, The Netherlands

Annual meeting: 'Experimental Plant Sciences', Lunteren, The Netherlands

\section{Seminars (series), workshops and symposia}

Seminar: Prof. dr. Jane Parker, 'Plant intracellular immunity: evolutionary and molecular underpinnings'

Symposium: Indonesia Scientific Expose (I-See), Wageningen, the Netherlands

Symposium: Wageningen Indonesia Scientific Exposure (WISE), Wageningen, the Netherlands

Seminar: Dr. ir. Fangming Xiao, 'Prf mediated immunity and PCN effectors (E3 ligase)'

Seminar: Dr. ir. Sander Schouten, 'Direct and indirect effects of a Fusarium oxysporum endophyte on the root-knot nematode, Meloidogyne incognita'

Seminar: Prof. dr. Martin J. Cann, 'The immune receptor Rx1 remodels chromatin and chromatin interactors in immunity'

Seminar: Dr. ir. Neima Briggs, 'Trichuriasis, vaccine discovery'

Seminar: Novel Developments in Fluorescence Microscopy,

Wageningen, the Netherlands

Seminar: Ir. Nicole van't Wout Hofland, 'The origin and evolution of the vascular regulatory dimer TMO5/LHW'

Seminar: Ir. Lieke Vlaar, 'Perception of the environment in cyst nematodes'

Seminar: Dr. Sebastian Eves van den Akker, 'Plant Immunity and development-altering "toolbox"of parasitic nematodes'

Seminar: Ir. Eliza C. Martin, LRR Predictor: a novel tool for the identification of LRR-motifs

13-14 Apr 2015

$11-12$ Apr 2016

$10-11$ Apr 2017

$9-10$ Apr 2018

8-9 Apr 2019

21 Jan 2016

28 Oct 2016

8 Mar 2017

20 Mar 2017

1 Jun 2017

11 Jul 2017

19 Oct 2017

0.1

20 Nov 2017

12 Apr 2018

0.1

21 Jun 2018

13 Feb 2019

9 Apr 2019

Seminar: Prof. dr. ir. Tina Kyndt, 'molecular plant nematode interactions in rice'

Seminar: Dr. Jiejie Chai, 'Structure, mechanism and biochemical insight of plant NLR proteins'

Seminar: Dr. Sophie Mantelin, 'Identification and functional characterisation of effectors derived from the potato cyst nematodes Globodera pallida'

Seminar: Dr. Erik Andersen, Comparative Genomics and Complex Traits in Caenorhabditis elegans

Seminar: Ir. Leendert Molendijk, 'Applied Nematology in the Polder'
14 Nov 2019

16 May 2019

30 Jan 2020
24 Oct 2019 


\section{Seminar plus}

Discussion flying seminar Prof. dr. Martin Cann

11 Jul 2017

- International symposia and congresses

Congress: 3rd Annual COST action SUSTAIN;

17 - 19 Feb 2016

Pathogen-informed strategies for sustainable broad spectrum crop resistance, Banyuls sur Mer, France

Congress: International Society for Molecular Plant-Microbe Interactions, XVII, Portland, United States

Congress: International Society for Molecular Plant-Microbe Interactions, XVII, Portland, United States

Congress: 4th Annual COST action SUSTAIN;

Pathogen-informed strategies for sustainable broad spectrum crop resistance, Bled, Slovenia

Congress: VISCEA, 3rd International conference 'Plant Biotic

Stresses \& Resistance Mechanisms', Vienna, Austria

Congress: International Society for Molecular Plant-Microbe Interactions XVIII, Glasgow, Scotland, UK

$17-21$ Jul 2016

$17-21$ Jul 2016

1-3 Mar 2017

2-3 Jul 2018

$14-18$ Jul 2019

- Presentations

Poster: 'Identification and Functional Characterization of Novel regulatory Components of the Potato CC-NB-LRR Immune Receptors Rx1 and Gpa2', 3rd Annual COST-action-SUSTAIN, Banyuls sur Mer, France

Poster: 'Ri-07 Potentiates Defence Against PVX by the Potato CC-NB-LRR Immune Receptor Rx1', TSL summer school, Norwich, UK

Talk: 'Identification and Functional characterization of host interactors of the Potato CC-NB-LRR Rx1', Lunteren, the Netherlands

Talk: User's committee meeting, STW (First Presentation)

Poster: 'Exploring the Role of RanGAP2 in Recognition by the

Potato CC-NB-LRR Immune Receptors Rx1 and Gpa2', IS-MPMI

18 Feb 2016

XVIII, Glasgow, UK

Talk: User's committee meeting, STW

24 Jun 2020

\section{3) In-Depth Studies}

\section{- Advanced scientific courses \& workshops}

PhD Course: Basic statistics, Wageningen, the Netherlands

Advanced Course: Microspectroscopy: Functional Imaging of Biological Systems, Wageningen \& Nijmegen, the Netherlands Advanced Course: The Sainsbury Laboratory Summer School: Plant Microbe Interactions, Norwich, UK

$\begin{array}{cc}\underline{\text { date }} & \underline{c p} \\ \text { 22 Jun - 1 Jul 2016 } & 1.5 \\ \text { 6-15 Sep 2016 } & 3.0 \\ \text { 31 Jul - 11 Aug 2017 } & 3.0\end{array}$




\section{Journal club}

Monthly Journal Club, hosted by the PNI Group, Laboratory of

\section{4) Personal Development}

- General skill training courses

Course: EPS Introduction course, Wageningen, the Netherlands

Course: Competence assessment, Wageningen, the Netherlands

Course: Project and Time management, Wageningen, the Netherlands

Workshops: WGS PhD Workshop Carousel, Wageningen, the Netherlands

Workshops: WGS PhD Workshop Carousel, Wageningen, the Netherlands

Course: Career Orientation, Wageningen, the Netherlands

Peer Consultation: Monthly PhD Meeting, hosted by PhDs of Laboratory of Nematology

- Organisation of meetings, PhD courses or outreach activities

Organisation of the EPS Get2Gether 2019, Soest, the Netherlands (Speakers)

Organisation of the EPS Get2Gether 2020, Soest, the Netherlands (Registration, Speakers)

Organisation and hosting of Nematology Student Symposium, Wageningen, the Netherlands

- Membership of EPS PhD Council

EPS PhD Council member (including Wageningen PhD Council Internal Secretary)

date $\quad \underline{c p}$

22 Sep $2015 \quad 0.2$

4 Nov $2015 \quad 0.3$

4 Nov - 16 Dec $2016 \quad 1.5$

8 Apr $2016 \quad 0.3$

7 Apr $2017 \quad 0.3$

2-23 Oct $2018 \quad 1.5$

$2017-2020 \quad 1.5$

$2018-2019$

$2019-2020$

12 Dec 2019

$2018-2020$

Herewith the Graduate School declares that the PhD candidate has complied with the educational requirements set by the Educational Committee of EPS with a minimum total of 30 ECTS credits.

${ }^{*}$ A credit represents a normative study load of 28 hours of study. 
The research described in this thesis was financially supported by the Dutch Top Technology Institute Green Genetics (5CFD051RP), Dutch Technology Hotel grant and the Dutch Technology Foundation STW and Earth and Life Sciences ALW (STW-GG 14529), which are part of the Netherlands Organization for Scientific Research (NWO).

Financial support from Wageningen University for printing this thesis is gratefully acknowledged.

\section{Cover design \& thesis layout}

Anne Morbach | schlaugemacht.net

\section{Cover image}

The front and back covers of this thesis include depictions based on the concept of an impossible cube, conceived by Maurits Cornelis Escher (1958).

\section{Printed by}

ProefschriftMaken | proefschriftmaken.nl 